
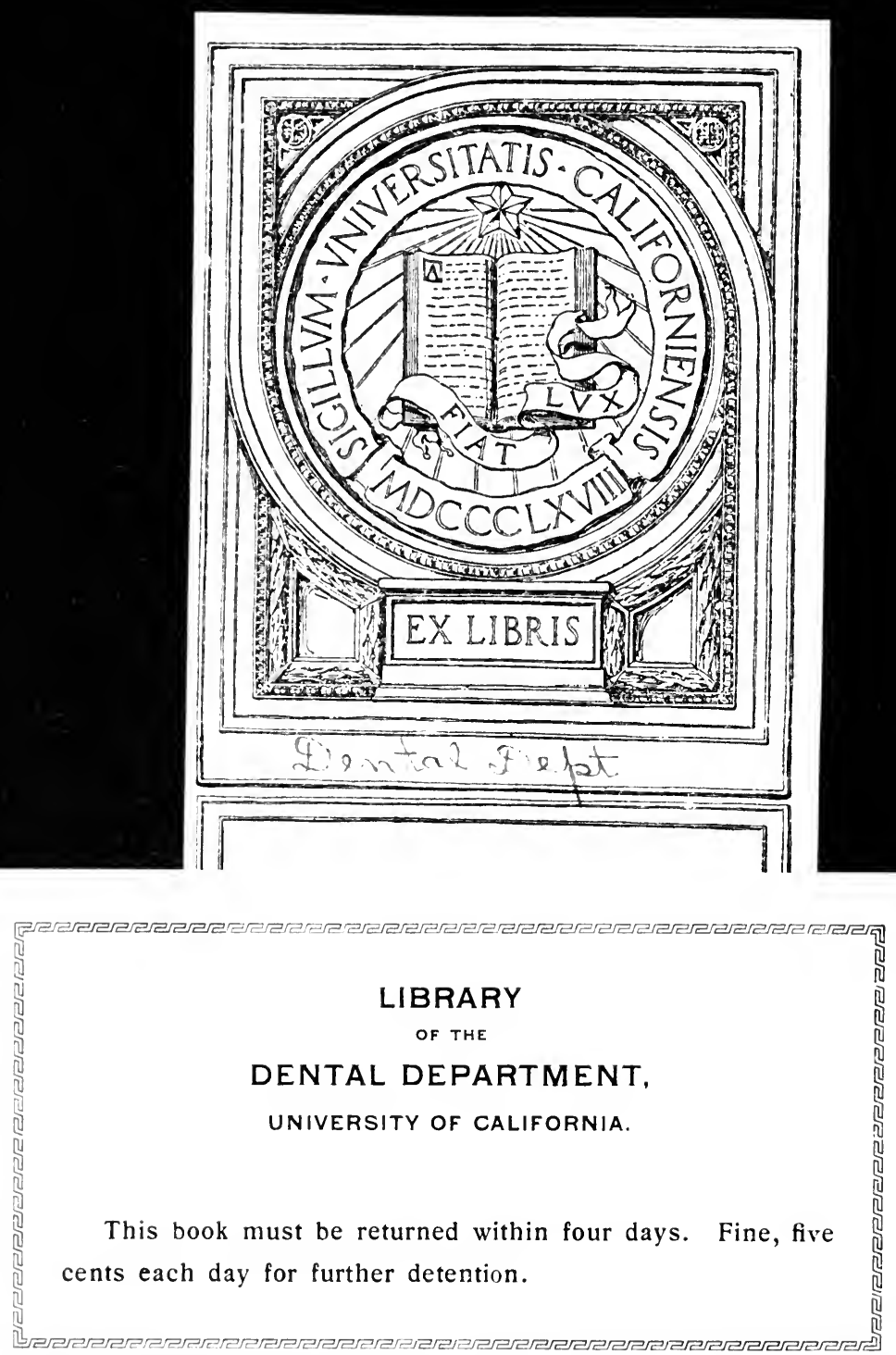






Digitized by the Internet Archive in 2007 with funding from Microsoft Corporation

http://www.archive.org/details/digestionmetabol00taylrich 



\section{DIGESTION AND METABOLISM}

\section{THE PHYSIOLOGICAL AND PATHOLOGICAL CHEMISTRY OF NUTRITION}

FOR STUDENTS AND PHYSICIANS

BY

ALONZO ENGLEBERT TAYLOR, M.D.

RUSH PROFESSOR OF PHYSIOLOGICAL CHEMISTRY, UNIVERTITY OF PENNSYLVANIA, PHILADELPHIA



LEA \& FEBIGER

PHILADELPHIA AND NEW YORK 


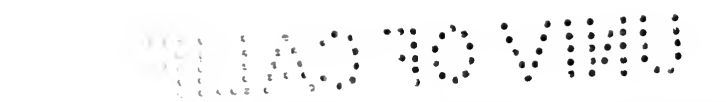

Entered accurding to the Acet of thingressin the $\because \mathrm{LE}=\mathrm{H}^{\circ} \mathrm{FE} \dot{\mathrm{B}} \mathrm{I} \mathrm{GE} \dot{\mathrm{R}}$

in the office of the Librarian of Congress. All rights reserved. 


\section{2}

QP141

T23

$\begin{array}{r}1912 \\ +2 \\ \hline\end{array}$

DEDICATED

TO

MADELEINE PECK TAYLOR 



\section{P R E F A E}

THE author is convinced from perusal of current medical writings in this country that there is need among American physicians for a work presenting the subjects of Digestion and Metabolism in a popular manner, without technical details, and from the standpoint of dynamics rather than from that of analytical statics usually occupied by textbooks of physiological chemistry. He has rewritten in the form of a concise and systematic treatise the substance of his lectures in this field. No effort has been made to prepare an encyclopedic survey or a methodic synopsis of the very extensive literature of these subjects, but rather to offer a practical interpretation of them in their present state of development.

The aim of the book is to describe the chemical changes in normal and abnormal digestion, and explain the known metabolic modifications that food materials undergo within the body. This understanding makes for comprehension of the pathology of diseases that may be termed metabolic-such as gout, diabetes, nephritis, autointoxication and the results of indigestion. In a word, the aim is to give the student and practitioner a working knowledge of just what is known to occur in the chemistry of the normal body and also of the changes concerned in many widespread and important diseases. Variations and findings in morbid states have been everywhere considered.

The student and practitioner of the art and science of medicine will gain the most definite idea of physiological processes and chemical functions if they view them as a moving picture; in other words, if they will consider them dynamically rather than statically. The experimental method alone has enabled us to acquire the larger portion of our present knowledge of digestion and metabolism. Ability to think in the terms of the experimental method is essential to an understanding of these subjects. The student and physician need more than facility in method, invaluable as this is. They need the dynamic concept of function on which to found a dynamic conception of disease. The definition of experiment is fundamental to the concept of function, 
which in turn is the foundation of the understanding of disease. The following pages attempt, with the minimum of technical details, to offer a presentation and interpretation of the broad functions of digestion and metabolism from the standpoint of chemical dynamics.

The proper introduction of references to literature is very difficult, for one cannot emphasize here and reject there without entering into the details of the several investigations in order scientifically to justify acceptance or omission of reported data. The first third of this work was written with full references to literature. It was then apparent that the references made up a third of the text. The direct references occupied much space, and the necessary discussion of them forced the inclusion of much technical detail. The work was then frankly recast into a direct interpretation of the subject matter, necessarily somewhat dogmatic and representing, in part, simply the judgment of the author. The method of procedure has been to let the facts point the way to theory, though in some instances it has seemed necessary to appeal to theory to aid in the interpretation of facts.

The newer nomenclature of ferments has been. employed. Without considerations of philological warrant, an arbitrary distinction has been made in the use of terms derived from glycogen and glucose. Thus "glycogenesis" and "glycolysis" mean respectively the formation of glycogen from glucose, and the cleavage of glycogen into glucose; "glucolysis" and "glucosuria" mean the destruction of glucose and the presence of glucose in the urine, respectively, etc. In the matter of chemical nomenclature, an extreme position has been avoided.

Philadelphia, 1912.

A. E. T. 


\section{CONTENTS}

CHAPTER I

The Composition of Foodstuffs .

CHAPTER II

The Theory of Ferment Action

CHAPTER III

Digestion

CHAPTER IV

The Carbohydrate Metabolism

CHAPTER V

The Fat Metabolism

CHAPTER VI

The Protein Metabolism. 370

CHAPTER VII

The Metabolism of Creatin-Creatinin and of Purins

CHAPTER VIII

Autointoxication

CHAPTER IX

Metabolism Considered as a Whole . . . . . . . . . . . . . 468

CHAPTER X

Production of Body Heat and Regulation of Body Temperature 509 



\title{
DIGESTION AND METABOLISM
}

\author{
C H A P'T E R I
}

\section{THE COMPOSITION OF FOODSTUFFS}

ThE foodstuffs are divided into three natural groups - carbohydrates, fats, and proteins. These are all energy-carrying materials, substances from which tissues are formed, with which they are maintained, and through whose combustion the heat necessary for the life of higher animals is derived. The inorganic salts are not to be regarded as foods, indispensable as they are to the life of cells. They combine with the organic components of tissues and they maintain physico-chemical conditions under which living cells display the chemical functions of life. While they are indispensable to tissue structure and to functiona-. tion, the term food would best be restricted to substances that carry energy into the organism. An elaborate chemical description of foodstuffs does not fall within the scope of this treatise, and only such description will be given as seems needed for the explicit purpose of this work.

\section{THE CARBOHYDRATES}

Under the term carbohydrates (meaning carbon combined with hydrogen and oxygen in the relations that hold in water) we include all forms of sugars, or saccharids as they are now termed. The saccharids are divided into four main classes: Monosaccharids, di- and trisaccharids, polysaccharids, and celluloses. The monosaccharids, primary sugars, are the simple substances from which all the larger carbohydrates are formed. When two, three, or even four molecules of primary sugar are combined, we speak of a di-, tri-, or tetrasaccharid. In the polysaccharid, an indeterminate though large number of molecules of primary sugar combine to form a. huge molecule. The celluloses are of still larger molecular dimensions. The monosaccharids are of small molecular dimension, very soluble, diffusible, not dissociated in the ordinary sense of the term, usually typically crystalloidal, and their solutions present low surface tension and high osmotic pressure. The disaccharids are also crystalloidal, very soluble, diffusible, and their solutions present low surface tension and high 
osmotic pressure. With the polysaccharids, or starches, the properties are very different. Starches are hydrophilic colloids; colloids that with water form emulsions in which the relations of the colloid to the water are expressed in degrees of viscosity. These colloidal solutions present high surface tension and low osmotic pressure, they diffuse little and possess no tendencies to crystallization. Celluloses are suspension colloids, they do not form emulsions with water, there is no relation between the colloid and water, they display little viscosity and no diffusibility. Cellulose suspended in water is in fact almost as inert as talcum.

Aldoses and Ketoses. - The primary sugars that are concerned in the bodies of higher animals are aldehyds or ketons of polyhydric alcohols; the aldehyd sugars are termed aldoses, the keton sugars being termed ketoses. The molecule of primary sugar is represented as having the several carbon atoms in chains, $i$. e., the alcohols are linked at the carbon atoms. Thus we speak of tetroses, pentoses, hexoses, containing respectively four, five, and six atoms of carbon. All primary sugars contain one or two primary alcohol groups $\left(\mathrm{CH}_{2} \mathrm{OH}\right)$, and one or more secondary alcohol groups $(\mathrm{CHOH})$, and the aldehyd group (CHO), or the keton group $(\mathrm{CO})$. 'The different primary sugars may all be synthesized from formaldehyd as the starting point. Though it is not possible to regard these chemical operations as illustrating the modus operandi in nature, it is still a fact that the natural starting point for the synthesis of sugars in plants is formaldehyd. In nature, however, we are able to observe only certain stages. Thus we are not able to observe any stage between the formaldehyd and the first natural sugar glycerose (a triose); from the triose to the pentose also we can observe no stage of transition. The hexose is the commonest form of primary sugar in nature, though pentoses are widely distributed in plants.

The simplest formulation for the reduction of carbon dioxid to formaldehyd is as follows:

$$
\begin{aligned}
& 2 \mathrm{CO}_{2}+2 \mathrm{H}_{2} \mathrm{O}=2 \mathrm{HCOOH}+\mathrm{O}_{2} \quad \text { Formic acid } \\
& 2 \mathrm{HCOOH}=2 \mathrm{HCOH}+\mathrm{O}_{2} \quad \text { Formaldehyd }
\end{aligned}
$$

It can be shown experimentally that chlorophyl isolated from leaves of plants is able in the presence of sunlight to generate formaldehyd from water and carbon dioxid, and the scheme corresponds perfectly with the respiration of plants. Light is essential to the demonstrable reaction. Electro-chemically, the reaction can be accomplished in the absence of any photo-chemical influence by the presence of ozone and hydrogen peroxid.

$$
\begin{aligned}
& 3 \mathrm{O}_{2}=2 \mathrm{O}_{3} \\
& \mathrm{O}_{3}+\mathrm{H}_{2} \mathrm{O}=\mathrm{H}_{2} \mathrm{O}_{2}+\mathrm{O}_{2} \\
& \mathrm{H}_{2} \mathrm{O}_{2}+\mathrm{CO}_{2}=\mathrm{H} . \mathrm{COH}+\mathrm{O}_{3}
\end{aligned}
$$


Glycollic aldehyd also appears in traces $\left(\mathrm{CHO} . \mathrm{CH}_{2} \mathrm{OH}\right)$.

The synthesis of formaldehyd in nature we may thus define as a photo-chemical reaction, occurring under the influence of chlorophyl as an accelerator, whereby carbon dioxid is reduced to formaldehyd.

The next step consists in the condensation of formaldehyd to a sugar. This is supposedly not a biose but a triose, probably glycerose. Glycerose has the equation:

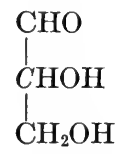

Isomerism.-We observe here one of the most fundamental properties of the sugars and of many other bodies-stereo-isomeric isomerism. It is this condition within the molecule upon which is founded the power possessed by sugars of rotating the plane of polarized light. This function rests in the condition of asymmetry in the bindings of the carbon atoms. Carbon is in the sugars tetravalent. When any two (or more) of the four bonds of a carbon atom are bound to the same element, group, or mass, the carbon atom is symmetric; but when all four bonds are bound to different elements, groups, or masses, the carbon atom is asymmetric. All compounds containing asymmetric atoms of carbon (unless this is in some way compensated for) rotate the plane of polarized light. Thus for glycerose:

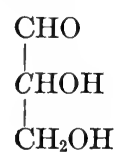

Obviously the centre carbon is asymmetric, and glycerose rotates the plane of polarized light. Since the bindings of two of the bonds of the centre atom of carbon are reversible, it follows that there are two forms of glycerose:<smiles>O=CC(O)CO</smiles><smiles>O=CC(O)CO</smiles>

one of which rotates the plane of polarized light to the right, the other to the left. If equal amounts of each form be mixed, these rotations will be compensated and the mixture is inactive, or rather balanced. The prefixes $\mathrm{l}$ and $\mathrm{d}$ were once applied to the direction in which the plane of polarized light was rotated; now they are applied to 
indicate the intramolecular groupings in the sugar molecule. Throughout this book, the asymmetric atoms of carbon in equations will be printed in italics.

Pentoses.-The smallest sugars concerned in the metabolism of plants and animals in a demonstrable sense are pentoses-sugars with five atoms of carbon. A pentose contains three asymmetric atoms of carbon; and as that number of stereoisomeric configurations of the molecule exist as are related to the number of asymmetric atoms of carbon in the proportion of $2^{\mathrm{n}}$ where $\mathrm{n}$ is the number of asymmetric carbon atoms, it is evident that there must be eight possible forms of aldo-pentose. Of these eight, six are of interest in biology: the two forms of arabinose, of xylose and of ribose.

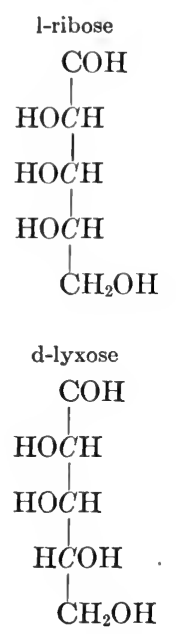
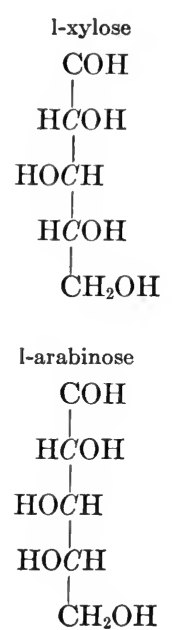
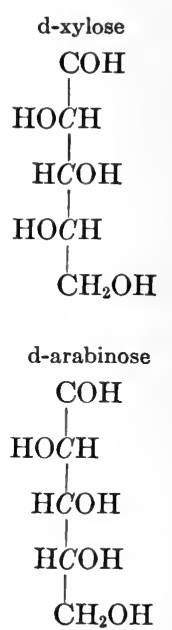

These pentoses occur free to but slight extent in plants, and under normal conditions never free in animals. They occur in both plants and animals in complex combinations.

Aldohexoses. - Of the sixteen possible aldohexoses, twelve of which have been found in nature or synthesized in the laboratory, but two are of importance here. They are d-glucose and d-galactose; d-levulose, a keton sugar, is the third common hexose.
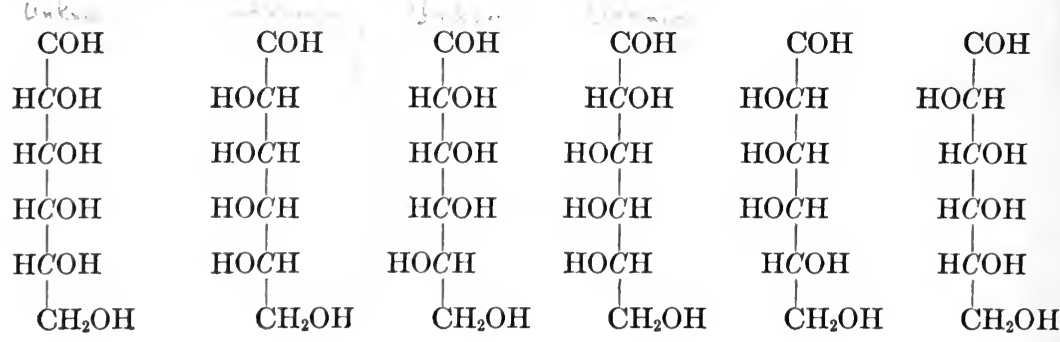


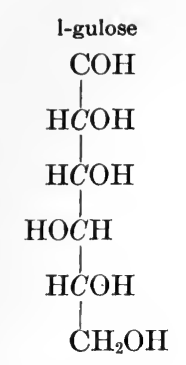

d-mannose



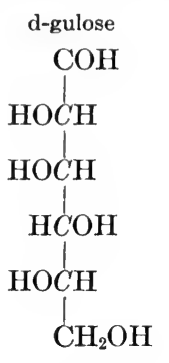
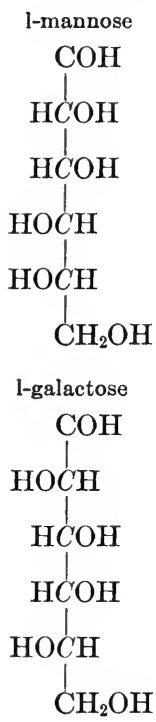
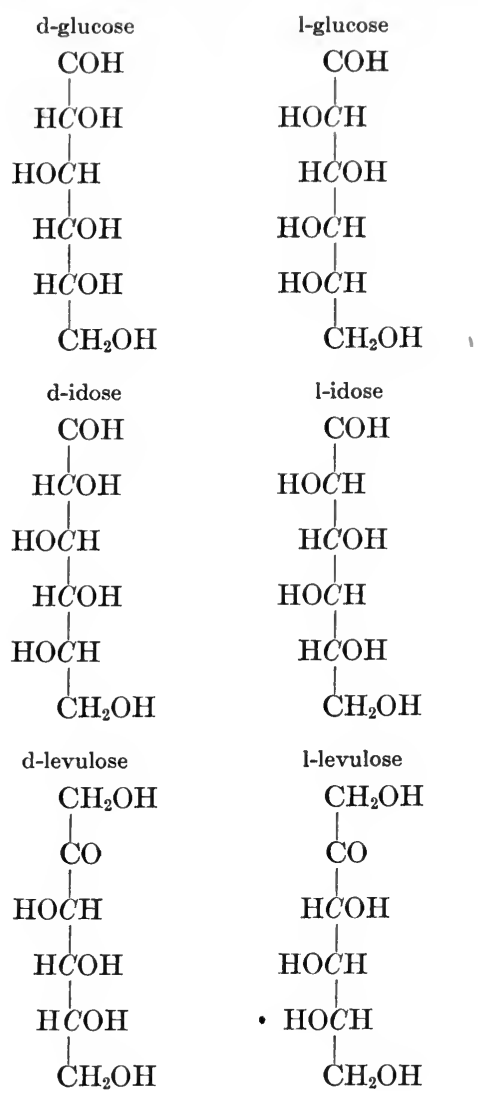

d-levulose and l-levulose are derivations of d-glucose and l-glucose, being the corresponding keton sugars. Each of these contains a primary alcohol group at the lower end of the chain, and the two levuloses also another at the upper end, replacing the aldehyd group that is situated at the upper end of the chain in the aldohexose. The glucoses and galactoses have four groups each of secondary alcohol, these containing the asymmetric atoms of carbon; the levuloses have only three, but contain the keton group. These different hexoses tend to become converted into each other by intramolecular re-arrangement, reactions that can be easily accomplished in the presence of dilute alkali. In a certain sense, the $d$-glucose seems to be the most stable configuration, the one toward which d-galactose and d-levulose tend. D-glucose and d-galactose rotate the place of polarized light to the right, respectively $(\mathrm{a})_{\mathrm{D}}=+52.5^{\circ}$ and $+81^{\circ}$. D-levulose (named $\mathrm{d}$ - on account of its relationship to d-glucose) rotates the plane of polarized light to the left, $(\mathrm{a})_{\mathrm{D}}=-94^{\circ}$. Very specific are the relations of these sugars to ferments and to physiological action, which properties are the expressions of intramolecular configuration. 
Glucosids.--Monosaccharids combine with alcohols, aldehyds, and aromatic bodies to form ether-like combinations that are termed glucosids. The alcohol with which combination is effected may be another monosaccharid, and thus the disaccharids are also to be regarded as glucosids. Whether the pentoses form disaccharids is not clear, and in any event it is of no importance to us here. The hexoses form stable disaccharids, of great importance as the common sugars, namely, cane sugar, malt sugar, and milk sugar. Maltose is a combination of two molecules of d-glucose, and is thus the glucosid of $d$-glucose. Cane sugar, or sacchrose, is a combination of d-glucose and $\mathrm{d}$-levulose, and is thus the fructosid of d-glucose. Milk sugar is a combination of $\mathrm{d}$-galactose with d-glucose, and is thus the galactosid of d-glucose. All such combinations of hexoses to form disaccharids follow the equation:

$$
\begin{aligned}
& \text { Hexose }+ \text { hexose }=\text { disaccharid }+ \text { water } \\
& \mathrm{C}_{6} \mathrm{H}_{12} \mathrm{O}_{6}+\mathrm{C}_{6} \mathrm{H}_{12} \mathrm{O}_{6}=\mathrm{C}_{12} \mathrm{H}_{22} \mathrm{O}_{11}+\mathrm{H}_{2} \mathrm{O}
\end{aligned}
$$

Conversely, when a disaccharid is split, the reaction is one of hydrolysis. The rotary powers of the disaccharids cannot be calculated from those of their components. Thus maltose rotates the plane of polarized light to the right $(\mathrm{a})_{\mathrm{D}}=+138^{\circ}$, saccharose $(\mathrm{a})_{\mathrm{D}}=+$ $66.5^{\circ}$, and lactose $(\mathrm{a})_{\mathrm{D}}=+52.5^{\circ}$. The configuration of the disaccharids is not understood. Some of the common reactions are also not clear. Thus maltose reduces copper in both alkaline and acid reaction, lactose only in alkaline reaction, while saccharose cannot effect reduction at either reaction. The importance of disaccharid to the human diet is evident when we recall that the cane-sugar production of the world is something like 15,000,000 tons per annum.

Polysaccharids.- The pentoses as well as the hexoses form polysaccharids. The polysaccharids of pentose are termed pentosans, widely distributed in plants and of great importance in the diet of herbivora. The polysaccharids of glucose are starches, glycogens, dextrins, lichinins, some native gums, and cellulose. The polysaccharids of levulose are termed fructans, and of these inulin is an illustration. The polysaccharids of $\mathrm{d}$-galactose are termed galactans, and many native gums belong to this group. The number of molecules of primary sugar in these polysaccharids is not known. The method of combination is identical with that of the combination of the primary sugars to form disaccharids-water is extruded. Thus we usually express the formation of a starch as follows:

$$
\begin{aligned}
& \mathrm{n} \text { glucose }+\mathrm{n} \text { glucose }=\mathrm{n} \text { starch }+\mathrm{n} \text { water } \\
& \mathrm{n} \mathrm{C}_{6} \mathrm{H}_{12} \mathrm{O}_{6}+\mathrm{n} \mathrm{C}_{6} \mathrm{H}_{12} \mathrm{O}_{6}=\mathrm{n}\left(\mathrm{C}_{6} \mathrm{H}_{10} \mathrm{O}_{5}\right) \mathrm{n}+\mathrm{n} \mathrm{H}_{2} \mathrm{O}
\end{aligned}
$$

The converse of this reaction, the cleavage of a starch into its component primary sugars, is obviously a reaction of hydrolysis. The pentosans, fructans, gums, and cellulose, while of importance to the diet of 
herbivora, are of little importance to man, and the same may be said of the lichen starches. It is the common starches and their derivatives that constitute the fuel of the race. The starch production of the world is something like $150,000,000$ tons per annum, or about ten times the production of sugar.

Caloric Values.- The caloric values of the carbohydrates are: Starch, 4.1 Cal.; disaccharids, 3.95 Cal., and hexoses, 3.75 Cal. per gram. 'They have the same heat values in the animal body as in the calorimeter, $i$. e., the body burns them completely.

\section{THE FATS}

The fats are esters, combinations of fatty acids with an alcohol. The common fats of plants and animals are combinations of palmitic, stearic, and oleic acids with the triatomic alcohol, glycerol (glycerin). Palmitic and stearic acids are members of the series $\mathrm{C}_{n} \mathrm{H}_{2 n} \mathrm{O}_{2}$, and have respectively the formulæ $\mathrm{C}_{15} \mathrm{H}_{31} \mathrm{COOH}$ and $\mathrm{C}_{17} \mathrm{H}_{35} \mathrm{COOH}$. A few of the lower members of this series occur in milk and in some plants; such are butyric acid $\left(\mathrm{C}_{3} \mathrm{H}_{7} \mathrm{COOH}\right)$; caproic acid $\left(\mathrm{C}_{5} \mathrm{H}_{11} \mathrm{COOH}\right)$; caprylic acid $\left(\mathrm{C}_{7} \mathrm{H}_{15} \mathrm{COOH}\right)$, and capric acid $\left(\mathrm{C}_{9} \mathrm{H}_{19} \mathrm{COOH}\right)$. They occur, however, only in traces, and cannot be said to possess any importance as foodstuffs. Oleic acid is a member of the unsaturated series $\mathrm{C}_{n} \mathrm{H}_{2 n-2} \mathrm{O}_{2}$, and has the equation:

$$
{ }_{\mathrm{H}}^{\mathrm{C}_{8} \mathrm{H}_{16} \mathrm{H}}>\mathrm{C}=\mathrm{C}_{\left(\mathrm{CH}_{2}\right)_{7} \mathrm{COOH}}\langle\stackrel{\mathrm{H}}{<}
$$

The fats of fishes and of marine mammals contain other fatty acids and also other alcohols than glycerol. The fats are formed largely if not entirely from sugar in the metabolism of plants. While it cannot be denied that plants may build up the higher fatty acids directly from the lowest member of the group, formic acid, the evidence is strongly in favor of their formation from sugar. In the germination of seeds, the fat contained in them is reconverted into glucose, and for plants the fat evidently represents a storage form of sugar, analogous to starch, though quantitatively of less importance. 'The fats of most plants consist largely of the esters of oleic and palmitic acids; stearic acid on the other hand is prominent in the fats of many animals.

In the combination of fatty acid with glycerol, each of the three hydroxyl groups of the alcohol binds a molecule of fatty acid by linkage with the carboxyl group, water being extruded.

\begin{tabular}{|c|c|c|c|c|c|c|}
\hline Glycerol & \multicolumn{4}{|c|}{ Palmitic acid } & Tripalmitin & Water \\
\hline $\mathrm{CH}_{2} \mathrm{O}$ & $\mathrm{H}$ & $\mathrm{HO}$ & OC. $\mathrm{C}_{15} \mathrm{H}_{31}$ & & $\mathrm{CH}_{2}$.O.OC. $\mathrm{C}_{15} \mathrm{H}_{31}$ & $\mathrm{H}_{2} \mathrm{O}$ \\
\hline $\mathrm{CHO} \mathrm{O}$ & + & $\mathrm{HO}$ & OC. $\mathrm{C}_{15} \mathrm{H}_{31}$ & $=$ & CH .O.OC. $\mathrm{C}_{15} \mathrm{H}_{31}$ & $-\mathrm{H}_{2} \mathrm{O}$ \\
\hline $\mathrm{CH}_{2} \mathrm{O}$ & $\mathrm{H}$ & $\mathrm{HO}$ & OC. $\mathrm{C}_{15} \mathrm{H}_{31}$ & & $\mathrm{CH}_{2}$. O.OC. $\mathrm{C}_{15} \mathrm{H}_{31}$ & $\mathrm{H}_{2} \mathrm{O}$ \\
\hline
\end{tabular}


When a fat is hydrolyzed, the reaction is just the reverse, water is added, and the fat split into the component fatty acids and glycerol. If the hydrolytic cleavage be accomplished through the agency of heat alone, as with the use of steam, or aided by the catalytic action of acids, the products are free; if the cleavage be accomplished through the action of alkali, soaps are formed. The soaps of ammonia, sodium, and potassium are soluble in water, those of calcium and magnesium are relatively insoluble. The soaps of triolein are more soluble than the soaps of tristearin.

Melting Point of Glycerids.-The melting points of the three glycerids are as follows: Triolein, $4^{\circ}$; tripalmitin, $62^{\circ}$; and tristearin, $72^{\circ}$. These are physical melting points. When the three glycerids are combined in a natural fat, the melting point of the mixture depends largely upon the relative amounts of triolein and tristearin. The greater the fraction of tristearin, the higher the melting point; the greater the fraction of triolein, the lower the melting point. The melting point of a natural fat is not a physical melting point at all, but rather something akin to a saturation point. The triolein acts as solvent for the other two glycerids, and the temperature at which the fat becomes fluid is the point at which the triolein present is able to hold the other two glycerids in solution. The melting points of the several natural fats are as follows: Horse fat, $60^{\circ}$ to $65^{\circ}$; mutton fat, $50^{\circ}$ to $55^{\circ}$; beef fat, $45^{\circ}$ to $50^{\circ}$; lard, $35^{\circ}$ to $40^{\circ}$; human fat, $35^{\circ}$ to $40^{\circ}$, and dog fat, $20^{\circ}$ to $30^{\circ}$. The melting point of the fat of the dog varies widely with the diet, as is the case with the fat of man; the fats of the other animals vary less. As will be pointed out later, the melting points of fats have a bearing upon their digestibility; other things being equal, the lower the melting point of a fat and the more soluble a fat, the greater the digestibility.

Caloric Values.-Fat is the most concentrated form of energy in a foodstuff. One gram of a fat of mean molecular weight of the three glycerids will yield 9.4 Calories of heat. When the degree of resorption of fat is normal this amounts to about 9 Calories per gram of fat ingested, and this is the figure usually used in diet calculations. This is obviously over twice the heat value of either glucose or protein. Since man is an animal endowed with locomotion, it is a fact of great practical importance that fuel is stored in the form of fat instead of in the form of starch.

\section{THE PROTEINS}

The composition and constitution of the proteins are of great complexity. The physical and chemical attributes of the different members of this large class are very manifold and are not to be grouped under rigid classification. Proteins may be defined briefly as condensation compounds of amino-acids. The physical attributes of these compounds have less bearing, at present at least, upon the processes of 
digestion and metabolism, than the chemical properties. To the former, therefore, we will devote less detail in description than to the latter. The details of the qualitative organic chemistry of protein are of fundamental importance to the interpretation of the metabolism of protein in the body.

Molecular Weight.-The molecular weights of proteins are large, but not accurately determinable. Various approximations for different proteins run from 3000 to 20,000 . Despite the fact that iron is combined in hemoglobin in definite proportions, despite the fact that proteins combine with many metals, despite estimations of the sulphur in native proteins, it has not been possible to fix the molecular weight. There is good evidence that proteins can exist in two states-as single molecules and as aggregates of molecules. To this fact are due many of the anomalous behaviors of proteins under different conditions of solution or suspension.

Colloidality.-The proteins are in general termed colloids. But this designation must be circumscribed. The colloidality of some proteins is very slight, of others marked. There are four stages to be outlined, the first of which approaches the true solution, the last of which borders on the mechanical suspension. Some proteins are quite soluble. Protamin is one of these. A solution of protamin sulphate is as truly a solution as is a syrup of cane sugar. All proteins in water may be regarded as having a fraction in true solution, though in the case of most proteins this fraction is small. The second stage is that of the hydrophilic colloid, and in this stage most of the proteins are found. In the hydrophilic colloids there is a variable relation between the colloid (solute) and the water (solvent), and this relation is expressed in the viscosity of the solution. The third stage is that of the aggregate or micelle. The molecules of protein in water unite to form aggregates that are optically visible in reflected light. There is for each protein a relation between stages two and three, reversible, that can be altered by dilution or concentration; for some proteins the conglomeration is favored by dilution, in others by concentration. The fourth stage is that of the suspension colloid, and many proteins tend to this state. Finally, some proteins of the connective-tissue groups are as inert in water as talcum. It is clear, therefore, that in the domain of proteins we find substances that range from true solubility to typical extreme colloidality - just as in the carbohydrates we have substances ranging from the true solubility of the di-, tri-, and tetrasaccharids, through the dextrins to starch and finally to cellulose. There has been a tendency to exaggerate the colloidality of proteins and to minimize their solubility. Most proteins in water are hydrosols, few are hydrogels.

The Tyndall test is of especial importance for the study of proteins in water. The dancing of dust particles in the air when a ray of light comes through a slit into a darkened room, is a typical instance of the Tyndall test. All proteins give this test, though in some it is slight. A colloidal solution appears white or opalescent in reflected light, for 
the reason that the particles reflect the light, which is in part polarized. It must be recalled that crystalloids of large molecular weight give the Tyndall test. A solution of protamin sulphate yields the test to but slight degree, no more than a solution of raffinose. From this point of view we may divide proteins again into four classes: In the first, the particles are visible to the naked eye and tend to settle out. In the second, the particles are visible on ultramicroscopic vision under all conditions of concentration and the particles so visible are termed submicrons. By special treatment, such as dilution, it can be shown that a third class exists, in which the particles are still smaller. These are termed amicrons, and these may be made to conglomerate to submicrons. Lastly, under certain conditions, particles may be encountered, whose movements indicate on calculation that they possess the dimensions of molecules. It is clear, therefore, that the results of the Tyndall test and of ultramicroscopic study lead to the same view, that proteins exist all the way in the scale from the large molecular crystalloid to the typical colloid.

Filtration and Diffusion.-The results of investigations on filtration and diffusion indicate that these are in part arbitrary processes, dependent upon the constitution of the filter and diffusion membranes; and in a classification of the behavior of proteins on filtration and diffusion, account must be taken of these facts. As a rule, proteins do not diffuse through ordinary membrane, though protamin sulphate diffuses with measurable velocity. But it is possible to so alter the diffusion membrane as to permit of the diffusion of many proteins. And, on the other hand, it is possible to so condense the fabric of filters as to retain even the finest colloidal particles. It is also possible to retain upon such filters large molecular crystalloids. And it is possible to so modify diffusion membranes as to make the diffusion of typical crystalloids slow and difficult. From the point of view of the filter, it seems to be simply a question of the size of the particles or molecules. With the diffusion membrane, however, there seems to be some relation of the composition of the membrane to the substance to be diffused; and for some apparently non-diffusible substances, the cause of their non-diffusion is precipitation in the interstices of the membrane. The cellular membranes are comparable membranes. These cell membranes are all protein-lipoid phases, and theoretically could not permit directly the passage of any substance not lipo-soluble. But amino-acids and sugar do pass into the cell walls; indeed, proteins pass in, and, of course, the lipo-soluble fats. To pass through this cell membrane, the substances must either be made lipo-soluble by complex chemical combination, or the membrane must be made permeable to their diffusion through adsorption of them by lipoids. Experimentally we have analogies for both processes.

It was once categorically stated that proteins could not present osmotic pressure in their solutions. 'This is not true. When the measurements are made with delicate and properly adjusted osmometers, it 
can be shown that pure proteins in solution exert a small but demonstrable osmotic pressure. Since the molecular weights of the proteins are known to be large and since the osmotic tension of solutions is inversely to the molecular dimensions of the solute, but small osmotic pressure would be expected in protein solutions. These run, as measured, from 3 to $30 \mathrm{~mm}$. of $\mathrm{Hg}$. In one sense it is possible to regard this osmotic pressure as evidence that a certain small fraction of the protein exists in water in true solution.

Crystallization.-Despite their colloidal character, many proteins display striking tendencies to crystallization. This is not only true for the hemoglobins (in which the crystallographic properties seem to vary with the biological stamp), but also for many of the ordinary forms of protein. Special conditions for crystallization must be attained, though these are often no more complex or difficult than are necessary in the case of some typical crystalloids.

Tension.-The more colloidal a solution of a protein, other things being equal, the more marked is its surface energy, or tension, compared with its volume energy. In such a solution, the particles of the colloid tend to surround themselves with a film of saturated solution of whatever may be present in the system. The relation works both ways of course, the protein may be either axis or periphery. It is possible to precipitate proteins from a solution and carry with them other bodies therein present; or it may be possible to precipitate a third substance and carry down with it the protein. Gases as well as salts, crystalloids and colloids may be held by adsorption, and future investigations may indicate that adsorptions play prominent rôles in animal functions.

Amphoterism.-As will be later described in detail, proteins contain both $\mathrm{NH}_{2}$ and $\mathrm{COOH}$ groups, and are, therefore, amphoteric, though with a greater basic or acid capacity in each case. Under these circumstances, it becomes clear why proteins combine as acids or as bases with other acids and bases. These combinations are usually very unstable, easily dissociated, and tend in particular to exhibit hydrolytic dissociation. It is when combined with acids or alkalies that the phenomenon of cataphoresis is displayed. When placed in the field of a galvanic current, the protein combined with acid migrates to the cathode, while protein combined with alkali wanders to the anode. If the acids or alkali be removed by dialysis or other mean, these migrations do not occur. Obviously, the particles of pure protein carry no charge, it is only the acid or alkali that endows them with electrical charges.

Nearly all proteins rotate the plane of polarized light. This is due to the presence of asymmetric atoms of carbon in the several aminoacids of which they are composed.

Gelification.-Certain proteins when brought into solution in hot water, on cooling pass into the state of gel. Certain carbohydrates possess the same property. This gelification is a reversible process; 
if the gel be warmed it becomes fluid, to solidify again on cooling. Heating, however, tends to destroy this property; for example, each hour's heating lowers the jelling point of gelatin $1^{\circ}$. Some gels melt at the same temperature at which solidification occurred; gelatin does so. Agar-agar, on the other hand, does not solidify on cooling until about $38^{\circ}$, but after it has set must be warmed to nearly $100^{\circ}$ before it will become fluid. The metallic hydrogels do not redissolve in water. The formation of a gel is considered to lie in a segregation, whereby two phases are formed, one rich in protein and poor in water, the other rich in water and poor in protein. The two phases can be distinguished with the microscope, and this demonstration is very suggestive for the student of physiological structure.

Coagulation.-Resembling gelification in many ways is the phenomenon of coagulation displayed by some proteins. We speak here of ferment coagulation-the clotting of milk, of blood, and of muscle plasma-not heat coagulation. The investigations to date seem to indicate that this process is due to the chemical transformation of the protein concerned into a para-stage, in which the protein combined with calcium, or other cation, is segregated into an insoluble phase. The process is irreversible.

Precipitation.-Colloids are often precipitable from their solutions in water by the addition of electrolytes, metallic salts. The amount of salt necessary to effect precipitation varies with the salt and with the protein. The proteins that wander to the anode are precipitated by the cations of the electrolyte; the proteins that migrate to the cathode are precipitated by the anions. The valency of the ions affects the flocking power; the trivalent ions are the most active, the bivalent next, the monovalent least. Proteins display often marked resistance or irregularities to these precipitations; obviously, the preëxistent salts would be expected to modify the results. And often it seems quite impossible to induce flocking in protein solutions. With the hydrophilic colloids, such as proteins, these flockings are reversible. The current theory of these precipitations is that it is an electrical neutralization process, and when the charge of the protein is just neutralized by the charge of the ions, precipitation occurs from the iso-electric medium.

Colloids sometimes precipitate each other, a behavior not commonly seen among the proteins. It is difficult in the instances where it does occur (as with protamin and albuminous solutions) to know whether we deal with a chemical combination or a flocking. The explanation for such precipitation of colloids is that colloids of opposite electrical charges precipitate each other. It is, however, not clear how adsorption can be ruled out. Proteins and other hydrophilic colloids have often the property of preventing the precipitation of suspension colloids by electrolytes. More than this, they prevent the physical denaturation of the suspension colloids. If a suspension colloid in water be evaporated to dryness, no colloidal solution will be formed on taking 
up the residue in water; but if the evaporation occur in the presence of a protein, a colloidal solution will reform on the addition of water. In like manner the presence of protein makes the filtration of a suspension colloid much easier. This protection of colloids by each other is probably a phenomenon of adsorption, the protecting colloid spreading like a thin film over the protected colloid, and modifying its physical and chemical properties. The use of colloidal states of iron and aluminum for the flocking of sewerage is an illustration of the practical application of this phenomenon.

Denaturation.-When solutions of proteins are carefully evaporated to dryness at low temperatures, well below the coagulation point, they form hard masses. These are usually soluble in water. But in some instances the process is not reversible and the protein refuses to again pass into colloidal solution. In all cases, as time passes, the resolubility becomes more and more difficult, to be finally lost. When proteins are precipitated by salting out, the precipitated and washed proteins are usually resoluble under appropriate conditions. But this is not always so. Some proteins are resoluble after precipitation with alcohol and tannic acid; others are not. In other words, in some cases precipitation means denaturation, in other cases not. The combinations with the heavy metals are very prone to cause denaturation. With some delicate proteins prolonged shaking produced denaturation; the proteins are conglomerated as definitely as churning conglomerates the fat of milk, and cannot be gotten back into solution.

A particular form of denaturation of proteins is coagulation by heat. This process is favored by salts and hastened also by acids and alkalies, although the denaturated proteins combine with the acid and alkali to form soluble compounds. These denaturations are irreversible. They carry with them also the loss of the specific biological properties of protein. We must, therefore, regard them as involving intramolecular transformations.

The physical and physico-chemical states of the colloids (the proteins, lipoids, and animal starches) are unquestionably of enormous importance in modifying and indeed in determining chemical reactions occurring in association with them, both in the quantitative and qualitative senses. Protoplasm may be defined as a system of heterogeneous phases, the water of solution forming multiphase systems with the inorganic salts, protein, and lipoids. In the fluid phases, the water is solvent and the ion-protein-lipoid solute; in the solid phase the protein or lipoid is solvent, the water and salts solute. The possibilities in such a multiphase system are enormous, both for chemical combination and physical adsorption. Concretely, we are not yet able to take account of these factors when dealing with the metabolism of a higher organism. Nevertheless, we must always make allowance for this factor, and never forget that the chemical reactions in the living body occur not in a homogeneous medium but in a two-phase system.

Origin of Proteins.-The origin of protein in the plant world, from which exclusively it is derived by higher animals, is well understood. 
Soils contain salts of nitric acid that has been derived from two sources; from ammonia originating in the decomposition of plant and animal tissues; and from the inert nitrogen of the atmosphere. The conversion of the inert nitrogen of the air into oxid is accomplished by bacteria, certain classes of which exhibit this faculty to such an extent as to make it their chief work; these are termed nitrifying bacteria, and with these are inoculated soils in which are growing higher plants upon whose roots they thrive well. There are on the contrary bacteria that denitrify, they set free gaseous nitrogen from nitrates; such bacteria are especially active in waters, both inland and oceanic, where the sewage of the world is ultimately disposed of. The oxidation of inert nitrogen to oxid of nitrogen can also be accomplished by electrochemical agencies. Thus nitric acid is formed in traces in thunder, and is being prepared commercially in large amounts by means of the electrical spark, the action of which is usually interpreted to be thermo-chemical, it being known that at high temperature nitrogen and oxygen unite. The oxid of nitrogen formed in the soil by bacteria is reduced to $\mathrm{NH}_{2}$ group in some manner as yet unclear, and these $\mathrm{NH}_{2}$ groups added to the fatty acids, whose origination has already been described, form amino-acids.

On paper we might assume that the nitrate is reduced to the nitrite, this then passes into $\mathrm{NH}=\mathrm{O}$, then combining with water to form hydroxylamin, $\mathrm{NH}_{2}=\mathrm{OH}$, which finally would combine with formaldehyd to form formamid $\mathrm{COH} . \mathrm{NH}_{2}$. There is no experimental evidence of this scheme. These syntheses are specific, since all amino-acids, except that of acetic acid (glycocoll), contain an asymmetric atom of carbon. With these amino-acids, plants form their many types and kinds of protein; and this plant protein is directly or indirectly the source of all protein in higher animals.

The Chemistry of the Proteins.-When proteins are completely hydrolyzed, the products consist of amino-acids. These vary in number in the different proteins, and the amounts of the several amino-acids also vary in the different proteins. Some proteins do not contain all the amino-acids that are to be found in certain members of the class; some typical proteins indeed contain but a few while others contain a great many amino-acids. It will be best to describe first the different amino-acids that are to be found in the products of the hydrolysis of proteins, after which the linkage of these bodies to form proteins will be described. Fundamental to our interpretation of the proteins as condensations of amino-acids is the fact that these exist preformed in the proteins. No matter in what manner and with what reagents (different acids in different concentrations, different alkalies in different concentrations, ferments, steam), the hydrolytic cleavages are accomplished, the end result in the yield of amino-acids is the same. In other words, the hydrolysis does not form amino-acids, it does not alter them, it does not convert one into another; it simply sets them free, their isolation thereafter depending upon particular chemical methods. In this point the reaction of the hydrolysis of proteins is 
like the cleavage of the fats and starches. The products are hexoses in the case of starches, fatty acids and glycerol in the case of the fats. The reactions of hydrolysis do not add to, substract from, or in any way alter the component bodies that existed preformed in the original substrates. The more the technique of the hydrolysis of the proteins is perfected, the more certain has it become that the component aminoacids exist preformed in the molecule of protein. The theoretical importance of this conception lies in the fact that we regard these amino-acids as the building stones of proteins, it is these that each organism puts together in accordance with the peculiar modus operandi of its species, forming therefrom protein structures of which the architecture is peculiar to the species. The amino-acids are not peculiar to the species, they exist preformed in all proteins, and are the common building materials of all plants and animals, just as brick, stone, timbers, boards, and plaster are the common building materials of all races. Just as the houses of different peoples vary greatly in size, in architectural design and conformation, so the proteins of different animals vary in construction.

The several amino-acids derived from proteins are all alpha aminoacids, $i$. e., the $\mathrm{NH}_{2}$ group is linked to the alpha carbon; in the case of the diamino-acids, there is no rule for the location of the second $\mathrm{NH}_{2}$ group. (It will be recalled that the carbons in the fatty acids are named in succession above the carboxyl, alpha, beta, gamma, delta, epsilon.) The presence of the $\mathrm{NH}_{2}$ and the $\mathrm{COOH}$ groups gives to these bodies basic and acid (amphoteric) characters; they combine with acids and bases to form salts, which being subject to a marked hydrolytic dissociation are in watery solution acid or alkaline in reaction. They combine with alcohols to form esters, which are bases; they combine with aldehyds to form methyl combinations that are acids. These may be illustrated for the reaction of glycocoll with ethyl alcohol and formaldehyd respectively:

$$
\begin{aligned}
& \underset{\mathrm{CH}}{\mathrm{CH}_{2} \mathrm{NH}_{2}}+\mathrm{C}_{2} \mathrm{H}_{5} \mathrm{OH}=\underset{\mathrm{COOH}}{\stackrel{C}{C} \cdot \mathrm{OH}_{2} \mathrm{NH}_{2}} \\
& \underset{\mathrm{C}}{\mathrm{C} \mathrm{H}_{5}} \\
& \underset{\mathrm{COOH}}{\mathrm{CH}_{2} \mathrm{NH}_{2}}+\mathrm{HCOH}=\underset{\mathrm{COOH}}{\mathrm{CH}_{2} \mathrm{NCH}_{2}}
\end{aligned}
$$

When $\mathrm{CO}_{2}$ is anchored to the amino-group, carbamino-acids are formed.<smiles>NCC(=O)CCCC(=O)C(=O)O</smiles>

The proteins and many peptids give the biuret reaction with copper sulphate in alkali. This reaction is produced by all substances in which two $\mathrm{CONH}_{2}$ groups are united to a carbon or nitrogen atom or to each other. One of the $\mathrm{CONH}_{2}$ groups may be replaced by a 
$\mathrm{CH}_{2} \mathrm{NH}_{2}$ or a $\mathrm{CSNH}_{2}$ group. The common peptid linking presents the appropriate combination. Most polypeptids, as will be described. respond to the biuret test, but there are striking exceptions to the rule, as in the case of the resistant antipolypeptid.

It is not contended that the list of amino-acids to be described is complete. Admitting, however, that others remain to be discovered, those at present known are certainly the most important. It is also not true that the amounts stated for the occurrence of the different amino-acids in the different proteins are even approximately correct; the figures are all too low. The methods do not recover the full yield, in nearly all cases the loss is 10 to 25 per cent.; in the case of some amino-acids, as serin, the loss is probably one-half. The quantitative findings will be given later in a summarized table.

Glycocoll (glycin)<smiles>O=C(O)CCCCCCC(=O)O</smiles>

This is amino-acetic acid. It is especially derived in the hydrolysis of proteins of the connective-tissue group; many proteins do not contain it. Possessing no asymmetric atom of carbon, it does not rotate the plane of polarized light. In the proteins that contain it, it seems to be firmly or centrally bound, and is not easily split off.

Alanin, $\alpha$-amino-propionic acid.<smiles>CC(C(=O)O)c1ccccc1</smiles>

This substance, while found in many proteins, is met with in large amounts in the connective-tissue albuminoids. Since the central atom of carbon is asymmetric, alanin rotates the plane of polarized light. The form found in proteins is dextrorotatory. Alanin is one of the most important of the amino-acids, less on account of itself perhaps than on account of other amino-acids that are derived from it. Such are: Phenylalanin, tyrosin, serin, cystin, tryptophan, and histidin.

Phenylalanin, phenyl- $\alpha$-amino-propionic acid.




Alanin in which a hydrogen in the methyl group has been replaced by benzene. It is present in the levorotatory form. Phenylalanin is present in small amounts in nearly all proteins.

Tyrosin, p-oxy-phenyl- $\alpha$-amino-propionic acid.<smiles>NC(C(=O)O)C(C(=O)O)C1CCCCC1</smiles>

Tyrosin is alanin in which an hydroxyl group is combined in the benzene neucleus in the para position to the fatty acid. Present in nearly all true proteins, it is absent from some of the albuminoids. It always exceeds alanin in amount. Tyrosin rotates the plane of polarized light to the left. Tyrosin is loosely or peripherally combined in the protein molecule and is set free early in the course of an hydrolysis. In this regard it is very different from alanin and phenylalanin, that are located in the molecule of protein in a manner more resistant to hydrolysis.

Serin, $\alpha$-amino- $\beta$-oxypropionic acid.

$$
\begin{aligned}
& \mathrm{CH}_{2} \cdot(\mathrm{OH}) \\
& ! \\
& C H \\
& ! \\
& \text { COOH }
\end{aligned}
$$

An oxidation derivative of alanin. It occurs in nearly all proteins, in larger amounts than usually determined on account of difficulty in isolation. As isolated from protein, it is either inactive (racemic) or rotates the plane of polarized light to the left. Serin is not easily split off in the hydrolysis of protein.

Cystin, $\alpha$-diamino- $\beta$-dithio-dilactylic acid. This is formed by the union of two molecules of a sulphur derivative of alanin, cystein, $\alpha$-amino- $\beta$-thio-propionic acid.<smiles>O=C(O)CCCCCCCCC(CS)C(=O)O</smiles>

These are linked by the two sulphur atoms to form the normal cystin of the protein molecule. 
<smiles>CC(CSCC(N)C(=O)O)C(N)C(=O)O</smiles>

Cystin is found in nearly all proteins, the protamins being free of it. It is partially broken down during hydrolysis, so that the real amounts are greater than those recovered. The cystein does not occur preformed in protein, it is always the double molecule. Cystin rotates the plane of polarized light strongly to the left. The cystin of protein and the cystin of renal calculi are identical. Cystin is derived in large amounts especially from the keratins. It is probably the sole form of sulphur in most proteins. In some proteins, however, sulphur seems to exist in another not fully identified state. There is some evidence that this may be $\alpha$-thio-lactic acid.



The cystin is closely bound in the molecule of protein and is not easily split off.

Tryptophan, indol- $\alpha$-amino-propionic acid. This complex substance has the constitution<smiles>NC(CC1C2NC3CCCC2C31)C(=O)O</smiles>

It exists in nearly all proteins, some keratins and protamin excepted. It is, like tyrosin, easily split off. It rotates the plane of polarized light to the left.

Histidin.-A somewhat related body is histidin. It is $\alpha$-amino$\beta$-imidazol-propionic acid.

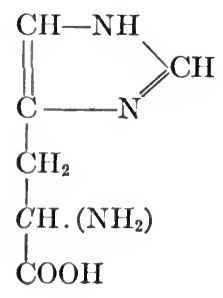


Histidin is present in nearly all the higher proteins. It is intimately bound in the centre of the protein molecule and resists cleavage. It rotates the plane of polarized light to the left. It is strongly basic.

Aspartic acid, $\alpha$-amino-succinic acid.<smiles>O=C(O)CC(C(=O)O)C(=O)O</smiles>

This occurs to a small extent in animal proteins, and to a much greater extent in plants. It is strongly acid. It rotates the plane of polarized light to the left. Another dicarboxylic amino-acid is

Glutamic acid, $\alpha$-amino-glutaric acid,<smiles>NC(C(=O)O)C(CCC(=O)O)C(=O)O</smiles>

It occurs to some extent in animal proteins, in large amounts in plants. It is strongly acid and rotates the plane of polarized light to the right. Both aspartic and glutamic acid occur widespread in plants in the form of the amids, asparagin, and glutamin.
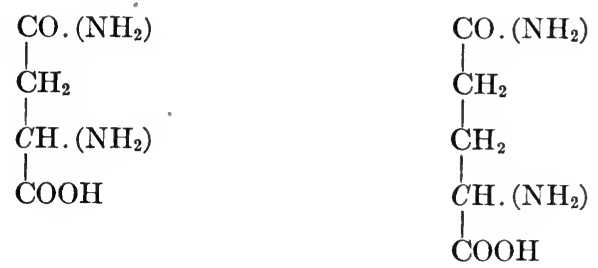

Whether these amids occur in the molecule of animal protein is not known. It is possible that some of the ammonia derived in the hydrolysis of protein is to be referred to them. Valin, $\alpha$-amino-isovalerianic acid.

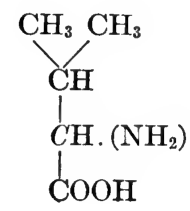


This substance occurs in small amounts in nearly all proteins. It rotates the plane of polarized light to the right.

Ornithin, $\alpha-\delta$-diamino-valerianic acid.<smiles>NCCCCCC(N)C(=O)O</smiles>

Ornithin does not exist preformed in protein, but only in combination with urea to form arginin. It is basic and rotates the plane of polarized light to the right.

Leucin, $\alpha$-amino-isobutyl-acetic acid.<smiles>CC(C)C(C(=O)O)C(N)C(=O)O</smiles>

Leucin is one of the most common cleavage products of protein, being present in all forms above protamin, and easily split off. . It rotates the plane of polarized light to the left. A somewhat similar substance is isoleucin. $\alpha$-amino- $\dot{\beta}$-methyl, $\beta$-ethyl-propionic acid:

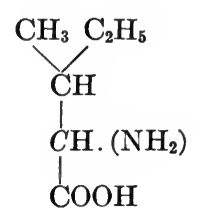

It occurs in traces in many proteins. It is dextrorotatory.

Prolin.-A heterocyclic amino-acid is prolin, $\alpha$-pyrrolidin-carboxylic acid.<smiles>O=C(O)C1CCCN1</smiles>

It occurs widespread, though in small quantities, as a rule, being present even in protamins. It is strongly levorotatory. An oxyprolin is also present, but its constitution is not understood. It is also strongly levorotatory. 
Lysin, $\alpha$-§-diamino-caproic acid.

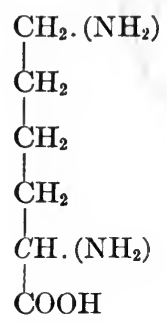

Lysin is widely distributed, nearly all proteins above the protamins contain it. It is strongly basic. It is dextrorotatory.

Arginin is a combination of the already described ornithin with a guanidin rest. This is illustrated in the equations:

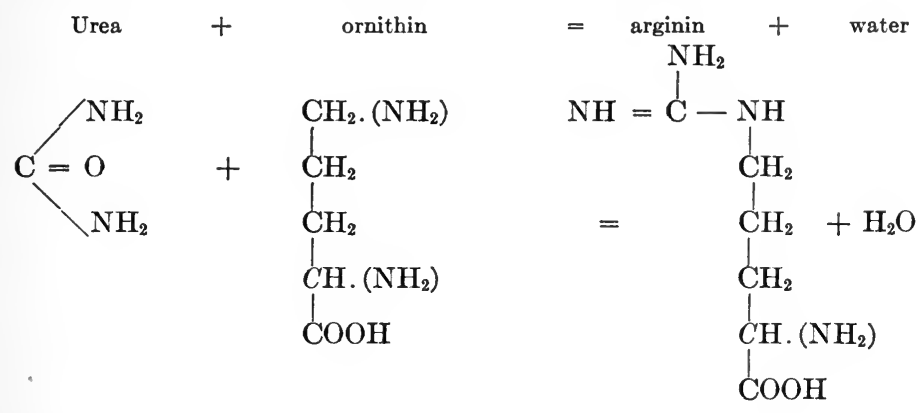

It is present in nearly all animal and vegetable proteins, and like lysin is strongly basic. It is dextrorotatory. Like lysin it is closely bound in the protein molecule, and is not easily split off.

Ammonia.-All proteins above the protamins contain small amounts of ammonia. Outside of arginin (and possibly the amids of aspartic and glutamic acids) we do not know of any preformed ammonia in the molecule. Yet it seems certain that it is not originated in the reactions of hydrolysis. Several other complex amino-acids have been isolated from digestion mixtures, but since their nature, as well as their constant occurrence, is entirely in doubt, they cannot concern us in a discussion of metabolism. Two points in particular require clarification: The nature of preformed ammonia and the nature of sulphur combinations outside of cystin. Bacteria produce ammonia from amino-acids and considerable ammonia is thus evolved in normal digestion, especially in the colon. There is no evidence that ammonia is formed from amino-acids of the usual type by hydrolysis.

The following table contains the figures for the content of the different proteins in amino-acids, all figures being as stated too low. Whenever figures are known, they are given; the positive presence of an amino-acid is indicated with a plus sign, its certain negative absence 
with a negative sign, while the empty spaces indicate that the question has not been determined. The great difficulty of adequate purification of the proteins submitted to hydrolysis adds to the complications.

\begin{tabular}{|c|c|c|c|c|c|c|c|c|c|c|c|c|c|c|c|c|c|c|c|c|c|}
\hline \multicolumn{4}{|c|}{ Proteins } & 离 & 思 & 步 & 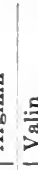 & & 蓉 & 妥 & 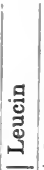 & 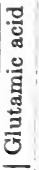 & 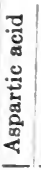 & 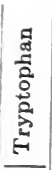 & 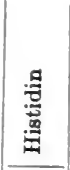 & $\begin{array}{l}\text { 要 } \\
\text { 总 } \\
\end{array}$ & 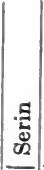 & 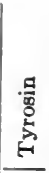 & 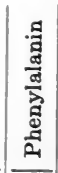 & 营 & \\
\hline bun & & & & + & -+ & $-t$ & t. & & + & 1 & 20 & & 3 & + & & 2.3 & 1 & 2 . & 3. & 3. & 0 \\
\hline & & & . & + & -2 & 2 & 2 & & + & 2 & 8 & & 2 & + & + & .2 & + & 1. & 4. & & \\
\hline & & & & $t$ & $+t$ & & $t-$ & & 1 & 4 & & 10 & 1 & + & + & + & + & 1. & 2. & 53. & V \\
\hline globu & & & 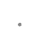 & + & & & + & f & . & 3 & 18 & 8 & 2 & + & & 1. & $\therefore$ & 2. & 54. & 2 & \\
\hline d & & & & + & & $\begin{array}{lll}1 & 1\end{array}$ & $1-$ & $t$ & 2 & 2 & 20 & 6 & 4 & + & 1. & + & 1 & 2. & 2. & 4 & \\
\hline & & & & + & - & 1. & . & + & . . & 3 & & 13 & 3 & + & & $\therefore$ & 1 & 2. & 4. & 4. & \\
\hline & & & -be & $\mathrm{n} 20$ & 0: & 3 & 5 & & .. & 4 & & 19 & 4 & + & 1.4 & $\ldots$ & .. & 2. & 4. & & \\
\hline & & & . &.. & & 5 & 5 & & .. & 6 & & 16 & 4 & & & & & 3. & 2. & 3. & 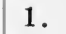 \\
\hline . & & & . & & 5 & 0 & $3-$ & & . & 2 & & 36 & 1 & 1. & 2 & + & + & 2 & 2. & 53. &  \\
\hline . & & & . & 3 & 3 & 0 & $1-$ & & 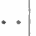 & 6 & & 18 & 1 & 0 & , & $\therefore$ & + & 3. & 5. & & \\
\hline ein & & &  & 5 & 50 & 0 & 2 & & . & 9 & & 33 & + & + & + & & + & 1. & 3. & 1. & 7 \\
\hline & & & 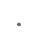 & 4 & 4 & 2 & & & 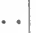 & 4 & & 23 & + & + & 8 & + & + & 4. & 2. & 5 & 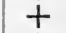 \\
\hline in & & &  & 1 & $1:$ & 3 & $6-$ & & & 3 & 11 & 6 & 3 & + & 3 & &.. & 3. & 4. & 4 & t \\
\hline & & & . & &  & & .2 & & 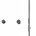 & 5 & & 18 & 4 & & & & & 1. & 3. & & 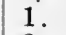 \\
\hline & & & . & t & & 4 & 31 & & & 4 & & 10 & 2 & + & + & & 1 & 3 . & 3. & & 3. \\
\hline , cow & & & . & & & & 5 & & + & 3 & & 11 & 1 & 1.5 & 3. & + & + & 5. & 3 . & & 0 \\
\hline his & & & & & & 71 & & & & 1 & 12 & + & & $\cdots$ & 2. & & & 5 & 2. & 3 & + \\
\hline $\mathrm{m}$ & I & bj & & & & 4 & 5 & & 1 & 2 & 29 & & 4 & + & 11. & .3 & 1 & 2. & 4. & & \\
\hline $\mathrm{in}, \mathrm{s}$ & $\mathrm{Im}$ & & & & & 08 & 72 & & 0 & 7 & & & 0 & 0 & 0 & 0 & 5 & 0 & 0 & 0 & \\
\hline & & & . & & & 6 & & & 0 & 0 & & & & 0 & 13. & 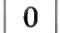 & 0 & 0 & 0 & + & \\
\hline & & & . & & & $0 \mathrm{~s}$ & 90 & & 0 & 4 & & & 0 & 0 & 0 & 0 & 0 & 0 & 0 & & \\
\hline roin & & & & 0 & + & & & & & + & & 0 & & .. & + & 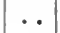 & 2 & 10 & 1. & 1 & \\
\hline & & & & & & t & 1 & & & 2 & 21 & + & + & . . & . & & & + & 4. & 6. & 2 \\
\hline & & & r. & & &  & & & & $=$ & & 18 & & .. & & & + & & 0 & & 14 \\
\hline & & & . & & & & 3 & & & 4 & & & & 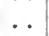 & 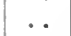 & & & 5. & 3 & 1 & . \\
\hline eratis & & & . & & & & & & & 4 & 12 & & & & & 8. & + & & 0 & 4 & 1. \\
\hline elatin . & & & . & & : & & S & & 3 & 5 & 2 & & & 0 & + & & 2. & 0 & .5 & 1. & 16. \\
\hline
\end{tabular}

Sugar Content of Proteins.-An important question concerns the sugar content of proteins. Do proteins contain preformed sugar, combined as an integral part of the molecule? As will be later described, there are many combinations between molecules of protein and molecules of carbohydrate. On account of adsorption, it is very difficult to free proteins of carbohydrate clinging to them. But as the materials are analyzed, it is becoming clearer that in the direct sense sugar is not usually a component of protein. So far as we know at present, there is but one form in which sugar is present preformed in the protein molecule, and that is in the amino-sugar glucosamin. Many proteins, especially the mucins, contain large amounts of preformed glucosamin that is bound in the molecule like the other amino-acids. Glucosamin, which is an amino derivative of glucose, bears a striking resemblance to lysin in configuration of the molecule. 


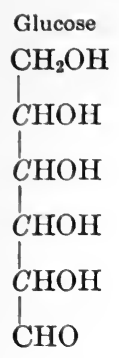



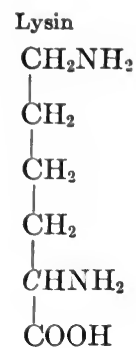

The molecule of glucosamin is to be regarded as directly bound in the protein molecule, being attached by its $\mathrm{NH}_{2}$ group to the $\mathrm{COOH}$ group of an amino-acid. Since it has itself, however, no $\mathrm{COOH}$ group, it cannot be regarded as linked, but must exist as a terminal group. The mucins and related proteins may contain as high as 30 per cent. of glucosamin. It is very doubtful if the stock proteins of the blood serum, serum albumin, and serum globulin, contain any glucosamin.

The Combination of Amino-acids in Protein.-The proteins being regarded as condensations of amino-acids, how are we to picture the internal constitution of the protein molecule? In other words, after what fashion are the amino-acids linked or bound? This question is pertinent not only to the problem of the purely chemical construction of protein, but also to problems of the digestion and metabolism of protein. The question has been approached from the inside as well as from the outside, through synthesis as well as through lysis, and the two sets of data are very harmonious and complementary. Important really are two phases of the question: (1) How are the aminoacids bound together, and (2) can we regard some as being nuclear and others peripheral in their location, some loosely bound and others firmly bound?

Method of Linkage. - With respect to the method of linkage of the amino-acids, our definite ideas were first drawn from the synthesis of the peptids. Under the term peptid we understand simple combinations of amino-acids; thus we have di-, tri-, tetra-, and polypeptids, substances containing two, three, four, or many molecules of aminoacid. These conceptions have been confirmed by investigations into native proteins, and what is now termed the peptid linking is known to be the method of combination that is for the most part present in native proteins.

This peptid linking may be explained as follows: Two molecules of glycocoll may be united to form a dipeptid termed glycyl-glycin. This peptid, like the glycocoll, has a free carboxyl and free aminogroup, it is amphoteric and presents all the group reactions of these two groups. Obviously, therefore, the combination of the two molecules may be regarded as a linking of the carboxyl group of one molecule of glycocoll to the amino-group of the other molecule of glycocoll. 
Thus:

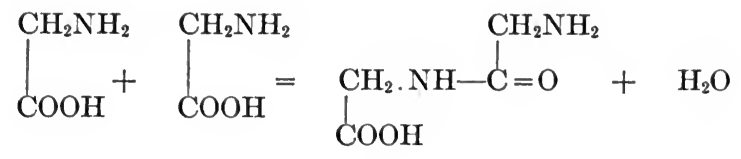

or to make the illustration linear:<smiles>CC(NC(=O)CCCCCCN)C(=O)O</smiles>

This may be illustrated for a tripeptid in the following equation: Leucyl-glycyl-phenylalanin.

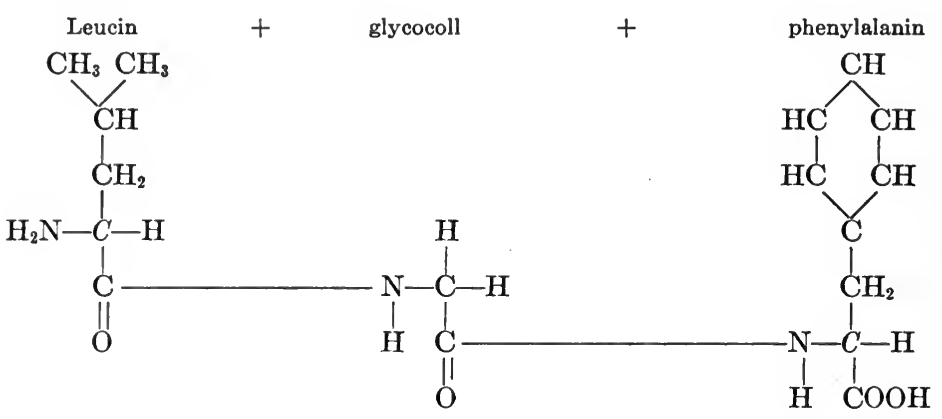

This tripeptid presents an $\mathrm{NH}_{2}$ group at one end and a $\mathrm{COOH}$ group at the other end, and in this respect acts just like an amino-acid. It is, of course, possible to have in a peptid larger members of amino and carboxylic groups when diamino and dicarboxylic acids enter into the peptid. This may be illustrated in a hypothetical tetrapeptid:




In this tetrapeptid there are two monamino-acids and two diaminoacids. The molecule contains, therefore, two free groups of $\mathrm{NH}_{2}$ and two free groups of $\mathrm{COOH}$.

The possibilities of this peptid linkage are very great, and no less than eighteen molecules of amino-acid have been synthetically linked into one chain, giving a polypeptid of the molecular weight of 1213 . At the same time it is obvious that the molecules of native protein can hardly be made up of such simple chains, and, indeed, other forms of linkage are known.

When urea and ornithin combine to form arginin, the $\mathrm{COOH}$ group of the ornithin does not combine with the $\mathrm{NH}_{2}$ group of the urea; it is the $\mathrm{NH}_{2}$ group attached to the $\delta$-carbon that combines with the carbon of the urea, so that the nitrogen binds two carbons, neither of which has a bond attached to oxygen.
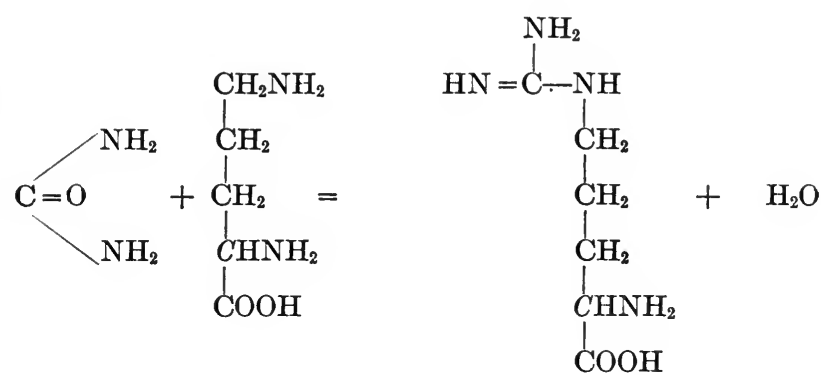

This type of linkage exists so far as known only in the union of urea and ornithin. It is a very firm binding, being resistant to the action of steam and acid, which split the peptid binding with ease. It is thus adapted to serve as the centre of a chain, as the keystone of an arch, as it were. In addition to this, arginin presents unusual opportunities for attachment of other amino-acids, since it has two amino and one carboxylic group.

Another form of binding is to be seen in cystin, which is formed of two molecules of $\alpha$-amino- $\beta$-thio-propionic acid.

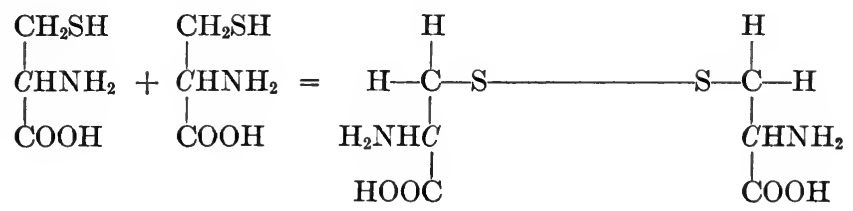

Like the binding between urea and ornithin, this linkage is obviously adapted to serve in the centre of a group, to either side of which two amino-acids could be attached.

These are the only form of linkages definitely known to exist in the protein molecule. But it were shortsighted to assume that the 
possibilities of combination are exhausted in these. The ammonia remains in arginin suggest another form of binding, nitrogen-tonitrogen, analogous to the sulphur-to-sulphur linkage in cystin. In phenylalanin the benzene ring is untouched save for the connection with the alanin; in tyrosin there is an hydroxyl combined with the ring in the para position to the amino-acid; in tryptophan we have indol attached to the fatty acid. It is certainly possible in the case of phenylalanin and tyrosin at least to imagine other bindings with the benzene ring; that there are other possibilities is shown in direct experiment. Peptids, like amino-acids, bind carbon dioxid and give the carbamino reaction. Now in the case of amino-acids it is only the $\mathrm{NH}_{2}$ groups that bind the carbon dioxid. In the case of peptids and proteins, however, not only the $\mathrm{NH}_{2}$ groups but also the nitrogens by which the amino-acids are bound together will add carbon dioxid. It is theoretically possible that ring formations of the type of piperazin may exist in the protein molecule. It is also possible that the oxyacids (serin and tyrosin) might form esters. Further investigation will make these clear and in all probability indicate additional relationships.

Groupings in Protein Molecule.-When now we come to consider the manner in which these several amino-acids are related or grouped in the huge protein molecule by the different bindings that have been described, we approach a question of great theoretical importance. Investigations in the disintegration of the protein molecule by steam, mineral acids, alkalies, pepsin, trypsin, and erepsin have taught us that certain amino-acids are early and easily split off; that others resist splitting; that some may be split off by acid and alkalies that cannot be split off by pepsin, or even by trypsin; that some amino-acids cannot be recovered until the protein is utterly disintegrated. These facts will be detailed under the appropriate headings. Here they interest us only because they prove that even if linked together in the same way, some amino-acids are refractory to hydrolysis, others yield to hydrolysis. Among those that are very easily split off are tyrosin, tryptophan, leucin, and ammonia. Later in the hydrolysis leucin, serin, cystin, aspartic acid and glutamic acid and a small fraction of the diamino bases are obtained. Last of all we recover glycocoll, prolin, phenylalanin and the rest of the diamino acids. These last are not recovered by tryptic digestion, and constitute what has long been known as the anti-peptone fraction. 'These facts suggest that certain aminoacids are, so to speak, peripheral and other amino-acids central in location within the molecule of protein.

There are two conceptions of these relations within the protein molecule. One corresponds to a branched chain; the other to linked groups or nuclei. The mere fact of the existence of the diaminoand dicarboxylic acids prevents us from regarding any protein as a straight chain, like the synthesized octadeca-peptid composed of 15 molecules of glycocoll and 3 of leucin. 'The double $\mathrm{NH}_{2}$ and $\mathrm{COOH}$ 
groups necessitate a branching. Aspartic and glutamic acid have each one $\mathrm{NH}_{2}$ group and two $\mathrm{COOH}$ groups; arginin and lysin have each one $\mathrm{COOH}$ and two $\mathrm{NH}_{2}$ groups; the other amino-acids in protein have one group of each. A diagram will illustrate the branched bindings. Let the sign A indicate the $\mathrm{NH}_{2}$ group, the sign $\mathrm{C}$ the $\mathrm{COOH}$ group. Assuming the centre of the chain to be composed of a group of glycocolls, the diamino-acids would cause branchings to the right while the dicarboxylic amino-acids would cause branchings to the left. This scheme is, of course, merely diagrammatic and has been made

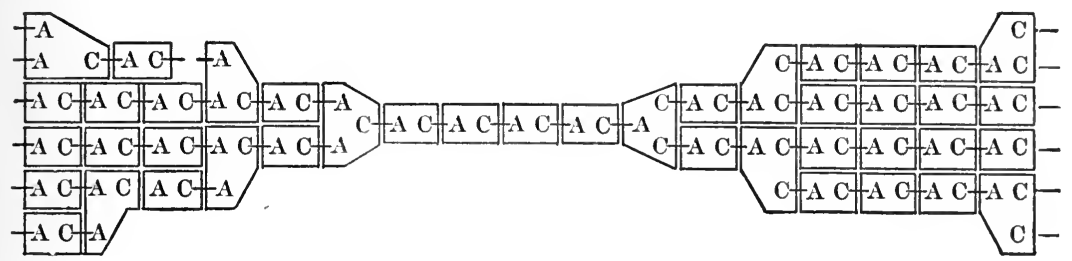

symmetric. It illustrates, however, the necessary branching that must occur as the result of the presence of amino-acids having two $\mathrm{NH}_{2}$ or $\mathrm{COOH}$ groups, and corresponds to the experimental fact that many proteins have a large number of free $\mathrm{NH}_{2}$ and $\mathrm{COOH}$ groups. A preponderance of the diamino-acids in the whole molecule would lead to a basic protein like protamin. The presence of a preponderance of the dicarboxylic acids would lead to an acid protein, like gliadin.

- It is more than doubtful if such a scheme corresponds to the full facts. The more proteins are studied, the more it appears that within the total molecule are definite groups, and it is the linking together of these groups that forms the completed molecule. This view is gaining ground for the reason that the existence of such groups is being demonstrated in different proteins. The point of view may be made clear in a contemplation of the protamins. The protamin of the salmon, salmin, contains according to our best evidence 12 molecules of arginin, 2 of serin, 3 of prolin, and 1 of valin. Now when this protamin is carefully digested, it will be found that there is a stage midway between the original state and complete cleavage, and that in this stage we have what are termed protons-smaller molecules than protamin, larger than the amino-acids. These protons are known to consist each of two molecules of arginin with one of monamino-acid. In other words, salmin when hydrolyzed first breaks up into six molecules of proton, and on further hydrolysis each molecule of proton breaks up into three molecules of amino-acids. Thus:

$$
\left(\begin{array}{c}
\text { Arginin } \\
! \\
\text { Serin } \\
! \\
\text { Arginin }
\end{array}\right)-\left(\begin{array}{c}
\text { Arginin } \\
\vdots \\
\text { Serin } \\
! \\
\text { Arginin }
\end{array}\right)-\left(\begin{array}{c}
\text { Arginin } \\
\mid \\
\text { Prolin } \\
! \\
\text { Arginin }
\end{array}\right)-\left(\begin{array}{c}
\text { Arginin } \\
\mid \\
\text { Prolin } \\
! \\
\text { Arginin }
\end{array}\right)-\left(\begin{array}{c}
\text { Arginin } \\
\mid \\
\text { Prolin } \\
! \\
\text { Arginin }
\end{array}\right)-\left(\begin{array}{c}
\text { Arginin } \\
\mid \\
\text { Valin } \\
\mid \\
\text { Arginin }
\end{array}\right)
$$


The first stage of hydrolysis consists in the cleavage of the bindings between the six diarginyls, setting these (the protons) free. Through hydrolysis the diarginyls are then split into the component aminoacids. This is quite analogous to the state of affairs in starch. The hydrolysis first splits the molecule of starch into a number of molecules of maltose; these are then split into molecules of d-glucose. Investigations with other proteins have yielded identical results.

Conception of Protein Molecule.-We may then formulate our conception of the molecule of protein as follows:

(a) Native proteins are to be regarded as linked aggregates of polypeptids, these forming nuclei or groups that are preformed and intact in the protein molecule, the connection of polypeptid to polypeptid being achieved through one of the described forms of linkage.

(b) Polypeptids are combinations of individual amino-acids, bound together by means of one of the described linkages.

The different polypeptids, as well as the molecule of native protein itself, are variable in their relations to pepsin, trypsin, and erepsin. When a molecule of native protein is digested, the groups of polypeptids are first separated, in part at least; these then react toward the ferment in accordance with their own properties, some being refractory, others cleavable. And when later we shall say of erepsin that it does not digest native protein, we mean that it cannot attack the bindings between the polypeptids, though it can separate the individual amino-acids in a polypeptid. A polypeptid may be formed as a straight or branched chain, as given in the first scheme. Several of these sets of branched chains (polypeptids) are then linked to form a molecule of protein, and the linkage between these polypeptids is one of relatively easy cleavage, so that when the native protein is submitted to hydrolysis, these are first separated. Following this occur the reactions within the several polypeptid groups.

This composition of protein makes clear the most extraordinary possibilities of isomerism. It is, of course, true that not all proteins contain all, or the same, amino-acids, and herein lies the basis of much individualism in proteins. When proteins contain the same aminoacids but contain them in different amounts, an additional basis for individualism exists. But even when proteins contain the same aminoacids in the same amounts, the possibilities of individualism through true isomerism are almost innumerable. In the simple protamin, salmin, composed of six diarginyls, a large number of isomers are possible. Serum albumins and serum globulins, in the higher animals, seem to have identical constituents; but they are very different substances. And finally, stereoisomerism affords still another basis of specialization. Bearing in mind the premises for chemical individualism in proteins - difference in amino-acids, difference in amounts of aminoacids, simple isomerism and stereoisomerism-let us consider two groups of biological facts that find therein their explanation. 
Disintegration of Protein in Digestion.-The diet of animals, be they carnivora, herbivora, or omnivora, contains many kinds of protein. These are disintegrated in the processes of digestion. From the numerous products of the digestion of these diverse proteins the body forms two stock proteins, the serum albumin, and serum globulin of the blood. The diet of the human race contains probably not less than thirty or forty different proteins, occurring in amounts large enough to be of importance in the diet. Yet from the digestion products of these (the building stones) the body builds the two stock proteins of the blood serum. No matter how varied the diet, so long as the products contain all the different amino-acids, the body builds of them serum albumin and serum globulin, employing the four factors above mentioned. The human body contains over twenty more or less well-defined proteins. 'These are all formed from the amino-acids contained in the two stock proteins of the blood. In the tissues concerned, these two stock proteins are split into their component amino-acids, and from these the tissues form the particular protein characteristic of this or that tissue; some amino-acids are rejected, others are massed, and all in all an internal arrangement is effected which has its outward manifestation in the striking variations displayed by the different tissue proteins of the body. The whole maintenance of growth rests simply upon the faculty of building specialized structures from common building stones-the synthetic function of tissues lies largely in design and not in material. It is this fact that indicates how a proper vegetarian diet is in every way a normal and competent diet. Plant proteins contain the same amino-acids as animal protein and all are there present in abundance. It is quite immaterial to the body whether it forms its two stock proteins from amino-acids derived from plant protein or from animal protein. Contrariwise, it is equally clear that animal protein is in nowise inferior to plant protein.

The specialism, however, goes much farther than this. Not only does the animal form many proteins from common building stones, it also places upon them, or rather in them, its biological stamp. It may be granted that the caseins, serum albumins, serum globulins, hemoglobins, and other proteins have the same percentage compositions in the higher animals, they are isomers. But they are very different substances nevertheless, as the now enormous literature on immunization and sensitation with foreign proteins positively testifies. Now the only conceivable basis for this biological specificity lies in isomerism. The striking differences in properties displayed by isomers is well illustrated in the hexoses. This is for the hemoglobins beautifully illustrated in their properties of crystallization, which varies for each species. The number of possible isomers in a substance like serum globulin runs into the hundreds of thousands. We may, therefore, feel assured that in the isomerism of the protein molecule, resting upon the intramolecular arrangement of the amino-acids, we have a fully adequate basis for the striking biological specificities displayed 
by proteins. Proved this hypothesis is not and cannot be until the intramolecular structure of the proteins is available for analysis and experimentation. But it is an hypothesis in which we may confidently believe.

Scheme for Protein Molecules.-If now we add to our knowledge of the protein molecule obtained by the study of the peptides the information derived by investigations into partial and completed hydrolyses under the influence of acids, alkalies, steam, and the several ferments, we are in a position to erect a scheme for the protein molecule. The first stage in hydrolysis, as commonly carried out, is acid protein or alkali protein. These compounds represent addition products; they are formed rapidly near the temperature of the coagulation point of the proteins, slowly at lower temperature. They are subject to high degrees of hydrolytic dissociation. Acids and alkalies in some way render the proteins, with which they are combined, open to the attack of enzymes. It is not necessary for ferment action, however, that proteins be combined with acid or alkali; the ferment can operate without them, though slowly. The next stage is that of the proteose. There have been many proteoses described, as there have been many dextrins described. But it is not possible to demarcate these bodies, since they are passing into each other. The highest proteose may be defined as the protein molecule minus one peptone. And the successive proteoses are apparently due to the successive splitting off of peptones. How many are in the series, we do not know, several certainly. The results of salting-out procedures do not and cannot define these proteoses, for the simple reason that the salting out of a particular proteose is modified by the presence of other proteoses and of peptone in the system. With the peptone is attained the stage when a neutral salting-out can no longer be accomplished. When the peptone is split, apparently polypeptids are set free. In a certain sense, the peptone may be defined as a polypeptid. The cleavage of the polypeptid sets free the di-, tri-, tetrapeptids and free aminoacids. The complete hydrolysis yields only free amino-acids. These polypeptids are not of one order, they are composed in part of different amino-acids, and they present very different degrees of resistance to chemical manipulations. Steam and acids split them all, erepsin and many bacterial ferments split them all, but trypsin is unable to split some of them and pepsin is able to split none of them. These resistant groups, the antipolypeptids, suggest themselves as the nuclei of the peptones, firmly bound and thus resistant to hydrolysis-the centres, so to speak, about which the other amino-acids are aggregated. They are not, however, present in all peptones, and thus we have in the peptones two orders of magnitude in the chemical and dynamic sense. This conception of the molecule may be put into a scheme, it being, of course, borne in mind that many of the details are arbitrary and that future investigations may easily result in pronounced modifications. 


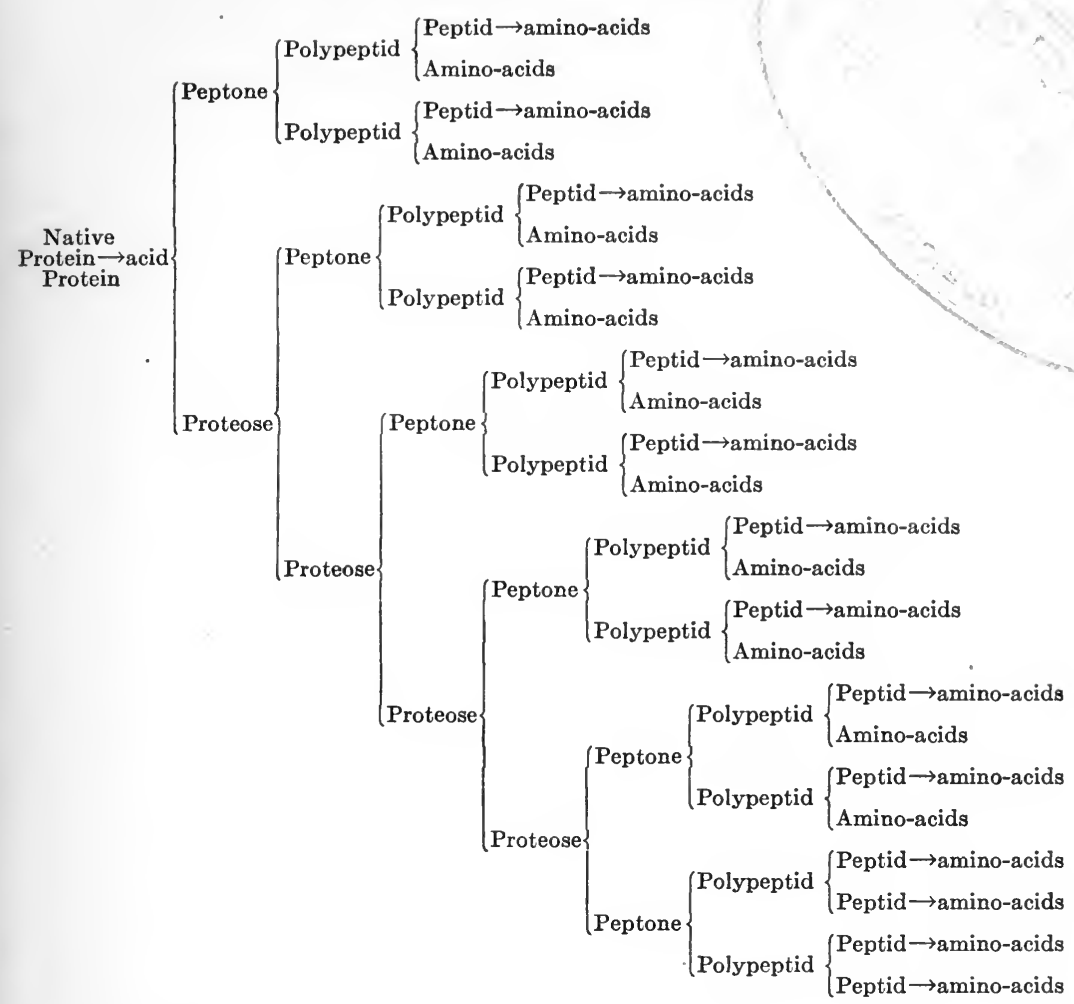

Concerning the natural peptids that are resistant to ferment action we possess data drawn from various sources. There are two groups resistant to the action of trypsin. One, which gives the biuret reaction, vields on hydrolysis after isolation prolin, glycocoll, alanin, phenylalanin, leucin, aspartic and glutamic acids. One antipolypeptid prepared from gelatin contains argenin, lysin, glycocoll, and glutamic acid. If proteins be carefully hydrolyzed with dilute acid, it is possible to check the process at a stage when resistant basic groups can be isolated. These have been termed kyrins. These kyrins are composed of arginin, lysin, histidin, glutamic acid and glycocoll. Belonging to this group of substances apparently is the long known oxyproteinic acid. This substance, which may be regarded as normal in the urine, has been shown on hydrolysis to yield alanin, leucin, phenylalanin, glutamic acid, and glycocoll.

In the products of the enzymic hydrolysis of protein many peptids have been found that are identical with peptids synthesized from amino-acids, a finding of the most impressive kind. In the buildingup of peptids large molecules have been constructed that give most of the reactions of peptones; they are digestible with trypsin and erepsin, some may even be salted out like proteoses. It is this success of synthesis, together with the isolation from digestion products of the same peptids that have been synthesized in the laboratory, that make us 
so certain that our present conceptions of the intramolecular constitution of protein are correct, and lead to the conviction that proteins in all respects identical with native proteins will ere long be synthesized in the laboratory. 'The peptids, known through synthesis, that have been recovered from digestion products include the following: Glycylalanin, glycyl-leucin, glycyl-tyrosin, glycyl-valin, alanyl-leucin, alanylprolin, alanyl-glycin, leucyl-glutamic acid, leucyl-alanin, and prolinphenylalanin.. In addition the following more complex peptids have been recovered: Glycocoll-alanin-tyrosin, leucin-tyrosin-glycocoll, cystin-tyrosin-glutamic acid, tryptophan-glutamic acid, and tryptophan-leucin-glutamic acid. In this connection it is interesting to note that the synthetic peptids that are digestible with trypsin in vitro, are completely digested and bound in the tissues when introduced intraveneously.

Tryptophan and tyrosin seem, in a certain sense, to render a protein susceptible to salting-out; at least, the proteoses seem to cease to be precipitable by salting-out in proportion to the splitting off of their tyrosin and tryptophan. On the other hand, there are relatively simple peptids containing these amino-acids in large amount that can be salted out from solution. Of the size of the peptones we know very little. One peptone isolated from silk fibroin seemed to consist of 2 glycocoll, 1 d-alanin, and 1 l-tyrosin. It is, however, as stated, certain that there are peptones of large size with peptid properties; while there are tripeptids with many of the qualities of proteose. The properties depend not. only on the size of the molecule, but also and largely, on the component amino-acids, and their linkage.

Certain amino-acids seem to be located or concentrated in one of the peptones of a protein; this holds especially for cystin and tryptophan. In some proteins, however, there seems to be two fractions of tryptophan, one is split off early while one appears late.

If we observe the qualitative march of an hydrolysis from day to day, we will observe a certain general order in the appearance of the amino-acids quantitatively. The earliest to appear are tyrosin, cystin, and tryptophan. Then gradually appear leucin, alanin, valin, aspartic and glutamic acids. Next appear the bases and histidin. Prolin and phenylalanin do not appear free in a tryptic digestion at all. They appear last of all in an ereptic digestion, with the final fraction of diamino-acids and glycocoll.

Caloric Value.-The caloric value of different proteins varies from 5.5 to $6 \mathrm{Cal}$. The heat value in the animal body is of course lower, the metabolizable energy of protein averaging 3.9 Cal. for plant protein, and $4.3 \mathrm{Cal}$. for animal protein.

\section{FOODSTUFFS IN THE DIET}

Do the descriptions of the foodstuffs from the standpoint of carbohydrate, fat, and protein exhaust the chemical possibilities of a diet? 
Are there other features of the foodstuffs that lie without the qualities of carbohydrate, fat, and protein? Can an organism subsist on pure sugar, fat, and protein? Four further factors must be considered, one quite obvious, the others as yet intangible. Inorganic salts are, of course, needed in a diet, since certain salts and ions are essential to the physico-chemical constitution of cells and tissues. The now apparently demonstrated relation between the occurrence of beri-beri and the consumption of polished rice supposedly furnished an illustration of the need of equilibrium, as well as plenty, in inorganic ions. The defect in the polishing of rice that is operative in the etiology of beriberi is, however, not inorganic but organic, diffusible and non-colloidal. The second factor lies in the state of conservation of the foods. Individuals nourished on conserved foods acquire scurvy. Infants fed on boiled milk do not maintain their nutrition normally. The use of fresh vegetables, especially potatoes and the citrus fruits, will prevent the occurrence of scurvy and cure it after it has set in. Under these circumstances it was inferred that certain thermolabile organic acids were in some way essential to metabolism. How labile were these assumed substances may be realized when it is recalled that the freezing of the potato robs it of its antiscorbutic properties. This point of view became untenable, however, when upon one of the Antartic expeditions the observation was made that fresh meat was as effective an antiscorbutic as fresh vegetables or lime juice. There is apparently some change that follows cooking, some circumstance attending the conservation of foods that disturbs an essential factor in normal nutrition. Thirdly, the nutrition of sterile animals fed on sterile food (in a word, digestion without bacteria) is not normal. We possess no concrete idea of this fact, we do not know whether bacteria contribute necessary substances, whether they in some way modify the processes of digestion or in what manner this apparently indispensable action is attained. Man is apparently a symbiotic organism, though it will hardly be possible to attain greatly advanced age through the consumption of any particular strain of microörganisms. Bacteria are plants and it has been inferred that possibly all they contribute could be found in raw plants. Vegetable fiber is indeed valuable, and plants probably contain all the chemical ingredients of bacteria. Yet the relation seems less tangible than this.

The final consideration affecting the total adaptability of a food or ration lies in the source of the foods. It will be made clear in the discussion of the minimum nitrogen input that proteins in different foods, even when containing all the requisite amino-acids, vary in their powers of covering the needs of the tissues. But more striking even are the variations to be noted in particular rations that are balanced in every chemical respect, both for protein and caloric values. Thus it has been shown by careful feeding experiments extending over several years, that a ration drawn solely from the wheat plant, including the grain, is not adapted to the normal growth and functionation of cattle, while 
an otherwise comparable ration drawn solely from the maize plant is fully adapted to all the needs. Heifers raised on the wheat plant are inferior in size and strength, the offspring are inferior and the milk secretion is below the normal. This could not have been due to the protein, the carbohydrates, fats, or to the ash; so far as can be determined, it lies in some as yet unknown factor. It is interesting to compare this observation in cattle with the widespread conviction that in man maize cannot be used to replace wheat, oats, or barley in the bread ration.

The three factors mentioned do not seem operative, in the experimental sense, in short diet tests. But they must never be overlooked in prolonged diet tests, or in the statistical attempt to estimate the diet relations of a group or race of people.

The following tables contain the food values for many raw and cooked foods. The figures are, of course, approximate only, and the analytical values will fluctuate with the grade of foodstuff and also with the method of cooking. (Modified from Atwater.)

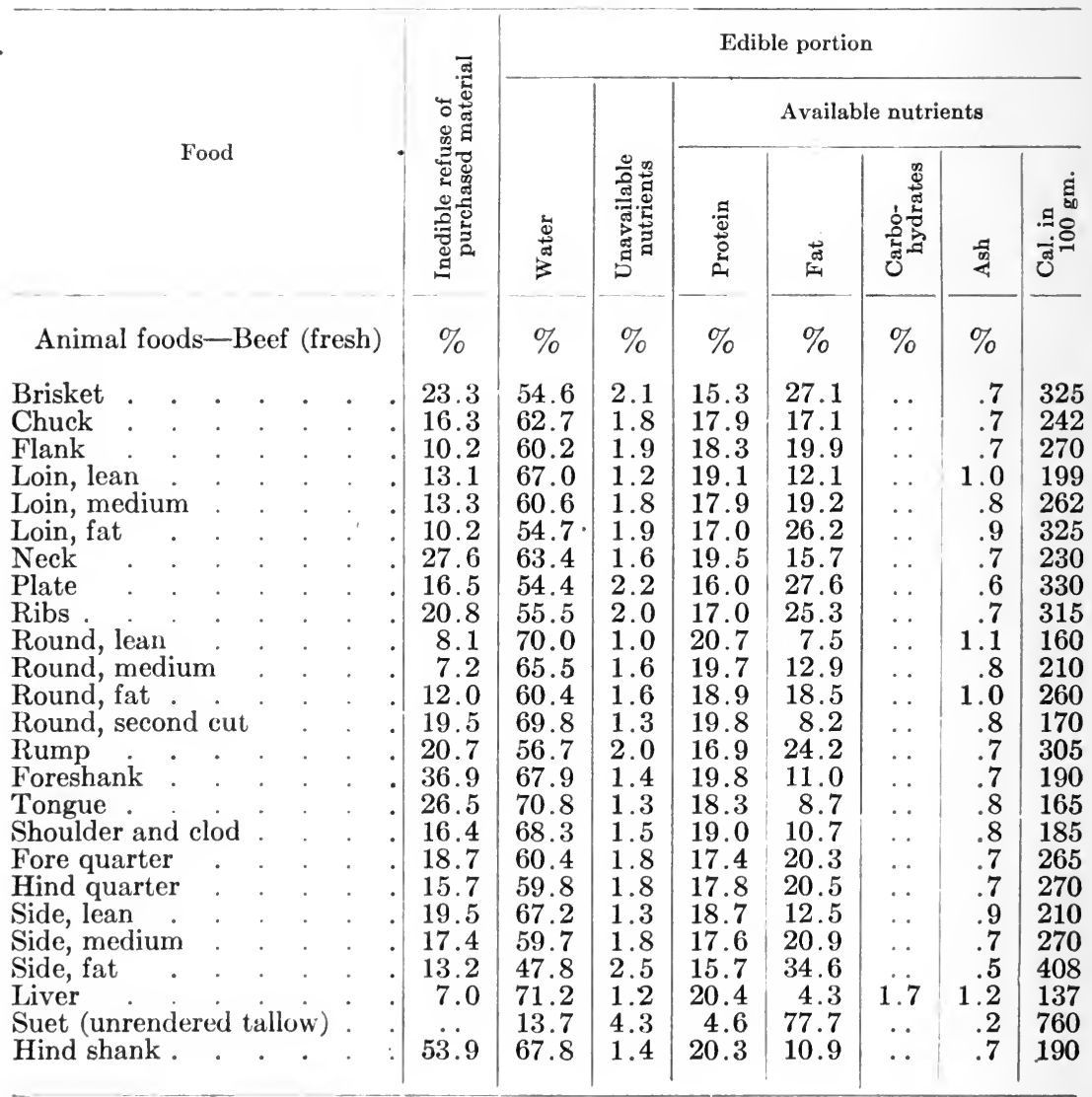




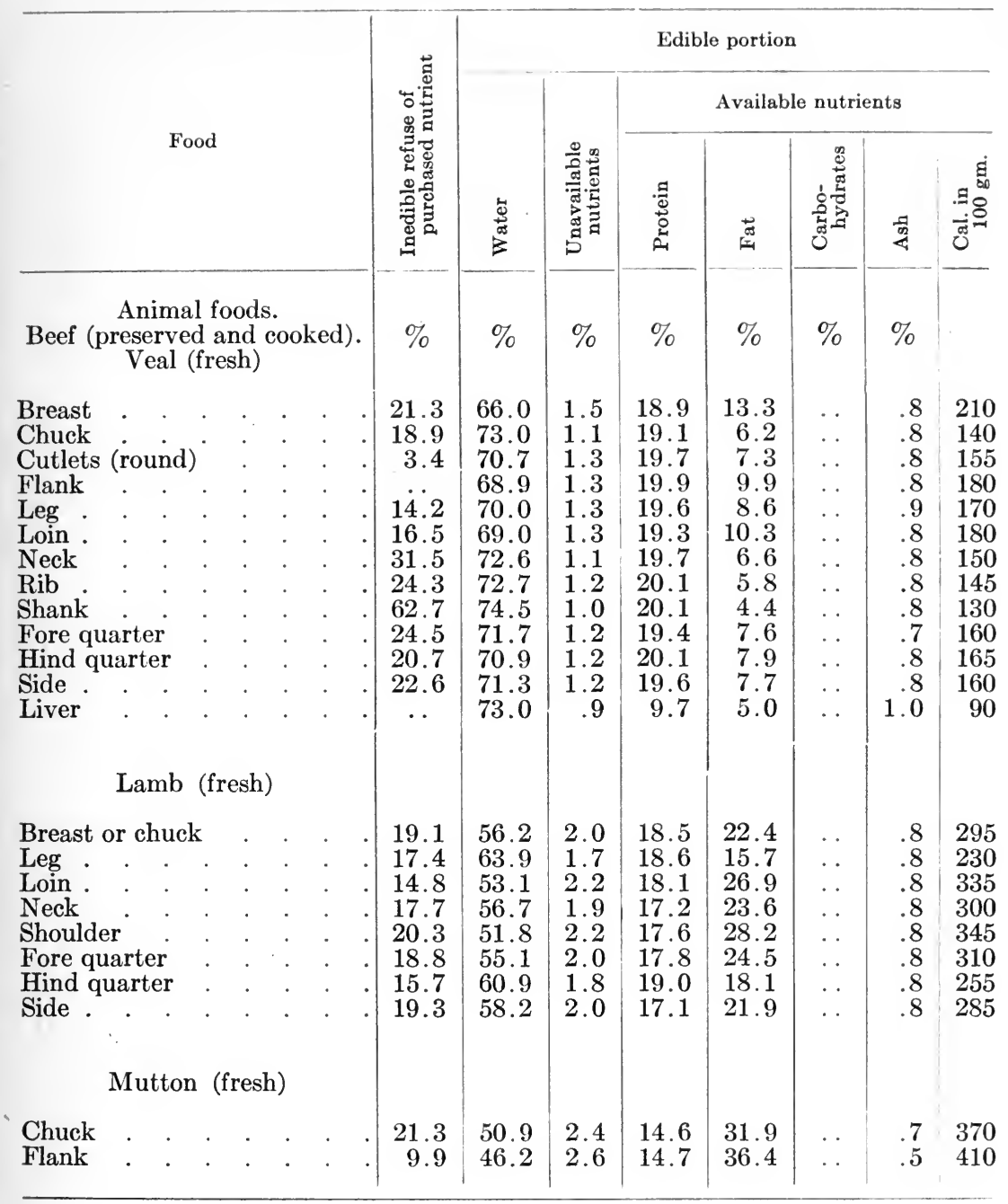




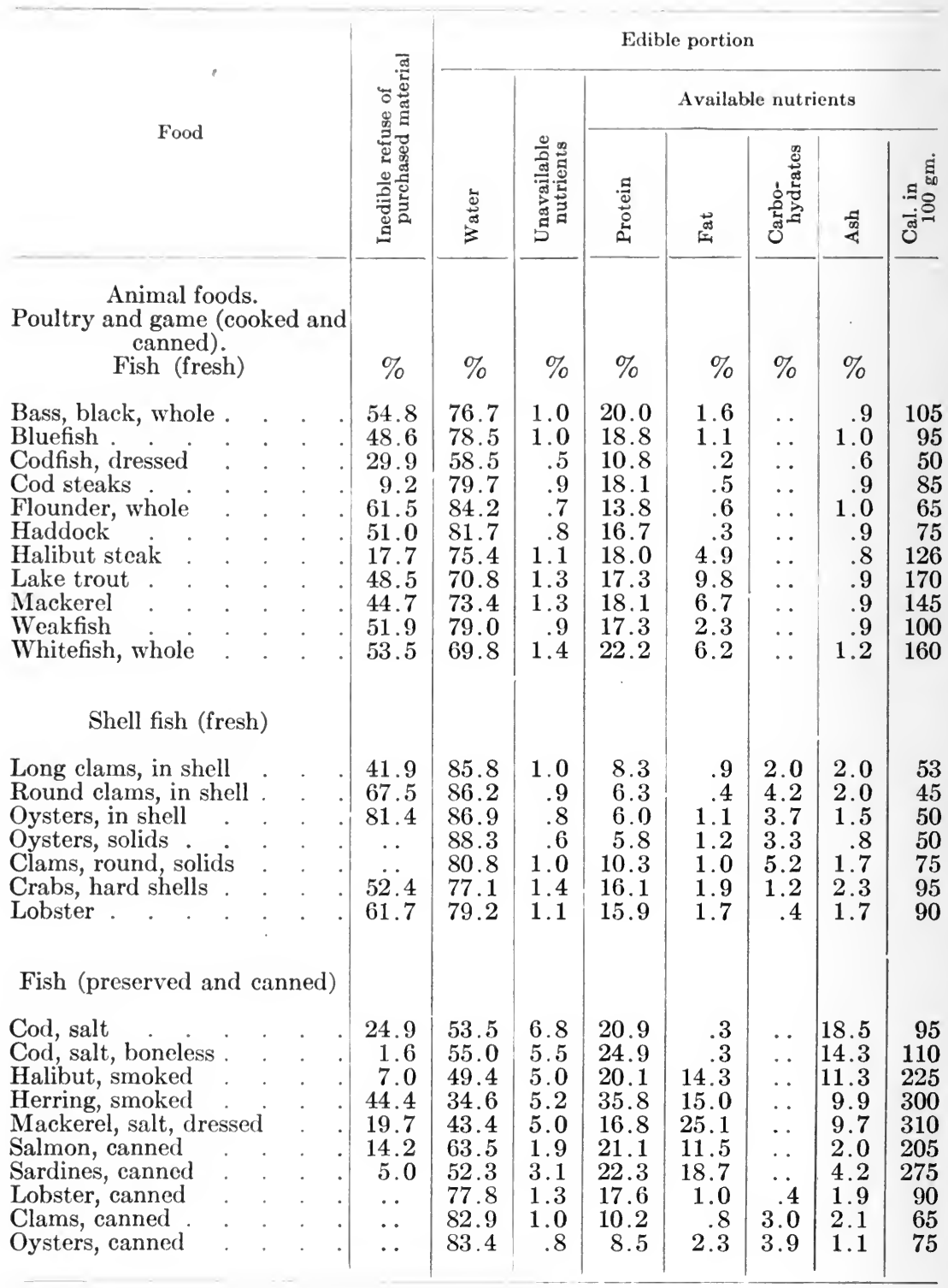




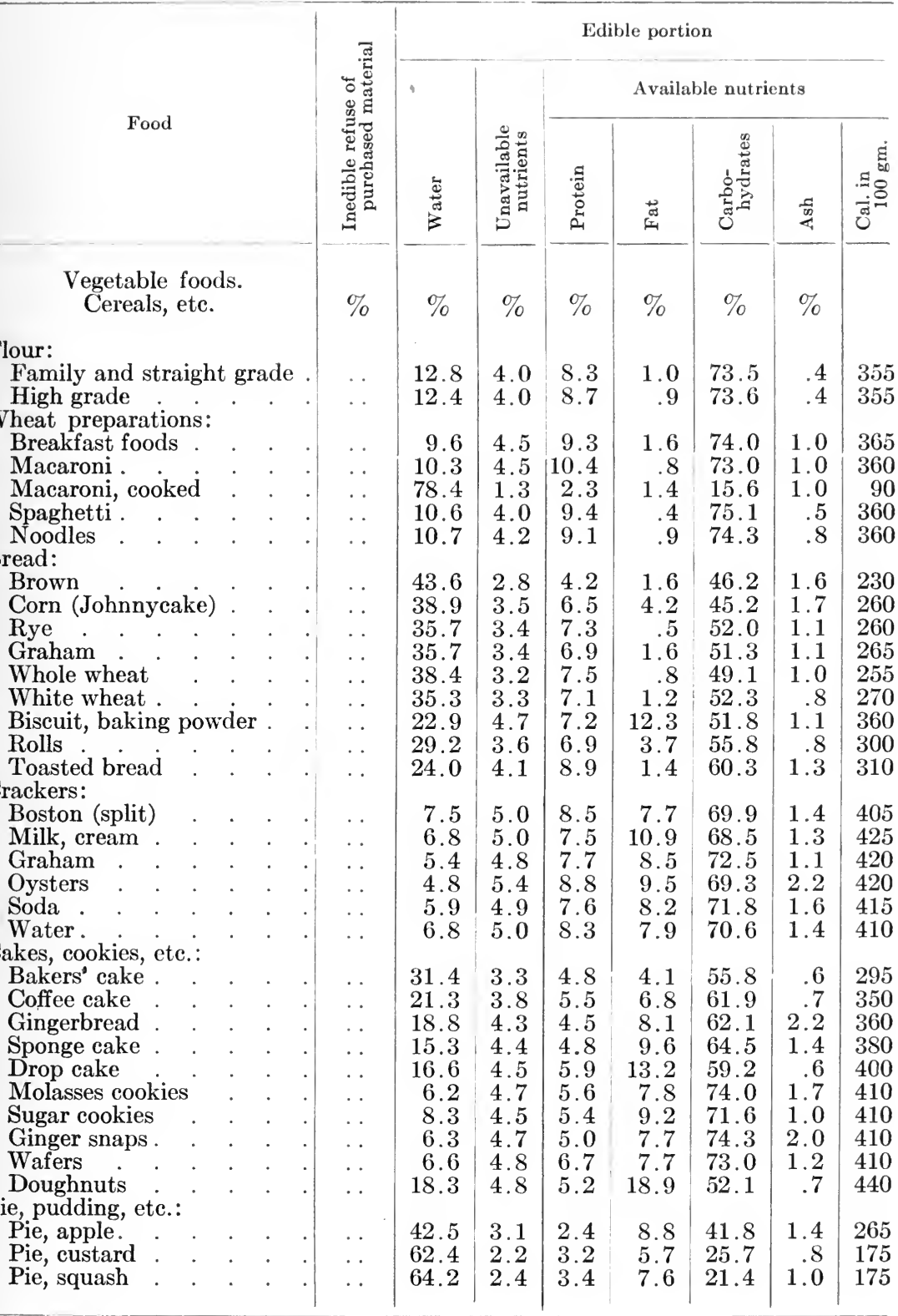




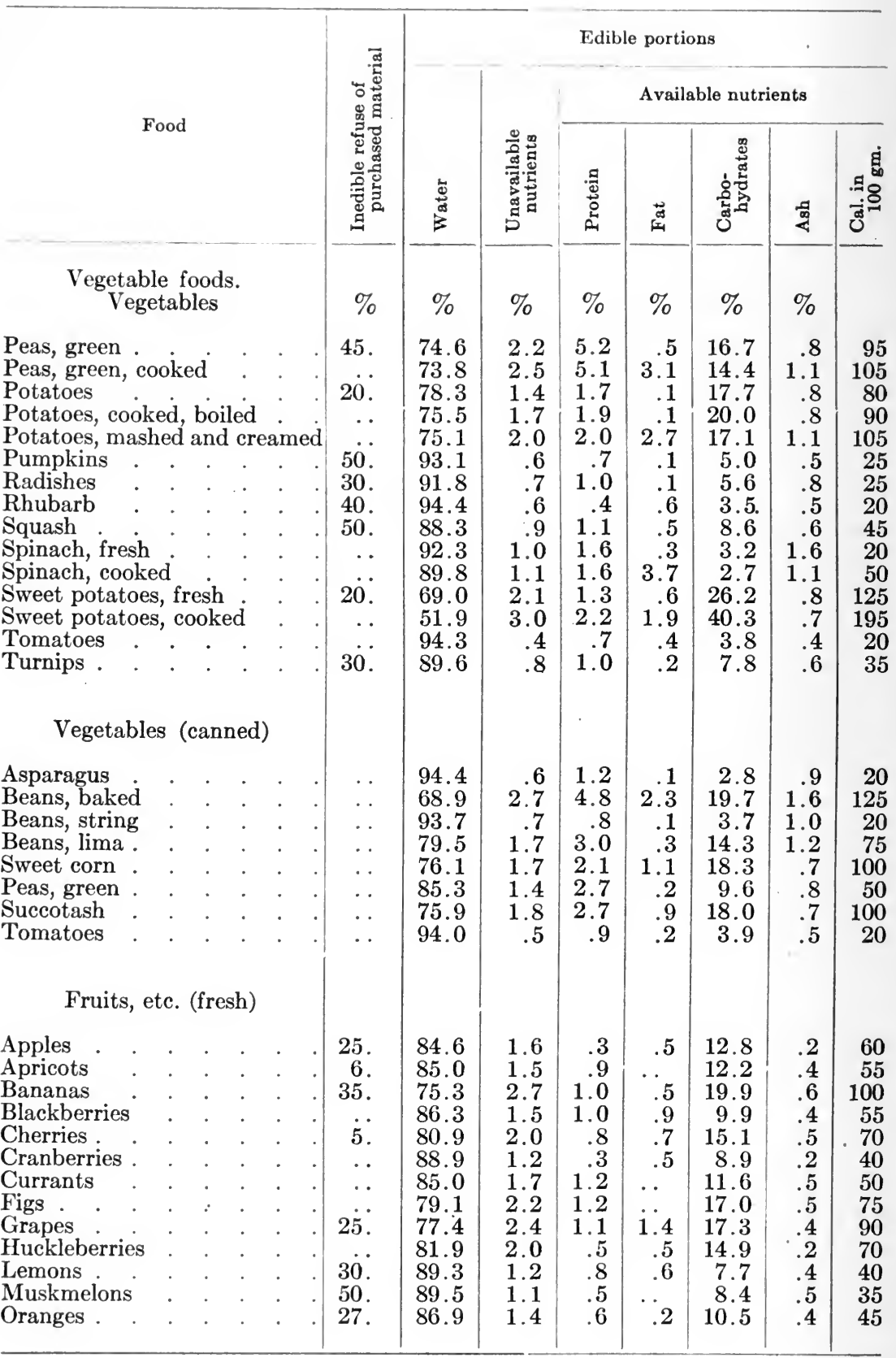




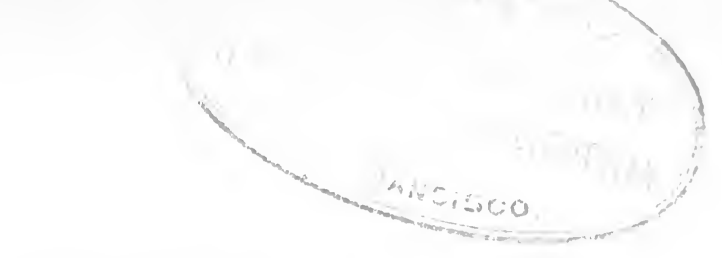

\section{H A P T E R I I}

\section{THE THEORY OF FERMEN'T ACTION}

Fermentations are to be regarded as accelerations of existing reactions. As accelerations of existing reactions they are classed with the reactions of catalysis. In every reacting system there is a driving force and a passive resistance to the reaction. Anything that will lower this passive resistance will accelerate the reaction velocity. The catalyzer is related only to the condition of passive or internal resistance. The positive catalyzer and increase in temperature both act by reduction in the internal resistance. The modus operandi of catalytic acceleration is in general defined as a succession of intermediary reactions - a definition that applies directly only to reactions in a homogeneous system. Fermentations are to be considered as limited and reversible reactions. There is no known essential difference between the action of an inorganic positive catalyzer and that of a ferment; there is no distinction in dynamics between the so-called formed and unformed ferments. Cells induce fermentations only through the agency of chemical substances elaborated by them. Fermentations tend to obey the laws of chemical kinetics; the experimental deviations are due to the multiplicity of adventitious variables and to difficulties in the definition of the units of measurement. The greater the control in the experiment, the closer the approximation to the theoretical law.

The experimental study of a fermentation may be formulated in specific terms as follows:

The primary reaction.

The relation of mass of substrate to reaction velocity.

The relation of mass of ferment to reaction velocity.

The relation of temperature to reaction velocity; temperature optimum.

The reversion of reaction by ferment action.

The relation of ferment to the products of reaction.

Auto-acceleration; zymo-excitors; zymo-depressors.

The inactivation of ferment.

Secondary reactions.

The nature of ferment; colloidal properties; relation of activity to history and method of preparation.

The control of the conditions - the purity of the reacting components and of the ferment, the temperature, the concentrations, and the absence of inhibition or inactivation of the ferment-will determine 
the reliability of the results. It is to be confessed that too often such control has not been attained. The more closely the conditions of the experiment approximate the conditions of an ideal chemical experiment, the more credible the results. 'To be entirely definite the results should be quantitative and capable of a mathematical interpretation. The problems of fermentations differ only in degree from the problems of catalytic reactions, directly in proportion to our inability to study all the aspects as above stated, to control the variables and to define the units of measurement. These conditions have most often been best attained in connection with the investigations of ferments derived from plants or lower orders of animal life.

The statement that fermentations are to be regarded as accelerations of existing reactions demands a certain restriction. The earlier chemists regarded catalytic reactions as reactions de novo; the present general currency of the opposite opinion is due largely to the influence of modern physical chemistry. We are now in possession of a large amount of experimental data confirmatory of this view. An apparent objection to the conception of fermentations as accelerated reactions is contained in the fact that a single substance will yield different products under the influence of different ferments. The fact is, however, capable of another interpretation, one harmonious to the theory, that the product represents a stage in the reaction, and that different ferments accelerate to different stages. Secondary and superimposed reactions are also to be considered. In special instances the addition of a positive catalyzer to a system in a state of chemical rest appears to inaugurate a reaction; and it is possible that this may be of not such infrequent occurrence in the domain of organic substances. For all the known fermentations occurring in nature, however, it is clear that fermentations represent accelerations of existing reactions. These auto-reactions progress as a rule at ordinary temperature with extreme slowness. In many instances, however, they have been studied and measured. This is true particularly for the aut-oxidations occurring in metals and the auto-hydrolyses noted in solutions of organic substances. We possess likewise accurate studies upon such reactions between gases. Indeed, studies upon the slow reaction between gaseous oxygen and hydrogen to form water at ordinary temperature were among the earliest investigations tending to indicate that catalyses are accelerated reactions and not reactions de novo.

\section{CHEMICAL REACTIONS OF FERMENTATIONS}

Fermentations are best studied and classified on the basis of the reactions involved. For every chemical phenomenon one may inquire: What is the reaction? And also: How and under what conditions does the reaction proceed? There are excellent illustrations of physicochemical studies of chemical processes of which the reactions are entirely 
unknown. The measurement of the relations between specific biological bodies and their antibodies is an illustration in point. For the enzymes, however, we possess actually more information upon the chemical nature of the reactions than upon the laws under which they proceed. The study of a fermentation ought to be carried out from the double point of view of organic and physical chemistry; the neglect of the latter by the organic chemist and the narrow formal treatment of the problem by the physical chemist have deterred the advance of knowledge.

The most simple reactions of catalysis are the intramolecular rearrangements. Many such instances are met with in organic substances. Aldehyd on standing passes into the paraldehyd. The transformation proceeds more rapidly at higher temperature, and is especially accelerated by the presence of acids. Thus for acetaldehyd:

$$
\mathrm{CH}_{3} \mathrm{CHO}+\mathrm{CH}_{3} \mathrm{CHO}+\mathrm{CH}_{3} \mathrm{CHO}<=>\mathrm{CH}_{3}-\mathrm{CH}\left\langle\begin{array}{l}
\mathrm{O}-\mathrm{CH}-\mathrm{CH}_{3} \\
\mathrm{O}-\mathrm{CH}-\mathrm{CH}_{3}
\end{array}\right.
$$

The polymerization into paraldehyd may be regarded as due to the conversion of the aldehyd

$$
\mathrm{CH}_{3} \mathrm{CH}=\mathrm{O} \text { into } \mathrm{CH}_{3} \mathrm{CH}=\mathrm{O}-
$$

following which three such groups join in a ring structure to form a saturated substance. The modus of action of the hydrogen ions in the acceleration of the polymerization is not known. The reaction is reversible, and tends to an equilibrium.

A very illustrative reaction of this type is the reversible formation of dianthracin from anthracin. This is a photo-chemical reaction, and tends to an equilibrium. The reaction of light is reversed in the dark, and thus the reaction is to be written

$$
\begin{array}{r}
\text { light } \\
2 \mathrm{C}_{34} \mathrm{H}_{10} \\
\text { darkness }
\end{array} \underset{\mathrm{C}_{28} \mathrm{H}_{20}}{\text { darks }}
$$

In all probability such photo-chemical reactions are numerous. It is possible that the formation of ozone from atmospheric oxygen is such a process.

Acetone slowly undergoes polymerization into di-acetone alcohol. The transformation is much accelerated by alkalies.

$$
\begin{aligned}
2 \mathrm{CH}_{3} \mathrm{CO} \cdot \mathrm{CH}_{3} & <=>\underset{\left(\mathrm{CH}_{3} \mathrm{CO} \cdot \mathrm{CH}_{3}\right)_{2}}{\mathrm{OH}} \\
\mathrm{CH}_{3} \mathrm{CO} \cdot \mathrm{CH}_{3}+\mathrm{CH}_{3} \mathrm{CO} \cdot \mathrm{CH}_{3} & <=>\left(\mathrm{CH}_{3}\right)_{2} \mathrm{C}-\mathrm{CH}_{2} \mathrm{CO} \cdot \mathrm{CH}_{3}
\end{aligned}
$$

The reaction is reversible. 
An excellent illustration for the aromatic series is afforded by the transformation of diazo-amino-benzol into p-amino-azo-benzol. Acids accelerate.

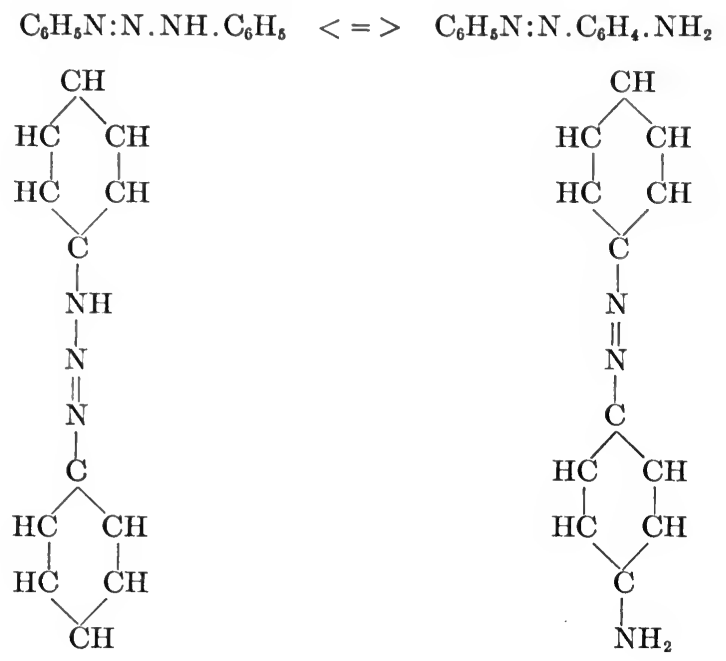

An exceedingly pretty illustration of a catalytic intramolecular re-arrangement is seen in the isomeric ketons $\mathrm{C}_{5} \mathrm{Cl}_{6} \mathrm{O}$.<smiles>C=C1C(Cl)C(Cl)C(Cl)C1Cl</smiles>

The reaction is reversible, and no matter from which keton one proceeds an equilibrium will be established in the system. Though the sole difference lies in the positions of the double chlorins, the two isomers present melting points as different as $31^{\circ}$ and $92^{\circ}$.

Natural fermentations of this type are not yet known.

In the next group of catalyses one substance is broken into two or more molecules, or for the reversed reaction two or more molecules combine to form one substance. Some of these reactions are of the simplest of association and dissociation. Such are:

$$
\begin{aligned}
& \text { Hydrogen }+ \text { oxygen }<=>\text { water } \\
& 2 \mathrm{H}_{2}+\mathrm{O}_{2}<=>2 \mathrm{H}_{2} \mathrm{O} \\
& \mathrm{Hydrogen}+\text { iodin }<=>\text { hydriodic acid } \\
& \mathrm{H}_{2}+\mathrm{I}_{2}<=>2 \mathrm{HI} \\
& \text { Sulphur dioxid }+ \text { oxygen }<=>\text { sulphur trioxid } \\
& 2 \mathrm{SO}_{2}+\mathrm{O}_{2}<=>2 \mathrm{SO}_{3}
\end{aligned}
$$


These are all greatly influenced by changes in temperature, are typically reversible, and are markedly accelerated by the members of the platinum group. Another good illustration is the following:

Dibrom-succinic acid $<=>$ bromo-malic acid + hydrobromic acid $\mathrm{COOH} . \mathrm{CHBr} . \mathrm{CHBr} . \mathrm{COOH}<=>\mathrm{COOH} . \mathrm{CBr} . \mathrm{CH} . \mathrm{COOH} .+\mathrm{HBr}$

Many fermentations are of this order.

$$
\begin{aligned}
& \text { d-glucose }<=>\text { ethyl alcohol }+ \text { carbon dioxid } \\
& \mathrm{CH}_{2} \mathrm{OH} .(\mathrm{CHOH})_{4} \mathrm{COH}<=>2 \mathrm{CH}_{3} \mathrm{CH}_{2}(\mathrm{OH})+2 \mathrm{CO}_{2} \\
& \text { Malic acid }<=>\text { lactic acid }+ \text { carbon dioxid } \\
& \mathrm{COOH} . \mathrm{CH}_{2} \mathrm{CH}(\mathrm{OH}) \cdot \mathrm{COOH}<=>\mathrm{CH}_{3} \cdot \mathrm{CH}(\mathrm{OH}) \cdot \mathrm{COOH}+\mathrm{CO}_{2}
\end{aligned}
$$

The acceleration of the reaction in the case of d-glucose may be accomplished by alkali or platinum, furnishing a good illustration of the identical natures of catalytic and fermentative acceleration. Glucose is subject to other fermentations.

$$
\begin{aligned}
& \text { glucose }<=>\text { two molecules of lactic acid } \\
& \mathrm{CH}_{2} \mathrm{OH} .(\mathrm{CHOH})_{4} \mathrm{COH}<=>2 \mathrm{CH}_{3} \cdot \mathrm{CH}(\mathrm{OH}) . \mathrm{COOH}
\end{aligned}
$$

This reaction is an intermediary stage in the fermentation of glucose to ethyl alcohol.

$$
\begin{aligned}
& \text { d-glucose }<=>\text { butyric acid }+2 \text { carbon dioxid }+ \text { hydrogen } \\
& \mathrm{CH}_{2} \mathrm{OH} .(\mathrm{CHOH})_{4} \mathrm{COH}<=>\mathrm{CH}_{3} \mathrm{CH}_{2} \mathrm{CH}_{2} \mathrm{COOH}+2 \mathrm{CO}_{2}+\mathrm{H}_{2}
\end{aligned}
$$

The fermentation of sinigrin by myrosin is possibly the most complicated of the known reactions of this group.

$$
\begin{aligned}
& \text { Sinigrin }<=>\text { allyl-sulphocyanid }+ \text { d-glucose }+ \text { acid pot. sulphate } \\
& \mathrm{C}_{10} \mathrm{H}_{12} \mathrm{NKS}_{2} \mathrm{O}_{10}<=>\mathrm{C}_{3} \mathrm{H}_{5} \mathrm{SCN}+\mathrm{C}_{6} \mathrm{H}_{12} \mathrm{O}_{6}+\mathrm{KHSO}_{4}
\end{aligned}
$$

Especially noteworthy is the formation of an inorganic electrolyte as one of the products.

Most of the ordinary fermentations involve four or more component molecules. The substrate combines with another molecule, and then divides to form two or more molecules. That our point of view begins with the body to be fermented is simply a result of historical development and of common experience with the phenomenon. Dynamically the two processes in the equation are of the same dignity. We use the term substrate to indicate the substance that in the ordinary sense of the term is the main component in the reaction-the substance to be fermented. The term product is applied to the substances that result from the reaction. Dynamically, what would be the products of the reaction in the one direction are the primary substances with the reaction in the other direction. Similarly, the second body in the 
ordinarily primary reaction, the substance that is added to the substrate, is not in common usage accorded the same dignity given to the substrate; but dynamically it is upon the same plane. For example: Ester + water $=$ alcohol + fatty acid. The latter are the products. But if we mix the alcohol and fatty acid, ester will be formed, and under such circumstances the ester is the product. Now, since both reactions are always taking place side by side in the system, the use of the terms substrate and products is simply a matter of convenience, based upon the fact that experiments at direct fermentation are common, while experiments as reversion are rare. The water, of course, is as essential a component in the reaction as the ester, and in the reversed reaction the water is as truly a product. Of these fermentations there are three large groups: Hydrolyses, oxidations, and reductions.

Hydrolytic Cleavage. - In these reactions water is added, and the product of the union is divided into two or more molecules of one substance or into two or more substances. In the reversions of these cleavage reactions, two or more molecules combine to form a larger molecule, with the extrusion of water. Hydrolytic cleavage seems to be universal in the world of organic substances; whenever these substances are dissolved in water, slow hydrolytic cleavages are inaugurated. These auto-hydrolyses have been demonstrated in a large number of instances. The thermodynamic demonstration of the nature of the phenomenon is contained in the fact that steam is a universal hydrolysing agent for these substances, and since the reaction occurs rapidly at high temperature it must occur to some extent at low temperatures. The agent in the auto-hydrolysis may be confidently assumed to be the hydrogen or hydroxyl ion of the dissociated water. A further proof of the occurrence of these auto-hydrolyses is contained in the fact that hydrogen ions are general accelerators of these reactions with these substances. In a certain number of these reactions, reversions have been demonstrated, both by catalytic and enzymic agents; and the occurrence of such reversions under appropriate conditions is postulated for all.

Of these hydrolyses many illustrations may be given. Thus:

$$
\begin{aligned}
& \text { Cellulose + water }<=>\text { hexose }+ \text { hexose } \\
& \mathrm{n}\left(\mathrm{C}_{6} \mathrm{H}_{10} \mathrm{O}_{5}\right)_{\mathbf{n}}+\mathrm{n} \mathrm{H}_{2} \mathrm{O}<=>\mathrm{n}_{6} \mathrm{H}_{12} \mathrm{C}_{6}+\mathrm{n} \mathrm{C}_{6} \mathrm{H}_{12} \mathrm{O}_{6}
\end{aligned}
$$

The hexose is d-glucose. The common bacterial fermentation of cellulose yields no sugar among the end products, but only gases and acetic and butyric acid, carbon dioxid being evolved in all cases but otherwise either hydrogen or methane. For starch and inulin similar relations hold.

$$
\mathrm{n}\left(\mathrm{C}_{6} \mathrm{H}_{10} \mathrm{O}_{5}\right)_{n}+\mathrm{n} \mathrm{H}_{2} \mathrm{O}<=>\mathrm{n} \mathrm{C}_{6} \mathrm{H}_{12} \mathrm{O}_{6}
$$

For starch the product is d-glucose when acids are employed as the catalyzer, maltose when diastatic ferment is employed; from inulin 
only levulose is secured. In the acid hydrolysis of starch the process passes through the stage of maltose and ends with the formation of the hexose; in the diastatic fermentation the process stops at the stage of the disaccharid. In all these hydrolyses of polysaccharids the reactions pass through many substages. The same reaction is noted for glycogen.

The disaccharids undergo similar cleavages. These follow the general type:

$$
\begin{aligned}
& \text { Disaccharid + water }<=>\text { hexose }+ \text { hexose } \\
& \mathrm{C}_{12} \mathrm{H}_{22} \mathrm{O}_{11}+\mathrm{H}_{2} \mathrm{O}<=>\mathrm{C}_{6} \mathrm{H}_{12} \mathrm{O}_{6}+\mathrm{C}_{\ell} \mathrm{H}_{12} \mathrm{O}_{6}
\end{aligned}
$$

In the case of saccharose the products are d-glucose and d-levulose; maltose yields d-glucose; milk sugar yields d-galactose and d-glucose. These reactions appear to occur directly, in the ordinary sense of the term.

Closely related to the splitting of the disaccharids are the hydrolytic cleavages of the glucosids. Glucosids are ether-like combinations of a hexose with an aromatic substance, as an alcohol or an aldehyd, instead of with another hexose as in the case of a disaccharid.

$$
\begin{aligned}
& \text { Helicin }+ \text { water }<=>\text { salicylic aldehyd }+ \text { d-glucose } \\
& \mathrm{C}_{13} \mathrm{H}_{16} \mathrm{O}_{7}+\mathrm{H}_{2} \mathrm{O}<=>\mathrm{OH} \cdot \mathrm{C}_{6} \mathrm{H}_{4} \cdot \mathrm{CHO}+\mathrm{C}_{6} \mathrm{H}_{12} \mathrm{O}_{6}
\end{aligned}
$$

There is a wide range of variety in the second components of these compounds. Thus arbutin yields hydroquinon; phloridzin, phloretin; tannin, gallic acid; gaultherin, methylsalicylic acid; while amygdalin yields, in addition to d-glucose, hydrocyanic acid and benzoic aldehyd.

For many of these hydrolyses of poly- and disaccharids, autohydrolysis has been demonstrated directly. To all of these reactions hydrogen ions act as positive catalyzers. For some of them the colloidal metals of the platinum group have been shown to act as accelerators. Ferments of the cytase type accelerate the hydrolysis of cellulose; ferments of the diastase type act positively for the group of polysaccharids; enzymes of the type of invertase accelerate the cleavage of the disaccharids and glucosids. It is a noteworthy fact that fermentation of polysaccharid, disaccharid, and glucosid is an act of hydrolysis, that of primary sugars is an intramolecular cleavage. There are possibly exceptions to this; thus there is presumed to be a direct fermentation of lactose into lactic acid. But, as a rule, the fermentation of the higher sugars is a hydrolytic cleavage, while the fermentation of the primary sugars is a direct intramolecular cleavage. Hydroxyl ions are prominent as catalyzers of the reactions of the primary sugars, hydrogen ions conspicuous as catalyzer for the hydrolysis of the poly- and disaccharids.

The cleavages of protein are all hydrolyses. The general reaction runs:

$$
\text { Protein }+ \text { water }<=>\text { amino-acids }+ \text { amino-acids }
$$


There are many substages in the process. The end products comprise a large number of differént amino-acids. These hydrolyses are also accelerated by hydrogen ions, and to some extent by colloidal platinum. The auto-hydrolysis has been experimentally demonstrated for several members of the protein group. Cleavage with steam was indeed one of the oldest methods employed for obtaining products of protein hydrolysis.

The fermentation of fat is likewise an instance of hydrolytic cleavage. All esters, both the synthetic esters and the natural fats, are hydrolyzed according to the general equations:

$$
\begin{gathered}
\text { Ethyl acetate }+ \text { water }<=>\text { ethyl alcohol }+ \text { acetic acid } \\
\mathrm{CH}_{3} \mathrm{CO} \cdot \mathrm{O} \cdot \mathrm{CH}_{3} \cdot \mathrm{CH}_{2}+\mathrm{H}_{2} \mathrm{O}<=>\mathrm{CH}_{3} \cdot \mathrm{CH}_{2} \cdot \mathrm{OH}+\mathrm{CH}_{3} \mathrm{COOH} \\
\text { Olein triglycerid }+ \text { water }<=>\text { oleic acid }+ \text { glycerol } \\
\mathrm{C}_{3} \mathrm{H}_{5}\left(\mathrm{C}_{18} \mathrm{H}_{33} \mathrm{O}_{2}\right)_{3}+3 \mathrm{H}_{2} \mathrm{O}<=>3 \mathrm{C}_{18} \mathrm{H}_{34} \mathrm{O}_{2}+\mathrm{CH}_{2}(\mathrm{OH}) \cdot \mathrm{CH}(\mathrm{OH}) \cdot \mathrm{CH}_{2}(\mathrm{OH})
\end{gathered}
$$

These reactions are typically and measurably reversible. The simple reactions are greatly accelerated by hydrogen ions, and to some extent by the colloidal metals of the platinum group. In many respects esters present the best opportunities for the study of enzyme action.

It is thus seen that the fermentation of the members of the three great groups of foods-protein, carbohydrate, and fat-are instances of the enzymic acceleration of hydrolytic cleavages. These hydrolyses are, under the conditions in which they occur in nature as well as under the circumstances of experiments, monomolecular reactions, at least in so far as the reaction in the direction of the right (the hydrolysis of the substrate) is concerned. The water of solution is so much greater than the water of combination that the mass of the water in the system is for practical purposes constant during the duration of the reaction.

Other interesting instances of fermentative hydrolyses may be described.

$$
\begin{gathered}
\text { Hippuric acid }+ \text { water }<=>\text { glycocoll }+ \text { benzoic acid } \\
\mathrm{C}_{6} \mathrm{H}_{5} \cdot \mathrm{CO} \cdot \mathrm{NH} \cdot \mathrm{CH}_{2} \cdot \mathrm{COOH}+\mathrm{H}_{2} \mathrm{O}<=>\mathrm{CH}_{2} \mathrm{NH}_{2} \cdot \mathrm{COOH}+\mathrm{C}_{6} \mathrm{H}_{5} \cdot \mathrm{COOH} \\
\text { Urea }+ \text { water }<=>\text { ammonia }+ \text { carbon dioxid } \\
\mathrm{CO}\left(\mathrm{NH}_{2}\right)_{2}+\mathrm{H}_{2} \mathrm{O}<=>2 \mathrm{NH}_{3}+\mathrm{CO}_{2} \\
\text { Arginin + water }<=>\text { urea }+ \text { ornithin } \\
\mathrm{NH}_{2} \mathrm{C} \cdot(\mathrm{NH})_{2} \cdot\left(\mathrm{CH}_{2}\right)_{3} \cdot \mathrm{CH} \cdot \mathrm{NH}_{2} \cdot \mathrm{COOH}+\mathrm{H}_{2} \mathrm{O}<=>\mathrm{CO}\left(\mathrm{NH}_{2}\right)_{2}+ \\
\mathrm{CH}_{2}\left(\mathrm{NH}_{2}\right) \cdot\left(\mathrm{CH}_{2}\right)_{2} \mathrm{CH}\left(\mathrm{NH}_{2}\right) \cdot \mathrm{COOH}
\end{gathered}
$$

The last two fermentations are accomplished by ferments contained in the liver. The cleavages may be accomplished as readily by the action of acids as by ferments. Interesting are the fermentations of the salts of vegetable acids. An illustration is afforded by calcium formate. 
Calcium formate + water $<=>$ calcium carbonate + carbon dioxid + hydrogen

$$
\begin{aligned}
& \mathrm{HCOO}
\end{aligned}>\mathrm{Ca}+\mathrm{H}_{2} \mathrm{O}=\mathrm{CaCO}_{3}+\mathrm{CO}_{2}+2 \mathrm{H}_{2}
$$

The hydrogen acts as an anticatalyzer.

Catalytic accelerations of hydrolyses are exceedingly common, not only in natural substances, but also in synthetic substances.

Thus:

Mon-chlor-acetic acid + water $<=>$ glycollic acid + hydrochloric acid $\mathrm{CH}_{2} \cdot \mathrm{Cl} \cdot \mathrm{COOH}+\mathrm{H}_{2} \mathrm{O}<=>\mathrm{CH}_{2} \cdot \mathrm{OH} \cdot \mathrm{COOH} .+\mathrm{HCl}$

Oxidation Fermentations.- Under oxidation fermentations we understand such accelerations in oxidation as occur under the influence of the presence of an enzyme. The steps in these oxidations are not well understood. It is not even known that oxygen is always added in these reactions, since oxidation can be effected by the withdrawal of hydrogen as well as by the addition of oxygen. Biologists have been inclined to group the fermentative oxidations under two headings, direct and indirect, according to whether hydrogen peroxid acted as a carrier or not. The data are not sufficient to justify such a distinction. We ought not to dogmatize upon the nature of these reactions, since we are well acquainted with so few. When the knowledge of the aut-oxidation of inorganic substances now being accumulated is applied to the study of these fermentations, we may expect light to break upon the subject. The best known instances divide themselves in two groups: Those in which a substance combines with oxygen to form a single product, and those in which water is split off. Obviously the reversion of the latter would constitute hydrolyses.

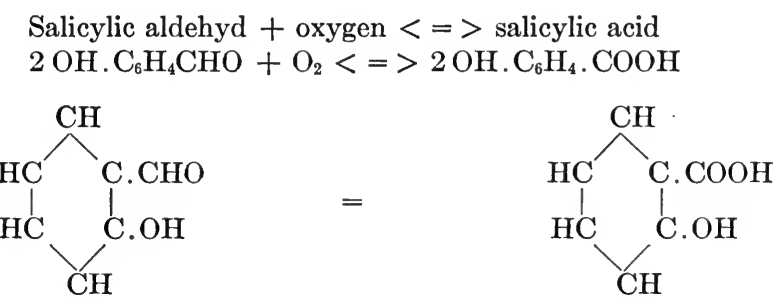

A soluble enzyme of mammalian tissues accelerates this reaction.

Hydroquinon + oxygen $<=>$ quinon + water

$2 \mathrm{OH} . \mathrm{C}_{6} \mathrm{H}_{4} \cdot \mathrm{OH}+\mathrm{O}_{2}<=>2 \mathrm{CO} \cdot \mathrm{C}_{4} \mathrm{H}_{4} \cdot \mathrm{CO}+2 \mathrm{H}_{2} \mathrm{O}$<smiles>O=C(O)C1CCCCC1</smiles><smiles>O=CC=CC=O</smiles> 
This fermentation, which is induced by the juice of the Japanese lac tree (laccase), and also by mammalian intestinal secretions, comprises, according to the modern conception of these substances, the transformation of a true aromatic substance into an alicyclic substance.

A good illustration is furnished by the acetic acid fermentation of ethyl alcohol, occurring in two stages.

$$
\begin{aligned}
& \text { Ethyl alcohol }+ \text { oxygen }<=>\text { acetaldehyd }+ \text { water } \\
& 2 \mathrm{CH}_{3} \mathrm{CH}_{2} \cdot \mathrm{OH}+\mathrm{O}_{2}<=>2 \mathrm{CH}_{3} \cdot \mathrm{COH}+2 \mathrm{H}_{2} \mathrm{O} \\
& \text { Acetaldehyd }+ \text { oxygen }<=>\text { acetic acid } \\
& 2 \mathrm{CH}_{3} \cdot \mathrm{COH}+\mathrm{O}_{2}<=>2 \mathrm{CH}_{3} \cdot \mathrm{COOH}
\end{aligned}
$$

Oxidation ferments are very active in the purin catabolism. One illustration will suffice. Xanthinoxydase converts hypoxanthin first into xanthin, then into uric acid.

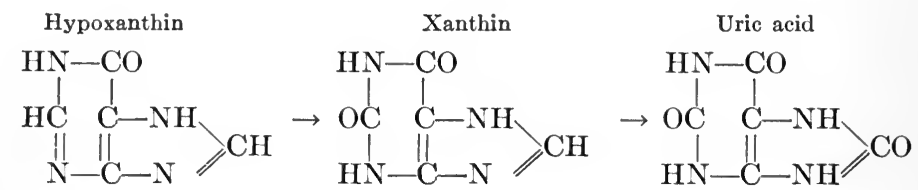

Two interesting fermentations are brought about by the Bac. xylinum.

$$
\begin{gathered}
\text { Sorbite + oxygen }<=>\text { sorbose + water } \\
2 \mathrm{C}_{6} \mathrm{H}_{14} \mathrm{O}_{6}+\mathrm{O}_{2}<=>2 \mathrm{C}_{6} \mathrm{H}_{12} \mathrm{O}_{6}+2 \mathrm{H}_{2} \mathrm{O} \\
\text { Glycerol }+ \text { oxygen }<=>\text { di-oxy-acetone + water } \\
2 \mathrm{CH}_{2}(\mathrm{OH}) \cdot \mathrm{CH}(\mathrm{OH}) \cdot \mathrm{CH}_{2}(\mathrm{OH})+\mathrm{O}_{2}<=>2 \mathrm{CH}_{2}(\mathrm{OH}) \cdot \mathrm{CO} \cdot \mathrm{CH}_{2}(\mathrm{OH})+2 \mathrm{H}_{2} \mathrm{O}
\end{gathered}
$$

These reactions have not been reversed. For all of them inorganic accelerators are known.

While inorganic accelerators of the oxidation of the inorganic salts of metals are numerous, fermentative acceleration of such oxidations are known certainly to exist only for the nitrites. The reaction:

$$
\text { Metal }-\mathrm{NO}_{2}+\mathrm{O}<=>\text { Metal }-\mathrm{NO}_{3}
$$

is a reversible reaction that is going on constantly in soils and waters under the influence of bacteria, certain microörganisms being especially active in the one or the other direction. Whether in the reduction hydrogen is added and then water extruded is not known; indeed, the mechanisms of the reactions have not been determined on account of the complexity of the conditions under which the phenomena occur. The oxidation of nitrite to nitrate has long been known to agriculture; the reduction of nitrate to nitrite has been learned more recently. Analogous fermentations have been described for the salts of sulphur.

Closely related to simple oxidations are the reactions with hydrogen peroxid. Hydrogen peroxid tends to a slow reduction, and the 
oxidations of substances by hydrogen peroxid are in general to be regarded as accelerations of this auto-reduction.

Hydriodic acid + hydrogen peroxid $<=>$ iodin + water

$2 \mathrm{HI}+\mathrm{H}_{2} \mathrm{O}_{2}<=>\mathrm{I}_{2}+2 \mathrm{H}_{2} \mathrm{O}$

Sulphurous acid + hydrogen peroxid $=$ sulphuric acid + "water

$\mathrm{H}_{2} \mathrm{SO}_{3}+\mathrm{H}_{2} \mathrm{O}_{2}<=>\mathrm{H}_{2} \mathrm{SO}_{4}+\mathrm{H}_{2} \mathrm{O}$

The oxidation of formic aldehyd occurs in two stages.

Formaldehyd + hydrogen peroxid $<=>$ formic acid + water

H. $\mathrm{COH}+\mathrm{H}_{2} \mathrm{O}_{2}<=>\mathrm{H} \cdot \mathrm{COOH}+\mathrm{H}_{2} \mathrm{O}$

Formic acid + hydrogen peroxid $<=>$ carbon dioxid + water

$\mathrm{H}$. $\mathrm{COOH}+\mathrm{H}_{2} \mathrm{O}_{2}<=>\mathrm{CO}_{2}+2 \mathrm{H}_{2} \mathrm{O}$

There is evidence tending to indicate that the reversion of these reactions represents the initial steps whereby carbohydrates are formed by plants, though the reactions are usually written with oxygen alone:

$$
\mathrm{CO}_{2}+\mathrm{H}_{2} \mathrm{O}=\mathrm{H} \cdot \mathrm{COOH}+\mathrm{O} \text { and } \mathrm{H} \cdot \mathrm{COOH}=\mathrm{H} \cdot \mathrm{COH}+\mathrm{O}
$$

The theory assumes the presence in the chlorophyl-containing cell of some enzyme accelerating the reductions, subject to the influence of light. Since carbon dioxid and water are universally present in the atmosphere, one has but to assume the removal or combination of the formaldehyd ( $i$. e., its condensation into sugar) in order to possess a firm physico-chemical basis for the continued reaction of reduction. The influence of light may possibly be interpreted to lie in a translocation of the station of equilibrium in the direction of the reaction of reduction. Investigations indicate that in the reduction of carbon dioxid to formic acid carbon monoxid may be formed, and that both ozone and hydrogen peroxid appear.

$$
\begin{aligned}
& 2 \mathrm{CO}_{2}<=>2 \mathrm{CO}+\mathrm{O}_{2} \\
& \mathrm{CO}+\mathrm{H}_{2} \mathrm{O}<=>\mathrm{H} . \mathrm{COOH} \\
& 3 \mathrm{O}_{2}<=>2 \mathrm{O}_{3} \\
& \mathrm{O}_{3}+\mathrm{H}_{2} \mathrm{O}<=>\mathrm{H}_{2} \mathrm{O}_{2}+\mathrm{O}_{2} \\
& \mathrm{CO}_{2}+\mathrm{H}_{2} \mathrm{O}_{2}<=>\mathrm{H} . \mathrm{CHO}+\mathrm{O}_{3}
\end{aligned}
$$

Formaldehyd would be thus derived in one or both of two ways. From formaldehyd sugar would be formed by condensation. The steps in the earlier stages of the condensation of formaldehyd are not known; it is only certain that amino-acids are not concerned. This general conception has been given a certain experimental foundation by investigations on the effects of the silent electrical discharge in a system containing carbon dioxid and water; reduction products were obtained that conform quite closely to the above scheme. 'Though a goodly amount of experimental work lies at the basis of these theories, 
it is apparent that the analytical demonstrations of traces of hydrogen peroxid and formaldehyd, appearing as transient stages in a reaction, must be a hazardous test. Nevertheless, it has been experimentally shown that chlorophyl in the presence of sunlight builds formaldehyd from carbon dioxid and water. A further biological difficulty lies in the great toxicity of formaldehyd, though this is obviated by the assumption of immediate combination or elaboration into higher carbohydrates.

The reactions of hydrogen peroxid are susceptible of acceleration by a large number of inorganic substances, especially by ferrous salts, colloidal metals of the platinum group, molybdic and tungstic acids. The acceleration of the reactions of formaldehyd and formic acid may be accomplished with plant extracts.

Fermentative Reductions.-Fermentative reductions, the acceleration of reactions of reduction, have not been long known. Indeed, the existence of such fermentations was once denied. Curiously enough, our present knowledge includes few instances of the fermentative acceleration of reductions of organic substances, most of the recognized reactions concerning metallic salts. The reduction of the nitrate to the nitrite mentioned in a previous paragraph is probably the most widely occurring reaction of the type known. This fermentation occurs also in mammalian juices. Very interesting are the reductions of the acids and salts of selenium and tellurium by certain bacteria. These reductions follow the regular types:

Selenous acid + sulphurous acid $<=>$ sulphuric acid + selenium + water $\mathrm{H}_{2} \mathrm{SeO}_{3}+2 \mathrm{H}_{2} \mathrm{SO}_{3}<=>2 \mathrm{H}_{2} \mathrm{SO}_{4}+\mathrm{Se}+\mathrm{H}_{2} \mathrm{O}$

Tellurous acid + sulphurous acid $<=>$ sulphuric acid + tellurium + water $\mathrm{H}_{2} \mathrm{TeO}_{3}+2 \mathrm{H}_{2} \mathrm{SO}_{3}<=>2 \mathrm{H}_{2} \mathrm{SO}_{4}+\mathrm{Te}+\mathrm{H}_{2} \mathrm{O}$

We do not know what the reducing body actually is that reacts with the selenous and tellurous acids (replacing the sulphurous acid in the written reactions) in these bacterial experiments, but the acceleration is very pronounced, and is observed with the -ous and -ic acids and their salts alike. In some instances the reduction may only be to a lower oxide. Similar reactions occur with arsenic, both in the arsenous and arsenic states.

Arsenic trioxid + hydrogen $<=>$ arsenuretted hydrogen + oxygen $2 \mathrm{AsO}_{3}+3 \mathrm{H}_{2}<=>2 \mathrm{AsH}_{3}+3 \mathrm{O}_{2}$

Arsenic trioxid + potassium acetate $<=>$ cacodylic oxid + pot. carbonate + carbon dioxid

$\mathrm{As}_{2} \mathrm{O}_{3}+4 \mathrm{KC}_{2} \mathrm{H}_{3} \mathrm{O}_{2}<=>\left(\mathrm{As}\left(\mathrm{CH}_{3}\right)_{2}\right)_{2} \mathrm{O}+2 \mathrm{~K}_{2} \mathrm{CO}_{3}+2 \mathrm{CO}_{2}$

These accelerations are produced in the culture media of certain bacteria; the steps and details in the reactions are not worked out. Of the fermentative reductions of organic substances we will cite two: 


$$
\begin{aligned}
& \text { Nitrobenzol }+ \text { hydrogen }<=>\text { anilin }+ \text { water } \\
& \mathrm{C}_{6} \mathrm{H}_{5} . \mathrm{NO}_{2}+3 \mathrm{H}_{2}<=>\mathrm{C}_{6} \mathrm{H}_{5} \mathrm{NH}_{2}+2 \mathrm{H}_{2} \mathrm{O}
\end{aligned}
$$<smiles>O=[N+]([O-])C1CCCCC1</smiles><smiles>NC1CCCCC1</smiles>

This reduction is accelerated by some substance contained in extracts of mammalian tissues and in extract of yeast. What the actual reducing component in the reaction is we do not know; it is certainly not hydrogen itself.

Aspartic acid + hydrogen $<=>$ ammonium succinate

$\mathrm{COOH} \cdot \mathrm{CH}\left(\mathrm{NH}_{2}\right) \cdot \mathrm{CH}_{2} \cdot \mathrm{COOH}+\mathrm{H}_{2}<=>\mathrm{COOH} \cdot \mathrm{CH}_{2} \cdot \mathrm{CH}_{2} \cdot \mathrm{COOH} \cdot \mathrm{NH}_{3}$

This reduction is accomplished by many of the common bacteria, such as the Bacillus coli communis. The component substance that reacts with the amino-succinic acid is not known.

Other fermentations are known that do not fit naturally into any of these groups. An illustration may be given in the deaminization ferments-substances that accelerate the replacement of the amino group by an hydroxyl group in the various amino-acids that are products of the hydrolysis of protein, and also the replacement of the amino group in guanin and adenin by hydrogen. Thus the action of guanase may be illustrated as follows:

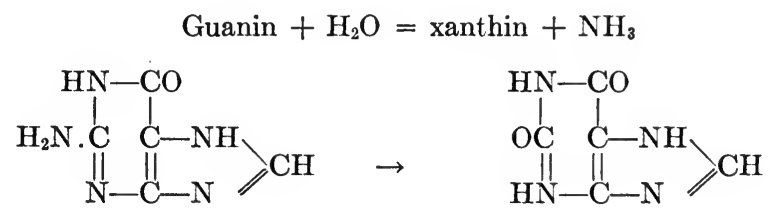

The deaminization of amino-acids are enzymic reactions of great importance. This process may be illustrated for alanin:

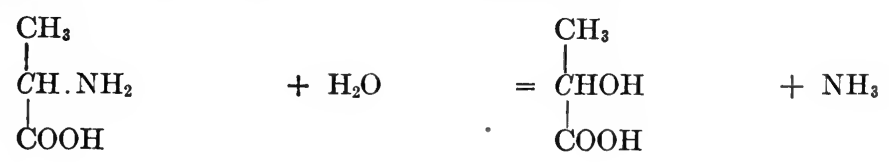

The amino-propionic acid is converted into lactic acid. This reaction directly viewed is, of course, an hydrolysis. But it is also an oxidation, since the oxy-acid is formed.

The formation of acetone from aceto-acetic acid gives an illustration of another unusual reaction. 




Similar reactions by splitting off of carbon dioxid are the formation of taurin from eysteinic acid and the derivation of p-oxy-phenylethyl-amin from tyrosin. It is clear that some hydrolyses constitute what especially from the physiological standpoint must be interpreted as oxidations; the splitting off of carbon dioxid is really a reduction.

General Features.-These illustrations will suffice to afford a general view of the final relations determined by the reactions of fermentation of different types. For nearly all these accelerated reactions the slow auto-reactions are known. For many of these processes reversions have been accomplished. For many of these reactions the influence of variation in temperature is known. These facts afford a natural presumption that the reactions of catalysis and fermentation are essentially identical.

In looking over these reactions one cannot fail to be impressed with certain general features. The heat relations in fermentations vary. In the common hydrolyses the products have approximately the same heat value as the substrate. The oxidations, on the contrary, are exothermic, the reductions endothermic reactions. Natural fermentations convert complex substances into simple substances, they convert colloids into electrolytes, electrolytes into simple gases and elements. Most of the substances that are commonly the substrates of fermentations are but slightly soluble in water, or even incapable of true solution but only of a colloidal suspension. Starches, cellulose, proteins, glucosids, fats, all form in water more or less colloidal suspensions and many display further the tendency to form hydrogels. When these substances are fermented, the products are substances of greater solubility, quite devoid of colloidal characteristics, with no tendency to the formation of hydrogels. Through the fermentation the system has been converted from the heterogeneous to the homogeneous state. The substrates of natural fermentations are usually substances of very large molecular weight; the products possess small molecular weight. These substrates are almost devoid of the power of diffusion, the products diffuse readily as a rule. The natural substrates exert little depression of the freezing point of their solutions, the products exert usually a marked depression; the substrates possess little osmotic pressure, the products marked osmotic pressure. The substrates are rarely crystalloidal, the products are usually crystalloidal. The substrates are substances that are not subject to electrolytic dissociation; the products often exhibit this property. The substrates are substances with little tendency to chemical reaction as 
compared to the products. There are, of course, exceptions to these statements; for instance, the properties of the higher fatty acids are in these respects but little different from their fats. The natural fermentations usually comprise the disintegration of complex substances that have been synthesized in the vegetable or animal organism. Since all fermentations are to be regarded as reversible processes, how obvious and attractive is the suggestion that the natural fermentations are simply the reversion of the reactions whereby these substances were formed. There are no thermodynamic reasons that plead against this proposition. The fact that fermentations of the natural order are exothermic reactions has been employed by some biologists in support of a teleological interpretation of the circumstances, according to which a synthesis could not be held to be accomplished by the action of a ferment. As will be pointed out, this interpretation is lacking in theoretical validity.

It must be conceded that the pendulum may swing too far and that fermentations may be accorded a too general scope in physiological and pathological processes. The theoretical possibility for such an overgeneralization lies in the very definition of fermentation. We have defined fermentation as the acceleration of some existing reaction by a substance formed in a plant or animal organism. When we reflect, further, that theoretically every reaction is capable of acceleration, the boundless application of the principle becomes apparent. It is, however, clear that chemical biology is in no greater danger of becoming simply a treatise on fermentations than are inorganic and organic chemistry in danger of becoming reduced to a treatise on catalysis, since the same principles apply to all. In each individual instance the question whether a particular phenomenon may be a fermentation (or a catalysis) is plainly a matter of concrete demonstration. And it is because this concrete demonstration must be so much more difficult in biological than in chemical questions, that there will be a tendency among biologists to overwork the principle. Standing bewildered before the apparently hopeless complexity of a biological problem, it is so easy to say "ferment action." This very tendency compels us to insist with the greatest objective strictness upon the precise demonstration of the occurrence of a fermentation. The safety of the investigator lies in close adherence to the laws of general chemistry connected with the kinetics of reactions. Not only do these serve as controls in our studies, they enable us to investigate in a proper and adequate manner the characteristics of a fermentation, once its identity as such has been established. The importance of the study of fermentations has been entirely underestimated by the biological world. While in the groupings of systematic biology it may have sufficed to know simply that a particular phenomenon was a fermentation, for the real study of the chemistry of the functions of animals and plants, that is simply the stating of the problem. The height of biological 
research is the reproduction of an act of nature. To attempt to reproduce the chemical functions of organized bodies, one must study fermentation from the point of view of the control of its several variables.

\section{APPLICATION OF LAWS OF CATALYSIS TO FERMENTATIONS}

We define a catalysis as an acceleration of an already existing reaction through the presence of another body that does not appear in the end products of the reaction. In the specific instance there are two criteria of catalysis. Every alteration in velocity not dependent upon alteration in concentration or of temperature indicates catalysis; and in the catalytic acceleration there are no stoicheiometric relations between the catalyzer and substrate or products. There is theoretically a catalyzer for every reaction, and every substance may act as a catalyzer. Certain classes of bodies possess to a high degree this quality of acceleration: The platinum group, colloidal metals, hydrogen, and hydroxyl ions, and the oxids and oxyhydrates of the elements of varying valency, such as iron, manganese, and nitrogen. The relations of energy involved in a reaction are not disturbed by catalytic acceleration; the result is achieved by a diminution of the chemical resistance. The existence of a large class of compounds in the natural state is dependent absolutely upon their chemical resistance, and were this materially diminished, these compounds would cease to exist. Chemical resistance diminishes with increasing temperature, and there is an optimal temperature for each concentration of a chemical system. Corresponding to the physical state of the system, we distinguish between catalyses in homogeneous and in heterogeneous systems. The current theory of physical states is that from the homogeneous to the heterogeneous state is a gradual transition. In fermentations we deal with bodies that present with water less of homogeneity than solutions of pure crystalloids, and usually less of heterogeneity than the typical suspension colloids. We shall see that the behavior of fermentations confirms this interpretation.

In our consideration of the kinetics of catalysis we are concerned in the first instance with the law of mass action, and especially as applied to a reaction of which the products may reunite to form the original substance to a measurable degree; and, secondly, with the relations observed when such a reaction is accelerated by the presence of a positive catalyzer or enzyme. Theoretically all reactions are to be looked upon as limited reactions and likewise reversible; but the point of equilibrium may be so slightly removed from the condition of a complete reaction as not to be analytically determinable; and it is not always possible to fix the conditions favorable to a reversion so that the theoretical result shall become experimentally apparent. What is about to be enunciated under these headings is specifically valid only for homogeneous systems; the relations involved when heterogeneous systems are concerned will be considered later. 
The fundamental proposition concerned in the kinetics of chemical reaction is that under constant conditions of experimentation the transformation in the unit of time is proportional to the mass of the reacting bodies. This proposition is directly analogous to the law for radiation of heat from a warm to a cold body; the radiation in time is proportional to the difference in temperature between the two bodies. The formulation may be expressed in another way in the statement that whenever a transformation is taking place, the rapidity of that transformation in a particular moment is proportional to the distance between the end point (the equilibrium) and the point in the course of the reaction attained at that particular moment. Applied to the concrete instance, say to the inversion of cane sugar, we mean that in each particular moment the amount of sugar inverted is proportional to the amount of uninverted sugar present in that same moment. 'This applies as stated only to monomolecular reactions-reactions in which the mass of only one substance is affected during the reaction. But the general proposition applies to bimolecular and trimolecular reactions, with the difference that the degree of reaction in time depends upon the concentration of the two or three active masses, instead of depending simply upon one. Now all the fermentations occurring in the biological world are monomolecular reactions, because the second body concerned, as water or oxygen, is present in such excess that alteration in its mass is of almost no consequence. If, for instance, in the reaction cane sugar + water $=$ glucose + fructose the amount of water present was approximate to the amount required in the reaction, that reaction would be treated as a bimolecular reaction; but as the experiment is carried out in dilute solution, where the water, as solvent, is present in a thousand times the amount required for the water as reagent, the reaction is equivalent to a monomolecular reaction. For this reason we will confine ourselves to the kinetics of the monomolecular reaction:

$$
\frac{\mathrm{d} \text { Conctr. }}{\mathrm{dt}}=\text { Const. Conctr. }
$$

This proposition is expressed in the differential equation:

$$
\frac{d x}{d t}=C(A-x)
$$

$\mathrm{A}$ is the original amount of substrate, $\mathrm{x}$ the amount of substance converted in the time $\mathrm{t}, \mathrm{C}$ is a constant. The equation holds only when the temperature and volume are held constant, and the system is in a state of certain dilution. When integrated and reduced to its simplest relations under the stipulation that when $\mathrm{t}=\mathrm{O}, \mathrm{x}$ also $=\mathrm{O}$, the equation becomes:

$$
C=\frac{1}{t} \log \frac{A}{A-x}
$$


In practice the equation is often used in a modified form, though directly derived from the one stated:

$$
\mathrm{C}=\frac{1}{\mathrm{t}_{2}-\mathrm{t}_{1}} \log \frac{\mathrm{A}-\mathrm{x}_{1}}{\mathrm{~A}-\mathrm{x}_{2}}
$$

This often gives a better concordance, as the first estimations are likely to be irregular. The constant expresses the work done under constant conditions. If, for example, we determine with a particular strength of acid in an inversion experiment that $\mathrm{C}=0.002$, that means that under constant conditions of temperature, volume, and concentration of acid, in a solution of sugar of the strength of a gram molecule in the liter, 0.002 gram molecule of sugar will be inverted in the first minute, and if we could add to the system in each minute without changing the volume 0.002 gram molecule of sugar, that quantity would be inverted regularly each minute.

This simple relation becomes more complicated when we deal with a reaction of active and measurable reversibility. Under these circumstances an equilibrium is established in the system when the opposing reactions just compensate each other. The reaction in the direction of the right becomes less each minute as the mass of substrate becomes less; the reaction in the direction of the left becomes greater each moment as the mass of the products of the other reaction increases. At a certain point these will be equal, and from this time no apparent change will be observable in the system. But it must not be supposed that the reactions have stopped; they continue as before proportional to the active masses of the respective components, but since they are balanced, the system is in equilibrium.

The reaction may be written in the following manner, using as an illustration the reaction ester + water $=$ alcohol + fatty acid.

$$
\text { Ester }+ \text { water }<=>\text { alcohol }+ \text { fatty acid }
$$

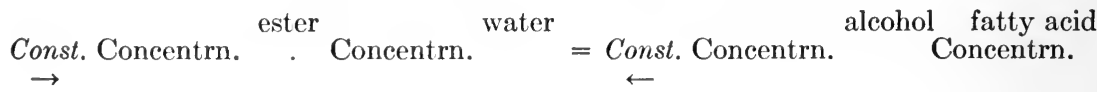

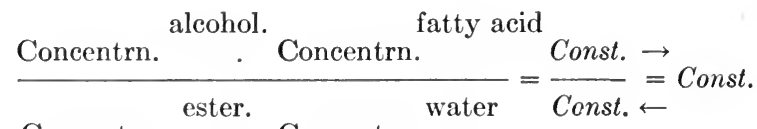

Const. stands for the constant of equilibrium

When a catalyzer is added to such a system under conditions of controlled relations in experiments with pure substances, nothing happens except that the time of the reactions is shortened. The accelerated reaction that naturally was practically a complete reaction remains practically a complete reaction; it is simply completed more quickly; and with different quantities of catalyzer the differences are 
simply those of degrees of rapidity. The accelerated reaction of measurable reversibility remains a reaction of reversibility and the point of equilibrium is not disturbed by the acceleration; the

$$
\frac{\mathrm{c} \rightarrow}{\mathrm{c} \leftarrow}=\mathrm{C}
$$

is simply reached more quickly. As stated, however, the conditions must be controlled. Concentration and volume must be held constant, in order that the relations of the active mass or masses are maintained; the temperature must be held constant because the point of equilibrium will vary with the temperature; and the products must not combine with the catalyzer, for in this manner also would the constant of equilibrium be disturbed. It is also very important that pure substances be employed, since traces of foreign bodies may exert a very great disturbance.

Let us now examine the conditions and variations somewhat in detail.

Initial Concentration of the Substrate.-This is not immaterial, and it is not true that at all concentrations of the substrate the reaction in any particular moment is proportional to the active mass of the substrate. This statement is true sometimes only of dilute solutions, just as the laws of electrolytic dissociation hold true only for dilute solutions. If one endeavors, in accordance with the current tendency, to rest catalytic reactions upon the theory of ionization, this fact becomes in a general sense intelligible. A further bearing upon the disturbing action of high concentrations of the substrate lies in the fact that under such circumstances the substrate participates in the rôle of solvent; the vapor pressure of the catalyzer may be thereby altered, since under such circumstances the solvent will be modified. Not only is the velocity of a reaction dependent upon a proper dilution of the substrate; the order of a reaction is likewise altered by excessive concentration. In a monomolecular reaction, under conditions of proper concentration, when the system is diluted the times of equal proportional transformation are not altered; doubling the concentration doubles the velocity. Under similar circumstances with a bimolecular reaction, when the system is diluted the times of equal proportional transformation are inversely as the initial concentrations; doubling the concentrations quadruples the reaction velocity. Now under conditions of high concentration these relations do not hold, and it is thus necessary to provide for proper dilution when determining the order of a reaction as well as when determining the velocity. The necessity of high dilution of the substrate is most urgent in the case of substrates of ponderous molecular weight.

Concentration of the Products.-This is likewise not immaterial. In general, the higher concentrations of the products, the more energetic the process of reversion. But in experimental catalysis it is some- 
times found that when the concentration of the system is high the resultant high concentration of the products disturbs the course of the reaction.

Influence of Temperature.-The acceleration of reaction velocities by increase in temperature holds good for catalytic reactions as well as for simple ones. For such work the simple formula of Arrhenius will usually suffice:

$$
\ln \frac{\mathrm{k}_{1}}{\mathrm{k}_{2}}=\mathrm{A}\left(\frac{\mathrm{T}_{1}-\mathrm{T}_{2}}{\mathrm{~T}_{1} \cdot \mathrm{T}_{2}}\right)
$$

A being the constant and $k_{1}$ and $k_{2}$ the velocity constants of the reaction at the absolute temperatures $\mathrm{T}_{1}$ and $\mathrm{T}_{2}$. The equation has been tested on many catalytic reactions. Corresponding very closely in its results to the above equation is the empirical rule of van't Hoff that for every $10^{\circ}$ increase in temperature the velocity of a reaction is doubled, $i$. $e$., the time required to do a unit of transformation is reduced one-half,

$$
\frac{V \text { at } T_{n+10}}{V \text { at } T_{n}}=2+
$$

For ferments the rule holds good in a restricted sense. The rule holds for most ferments from about $15^{\circ}$ to $35^{\circ}$; above this the increase in velocity is often much more than predicated by the rule, while above $45^{\circ}$ there is a rapid fall to zero. The fall is the result of the destruction of the ferment, a condition not contemplated in the rule. As a whole, however, in consideration of the complexities attending the experimentation, the concordance of the facts with the theory is quite good.

It is a common error to suppose that a temperature optimum is peculiar to ferment action. Every reaction has a temperature optimum, and this optimum is usually altered (lowered) in a catalytic acceleration. The system $\mathrm{SO}_{2}+\mathrm{O}=\mathrm{SO}_{3}$ has its temperature optimum about $900^{\circ}$; at $1000^{\circ}$ the system is in equilibrium, the velocity of the reactions being, however, very slow. In the presence of platinum the temperature optimum is lowered to about $450^{\circ}$, the velocity is rapid, and as the reversed reaction (the dissociation of $\mathrm{SO}_{3}$ ) does not occur to any material extent below $500^{\circ}$, the acceleration of the reaction is very effective. The catalytic acceleration of the formation of water from oxygen and hydrogen also displays a sharp optimum. Ferments are notable simply for the lowness of the temperature optimum, and for the narrowness of the optimal range. The latter condition has lost much of its significance, since we have learned that the sharp descent of the curve from the optimal temperature is due in large part to the destruction of the ferment.

Relations of the Catalyzer.-The typical positive catalyzer simply accelerates the velocity of a reaction without altering the nature of 
the reaction, the order of the reaction, the character or yield of the products, the point of equilibrium, and without being itself altered in the process. It is just as though there were a certain resistance to the progress of the reaction, and the presence of the catalyzer diminished this resistance, like a lubrication. These conditions are often but not always maintained in inorganic catalyses, are as a rule not fully maintained in the acceleration of reactions involving organic substances, while they may be said to be rarely realized in fermentations. Since the presence of the catalyzer simply accelerates the reaction, it ought in general to do so proportionally to the quantity of the catalyzer employed, and this is usually true. Especially for the catalytic action of hydrogen ions has this relationship been shown to be true; the catalytic action of acids is related to their electrolytic dissociation. The same holds true for hydroxyl ions. For other catalyzers the same rule has in general been found to hold good. There are, however, several well-studied reactions, for which the catalytic acceleration is not proportional to the quantity of catalyzer. Thus, in the reaction between stannous chloride and ferric chloride the catalytic acceleration of acids is proportional to the square of the hydrogen ions; and in the catalytic reduction of hydrogen peroxide by colloidal platinum the acceleration is not proportional to the quantity of platinum.

The relation of the mass of ferment to the acceleration of the reaction may be usually expressed in the equation of Bredig:

$$
\frac{\mathrm{C}_{1}}{\mathrm{C}_{2}}=\left(\frac{\mathrm{F}_{1}}{\mathrm{~F}_{2}}\right)^{\mathrm{m}}
$$

The $\mathrm{C}$ is the constant of velocity for the ferment concentration of $\mathrm{F}$, $\mathrm{m}$ is constant. For the common catalyzers $\mathrm{m}=1$ and this holds for many ferments. The proportionality holds for low concentrations only.

To the statement that the order of a reaction is not altered by the presence of a positive catalyzer, exceptions seem to exist. The reaction $\mathrm{H}_{2} \mathrm{O}_{2}+2 \mathrm{HI}=2 \mathrm{H}_{2} \mathrm{O}+\mathrm{I}_{2}$ depends upon the concentration of the two reacting bodies; that is, it is a bimolecular reaction. Under the influence of molybdic acid and iron sulphate, the velocity of the reaction is a function of the concentration of hydrogen peroxid, that is, the presence of the catalyzer had converted the reaction from one of the second to a reaction of the first order. There exist also other studies suggesting a similar shifting in the order of the reaction, though in none have the relations been so carefully worked out. The alteration is unquestionably to be explained upon the basis of intermediary reactions.

The statement that the station of equilibrium is not translocated by the presence of a catalyzer seems to hold absolutely good unless the catalyzer is altered in the progress of the reaction, and there is for this statement experimental evidence as well as thermodynamic 
theory. The acceleration of the catalyzer per se simply multiplies the $\overleftarrow{c}$ and $\vec{c}$ of the equation, the $\mathrm{C}$ is not affected by the acceleration. When, however, the catalyzer is altered during the course of the reaction, it means that the substrate, the products or the solvent has entered into reaction with the catalyzer, as a consequence the concentration of one or other member of the system has been altered, and as a result of this the station of equilibrium has been shifted.

The remarks previously made upon the necessity of adequate dilution hold also for the catalyzer. When to a system is added an excess of a catalyzer, bizarre and irregular results may be expected. For such catalyzers as act through ionization this behavior is quite intelligible. It is here again possible that at a high concentration of the catalyzer it will in part participate in the solution of the substrate. Under such circumstances the solvent is not identical with that in a system at high dilution, and since the station of equilibrium may be shifted very materially by alterations in the solvent, irregular results might be expected for this reason alone. Furthermore, the active mass of the substrate cannot be assumed to be the same in the changed state of the solvent. There is in any event no necessity for the use of high concentrations of the catalyzer, since one of the most striking aspects of these accelerations is the minimal amount of catalyzer required to effect a notable acceleration.

Inactivation.- Under the term inactivation are understood several different relations. The slowing of the reaction under the influence of concentration of the products is a manifestation of the mass law, and ought not to be referred to as an inactivation of the ferment. The ferment may be inactivated by union with some extraneous body, or even by its mere presence; in a purely chemical inactivation the ferment will be restored to power when the offending condition is removed. Lastly, the ferment may be inactivated by hydrolysis or oxidation, in which it is destroyed as a catalytic agent.

Under certain conditions the presence of a substance not itself a catalyzer will enhance the acceleration of a known catalyzer. A good illustration of this fact is contained in the observation that the presence of a trace of cupric sulphate, in itself inactive, will increase enormously the acceleration of the reduction of hydrogen peroxid by ferrous sulphate. The mechanism of such zymo-excitation is unclear.

As there are accelerating substances, so there are retarding substances. We must here distinguish between negative catalyzers and anticatalyzers. A negative catalyzer would be a substance that retards the auto-reaction. Anticatalyzers are substances that inhibit in part the accelerating action of a positive catalyzer. Without denying that genuine negative catalyzers exist, it is certain that the bodies described as such have been anticatalyzers. In many inorganic reactions the most trifling amounts of foreign substances may act as pronounced anticatalyzers, and there can be no doubt that in the domain of ferments such influences are still more numerous and potent. 
Station of Equilibrium. - The station of equilibrium in a reaction of reversible character is dependent upon certain conditions. In the ideal sense it applies to a system at constant temperature in a high and constant dilution. It is possible experimentally to alter the station of equilibrium in several ways. More of the substrate may be added, in which event the reaction in the direction of the right will again assume the leadership until a new balance is established. Some of the substrate may be removed, in which event the reaction in the direction of the left will be accelerated until a new balance is established. The products may be removed or more of those bodies added, with the resultant accelerations in the respective reactions until new equilibria are established. Or only one of the products may be added; thus one can add acetic acid to the system ethyl acetate + water $=$ acetic acid + ethyl alcohol until the equilibrium is established in the sense that there are but two bodies present, ethyl acetate and acetic acid. The station of equilibrium may also be altered by dilution or concentration of the entire system. Alteration in temperature may also bring with it an alteration in the station of equilibrium, must indeed if the reaction be endo- or exothermic. To these we must add two final procedures for the translocation of the point of equilibrium-reactions between ferments and components, and reactions between solvent and product. These considerations of alterations in the station of equilibrium are of great importance in the study of fermentations, and much of the reigning confusion has been due to neglect of them.

Purity of the Reacting Bodies.-This is generally of great importance for the regular progression of catalytic accelerations. The simple presence of foreign bodies is not itself of necessity a disturbing factor; extraneous substances may be divided into active and inactive. A trace of an active substance may seriously disturb the reaction in an otherwise flawless catalysis; a good illustration is afforded in the inhibition that may be exerted upon a large mass of platinum by a trace of arsenic in the contact method for the manufacture of sulphuric acid. On the other hand, the mere presence of one or a number of inert substances may have little or no disturbing effect. We shall later see that it is sometimes possible to secure good quantitative results in fermentation experiments conducted with materials known to be very complex. In other instances good results are not secured, the difference depending not upon the presence of foreign substances, but upon the presence of particular foreign substances that are active in their chemical relations to some member of the system under investigation. In various chemical investigations of this matter it has been acids, alkalies, and inorganic salts in particular that have produced disturbances in the progress of catalytic accelerations. A priori, we must understand that since we conceive the modus operandi of catalytic acceleration to lie in successive intermediary reactions, there must be for each type or group of reactions certain substances whose introduction into the system would lead to different reactions, that would proceed more or 
less at the expense of, and certainly to marked disturbance of the quantitative progression in, the original reaction. Just as there are certain substances and conditions that prevent a good yield in the synthesis of organic substances, and these conditions and substances vary for the different synthetic procedures; so there are conditions and substances that disturb or prevent the regular march of a catalytic acceleration, and these conditions and substances will vary with the different reactions whose accelerations are being studied.

\section{APPLICATION OF LAWS OF REACTIONS TO FERMENTATIONS}

Concentration of the Substrate.-This is in fermentations often difficult to adjust and determine. Most of the materials that are employed as the substrate of fermentations are natural substances and, therefore, never pure. Furthermore, it is often not possible to determine the quantitative degree of impurity. Let us take starch for illustration. Starch contains a certain amount of ash, composed of various inorganic bodies. It contains also organic bodies, traces of sugar, fats, protein, and what not more. These bodies cannot be separated from starch, and the only way of determining the actual amount of starch is to hydrolyze completely with acid and then determine the sugar present, and even this method would neglect the preformed sugars. When, therefore, one prepares a 1 per cent. solution of starch, one does not know with just how much less than a 1 per cent. solution one is actually working. But more, starches vary in their resistance to hydrolytic cleavages, depending upon their origin, mode of preparation, age, and method of preservation. A certain starch might be already partially hydrolyzed to as much as 10 per cent., depending on the method of preparation and preservation. Let us further consider albumin. All that has been said of native starch holds true of native albumin to still greater degree. Not only are foreign substances present to greater extent and in greater variety than in starch, but the tendency to -alteration in the albumin itself is more pronounced. Furthermore, an albumin is usually composed of several proteins, and unless one works with a pure protein like casein the experiment actually involves the digestion of several proteins, of possibly widely varying resistance to hydrolysis. While it is possible in some instances to carry along in the same solution two catalyses of different materials and have each maintain its own proper velocity (the inversion of sugar and the cleavage of methyl acetate by acids), it would not be possible to do so under circumstances where the products of the one reaction make the measurement of the other reaction uncertain or futile. Yet this is what is attempted when egg albumin is employed as substrate. It is obvious, therefore, that it is often not possible to fix the concentration of and to exclude plurality in the substrate. Now since the concentration of the active mass of the substrate is a fixed requirement, it is clear that at the very outset in a fermentation we are confronted 
with an inability to define properly the conditions of the experiment. In the early part of the fermentation this indefinite minus in the concentration will not disturb the results so seriously, but toward the close of the reaction the influence must be marked. This state of affairs is, of course, not general; we can prepare cane sugar, any of the hexoses, alcohols, and the synthetic fats with a high degree of purity, and it has been along these lines that much of the best work has been done. One ought always to attempt the purification of materials; but very often such attempts at purification only lead to some denaturation of the substance, so that nothing is gained.

Experience with fermentations teaches that there are three zones of concentration with particular behaviors. In the zone of high concentrations, variations lead to no changes in velocity. In a system with an excessive concentration of substrate, the velocity depends on the concentration of ferment alone; within certain limits, variations in the substrate have no result. Where both substrate and ferment are in excessive concentration, irregular and bizarre results will be obtained. In the zone of medium concentrations, the velocity of the transformation is a function of the mass of substrate, and the intensity of the acceleration is a function of the concentration of ferment, but there is an interdependence. The intensity of the fermentative acceleration accomplished by a constant concentration of ferment will vary to some extent with the concentration of the substrate. For different concentrations of substrate the ferment seems to set a different pace, a condition that suggests a sort of a stoicheiometric relation. Most of the experiments with ferments have been conducted within this zone of concentrations. In the last zone of concentration, that of high dilutions, the velocity of the transformation is the function of the substrate concentration, the intensity of the acceleration is the function of the mass of ferment and these are independent, just as they should be in a pure catalysis. When operating within this zone, dilution of the entire system (in monomolecular reaction) has no effect upon the proportionalities of the reaction, while the contrary is observed on dilution of the system in the other zones of concentration. If the proper conditions can be attained, this zone will be found in most fermentations. It has been demonstrated for invertase, lactase, maltase, trypsin, erepsin, lipase, and catalase. For the study of fermentation from the kinetic point of view, this zone of concentration is obviously the one to be sought.

It must be insisted upon that the failure of the law of mass action to hold within wide limits of concentrations is not peculiar to the phenomena of fermentations. For many of our best known physicochemical laws the conformity of fact to theory is confined to narrow limits of conditions. The general equation for simple bimolecular reactions:

$$
\frac{\mathrm{dC}_{1}}{\mathrm{dt}}=\mathrm{kC}_{1} \mathrm{C}_{11}
$$


is valid only for very high dilutions, practically tenth normal. The law of electrolytic dissociation applies only to conditions of high or theoretically infinite dilution, and the variations that are induced in the system by the presence of undissociated molecules are well known. Similar relations are naturally to be expected in fermentations, and the multiplicity of the conditions should lead us to expect the relations here to be still much more complex.

Measurement of the Reaction.-Frequently we are not in a position to measure in an accurate analytical manner the products of the reaction. It is rare that we have the opportunity to measure the products with a polariscope, by a direct accurate titration, or by a determination of conductivity. More often we need to undertake a formal isolation of the desired product and then identify it and measure it by means of chemical behavior. In some instances such a procedure may yield accurate results, but it may not be feasible to do this in the number of determinations necessary in a measurement of reaction velocity. Sometimes the product may not be accurately estimated in any way, as in the case of the digestion of protein.

A more serious complication arises with a great many fermentations in which the reaction occupies not one but many stages. These mediated catalyses exceed the direct catalyses in the organic world. We have substrate + water $=$ product $_{1}+$ product $_{1} ;$ product $_{1}+$ water $=\mathrm{p}_{2}+$ $\mathrm{p}_{2}$, etc.; and finally $\mathrm{p}_{\mathrm{n}}+$ water $=$ end product + end product. These really represent a series of successive catalyses. The reactions, however, do not progress in complete stages; that is, all of the substrate is not first converted into product ${ }_{1}$; and all of product ${ }_{1}$ then converted into product, and so on until the end products are reached. On the contrary, several of the products may be found in the mixture in a particular moment. Each of these stages represents work. Now in a fermentation experiment under such conditions, what shall one measure? Obviously if one wishes to measure the transformation of the substrate, one must measure product ${ }_{1}$, which cannot be done because the stage is not completed en bloc. If one measures the end product, what one really measures is the appearance of the end product and not the conversion of the substrate. And yet the measurement of the end product may be the only possible measurement. Under these circumstances we employ the equation

$$
\mathrm{C}=\frac{1}{\mathrm{t}} \log \cdot \frac{\mathrm{A}}{(\mathrm{A}-\mathrm{x})}
$$

so that under $\mathrm{A}$ we understand the quantity of end product when the reaction is completed and under $\mathrm{x}$ the quantity of end product formed in the time t. Under these circumstances it becomes possible to work with fermentations involving reactions in many stages, though in some instances the results are very irregular. It is apparent that while a positive result might indicate that the law holds good for the particular 
fermentation, a negative result would not indicate the converse; it would indicate nothing, beyond that the conditions of experimentation were too uncontrolled to yield results susceptible of interpretation. The majority of the fermentations of great biological importance belong to this class of mediated catalyses; no highly organized body like cellulose, starch, or protein may be hydrolyzed into simple crystalloid end products in a single main reaction. It must be realized that this constitutes not an analytical but a fundamental difficulty. The equation

$$
\frac{d x}{d t}=C(A-x)
$$

presupposes that the substance undergoing transformation exists in each moment in the system either in the form of unaltered substrate $\mathrm{A}$ or of product $\mathrm{x}$. The intermediary reactions from the state $\mathrm{A}$ to $\mathrm{x}$ are not held to occupy measurable time. If now the substrate in the moment of analysis exists in part in the state of e, f, or more states, and not either as $\mathrm{A}$ or $\mathrm{x}$, the equation cannot apply. This is precisely what occurs in the digestion of complex substances like protein and starch; and under these circumstances one cannot be surprised that the fermentations of these substances do not follow closely the law of mass action as expressed in the equation.

Reversion of the Reaction.-'The equation for the simple monomolecular reaction does not contemplate a reversion of the reaction. For fermentations, however, we postulate theoretically such a reversion. If such reversion occurs with anything like measurable rapidity; the constants obtained in series tests with the use of the equation cannot be identical and an entirely different mathematical formulation will be necessary. That a certain degree of reversion occurs in actual fermentations has been practically assumed on the basis of three facts: the substrate is never completely fermented; removal of the products increases the completeness of the fermentation and reinaugurates it after it has ceased; and the removal of the products increases the rapidity of the fermentation. It must, however, not be overlooked that two of these results could be due to chemical influence of the products upon the ferment. As a matter of fact, the results of the many known experiments in reversion by ferment action (in which the ferment has been mixed with the products of the reaction) has been to indicate that reversions occur with great slowness as compared to the reaction of cleavage. If one attempts to incorporate into the equation of a monomolecular reaction as a conditioning factor that rapidity of reversion experimentally observed in direct tests, the deviation will be almost nil. In addition to this, the station of equilibrium is for most substrates at high dilution so near to a complete reaction that reversion could scarcely be held to modify to any appreciable extent the mathematical expression of a ferment reaction. Nevertheless, the actual determination of the extent of this variable lies not in mathe- 
matical considerations, but in the direct experiment. This may be one of the reasons why the experimental velocities in fermentations do not always agree with the mathematical predications.

Concentration of Ferment.- It is as difficult to obtain and maintain a constant concentration of the ferment as of the substrate. The activities of ferments as well as their stabilities seem to depend to a marked degree upon the methods of preparation and conservation. All ferments contain more or less extraneous matter; probably in most instances the percentage of the actual ferment is but a small fraction of the weight of the preparation employed. Under such circumstances it is not possible to define the exact initial concentration of ferment, and this results in an indeterminate error in the course of series of experiments.

The lack of regularity between the chemical composition and enzymic activity of a ferment is a condition not peculiar to ferments, but is an unfortunate quality of all colloids; for its designation we may amplify the use of the term hysterisis. The age, origin, method of preparation, in short every incident in the history of a colloid tends to influence its chemical qualities. Further than this, once prepared and conserved under constant condition, colloids tend slowly to alterations, a denaturation that must be conceived to lead to a reduction in the dynamically active mass of the colloid.

Of far greater practical importance than the difficulty in determining the initial concentration is the impossibility of maintaining the concentration. Ferments as a class have been held to differ from inorganic catalyzers in that the latter emerge from the completed reaction unchanged. This is not true, for the colloidal metals are subject to similar reductions in their catalytic properites; in some instances the inactivation is accompanied by precipitation in a granular amorphous form, but in other instances the appearances of the colloid undergo no change. The inactivation proceeds more rapidly at high temperatures, and seems to affect old suspensions more than fresh preparations.

All ferments are more or less altered during the course of a fermentation. Upon the supposition that we are dealing with pure conditions, there are apparently three conceivable sources for these alterations, namely, reactions with the substrate, the products, the solvent. We have no knowledge of reactions between the substrate and ferment that would result in inactivation of the ferment. There is a good illustration of this relation in the acceleration of the reduction of hydrogen peroxid by colloidal silver; the substrate combines with a portion of the silver to form a compound that is catalytically inactive. We have little chemical knowledge of the inactivation of ferments by reactions with the products. Trypsin is more rapidly destroyed in a solution of amino-acids (products of tryptic digestion) than in simple watery solution. Vegetable lipase is somewhat sensitive to acids, and is destroyed more rapidly in even moderate concentrations of acetic 
acid than in distilled water. Ferments, however, often combine with the products of the reaction. In all probability, however, the chief reaction resulting in the destruction of the ferment is hydrolysis upon the part of the solvent. Just as the albumin or ester constituting the substrate, on suspension in water undergoes a slow hydrolytic cleavage of which the digestion is but the acceleration, so ferments on suspension in water undergo hydrolysis; and as the products are not active in the catalytic sense, the active concentration of the ferment is thus steadily reduced during the progress of the experiment. The two hydrolyses, of the substrate and of the ferment, proceed side by side and probably entirely independently. The hydrolysis of the ferment is, of course, accelerated by increase in temperature, and seems to follow the usual rule for such increase. The destruction of vegetable lipase proceeds closely according to the rule, while the destruction of trypsin proceeds rather more rapidly. The possible extent of such an inactivation will be understood when one realizes that in some instances a solution of trypsin may be inactivated one-fifth in an hour; and the degree of disturbance that must follow becomes apparent when we consider that some half dozen hours might be required for a digestion experiment with such a solution. In their resistance to hydrolysis ferments, however, vary widely. The inactivation of the ferment proceeds usually more rapidly in simple solution in water than during the course of a fermentation experiment; this has been shown to be true for amylase, invertase, trypsin, vegetable lipase, and zymase. The natural interpretation of this phenomenon is that during the course of the fermentation the ferment is passing through intermediary reactions with the substrate, and that when thus combined its hydrolysis is suspended. The free ferment adds water to undergo cleavage; in the complex substrate-ferment this reaction does not occur.

Inactivation of the ferment may be produced also by reactions with extraneous substances. Theoretically it ought always to be possible to distinguish between inactivation and destruction of a ferment; practically this may not be always possible. 'The phenomenon is very frequent in actual experimental work, and may also be provoked in inorganic catalyses. The accelerating action of iron salts may be abolished by the presence of acetic or oxalic acid; the accelerating action of colloidal platinum is depressed by hydrocyanic acid or a thiosulphate, as well as by alkalies, etc. These influences are not all of one nature; in some the ferment or catalyzer is destroyed; in others the action is inhibited though the substance is not altered, for when the depressing body is removed the original activity of acceleration returns. Colloidal platinum again affords a striking illustration; the inhibition of its catalytic acceleration by hydrocyanic acid, carbon monoxid or phosphorus will pass away with the removal of these substances while the inhibition following the addition of sulphuretted arsenic, iodin, or mercuric chlorid remains after the removal of those bodies. For the natural ferments the negatively catalytic influences 
are exceedingly numerous, and in nearly all instances they effect a permanent inactivation. Many of these substances act by accelerating the hydrolytic cleavage of the ferment, that is, they are positive catalyzers to the hydrolysis of the ferment. Such is almost certainly the nature of the influence of acids and alkalies.

The combination of ferment with the products of the reaction is a disturbing factor in many fermentations. The so-called rule of Schütz is an expression of this disturbance; the enzrme combines with the reaction products and the active mass of the ferment is inversely proportional to the mass of the reaction products.

Stimulation of the ferment by the presence of substances not in themselves accelerators is very frequently observed in connection with fermentations. Thus a trace of acid aids the action of invertase, vegetable lipase, and of the ferments of the pepsin group; a trace of alkali aids many reductions and also the fermentations of the trypsin group. Many salts have similar actions, as have innumerable other substances. These zymo-excitors seem to have two things in common: An optimal concentration and an optimal temperature. A good illustration of these facts (and one that has the further value that it also illustrates the identity of the conditions in the organic and inorganic worlds) is to be found in the zymo-excitation by alkali of the reduction of hydrogen peroxid by colloidal platinum and oxidase of animal origin; for both of these reactions one may obtain a curve of the influence of increasing alkali content with a well-defined maximum, and for different alkali content also a curve of temperature influence with a well-defined maximum. Some instances if zymo-depression and zymo-stimulation will be considered in detail in connection with the discussion of particular fermentations.

Despite all extraneous factors, working with a proper constant concentration of substrate the velocity of reaction has been shown to vary proportionately with the mass of ferment in the case of trypsin, erepsin, lactase, invertase, lipase, rennin and hemase.

Station of Equilibrium.- We have to deal here with a most interesting phase of the question of fermentations. Pure positive catalyzers do not usually bring about any translocation of the station of equilibrium. When such a thing occurs in an inorganic cataly'sis it is because some one of the possible factors mentioned has intervened. But in the domain of fermentations we encounter a new state of affairs, namely, that reactions in themselves practically complete and unlimited, and which remain practically complete reactions when accelerated by inorganic catalyzers like acids, seem to become limited reactions when accelerated by ferments. Many ferments seem practically unable to carry through their accelerations to the point of complete conversion of substrate into products that is observed when inorganic catalyzers are employed. 'This is not true of all ferments, but of a great many, and the phenomenon is especially observed in the hydrolysis of starch, glucosids, and protein. 
The facts are as follows: A certain reaction when accelerated with hydrogen ions is practically a complete reaction. Let us say the condition of the system may be represented by the relations substrate 1 : products 99 . At this point the reactions in each direction are equal; the tendency to combination upon the part of the products is very slight since with all the mass of products the combination is only able to balance the cleavage of the substrate when its mass has fallen to 1 per cent. When this same reaction is accelerated by a ferment the cleavage will not be nearly so complete, and at the close the relations may be expressed something like substrate 15 : products 85. Does this apparent shifting in the point of equilibrium correspond to a real translocation of the station of equilibrium, to a change in the constant of equilibrium? 'There are many facts which tend to indicate that such may really be the case. 'The addition of more substrate to the system after the reaction has ceased to progress will serve to reinagurate the reaction. When products of the reaction are added early in the course of the experiment, the reaction will cease sooner than otherwise, and cease sooner proportionately to the quantity of products added. On the other hand, when the products are removed from the system, the reaction will be reinaugurated. Concentration or dilution of the volume will also disturb the apparent equilibrium, and an increase of temperature will cause the reaction to recommence in a system that had become quiescent. Finally, the addition of further ferment may reinaugurate the reaction, although it can easily be shown that an abundance of active ferment is still present in the mixture. The only possible direct interpretation of the last fact is that the ferment is reacting with the components of the system, probably with the substrate, and in a relation of proportion. By repeated additions of ferment it may be possible in some instances to eomplete the reaction, provided only that the initial concentration of the system was sufficiently diluted. These various facts are identical with those that hold for a translocation of the station of equilibrium in a true reaction of measurable reversibility, except that in these cases the further addition of catalyzer will not shift the point of equilibrium. The least difficult interpretation is that the ferment has entered into reactions with the components of the original system and that the station of equilibrium has been shifted thereby. The equilibrium is not determined by the substrate and the products alone, but by the substrate, the products, and the ferment. The observation has not been confined to ferments; the condition occurs with inorganic catalyzers.

\section{REVERSION OF FERMENT ACTION}

Theoretically it is possible to reverse every reaction if the appropriate conditions be secured. And, of course, this applies to catalytically accelerated reaction. The catalysis has only to do with the speed of the reaction, not with the direction. The direction of the reaction is 
related to the concentrations of the reacting masses, not to the catalyzer. Just as lubrication will make a locomotive run better either backward or forward, so a catalyzer will make a reaction proceed faster no matter in which way the reaction is proceeding. All this applies without qualification to enzyme reactions. But the attainment of the appropriate conditions for reversion of reactions is often very difficult and usually practically impossible. These difficulties are no more inherent in fermentations than in reactions between inorganic or organic substances. To reverse the reaction for the formation of hydrogen gas by the action of sulphuric acid on zinc, over sixteen atmospheres pressure of hydrogen gas is necessary. That in practice it has been impossible to reverse more than a few ferment reactions in nowise reflects upon the doctrine of enzymic reversion of reaction. When added to the inherent difficulties of the subject, we add the extremely unfavorable property of lability possessed by ferments in general, the wonder is not that so few ferment reactions have been reversed; the real wonder is that they have been reversed at all. The usual expression-reversed ferment action-is a misnomer. It is not the ferment action that is reversed; it is the direction of the reaction that is reversed and the accelerating action of the ferment makes this analytically demonstrable.

It is in the domain of the esters, the fats, that the most successful reversions have been accomplished. Here the chemical relations are relatively simple. Through the accelerating action of lipases of both plant and animal origin, many different esters, both natural and synthetic, and natural neutral fats have been formed in such amounts and under such conditions of experimentation as to exclude any other interpretation than reversed reaction made demonstrable by the accelerating influence of lipase. If these fats were not formed through the action of lipase, then fats have never been digested through the action of lipase; the procedures are identical except for the variation in the relations of concentration of the reacting bodies, and the results are indubitable.

Results have been attained also with carbohydrates, but the relations are very complex. Under influence of maltase it is possible to form a disaccharid from glucose. But the disaccharid is not maltose, it is isomaltose. Now isomaltose is a substance that may be formed from starch by acids and diastatic ferments. It may also be formed from glucose by acids. The formation of isomaltose instead of maltose by ferments is, therefore, not peculiar to the enzyme factor, since acid does the same thing. It is peculiar to reversion, under the conditions employed up to the present, but not peculiar to the accelerations of the reversion. It is incorrect to blame the ferment for the deflection, whose cause lies in some unknown conditions within the reaction. At the same time it is possible for a catalyzer or enzyme to modify the products of a reaction it is accelerating. For us the important fact is that under the influence of ferment, disaccharid is formed from 
primary sugar. Under the action of acids and ferments polysaccharidlike bodies have also been formed from hexoses. But the analytical findings are too inconclusive to mean much. The formation of amygdalin from mandel-nitril glucosid and d-glucose has been accomplished through the action of maltase.

Little success has been attained with proteins. The formation of plastein by pepsin cannot be regarded as a reversion. It has been possible with a trypsin to form from the products of the digestion of protamin a substance of the percentage composition, and general reactions of protamin. There is at present no way of knowing whether this substance be identical with native protamin or an isomer. By the action of pepsin certain of the digestion stages of casein have been reversed. Beyond this, all experimental investigations have yielded failures.

It is necessary in judging investigations of enzyme reversions to bear constantly in mind what is a general experience in organic chemistry. In the building up of complex substances, in syntheses, it is easy to develop side reactions; there are so many opportunities for reactions with materials that are so liable. In the disintegration of complex substances on the other hand, the tendency to side reactions is much less pronounced, the reactions tend to proceed with little modification to the components. Naturally these tendencies would hold with catalytic or enzymic accelerations of the same reactions.

\section{CATALYSIS IN HETEROGENEOUS SYSTEMS}

What has been said heretofore applies specifically only to homogeneous systems. Now in many reactions of fermentation, as well as in many organic catalyses, we have to deal with heterogeneous systems, and very important physical deviations are here presented. We have furthermore to deal with different combinations of conditions. The substrate may be solid, the solvent fluid and the catalyzer fluid. Or the substrate may be in homogeneous solution, while the catalyzer is solid. There are even conditions in which the products may be solid, as in the enzymic reaction in which amorphous sulphur is produced from hyposulphite. And, lastly, we have the condition, common in the world of living matter, of a suspended colloidal catalyzer accelerating the reaction of a colloidal substrate suspended in water.

Let us first consider the relations when the substrate is colloidal or solid, the catalyzer fluid. When such a body is suspended in water, the same relations hold that apply to the conditions of solution of a substance in water. Each particle of the solid is to be conceived as surrounded by an infinitely thin film of saturated solvent; and if the general bulk of the solvent be kept homogeneous by proper stirring, the velocity of solution will be proportional to the difference between 
the concentration of a saturated solution and of the particular saturation present in the particular moment, in accordance with the formula:

$$
\frac{d x}{d t}=k(C-c),
$$

$\mathrm{C}$ being the concentration of saturation and $\mathrm{c}$ that concentration actually present in a particular moment. The surface of the solid must be constant.

If, on the other hand, the substrate is fluid or soluble and the catalyzer solid the relations will be, so to speak, reversed. The reaction must be conceived to occur only at the film of contact of the particle with the solution, and the substrate must be brought to this film and the products removed.

Systematic authors have in general applied the law of mass action and the theory of the order of a reaction to heterogeneous systems, the formulæ being modified to meet the complicated conditions. Under such circumstances the progress of the reaction is held to take place only in the film of contact between the two phases, and is there proportional to the dimensions of the surface of contact; but otherwise it follows the general law and is proportional to the mass of the reacting body or bodies, it being assumed that the homogeneity of the general bulk of the solvent is maintained. It was assumed that the time required for the diffusion of the reacting bodies to and from the film of contact was rapid in comparison to the time required for the reaction. Obviously the conditions would vary depending upon whether the dimensions of the surface of contact are constant, increase or diminish. For metallic colloids the suspended particles may be assumed to be symmetrical, probably globular, and under these conditions there is a definite relation between quantity and surface; for ferments, however, the shape of the particles is probably amorphous and of no regular symmetry, so that here the alteration in the dimensions of the surface of contact with the progress in the reaction cannot be even surmised.

Under present conceptions of the nature of suspensions of the so-called stable colloids, such a colloid suspended in water forms two phases-a water-poor and a water-rich phase, in short, an aqueous and a colloidal phase. When a third substance is dissolved in such a two-phase system, it is distributed between the two. The substance will be taken up in two ways, partly by adsorption at the film of the colloidal phase, partly in solution in both phases. Now a portion of the substance that has entered into the colloidal phase is irreversibly bound; a larger portion, however, may be withdrawn. The coefficient of distribution of the substance in the two phases will depend in part upon the concentrations and in part upon the chemical relations that determine for different substances the extent of the irreversible combination. If, now, the colloid happens to be a ferment, and the dissolved substance the substrate of a fermentation, it is clear that the law of 
mass action cannot be applied to the reaction under the simple assumption that the velocity of the reaction is proportional to the active mass of the substrate and to the dimensions of the film of contact: In such an experiment, account must be taken of the factor of the coefficient of distribution, and also of the velocity with which this distribution is effected, since with the progress of the reaction, this would be of influence. If both the substrate and the ferment be stable colloids, the situation would be only the more complicated. The difference between the stable (hydrophilic) colloids, like starch and protein and the unstable (suspension) colloids, like the metallic suspensions, must never be overlooked; and they are associated with such pronounced differences in physical behavior that we are not permitted to apply directly to the stable colloids the results of investigations with unstable colloids. While, therefore, it must be conceded that the law of mass action cannot be applied to fermentations in the full theoretical sense, the actual question is: To what extent do the factors of the coefficient and velocity of distribution produce deviations in the operation of the law of mass action? This is a question for experimentation, and there is very little data bearing upon it. It is certain that the relations are different in different fermentations.

In many instances, however, it is clear that the velocity of reaction at the film of contact is much more rapid than is the diffusion of the reacting bodies to and of the products from the film. We may state this point of view in the contemplation of the velocity of reactions in heterogeneous systems, by amplifying the principle of the solution velocity to include the apparent velocity of reaction, and to define just what an experimental velocity in a heterogeneous system really means. There are obviously three main processes in such a reactionthe passage of the substance through the surface of contact between the two phases, the boundary film; the chemical reaction in one of the two phases; and diffusion to and away from the boundary film. The first process occurs with such rapidity as to have no influence upon the relations. If the reaction be rapid as compared to the diffusion, equilibrium will exist at the surface of contact; and if proper mixing be provided, the velocity of reaction represents simply the velocity of diffusion of substances to and from the surface of contact.

The point of view is best stated in the words of Nernst: "Many facts have led to the assumption that equilibrium is established with extraordinary rapidity at the surface of contact of two phases. Such a condition is indeed a theoretically natural assumption, because at the surface of separation of two phases, as infinitely approximated points, marked differences in chemical potential would appear, and these would obviously produce much chemical energy and lead to great rapidity of reaction. This means nothing more than in each moment the equilibrium is established very rapidly in the immediate neighborhood of the surface of separation. If one assumes, what is mathematically more probable, that the surface of contact is not a 
mathematical point but rather a narrow area of transition, we are nevertheless still concerned with dimensions of the order of the sphere of activity of molecular potentials; and though we can then no longer speak of an infinite velocity of reaction, we will still be dealing with such velocities as are for practical purposes infinite. When we consider a chemical reaction from this point of view, for example, the solution of magnesia in dilute acids, we assume that the magnesia is in each moment in equilibrium with the solution, $i . e_{\text {., }}$ the solution in immediate proximity to the magnesia is saturated and, therefore, alkaline. The diffusing acid will be entirely neutralized at the surface of contact; the velocity of solution of the magnesia depends solely upon the velocity with which the acid diffuses to the layer of contact of solvent and magnesia.

"In recent time the van't Hoff theory of the order of a reaction, $i . e$., the deduction of the number of reacting molecules from the progression of a reaction, has been often applied to reactions in heterogeneous systems. When one considers that this theory rests upon the calculation of the probability of the kinetic collision of two or more molecules in the gaseous state or in dilute solution, it becomes clear that there is no sense in its application to heterogeneous systems, or at least that there is for such application no theoretical foundation extant. The above-mentioned considerations, however, teach us that the application of the van't Hoff theory to heterogeneous systems is not only without direct foundation, but, indeed, entirely improper, because in reactions in a heterogeneous system, in so far as the reactions occur only at the surface of contact of the two phases, the velocity is partly or entirely dependent upon the velocity of diffusion, which has no connection with the order of reactions.

"A special instance of heterogeneous chemical reactions is afforded by the accelerations of reactions by colloidal catalyzers such as platinum-asbestos, Bredig solutions, etc. Since these reactions probably progress solely upon the surface of the catalyzer, the velocity will in no way depend upon the mechanism of the particular reaction; if the catalyzer maintains its integrity through the course of the reaction (which cannot be foreseen in advance), and carries through its reaction on the surface of contact with practically infinite rapidity, the velocity will depend upon the diffusion of the reacting bodies to the catalyzer."

That this reasoning need not hold true in chemical reactions of this type is illustrated by the electrolytic reduction of nitrobenzene; the reaction at the surface of the electrode was found to be slow compared with the velocity of diffusion to the electrode. As contrasted with the total denial of the application of the kinetic theory of the order of a reaction to a heterogeneous system, it may be pointed out that the Brownian movements in the particles of a colloid are proportional to the molecular movements postulated in a homogeneous solution by the kinetic theory. 'The general proposition that chemical reac- 
tions must occur with infinite velocity in the film of contact of two phases has also been denied on thermodynamic and experimental grounds.

While it is too early to pass judgment upon this theory, whose fundamental import to biology must be apparent, several general considerations may be pointed out. One is that the relations in fermentations will be much more complex, because we have here often a colloidal substrate and a colloidal ferment suspended in water instead of having a heterogenicity dependent upon only one member of the system. Since in the case of a colloidal substrate the reaction is held to occur on the surface of its particles, and in the case of a colloidal catalyzer the reaction is held to occur on the surface of its particles, when these conditions are united in one system, the reaction ought to be very slow; experience, however, has taught us that some of these reactions are quite rapid. Secondly, the reasoning is based upon the assumption that the solid body is so slightly soluble in the medium of the reaction that it does not participate appreciably in the diffusion. This condition will in all probability be found not to hold good for many of the pseudocolloidal substrates employed in fermentations. Thirdly, the theory assumes that the catalyzer is not altered in the course of the reaction, whereas in most fermentations the contrary is the case.

An argument against this theory lies in the temperature coefficient of fermentations. The velocity of chemical reactions is greatly accelerated by increase in temperature; the velocity of diffusion only slightly. Now in the case of most ferments an increase in temperature is followed by the increase in velocity that would be expected in a chemical reaction-it is usually more than doubled in $10^{\circ}$-far more than would be expected were a process of diffusion alone concerned. This suggests that there is a small portion of the colloidal substrate and colloidal ferment in true solution, and that the reaction is concerned with these.

Another objection is that proportional increases in viscosity in the fermenting system, due to the addition of different substances, ought to exert proportional retardations of velocity if that velocity express simply the diffusion velocity. This is not the case. Similarly, in heterogeneous reactions of known chemical nature, variations in concentration and viscosity ought to produce the same variations in velocity as in fermentation experiments; but this has not been observed to be true.

Lastly, it must be again pointed out that the term colloid does not correspond to a fixed quality, but to a tendency to physical qualities that is more or less pronounced in different substances. For Graham the colloid was the non-diffusible body, the crystalloid the diffusible body. Colloids confer upon their solutions or suspensions a very slight depression of the freezing point or elevation of the boiling point, and possess a very low osmotic pressure, all of which indicate that the work necessary to effect the separation of the colloid from the medium 
is small; with crystalloids the contrary is true. Like all suspensions, even the hydrophilic colloidal solutions display peculiar conditions of precipitation and coagulation, and are very active in all that relates to surface tension and adsorption. But it is not at all true that all chemical bodies belong to one or to the other of these classes. On the contrary, there are innumerable intermediary conditions, corresponding to all conceivable gradations from the typical crystalloid to the typical colloid. Not only this, many substances display attributes quite extreme; thus protamin will not crystallize, but does diffuse well and will trainsport an electrical current, while some higher proteins will crystallize and yet not diffuse with measurable rapidity; and some are quite insoluble in the true sense, while others are quite soluble. Under these circumstances, dealing with bodies displaying all degrees of gradation from the typical crystalloid to the typical colloid, it is difficult to foresee to what extent this theory based upon the qualities of practically pure heterogenicity in the system will bear the test of experimental application to fermentations. The hydrophilic colloids with which we are dealing in fermentations are in many respects different from the metallic suspensions to which the theory finds direct application. They react differently to electrolytes, resist precipitation by them, are indeed in some instances protected from precipitation by them, and they have the power to protect genuine colloids from precipitation by electrolytes. Under ultramicroscopic examination, the stable hydrophilic (or pseudo) colloids are seen to possess much smaller particles than the true or suspension colloids and corresponding to this they tend to display crystalloid tendencies-power of diffusion, osmotic pressure, etc. In fact, some of the proteins have as pronounced crystalloid properties as many dyestuffs (which are commonly considered as crystalloids), and scarcely more measurable colloidal properties. Obviously the hypothesis cannot be applied to such bodies with complete theoretical validity. That the conditions upon which this theory is based will often be of great influence in the progression of reactions must be conceded in advance; it is indeed a priori probable that in many instances the experimental velocity will be the result of both a true reaction velocity and the diffusion velocity; but whether the theory will afford an adequate explanation of all the phenomena is very much to be doubted so far as biological fermentations are concerned. The results in many instances correspond very closely with the requirements of the older law. In other instances, however, such conformity has not been obtained, and it may be precisely in these cases that the conditions stated have played predominating rôles. The velocity of cleavage of an insoluble fat by lipase represents a probable illustration of a diffusion velocity.

Another factor which must modify the relations in the complex fermentations of biological order is the relation of colloids to each other. The adsorption of stable colloids by one another is not solely a function of surface tension; it is in part at least a function of composi- 
tion. The adsorptions of a particular colloid by two different colloids even of the same surface tension are not identical. It is the usual teaching that colloids cannot diffuse, but the phenomena of adsorption throw grave doubt upon this statement. Colloidal hydrosols will penetrate hydrogels in a progressive linear manner. That this may not be a true diffusion, but rather a co-solution, a relation of the coefficient of distribution, possibly even a chemical combination with the formation of a new colloidal complex, is freely granted. But the fact will no less indicate that the phenomenon under whatever name it passes must constitute a variable in the reactions in such a system.

It is necessary further to consider the nature of the relations between the substrate and the ferment. We know on the one hand that colloids enter into complex chemical combinations with other substances. We have evidence that complex combinations of this sort occur in the body; such are the sugar-protein, the protein-fat, the nucleoprotein, the lecethin-protein complexes. These are destined to play a most important rôle in the chemical physiology of the future. As chemical combinations these complexes are subject to the laws of mass action, equilibrium, and partition. On the other hand, we know that colloids form with other substances physical equilibria that are termed adsorption compounds. Recent investigations seem to indicate that for the typical unstable colloids (metallic hydrosols, silicate, etc.) the laws of mass action, equilibrium and partition do not hold. For the atypical hydrophilic colloids (protein, starch, etc.) these laws tend to hold. It is fairly certain that for some reactions $(e . g$., the system dye-cellulose) the early occurrence of secondary reactions affecting the one component (the dye) is responsible for the non-fulfilment of these laws and the cause of the irreversibility of the reaction; such a phenomenon, however, does not constitute a fundamental exception to the laws. Concerning these matters we possess as yet so little quantitative data that definite conclusions are not warranted. Nevertheless, until we can conceive that two substances meeting in a thin film on the surface of a third indifferent substance react with infinite velocity, we shall be compelled to consider the combination of ferment-substrate to be of the nature of a chemical complex rather than of a physical adsorption compound.

In its broadest application, the fact that reactions occur with great velocity at the boundary of contact of two phases must appeal to everyone as a fact of the deepest biological significance. When we consider that the cells of the body in their relations to the circulating fluids, indeed the fibrillar and granular parts of cells in their relations to the intercellular fluids, represent precisely just such two-phase systems, we realize the magnitude of the factor with which we are dealing. From the physical point of view, the cellular constructions are colloidal phases, hydrogels; the body fluids watery phases, hydrosols. The magnitude of the dimensions of the boundaries of contact of the two phases in a human body is almost inconceivable; and the influence 
upon the velocity of a reaction under these circumstances contrasted with the velocity of a reaction in a homogeneous system of the same bulk must be enormous. Though it is a pure speculation, one cannot refrain from entertaining the thought that to this factor may be ascribed, in part at least, the great difference in velocity everywhere to be observed between reactions in vivo and in vitro.

\section{THE NATURE OF FERMENTS}

We know very little of the chemical nature of ferments. They are always associated with numerous bodies derived from the cells to which they owe their origin, and they are so labile that isolation is attended with denaturation and decomposition. We may say that ferments are proteins, or closely resemble them. This general conclusion is based upon the facts that ferments are usually coagulable; they respond to the color tests and reactions for protein; they are precipitated by the ordinary salts, and they yield on hydrolysis or digestion, to which they are all more or less susceptible, amino-acids. These observances are, however, alike in the case of no two ferments. Their physical properties likewise vary. Some are very colloidal, others diffuse. Some rotate the plane of polarized light, and are thus known to contain asymmetrical carbon; others are optically inactive. Some contain a carbohydrate moiety, and thus appear to resemble glycoproteids. Others contain a large amount of phosphorus in organic combination, on digestion yield purin bodies and thus appear to resemble nucleoproteids. In fact, the best studied of the animal ferments, pepsin, exhibits these qualities. Other ferments contain no nuclein or carbohydrate, are not coagulable, and resemble proteose. Thus, only the most tentative opinions may be passed upon the chemical nature of these bodies.

\section{THE MODUS OPERANDI OF FERMENTATION}

A discussion of the chemical basis of enzymic acceleration must in the nature of the phenomenon be based primarily upon a study of the nature of catalytic accelerations in general, and upon the demonstration of analogies and reactional relations existing between them. Since it seems certain from the thermodynamic point of view that a catalyzer or ferment acts only by direct or indirect reduction of the internal resistance, and not by any increase in the driving force of the reaction, all investigations must be directed to the internal physical and chemical resistance.

In the consideration of the modus operandi of the catalytic acceleration, we thus face directly the question of the nature of the internal resistance to chemical reactions that is a property of all substances. Since a catalytic acceleration is defined as an acceleration due to the 
lowering of the internal resistance of the substance, we must attempt to define a conception of chemical resistance. There has been little study of this aspect of the question. Of the two factors in every reaction-the driving force and the internal resistance-nearly all the physico-chemical research has been directed to the driving force. That the constitution of organic molecules is associated with variations in the resistance to reactions is, of course, well known. But the same factor of resistance resides in the most simple inorganic substance. In any event, it is certain that the term internal resistance stands not for one thing, but may stand for many things that are varied from case to case. Under these circumstances, therefore, the means whereby a catalytic agent lowers this resistance may vary from case to case, and must be studied anew for each individual reaction. One of the future achievements in chemistry will be to define an Ohm's law for chemical reactions.

There are several ways in which the presence of a catalyzer might accelerate the velocity of a reaction. Firstly, it might reduce the chemical resistance of a substance just as temperature does. Secondly, it might reduce the number of intermediate stages that occur naturally in the reaction. An illustration of this in the domain of inorganic chemistry is to be seen in the action of a cobalt salt on the reaction between $\mathrm{NaOH}$ and $\mathrm{Cl}$. This would amount to a short cut to the final product, and if the velocity of each step were no greater than in the ordinary reaction, the total velocity would be greater. Thirdly, new intermediary reactions might be introduced, of greater velocity, so that the sum of their velocities would be greater than the velocity of the original reaction. A quantitative illustration of this is to be found in the acceleration of the reduction of hydrogen peroxid by hydriodic acid under the catalytic influence of molybdic acid.

Theory of Intermediary Reactions.-The theory of intermediary reactions is not only the oldest theory, it has also in its favor a large amount of experimental evidence. The theory is in brief that a catalyzer accelerates the velocity of a reaction by the introduction of intermediary reactions with the formation of unstable products, usually of the type of addition products; that these products are themselves so unstable that they disintegrate of their own accord or they are disintegrated by the action of other bodies in the system; and that the sum of the velocities of the several reactions is greater than the velocity of the primary reactions. The theory has been tested solely upon catalyses of pure reactions, usually of inorganic nature. Thus far the evidence is almost entirely qualitative; in investigations upon the acceleration of the reaction between hydrogen peroxid and hydriodic acid by molybdic acid, however, the kinetic relations were worked out in such a manner as to demonstrate that the sum of the velocities of the several reactions is greater than the velocity of the natural reaction.

It is usually assumed that even the simplest reactions are not accomplished directly; they, too, pass through intermediary stages; and thus 
the catalyzer by the introduction of other intermediary stages does not in the least alter the general nature of the process of reaction, but by introducing intermediary reactions with lower chemical resistance effects a short cut to the stage of equilibrium. Experimental researches indicate that while in some of these accelerations the number of intermediary reactions is increased, instances are known in which the number of intermediary reactions has been diminished. In any event, be the number in the catalytic series greater or less, the theory assumes that the sum of their velocities is greater than was the velocity of the original reaction. Obviously the theory can be properly tested only upon a reaction for which we can determine the intermediary reactions with and without the catalyzer. These intermediary reactions are to be studied from the standpoint of the Ostwald law of reaction stages. In all chemical reactions the most stable condition is not attained at once, but either the nearest reaction is attained or among several possible reactions the most unstable, etc.

Thus, $\mathrm{CuSO}_{4}+2 \mathrm{KOH}=\mathrm{K}_{2} \mathrm{SO}_{4}+\mathrm{CuO}+\mathrm{H}_{2} \mathrm{O}$ passes through one intermediary stage.

$$
\begin{aligned}
& \mathrm{CuSO}_{4}+2 \mathrm{KOH}=\mathrm{K}_{2} \mathrm{SO}_{4}+\mathrm{Cu}(\mathrm{OH})_{2} \\
& \mathrm{Cu}(\mathrm{OH})_{2}=\mathrm{CuO}+\mathrm{H}_{2} \mathrm{O}
\end{aligned}
$$

When to such a system an appropriate positive catalyzer is added, new intermediary stages are introduced, termed for a certain large class of reactions, stages of primary oxids; the primary oxids have some of the behaviors of peroxids; they are stronger oxidizers than the highest stable oxidation stage and stronger reducers than the lowest oxidation stage.

$$
2 \mathrm{H}_{2} \mathrm{O}_{2}=2 \mathrm{H}_{2} \mathrm{O}+\mathrm{O}_{2}
$$

In the presence of ferric oxid this reaction is very rapid.

$$
\begin{aligned}
& 2 \mathrm{H}_{2} \mathrm{O}_{2}+\mathrm{Fe}_{2} \mathrm{O}_{3}=\text { iron primary oxid }\left(\mathrm{Fe}_{2} \mathrm{O}_{3} \mathrm{O}_{2}\right)+2 \mathrm{H}_{2} \mathrm{O} \\
& \mathrm{Fe}_{2} \mathrm{O}_{3} \mathrm{O}_{2}=\mathrm{Fe}_{2} \mathrm{O}_{3}+\mathrm{O}_{2}
\end{aligned}
$$

A reaction in more stages is as follows:

$$
\begin{aligned}
4 \mathrm{NaOH} & +2 \mathrm{Cl}_{2}=4 \mathrm{NaCl}+2 \mathrm{H}_{2} \mathrm{O}+\mathrm{O}_{2} \\
24 \mathrm{NaOH} & +12 \mathrm{Cl}_{2}=12 \mathrm{H}_{2} \mathrm{O}+12 \mathrm{NaCl}+12 \mathrm{NaClO} \\
12 \mathrm{NaClO} & =8 \mathrm{NaCl}+4 \mathrm{NaClO}_{3} \\
4 \mathrm{NaClO}_{3} & =\mathrm{NaCl}+3 \mathrm{NaClO}_{4} \\
3 \mathrm{NaClO}_{4} & =3 \mathrm{NaCl}+6 \mathrm{O}_{2}
\end{aligned}
$$

Here there are three stages of primary oxids, and each more "primary" than the succeeding one. When a cobalt salt is added the reaction is accelerated.

$$
\begin{aligned}
& 24 \mathrm{NaOH}+12 \mathrm{Cl}_{2}=12 \mathrm{NaCl}+12 \mathrm{H}_{2} \mathrm{O}+12 \mathrm{NaClO} \\
& 12 \mathrm{NaClO}+\text { cobalt salt }=12 \mathrm{NaCl}+\text { cobalt primary oxid } \\
& \text { Cobalt primary oxid = cobalt salt }+6 \mathrm{O}_{2}
\end{aligned}
$$


Here all the $\mathrm{NaCl}$ are formed in the first and second stages; that is, the number of intermediary reactions is reduced.

The reaction of potassium permanganate with hydrochloric acid has its direct expression in the following formula:

$$
\mathrm{KMnO}_{4}+8 \mathrm{HCl}=\mathrm{KCl}+\mathrm{MnCl}_{2}+4 \mathrm{H}_{2} \mathrm{O}+5 \mathrm{Cl}
$$

Platinic chlorid accelerates this reaction in the following way:

$$
\mathrm{KMnO}_{4}+4 \mathrm{H}_{2} \mathrm{PtCl}_{6}=\mathrm{KCl}+\mathrm{MnCl}_{2}+4 \mathrm{PtCl}_{4}+4 \mathrm{H}_{2} \mathrm{O}+5 \mathrm{Cl}
$$

The platinic chlorid having combined with the hydrochloric acid to form the chloroplatinic acid $\mathrm{H}_{2} \mathrm{PtCl}_{6}$, which is more rapidly reacted upon by permanganate than is hydrochloric acid.

The reaction between hydrogen peroxid and hydriodic acid is expressed in the following equation:

$$
\begin{aligned}
& \mathrm{H}_{2} \mathrm{O}_{2}+2 \mathrm{HI}=2 \mathrm{H}_{2} \mathrm{O}+\mathrm{I}_{2} \\
& \mathrm{H}_{2} \mathrm{O}_{2}+\mathrm{I}^{-}=\mathrm{H}_{2} \mathrm{O}+\mathrm{OI}^{-} \\
& \mathrm{OI}^{-}+2 \mathrm{H}^{+}+\mathrm{I}^{-}=\mathrm{H}_{2} \mathrm{O}+\mathrm{I}_{2}
\end{aligned}
$$

When molybdic acid is added to the system we have, expressed in its simplest terms:

$$
\begin{aligned}
& \mathrm{H}_{2} \mathrm{O}_{2}+\mathrm{H}_{2} \mathrm{MoO}_{4}=\mathrm{H}_{4} \mathrm{MoO}_{6} \\
& \mathrm{H}_{4} \mathrm{MoO}_{6}+2 \mathrm{HI}=\mathrm{H}_{2} \mathrm{MoO}_{4}+2 \mathrm{H}_{2} \mathrm{O}+\mathrm{I}_{2}
\end{aligned}
$$

The sum of the velocities of these reactions is very much greater than in the original reaction.

When hydrogen peroxid acts as an oxidizing agent it first combines with the substance to form an unstable peroxid-like body.

Thus,

And

$$
\begin{aligned}
& \mathrm{H}_{2} \mathrm{SO}_{3}+\mathrm{H}_{2} \mathrm{O}_{2}=\mathrm{H}_{4} \mathrm{SO}_{5} \\
& \mathrm{H}_{4} \mathrm{SO}_{5}=\mathrm{H}_{2} \mathrm{SO}_{4}+\mathrm{H}_{2} \mathrm{O}
\end{aligned}
$$

$$
\begin{aligned}
& \mathrm{Ag}_{2} \mathrm{O}+3 \mathrm{H}_{2} \mathrm{O}_{2}=\mathrm{H}_{4} \mathrm{Ag}_{2} \mathrm{O}_{6}+\mathrm{H}_{2} \mathrm{O} \\
& \mathrm{H}_{4} \mathrm{Ag}_{2} \mathrm{O}_{6}=2 \mathrm{Ag}+2 \mathrm{H}_{2} \mathrm{O}+2 \mathrm{O}_{2}
\end{aligned}
$$

Theory of Induction.-Closely related to the theory of intermediary reaction is the theory of induction. When two reactions are going on in the same solution, the presence of one may accelerate the velocity of the other. On close analysis it is seen that the actual process is one of intermediary reaction. All instances of reaction by induction are, of course, not catalytic, but many of them cannot be otherwise defined on account of the existence of a slow primary reaction. Theoretically, induced reactions may be divided into two groups: Those in which the intermediary products are stable, and those in which they are labile. In catalyses we have apparently to deal with those in which the intermediary reactions are labile. The two bodies in the reaction 
whose presence results in the acceleration (the primary, voluntary reaction) are termed the inductor and the actor, while the body whose reaction is induced to an acceleration is termed the acceptor, the actor being the same in each reaction. The intermediary body may be either a combination of the actor with the inductor, or it may be an unstable addition product of the actor or of the inductor. Good illustrations may be given from the group of oxidations, though the phenomenon is not at all confined to oxidations; it is, on the contrary, in all probability a phenomenon of widespread occurrence and importance. The simplest type is where the acceptor $\mathrm{D}$ is slowly reacting with the actor $\mathrm{A}$; when the inductor $\mathrm{I}$ is added it also reacts with the actor $\mathrm{A}$, and then the product reacts with the acceptor $\mathrm{D}$, as a result of which the actor $\mathrm{A}$ associated with $\mathrm{I}$ is transferred to $\mathrm{D}$; that is, I induces more of $\mathrm{A}$ to react with $\mathrm{D}$ than before.

$$
\begin{aligned}
& \mathrm{D}+\mathrm{A}=\mathrm{DA}-\text { very slow } \\
& \mathrm{I}+\mathrm{A}=\mathrm{IA}-\text { very rapid; then } \\
& \mathrm{IA}+\mathrm{D}=\mathrm{I}+\mathrm{DA}
\end{aligned}
$$

so that the velocity of the formation of $\mathrm{DA}$ is increased.

The reaction $\mathrm{SO}_{2}+\mathrm{O}=\mathrm{SO}_{3}$ is very slow. When in the system, however, the reaction ferrous salt + oxygen $=$ ferric salt is going on, the combination of the sulphur dioxid is greatly accelerated. The reaction between the iron salts acts as the carrier of oxygen; as fast as the ferric oxid is formed it is reduced by the sulphur dioxid.

$$
\begin{aligned}
\mathrm{SO}_{2}+\mathrm{O} & =\mathrm{SO}_{3}-\text { slow } \\
2 \mathrm{FeO}+\mathrm{O}_{2} & =2 \mathrm{FeO}_{2}\left(\text { possibly } \mathrm{Fe}_{2} \mathrm{O}_{5}\right) \text { rapid; then } \\
\mathrm{SO}_{2}+\mathrm{FeO}_{2} & =\mathrm{SO}_{3}+\mathrm{FeO}
\end{aligned}
$$

The reaction from the ferrous to the ferric salt must be kept going by an appropriate catalyzer. Many of the induced reactions are not so simple, in that there is no reaction between the product of the second reaction and the acceptor. For example, chromic acid, or its salts, is not able to oxidize tartaric acid with measurable velocity; it oxidizes arsenous oxid with great rapidity. When the two reactions are accomplished in the same system the tartaric acid is also oxidized.

\footnotetext{
Chromic acid + tartaric acid = formic acid and other acids - very slow

$\{$ Chromic acid + arsenous acid $=$ arsenic acid - very rapid

$\{$ Chromic acid + tartaric acid $=$ formic acid - rapid
}

Here the product of the primary reaction, arsenic acid, is stable; when the reaction is completed the arsenous acid is entirely oxidized, the tartaric acid in large part. 'The relations have, therefore, not been those of an oxygen carrier, as in the first illustration. 'The explanation is that some intermediary stage in the reaction chromic acid + arsenous 
acid provides the point of departure for the impetus of the second reaction. This may be represented as follows:

Chromic acid + arsenous acid $=$ intermediary product $=$ arsenic acid

Intermediary product + tartaric acid $=$ formic acid, etc.

Another illustration is furnished by bromic acid, which does not act upon arsenous acid, but reacts rapidly with sulphurous acid; when the reactions are associated, the arsenous acid is also oxidized.

As the subject of inductions is investigated, it becomes apparent that many of the accelerations by intermediary reactions are of this nature. All the activations of oxygen, in which the formation of peroxid-like bodies is probable, belong to the simpler reactions by induction. Indeed, the coupled reactions should be a subclass of the transformations by intermediary reactions. It will, on the contrary, not be possible to class all the catalyses as induced reactions, numerous and important as these certainly are. The process seems to follow one of two relations, depending upon whether the intermediary stage is stable or labile. And of the latter, the intermediary stage that acts as the accelerator to the induced reactions may be either a combination of the actor with the inductor, or a higher oxidation stage of the actor or the inductor.

A closely analogous condition in the organic world seems to lie in the phenomenon of the action of acids upon the formation of isomers of cinchonin. When cinchonin is exposed to the action of hydrochloric acid (or other halogens), one isomeric base is produced, the $a$-i-cinchonin, and the $\mathrm{HCl}$ addition product of cinchonin.

$$
\text { Cinchonin }+\mathrm{HCl}=\left\langle_{a-i \text {-cinchonin (transformation reaction) }}^{\mathrm{HCl} \text {-cinchonin (addition reaction) }}\right.
$$

Secondary reactions: $a-i$-cinchonin $+\mathrm{HCl}=\mathrm{HCl}-a$-i-cinchonin

The first idea would naturally be that the addition product represented the intermediary stage. However, it may be shown that the addition product with $\mathrm{HCl}$ is not to be converted into $\mathrm{HCl}$ and the isomeric base under the conditions of the experiment. When $\mathrm{HCl}$ and cinchonin are brought into a system two reactions occur, probably in definite proportions and in accordance with the law of mass action; the end products of the two reactions are, firstly, the $\mathrm{HCl}$ addition product of cinchonin, and secondly, the isomeric base, $a$-i-cinchonin. The first reaction or product acts in some way as the catalyzer for the second reaction, the transformation into the isomeric base. It is certain that the concentration of the hydrogen ions does not determine the velocity of the formation of the isomeric base. The accelerating influence of the side reaction of addition upon the reaction of transformation may be regarded as one of two procedures. Either some intermediary stage of the addition-reaction constitutes an intermediary stage of 
the transformation-reaction, $i$. $e$, in the series of intermediary stages of the addition-reaction is a point where the process may go on to the reaction of transformation, a point where the line of least chemical resistance lies in the direction of the transformation-reaction; or the two lines of direction are early separated and some product of the addition-reaction acts as a catalyzer to the transformation-reaction and produces with this reaction intermediary states that carry with them a heightened velocity of this second reaction. Most probably the first reaction alone, the addition-reaction, is an auto-reaction; the second reaction is not one that exists per se and is simply accelerated by the first reaction, the first reaction or its products actually calls the second reaction into being.

We meet here with an apparent contradiction of the statement that a fermentation is an acceleration of an already existing reaction. If in a catalysis or fermentation the end product is different than that yielded in the unaccelerated reaction, the relations suggest a reaction de novo. When the relations are carefully scrutinized, however, it seems clear that we are dealing not with a contradiction, but with an extension of the principle. Even though in some instances the end product be different in the accelerated and unaccelerated reactions, it is the existence of the primary unaccelerated reaction that makes possible the secondary reactions in the process of acceleration that yields the end products. In the domain of organic substances lability is so great and the possibilities of reactions so numerous that many possibilities for the installation of side reactions are presented in the catalyses and fermentations of such substances. This may be illustrated in the following scheme:

$$
\begin{aligned}
& \text { Substrate }+ \text { water } \rightarrow \mathrm{p}_{\mathrm{a}} \rightarrow \mathrm{p}_{\mathrm{b}} \rightarrow \mathrm{p}_{\mathrm{c}} \rightarrow \text { end product } \mathrm{A} \text { (auto-reaction) } \\
& \text { Substrate }+ \text { water }+ \text { ferment } \\
& \qquad \mathrm{p}_{\mathrm{r}} \rightarrow \mathrm{ps}_{\downarrow} \rightarrow \mathrm{p}_{\mathrm{t}} \rightarrow \mathrm{p}_{\mathrm{u}} \rightarrow \text { end product A (accelerated reaction) } \\
& \qquad \rightarrow--\mathrm{p}_{\mathrm{y}} \rightarrow \mathrm{p}_{\mathrm{z}} \rightarrow \text { end product } \mathrm{B} \text { (side reaction) }
\end{aligned}
$$

Obviously the silde reaction is not a reaction de novo, but is as essentially an acceleration (and deviation) of the auto-reaction as is the accelerated reaction that yields the same end product as the autoreaction. And were the entire trend of the reaction to take the side path and product $B$ appear as the sole end product, that fact would hold just as true. In many of the cases we are dealing with incompleted or superimposed reactions. Thus sugar may apparently be fermented to alcohol and to acetic and lactic acid. Now there can be little doubt that the lactic acid fermentation consists in the reaction as described for alcoholic fermentation checked at the stage of lactic acid; and the acetic acid fermentation is an oxidation fermentation of alcohol. Up to the present, therefore, we have no data tending to indicate that natural fermentations are ever reactions de novo.

In organic reactions intermediary products have been less often demonstrated, on account of the greater complexity of the relations. 
Some are, however, known. The first demonstrated instance (which is now known to be susceptible of marked catalytic acceleration) was contained in the formation of ether from alcohol through the action of sulphuric acid, ether-sulphuric acid being shown to represent the intermediary stage.

$$
\begin{aligned}
& \mathrm{H}_{2} \mathrm{SO}_{4}+\mathrm{C}_{2} \mathrm{H}_{5} . \mathrm{OH}=\left(\mathrm{C}_{2} \mathrm{H}_{5}\right) \mathrm{H} \mathrm{SO}_{4}+\mathrm{H}_{2} \mathrm{O} \\
& \left(\mathrm{C}_{2} \mathrm{H}_{5}\right) \mathrm{H} \mathrm{SO}_{4}+\mathrm{C}_{2} \mathrm{H}_{5} . \mathrm{OH}=\left(\mathrm{C}_{2} \mathrm{H}_{5}\right)_{2} \mathrm{O}+\mathrm{H}_{2} \mathrm{SO}_{4}
\end{aligned}
$$

When propyl alcohol is heated with sulphuric acid, a molecule of water is withdrawn; thereupon another molecule of water is added, though in a different way, so that isopropyl alcohol is formed.

$\mathrm{CH}_{3} \cdot \mathrm{CH}_{2} \cdot \mathrm{CH}_{2} \mathrm{OH}-\mathrm{H}_{2} \mathrm{O}=\mathrm{CH}_{3} \cdot \mathrm{CH}: \mathrm{CH}_{2}$ (propylen)

$\mathrm{CH}_{3} . \mathrm{CH}: \mathrm{CH}_{2}+\mathrm{H}_{2} \mathrm{O}=\mathrm{CH}_{3} \cdot \mathrm{CHOH} \cdot \mathrm{CH}_{3}$ (isopropyl alcohol)

The formation of acrolein from glycerol by heating is illustrated in the following series:

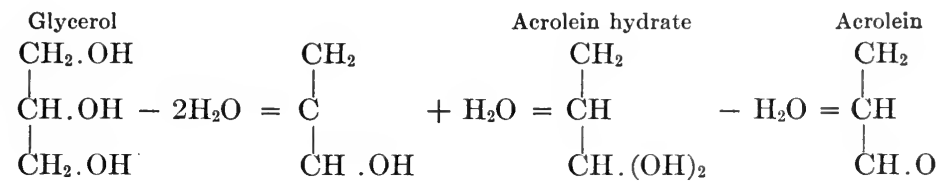

According to the now accepted theory, when water and carbon dioxid are in contact in the presence of sunlight formaldehyd is slowly formed, and the acceleration of this reaction is assumed to constitute the first step in the assimilation of carbon by plants. The reaction may be regarded as passing through the intermediary stage of formic acid.

$$
\begin{aligned}
& \mathrm{H}_{2} \mathrm{O}+\mathrm{CO}_{2}=\mathrm{H} . \mathrm{COOH}+\mathrm{O} \\
& \mathrm{H} . \mathrm{COOH}=\mathrm{H} . \mathrm{COH}+\mathrm{O}
\end{aligned}
$$

An interesting catalytic intramolecular transformation affords another good illustration. The ketone of $\mathrm{C}_{5} \mathrm{Cl}_{6} \mathrm{O}$ presents several isomers, and two of these in particular tend always to pass into each other and to establish an equilibrium in the mass. The reaction is apparently not direct, and although the intermediary body has not been isolated, the evidence seems to indicate that the reaction follows the following equation:


An additional illustration is furnished in the equations for the fermentation of $\mathrm{d}$-glucose to alcohol and carbon dioxid. There the one 
certain intermediary product is lactic acid, which has been confirmed; while none other is certainly known, a methyl-glyoxylic body seems recently to have been identified in the intermediary series.

Apart from the considerations adduced for alcoholic fermentation (and for the oxydases and peroxydases) there have been few studies of fermentations from the point of view of intermediary reactions. As a rule, the conditions are so complex and uncontrollable that we do well if we are able to estimate the march of the reaction and the nature of the final products, without even attempting the isolation of intermediary stages. In the fermentations of the hexoses, in the platinic accelerations of cleavages of carbohydrates, and in the reactions of hydrogen peroxid with organic bodies the relations promise soon to be sufficiently clear to permit of investigations from this point of view. We know that ferments are very labile bodies, that precisely such labile bodies have the tendency to enter into unstable combinations, and we may infer that in this very quality lies their adaptability to the acceleration of slow reactions. But this very lability makes the intermediary products very elusive and difficult of isolation, so that qualitative results are probably all that may be hoped for in the near future. Schoenbein spoke of the reactions of hydrogen peroxid as the "Urbild aller Gaehrung," and this terse sentence is yearly becoming more impressive. There was a time when the reactions of hydrogen peroxid were as little understood as are those of the common ferments today; and it is not too much to hope that as much progress may be made with the latter within the next decade as has been made during the last three decades upon the study of the catalyses with hydrogen peroxid.

Although we are not as yet able in concrete instances of fermentation, apart from alcoholic fermentation, to point out the intermediary reactions, we have an indirect argument for this theory in the fact that the ferment is known to combine with the substrate. While it is true in the general sense that the action of catalyzers is peculiar in this, that there is no stoicheiometric relation between the catalyzer and the substrate, it is, on the other hand, equally true that on the theory of intermediary reactions, during the moment of reaction there must be a stoicheiometric relation between them. The statement that there is no stoicheiometric relation between the catalyzer and the reaction it accelerates is true only in the relative sense that there is no stoicheiometric relation between the mass of the catalyzer and the mass of initial substrate or the final products. But so long as we locate the modus operandi of catalytic acceleration in intermediary reactions, there must obviously be a stoicheiometric relation between the catalyzer and the substrate in the moment of reaction. This is as true of colloidal platinum as it is of ferrous sulphate. It is the rapidity of the intermediary reactions, the putting-on and casting-off of the reaction, so to speak, that gives the gross appearance of absence of a stoicheiometric relation. Only on such a basis can the relation of degree of accelera- 
tion to mass of catalyzer or ferment be explained. It is most probable that when the common fermentations, the reactions of monomolecular order, are carefully studied, it will be found that in all the velocity of acceleration is proportional to the mass of the ferment. 'This is just what we should expect, since the only relations in the reactions are the masses of the substrate and the ferment. It might be assumed that the molecules of ferment $f$ each combined with one of substrate $s$. This would give us $f+s=f s=$ intermediary product (one or more) $=$ end product $+f$. This $f$ would then combine with another molecule of substrate, and the process be repeated. On the basis of this scheme, the degree of acceleration would naturally be proportional to the number of $f$, at least, within certain limits of relations of concentration.

There have been several other considerations urged to explain the accelerations of catalysis and fermentation. These theories do not exclude the proposition of intermediary reactions. The theory of ionization has been applied to this entire group of reactions. This hypothesis, though unsupported by much experimental evidence, is founded upon solid general considerations and regards the action of the catalyzer as a positive influence on the magnitude of ionization of the reacting bodies. The presence of the reacting body is considered to so alter the concentration of the active ions that the reaction is hastened. "Chemical catalyses depend upon alterations in the concentrations of one or more of the molecules that carry on the unaccelerated reaction, $i$. $e$. (by the application of the electro-chemical principle to the general field of chemistry), upon an increase (or a decrease) of the ions that participate in the reaction." (Euler.)

But, it cannot be believed that this alone can explain catalysis or fermentation, independent of the existence of intermediary reactions.

The views drawn from the studies of the catalytic action of colloidal suspensions of metals have tended to exaggerate the physical aspect of the subject. Granting unreservedly the accessory influences that the physical properties of colloidal suspensions (their enormous surface tension, etc.) may possess upon a reacting system, the fact remains that the catalytic influence of colloidal metals must, too, be attributed to intermediary reactions. The colloidal state must be held to endow the metal with activity in the chemical sense, to activate it in the mass sense. A gross suggestion of such a process is contained in the proposition that if a metal were chemically active in itself, the finer the subdivision of the metal in the system, the greater the surface exposed for contact. Thus copper and platinum are slightly catalytic in sheet form, enormously active in colloidal state. Whatever the process may be, we may be sure that the catalytic property of colloidal suspensions lies in the chemical activity of the substance in that state. The physical properties of colloids, especially of the stable organic colloids, are indeed difficult of definition and characterization, and almost impossible of control; but that is no reason why the "colloidal 
properties" should be blindly invoked as an explanation of whatever may appear obscure. The fundamental fact in the phenomenon of fermentation is a chemical act; and howsoever the physical conditions of the reacting substances and the system may modify that reaction in one direction or another, they cannot supplant the chemical reaction as the fundamental fact of the phenomenon.

Lastly, the specificity of enzyme action is in obviously harmonious relation to the theory of catalytic acceleration through intermediary reactions. In a word, both the occurrence of the fermentation per se and the specificity of this action are founded upon intermediary reactions.

\section{THE SPECIFICITY OF FERMENT ACTION}

In many text-books on physiology one meets with the statement that the specific limitations of the power of ferments, the ability to ferment but one or at most nearly allied bodies, constitutes a distinction between ferments and inorganic catalyzers. This statement is incorrect. There are many instances of quite specific action among inorganic catalyzers. For example, iron salts act as good catalyzers for the oxidation of potassium iodide by a persulphate; but they will not accelerate the reaction of the same persulphate upon sulphurous acid. Wolframic acid is an active catalyzer for the oxidation of hydriodic acid by hydrogen peroxid, but it will not accelerate the same oxidation by a persulphate. Platinum black is a good accelerator for the hydrolysis of esters of the simple alcohols, but it has no appreciable effect upon the hydrolysis of esters of glycerol. Laccase will accelerate the oxidation of many aromatic bodies, and the reduction ferments will accelerate many different reactions of reduction. A simple contemplation of the chemical relations concerned leads to the view that since these accelerations are to be regarded as founded upon intermediary reactions, whether a catalyzer or ferment acts or not will depend solely upon the particular reaction involved. The dissociated hydrogen ions are indeed quite general catalyzers for reactions, but they do not share this generality of action with many inorganic catalyzers. An interesting exception to the rule that hydrogen ions act as general catalyzers of hydrolyses is found in the observation that acids are not able to convert adenin and guanin into xanthin and hypoxanthin, though these are reactions of hydrolysis. All catalyzers may be said to be more or less specific; the inorganic catalyzers are less specific $(i . e$., have a wider range of availability in the inauguration of intermediary reactions) than the organic ferments. The specificity itself must theoretically be vested in the chemical relations of the intermediary reactions.

In a discussion of the specificity of ferment action we must distinguish between quantitative and qualitative specificity. By quantitative specificity we mean whether a ferment does or does not accelerate a certain reaction. By qualitative specificity we mean that a ferment 
not only accelerates a reaction, but so modifies it as to determine the chemical nature of the products.

In the beginning it must be pointed out that the fermentability of a certain body is only a relative term with a time limitation. When we say that a certain ferment will not act upon a certain substance, we usually mean that a test of several hours or days is made and the results then determined with a certain analytical precision. The accuracy of the observation depends upon the purity of the reacting bodies, the stability of the ferment, the length of time permitted, and the delicacy of the analytical procedures used to determine the occurrence of the reaction. It is apparent that a ferment could act in a positive manner, but that the acceleration might not be measurable under the chosen or necessary conditions of the experiment. In a strict sense one ought to demonstrate that the velocity of the reaction with the ferment of supposed inactivity is identical with the velocity in the simple system without ferment. For example, pentoses are not fermentable with zymase; but an appreciable quantity of alcohol and carbon dioxid could appear in such an experiment, derived from the glycogen contained in the extract of the yeast. Reviewing the reported work one is impressed with certain facts: As a rule, the time has been too short; the analytical methods for the determination of the occurrence of a reaction have often been crude; and the reacting bodies and ferments have rarely been pure enough to insure an unequivocal interpretation of positive or negative results. If the successful reversions of ferment action had been done in the routine manner of testing for ferment action, not a single one of the now demonstrated reversions would have been demonstrated.

Many ferments have but a limited range of activity; they are able to ferment but certain few substances. When we say that a ferment is able to act upon a certain body, we mean to a measurable degree. For example, trypsin is able to ferment protamin; pepsin is not able to ferment it. By this we mean that in a test of several weeks no demonstrable quantity of arginin may be recovered from the system. For many other ferments, however, the situation is different, in that a very slow fermentation occurs. Thus pepsin ferments reticulin with difficulty, trypsin with still greater difficulty, so that the current statement is that reticulin cannot be fermented with trypsin. In all probability the true statement would be that outside of the fermentations of the carbohydrates, which have been best studied, all statements of non-activity applied to ferments usually mean that under the conditions of the experiment, in the short life of the ferment, no appreciable reaction occurred; and it is not equivalent to the physico-chemical statement that such and such a ferment is not a catalyzer for such and such a reaction.

In the case of the sugars, however, the experimental data are much greater in amount, and of good quality. The fermentability of sugars rests upon their own stereoisomeric configuration and upon an appropriate assumed stereoisomeric configuration upon the part of the mole- 
cule of ferment. Certain yeasts will ferment only certain sugars and identical results are obtained with powdered yeast cells or their expressed juices. To make the experiments convincing, the configuration of the sugars under study must be undoubted, and to fill this requirement one employs for crucial experiment synthesized sugars.

The fermentability of a sugar depends, according to the now accepted hypothesis, upon the stereoisomeric configuration of its own molecule and of the molecule of the ferment. Many facts in the chemistry of the sugars indicate that the resistance to reactions and the reaction ability is allied not solely to the structural, but also to the stereo-isomeric configuration. 'The different hexoses present widely varying relations to the different compounds of hydrazin; the different osazones and hydrozones vary widely in their solubility, velocity of formation, stability, crystal properties, etc. The resistance of different sugars toward simple reagents like acids display also variations. Thus, maltose is most easily hydrolyzed by acid, cane sugar next, and lactose most difficult of all. Two molecules of d-glucose unite to form a disaccharid, maltose; but two molecules of d-levulose or of d-galactose do not unite to form disaccharid, though each of them will unite with d-glucose to form disaccharids, they do not unite with each other. From the point of view of fermentations as accelerations through intermediary reactions, the hypothesis is very feasible, since the configuration might naturally be supposed to be of dominating influence in such intermediary reactions. The specificity of the ferment lies in the coadaptation of the configuration of a particular ferment to certain sugars.

For the members of the benzol series a large number of instances are known in which reaction affinity is dependent upon or associated with a certain configuration. The location of radicals in a benzene ring determines often the resistance to chemical reaction displayed by that body, in that the substitution of hydrogen is not effected with the same readiness when the radicals occupy different positions. Thus substitution by sulphur radicals is easy in meta-xylol, less ready in ortho-xylol, and difficult in para-xylol. In the case of substitution by nitric acid on the contrary, as for instance in the action of nitric acid upon the isomeric nitrotoluols, the reaction is most easy in orthoand most difficult in meta-nitrotoluol. The oxidation of a lateral chain to a carboxyl group is likewise related to the configuration; ortho derivatives resist the action of chromic acid entirely, while para derivatives are quite susceptible; thus ortho-brom-benzylbromid is entirely refractory to chromic acid, while the para-brom-benzyl-bromid is easily oxidized. Salicylic acid is a much stronger acid than the paraor meta-oxybenzoic acid. Para- and ortho-xylol are easily oxidized, meta-xylol only with difficulty. Similar relations exist for the splitting off of the carboxyl group; in ortho- and para-oxybenzoic acid this may be accomplished by hydrochloric acid, which will, however, fail with the meta-oxybenzoic acid. In the case of the chlorhydrates of nitro-anilins, the dissociation varies; at ordinary temperature the 
ortho derivative is dissociated to 10 per cent., the para to 5 per cent., and the meta derivative to but less than 1 per cent. In an analogous manner, the catalytic action of metals in synthetic reactions with aromatic bodies illustrates a certain specificity; thus in the sulphuring of anthrachinons in the presence of the salts of mercury, sulpho acids of the $a$ series are formed in large part, which is not true in the presence of other heavy metals. Another illustration is the action of boric acid in the synthesis of poly-oxy-anthrachinons. Not only is there a relation of specificity between the configuration of the reacting aromatic body and the metal, there is also a specificity in the resulting product, and in a general sense under these circumstances these metals might be spoken of as catalyzers that not only accelerate the velocity of the reaction but also modify the products. Illustrations may be adduced from the chemistry of the benzene series in which the interreaction of two ring compounds is dependent upon an appropriate configuration of the two molecules.

A very striking illustration of the relations of reaction acceleration to configuration is to be noted in the recent studies in photochemistry. The sensibility to light upon the part of aromatic bodies is noted only in such bodies as possess a nitro group in the ortho- position to a $\mathrm{CH}_{2}$ group. Now many of these bodies are fermentable, and the fermentative accelerations bear similar relations to the configuration. Thus laccase will accelerate the oxidation of hydrochinon (para-dioxybenzol), but will not ferment the ortho- (pyro-catechin) or the meta-dioxybenzol (resorcin). Tyrosinase, furthermore, will ferment metatoluidin, but not the ortho- or para-toluidin, while it will ferment all three of the xylenes.

Other suggestive illustrations of the relations between configuration and reactionability are to be seen in the esterfication of different benzoic acids. The replacement of the hydrogen atoms in the ring results in a reduction of the tendency to the reaction due to the absence of the hydrogen, which accelerates reaction. The measurement of the reaction is accomplished by introducing the benzoic acids into an excess of methyl alcohol saturated with hydrochloric acid. But the relations of the different hydrogens are not identical. The carboxyl group being placed in the position of 1 in the ring, if the hydrogens are replaced from 2 and 4, or from 3, 4, and 5, over 95 per cent. of ester will be formed; while if the two hydrogens adjacent to the carboxyl group are replaced, at 2 and 6 , or at 2,4 , and 6 , almost no ester, less than 5 per cent., will be formed. Of influence further is the mass of the radicle that replaces the hydrogen in the ortho position to the carboxyl group; thus bromin and iodin with their heavy molecular weights depress the esterfication, while methyl has but slight inhibitory effect. Fumaric acid does not tend to the formation of the anhydrid while the isomeric maleic acid does tend to the formation of the anhydrid; this may be ascribed to the fact that in maleic acid the carboxyl groups are adjacent, while in fumaric acid they are separated. This relation of the tendency to anhydrid formation to the relative situations of the carboxyl groups seems to hold in many compounds. 
Since internal configuration of the molecule is of such striking influence on reaction properties, this will apply to two molecules in reaction. In other words, that which is true of the substrate must hold true for the ferment. The molecule of ferment we know to be very complex. That it has the same relations of reaction to internal configuration, we cannot doubt. And for both ferment and substrate, the reaction properties may lie either in a relation of the configuration to the internal resistance or to the factor of chemical affinity; for the acceleration of the reactions of the substrate by the ferment, however, we must hold that the specificity of the ferment lies in a reduction of the internal chemical resistance of the substrate through the introduction of specific intermediary reactions.

Many substances present in addition a particular form of variation in the intramolecular configuration, namely, stereo-isomerism, due to the presence of asymmetric atoms of carbon. The sugars are such bodies, also the amino-acids found in proteins, with the exception of glycocoll. The ferments are protein-like in nature, and are certainly stereo-isomeric.

Among the reaction possibilities of an asymmetric substance, we might most reasonably suppose that some reaction tendencies as well as resistance to reaction might be vested in the stereo-isomeric configuration, and this would also correspond to the stereo-isomeric configuration of the second reacting molecule. This relation of the two reacting molecules Fischer compared to the relations between a lock and key; and as a purely symbolic illustration he compared the lateral recessions of the lock and the lateral projections of the key to the lateral arrangements of the elements upon the carbon chain of a sugar molecule. This means simply a correlation favorable to interreaction in the purely chemical sense.

Certain configurations endow the molecule with a marked resistance to the reaction; the ferment modifies this internal resistance.

There are sixteen possible stereo-isomerids of hexose. Of these twelve have been isolated from natural sources or synthesized. Of these but four are susceptible of alcoholic fermentation by symase: d-glucose, d-mannose, d-levulose, and d-galactose. For purposes of illustration, the stereo-isomeric configurations of these will be given, together with that of d-talose, which is not fermentable.
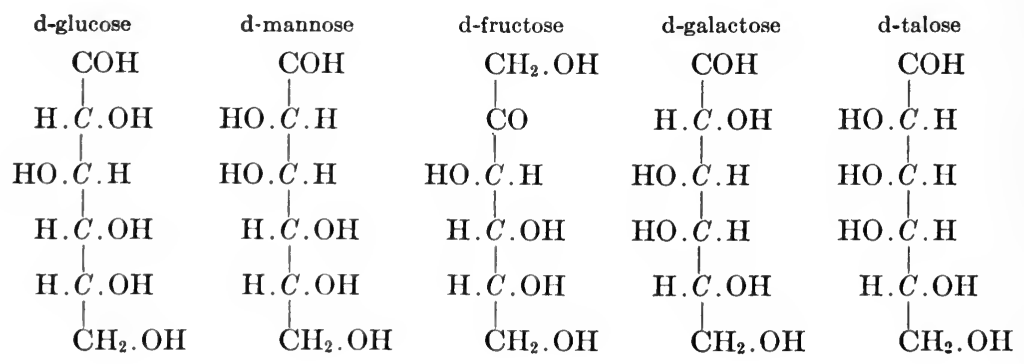
The d-glucose, d-levulose, and d-mannose are identical in this, that the relations of the three asymmetric atoms of carbon that are common to them all are alike. The other asymmetric atom in the d-glucose and d-mannose seems to be of no determinable influence. In the molecule of the d-galactose there is the difference from the other three, that in the centre one of the three asymmetric atoms of carbon common to them all, the centre carbon has the relations of hydrogen and hydroxyl reversed laterally. The upper asymmetric carbon is identical in its relations with the corresponding atom of the d-glucose, but has the reverse relation of lateral attachments possessed by the d-mannose. Nevertheless, these differences are not determinating, since d-galactose may be fermentable by the same yeasts, though some yeasts cannot ferment it at all, and all do so with slower velocity. It is, therefore, not the situations of individual hydroxyls that determine the fermentability, but the total combination. Thus, d-talose, which is non-fermentable, resembles d-mannose in the relations of the upper asymmetric carbon, d-galactose in the relations of the two middle asymmetric carbons, and all four in the relations of the lower asymmetric carbon. A direct rule is obviously not contained in these facts, but somewhere in the series of asymmetric carbon atoms of d-talose is a high passive resistance not encountered in the others, while somewhere in the series of asymmetric carbons in d-galactose is a certain degree of passive resistance not met with in the other three. There can be no doubt that the facts suggest a quantitative rather than a qualitative difference.

It has been suggested that the fermentability of the hexoses lies in their being convertible into d-glucose; only d-glucose is fermentable, and those sugars only subject to fermentation that are convertible into d-glucose. That these hexoses may be easily converted into each other through the action of alkali has been shown. The formation of $\mathrm{d}$-levulose from $\mathrm{d}$-glucose is according to recent investigations not confined to experimental conditions but occurs in every hydrolysis of starch, arising not from the starch directly but by derivation from d-glucose.

Clearer relations are secured in the fermentation of natural and synthetic glucosids. When aldo-hexoses are heated in alcoholic hydrochloric acid, glucosids are formed. Of both the d- and l-sugars two isomers are formed, termed the $a$ and $b$ series. Thus for methyl alcohol we have the two isomers of d-glucose:

The glucosids of the l-series of glucose, mannose, and galactose are not fermentable at all. The glucosids of the $d$-series of glucose, mannose, and galactose are fermentable, and to a noteworthy degree specifically. The $a$-glucosids of these d-hexoses are fermentable with yeast, the $b$-glucosids are fermentable with emulsin. The natural glucosids are $b$-glucosids, so that the concordance between the natural and the synthetic glucosids is complete. It is not the zymase in the yeast that accelerates these reactions; it is the maltase. The data bearing on these synthetic glucosids is very positive, and suggests strongly the validity of specificity.

Very instructive is the study of the tryptic digestion of synthetic peptids. Of the synthetic peptids that have been prepared many are digestible with trypsin. When racemic peptids are digested with trypsin, the cleavage is an asymmetric one; and the amino-acid that is split off is the same active amino-acid that is to be found among the products of the digestion of natural protein by trypsin. There are two racemic alanyl-leucin peptids, and they include the four possible combinations of the two components. Thus, alanyl-leucin A is d-alanyl-l-leucin + l-alanyl-d-leucin; while B is d-alanyl-d-leucin + l-alanyl-l-leucin. Only A is digestible, and of the compound only the d-alanyl-l-leucin is split off, and the two components separated. These facts have all the more weight, because they have been obtained with a class of compounds totally different from the sugars.

According to the theory, since the velocity of reaction under the influence of a ferment depends upon the stereo-isomeric configuration of the ferment molecule, the influence of organic acids upon the same reactions might depend upon similar relations. But when cane sugar is hydrolized by d-and l-camphoric acid, the acceleration is identical for the two, $i$. e., the acceleration depends upon the electrolytic dissociation, and not upon the optical isomerism.

Another interesting point from which to view the theory lies in the fermentation of racemic acids. Both the $\mathrm{d}$ - and l-acids are fermented, but with different velocities. In some few instances the reactions upon the two enantiomorphic bodies are quite equal, in other instances there is a distinct predominance; in a few instances the reaction with the one is marked, with the other very slight. The greater velocities are in the direction demanded by theory of specificity. The temperature optimum is different for the $\mathrm{d}$ - and l-acids. If these results may be applied to the fermentation of sugars, it indicates that the specificity may be only one of degree; not a question of reaction or no reaction, but of slight reaction as against pronounced reaction.

Finally, one may study the cleavage of optically inactive racemic synthetic esters by lipase. The products are found to be rotary in one direction, and the residual ester is rotary in the opposite direction. In a word, the cleavage is an asymmetric process. But it is only a difference in velocity, not a qualitative differentiation. The fact corresponding to this is to be found in the observation that two optically 
opposite active acids do not form ester with an optically active alcohol with the same velocity. The unequal velocities may be explained as follows: When optical isomers combine separately with the same structurally asymmetric substance, they may do so with unequal velocities; and conversely, the products formed by such reactions, since they are no longer optical opposites, might be expected to undergo further changes at unequal velocities. If the enzyme be supposed to be dextrorotary and the components of the racemic acid be represented by $+\mathrm{S}$ and $-\mathrm{S}$, the additive compounds formed by the union of the ester and the ferment would be $(+\mathrm{e}+\mathrm{S})$ and $(+\mathrm{e}-\mathrm{S})$. These two compounds are obviously not enantiomorphic (opposite compounds would be respectively $(-\mathrm{e}-\mathrm{S}$ ) and $-\mathrm{e}+\mathrm{S}$ ), and might therefore be expected to be formed and undergo changes at different velocities. Ferments are apparently sometimes active, sometimes racemic-substances. If a racemic ferment react with a racemic substrate, we would expect the formation of enantiomorphic complexes of ferment-substrate, and under these circumstances we should expect the reaction relations to be identical. Obviously, therefore, we should expect an active acid to act in a different manner from a racemic or an inactive acid.

The reason a ferment is elective must be ascribed to the fact that configuration means resistance to the reaction or that it means resistance to the catalyzer.

The question of qualitative specificity of ferments has a narrower interest, though it is one of importance. Do the different accelerators of a reaction yield the same products, or may they yield different products? It is obvious that with a pure catalyzer, a body that simply diminishes the chemical resistance of a substance, the nature of the products would not be altered. But in the frequent atypical fermentations, where the ferment is often more or less altered in the course of the reaction, some alteration in the products might be expected. Such alteration might, however, not always be properly attributed to the catalytic acceleration. For example, a certain reaction constitutes a hydrolysis, with the product $z$. The ferment enters into reactions with $z$, and as a result $y$ is produced. Here we have a new reaction, one not connected with the acceleration, but one that would have occurred were we to mix $z$ and the ferment. The reaction constitutes a new reaction, a secondary reaction in the system. An illustration of this is seen in the transformation previously described of cinchonin into the isomeric base, $a$-i-cinchonin under the action of hydrochloric acid. The product slowly adds hydrochloric acid; this is a secondary reaction having no relation to the accelerated transformation of the cinchonin into the isomer, but simply a reaction between the $a-i$ cinchonin and $\mathrm{HCl}$ such as would occur were these two mixed in a solution. A further possibility lies in the fact that the products may react among themselves to form new bodies, which are, of course, not to be classed as products of the fermentation. For example, arginase 
accelerates the hydrolysis of arginin to urea and ornithin. The fermentation proceeds well at a slightly alkaline reaction. Now the alkali accelerates the hydrolysis of urea to carbon dioxid and ammonia. The appearance of ammonia in the fermentation of arginin by arginase is, therefore, not a product of this fermentation. We must thus bear in mind the possibilities of secondary reaction-reactions between the product and ferment, between product and solvent, between products and some extraneous substance, and between products and products. An excellent illustration of the relations to an extraneous body is furnished by experiments on the chemical fermentation of d-glucose. When glucose is exposed to sunlight in the presence of a trace of sodium hydroxid, ethyl alcohol is produced; when calcium hydroxid is employed lactic acid is produced. Now, since the accelerator is the hydroxyl ion, which is the same in each system, the difference in product must be due to an action of the calcium as against the sodium. It is clear, therefore, that only under conditions of experimentation with pure substances can we determine whether different ferments produce different products. Practically all the studies in connection with animal ferments may be regarded as worthless from this point of view.

Now theoretically a ferment must be conceded the power of modifying the reaction that it accelerates, of accelerating side reactions as well as direct reactions. There are undoubted instances in which ferments modify the course of the reaction in the qualitative sense. The illustrations that at once come to mind are the different results that may be secured with fermentations with pure cultures of microorganisms under different conditions. But these instances cannot be directly quoted in a chemical discussion, because of the complexity of the relations. We have definite chemical illustrations. The hydrolysis of hydroxylamin in alkaline solution follows the equation: $3 \mathrm{NH}_{3} \mathrm{O}=\mathrm{NH}_{3}+\mathrm{N}_{2}+3 \mathrm{H}_{2} \mathrm{O}$, while the acceleration of the reaction with platinum follows the equation: $4 \mathrm{NH}_{3} \mathrm{O}=2 \mathrm{NH}_{3}+\mathrm{N}_{2} \mathrm{O}+3 \mathrm{H}_{2} \mathrm{O}$. The reactions of hydrazin also illustrate the modifications that may be effected by other relations in the system. Thus in simple watery solution the reaction with platinum runs: $2 \mathrm{~N}_{2} \mathrm{H}_{4}=2 \mathrm{NH}_{3}+\mathrm{N}_{2}+\mathrm{H}_{2}$. while in the presence of an alkali the reaction is: $3 \mathrm{~N}_{2} \mathrm{H}_{4}=2 \mathrm{NH}_{3}+$ $2 \mathrm{~N}_{2}+3 \mathrm{H}_{2}$. Illustrations also are to be observed in the ferment and acid reversions. When lactase is allowed to act upon equal parts of $\mathrm{d}$-glucose and d-galactose a disaccharid is formed; this is not lactose, as would be expected since the synthesis is the ferment reversion of lactose cleavage; the sugar is the isomer isolactose. The same fact holds true for the synthesis by ferment action of disaccharid from $\mathrm{d}$-glucose by maltase; the sugar is not maltose, but the isomer isomaltose. These modifications are best explained as the result of qualitative alteration of the acceleration by the ferment. 


\section{CH A P T E R I I I}

\section{DIGESTION}

Tue functions of digestion are twofold: the conversion of large non-diffusible molecules into small diffusible molecules, and the cleavage of complex molecules into simple bodies in order that these may be utilized in the various metabolic processes of the body. To pass the wall of the alimentary tract a substance must be diffusible. The starches, fats, and proteins are non-diffusible; the disaccharids and primary sugars, that are constituents of a natural diet, are, however, diffusible. On digestion the starches are converted into the component sugars, which are diffusible. Following digestion of the fat, the component parts, glycerol and the fatty acids, are diffusible. On digestion of the protein the resulting amido-acids and peptids are diffusible. It is immaterial to the present argument whether the velocity of the processes of diffusion of these several substances can be explained at present wholly on physical grounds; it is, however, practically a fact that a non-diffusible body does not pass the wall of the alimentary tract. It is not to be denied that under certain conditions, a colloid may pass the intact intestinal wall; but this does not occur to any demonstrable extent in the course of digestion. The second function of digestion is of especial importance to the metabolism of protein. The common vegetable fats are glycerids of the same fatty acids as exist in the animal body; there is no chemical peculiarity known there. The several carbohydrates of the diet are converted in the body into one form of sugar, d-glucose, which is identical with the sugar of the blood. The proteins must be dismembered in order that the body may build of them its own peculiar proteins. In a certain sense, the metabolic acts of the human body are not neo-constructions but re-constructions; and for these re-constructions it is necessary that the foods be reduced to simple units. These units are relatively small and diffusible substances, and from them the body builds its tissues. For the heating of the body the same general truth holds, the body does not burn the polysaccharid, the protein, or the fat; these must first be split. And looking at the matter by and large, the forms in which these are available for the combustions of the body are identical with the states in which they are absorbed following digestion in the alimentary tract.

From a contemplation of the chemical processes that occur in the cleavage of proteins, carbohydrates, and fats into their component bodies, it is clear that digestion is a reaction of hydrolysis. All the ferments of the alimentary tract are ferments of bydrolysis. It will 
not be denied that enzymes of oxidation or reduction occur in the juices of the alimentary tract (though such have never been properly demonstrated); but if present they have no known function in the reactions of digestion.

Of great importance in the physiology and pathology of digestion are the presence and metabolic activities of bacteria. The relation of abnormal bacterial infection to the etiology of alimentary disease requires no elucidation here. The question whether bacteria are physiologically of advantage in the alimentary tract has been attacked by direct experiment. The guinea-pigs that were first brought aseptically into extra-uterine life and kept thus, were apparently normal during the two weeks of observation. Later experiments on fowl and frogs, extending over long periods of time, seem to have shown that growth and nutrition are not normally maintained with a sterile alimentary tract. The under-nourishment of the animals quickly subsided when bacteria were added to their food. 'The function of bacteria in this connection is not clear. It surely does not lie in the direction of the chemical reactions of digestion. It is possible that it lies in the same direction as the freshness of foods, so necessary to proper nutrition in man, in the sense that bacteria elaborate the same substances that are present in fresh vegetables and fresh meats. The whole question is, however, in such a state of obscurity that discussion is not possible; it is only necessary to mention the experimental fact that normal nutrition is apparently not possible with a sterile alimentary tract.

\section{DIGESTION IN VITRO}

Digestion experiments in glass present, in addition to obvious conveniences, the direct advantage over digestion tests in a stomach or loop of intestine, that the products of digestion are not removed from the system and are thus arailable for analysis. This objection does not negate gastric tests as much as intestinal experiments, since the processes of resorption are minimal in the stomach and very active in the intestine. It is, however, this retention of the products of digestion in the system in glass that is responsible, in part at least, for the low quantitative yield commonly observed in such tests. The accumulated products of the reactions check the action of the ferments. It can be shown in direct experiment in the digestion of synthetic peptids with trypsin that the presence of amino-acids checks the digestion. On the other hand, experiments in glass are at a disadvantage in that it is impossible to duplicate in the test all the natural conditions that surround an act of digestion. The psychic concomitants of digestion are of great influence and importance, not only as to the rate and amount of the secretion of the juices of digestion, but also as to the composition of the juices of digestion. To procure pure juices of the digestive glands, we must, in one form or other, practise mock 
feeding; and there is no question that juices so secured cannot be held to even approximately correspond to those formed and operative in normal feeding and digestion. There are, furthermore, great personal variations, which naturally effect the results in tests in glass more than in the body. "Attempts to make the tests in the animal body directly comparable to those in glass by preventing resorption (as by ligation of the orifices of the stomach and of its bloodvessels) are totally futile. On the whole, we may say that the results of experiments in glass are qualitatively comparable to those in the body, but quantitatively they are very defective.

The results of digestion tests in glass have for the most part given direct and indubitable results for the fermentations of carbohydrates and fats-in which the results were of lesser importance. In the case of the digestion of proteins, which is of the greatest practical and theoretical importance, it has taken much time and elaborate experimentation to yield data that are adapted to the solution of the numerous physiological problems related to the subject. To the present fairly satisfactory state of knowledge, the study of the digestion of synthetic peptids has contributed greatly. We will here review concisely the fermentative activities of the different enzymes, leaving the discussion of the conditions of activity, that are of importance to an understanding of the processes of digestion, to the section devoted to digestion in vivo.

The salivary ferments are two, amylase and maltase. Amylase acts to accelerate the cleavage of starch into maltose, the reaction passing throughout various stages of dextrins. All starches are susceptible to the action of salivary amylase; the different degrees of resistance noted experimentally are probably due more to differences in methods of preparation than to differences in origin of starches. Raw starch is hydrolyzed with extreme slowness. After the polysaccharid is converted into maltose under the action of the amylase, it is then converted rapidly into glucose under the influence of the maltase. The two ferments are entirely distinct, the substrate of the reaction of the maltase is the product of the reaction of the amylase. Neither ferment has any qualitative influence on the sugars, these are simply set free, as they existed preformed, by the reactions of hydrolysis accelerated by the ferments amylase and maltase.

The stomach has no ferments for the digestion of carbohydrate. In the secretion of the pancreatic juice are amylase and maltase, that in all respects compared with those of the saliva. The juice of the pancreas also contains lactase but no invertase. These accelerate respectively the cleavage of the disaccharids cane sugar and milk sugar, into their component hexoses. All the four ferments are contained in the succus entericus, which in all known ways operate precisely as do the ferments of the pancreatic juice. For all these ferments it is known that they are able to accomplish no qualitative changes in the sugars, they simply set free, by acceleration of the reactions of hydrolysis, the sugars that exist preformed in the disaccharids and 
polysaccharids. The alimentary tract contains no enzymes in its secretions that ferment or act in any way upon the primary sugars; the simple conversion of polysaccharids and disaccharids into primary sugars is the full chemical function of amylase, maltase, invertase, and lactase.

The gastric secretion contains a lipase. When this is allowed to act in a faintly alkaline reaction, it accelerates the cleavage of neutral fats, and of the complex phosphatids, into glycerol and the fatty acids. The pancreatic juice and the succus entericus contain each a lipase, of identical properties. That these juices do not operate very well in tests in glass is due in part to the lack of zymo-excitors contributed by the bile in intestinal digestion. These ferments in no way modify the chemical nature of the products of the reaction they accelerate, they simply set free the glycerol and fatty acids that exist preformed in the neutral fats and phosphatids whose hydrolysis they accelerate. It is ferments of the type of lipase, amylase, and invertase that closely follow in their manifestations the laws known to exist for catalytic acceleration in general.

Reverting to the scheme for the internal constitution of the protein molecule, the catalytic activities of the several proteolytic ferments may be briefly stated. It is easily possible that future studies may radically modify our present conceptions.

Pepsin.-Pepsin digests all the native proteins with the exception of the protamins. It splits them, however, only to the stage of the peptone. It cannot cleave peptones, it cannot set free amino-acids. The synthetic peptids are not split by pepsin. So far as is to be seen, the cleavage of protein by pepsin is exactly like that of acids as far as the stage of peptone. Different native proteins present different degrees of resistance to pepsin. Thus keratin is very resistant. But differences may exist even between nearly related proteins; thus serum globulin is much more difficult of digestion by pepsin than serum albumin. Coagulation increases the resistance of some proteins to peptic digestion. Dried protein is very resistant to digestion by pepsin.

Trypsin.-The secretion of the pancreas digests nearly all native proteins, including the protamins. Collagen and reticulin are refractory, and keratin, native serum globulin, and egg albumin are very resistant. Coagulation does not increase the resistance of proteins to tryptic digestion. Peptones are easily digested, at least certain ones; and amino-acids, especially tyrosin, appear very early in the course of a tryptic digestion, at a time when the larger portion of the mass is still in the stage of proteose. The digestion of protein by trypsin goes completely to the stage of polypeptids. Beyond this, while many polypeptids are completely digested, others are refractory; these have proceeded from what used to be called the antipeptone that has long been known for its resistance to trypsin. The fraction of polypeptid that resists tryptic digestion varies with different proteins, ranging from 20 to 40 per cent. of the whole. This fraction itself is, 
however, not a unit, but consists of two parts, one relatively and the other absolutely resistant. One may digest a protein a week with trypsin, and at the end of this time find 50 per cent. of the nitrogen in the form of polypeptid. If this digestion be prolonged for several months, it will be found that this will be reduced to a fifth or tenth of the total, but beyond this point no further digestion can be accomplished. 'These antipolypeptids are different for different native proteins; but when split by treatment with strong acid, they yield in particular glycocoll, prolin, and phenylalanin, usually with glutamic and aspartic acids, lysin, and arginin. (Cf. page 47.)

A tryptic digestion that is preceded by a peptic digestion seems to yield in part different results than those observed in a direct digestion. If a preliminary digestion with pepsin has preceded the action of trypsin, some of the amino-acids that are not set free in a direct tryptic digestion will be set free in part. There will be more tryptophan, glutamic acid, lysin, and arginin set free; and of the total content of prolin, which is not set free at all in a direct digestion, some will be found free. The difference, however, is largely a quantitative one. Evidently the pepsin, that in itself cannot digest peptones, in some way modifies some of them so as to afford to trypsin an entrance into groups that otherwise are refractory to it.

Trypsin digests many synthetic peptids. The relations are very complex. The cleavage is asymmetric. Such peptids as are split by trypsin always contain the two or more components of the same stereoisomeric configuration in which they exist naturally; if one or more of the component amino-acids presents a stereoisomeric configuration that is not to be found in a naturally existing amino-acids, the peptid will not be split. But the converse does not hold; peptids composed only of amino-acids present in the stereoisomeric configuration seen in natural amino-acids are not all digestible by trypsin, some of them are digestible, others are not. Another fact in this connection indicates that analogous relations hold in the digestion of native proteins by trypsin. It is possible to racemizate asymmetric amino-acids within the protein molecule by the action of alkali, in fact, the conversion of the $\mathrm{d}$ - or $\mathrm{l}$ - form into the racemic state occurs more easily when the amino-acids are bound in protein than when free. Proteins in which the amino-acids have undergone racemization are either resistant or entirely refractory to tryptic digestion.

Trypsin can only split the peptid binding; it cannot split the two molecules of amino-thio-propionic acid that form cystin, it cannot split arginin into ornithin and urea. Neither can it split the analogous binding of glycocoll to benzoic acid in hippuric acid.

Erepsin.-This proteolytic ferment is secreted in the succus entericus. It is inactive upon native protein, though casein and the histones are slowly digested, protamin also. It is very active in the acceleration of the cleavage of peptones. These it can split to the component amino-acids, though the antipolypeptid that is so resistant to trypsin 
presents difficulties here also. The amino-acids of the resistant polypeptid that are set free in an acid hydrolysis are also set free by erepsin. The activity of erepsin is as well displayed on the products of a peptic digestion as on the products of a tryptic digestion; in the latter case the cleavage of the peptids is completed most rapidly in the case of a preliminary peptic digestion. In the superimposition of the action of the two ferments pepsin-erepsin, trypsin-erepsin, we have a mechanism by which proteins are broken into their component amino-acids. Erepsin digests the same synthetic peptids that are digestible by trypsin. Like trypsin it cannot split eystin, arginin, or hippuric acid.

Pepsin and trypsin split all the conjugated proteins, erepsin does not. Once split, the protein portion is digested, and the conjugated protein is thus destroyed, not to be recovered in the resorbed products.

None of the proteolytic ferments modify in any way the aminoacids of the proteins they hydrolyze; these exist in the digestion products as they existed preformed in the molecule of protein.

\section{DIGESTION IN VIVO}

A discussion of the derivation of the ferments and of the modus operandi of their secretion from the glands in which they were formed does not lie within the scope of the present treatise. The formation of the ferment is an act of organic synthesis, a problem of organic chemistry. The ferments are currently regarded as highly specialized proteins. This assumption is based upon the facts that they, in the purest state in which they have thus far been isolated, give the common test for protein, respond to many of the group tests for known amidoacids, on cleavage yield amido-acids, and contain about the same percentages of nitrogen and carbon as the well-known proteins. It must, however, be noted that the more preparations of ferments are purified the less definite become these tests. It is furthermore proper to state that the analytical data at our disposal is not in disaccord with the idea that the ferments may be closely associated with proteins in the form of complex chemical combinations or of adsorption compounds; that of a preparation commonly termed a "ferment" the larger fraction may be protein and the real ferment present only as a trace, the actual chemical nature of the ferment itself being unknown. In our consideration of the processes of digestion, however, we accept the digestive ferments as organic substances, probably proteins formed by synthesis from the common proteins of the blood, and having the property of a catalytic substance in relation to the reactions of the hydrolysis of carbohydrates, proteins, and fats. Present in the contents of the digestive tract are oxidation and reduction ferments; since these reactions have, so far as known, no integral part in the processes of digestion, they can receive no consideration here. It is not to be understood from this that the possibility of reactions of oxidation and reduction being present as intermediary stages in the 
hydrolysis of the carbohydrates, fats, and proteins is to be denied; quite to the contrary. But we have no data bearing upon such an aspect of the subject.

The secretion of the ferments, the process by which the formed ferment is discharged from the glandular cells through their lining membranes into the alimentary tract, is a physico-chemical problem. For the purposes of the present treatise the problem begins when the food meets the digestive juices in the lumen of the alimentary tract.

\section{SALIVARY DIGESTION}

The secretions of the salivary glands contain ferments of one type only (apart from oxydases) - such as act upon carbohydrates. It is the current teaching that these ferments are secreted by the parotid and submaxillary glands, not by the sublingual gland. The salivary secretion of the strictly carnivorous animals is commonly stated to be devoid of ferment; certainly this holds for the dog. The salivary secretion of the frugivora and herbivora is rich in ferment, that of the ruminantia surprisingly poor. Human saliva is rich in ferment.

Amylase.-Under this term we include such ferments as accelerate the cleavage of starch and glycogen to maltose, the hydrolysis of the polysaccharid to the disaccharid. The older terms "ptyalin" and "diastase" should be abandoned in favor of the term amylase, which is in harmony with the newer and generally accepted system of naming ferments. The old conception that a polysaccharid could by a single act of fermentation be converted into the simple hexose has been abandoned; it is known that the reaction proceeds only to the stage of the disaccharid. Thus the cleavage of starch to maltose is one act of fermentation; the cleavage of maltose into glucose is another act of fermentation. All the known starches of plant or animal origin (glycogens) are to be regarded as polymerizations of maltose; the hydrolysis of starches yields maltose alone. Whether all the different amylases of plant or animal origin are identical is at present a question of secondary importance, and, furthermore, one of greatest intricacy. In the literature of the technical industries we read much of the "liquefaction ferment" as distinct from the "saccharification ferment." In the case of malt, for example, the two seem quite distinct, and possess very different temperature optima. In human saliva the distinction is of little importance, since our saliva possesses feeble powers in the direction of the solution of starch. Human saliva does digest raw starch, and liquefaction must occur, since we know that it always precedes saccharification; but the velocity of the reaction is low.

The amylase is secreted in an inactive state, the proferment, in this case, therefore, proamylase. This is rapidly changed into the active enzyme. According to our present conception, this conversion represents an act of hydrolysis, as illustrated in the equation: 
The velocity of this reaction is accelerated by acids, alkalies, by salts, and by animal extracts. In the present instance, bacteria, through the medium of substances they elaborate, seem to be very active.

Qualitative Reaction.- The reaction of the hydrolysis of starch to maltose is probably best represented as follows:

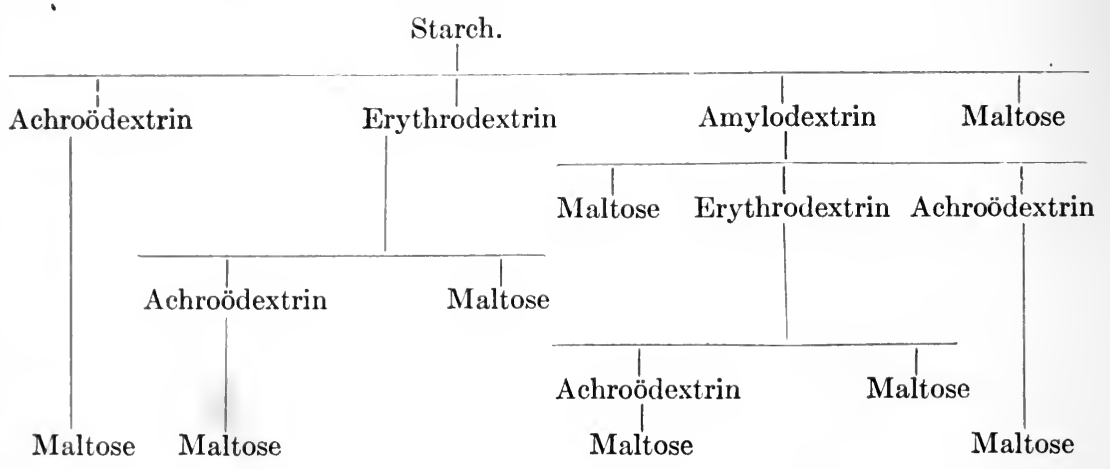

The hydrolysis of glycogen follows evidently analogous intermediary reactions, as indicated by the qualitative tests; but less definite knowledge is here at hand than in the case of starch, for which confessedly the steps given above have not been definitely proved.

Conditions of Favorable Action.-Amylase acts best in a low concentration of acid, not over a $\frac{\mathrm{N}}{10000}$. The fresh saliva is quite neutral, but becomes faintly alkaline as the carbon dioxid is dissipated, later to become again acid through bacterial action. Within these normal variations the amylase is quite regularly active. In alkaline reaction of notable degree the reaction is depressed. An acid reaction of $\frac{\mathbf{N}}{100}$ inhibits the action. The presence of the common neutral salts in more than traces hinders the action of the enzyme, higher concentrations tend to inhibit. No zymo-excitor for this ferment has ever been described; traces of sodium chlorid, of a neutral phosphate, of peptone, of the double tartrate of potassium and antimony, amino-acids, and other substances have been stated to aid the action of the enzyme, but only in an incidental manner, and not after the fashion of a true zymoexcitor. Alcohol, even in small concentrations, seems to be injurious, as are the antiseptics with the exception of toluol and chloroform.

Quantitative Relations of Reaction.-The secretion of saliva varies in amount and in enzymic strength; the variations occur not only in different individuals, but also in the same individual at different times. These facts are well illustrated in class-room experiments with saliva, in which each student uses the individual saliva. The total secretion varies in different individuals from one to two liters per day. The secretion of water in the saliva thus equals or may exceed that of the kidneys and of the skin under ordinary circumstances. This water is, of course, again resorbed into the circulation, otherwise the secretion 
would seriously disturb the equilibrium of the body. The saliva of the periods of mastication is rich in ferment, that of the periods of rest poor in or even devoid of ferment. 'To what extent secretion passes into the mouth from the salivary glands proper during periods of rest has not been determined. The act of mastication of food is a direct and powerful stimulant to the secretion of saliva and persons who masticate their food carefully convey into their stomachs much more of the amylase than do the individuals who, through habit or on account of the character of the diet, swallow their food with little mastication. There is some evidence tending to indicate that the quantity of saliva and possibly its composition may be dependent upon the character of the diet.

The enzymic activity of the saliva cannot be estimated in operation in the mouth; it has not been properly measured in the stomach. There is, however, evidence that it operates more actively than in digestion experiments in vitro. If the velocity of the digestion of starch could be measured in the stomach of an individual suffering from achlorhydria without abnormal fermentation, the direct evidence would be at hand. The experiment would, however, suffer from the presence of three uncontrollable variables - the resorption of sugar by the gastric mucosa, the secretion of water by the stomach, and the passage of contents through the pylorus. The last could in all probability be estimated, but the resorption of sugar by the stomach, though not heavy, could not be measured and the secretion of water could not be controlled. So far as I am aware, no such experiment has ever been done, so we must judge alone by observations of digestion in glass. If a 1 per cent. suspension of soluble starch (so-called, meaning starch in the gel state) be digested with half its volume of saliva obtained by the mastication of an inert substance like paraffin or a gum, maltose will be detectable within ten minutes, and the larger portion of the substrate will be split within four hours. If the mass of the starch be larger, the progress of the reaction will be delayed. If bacteria be present, the activity of the ferment will suffer a notable depreciation after ten hours; working under conditions of sterility, as secured by the presence of toluol, the amylase will retain its activity for several days, though naturally with progressive loss. Judging from such digestion experiments in glass, the day's secretion of saliva would not be able to digest the day's average ration of starch, set at 250 grams. Occasionally in class-room work one encounters a specimen of saliva so rich in enzymic strength that this would be possible; on the other hand, however, one will meet with other specimens of saliva, which in the quantity of the day's secretion would not be able to digest over one-tenth the day's ration of starch. Certainly the amylase of the salivary secretion is a much less potent accelerator of the digestion of starch than is the amylase of the pancreas or of the small intestine. In the mouth very little action can occur, the time is too short. In the stomach, the opportunity for action depends largely upon the time 
of appearance and the concentration of the hydrochloric acid. In those individuals in whom the hydrochloric acid appears in large amounts within a quarter of an hour after the ingestion of a meal, the digestion of starch in the stomach cannot be extensive; in other persons, in whom the secretion of hydrochloric acid is delayed for an hour and is of low concentration, a large digestion of starch may be accomplished in the stomach. 'The amylase is undoubtedly less sensitive to the action of hydrochloric acid than has been currently stated, and a notable degree of digestion of starch has been observed in gastric contents presenting concentrations of hydrochloric acid of $\frac{N}{100}$ to $\frac{N}{200}$. From one-half to three-fourths of the starch of the Ewald test breakfast will be dissolved in the stomachs of many individuals within fifteen minutes after the ingestion of the meal. This digestion, however, is largely confined to the reaction of liquefaction, and goes scarcely beyond the stage of the higher dextrins; little maltose is formed. But it must also be realized that some little time elapses before the gastric juice becomes intimately mixed with the ingesta, during which time the amylase is active. Strongly acid gastric contents, as $\frac{N}{50}$, inhibits the action of the amylase. It is safe to state that in normal individuals not over one-fourth of the starch of a normal ration is digested in the mouth and stomach by the salivary amylase, and that often the amount will be as low as 5 per cent. It is not likely that the ferment normally passes in an active state into the small intestine. With lesser degrees of gastric acidity it is probably, as has been reported, that the amylase is only inactivated in the stomach, not destroyed, and becomes active again at alkaline reaction. It can be shown in direct experiment that salivary amylase is destroyed by a pronounced peptic digestion; and in all probability this process in the normal stomach completely destroys the amylase. Rapid digestion of starch in the stomach would be self-limited, in the absence of the resorption of the sugar, since amylase is practically inactivated in a concentration of from 3 to 5 per cent. of glucose.

A consideration of these facts leads to the conviction that the scope of activity of the salivary amylase is comparatively limited, even under favorable conditions. The mouth, with its accessory glands, is essentially an organ of trituration rather than of digestion; and the function of the saliva is allied rather to the relations of admixtion and solution than to actual digestion. Whether the extirpation of the salivary glands would lead to indirect effects upon the lower digestive secretions is not known. One might be tempted to invoke the analogy of the secretin, but this ought not to be done in the absence of direct experimentation.

Variations in Diseases of the Mouth, Salivary Glands, and Stomach.The data upon this subject are for the mouth and salivary glands quite fragmentary; for the stomach the facts seem clear. Inflammations of the mouth that do not involve the salivary glands operate to disturb salivary digestion by the establishment of bacterial conditions 
that are detrimental to the life and action of the ferment. Thus in case of stomatitis, the buccal fluids may inactivate the amylase, probably through the action of bacteria or of products elaborated by them. Through the drying up of the secretions of the mouth, the action of the saliva may be also retarded to some extent. The importance of these conditions is in all probability slight; they are in practice far outweighed by the fact that in stomatitis mastication is usually avoided by the subject.

In inflammations involving the salivary glands, two different periods of disturbance may be noted. Early, inflammation is associated with excessive secretion, the quality of which may at first be normal, though later the ferments are present in but sparse amounts. Long-continued inflammation is often associated with a deficiency in the mass of saliva secreted and with a reduction in the enzymic strength. Rarely are all the glands involved, usually only one; thus the total function may be maintained normally, or at least fairly well, by the uninvolved glands. Prolonged inflammation may lead to the complete destruction of the secreting cells.

Disturbances in the nervous innervation lead to marked variations in the secretion of the salivary ferments. The paralytic secretion observed in some instances of organic disease involving the nerves related to the glands is usually very watery and feeble in enzymic power. The same may be said of the functional forms of excessive secretion. In the salivations due to the action of such substances as mercury and pilocarpin, the secretion is after the first stages deficient in ferments. As a rule, it may be stated that the results observed in stimulation experiments hold for conditions of disease; in prolonged. stimulation, the quality of the secretion is at first maintained, later the fluid is poor in ferment and may become inert. Whenever the conditions of secretion are such as to exhaust the secreting cells the result is an inactive secretion. Vicious habits, like the vulgar chewing of tobacco or gum, keep the salivary glands constantly overworked, and the results are as bad physiologically as the practices are reprehensible to good taste.

In diseases of the stomach, the relations are in the main clear, subject, of course, to the individual variations that are to be observed in all the domains of physiology. Hydrochloric acid and excessive and abnormal bacterial activity operate to inhibit the action of amylase and destroy it in the stomach. In those conditions of hypo- or achlorhydria not associated with abnormal and excessive fermentation of bacterial origin, the amylase will retain its normal activity and pronounced digestion of starch will occur in the stomach under such circumstances. If, however, the deficiency of hydrochloric acid be associated with abnormal and excessive bacterial fermentation, the salivary enzyme may be inactivated and the digestion of starch will be slight. Frequently, however, the conditions do not operate in this manner; despite excessive bacterial action, the amylase will not be 
inactivated and the maltose resulting from its operation will be promptly fermented by the bacteria after the cleavage into glucose. This leads in practice to the separation of two classes of achlorhydria; those in which the presence of starch in the diet is followed by the formation of large amounts of carbon dioxid and fatty acids; and those in which such fermentation occurs with the use of sugar but not of starch. The distinction must be based upon the simple fact that certain cultures of the common yeasts and bacteria destroy amylase, while others do not; the value of the distinction in dietetics is apparent. 'There are, of course, bacteria that ferment starch, and in some instances of gastritis with dilatation, such are present in large amounts and are very active, as may be observed in the expressed gastric contents. As a rule, however, such bacteria do not operate with a rapidity to be compared with the velocity exhibited by amylase; and the digestion of starch in cases of chronic gastritis with achlorhydria will be found more often to be due to saliva than to the action of starch-splitting bacteria.

The excessive secretion of hydrochloric acid operates to reduce the salivary digestion in the stomach to the minimum, or to inhibit it entirely. This is obviously but an exaggeration of the normal relation, and is in all probability of no serious detriment to the subject. So far as the mere absence of the salivary digestion of starch and disaccharid is concerned, there is no evidence that the complete cessation of the function is of the slightest injury to the subject, provided the act of mastication is properly performed; the motor function of the mouth is paramount to its chemical function.

As a rule, in plants and animals amylase is accompanied by ferments for the cleavage of disaccharids. This is the case with the saliva. The converse is not always true in the lower plants, many of which contain such ferments without amylase. The technical separation of the two ferments is possible, and one can now procure upon the market preparations of amylase that are free of any ferment for disaccharid, and yield only maltose as the result of their action upon starch.

Maltase.-Under this term are included all ferments that accelerate the cleavage of maltose to glucose. The general groups of ferments that act upon the cleavage of the disaccharids we term disaccharidases, three of which are of great importance in higher physiology. These ferments are not general but specific in their action; maltase acts upon maltose alone, not upon cane sugar or milk sugar. The presence of maltase in the saliva was long overlooked, the total reaction from starch to glucose being held to the result of the action of the amylase. Even now we know little more than that there are two ferments concerned; beyond the simple knowledge that a maltase is present we have little data. It is secreted by the same salivary glands as is the amylase. The two secretions seem to run parallel. In the study of salivary digestion one realizes that the stage of maltose is transient, the disaccharid is split into the two molecules of diglucose almost as soon as formed. In general, the cleavage of a disaccharid is a simpler 
and more rapid reaction than is the cleavage of polysaccharid to the disaccharid; and in working with a digestion that includes the two superimposed processes, one gets the impression that the conditions of action of the maltase are very favorable, that each molecule of maltose is split practically as soon as it appears in the system. There is no known investigation in which a separation of the maltase from the amylase has been undertaken with human or mammalian saliva. Under these circumstances we know little or almost nothing of the properties of the salivary maltase. Of indirect evidence we have not a little. A large number of experiments have been done to determine the nature of the products of salivary digestion of starch. Some investigators have found the product to be largely maltose; others maltose and isomaltose; others maltose and more or less glucose; others largely glucose. In my own experience, with proper dilution of the total system and prolongation of the time of digestion, the form of sugar has been found to be largely glucose. This can be shown in an experiment that can be done in every laboratory. Starch in dilute suspension ( $\frac{1}{2}$ per cent.) is digested with such volumes of saliva as will fully digest the substrate in three or four hours. At the end of each hour, samples are removed and treated as follows. The fluid is made alkaline and the rotation of the plane of polarized light measured. The starch and dextrins are then precipitated with methyl alcohol, the filtrate faintly acidulated with acetic acid, the alcohol removed by evaporation at low temperature, the volume restored to the original and the rotation again measured, following which the fluid is acidulated with hydrochloric acid, heated for several hours on a water bath, the fluid again made alkaline, the volume restored and the rotation again measured. It is my experience that under such circumstances, the third rotation will not be much less than that observed in the second, often no less. From this we conclude that the sugar present is largely glucose rather than maltose. Naturally the experiment has a large range of error, as the dextrins are not entirely insoluble in methyl alcohol, and there are numerous manipulations in the experiment. From the experiment, furthermore, we may also conclude that the maltose is split into glucose almost as rapidly as it is formed. When we consider that the velocity of the reaction is proportional to the concentration of the substrate, and realize how low is the concentration of the maltose in the experiment described, the conclusion follows that the ferment must be one of great activity. The reaction of the cleavage of maltose is, of course, a simple one in comparison with that of the cleavage of starch; and as a matter of experience maltases operate, as a rule, more rapidly than do amylases, though to this there are exceptions. Beyond the fact, therefore, that the saliva contains maltase as well as amylase and that the maltase is probably a ferment of great activity, we know little. The idea that the saliva contains a third ferment that is able to convert starch directly into glucose, is devoid of foundation. 
Saccharase and Lactase.-The saliva contains no ferments capable of accelerating the hydrolysis of cane sugar or milk sugar. At least, this is true in the adult. The assimilation limit for cane sugar has been reported reduced in animals by extirpation of the salivary glands. The fact, if confirmed, would be noteworthy; but it does not lead to the inference that the salivary secretion is necessary for the digestion (the cleavage) of cane sugar.

\section{DIGESTION IN THE STOMACH}

The amount of the daily gastric secretion varies from a few hundred cubic centimeters up to a full liter or more. Like the salivary glands, therefore, the stomach is an active organ for the secretion of water. The gastric secretion contains mucus, traces of salts and of organic matters of unknown nature, hydrochloric acid, and three ferments-a lipase, a proteolytic enzyme termed pepsin, and a curdling ferment called chymosin. The hydrochloric acid is supposed to be secreted only in the fundus of the organ, not from the pyloric extremity; the ferments are secreted from all parts of the stomach, but most actively from the pyloric cavity; the mucus is secreted from all parts. The importance of the mucus is difficult to estimate, but it must not be overlooked that it normally combines with one-fourth of the hydrochloric acid secreted.

In a general sense, the gastric secretion behaves as a unit, the secretion containing all of the constituents. A more exact statement would be that the secretion of the three ferments is in a sense secondary to the presence of the acid. This is not strictly true, the ferments can be secreted in traces in the absence of the hydrochloric acid, but there is much evidence that the acid acts as a powerful stimulant to the secretion of the ferments. The secretion of the ferments does not, however, follow pari passu; wide variations are observed. The lipase does not seem to follow closely the other ferments, but the curdling ferment seems to run quite parallel to the pepsin. The relations are different with secretion into the empty and into the full stomach; under the former circumstances the secretion behaves more as a unit. A large number of factors are known to influence the secretion of the gastric juice, both in quality and quantity; the line between the physiological and the pathological relations is difficult to draw. Most of the facts to be quoted in this connection have been learned through investigations on dogs.

The mere act of mastication has some influence upon the gastric secretion, but it probably would not be marked if the senses of taste, touch, and smell were excluded from the experiment. The several experiments that are done to elucidate the different factors in gastric secretion are best performed upon dogs who have two fistulæ, one out of which the esophagus empties and a second at the pyloric end 
of the stomach. By means of the first fistula, the animal can perform what may be called mock or fictitious feeding; the food is masticated as in the normal and swallowed but passes out of the esophageal fistula and not into the stomach. By means of the gastric fistula, the gastric secretion may be removed directly; and by this means the animal may be fed without any contact of the food with the mouth and removed from the senses of sight, taste, touch, or smell. If a dog be given such a mock feeding there is no chemical, mechanical, tactile, or thermic stimulation of the mucous membrane of the stomach; nevertheless, within a few minutes, the stomach will begin to secrete a normal juice, containing all the constituents, and this purposeless secretion will continue for a couple of hours. It is probable that this cephalogenous secretion, as it is called, is dependent upon the act of mastication to a slight though indeterminable extent. Section of the vagi prior to feeding will inhibit the reflex secretion; section of the vagi after the secretion has begun will not check it, however, though it may reduce the enzymic strength of the secretion. It is believed that the strongest factor in this reflex secretion is the psychic state of appetite, that the sensations of taste, touch, and smell have less effect. Dogs proverbially bolt their food; their chief concern seems to be to fill the stomach, the sense of taste is given little consideration by them. In human beings, on the contrary, the sense of taste in the act of eating is highly developed. A repetition of the experiments on persons with gastric fistulæ, such as not infrequently occur, has led to the conclusion that the results obtained with dogs do not hold with men; that the factors of taste, smell, and mastication have a marked influence upon the secretion of the gastric juice, and the factor of appetite is not predominant as in the dog. Whether an individual be fond or not of a dish has a marked influence upon the extent of reflex gastric secretion. In this state of being fond of a food, the factors of appetite, taste, and smell, as well as sight, all share. In any event, it is not clear how even in dogs the senses of taste, touch, sight, and smell are to be separated from the appetite, and to what extent we can frame a conception of appetite independent of these sensations. That the appetite in a $\operatorname{dog}$ is of influence even when the $\operatorname{dog}$ is fed, is shown by the following experiment: One dog is fed meat through its gastric fistula; the second dog, following mock feeding, is given the same amount of meat through its gastric fistula. In the second dog the gastric secretion is more abundant. That the appetite is of enormous influence upon nutrition has, of course, been always known; to attach a teleological inference to the fact is, however, unjustified.

Mechanical stimulation of the stomach provokes feeble secretion. Electrical stimulation is effective to some extent. Thermic stimulation is of some effect; both heat and cold increase the stimulating action of a food. Most marked of all is the effect of chemical stimulation, under which we understand the stimulation of foods-the endogenous secretion. 'To what extent the data collected for the dog is applicable 
to man is not known. There is, however, confirmatory evidence at hand.

Viewed from the standpoint of the nervous mechanism, we have two forms of gastric secretion-cerebrospinal and sympathetic. Under cerebrospinal secretion, the impulse to which is transmitted via the vagi, are apparently to be classed all the psychic, reflex, and natural secretions of gastric juice. The idea of a secretion dependent upon the sympathetic system rests upon the experimental fact that after section of the vagi and all other direct central nervous connections, if a portion of the stomach be isolated from the rest of the organ and this restored to its continuity with the esophagus and duodenum, a feeding into the restored stomach will be followed by a secretion into the artificial second stomach, though the latter is devoid of all nervous connections except by the sympathetic nerves in the walls of the bloodvessels. 'This experiment today, in the light of our knowledge of the secretion of the pancreatic juice under the influence of secretin, cannot, however, be held to prove the occurrence of a secretion of sympathetic type.

The normal secretion is a combination of the cephalogenous and endogenous secretions. If a set of curves of cephalogenous and endogenous secretions be secured, the first by mock feeding, the second by concealed feeding through the gastric fistula, it will be found that these when superimposed give a curve very much like that obtained after normal feeding. In man mock feeding gives secretion and acidity that are approximately constant for different foods-meats, bread, milk, and sugar.

The amount of the total secretion is in a general way proportional to the mass of the food ingested. The less adapted the food is to gastric digestion and the longer it remains in the stomach, the larger the secretion. Withdrawal of water of ingestion leads to a pronounced reduction in the secretion. The acidity of the secretion of the acidsecreting cells is believed to be constant for each individual; the variations in the acidity of the gastric secretion being, therefore, dependent upon variations in the secretion of water. Extract of meat is very stimulating, milk is but little less so; even water is quite active. The most active juice in the digestive sense is obtained following the ingestion of bread, less with meat, least with milk. 'These variations are due to variations in the secretion of the ferment; the secretion following the ingestion of meat is the most acid, there is less acid after the use of milk, while after the ingestion of bread, where the most active juice is secured, the acidity is lowest. Fats depress the gastric secretion.

The time relations also vary. Following the ingestion of meat, the maximum is not noted for several hours. Widely current is the attempt to place a teleological interpretation upon these relations. Thus it has been assumed that a low acidity is present in the digestion of bread in order that the salivary digestion of the starch should proceed as long and far as possible. To read some of the published descrip- 
tions, one might imagine that the conscious reasoning stomach, on observing the nature of the meal placed within it, sends to the central nervous system an order for so and so much hydrochloric acid, for which excess of vitalism the investigations are not to be held responsible. When the facts are known in detail, it will be surely found that they are to be naturally and logically explained upon the basis of physical and chemical relations. There are many facts that suggest that the variations in the secretion of hydrochloric acid in the stomach are related to the law of mass action, but the available data do not now permit of the calculation necessary to test the hypothesis. What is needed are quantitative data of a type that the experiments to date were not designed to furnish.

The action of the gastric juice upon the contents of the stomach depends in part upon the physical state in which they were ingested. As a rule, it may be said that the stomach begins to discharge its contents into the duodenum at the end of an hour; and this process of discharge continues from four to six hours, sometimes even longer in normal individuals. Those portions of the ingesta that remain longest will, of course, suffer the greatest degree of digestion; those that pass early into the intestine will have undergone little digestion. If the food has been well masticated and is of such a nature as to remain in a condition of fine subdivision and there is an abundance of water present, the movements of the stomach will be able within a short time to mix the ingesta thoroughly with its own secretions and to form an evenly suspended chyme, thus bringing about conditions favorable for the digestive action of the ferments. Under these circumstances, the discharge of the contents into the duodenum is apt to begin sooner than otherwise. If the food be not well masticated, proper admixtion does not occur, the gastric ferments will have less opportunity to act and portions of the ingesta may easily pass into the duodenum without having been in contact with the gastric juice at all. These facts have a direct bearing upon the validity of the results of the chemical examination of the gastric contents. If the expressed contents be not soft and of a chylous nature, the results of the chemical examinations will have little value and cannot be considered representative. Throughout the study of gastric digestion, many facts place emphasis upon gastric motility as the controlling variable in this domain.

Hydrochloric Acid.-The secretion of mineral acid is a widespread phenomenon in the animal kingdom. Hydrochloric acid is found in the stomach in all the known mammals and birds, and in many of the lower invertebrates. Sulphuric acid is also an animal secretion; in several varieties of marine snails, sulphuric acid is secreted by glands lying in the folds of the skin surrounding the pharynx and the stomach, in the last-named situation accompanied by hydrochloric acid. The secretion of organic acids of the fatty series has also been described as occurring in the stomach; but this is doubtful, since bacterial activity 
cannot be absolutely ruled out. Before proceeding to the discussion of the relations of the hydrochloric acid in the digestion of protein in the stomach, two questions must be considered. First, is hydrochloric acid to be regarded as a cellular secretion? Second, under the chemical and physical conditions that are known to hold within the body, what physico-chemical theory may be advanced to explain the formation of the hydrochloric acid?

Formation of Hydrochloric Acid.-Until recently, it has been supposed that the hydrochloric acid was formed within cells of the gastric mucosa; in fact, the older histologists regarded certain cells as the "acid-secreting" cells. For this specific derivation of the acid from particular cells, the demonstration was as a matter of fact wanting; but beyond that, no real doubt was raised as to the formation of the acid within the cells until the hypothesis of Köppe appeared. According to this hypothesis the hydrochloric acid is formed not in the cells lining the stomach, but in the lumen of the stomach itself, upon the surface of the mucous lining. For the purpose of this hypothesis we regard the mucosa of the stomach as a semipermeable membrane. Sodium chlorid, and to a smaller degree other chlorids, are present in the stomach, derived in part from the saliva and in part contained in the food. 'The undissociated molecule is diffusible, but the chlorion is not diffusible through the gastric mucosa. The cation sodium could not diffuse outward from the stomach unless another cation diffused into the stomach. Let us assume that this reciprocity occurs between the sodium ions of the gastric contents and the hydrogen ions of the blood; sodium ions pass from the lumen of the stomach into the blood and hydrogen ions pass from the circulation into the lumen of the stomach, the result being, of course, the formation of hydrochloric acid in the cavity of the stomach. The result in the blood would be the formation of sodium bicarbonate. These bicarbonates are then supposed to be secreted by the pancreas, to combine in the duodenum with the hydrochloric acid of the discharged gastric contents, following which the resultant sodium chlorid would be resorbed, the intestinal mucosa being held to be permeable for both the sodium and the chlorin ions.

This hypothesis must be rejected. The formation of hydrochloric acid is not dependent upon the presence of chlorids in the contents of the stomach; one can secure the secretion of strongly acid gastric juice in the entire absence of any chlorid in the lumen of the organ, nor is the secretion under any circumstances parallel to the chlorin content of the viscus. Contrary to the earlier ideas, it is possible under proper arrangement of the experiment to secure the secretion of hydrochloric acid in the dog on a pure carbohydrate or sugar diet. It has been shown that psychic stimulation of the appetite, without feeding or with mock feeding, both in the case of the isolated stomach, will lead to the secretion of notable amounts of hydrochloric acid. In the case of the acid-secreting snails, the $\mathrm{SO}_{4}$ ion is not derived from the 
medium in which the animal is immersed, but from the circulation. It is therefore clear that the hydrochloric acid is formed by the cells of the stomach from the chlorids of the blood. Chlorid starvation leads to reduction in the gastric secretion, the stomach striving to maintain the acid concentration. In severe and prolonged chlorid starvation, the gastric secretion may be practically suppressed.

What physico-chemical explanation can be given for the formation of hydrochloric acid by the stomach? None today, based upon quantitative work. In the blood are hydrogen ions derived from several sources. 'The blood is charged with carbon dioxid, from whose acid hydrogen ions are present through electrolytic dissociation. The blood contains bicarbonates, which are subject to both electrolytic and hydrolytic dissociation. The water of the blood displays, of course, the usual minute degree of dissociation. The reaction of native blood is neutral. In this respect it resembles a weak solution of sodium bicarbonate. If a weak solution of this salt be prepared, it will be found to have a slight alkaline reaction, due to hydrolytic dissociation. If the solution be placed in a closed system under pressure of carbon dioxid a point will be reached where the reaction of the solution will be neutral. If now the solution be placed in the open, carbon dioxid will pass off and the solution will return to its original faint alkalinity. But more, on further exposure to the air a part of the bicarbonate will be dissociated, the carbon dioxid will be dissipated, and a stronger alkaline reaction will be presented. An equilibrium will be finally established in which the concentrations of sodium carbonate, bicarbonate, and hydroxid and carbon dioxid will participate. In the state of neutrality in which the blood exists, it is clear that hydrogen ions are available to the degree required for the formation of hydrochloric acid, since those withdrawn would be promptly replaced by dissociation processes coupled with the regular production of carbon dioxid in the tissues. For the derivation of the chlorin ions we are much more in quandary. Chlorids under pressure of carbon dioxid are dissociated to some extent with the formation of hydrochloric acid; this can occur in the blood to scarcely more than infinitesimal extent. A mixture of equivalent parts of sodium hydroxid and hydrochloric acid will yield sodium chlorid and water, with the faintest traces of hydrochloric acid and sodium hydroxid; in other words, the hydrolytic dissociation of sodium chlorid is so slight as not to be measurable. It does not seem possible that these traces of hydrochloric acid could be eliminated by the epithelial cells of the gastric mucosa. Unless we can conceive of some condition whereby the station of equilibrium in these equations can be widely shifted, we are unable to explain the formation of hydrochloric acid in accordance with the laws of affinity and mass action. What to the phrsical chemist is mathematically difficult, seems to the vitalist easy. 'To read the text-books of physiology written under the spell of vitalism, one might imagine that the acid-secreting cells are endowed with tongs wherein they 
reach into the circulating blood, pick out the stray and wandering atoms of chlorin and hydrogen, pull them into their protoplasm, there unite them, and then cast the hydrochloric acid into the lumen of the stomach.

Recently a very interesting hypothesis has been suggested for the physico-chemical formation of hydrochloric acid, namely, cataphoresis. The conditions of electrolytic dissociation disturb so seriously the phenomenon of electrical osmosis that it has not yet been possible to study this hypothesis experimentally.

Chemical Relations.- The hydrochloric acid of the gastric contents presents six chemical relations: (a) Direct hydrolysis of protein, the action as an inorganic catalyzer; $(b)$ secretion and activation of the ferments; $(c)$ action as zymo-excitor for the pepsin; $(d)$ inhibition of the activity of the gastric lipase; $(e)$ inhibition of the activity of the salivary ferments, and $(f)$ inhibitory action upon bacterial fermentation. The action of the acid upon the duodenal mucosa, that operates to keep the pylorus closed, will be described under the section of gastric motility, also its relation to the formation of secretin; the properties under $d, e$, and $f$ are discussed under their appropriate headings.

The hydrolysis of protein by hydrochloric acid is the classical method for the complete cleavage of a protein into its component amino-acids. In the quantity in which the acid is present in the stomach, at the temperature there maintained and during the limited time available, the hydrolysis of protein attained in the stomach under the catalytic action of hydrochloric acid is demonstrably very small, so small indeed that it may be omitted from our consideration.

The action of the hydrochloric acid in stimulating the secretion of pepsin and chymosin by the glands of the stomach is not well understood, and is not susceptible of experimental investigation in a satisfactory manner. The activation of the zymogens of the proteolytic and coagulating ferments is to all appearances normally accomplished by hydrochloric acid. This action is simply that of a catalyzer, the actual reaction lies between the zymogen and water.

$$
\text { Pepsinogen }+ \text { water }=\text { pepsin. }
$$

The action of the hydrochloric acid lies in a pronounced acceleration. It is known that the unit of pepsin formed per minute from pepsinogen is roughly proportional to the mass of the pepsinogen; that the activation within certain limits is accomplished more rapidly at higher temperature; and that it is proportional, within certain limits, to the mass of acid in the system. Inferentially the same relations are assumed to hold for the chymosin. In the absence of hydrochloric acid, pepsinogen if secreted would be activated by organic acids elaborated by bacteria or by acid salts of the diet.

The relation of the hydrochloric acid to the enzymic action of pepsin is a complicated subject. 'The optimum of the zymo-excitory influence of hydrochloric acid is attained when there is present in a digestion 
system a certain amount of the acid in excess of the minimal combining power of the protein there present, the most favorable degree of this so-called free acidity being variable with different preparations of pepsin. Since hydrochloric acid shares this property with the other acids, the natural thought would be to relate it to the hydrogen ion. Investigations do not confirm this inference. The zymo-excitory actions of hydrochloric, sulphuric, phosphoric, and lactic acids do not run parallel to their electrolytic dissociation and are not, therefore, functions of the concentrations of the hydrogen ions. The exceptional activity of hydrochloric acid might be further supposed to be due to an action of the chlorion, a view that is not sustained by experiments with trichloracetic acid. A rather widely held opinion relates the activity of the hydrochloric acid to the substrate rather than to the ferment in the direct sense. The pepsin is supposed to act not upon the protein, but upon acid-protein. According to this view, we would have the general scheme of reaction: protein + acid + water + pepsin $\rightarrow$ protein-water-acid-pepsin $=$ proteose + peptone + acid + pepsin - . . etc. This point of view suggests another explanation of the varying activities of the different mineral acids. The combination protein-acid is one subject to a very high degree of hydrolytic dissociation; thus the combination of serum albumin and hydrochloric acid is dissociated to over 80 per cent. The hydrolytic dissociation of sulphuric-acid-protein and of phosphoric-acid-protein are not identical with the dissociation of hydrochloric-acid-protein; and it might be possible to connect to this variable of hydrolytic dissociation the varying relations of acids to the action of pepsin. This whole point of view, however, does not agree well with the experimental fact that the optimal concentration of the acid is one in excess of the combining power of the protein present in the system. Experimentally, the subject is extremely complicated; practically it is of little importance; while theoretically the data at our disposal are not such as to permit us to draw even approximate conclusions.

Quantitative Relations.-The quantitative secretion of hydrochloric acid is subject to a number of variables. In the empty isolated stomach of the dog, the concentration of the acid in the secretion is quite constant, though the volume of secretion may vary widely; the variation in different dogs is surprisingly small. With the presence of food and of different diets in the stomach, conditions are changed. The stomach does not present in the contents with different diets, or even with varying amounts of the same diet, the same constant concentration of bydrochloric acid. Obviously the variables for the secretion of water are not related to the secretion of the acid. Under the discussion of the secretion of water by the stomach, several variables of water secretion are defined; the secretion of water with the hydrochloric acid, with the ferments, with the mucus, and alone, to which must be added the water arbitrarily present in the food and water of the saliva. To these variables the function of simple secretion of water seems to be 
compensatory, the stomach obviously strives to secure in general a fairly constant water content in the chyme, the secretion of water being in an approximate sense proportional to the solid mass of the diet. 'The total secretion of hydrochloric acid is, on the contrary, not related to the total mass of the diet, but in an approximate sense to the protein in the diet. It is greatest with a protein diet, least with a diet of carbohydrate, though present even if the diet contain no protein at all, as in the case of a meal of syrup. There is, however, an unquestioned tendency for the establishment of a fairly constant so-called free acidity, in the same sense as there is the attempt at the establishment of a fairly constant water content in the chyme. This fact, that has been given a highly teleological explanation, admits of a simple physiochemical interpretation. Let it be assumed that at a certain concentration of hydrochloric acid the formation of the same is normally inhibited; it will be apparent that the formation of hydrochloric acid would necessarily run parallel to the mass of protein (with which the acid combines) in the meal.

These general facts, which have been determined for the dog, hold for human beings only in a restricted sense. There can be no question that for healthy men and women living upon mixed, general, and daily varied diets, the facts as determined for the dog tend to hold. But they will often not hold for individuals, because the power of adaptation is limited in many individuals. If to a thin woman of the working classes, who naturally lives upon bread, tea, a few vegetables, and little meat, a meal of fifty or sixty grams of protein be given, the stomach will not be able to secrete enough acid to combine with all the protein. If the diet be persisted in, the stomach will in a variable space of time adapt itself to the diet and present much higher secretions of hydrochloric acid. On the other hand, if to an athlete in training, consuming large amounts of meat daily, a meal low in protein be given, the secretion of hydrochloric acid will exceed the combining power of the protein, and the acid will be far in excess of the character of the meal. This is only a physiological illustration of the pathological fact that nervous hyperchlorhydria cannot be cured by reduction in the protein of the diet. If the diet low in protein be persisted in with the athlete, the secretion of hydrochloric acid will gradually fall to that normally seen with the diet under consideration. It may be replied that such variations are in the strict sense not physiological, but pathological; if such a point of view be tolerated, we have then no means of defining the physiology of human digestion.

The total secretion of hydrochloric acid in the human stomach is such as to present in gastric contents concentrations running usually from the tenth to the twentieth normal, though higher and lower concentrations are, of course, seen. When one measures the total contents, it will be found that the total secretion of hydrochloric acid for the day may vary from five to twenty grams.

Of the hydrochloric acid in the stomach two states exist-free and 
combined with protein. The so-called free hydrochloric acid is present normally but in small amount. It is, however, not clear just what this fraction really consists in. For the older writers, the free hydrochloric acid was that fraction that could be separated with certain color tests, such as Congo red and phloroglucin vanillin. For the modern chemist, the free hydrochloric acid ought to be defined as the fraction of the acid that is dynamically free, revealing itself in the electromotive potential and measurable by its work as a catalytic agent. Estimated in this way, the free acidity is not high. Unfortunately, the conditions of hydrolytic and electrolytic dissociation are here superimposed so that the arrangement of experiments becomes difficult. The acid protein is subject to a very high degree of hydrolytic dissociation. The fraction of the acid beyond the combining power of the protein is presumably dissociated electrolytically. The greater the amount of acid present beyond the amount necessary to combine with the protein, the lower the absolute hydrolytic dissociation of the acid-protein, since the dissociation is opposed by the excess of the one component. Furthermore, there is evidence that with large excess of acid, the relations of combinations between protein and acid are altered; the higher the concentration of acid in a given system, the greater the amount that will be combined with the protein. When gastric contents are studied with the gas chain, it will usually be found that the true dynamic potential of hydrogen ions is not greater than is to be found in a hundredth normal acid; often results as low as a thousandth normal acid are encountered. Occasionally a result will be observed that is entirely bizarre and susceptible to no explanation. When working with gastric contents of moderate strength, the result of the Congo-red test often agrees roughly with that of the electropotential measurement; working with contents weak in acid, however, the paper test and the Günzberg test as well give much too high results and cannot be relied upon. Practically the point is of little importance, since the examination of the degree of acidity of gastric contents is of diagnostic importance only, the chief point being whether free acidity be present or not; whatever the physico-chemical error of the color-test methods, the results are comparable with each other and as such may be safely relied upon.

Variations in Disease.--These are especially of diagnostic importance. The variations may be divided into two large groups-variations occurring in conditions of organic disease of the stomach; and variations occurring in the organically sound viscus.

Systematically we may distinguish variations in the quality and in the quantity of the gastric secretion. Variations in the quality of the secretion may be in the direction of excess or of diminution. It is uncommon to find an increase in the acid in a pure gastric juice. Usually the variation occurs only in connection with digestion. The contrary variation, decrease in the acid, is common. 'The pepsin usually follows the acid. Variations in the quantity of the secretion may be divided 
into supersecretion and subsecretion. Of supersecretions we have transitory and continuous forms, periodic and occurring in exacerbations. In some instances the excessive secretion occurs only in the course of digestion; in others it persists during the periods of empty stomach. Subsecretion may be either transitory or continuous. Complete cessation of secretion is termed achylia gastrica, and is, as a rule, absolute for both acid and ferments, though ferments will usually be secreted if acid be administered. The different forms of supersecretion may be associated with qualitative variations; thus hypochlorhydria is frequent in supersecretion, and though normal acidity in concentration is possibly the more common, true hyperchlorhydria does occur often with excessive secretion. With subsecretion hypochlorhydria is the rule. We are acquainted also with a peculiar state of asthenia of the secreting mechanism; when food is ingested there is active secretion for a short time, then sudden cessation. Systematic classifications of secretory disturbances of the stomach are of little value because so variable and so dependent upon the conditions of diet and methods of investigation.

The variations occurring in conditions of structural disease of the stomach walls are to be divided into two groups, depending upon whether the secretion be increased or decreased. Increased secretion of hydrochloric acid associated with organic disease of the stomach is not very common. It is observed as the rule in simple ulcer of the stomach; is seen sometimes in cases of gastric carcinoma, especially in the variety that has originated in the scar of an ulcer; is seen sometimes in early inflammation of the gastric mucosa, especially of the toxic variety and is sometimes seen in cases of chronic gastritis, even with dilatation and excessive fermentation. In these several diseases the hyperchlorhydria may be intermittent or continuous; and may be associated with excessive secretion of water or not, usually not. The degree of hyperchlorhydria is not usually excessive, though there are exceptions to be noted in all the classes above enumerated; rarely is the acidity over double the normal.

Much more common is hypochlorhydria in association with organic disease of the stomach. As a rule, it may be held to be roughly proportional to the intensity of the disease, though it cannot be taken as indicative of the integrity or restitutionability of the tissue. It is the rule in carcinoma of the stomach, as it is also in advanced atrophic gastritis, in simple atrophy of the mucosa and in conditions of extreme cachexia. In simple chronic gastritis of any type hypochlorhydria is often seen. The defect may in the more grave cases pass to complete failure of the secretion, though this is rare except in the latest stages of the diseases. Usually there is some acid secreted; but free acidity is not attained though the protein in the diet be as low as the normal minimum, one gram per kilo body weight per day. In a certain sense the defect has diagnostic value, though exceptions are very common. Proper cleansing of the viscus and care in diet will sometimes be followed 
by partial restoration of the secretion. In a large percentage of instances of achylia gastrica there are disturbances in intestine digestion; such are also to be observed in many cases of hyperchlorhydria. Constipation or diarrhea may be present in either type, and it is not clear upon what these concretely depend.

Variations in the secretion of hydrochloric acid occurring as secretory anomalies independent of organic disease of the gastric tissues are very common. A slight decrease in the secretion is seen in many diseases not involving the stomach organically, as in chlorosis, nephritis, etc. There are, however, gastric neuroses that offer this as their chief symptoms. Thus in the condition known as achylia gastrica, a normal stomach will for days or weeks at a time secrete neither water, acid, pepsin, nor chymosin. Occasional instances of this sort, not always complete and usually intermittent, are to be met with in the hysterical and neurasthenic. There are experiments in animals indicating that increased bacterial action in the intestine, leading to the formation of large amounts of fatty acids, provoke in some way an increased secretion of hydrochloric acid in the stomach.

Much more common is functional hyperchlorhydria. Seen often in diseases not involving the stomach, such as chlorosis, it occurs more frequently in nervous dyspepsia, hysteria, neurasthenia, and as a disease sui generis. Hyperchlorhydria of this type may be intermittent or continuous; it may occur for months at a time or may take place in veritable crises that almost overwhelm the sufferer. It may be associated with hypersecretion of water or not; indeed, the relations between it and the secretion of water may vary at different times in the same case. 'The degrees of acidity that may be seen are very striking. Rarely will the concentration of the acid be higher than fifth normal; but when to this is added heavy hypersecretion, the amount of acid that may be secreted in the day is very large. Cases are seen in which the hypersecretion occurs only on the taking of food; in other and numerous cases, the secretion persists in the empty stomach. That some of these cases develop ulcer of the stomach or duodenum is certain; that most of them bear the condition without consequent organic lesion seems equally certain.

According to theory, the stomach ought to empty itself more quickly than normal in subjects with hypochlorhydria, and to retain its contents longer than normal in subjects with hyperchlorhydria. In clinical practice such results are not regularly observed. Indeed, some of the most distressing cases of hyperchlorhydria are those in which the highly acid contents are discharged precipitously and rapidly into the duodenum, with the production of great pain.

The Antiseptic Action of Hydrochloric Acid.-Normally the stomach is a poor culture tube for bacteria. This has usually been ascribed to the hydrochloric acid. It can be shown in culture experiments that the concentrations of hydrochloric acid present in the stomach are repressive to bacterial life. On the other hand, it is probably only 
to certain flora that hydrochloric acid is bactericidal. Every experienced specialist in diseases of the stomach has seen cases of chronic gastritis with hyperchlorhydria associated with excessive bacterial fermentation.

The action of hydrochloric acid upon the intestinal flora is largely indirect but nevertheless potent. In the first place, a normal gastric chyme, poor in bacteria, makes for few bacteria in the intestine. Secondly, a good secretion of hydrochloric acid results in a good secretion of pancreatic juice, so that the chyme is promptly digested. The predigestion of the proteins of the diet by pepsin- $\mathrm{HCl}$, makes intestinal digestion most rapid. Free secretion of gastric juice means free secretion of pancreatic and intestinal juice and this leads to rapid absorption. Anything that accelerates the velocity of the intestinal digestion and absorption of protein lessens the opportunity for bacterial action.

Pepsin.-The secretion of the stomach contains a proteolytic ferment in an inactive state, as is easily shown by collecting the separated secretion of a cul-de-sac formed in the pyloric end of the stomach. This proferment, propepsin, is activated through hydrolysis, the catalyzer for the reaction being hydrochloric acid. Experiments, however, indicate that tissue juices and bacterial extracts are able to activate the proferment. This reaction we now consider to run as follows:

Propepsin + water $=$ pepsin

the catalyzer being as stated hydrochloric acid. That other factors operate in life as well as in the test tube is shown by the fact that it is not rare in the clinic to find small amounts of active pepsin in gastric contents that are devoid of hydrochloric acid.

The march of the hydrolysis of protein that is accelerated by pepsin, cannot be given except crudely, as in the case of starch; there are many substages, for which we know as yet but few indefinite chemical or physical characteristics. The action of pepsin is a limited one, its accelerating influence is practically restricted to the upper stages of the reaction, to the stages of peptones. Traces of amino-acids occur rarely in peptic digestion tests; the bulk of the material is not hydrolysed beyond the stage of peptone. While it is true that successive additions of fresh pepsin will intensify the reaction and lead to the formation of more notable amount of amino-acid, the fact remains that the large amounts of different amino-acids that are secured following the action of trypsin or erepsin are not formed in digestion experiments with pepsin.

Under proteoses we understand simply proteins that have lost the power of coagulation by heat, have smaller molecules than the original proteins, and are precipitated by ammonium sulphate, zinc sulphate, tannic acid, and alcohol. The peptones have still smaller molecules, possess measurable osmotic pressure, are not precipitated by ammonium sulphate, but are still precipitated by tannic acid and alcohol. In the 
earlier days in physiological chemistry, an enormous amount of work was done in the fractionation of the albumoses by different salts and by different concentrations of the same salts. It is now clear from modern knowledge of physical chemistry, that these procedures do not lead to the separation of different chemical individuals; and that the older designation of several albumoses was purely fictitious, so far as the methods used were concerned. Many albumoses there are undoubtedly, just as there are many dextrins; this we are able to state from our general knowledge of the step-like progression of the reaction of cleavage; but just what they are, remains for the future to determine. Several peptones also exist but we know little of them, except that they contain different groupings of amino-acids. The peptone is certainly the physiological end product of the action of pepsin. All the conjugated proteins are split in the stomach and the protein fraction hydrolysed to the peptone stage. (Cf. page 47.)

The question of the quantitative action of pepsin on protein is not identical with that of the scope of its action within the stomach. Under the most favorable circumstances, the gastric juice is in contact with the food for not over five to six hours, which time will be shortened if the admixture of the secretion with the ingesta be delayed. Within this time, in tests in glass, pepsin will not be able to do more than convert native protein into primary proteose. Careful analyses of gastric contents indicate that little more is accomplished in the stomach; in this case the digestion in vivo does not exceed the digestion in vitro. If the pylorus of the dog be tied and the blood supply of the stomach cut off after a digestion is in progress, it will be found, after ten or more hours, that traces of tyrosin can be secured from the gastric contents, while much of the substrate can be precipitated with ammonium sulphate. In the human stomach, amino-acids are not to be met with at all during the course of a normal digestion, nor is much peptone formed. The reaction is, in the time available, confined largely to the cleavage of the protein into proteose, and this may not be fully accomplished. Toward the close of the period of gastric digestion, the reaction is naturally more advanced; the portions first passed into the duodenum often contain still coagulable protein. If the fat in the diet be low, digestion will proceed more rapidly than if the meal be rich in fat; for reasons not yet clear, fats depress the processes of gastric digestion, in part possibly through interference with solubility. If one were to attempt to measure the extent of gastric digestion, from the point of view of the total work, one would be safe in the statement that from one-tenth to one-fourth of the work of the digestion of protein is accomplished in the stomach under normal conditions. For casein in particular is it true that little digestion beyond coagulation is accomplished in the stomach.

Under these circumstances the question arises: What is the actual scope of the chemical action of pepsin in the total process of digestion? Has it beyond its direct action, any indirect influence? For the last 
question we have a positive answer; pepsin is of marked aid to the intestinal digestion of protein. Erepsin, which is quite inactive upon proteins in their original state, is an active ferment when added to these proteins after the action of pepsin. Pepsin aids the later action of trypsin. A controlled experiment may be done to illustrate this. To a certain amount of a solution, let vs say of blood serum, a known amount of trypsin is added under appropriate conditions of alkaline reaction. 'To a second identical amount of solution of serum, pepsin and hydrochloric acid are added, and after the lapse of six hours, the solution is made alkaline to the same degree as in the first test, and the same amount of trypsin added. Not only will the total digestion in the second test exceed that in the first test, but the velocity will be greater. The gain lies partly but not entirely in the fact that the trypsin has an increased field for its action, since the number of molecules of peptone is much greater than of the native protein. How important this may be in actual life is not known. That it is dispensable is shown by the fact that the digestion of protein in usual amounts is normal in individuals from whom the stomach has been removed, provided the diet be properly arranged as to administration and consistency. There is no evidence that the presence of pepsin has any other action upon the intestinal secretion or processes than through its preliminary action upon the protein. It is clear, therefore, that the estimation of the peptic secretion of the stomach is merely an estimation of the secretory state of the stomach, and not at all an estimation of the power of digestion of protein, on the part of either the stomach or the intestine. When one considers the importance of the hydrochloric acid in the stomach in connection with its action upon the pancreatic secretion it seems likely that the acid is the more important factor of the two, and from the standpoint of adaptability the least dispensable.

Relations to Hydrochloric Acid.-The relations of the activity of pepsin to the hydrochloric acid of the stomach are of importance. The relation of hydrochloric acid to the action of pepsin must be held separate from its relation to the secretion of pepsin. The presence of hydrochloric acid in the stomach acts as a pronounced stimulant to the secretion of pepsin. The older statements that the two ran parallel and that in the absence of hydrochloric acid no pepsin would be secreted are not strictly true; but the fact still remains that the secretion of pepsin in a stomach devoid of hydrochloric acid will always be slight, and usually it is entirely wanting. The action of the hydrochloric acid in the activation of the pepsinogen has been already referred to. While the secretion of pepsin is to a large extent dependent upon the presence of hydrochloric acid in the stomach, beyond that point there is no quantitative parallelism; high acid secretion is not necessarily associated with high peptic secretion.

The relation of the hydrochloric acid to the enzymic activity of pepsin is also striking. 'The acid itself, of course, acts as a catalyzer 
for the hydrolysis of the proteins; but in the concentration and in the time and at the temperature employed, little measurable action will occur. Pepsin will digest feebly at a neutral reaction. On the addition of a slight amount of hydrochloric acid, the curve of action rises. On further additions of the acid, the curve continues to rise, finally reaches a maximum and then falls rapidly on excessive addition of acid. With most pepsins, the top of the curve, the optimal concentration of acid, will be found to run from tenth to twentieth normal. At first sight one might be tempted to ascribe this zymo-excitor action to the hydrogen ions; investigations, however, have shown, as already stated, that it is not a function of that variable alone, the different mineral acids do not act in proportion to their electrolytic dissociation. While the acid operates to excite the enzymic power of the pepsin, it also acts as a catalyzer for the hydrolysis of the ferment; the descent in the curve from the optimum is due in large part to the destruction of the ferment by the higher concentrations of acid. In other words, the curve of action is the result of two superimposed processes; and the optimum in the curve is the point where the zymo-excitory action of the acid is not appreciably overlapped by the destructive action of the acid upon the ferment. According to recent investigations the mucous membrane of the stomach possesses the property of antagonizing the action of pepsin, and to the hypothetical substance the name anti-pepsin has been given. The characteristics of this substance as stated in the researches devoted to it are rather intangible and while anti-pepsin may serve as a basis for speculation, it cannot yet serve as a basis for physiological or pathological inference.

Action of Pepsin in the Intestinal Tract.-The action of the pepsin is for practical purposes confined to the stomach. Pepsin does not digest protein to any extent in the presence of bile, even at an acid reaction. At a neutral reaction, a precipitate may form when bile and gastric juice are mixed. The pepsin, furthermore, is practically inactive in the faintly alkaline reaction of the upper small intestine. Lastly, the pepsin is rapidly digested by the trypsin. The result of these combined influences is to limit sharply the action of the pepsin to the cavity of the stomach. This would not hold so true if the contents of the stomach were discharged en bloc into the intestine. But the contents of the stomach are discharged into the duodenum in small portions and the concentration of hydrochloric acid there is thus never permitted to reach any appreciable degree; and as the projected portion of gastric contents is soon mixed with the abundant pancreatic and intestinal secretions, the destruction of the pepsin is promptly accomplished.

Variations in Disease.-The question of the variations of the secretion of pepsin in conditions of disease has not received the same attention that has been accorded the variations in the secretion of hydrochloric acid. This has been duc in part to the fact that the variations in the concentration of pepsin are not readily determined; and by the general 
assumption that the two secretions, as a usual fact, go hand in hand. The difficulty in the quantitative estimation of pepsin has not been overcome. The figures obtained by the Mett method are not to be relied upon, partly because of the errors inherent in the method itself, and partly because the results are usually calculated according to the rule of Schütz. Now this rule is not a rule of ferment action at all, but simply expresses a relationship between the ferment and the products of the reaction under certain conditions of concentration. One could found a method for the approximate quantitative estimation of pepsin in the following manner. The test meal, to be given on a carefully emptied and cleaned stomach, should always be of the same kind and amount, both in solids and fluids. The time allotted to the test meal in the stomach should always be the same. 'Then one would take a fixed portion of the expressed contents and observe its action upon a standard solution of a protein with a fixed concentration of hydrochloric acid at a fixed temperature. The standard solution of protein could be for example, a 1 per cent. solution of serum albumin. The measurement of the velocity of the reaction could lie in the gravimetric estimation of the coagulable protein. Such a method, which alone would fulfil the minimal methodic desiderata, would be obviously arduous and complicated. But beyond this, the validity of the result would be made questionable by several uncontrollable variables. The secretion of water in the stomach is not parallel to that of pepsin. One stomach will secrete much, another little water with the same test meal, and these variations might be most pronounced in conditions of disease. The fixing of the volume of the test meal does not result in the control of the total volume of the gastric contents, upon which rests in the test the fundamentally important concentration of the ferment. This variable might possibly be controlled by an estimation of the total mass of the gastric contents at the time of expression. But one can never be sure that none of the contents have passed into the duodenum, unless this fact can be determined by calculation with figures obtained for the total contents of the stomach, if the meal contain some ingredient that can be estimated. Another error would lie in the time fixed for the expression of the contents, at the end of an hour or an hour and a half. The relaxation of the pylorus is in part related to this variable, in part not. At any fixed time, therefore, the total secretion of the stomach will not have been attained in some cases; while in others a goodly portion of the contents will have been already discharged into the duodenum. A third difficulty would lie in the concentration of hydrochloric acid to be used in the tests, since the optimum concentration of hydrochloric acid varies with different specimens of gastric contents. Should all these variables be under control, the result would still be relative only. And it is clear that the results would not be of theoretical or practical value proportional to the labor of the procedure. The data already at our disposal permit of general approximate statements. 
In conditions of disease attended with excess of the secretion of hydrochloric acid, the secretion of pepsin is usually abundant, without showing such excess as is to be observed in the case of the acid. This is especially true when the hyperchlorhydria is of the intermittent type. Defective secretion of pepsin does, however, occur in hyperchlorhydria. When the condition is continuous, a different result will often be noted. The glandular cells of the stomach form the pepsinogen from the proteins of the circulation. This is a process requiring time and it is during the period of gastric rest that this storage of the cells with pepsinogen occurs; on the ingestion of food, the cells discharge this inactive ferment and are then quite empty. A prolonged gastric secretion will therefore lead to the formation of gastric contents weak in pepsin; just as in the case of the parotid gland, prolonged stimulation leads after a time to the secretion of a saliva feeble in enzymic strength. In cases of prolonged hyperchlorhydria, therefore, one may encounter high concentrations of hydrochloric acid with low concentrations of pepsin. This may be seen not only in the hyperchlorhydria of nervous origin; but sometimes also in cases of gastric ulcer, one may see intermittent hyperchlorhydria with low concentrations of pepsin. The quantitative maximum is always lower for the ferment than for the hydrochloric acid; the formation of the acid is obviously much more easy than is the synthesis of the proferment.

In conditions attended with reduction in the amount of hydrochloric acid, the rule observed is that the secretion of pepsin sinks roughly in proportion. To this rule there are many exceptions. Especially in those cases where the secretion of the hydrochloric acid is low but still enough to combine with the larger part of the protein of a test meal, do we often find the secretion of pepsin normal. The corollary of this fact is to be noted in conditions of nervous achylia; the administration of a small amount of hydrochloric acid, far less than the amount of the normal secretion, may be followed by the secretion of pepsin in normal amount. In other words, the secretion of pepsin is not solely a function of the concentration of the hydrochloric acid. When the secretion of the acid is very low the secretion of pepsin is usually low. When the secretion of the acid is abolished, there is usually no pepsin secreted. 'The cases of abolition of secretion may be divided conveniently into two classes; those in which the secretion of pepsin may be reëstablished by the administration of hydrochloric acid; and those in which no secretion of pepsin follows the administration of the acid. In achylia gastrica of nervous origin, the secretion of pepsin can usually be provoked by the administration of a goodly dose of hydrochloric acid. But in the group of achyliæ of grave organic disease, in advanced cachexia, in carcinoma of the stomach and atrophic gastritis, the administration of hydrochloric acid may have no effect-obviously because the secreting structures are quite completely destroyed. In a certain sense, therefore, the results of the administration of hydrochloric acid upon the peptic 
secretion yield a diagnostic indication of the anatomical state of the peptic cells of the gastric mucosa. As will be explained in another section, the cessation of the peptic secretion has no necessary consequences of practical importance upon the digestion of protein; if the motor functions of the stomach are preserved and the food is not allowed to fall a prey to bacterial action within the stomach, the intestinal digestion of protein in carefully fed individuals without gastric secretions is entirely normal.

Chymosin.-Chymosin (rennet) exists in the stomach of all carnivora and herbivora. It is not present in the human infant during the first few days after birth, but the secretion soon becomes established. It is secreted in the form of a proferment, termed chymosinogen, which on standing in water is slowly converted into the active rennet through a reaction of hydrolysis. Hydrogen ions accelerate this conversion into the active ferment, and all acids are, therefore, activators. Alkalies do not activate, but destroy the proferment, and the ferment as well.

The views as to the individuality of chymosin are divided, some holding that it is identical with pepsin, others that it is an individual ferment. In the opinion of the author, the present status of evidence is in favor of the view that rennet is an individual ferment. The action of chymosin appears to lie in the conversion of casein into an isomer, paracasein. The idea that paracasein is a product of the hydrolysis of casein has failed of direct demonstration, and for the present we must regard paracasein as an isomer. In this alone lies the action of the ferment. The physical phenomenon of coagulation is due to the fact that the calcium salt of paracasein is insoluble, whereby the formation of the curd occurs. This action of the chymosin takes place only on neutral or acid reaction. Since, however, the stomach is always acid, either from hydrochloric or organic acids, the conditions of favorable action are always present. The action of the ferment upon the casein is quite rapid; within an hour at the outside, often within a quarter hour, the conversion is complete. Alkalies destroy gastric chymosin rapidly. Trypsin also destroys rennet, and it is, therefore, very doubtful if it has any action within the intestinal tract. It is also very sensitive to bile and to bacterial products.

Rennet is a very active ferment. Of the prepared chymosins, one part will coagulate a half million parts of milk; of normal gastric contents one part will coagulate from one hundred to one thousand parts of milk. Viewed casually, the phenomenon of coagulation seems a very precipitous one. For some time after the addition of the rennet to the milk there is no sign of any action; then, quite suddenly, coagulation will set in and proceed rapidly to completion. This is due to the fact that casein, like any other protein, acts as a solvent for the calcium combination with paracasein; it is only when the casein is nearly all converted into paracasein that the physical phenomenon of coagulation appears. It is for a similar reason that colostrum does not coagulate; its high content of albumin holds the paracasein in solution. When 
the great activity of chymosin is understood, it will be apparent that the day's secretion is more than sufficient to coagulate the body weight of milk, even in the adult. Very pronounced reductions in the secretion occur before the physiological limit of action of the ferment is reached.

Variations in Disease.-These in general run parallel to those of pepsin. Rarely, however, one will see gastric contents in cases of advanced carcinoma of the stomach that will digest protein but will not longer coagulate milk. As a rule, gastric secretions that are so attenuated as to scarcely digest protein, are still able to coagulate milk quite normally, as so little of the ferment is needed for a few hundred cubic centimeters of milk such as would be contained in a meal of milk. A marked reduction in the coagulating power of milk is generally regarded as possessing more diagnostic importance than a heavy reduction in the secretion of pepsin, as indicating a greater destruction of gastric mucosa. In children, especially, the failure of the secretion of the stomach to coagulate milk is considered ominous. The failure to coagulate milk is of no loss to the organism, so far as is known, since the trypsin is able to digest casein as well as paracasein; and in any event, the pancreatic juice contains a chymosin.

Amylase, Saccharase, Maltase, Lactase.-Ferments of carbohydrate digestion are not secreted by the mucous membrane of the stomach of carnivora. In the herbivora such ferments are secreted in the second stomach, but the anatomical variations are so great that the comparison is of little meaning. The stomach contains, of course, the amylase and maltase of the saliva. Concerning the activity of these ferments in the stomach mention was made in the chapter devoted to salivary digestion. The general conclusion was that under normal conditions, the salivary digestion of carbohydrates in the stomach was of subordinate extent and importance; that the larger function of the saliva for the purposes of the total process of digestion lay in trituration, admixtion, and solution rather than in the saccharification of starch or the cleavage of maltose. This conclusion is directly in line with the opinion that the motor functions of the stomach have a higher importance than the chemical functions, in the sense that adaptation to the loss of the gastric chemical functions is much more easily attained than is adaptation to the loss of the motor functions of the stomach. In the judgment of the importance of a given function, its relations to the faculty of adaptation constitutes a sound guide. The application of this rule teaches us that the chemical functions of the saliva in the mouth and stomach are of subordinate importance.

Lipase.-The existence of lipase in the secretion of the stomach has been until recently the subject of disagreement. The contradictions now in the literature are possibly due to the differences in the gastric secretions of the dog and of man. The gastric contents of the dog does not seem to contain lipase unless there has been a backward flow of duodenal secretions into the stomach. When to a dog a heavy meal, 
rich in fats, has been given, this backward flow of duodenal juice seems to be frequent, if, indeed, not of regular and periodic occurrence. The presence of bile affords a ready test of the exact relations. On the other hand, the gastric contents of man presents a lipolytic activity under conditions where the admixture of duodenal contents seems excluded. 'The gastric contents expressed an hour following the ingestion of the Ewald test meal on an empty stomach exhibit lypolytic activity, will not digest protein at an alkaline reaction and give no chemical or spectral signs of bile. The obvious conclusion is that the gastric mucosa, in man at least, secretes a lipase; since it is not known in what state this ferment is secreted, inferentially it is in the state of proferment.

The reaction is simply the cleavage of the fat or ester into its two components, the fatty acid and the alcohol, an act of hydrolysis. Gastric contents have been tested upon triacetin, ethyl acetate, and triolein, in all cases with positive results. As to the comparative activity of gastric lipase upon the different esters, no published investigations exist.

The gastric lipase acts to but slight extent in the stomach. Since it is inactive in an acid reaction and its secretion in the stomach does not precede that of the hydrochloric acid, it has practically no field of action there.

The gastric lipase acts in a neutral or slightly alkaline medium. Since in a digestion of fat free fatty acid is being constantly set free, it is clear that the alkali would be soon combined, and after that time the reaction would be very faintly acid; such an acidity does not seriously disturb the action of the ferment, as does the much greater inorganic acidity of the gastric contents. The failure of the lipase to digest fat in the stomach is not due entirely to the acid reaction, but partly to the absence of the proper state of emulsification. In the chyme mixed with bile, emulsification is much better attained, and under such conditions the action of the lipase is greatly intensified.

Variations in Disease.-Of these, little is known. In profound organic disease of the gastric mucosa, the ferment ceases to be secreted. Important probably is the persistence of the ferment in cases of obstruction of the pancreatic duct. In many of these cases the digestion of fat suffers little, sometimes not at all; in all cases, with the presence of bile or in the event of the administration of the fat of the diet in a proper state of emulsification, a goodly digestion of fat is often to be secured. For this, the gastric lipase, together with that of the intestine, must be held responsible.

Pancreatic Juice and Bile in the Stomach.-Careful experimental investigations on dogs have recently indicated relations between the contents of the stomach and duodenum which, if confirmed for human beings, promise to throw light on obscure questions of the physiology and pathology of the digestive tract. In dogs the passage of duodenal contents into the stomach is not seldom, but frequent, and follows 
certain stimuli and conditions. The presence of an excess of fat or fatty acids results in a prompt transfer of duodenal contents into the stomach. While there can be little question that in man a lipase is secreted in the stomach, it may well be that in cases of excessive ingestion of fat, cleavage does occur in the stomach as the result of the presence there of pancreatic lipase. The same transfer of duodenal contents into the stomach may be observed in association with hyperchlorhydria, with resultant reduction in the acidity. There are indications that this may occur in the human stomach. It has been suggested that in some such way hyperacidity may be physiologically corrected, and that hyperchlorhydria may depend upon a non-functionation of this physiological check. On the other hand, it is possible that some cases of hypochlorhydria may be due to an excess of alkaline duodenal contents in the stomach. There seems also to be a third form of such discharge of duodenal contents into the stomach. When the stomach is empty, the pancreas and duodenum are engaged in periodic secretion. The stomach is empty and the pylorus often open. Not infrequently in the dog, this duodenal mixture of ferments is discharged into the stomach. The importance of such a situation for the question of the etiology of ulcer of the stomach is clear. It is indeed possible that the self-digestion of the stomach, which is currently assumed to be one stage in the formation of gastric ulcer, may be due to pancreatic trypsin rather than to the gastric pepsin. It is also possible that future investigations may indicate that certain forms of indigestion are due to the action of such duodenal secretions and ferments in the stomach.

The Secretion of Water.-A widely varying amount of water is introduced into the stomach with the food. Depending upon the habits of the individual, different amounts of water are introduced in the form of saliva, varying from 50 to 500 c.c. per meal. In the stomach itself there are four states of secretion of water-with the ferments, with the mucus, with the hydrochloric acid, and directly; it can be shown that these four avenues of water secretion are independent. Apparently the amounts of water that are secreted with the acid, mucus, and ferments are roughly constant for a fixed diet, the secretion of water with the mucus probably varying most of all. Least constant is the simple secretion of water, which is a variable compensatory, so to speak, to the other secretions of water and, of course, to the amounts introduced with the food. Careful investigations on the dog have indicated that under normal conditions there is a relation between the mass of the diet and the water, the water content of the chyme, in other words, is quite constant. Observations upon the chyme of human digestion confirm this conclusion. If the amount of water introduced plus that secreted with the acid, ferment, and mucus are sufficient to produce a certain consistency, no water will be secreted directly by the gastric mucosa; if the amount be insufficient, more water will be secreted. Thus, in dogs of a certain weight, it has been found that 
for a certain fixed large mixed meal, the total water secreted would be about 700 c.c., this to be followed by the secretion of some $50 \mathrm{C}_{\text {, c.c. }}$ of pancreatic juice; with a small test breakfast, the total water secreted by the stomach would be from 150 to 200 c.c., to be followed by the secretion of some 200 c.c. of pancreatic juice. The converse does not hold; if an excess of water be ingested, none will be absorbed by the stomach, the chyme will be abnormally fluid. As will be later elucidated, the motor functions of the stomach are the most important of its actions, and to the motor functions the water secretion is directly related.

According to certain pseudo-scientific cults now prevalent, the drinking of water with meals is held to be injurious. In so far as the drinking of water with the meals causes the individual to bolt the food and to neglect proper mastication (the drier the food the greater the flow of saliva), the practice of using water with the meals would be harmful. But apart from this it cannot be injurious. If the individual does not take water with the meals it must be taken between the meals, absorbed by the intestine and then secreted by the stomach during the period of gastric digestion. This work may be spared by the consumption with the food, between the acts of mastication, of a moderate amount of water, say half a liter. This is a natural practice, is rarely abused when plain water is used, and not only cannot be harmful but must be regarded as advantageous. The use of excessive amounts of water or of other beverages is harmful, because it imposes upon the stomach an abnormal labor, and upon the intestines and kidneys also an unnecessary labor. Herein lies the greatest harm in the use of beer and light wines, which are often, indeed usually, consumed in excessive amounts.

Variations in Disease.-Rarely the secretion of water is reduced or abolished as a functional neurosis, as in achylia gastrica. Reduction in the secretion is often observed in grave organic disease of the stomach, as in carcinoma, atrophy of the gastric mucosa, and chronic gastritis. It is not the rule, however. The secretion of water is apparently the last function lost in obliterative disease of the gastric mucosa.

Very frequently is the secretion of water increased in disease. As a rule, it may be said that irritation results in excessive secretion of water. Such hypersecretion often accompanies the secretion of excessive amounts of hydrochloric acid; nevertheless many cases of hyperchlorhydria without total hypersecretion are observed. Hypersecretion of water with normal acidity is also often observed, especially in chronic gastritis. In many cases the acidity is very low. This may be noted in carcinoma of the stomach, in atrophy of the mucosa and in extreme dilatation. Some of the most pronounced cases of total hypersecretion occur with dilatation of the stomach, the epithelium of special type being quite totally destroyed. 'This is comprehensible when it is recalled that in the atrophies of the gastric mucosa the special epithelia are replaced by cells of the common intestinal type, which are able to 
secrete mucus and water. The excessive secretion of water forms one of the serious features in the pathology of dilatation of the stomach.

Secretion of Mucus.-Whether the protein contained in the gastric secretion be a true mucin (a glycoprotein) or a nucleoprotein is not material to us here. The normal gastric secretion contains a large amount of mucus, how large may be realized when it is stated that one-fourth of the normal amount of hydrochloric acid is combined with it, $i$. e., the mucus secreted per day, may amount to some 20 grams. The mucus secretion of the stomach is independent of the secretion of hydrochloric acid and the ferments. Thus high temperature and electrical irritation provoke in the dog the production of a neutral mucus secretion. Unlike the other gastric functions, the secretion of mucus cannot be provoked by psychic or reflex factors, but is always a reaction to conditions within the viscus. The mucus has an unquestioned physical function in digestion, which it shares, of course, with that contained in the salivary and buccal secretions. The colloidal and viscous properties of the mucus aid in the proper formation of the chyme, in the same manner that gumma arabicum aids in the preparation of a permanent suspension and emulsion. If two portions of bread in a state of fine subdivision are shaken respectively with a fixed amount of water and with the same volume of water plus mucus, it will be soon noted that in the case of the latter a totally different chyme is produced. Whether the mucus has other functions is not known.

Variations in Disease.-Rarely is the secretion of mucus reduced, except in achylia gastrica and in the most advanced stages of organic disease, when the lining of the stomach consists largely of connective tissue. Like the secretion of water, the secretion of mucus persists late in degeneration, atrophy, and obliteration of the gastric mucosa. On the other hand, the secretion is often excessive. In chronic gastritis, with or without dilatation, in carcinoma of the stomach, in the functional hypersecretions with or without hyperchlorhydria, the secretion of mucus is often very excessive. Not only is the amount excessive, but the substance presents an excessive tenacity and colloidality, the result probably of the action of organic acids or bacteria upon it.

Resorption by the Stomach.-Great advances have been made within recent years in our knowledge of the relations of the stomach to resorption. Experimentations by means of the Pawlow methods have indicated that the inferences drawn from experimentation with drugs and upon the unisolated stomach could not be controlled, and were in the larger number of instances in error. That many chemicals on administration are absorbed from the stomach is, of course, true. The facts in pharmacology, however, have necessarily no bearing upon those of the physiology of digestion. We are now in the possession of sufficient data to enable us to indicate quite accurately the facts of gastric resorption in digestion. 
The fats are not digested in the stomach, and there are, therefore, no products to be absorbed. Experiments directed to the elucidation of the absorbability of the higher fatty acids and glycerol from the stomach, if they existed, would possess but secondary interest, of importance only to the physician engaged in the administration of predigested food. The available data tend to indicate that the higher fatty acids would not be absorbed from the stomach to any appreciable extent, the acid reaction would cast them out of solution.

The most recent investigations indicate that starches, cane sugar, maltose, milk sugar, and glucose are absorbed by the stomach to but the slightest extent in the dog. This work has not been confirmed for man, and in the nature of the methods employed cannot be confirmed except through accidental circumstances. That sugar will be absorbed from the dilated and ligated organ is true; but that fact has little bearing upon the stomach under physiological conditions. The general experience with test meals, when properly interpreted, tends to confirm for man the conclusions that are known to hold for the dog.

The proteins are digested to but a slight extent in the stomach of man normally. About three-fourths of the protein of a normal meal is converted in the non-coagulable state in the stomach; some secondary proteoses appear, peptone only in traces if at all, amino-acids not at all. It has been demonstrated for the dog that none of the products of the gastric digestion of protein are absorbed from the stomach. Our best evidence in man tends to confirm this conclusion.

The more finely foods are subdivided, the more rapidly is the chyme formed, and as a consequence the stomach is quickly emptied and digestion in the stomach is slight. The more coarse the food, the longer will it remain in the stomach, subject to gastric digestion.

The salts of the diet are largely absorbed by the stomach.

The final conclusion, therefore, is that the stomach is not to be regarded as an organ of resorption so far as the processes of normal digestion are concerned. That it does not normally absorb water, but on the contrary secretes water, has been pointed out in detail.

The Motor Functions of the Stomach.-The motor functions of the stomach are twofold; the mixing of the contents, the physical preparation of the chyme; and the discharge of the contents into the duodenum. There has been a great deal of controversy as to the modus operandi of the gastric movements. It suffices for us that in the normal stomach the contents have within an hour after ingestion of the food been mixed into a thin viscid chyme, in which the solid particles are in uniform suspension. 'The relations of the secretion of water and of mucus to the preparation of the chyme have been already elucidated. The water secretion of the stomach is such as to produce chyme of quite constant consistency; and the mucus aids in the establishment of a minute suspension of the solid particles. At the end of an hour or a little longer in the normal stomach, the pylorus is relaxed and a 
small amount of the gastric chyme, probably in man not over 30 or 40 c.c., are ejected into the duodenum, following which the pylorus is again tightly closed. The pyloric muscles are under duodenal, rather than gastric control; and the determining factor in the reflex appears to be the hydrochloric acid. The relaxation of the pylorus is apparently related to the presence in the duodenum of the alkaline pancreatic juice. So soon as the hydrochloric acid has rendered acid the contents of the upper duodenum, the pylorus is closed. Following the neutralization of the hydrochloric acid by the alkali of the pancreatic, biliary, and intestinal juices, the pylorus is again relaxed, and another small portion of chyme is discharged into the duodenum. These successive reactions are repeated until the stomach is emptied - a process requiring from three to eight hours, depending upon the amount and character of the diet. Our data indicate that this represents the fundamental and most important function of the stomach. As will be elucidated under its proper heading, when the gastric chyme is discharged into the duodenum, the intestinal digestion and resorption proceed with such rapidity that there is practically no accumulation of contents in the intestine.

Variations in Disease.-Reduction in the motor power of the stomach occurs sometimes as a functional neurosis, but rarely leads to serious results. Reduction of the motor function of the stomach occurs, apart from pyloric obstruction, in connection with chronic gastritis, atrophy of the mucosa, muscular degeneration, and in dilatation of the stomach, however produced. Pyloric obstruction has for the process of digestion the same meaning as abolition of motor power. The result is that the food instead of being discharged into the intestine, lies in the stomach, the prey of bacterial processes. Excessive secretion of water often occurs in these conditions, whereby the burden of the stomach is only increased. The results are inanition and water starvation, leading to profound asthenia. Since hypochlorhydria is often seen in these cases, the digestion of carbohydrates by the salivary enzymes continues; as the sugar is not absorbed but fermented by bacteria, the result is only further loss. The peptic digestion is usually depressed, so that in this direction also digestion suffers. Instead of being an organ for the preparation of the food for intestinal digestion, the viscus under these circumstances becomes converted into a fermentation tank.

As a general rule, to which there are many exceptions, excess of hydrochloric acid leads to prolonged retention of the chyme in the stomach, and deficiency of acid leads to early discharge into the duodenum. Excess of motor function, which is common, has a meaning only when the pyloric reflex is not maintained, and the hyperactive stomach is enabled to discharge its contents precipitously and in too large amounts into the duodenum. If this abnormality be pronounced, it may lead to diarrhea and enteritis, more especially if, as will some- 
times be the case, hyperchlorhydria be present. More often there will be much intestinal pain, but the work of digestion will be normally accomplished in the intestine. Much depends upon the diet in these cases, since the stomach will discharge the food into the duodenum before a proper chyme has been prepared.

General Consideration of the Functions of the Stomach.-From the detailed descriptions, it is clear that the human stomach has three functions; the digestion of protein and carbohydrates; the secretion of water and mucus and the preparation of the chyme; and the discharge of the chyme into the duodenum. When contrasted with the demonstrable importance of the second and third functions, the following points in regard to the first function must be borne in mind. There is no digestion or absorption of fat occurring in the stomach at all; probably half the starch of a normal diet is liquefied and a quarter saccharified, none is absorbed; three-fourths of the protein of a normal diet is converted into the non-coagulable state, there is no formation of peptone or of amino-acids and none are absorbed; finally all the digestion of carbohydrate can be easily accomplished in the intestine, and the gastric digestion of protein is of value only as a preparation for the intestinal digestion and can be easily dispensed with. The conclusion is positive that the primary and most important functions of the stomach are motor, and the chemical functions are secondary. In a broad way, the stomach of the carnivora is not so different from the first two stomachs of the herbivora or the crop and gizzard of birds. The chief function is the preparation of the food for intestinal digestion and the regulated discharge of the prepared contents into the intestine. Modern experience of surgeons supports this conclusion completely. There are now enough cases of complete extirpation of the stomach to convince all that the stomach is to an absolute extent a dispensable organ; such individuals remain in perfect health and nutrition, performing normally all the processes of digestion if in the culinary preparation of their food and in its frequent administration the motor functions of the stomach are supplanted. Tests in subjects with achylia gastrica have indicated that the intestine is alone not well able to digest connective tissues, but this is a matter of small importance. With this conclusion experiences with pyloric obstruction and gastric dilatation before and after gastro-enterostomy are in perfect accord. The possession of a stomach enables the carnivora and omnivora to ingest in one, two, or three feedings per day, the quantum of food necessary for the entire day, just as the fore stomach and psalterium enable the herbivora to consume the enormous quantities of grass necessary for their nutrition; in each instance the stomach, largely through its motor functions, prepares the food for chemical digestion and resorption by the intestine. For the physician it is especially important that the relative value of the chemical and motor functions of the stomach be understood. 


\section{DIGESTION IN THE INTESTINE}

The small intestine is the chief organ of digestion and practically the sole organ of resorption of the products of digestion. The small intestine is capable alone of maintaining the entire function of digestion; and under normal circumstances over three-fourths of the chemical work of digestion is accomplished there. Three important agencies in digestion operate in the small intestine; the pancreatic juice, the bile, and the succus entericus. The juice of the pancreas and the succus entericus each contain practically complete sets of enzymes; in a sense they are duplicate plants. The bile contains no enzymes, but contains important activators and zymo-excitors. The juices of the pancreas and intestine also contain activators. The relation of these three agencies to the stomach and to each other present, therefore, interdependencies. The functionation of the pancreas and of the secretory activity of the small intestine are dependent upon the secretions and the motor functions of the stomach. The secretions of the pancreas and of the small intestine are to a certain extent dependent upon each other. On the other hand, physiological experimentation and observations of states of disease have taught us that there is an enormous power of adaptation and compensation in the several functions of digestion; and that if the membrane of resorption be preserved, the chemical work of digestion can be accomplished quite independent of the relations of dependency that are to be described. An important part of our knowledge of intestinal digestion has been obtained by experimentation upon animals, largely upon the dog. In this presentation, it is assumed that except where contradictory data exist, the facts thus determined hold for man.

\section{THE PANCREAS}

From the standpoint of the constituents of its secretion, the pancreas is the most complex gland in the body. Its secretion contains no less than six different and separable ferments, important activators for the intestine and a varying amount of alkali, largely in the form of bicarbonates.

The pancreatic secretion of alkali is reciprocal to the gastric secretion of acid. Leaving aside the question of the modus operandi of the formation of hydrochloric acid, the fact remains that in the mucosa of the stomach sodium chlorid is split into its component ions, the chlorion passes down the alimentary tract as hydrochloric acid, the sodium is eliminated in large part by the pancreas and in small part by the intestine as sodium bicarbonate. In the lumen of the small intestine, they recombine to form sodium chlorid, which is resorbed to reënter the circulation. It has been the current teaching that during the period of secretion of hydrochloric acid the alkalinity of the blood 
is increased. While this might be expected, since the sodium that is to be eliminated by the pancreas must be carried there by the circulation, as a matter of experimental demonstration it has not been possible to prove this. The reaction, or electromotive potential, of the blood is at all times, during digestion and during fasting, quite neutral. Evidently the sodium is carried in part in some complex undissociated combination (ion protein) to the pancreas, in part as bicarbonate; and this together with the tension of carbon dioxid in the blood results in the maintenance of a neutral reaction. The reaction of the pancreatic juice is, of course, alkaline; the cells of the pancreas possess, therefore, the power of splitting the postulated complex sodium protein combination just as the cells of the stomach possess the power of separating the chlorions from the sodium. In each instance the process is believed to rest upon a physico-chemical basis. Since many of the functions of the pancreas can be shown to be dependent upon the acid secretion of the stomach, it might be questioned whether they could be the result of the action on the pancreas of the alkali eliminated as the result of the acid secretion of the stomach. This is, however, not the case. Hydrochloric acid introduced into the stomach has the same results upon the secretion of the pancreas as does acid secreted in the stomach, except that the pancreatic elimination of alkali is in the first instance much less. When acid is introduced into the stomach, this is in the intestine (to the extent that it is there neutralized) neutralized largely by alkali secreted by the intestinal mucosa as an abnormal compensatory process. Normally very little more alkali is secreted into the intestinal tract than necessary to neutralize the acid of the stomach-the alkalinity of the intestinal contents is very slight, not over $\frac{\mathrm{N}}{10000}$.

Relations and Variations in Pancreatic Secretion.-The relations and variables of the total secretion of the pancreas are known in but a fragmentary manner. Acidity in the gastric secretion or in the food promotes secretion; alkaline reaction depresses or inhibits. The free use of water tends to increase the volume of secretion. In the herbivora the secretion is quite constant; in the carnivora and in man the secretion is intermittent. There are psychic influences that may operate in a positive direction. If to a dog with esophageal, gastric, and pancreatic fistulæ a mock meal be given, it will be found that a secretion of the pancreatic duct appears some time before that of the stomach, and therefore independent of the latter. Variations in the total volume of the secretion are not known to rest upon variations in the diet except in so far as these influence the concentration of acid in the stomach. Qualitative variations, however, are known to correspond to variations in the diet. Thus a meat meal causes a secretion very strong in ferment (in all the ferments), bread leads to the formation of a weaker secretion, milk to the fecblest secretion. It is necessary to note that there is no apparent tendency for a food to stimulate the secretion of the ferment necessary for its digestion; on the contrary, 
stimulation of one means stimulation of all. With pure pancreatic juice, the proteolytic activity is quite constant for the unit of secretion, independent of the quality or the type of food within the stomach. Fats exert an undoubted positive stimulation of the secretion. Extractives and condiments introduced into the stomach are without effect except through the secretion of hydrochloric acid that they may occasion. Alcohol depresses the enzymic activity of the secretion, though the total volumn is somewhat augmented.

The gastric acid is apparently the chief influence on the pancreatic secretion. Up to a certain point, the secretion of the pancreas produced through the action of hydrochloric acid in the stomach displays augmenting volume and increasing activity with increasing concentration of the acid. Beyond this point, the secretion will be very large in amount and strong in alkali, but poor in ferments. It is clear that the secretion of water, of alkali, and the formation of ferments are not parallel functions. When acid is introduced into the stomach, there is a latent period of a few minutes, following which the pancreatic secretion begins to flow. The influence of hydrochloric acid is not a function of the chlorion but of the hydrogen ions, and is, therefore, common to all acids. The action upon the pancreas can be shown not to be due to the withdrawal of the chlorions from the circulation or to the presence of the relative excess of sodium in the blood. There is obviously some form of circuitous action proceeding from the lumen of the stomach and terminating in the cells of the pancreatic gland. That the older designation of reflex nervous action affords no elucidation of the phenomenon, is clear. It has been possible, however, in part at least, to trace this influence in a chemical manner.

If an extract of the resting intestinal mucous membrane be prepared and injected into the circulation, no secretion of the pancreatic gland is to be noted. If, on the contrary, the extract be prepared from a section of intestinal mucous membrane that has been in contact with hydrochloric acid, injection of this extract will be followed by vigorous secretion of the pancreas. It is clear that as the result of the action of the acid upon the cells of the mucosa, a substance is formed that, like pilocarpin, is a stimulant to the gland. It can be shown by the ablation of all nervous connections of the pancreas that the action of the extract is independent of the central nervous system. Direct injection into the pancreatic artery gives also a positive result. The same result may be secured with an extract of the mucous membrane of the stomach. 'To this hypothetical substance the name secretin has been given. It is soluble in alcohol, and is thermostable. It is, therefore, not a protein. But since it resists ordinary dialyzation, it cannot be crystalloid. It was originally held to exist in the mucosa in an inactive state, the activation being the result of the action of the acid. Secretin is not peculiar to the species, but derived from one species is active for all animals. It has been shown in more recent work that lower peptones and polypeptids have a similar power of stimulating 
the action of the pancreas. These cannot, however, be held responsible for the early secretion of the pancreas that occurs during the course of gastric digestion. The chemical nature of secretin is thus as yet unknown. But it is clear that we have to deal with a striking type of phenomenon, so far as the modus operandi is concerned; the pancreas is stimulated by a substance from the alimentary mucosa that is carried to it in the circulation.

The formation of secretin is not solely limited to the action of hydrochloric acid. There is evidence that the action of the bile upon the intestinal mucosa tends to stimulate the secretion of the pancreas. This is not an experimental fact, but simply a conclusion drawn from the observation that bile tends to accelerate the pancreatic digestion of protein and starches as well as of fats, in whose resorption bile is directly concerned. As a matter of fact, however, the secretion of the pancreatic juice is not dependent upon the formation of secretin, unless this formation is brought about in more ways than are at present known. This is made certain by the fact that the secretion of the gland occurs and continues in the animal body deprived of gastric juice and bile. Either secretin is not necessary for the secretion of the pancreas, or else hydrochloric acid and bile are not the sole agents that provoke the formation of secretin. The products of bacterial action suggest themselves as possible agents. Chloral hydrate, in the total absence of acid, acts upon the intestinal mucosa in such a way as to stimulate the secretion of pancreatic juice. Maceration of the intestinal mucosa in alkali yields also an extract quite active as secretin.

Under the term secretion of the pancreas we understand the discharge of the secretion from the ducts, not the formation of the secretion. In the carnivorous animal, the formation of the ferments occur during the period of rest, following a digestion. The protoplasm of the cells of the acini becomes distended with granular appearing material, the product of the synthetic action of the protoplasm. This granular material represents the raw state of the proferments. The act of secretion consists in the liquefaction (using the word in the ordinary sense) of these granules and their discharge through the lining membranes of the cells into the ducts.

When the secretion is drawn from the common ducts of the gland, it is devoid of digestive power, $i$. $e$., the ferments are in the state of the zymogen. If a fistula be so constructed that the outflowing secretion does not come in contact with the duodenal mucosa, the juice will be found to be inactive. Not infrequently (possibly always) a slight activity may be noted, due possibly to the action of the lining cells of the ducts, since all tissues, practically, on maceration yield extracts that activate the enzymes. This activation of the ferments is here, as elsewhere, due to acceleration of an autohydrolysis by the presence of some catalytic substance. The proferments on standing in water slowly undergo hydrolysis, being thus converted into the active ferments. 
Positively catalytic to these reactions of hydrolysis whereby the pancreatic proferments are activated, are hydrochloric acid, the biliary salts, bacterial products, and enterokinase, of which particular mention will be made later. Since bacterial products are always present in the intestinal contents, it follows that activation of the ferments, though possibly slow, could never fail, even in the absence of bile, gastric contents, 'or intestinal juices. On the other hand, it is clear that the introduction into the pancreatic duct of intestinal contents or bile might result in the activation of the ferments within the ducts of the gland; and one of the causes of disease of the pancreas is quite certainly such abnormal activation of the ferments within the ducts.

The function of the adrenal bodies is in some unclear manner connected with the secretion of the pancreas, and epinephrin can be shown to act as a stimulant to the secretion. Many other organs have been tested, most of all the spleen, for such problematical action, but with negative results. The total volume of pancreatic secretion in man is not known definitely, as there are few recorded instances of measurements in cases of accidental or surgical fistulæ. It may probably run as high as half a liter per day. Judging by animal experimentation, hyperchlorhydria and the free drinking of water would result in large secretion.

Our knowledge of the action of the several ferments of the pancreatic secretion is, for obvious reasons, less extensive than in the case of the ferments of salivary or gastric secretion. Many of the studies of the pancreatic ferments have been carried out with dry preparations of the glands, and such investigations cannot be expected to yield final data.

Amylase.-The pancreatic juice contains an active amylase, a more powerful ferment than is found in the saliva, in particular more active in the hydrolysis of raw starch. It is secreted in the inactive state and is activated by the bile, the succus entericus, and possibly also by bacterial products and by acids. It is not possible, with the use of either starch or glycogen as the substrate, to determine that the qualitative action of the ferment is in any way different from that of the saliva. The amylase of one secretory period of the pancreas is able within a few hours to convert into sugar several hundred grams of cooked starch, aided, of course, by the preceding action of the salivary amylase and the accompanying action of the intestinal amylase; the reaction is usually complete, $i$. $e$., the feces contain no undigested starch. Were the action of these ferments not complete, bacteria would in all probability, in the absence of diarrhea, often ferment the undigested starch. It is a common experience of those engaged in the analyses of stools to find occasional particles of undigested starch. Such are usually due to improper cooking or to imperfect mastication. On the other hand, how effective may be the digestion of uncooked starch, when the foods are properly masticated, is illustrated by the normal digestion to be noted in the adherent of the sects who, like 
the herbivora, consume only raw food. We read so much of the proper baking of bread and of the completed cooking of cereals; yet there are whole colonies of diet fadists, adults and children, who masticate and digest raw grains.

The conditions for the favorable action of the amylase have not been worked out. The reaction optimum is a very slight degree of alkalinity, about $\frac{N}{10000}$. Good fermentation is to be noted at neutral reaction, and even some action at slight acidity. High alkalinity depresses strongly, though excess of alkalinity is better borne than excess of acid. In each instance the depression is due to destruction of the ferment, not to inhibition of the activity. So far as known, none of the variations in the reaction of the normal digestive tract could disturb appreciably the activity of this ferment. The normal stools do not usually contain amylase that can be shown to be separated from the bodies of the fecal bacteria. Contrary findings are, however, often noted, especially with diarrhea or purgation. Apparently the bacterial processes in the lower intestinal tract, probably in the colon, destroy the ferment. In the presence of starch, the ferment is quite resistant; in the absence of starch, which is the normal condition in the colon, the ferment is sensitive. This fact is of importance, since it teaches that we cannot count upon amylolytic action in the colon in the use of rectal alimentation. The presence' or absence of amylase in the stools cannot be employed as a reliable test of the presence or absence of pancreatic secretion in the intestine.

Maltase.-Of the maltase of the pancreatic juice still less is known. The existence of maltase in the pancreatic juice has indeed been denied; in all events it is subordinate to the intestinal maltase in importance. Unlike the amylase it is not of high activity. The utilization of maltose proceeds apparently quite as rapidly as that of glucose, so that the digestion of starch, in which, of course, the pancreatic ferment plays only one part, must be very rapid. What little is known of the favorable conditions of action correspond to what has been stated for amylase. It is apparently very easily activated, since under the conditions of procurement in which the pancreatic juice is obtained inactive as to trypsin and lipase, the maltase is active, yet it can be shown to be secreted in the inactive state. It is a ferment very sensitive to untoward chemical influences. The normal stools contain no maltase not connected with fecal bacteria-important again in relation to rectal alimentation.

Invertase and Lactase.-Invertase is not present in the pancreatic juice-all searches have been negative. The digestion of cane sugar is, therefore, outside the scope of the pancreatic secretion. This fact holds for herbivora as well as for carnivora, and corresponds to the fact in the salivary secretion.

Whether lactase exists in the pancreatic juice of the adult is doubtful. Nearly all investigations have led to negative results. A few have found indications of the cleavage of milk sugar with the use of the 
pancreatic juice for young animals after a period of milk diet, but even here the experimental results have not been clear-cut. 'The widespread occurrence of invertase and lactase in bacteria renders the question difficult of study. For the present, it will be safest to state that the cleavage of cane sugar and of milk sugar are functions of the succus entericus alone.

Emulsin.-There is no question that glucosids are hydrolyzed in the intestinal tract of man and of the higher animals. This might be due to an emulsin in the pancreatic juice, in the succus entericus, or to a ferment derived from bacteria, many of which have this function. It now seems clear that the pancreatic juice contains no emulsin-this is contained only in the succus entericus. The term emulsin is used generically, since the faculty of the intestinal tract has not been systematically tested upon glucosids.

Lipase.-To this ferment belongs one of the most important properties of the pancreatic juice, the cleavage of fat. The ferment is secreted in an entirely inactive state. It is activated by bile, by the succus entericus, and by bacterial products. Gastric contents also have the property of activation, though the hydrochloric acid is destructive to the ferment. The ferment is a very active one. Its action in the intestine is, however, augmented by the presence of zymo-excitors. The ethereal extract of the bile is slightly active in this direction. The bile salts are very active, the cholic acids being the potent substances. Thus the presence of bile can be shown in vitro to quadruple the velocity of the hydrolysis of a constant substrate of fat acted upon by a constant mass of lipase. This constitutes in fact the best-known illustration in the body of the action of a zymo-excitor. Acting under these circumstances, despite the low solubility of the fats and the dependence of the reaction velocity upon the attainment of a proper emulsion, in actual digestion the cleavage of fat is a reaction of large extent. Within a few hours a hundred grams of fat may be entirely split, this accomplishment being, of course, the united work of the intestinal and the pancreatic lipases. That the activation and zymo-excitation of the bile are not indispensable is shown by the excellent digestion of fats to be noted in many cases of complete absence of the bile from the intestine. As will be described later, the bile has not only active relations to the cleavage of the fats by lipase, it has also important relations to the solubility of fats. And since it is not possible in vivo to separate these two factors and to evaluate them, we are not in a position to state to which of the actions of the bile the loss is to be ascribed in those cases in which the absence of bile carries with it a reduction in the digestion of fat. Certain it is, however, that in vivo the pancreatic lipase is able to exhibit a proper activation and a satisfactory acceleration of the hydrolysis of fats in the absence of the bile.

The lipase of the pancreatic juice accelerates the hydrolysis of esters as a class, not only the higher fats, but also the lower esters of the natural fatty acid series, and all manner of complex and synthetic esters. 
The enzyme is, for a ferment, very stable, the vegetable lipases being probably the most stable of all ferments. The ferment acts well in either acid or alkaline medium of moderate concentration. Accurate investigations are not easily carried out, since with the progress of the hydrolysis of a fat, acid is being constantly set free to combine with alkali. Rather high concentrations of the product (fatty acid), are tolerated, even in the case of the more highly dissociated lower fatty acids. In fact, up to a certain point, it is a question if the fatty acid does not indeed contribute an auto-catalytic influence with the result of increasing the velocity of cleavage. The ferment is fairly resistant to bacterial action, particularly in the presence of fat. Lipase may be found in the normal stools, though not invariably. This finding, different from that noted for the fermentation of the sugars, is explained in part by the fact of the greater resistance of the ferment; in part, however, by the fact that unaltered substrate, which protects the ferment from destruction, is always present in the stools. The presence of lipase in the stools cannot be used as a test of the function of the pancreas, since the succus entericus contains a lipase. In any event, its absence from the stools would not indicate that it had not been secreted.

Included in the diet are many complex lipoids, termed phosphatids and sterins, the chemical constitution of which will be described, so far as that is possible, on another page. 'The behavior of these lipoids in digestion is not well understood. For the sterins (cholesterols) the present idea is that they are largely rejected with the feces. It is, however, not proper to state that they cannot be and are not resorbed. Though they are very insoluble in water, they are to a slight extent soluble in triolein, and thus the chemical possibility of their resorption is established. 'The fate of the phosphatids, if we may judge them at all by common lecithin, is hydrolysis through the action of the lipase of the pancreatic duct and intestine. Beyond that we know nothing. Whether the components, especially the glycerol-phosphoric acid and the cholin, are utilized in the body following their resorption, is not known. That the body possesses the power of forming glycerol-phosphoric acid from the two components is known; the organism is, therefore, not dependent upon the phosphatids of the diet. To the writer the most reasonable assumption is that the body forms its phosphatids de novo, and is in no way dependent upon the glycerol-phosphoric acid and cholin of the diet.

Chymosin.-Within recent years it has been demonstrated that the pancreatic juice is able to coagulate milk. This faculty can be shown to be in all probability independent of the ferment trypsin. The properties of the pancreatic rennet seem to be identical with those of the gastric rennet, except possibly in a greater sensitiveness to acid and a greater resistance to alkali. The reaction of curdling is accomplished in either acid, neutral or alkaline reaction, the appearances and properties of the curd differ in nowise from those noted 
in curdling by gastric chymosin. There is one difference in the two secretions, however: the gastric juice contains more rennet than does the pancreatic juice, and less of the secretion is required to coagulate a given volume of milk. The function is obviously non-operative in ordinary digestion, since after the action of the gastric juice upon milk no further coagulation is possible.

Trypsin.-The proteolytic ferment of the pancreatic juice is termed trypsin. It is secreted in an inactive state. On reaching the duodenum the zymogen becomes activated by the suecus entericus. Since it can be shown that this property of activation is not common to all forms of intestinal secretion, it is credited to a special substance that has been named enterokinase. The duodenal mucosa presents enterokinase in its juice only following the discharge of the inactive pancreatic juice into the duodenum. In other words, it is the presence of the inactive pancreatic juice (activated juice will have the same result) in the duodenum that stimulates the mucous membrane there to the secretion of the enterokinase. The mere presence of food or water or even of boiled pancreatic juice has no such result. No enterokinase will be found in the succus entericus of an animal whose pancreatic juice is discharged outside the body. This enterokinase activates in direct portional to its mass, and is to be regarded as a catalyzer. The reaction of the activation of the trypsinogen is a reaction of hydrolysis, and can be shown to be an autoreaction:

\section{Trypsinogen + water $=$ trypsin}

The enterokinase accelerates this reaction. While on the one hand the secretion of the pancreatic juice and its presence in the duodenum is the sole occasion for the secretion of enterokinase, the activation of trypsinogen is not limited to enterokinase. The salts of calcium are quite active activators, and it is to its calcium content that the bile owes its demonstrable powers of activation. Bacterial products and extracts of tissues (apparently of any of the internal glands or tissues) are also activators. Apparently, therefore, the activation of the pancreatic trypsin would occur even in an intestine exhibiting no secretion. Under normal conditions the reaction of activation is one of great rapidity. Enterokinase is thermolabile, but soluble in alcohol.

Trypsin is a ferment of comparative stability and of great activity. As previously described, experiments in glass would seem to indicate certain limitations. The early stages of digestion (decoagulation and transformation into proteoses) it does not accomplish as rapidly as does pepsin; egg albumin and serum albumin are digested by it with difficulty, and some of the proteins of the connective-tissue groups seem entirely resistant to it. On the other hand, it is not able to complete the cleavage of the resistant polypeptid nucleus, and to set free the contained amino-acids. The last function is, however, executed by the erepsin. As to the first-mentioned limitations, experience in intestinal digestion without gastric juice does not indicate any appre- 
ciable delay or limitation in the digestion of proteins under such circumstances. All of the work of the gastric digestion of protein in the stomach is easily and unnoticeably accomplished in the small intestine. And since it seems clear from investigations with erepsin that it cannot be held responsible for the reactions in the earlier stages, either the limitations of trypsin observed in experiments in vitro do not hold in the intestine, or the duodenum must secrete another proteolytic ferment. For the latter suggestion some experimental work may be held to speak in a provisional manner. It will for the present, however, be safest to enlarge our concept of the function of trypsin. Apart from the proteins of the connective-tissue group, all the chief proteins of plant or animal origin are easily digestible by trypsin. The secretion of a day can be shown to be capable of digesting several hundred grams of protein. This digestion, shared with the erepsin, must be regarded as a most thorough one; that is, all the protein of a diet is digested at least to the polypeptid stage, the larger amount passing completely to amino-acids. (Cf. page 47.)

To what extent quantitatively the cleavage of protein in digestion normally proceeds, is impossible of accurate determination. On the one hand, there is no question that peptones have the power of diffusion. Proteoses and peptones, native proteins indeed, seem to be resorbable from isolated loops of intestine. That this resorption, however, is not simple in the sense of a parenteral resorption, is shown by the fact that a precipitin reaction is not established by such intestinal resorption. On the other hand, it seems clear that peptones cannot be used as building stones for protein. Even should peptones be resorbed, it is most likely that they would be split within the intestinal wall. Nitrogenous equilibrium and the formation of flesh can be accomplished by the split products of the digestion of protein-aminoacids. This fact is obviously in itself no direct proof that normally digestion proceeds thus far. The data bearing upon anaphylaxis make it certain that the reaction of hydrolysis proceeds to the point of obliteration of the specific biological properties of protein. Whether this cleavage occurs within the lumen or partly within the wall of the intestine is not known. There are observations tending to connect the sensitizing property of native protein with the antipeptone fraction. If this be true, protein digestion must be a very complete one. From the point of view of the syntheses of the blood proteins, it is evident that complete or nearly complete cleavage of the protein molecule is necessary to make possible the formation of the blood proteins from the diverse proteins of the diet. Some of the amino-acids required in the synthesis of the blood proteins are in the last resistant fraction of antipeptone, and it cannot be believed that this resistant fraction is a unit common to all proteins; it too is a biological entity. From every point of view we are led to the assumption that in the digestion of protein, accomplished finally by trypsin and erepsin, the cleavage of the protein molecule is practically complete. 
The conditions of favorable action of trypsin are rather wide. It exhibits a notable resistance to hydrochloric acid, which enables it to resist destruction by the gastric contents pending the neutralization of the acid by the pancreatic alkali. It is able to act in a slightly acid medium. It tolerates rather high concentrations of alkali, though the reaction optimum can be shown in the case of the dog to be about $\frac{\mathrm{N}}{10000}$. The concentration of bacterial organic acids in the normal intestinal tract does not inactivate trypsin. Such high concentrations of hydrochloric acid as are sometimes seen in exaggerated instances of hyperchlorhydria would certainly operate destructively to the trypsin were it not for the fact that in such cases the pancreatic juice is in a proportional manner excessively alkaline, so that in the end the reaction within the intestine is not different from the normal-there is simply more sodium chlorid present. The favorable action of alkali upon the process of digestion by trypsin may be regarded as due to combination with the ferment. The enzyme is to be regarded as a weak acid, and a salt with the alkali would be subject to a greater dissociation than the acid itself, thus leading to a greater catalytic activity.

Trypsin is often present in the stools. This is again a fact to be remembered in connection with rectal alimentation. When absent, this is probably the result of bacterial putrefaction. In the case of the destruction of amylase, maltase, and lipase it is possible to picture them as digested by the trypsin and erepsin. In the case of these, however, bacterial action is to be invoked in explanation of their destruction. One factor of importance in determining the occurrence of active trypsin in the feces is the period of retention of the feces in the colon, which varies in different individuals from twenty-four to sixty hours, rarely to ninety hours. The longer the retention of the feces in the colon, the more opportunity is afforded bacteria to destroy the ferment.

The Pathology of Pancreatic Secretion.-The variations in the secretion of the pancreatic gland, under systematic classification, would fall under two heads-excessive secretion and deficiency or total lack of secretion.

Excessive Secretion.-Excessive secretion could involve the water, the ferments, or the alkali. Of the total secretion of the pancreas so little is known that we lack a norm with which an accidentally measurable secretion could be contrasted. That such a state exists as a hypersecretion of the pancreas, comparable with hypersecretion of the stomach, is not known. Judged by the known facts for the parotid gland, such a state could result from abnormal reactions in the nervous connections and might result also as an acute condition from inflammation. A prolonged excessive secretion would be a hypersecretion of water, and not of the ferments and alkali, as is true of the chronic hypersecretions of the parotid gland. Excessive secretion of alkali, except as reciprocal to hyperchlorhydria of the stomach, is unlikely if not impossible. While it is possible to withdraw cations through 
the agency of acid introduced into the system, there is no known possibility of the spontaneous elimination of alkali.

Deficient Secretion.-Much more likely to occur is deficient secretion. From what is known of the diseases of the stomach and salivary glands, chronic disease involving the secretory apparatus might be expected to result in a reduction of the secretion. But the chronic diseases of the pancreas are concerned largely with sclerosis, and usually involve the acini to but slight extent. Acute disease of the pancreas attended with destruction of tissue, as in septic and hemorrhagic pancreatitis, surely leads to pronounced reduction or complete abolition of the secretion. But there is no simple way of measuring the pancreatic secretion except by observation of the functions of digestion of fats and protein, and in this way only in a conjectural manner. Such observations, revealing sometimes profound reduction in the digestions, have been made in instances of chronic disease; the tests are out of the question in acute diseases of the pancreas. With intact functions of the stomach, bile and intestinal juice, the complete cessation of pancreatic secretion might lead to no reduction in the efficiency of digestion. Low indicanuria was once supposed to suggest deficient pancreatic digestion; this is not true in fact, and the suggestion is faulty in theory, since the tryptophan from which the bacteria form the indican, is set free by erepsin as well as by trypsin. Cessation of pancreatic secretion, or complete obstruction of the pancreatic duct or ducts, is often followed by some distinct reduction of the powers of digestion, involving most the fats. When such a patient is placed upon a known diet, it is found that the residual nitrogen of the stools is much increased, from possibly 10 per cent. of the input up to 30 per cent.; in pancreatic diabetes the azotorrhea may be still higher. If the subject is on a minimal protein ration, this may not be at all apparent, but can be provoked by placing the subject upon a higher protein input. With a known fat input the residual neutral fat of the stools may exhibit a pronounced increase. This fat will usually be neutral fat, indicating failure of enzymic cleavage and not failure of resorption. If the subject be on a low fat ration, this may not be apparent, but can be provoked by the ingestion of amounts of fat that are larger though well within the normal range. It is remarkable in experiments with dogs, how frequently a normal digestion will be maintained in the properly fed animal following ligation of the pancreatic ducts; but if the gland be extirpated, the results upon digestion are often much more pronounced. Applying these results to human beings, it may be said that a pronounced reduction in the cleavage of fat and in the resorption of protein suggests strongly the cessation of pancreatic secretion or obstruction of the ducts; but a normal digestion does not prove normal secretion or patulous pancreatic ducts. Different animals react differently, thus the rabbit reacts to the absence of pancreatic juice much less than the dog. 'This warns us to apply to man with circumspection the knowledge obtained by tests with animals. 
Within recent years methods have been developed to secure duodenal contents from human beings for the purpose of direct determination of the presence of pancreatic juice. Following the ingestion of a meal rich in fat, regurgitation of duodenal contents into the stomach occurs with such frequency as to afford a basis of examination. Methodically preferable, however, is direct sampling of the duodenal contents by means of the Einhorn bucket. In the nature of things, the evidence to be obtained from a direct examination of duodenal contents must surpass any indirect method of estimation of the presence of the pancreatic enzymes in the intestinal tract.

Reduction or cessation of the alkali secretion of the pancreas must be regarded as strictly reciprocal to the secretion of hydrochloric acid in the stomach. In achlorhydria, there must be practically an absence of alkalinity in the pancreatic juice. The influence of this upon the processes of intestinal digestion can only be conjectured. Since, however, it is well known that achlorhydria need carry with it no disturbance of digestion at all, it is clear that the pancreatic alkali is dispensable in the intestine. The figures given for the optimal reactions of the several ferments operating within the intestine indicate how little alkali is required for them. Test-tube experiments indicate also how little alkali is necessary to produce good emulsifications with neutral fats and fatty acids, especially in the presence of bile. That higher concentrations of alkali increase the solubility of the fatty acids is, of course, true; but the surface of resorption is so large that apparently the intestine can complete absorption from low concentration. In the case of obstruction of the pancreatic duct, if the secretion of hydrochloric acid in the stomach be continued in the usual manner, the alkali secretion that normally passes through the pancreas must be eliminated by the small intestine.

Activation of the ferments of the pancreas within the organ, in particular of lipase and trypsin, would have the result of self-digestion of the gland and possibly of the surrounding tissues. There is no question that this does occur; this and septic infection probably comprise nearly all the cases of acute pancreatitis. The activation of the ferments within the ducts may be caused by bile, hydrochloric acid, intestinal juice, and possibly also by bacterial products. Just as septic infection will be added to self-digestion, so self-digestion may be added to septic infection. The products of such self-digestion are very toxic. If into the sterile peritoneal cavity of a dog, the sterile, freshly macerated pancreas of a second dog be introduced, the result will be death within twenty-four hours as a rule. This illustrates what will happen if the self-digestion occurs within the animal's own pancreas, except that resorption of the toxic products will in such a case be slower and death longer deferred. This experiment indicates the necessity of drainage in such a case in man; how one who survives such an attack with operation is to continue life, will depend upon the extent of destruction of the pancreas and upon other circumstances. 
One difficulty, it must be pointed out, lies inherent in the investigation of abnormalities of pancreatic secretion. As will be later described, the pancreas possesses a most important inner function. Derangements in this inner function lead to profound disturbances in metabolism, $i$. e., diabetes. The separation of these from the effects of digestive derangements that might result from suspension of pancreatic secretion (or obstruction of the ducts) may be impossible. A lesion might involve only one function, secretory or internal; a lesion might affect both functions. Diabetics usually present normal intestinal digestion; in some cases it is seriously disturbed. Obviously the relations may be obscure and a clear picture of the facts, morphological and chemical, may not be obtainable. The experimental data are contradictory. On the one hand are reports that indicate that ligation of the ducts leads to little or no injury to the processes of digestion. One explanation that was suggested to elucidate this result was that the ferments were resorbed into the circulation, carried to the intestine, and eliminated into the lumen, there to meet the food. This explanation, however, fell to the ground as soon as it was found that the identical result was secured in the dog with pancreatic fistula, in which the intestine was deprived of its pancreatic ferments completely. Extirpation of the pancreas in these cases was followed by profound reduction in the processes of digestion, $i$. $e$., in some internal way the pancreas acts upon the processes of digestion. In harmony with this interpretation is the fact that feeding with pancreas or the injection into the duodenum of pancreatic juice, has little result on digestion in animals that have suffered extirpation of the gland, as in this way only the externally active substances of the gland are introduced. In the rabbit and apparently in the dog, it is possible to have the pancreas atrophy after ligation of the ducts with little or no disturbance of digestion, at least with no more than a reduction in the limit of assimilation of sugar. In such a rabbit, autopsy has indicated the complete atrophy of the acini, with retention of the islands of Langerhans. On the other hand, opposed to all these findings, are the facts that not seldom in dogs with diabetes following removal or atrophy of the pancreas, the digestive processes are very well maintained. And in some dogs, in whom a portion of the pancreas has been retained, digestion is greatly disturbed. These results are interpreted to mean that the sole influence the pancreas has on the processes of digestion is through the pancreatic juice; and whether digestion in the absence of this juice is well or poorly accomplished, depends upon the powers of adaptation of the other secretions of the digestive tract.

\section{THE SECRETIONS OF THE INTESTINES}

The secretions of the intestine contain substances of four groups: ferments and allied bodies; salts; end products of metabolism; and 
proteins. The proteins belong to the mucin group and are secreted, as in the stomach, in considerable amounts, probably as much as 10 to 15 grams per day. Their function is to aid in the processes of emulsification of the intestinal contents. End products of metabolism are here eliminated in but small amounts; a consideration of these will be undertaken in another place. The salts eliminated by the intestine include all those subject to elimination by the body, but concern especially the salts of iron, lime, and phosphoric acid. In the elimination of chlorids and sulphates, and of the cations sodium, potassium, and ammonium, elimination by the intestine seems to be in a sense incidental. But in the elimination of iron, calcium and phosphoric acid, the intestinal elimination is specific. 'The larger fraction of ferric elimination is accomplished by the small intestine, and of the calcium and phosphoric acid, half is frequently eliminated here.

The small intestine displays apparently two types of secretionone associated with digestion, one independent. When the stomach of the dog is empty and the dog is starving, a periodic secretion of succus entericus occurs about every two hours. The secretion is rich in mucin and poor in ferment. It is this secretion (including the bile) that forms the stools in starvation. When now food is in the stomach, this periodic secretion seems to be inhibited. When food is discharged from the stomach into the intestine, rapid secretion of strongly enzymic juice is inaugurated. In this digestive secretion several factors seem to be operative; the presence of the bulk of the chyme (distention) in the duodenum; the action of the pancreatic juice and possibly the action of secretin, as in the case of the pancreas. The bile has apparently no effect upon the secretion of the intestine, nor has the hydrochloric acid any demonstrable direct effect. The secretion of the intestine is most marked in the duodenum, and descending the tract lessens.

Not a little alkali is eliminated in the succus entericus. Contrasted even with the amount eliminated in the pancreatic duct, the fraction is considerable. Although no analyses are possible, it is clear that it must be largely in the state of bicarbonate. It is sufficient to be of determining importance in the solution and resorption of fats and their products of digestion, as is to be witnessed in those not infrequent instances in which, with the exclusion of the pancreatic secretion from the intestine, the digestion and resorption of fats proceeds in a fairly or indeed entirely normal manner. It is, however, possible that in the event of non-secretion by the pancreas, the alkali elimination of the succus entericus is increased; certainly this is to be taken for granted in the event of normal secretion of hydrochloric acid in the stomach in such cases. In infants the presence of abnormal amounts of acid in the intestine may result in the elimination of excesses of alkali, and in the consequent depletion of the cations of the body.

Very little water is eliminated by the intestine, probably not over 200 c.c. per day. The secretions are not watery, but thick and viscid under normal conditions. When one contrasts the water content of the normal feces with the amount of water that enters the duodenum, 
the water ingested in food and beverage, the water secreted by the salivary glands, by the stomach, by the pancreas and in the bile, the extent of water resorption by the intestine becomes clear. We may approximate this water as amounting to some six liters per day. This is the water that serves as the solvent for the products of digestion in their resorption. The dependence of the normal processes of digestion and resorption upon this volume of water in the intestine must be very direct. And the diet fad that attempts to restrict water input to the lowest possible limits is operating contrary to sound physiological sense.

Ferments of Intestinal Juice.-The succus entericus contains eight ferments, the intestine being the one tissue that forms all the different ferments of the alimentary tract. These are amylase, maltase, invertase, lactase, emulsin, erepsin, lipase, and chymosin. It is the occurrence of all these ferments in the secretion of the intestine that explains the unquestioned fact that with the removal of the functions of the salivary glands, stomach and pancreas from the processes of digestion, these can under favorable conditions be fully accomplished by the small intestine alone. It is incorrect to state that with the loss of the mentioned secretions, one or all, the small intestine vicariously assumes their functions; in truth, it possesses all these faculties naturally and exercises them normally. We possess, therefore, in digestion what in the technical trades would be called duplicate plants. Of the advantage of this duplicate installation, the study of pathology furnishes many illustrations.

Of these ferments little may be stated in a definite sense. It is so difficult to secure succus entericus for study and so difficult to exclude bacterial processes; that investigations have been few and the results have been meagre. The rennet, lipase, and erepsin seem to be secreted only in the very uppermost portion of the tract, in the duodenum and upper jejunum; the amylase and the ferments for the disaccharids, however, seem to be secreted also in the ileum. None are secreted in the colon. Concerning the properties of the amylase and maltase we know nothing that would distinguish them from the amylase and maltase of the pancreatic juice. There is a form of dyspepsia known as intestinal fermentative dyspepsia, assumed to be due to defective digestion of starch, the result of defective action of the intestinal amylase; it is not clear how the relation of the pancreatic amylase is to be excluded. 'The invertase and lactase are ferments peculiar to the intestine, as is also probably the emulsin, whose importance is greater to the pharmacologist than to the physiologist. The invertase, which splits cane sugar, is an active ferment and in the amounts secreted per day is able to hydrolyse several hundred grams of sugar. The invertase of bacteria and yeasts are usually very sensitive to bacterial action, a quality evidently not shared by the intestinal invertase. The lactase is usually not a prominent ferment in the intestinal juice, since it has been often missed. It is most easily demonstrated in young animals. The intestinal juice of adult herbivora lacks it, while it is sometimes 
present in the intestinal juice of adult carnivora and in man. It is absent at all ages in reptiles and birds. The rennet is secreted only in the upper duodenum, is not prominent, and can be easily overlooked. The lipase is a moderately active ferment; the hile seems not to be necessary to its activation.

The erepsin is a peptolytic ferment that is especially prominent in the cleavage of peptone and polypeptids into the amino-acids, $i$. $e$, it operates in the lower part of the scale of the hydrolysis of protein. When a protein is submitted to prolonged digestion by trypsin, it is found that from 10 to 20 per cent. of the nitrogen remains partially hydrolysed, in two fractions of resistant polypeptids-one the so-called abiuret peptone and the other a fraction of diamido-acids combined with monamino-acids. These groups erepsin is able to hydrolyse. In the test-tube erepsin is able to digest casein, fibrin, protamins, and histons, but has little effect upon the common native albumins, globulins, and muscle proteins. It is also able to digest all the synthetic polypeptids formed of naturally occurring amino-acids. It is apparently active when secreted. Erepsin acts only at neutral or alkaline reaction, and in the test-tube behaves as though very sensitive. All this to the contrary, however, the erepsin is able alone to carry on the total digestion of protein in the body. This it is able to do only under favorable conditions, and it is possible that digestion could not be indefinitely maintained with this ferment alone. The superimposed digestion of protein by pepsin, trypsin, and erepsin yields by far the greatest velocity of reaction, though digestion by trypsin-erepsin is not greatly inferior.

The enterokinase of the succus entericus was described in connection with the activation of trypsin.

Exogenous Ferments. - The foods consumed in a raw state contain ferments of different kinds. The bacteria of the alimentary tract likewise contain ferments. Have these any rôle in digestion? For the carnivorous animal and for man, probably not. The velocity of the processes of autolysis are so low that during the period of time concerned in digestion little could be accomplished. 'The fermentative action of bacteria is much more pronounced. Plants contain amylase, invertase, and maltase; many also lipase. Much more prominent, however, are usually the alcoholic and acetous fermentations; and were the amylase, maltase, and invertase to act with a notable velocity, the sugar formed would fall a prey to these fermentations rather than be absorbed. The bacterial fermentation of fats is usually very slow. The bacterial fermentations of protein are rather of the nature of putrefaction than of simple hydrolysis, of which products are available for use following resorption. In any event, the putrefactive processes are very slow compared with the velocities of the processes of proteolytic digestion. It may be regarded as certain, therefore, that in man the activities of the ferments contained in the foods or elaborated in the alimentary tract by bacteria do not contribute to the chemical processes of digestion to any appreciable degree. 
In the case of herbivora, however, the facts are different. The digestion of the grasses and coarse plants, especially of the cellulose they contain, is to some extent aided by the preformed ferments and by the action of bacteria. While it is true that the intestinal secretions of herbivora are able to hydrolyse cellulose, the bacterial ferments are surprisingly active in this direction. To what extent they contribute in the digestion of this extremely resistant substance can only be conjectured; but the action is not to be questioned. In man the digestion of cellulose is practically nil.

In carcinoma of the stomach, in the stage of ulceration, a ferment of trypsin type is found in the stomach, evidently the secretion of the neoplasm. This ferment is able to split peptones, and tyrosin and tryptophan are quickly set free. It is also able to split synthetic polypeptids, as glycyl-tryptophan. It operates at neutral, faintly acid, or alkaline reaction. Whether this ferment be different from the common type of intracellular trypsin is not known. If it is present early in carcinoma, it is possible that it may become a sign of decided diagnostic value.

\section{THE BILE}

The bile represents the mixed secretions of the liver cells, the lining cells of the biliary ducts, and the lining cells of the gall-bladder. It is to be regarded partly as a secretion, in part as an elimination. It is a continuous secretion, persisting during inanition, presenting, however, periods of increase following the ingestion of food, and in part dependent upon the character of the diet. The amount of secretion probably varies to some extent, though not susceptible of measurement except in the event of an external fistula of the common bile duct, under which circumstances, however, the secretion may display marked abnormalities. The total amount may be judged to vary from 500 to 1000 c.c. per day. There is a continuous secretion of bile. Under the influence apparently, of secretin, a larger flow of bile occurs during the period of intestinal digestion. The nitrogen and sulphur content of the bile rise following the digestion of a meal rich in protein. The secretion of bile continues during starvation, though to less extent. The reaction is alkaline, due to traces of carbonate; on titration it gives the appearance of a much greater alkalinity, due to the presence of bicarbonates. 'The general composition may be seen from the table, a composite of several analyses of human bile, taken from the ducts of the liver. The figures are in one thousand parts.

Water

Solids

Protein

Biliary salts

Pigments

Lipoids

Cholesterol

Inorganie salts

Diverse organic substances .
950.0 to 975

25.0 to 50

3.0 to 5

8.0 to 20

1.0 to 1

0.5 to 1

0.5 to 1

0.5 to 1

$\therefore 0.5$ to 1 
The bile of the hepatic ducts is much richer in water than the bile of the gall-bladder, where inspissation occurs and mucus and cholesterol are added. The alterations are, however, more than thus stated. The solids of liver-duct bile average about 3 per cent.; the solids of gall-bladder bile run from 10 to 17 or even 20 per cent. As the bile is concentrated by inspissation in the gall-bladder, the inorganic salts are in part absorbed, so that the gall-bladder bile is poorer in inorganic salts and richer in organic solids than is the liver-duct bile. Of the total solids of liver-duct bile, one-fourth to one-half are inorganic salts; of the total solids of gall-bladder bile only 4 to 6 per cent. are inorganic salts. The biliary salts take part in a curious lesser circulation; a small fraction is absorbed from the intestine, carried in the portal circulation to the liver, to be again eliminated in the bile. It is regarded as certain that the biliary acids and pigment are formed in the body exclusively in the liver.

There are two proteins in human bile, a mucin and a nucleoproteid, the latter being present in larger amount. The mucin is probably secreted by the lining cells of the bile ducts; the nucleoproteid is secreted by the lining cells of the gall-bladder. The bile of herbivora contains nucleoproteid only.

Sulphur Compounds in the Bile.-The richness of the bile in sulphur compounds impressed the earliest observers. The total content of sulphur runs from 2 to 5 per cent., according to the animal, with moderate individual variations. The biles of man, primates, swine, kangaroo, and hippopotamus present low values; the bile of the common herbivora yield medium values; the highest values are found in the bile of geese, canines, marine animals, and some fishes. The total sulphur content and the taurin run parallel. Human bile from the gall-bladder contains from 2.5 to 5 per cent. The bile flowing from a fistula of the gall-bladder has been found free of organic sulphur, a markedly pathological condition. There is no relation between the diet of a species and the sulphur content of the bile; the diet and bile of fishes are both rich in sulphur, but the carnivorous animals, whose diet is rich in sulphur, have of ten less sulphur in the bile than is to be noted in herbivora whose diet is poor in sulphur. The sulphur compounds in the bile are of four types: preformed sulphates; ethereal sulphates; sulphophosphorized lipoids, and taurin and allied compounds. Preformed sulphates are present only in traces. Ethereal sulphates are contained in all biles, the amounts running from 5 to 15 per cent. of the total sulphur. They yield no phenols on distillation, thus resembling the lesser fraction of human urinary conjugated sulphates. It is known that ethereal sulphates are conjugated in the liver; apparently a fraction passes into the bile, the larger fraction into the venous circulation. Possibly the amount in the bile may stand in some relation to the intensity of intestinal putrefaction. The sulphophosphorized lipoid is present in but a trace, it is soluble in alcohol and ether, free from carbohydrate but yet reduces metallic 
salts, contains phosphorus and nitrogen as well as sulphur, thus a sulphophosphatid of unknown constitution and derivation.

Taurin.-The last and the most important sulphur compound in the bile is the taurin. Human bile contains two so-called biliary acids, combinations of cholic acid with glycocoll and taurin respectively, eliminated as salts of sodium, calcium, and potassium. Taurin is present in the bile of practically all mammals, reptiles, birds, and fishes. In certain fishes no taurin is to be found, instead are complex esters, sulphocholesterols, which take its place. While taurin is, therefore, a substance of almost universal occurrence in bile, quantitatively in most biles the amount is not large, and the glycocholate forms the larger fraction of the biliary acids. In the polar bear, the dog, some fishes, and in the goose the bile is practically free of glycocholic acid, $i$. $e$., the amount of taurin being high and the amount of cholic acid being low; the taurin is present in amount sufficient to combine with all the cholic acid. The chemical fact seems to be that glycocholic acid is found only when cholic acid is formed in the liver in excess of the taurin, which is the case except in the instances mentioned. In man the ratio of glycocholate to taurocholate varies from $5-10: 1$.

Cholic acid has the elementary formula $\mathrm{C}_{24} \mathrm{H}_{40} \mathrm{O}_{5}$. Closely related to it are two companion substances present in traces in many biles, choleic acid $\left(\mathrm{C}_{24} \mathrm{H}_{40} \mathrm{O}_{4}\right)$, and fellic acid $\left(\mathrm{C}_{23} \mathrm{H}_{40} \mathrm{O}_{4}\right)$, equally obscure in chemical composition. The separation of the cholic acid from the glycocoll and taurin is a simple act of hydrolysis. Thus for taurocholic acid:

$$
\mathrm{C}_{26} \mathrm{H}_{45} \mathrm{NSO}_{8}+\mathrm{H}_{2} \mathrm{O}=\mathrm{C}_{2} \mathrm{H}_{7} \mathrm{NSO}_{3}+\mathrm{C}_{24} \mathrm{H}_{40} \mathrm{O}_{5} .
$$

Cholic acid is known to be a monobasic alcoholic acid, containing two primary and two secondary alcoholic groups. Since it has been shown to be a derivative of cholesterol, known facts for other derivatives of cholesterol and the stated facts for cholic acid suggest as the probable constitution the following:

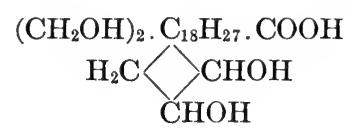

Cholic acid is formed in the body only in the liver, and its conjugations with glycocoll and taurin are so far as known accomplished in the body only in the liver. Experimental investigations, both in physiology and pathology, have made it most probable that in the liver it has but one source of derivation, the cholesterol of the red-blood corpuscle. The erythrocytes have a limited life of possibly only a few weeks' duration, the daily depletion of the circulating blood by their death being made good by a corresponding daily regeneration, the formation of new cells in the red bone marrow. The disintegration of these erythrocytes, in its final stages at least, occurs in the liver; and from the 
cholesterol, which is a prominent constituent of the stroma of these cells, the cholic acid is formed. Cholic acid we may, therefore, term an end product of the catabolism of erythrocytes, the expression of a special metabolic function of the hepatic cells. If the liver of a reptile be extirpated, no cholic acid is to be found in the body, where normally it occurs. If the common bile duct be ligated in the dog, the biliary acids are soon found in the blood; if, however, the mouth of the common thoracic duct be ligated also, no biliary acids are to be found in the blood. Apparently, therefore, in two as widely separated species as the frog and dog, cholic acid is not formed outside the liver, and the sum total of the available data justifies the conclusion that the formation of cholic acid is an exclusive function of the liver and that probably the chemical source of the cholic acid lies in the cholesterol of the red corpuscles undergoing cytolysis in the course of daily cellular metabolism. It is not to be inferred from this that the conversion of cholesterol to cholic acid is a total reaction. On the contrary free cholesterol is present in the bile and pathological erythrolysis increases the cholesterol content of the bile.

Of the constitution of the two amino-acids whose combinations with cholic acid form the biliary acids, glycocoll, and taurin, we are much better informed. The glycocoll, simple amino-acetic acid, is derived from the common catabolism of protein, all the common proteins yielding on hydrolysis varying amounts of glycocoll. It is, therefore, present in the liver at all times. Glycocoll is also formed in the liver in the catabolism of erythrocytes, whose protoplasmic protein undergoes hydrolysis there.

Taurin is a thio-amino-acid, derived from cystein according to the following equation:

Cystein plus oxygen $=$ cystenic acid minus $\mathrm{CO}_{2}=$ taurin .

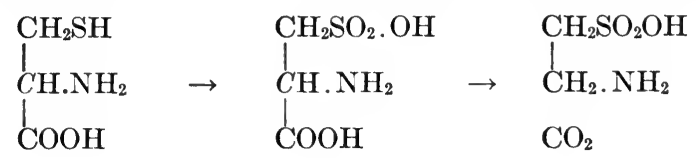

Like glycocoll, cystin is available in the liver from two sources: derived from the general catabolism of protein, since it is the sole or chief thio-amino-acid contained in protein, and from the special catabolism of the protein of the protoplasm of the red corpuscles that is localized in the liver. A very pretty hypothesis may be stated in the proposition that the end catabolism of the erythrocytes is localized in the liver and the three chief end products (cholic acid, taurin, and bilirubin, as will be described) are eliminated in the bile. This hypothesis rests upon the assumption that just as the cholic acid is derived from the cholesterol of the erythrocytes, so the taurin is derived from the cystin of the protein of the erythrocytes, the cystin derived from the common protein being eliminated in the urine in more or less 
completely oxidized sulphur compounds. Indirect evidence supports this hypothesis. If taurin be administered by the mouth it will be recovered in large part in the urine in three states: unchanged, as a substituted thio-urea, and in part completely oxidized to sulphuric acid. Feeding with taurin or cystin leads to no increase in the taurocholic acid of the bile, obviously because there is no further available cholic acid. When, however, taurin and sodium cholate are fed together, an increment of sodium taurocholate is found in the bile. It is, therefore, clear that the liver can conjugate cholic acid and taurin brought to it in the portal circulation, as well as when formed within its own tissues. Unless, therefore, as a matter of mere chance, it should happen that the taurin derived from the cystin formed in the liver from the catabolism of erythrocytes were enough to combine fully with the cholic acid formed there from the cholesterol of the erythrocytes, it might be assumed that the liver would draw upon the cystin formed in the liver and elsewhere in the catabolism of common protein and with taurin derived therefrom combine the excess of cholic acid. Yet the fact remains that the liver does not so utilize the cystin from the catabolism of common protein; this is eliminated more or less oxidized in the urine, while the larger fraction of the cholic acid (in man) is eliminated as glycocholate in the bile. Albumin of blood serum contains nearly 2 per cent. of sulphur, all of which is converted into cystin. Now on a basis of a protein ration of 100 grams per day and a sulphur percentage of only 1 per cent., it is clear that the liver would have presented to it during the day for conjugation with the cholic acid more than 5 grams of cystin from which taurin could be formed. This is many times the amount of cholic acid formed in the day. Yet the liver refuses this cystin from the common protein catabolism, and eliminates the larger fraction of cholic acid combined with glycocoll. This speaks for the hypothesis that the taurin (and the glycocoll with the same probability) eliminated in the biliary acids is derived in the catabolism of erythrocytes alone, and not drawn from the products of the common catabolism of protein. Thus we have the hypothesis of an isolated metabolism in the liver; the erythrocytes autolyzed there and the end products (cholic acid, glycocoll, and taurin) eliminated in the bile so long as the cholic acid suffices, any excess of taurin and glycocoll being sent into the circulation to join there the aminoacids formed from the common catabolism of protein.

Normal stools contain no biliary acids, but only traces of taurin and of cholic acid, the original acids having been split by bacterial action. In the meconium the unaltered biliary acids are present.

The salts of the bile include those of sodium, potassium, and calcium; chlorids, sulphates, and phosphates.

The color of the bile of the liver ducts is a yellow or yellowish brown. The bile of the gall-bladder may be tinged with green, a deep green, or almost back. 
Bilirubin.-In the bile of the liver ducts is to be found one pigment, termed bilirubin. This originates from hematin (a tetrapyrrol) derived from hemochromogen which is formed when hemoglobin is split into its two groups components, hemochromogen and globin. It is not to be inferred that these are the sole stages in this reaction; we know very little of the chemistry of these bodies. The formation of bilirubin from hematin is probably an exclusive function of the liver cells; the formation of the hematin from hemochromogen on the contrary can apparently occur in any tissue. The splitting off of the iron, which is the chief fact in the formation of bilirubin from hematin, devolves upon the liver cells. Under pathological conditions, in connection with extravasations of blood, iron-free pigments are formed that resemble bilirubin. Particular examination has, however, demonstrated that these pigments are not identical with bilirubin; and biliverdin cannot be derived from them. The bile of the gall-bladder may contain not only bilirubin and biliverdin but also urobilin, whose presence is most reasonably to be ascribed to bacterial action within the gall-bladder.

Recent investigations have thrown much light on the chemical nature of the blood pigment and its derivatives. The iron-bearing component of hemoglobin is hematin. Hematin is a complex of four pyrrol groups, linked together with iron.

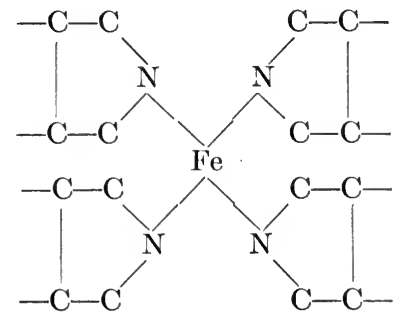

To the four pyrrol groups are attached ethyl and methyl groups; and different peripheral linkages have the result that the four are not identical, two reacting in one direction and the other two in another direction. The iron is combined by replacement of the imid-hydrogens of the nitrogen atoms. The iron is in the ferro state, and the binding with oxygen is to be interpreted either as operative through the ferri state or by the formation of peroxids. From the hematin is derived bilirubin; apparently there is cleavage of the molecule in the liver, hematoporphyrin is first formed, and from it bilirubin. One molecule of hematin will yield two molecules of hematoporphyrin; and one molecule of the latter will yield one molecule of bilirubin, which, therefore, contains two pyrrol groups. Hematoporphyrin is an acid normally present in the liver, and may be regarded as a condensation of two molecules of a hemo-pyrrol-carboxylic acid: 


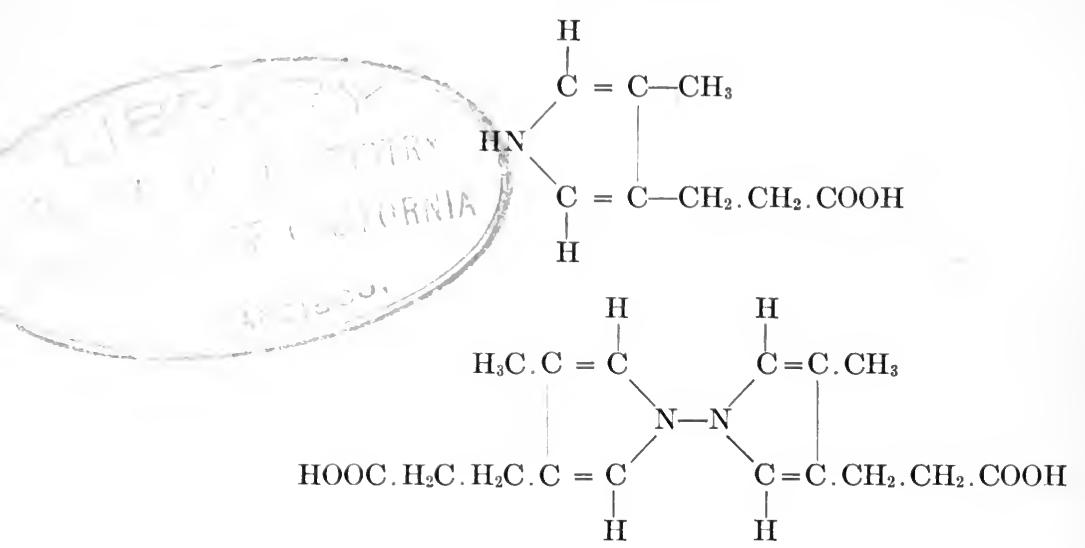

Bilirubin is an isomer of hematoporphyrin. Under different conditions of disease and intoxication (as a result of sulphonal, for example) the conversion of hematoporphyrin into bilirubin is in part checked, and hematoporphyrin appears in the urine. Urobilin is a reduction compound of bilirubin, but apparently contains four pyrrol groups, $i$. e., in the reaction of reduction a polymerization occurs. All conditions leading to erythrolysis result in the formation of excessive amounts of bilirubin, the end product of hemachromogen. Strikingly erythrolytic are certain venoms, bacterial poisons, phosphorus and arsenic, and poisons such as toluylendiamin, pyrogallic acid, chlorates, chromates, and phenylhydrazin.

Of particular interest is the fact that the chlorophyls are closely related to the hematins, being pyrrol compounds in which manganese takes the place of iron. It is to be expected that continued investigations into the constitution of these bodies will yield valuable information to physiologist and pathologist. As will be pointed out in another connection, our present knowledge of the constitution of hematin leads us to infer that it may be a source of tryptophan.

In the gall-bladder, bilirubin may be converted into biliverdin through oxidation. In general, the equation runs:

$$
\mathrm{C}_{16} \mathrm{H}_{18} \mathrm{~N}_{2} \mathrm{O}_{3}+\mathrm{O}=\mathrm{C}_{16} \mathrm{H}_{18} \mathrm{~N}_{2} \mathrm{O}_{4}
$$

There are several oxidation stages of bilirubin known; of these the chemistry and properties are very unclear, and they will not be further discussed. The bile of the gall-bladder usually contains some biliverdin, but this is not invariable. In any event, it is present in but small amount, as most of the bile pigment enters the intestine in the state of bilirubin. Within the intestine it is exposed to the action of bacteria, and is there in part reduced to urobilinogen. 'The extent of this reduction depends upon the activity of the intestinal flora, the duration within the intestine and apparently to some extent upon the 
state of digestion. Feces normally contain no bilirubin; they contain some urobilin; and they may contain biliverdin, though oxidations are unusual in the intestine where reduction reactions predominate. The freshly passed stool contains largely urobilinogen, which on exposure to the air is oxidized to urobilin.

Urobilin.-The urobilin formed by reduction of the bilirubin is in large part absorbed, in part excreted in the stools. Reduction of the bilirubin to other related pigments as stercobilin, hydrobilirubin, has been described; but until something definite is known of these supposed substances, we will class them all as isomers and homologues of urobilin. The urobilin absorbed from the intestine is carried to the kidneys and eliminated in the urine, being one of the chief normal pigments. In the urine the urobilin is supposed to coexist with another yellow pigment, termed urochrome; whether they are identical, whether isomers or homologues, we do not know. Important, however, is the fact that all the urochrome and urobilin of the urine are of intestinal origin; none are normally formed within the tissues. How much of the urobilin formed in the intestine is absorbed and how much eliminated is not known, there being no quantitative method available for its estimation. The more bulky the diet and the more frequent the evacuation of the bowels, the more bilirubin and urobilin are to be found in the stools. Conversely, constipation is apt to be attended with highly colored urine, since the prolonged stay of the feces in the colon permits of more complete absorption. The individual variations are very wide.

Urobilin is formed in the aseptic autolysis of the liver, and may be present in the bile within the liver. This fact may make it incorrect to attribute the urobilin of the urine solely to the intestinal reduction of bilirubin. Pathological urobilinuria might be of hepatic as well as of intestinal origin.

The Phosphorus Compounds of the Bile.-The bile of herbivora is poorer in phosphorus than that of the carnivora. The percentages run from less than $\frac{1}{10}$ up to 1 per cent., human bile standing midway in the scale. Fish bile is almost devoid of phosphorus; the content is very low with most marine animals; that of the polar bear is very high. Three forms of organic phosphorus are met with in human bile; one the sulphophosphatid described under the sulphur compounds; one a lipoid behaving like a lecithin; and one a very complex phosphatid. In view of the darkness surrounding the chemistry of the complex lipoids, all this is to be regarded as provisional. It is so difficult to separate the organic sulphur from the organic phosphorus compounds in the bile, that one is not in a position to determine whether the combinations are of the nature of chemical compounds or physical adsorption compounds. From the phosphorus content it may be calculated that dried human bile contains from 10 to 20 per cent. of these lipoids. Bile contains neutral fats and fatty acids. Whether the fatty acids are derived from cleavage of the neutral fats, from cleavage of the phosphorized lipoids, or exist preformed in the secretion of the liver 
cells, cannot be determined. In the bile these fatty acids exist, of course, in the form of the soaps of sodium, potassium, and calcium.

Lipoids.-Under the term lipoid then we comprise the neutral fats, the fatty acids, the complex thio- and phosphorized fats, and with these may be grouped cholesterol. In this classification we make no distinctions between the lipoids in which the phosphoric acid is loosely combined as glycerol phosphoric acid and those in which the phosphoric acid is firmly bound in the nucleus of the lipoid. These lipoids are all constituents of tissues in general. Whether their presence in the bile represents an act of secretion (regarding them, therefore, as lipoids of bepatic origin) or an act of systematic elimination is not known. If the latter view be favored, then the liver is to be regarded as the gland through whose elimination the end lipoids of the body are discharged. Protoplasm contains a lipoid-protein complex. When the cell undergoes autolysis, the end products of the hydrolysis of the protein appear in the urine; and under the terms of this hypothesis, the end products of the lipoids appear unoxidized in the bile in the form of the substances stated. If they are held to originate in the liver, their source may reasonably be sought in part in the red-blood cells with whose catabolism the liver is so closely identified. For the cholesterol especially, a local origin has been usually predicated on the hypothesis that the formation of gallstones is not associated with excesses of cholesterol in the body, and must, therefore, have been due to an excessive formation of the substance in the liver. Now it is as a matter of fact quite certain that cholesterol is secreted by the mucous membrane of the ducts and gall-bladder. When, however, one considers how slowly gallstones are formed, bears in mind the weights of gallstones and the amount of cholesterol in normal bile, it is clear that the formation of gallstones may be adequately explained as due to abnormal conditions of crystallization and precipitation, and requires no assumption of any increased formation of cholesterol. The causes of the abnormal crystallization and precipitation are now believed to lie in the presence of nuclei consisting of bacteria or epithelial cells, which favor greatly the crystallization of the cholesterol.

Lastly, the bile contains traces of urea, ammonia, and other organic substances, whose presence is incidental. 'Traces of iron are also present. It is probable, however, that the larger fraction of the iron split from hematin in the liver in the reaction of the formation of bilirubin is eliminated by the small intestine and not by the bile, where its presence is to be regarded as incidental.

Our knowledge of the function of the formation of the bile is very incomplete, and the variables are scarcely known, since the experimental difficulties in investigations are extreme. It is incorrect to study the secretion by means of a fistulous opening from the gallbladder; the fistula must be made by transplantation of the opening of the common bile duct in the duodenum. It is also questionable to what extent the results of experiments with biliary fistulæ in the 
dog can be applied to man, or even to the normal dog, since to some extent fistula bile is different from bladder bile or liver-duct bile. Following the ingestion of food, the flow of the bile is increased; in other words, the secretion of bile, like that of the pancreas, is related to gastric digestion. Meat produces the largest secretion, fats the next; carbohydrates in the diet have little influence on the secretion of bile.

Functions of the Bile.-Leaving now the constituents of the bile, and disregarding all questions concerning the secretory or excretory nature of the several constituents, what are the functions of the bile? It is not a digestive juice in the direct sense, since it contains no ferments that accelerate the hydrolysis of carbohydrates, fats, or proteins. It is, however, an important accessory to intestinal digestion. Not only does it influence the action of the ferments of the pancreas and intestine, it promotes conditions of solubility and emulsification.

Two general functions have long been popularly ascribed to the bile: stimulation of peristalsis; and antiseptic action. It is difficult to show directly that the bile stimulates intestinal peristalsis. The frequent and prolonged observations of the bowel movements of jaundiced individuals have led to conflicting conclusions. Of much importance it is not.. The long-assumed antiseptic or bacterial action of bile is practically nil. There is no evidence that it normally checks or in any way controls the activity of the intestinal flora. Practically it may be said that if the diet of a person with obstruction of the common bile duct be so arranged as to leave no excess of undigested food, especially of fats, to act as culture media for the intestinal bacteria, no exaggeration of intestinal bacterial activity is to be observed. Intestinal indigestion and excessive bacterial intestinal fermentation are more easily aroused in the jaundiced than in the normal individual; but this is due to the lack of the bile in the processes of digestion, and not to the lack of an antiseptic action of the bile. The gall-bladder is known to be a good culture tube, and may harbor a culture of the typhoid bacillus for years.

The bile has little relation to the digestion of carbohydrates in the intestine. While it has a slight power in the activation of the pancreatic amylase and maltase, this is wholly subordinate.

The influence of the bile on the digestion of protein in the intestine is slight though positive. 'The important activators for the proteolytic ferments do not lie in the bile. Nevertheless the presence of bile results in acceleration of tryptic digestion. Its action in checking digestion by pepsin when the gastric contents are passed into the duodenum, has been greatly exaggerated. If the bile be mixed with a peptic digestion mass, a precipitate is formed that includes the pepsin. But in all the experimental work on digestion with fistulæ, no such precipitation has ever been observed in the duodenum when the gastric contents encountered the biliary, pancreatic, and intestinal secretions. The alkaline reaction of the duodenum and the action of the trypsin (which 
digests pepsin with avidity) suffice to check peptic digestion in the duodenum.

Important are the actions of the bile on the digestion and resorption of the fats. These actions are several. Bile activates the pancreatic and intestinal lipases. The bile contains also a zymo-excitor for the pancreatic lipase. This property lies for the most part in the biliary acids; some activity is, however, displayed by the lipoids of the bile. The bile aids greatly in the solution of the neutral fats, fatty acids and soaps, especially of triolein, which then in turn acts as solvent for the fats of higher melting points. In a large measure, the action of the bile on the solution of fat is dependent on the presence of triolein or oleic acid. If a pure tristearin be ingested, it is very incompletely resorbed. This result is commonly ascribed to the high melting point of the tristearin. This is, of course, important; but especially favorable is the solvent action of the triolein. This increase in solubility has the certain result of enlarging the field of action of the lipases. It has also the result of increasing enormously the velocity of the resorption of the fatty acids, a feature too often overlooked. Finally, the bile aids in the emulsification of the intestinal contents, whereby the velocity of the cleavage of fats into fatty acids and glycerol is augmented. These influences of the bile on the digestion of fats are demonstrable in test-tube experiments as well as in the living intestine. From this description of the data, it is clear that the function of the bile in digestion is much more important in the carnivora, who consume much fat preformed, than in the herbivora who consume little fat. In fact, in the ruminantia the bile is to be regarded practically as an excretion.

Pathological Variations.-An excess of the secretion of bile is unknown; a deficiency in the secretion is hypothetical. In diarrhea biliary acids appear unsplit in the feces. Unaltered bilirubin is also present in the feces in diarrhea. The absence of bile from the digestive tract is a frequent pathological condition, the result of obstruction of the common bile duct. Of this condition there are two distinct aspects: the state of intestinal digestion in the absence of bile; and the resorption of the bile into the general circulation, followed by deposition in the different tissues of the body. Jaundice, with its manifestations, is to be considered as a true auto-intoxication. We will consider here only the conditions of intestinal digestion in the absence of bile. In practice one sees this, of course, in connection with jaundice from obstruction. But the conditions are to be studied more clearly and with fewer complications in instances of biliary fistulæ, in which the bile is discharged outside the body instead of into the duodenum, the body tissues, however, being free of bile.

After what has been said of the influence of the bile on the digestion of carbohydrate and protein, little disturbance in these functions would be expected, and, as a rule, none is to be noted. On the contrary, positive disturbances in the digestion of fat would be expected. Such, 
however, are often not to be observed. Whether disturbances in the digestion or absorption of fats occur seems to depend in large part upon the diet of the subject. A well-selected diet, relatively poor in fat, is usually digested in a normally efficient manner. When first seen, the subject may complain of symptoms of indigestion, that disappear when the diet is corrected. Three abnormalities may occur: the undigested residue of neutral fat may be excessive; the resorption of the fatty acids may be reduced; and bacterial fermentation in the intestine may be abnormally active. If the fat in the diet be reduced to 50 grams, and the fuel needs of the body supplied by easily digested carbohydrate, the digestion of fat and its resorption become normal and the excessive bacterial fermentation disappears. In some instances large amounts of fat are perfectly digested and absorbed. In other words, the usual load of the fat digestion can be carried by the intestinal and pancreatic juices, but an overload is not tolerated. The deprivation of the bile, therefore, reduced the range of adaptation of this function. It will be rarely found that a normal minimal ration of fat cannot be digested without the bile. With increasing amounts of fat in the diet, the defect first to appear is faulty resorption (fatty acids in the stools), and this is under nearly all circumstances in these cases, much more prominent than incomplete cleavage. Apparently the activation of the pancreatic lipase is fully accomplished in the absence of bile. Reduction in the solubility of the fats and fatty acids and imperfection in emulsification may result in a lowering of the resorption, and may indeed lead to defective cleavage of the fats. But, as a rule, disturbances appear only in the event of a heavy ration of fat in the diet, and they are then more likely to appear with the use of fat of high melting point than with the use of fat of low melting point. This indicates in another way that the defect concerns the function of solution and resorption rather than that of cleavage.

- When all is said and done, observation of cases of chronic biliary fistula, in which for anatomical or pathological reasons it is impossible to return the stream of bile to the intestine, has taught that a state of ill-defined poor health is very prone to develop. It is, of course, possible that this may be due to the absence of some unknown influence of the bile within the intestine. Most of these cases, however, suffer from septic infection, cirrhosis or degenerative changes of the liver, or from other diseases; and to these the states of ill-health observed are more reasonably to be ascribed. Rarely in severe organic disease of the liver the bile may be free of pigment, the biliary acids, however, being present.

\section{THE MASS RELATION OF THE DIET}

A diet contains, apart from the salts, condiments and flavoring additions, three ingredients: water, the digestible solids, and the indigestible solids. The indigestible solids are of little importance 
to the chemical functions of digestion, but are of importance to the motor functions of digestion. It is astounding how grossly inaccurate is the average personal conception of the masses involved in mixed diets. Only one who has participated in a metabolic experiment really has a personal sense of perspective in this matter. It is one thing to know that so and so much of this or that article of diet has such and such a percentage of solids, of starch, fat, or protein; it is a very different matter to know what a certain unit of food means on the table. The most striking variables-from person to person, and in the same individual from day to day and from season to season-are displayed in the unconscious selection of the masses of the diet. The appetite is commonly supposed to record in some way the needs of the body. In laboratories where long-continued and extensive investigations into the digestion and nutrition of dogs are carried out, the conviction prevails that to a goodly extent the appetite of dogs is an expression of the needs of their nutrition. A dog, not under daily exercise, will eat less of a stated food than will a dog under daily exercise, even if the feedings be held prior to the time of exercise. Dogs refuse on warm days what will be eagerly eaten on cold days. In the primitive state, before the development of the culinary art, this may have held true in man. Today the needs of the body in diet are indeed more honored in the breach than in the observance. By this it must not be understood that the desire for food does not in civilized beings present indications of the needs of the body. But we have been so modified by the development of culinary art and gustatory tastes that the average cultivated man is usually quite uniable correctly to interpret the meaning of his appetite. The average individual does not know how much he eats; he does not know whether the promptings for food are appetite in the animal sense, or the expression of a highly cultivated esthetic desire for pleasant sensations of the gustatory nerves. One of the most striking illustrations of this fact is the total lack of relationship between the salt needs and the salt tastes of civilized people, the latter exceeding the former many fold. The range of the individual variations in diet, dependent upon age, sex, occupation and physical type, and multiplied by individualism in gustatory taste and experiences, is still further diversified by the currency of numerous theories and fads of diet. A menticulture founded upon the ostensible denial of the existence of matter, and co-existing in an individual with a personal hypothesis of nutrition, constitutes a striking illustration of the combination of lack of sense of humor and of logic developed in a popularized striving for individualism.

A study of diets of all kinds indicates that within the widest range of choice or economic compulsion, the values fall within the figures determined by scientific experimentation. Scattered through the literature are to be found careful studies of many diets, including the following: The diet of the lower coolie classes in Japan; the diet of the working classes of India; the diet of the negroes of our southern 
States in summer and in winter; the diet of the so-called "poor white trash" in our southern States; the diet of the lower classes of Italian peasants; the diet of the poorer German peasants; the diet of the inhabitants of the polar regions; the diet of tropical tribes; the diet of several barbaric or aboriginal tribes; the diet of students of American colleges; the diet of the so-called upper classes in Great Britain and the United States; the diet of vegetarians and fruitarians; the army rations of all the civilized nations; the rations in civic institutions of all kinds, asylums, prisons, hospitals, etc. Under these widely varying circumstances, the amounts of protein, fat, and carbohydrate and the sources in the several foodstuffs of the protein, fat, and carbohydrate are variously distributed. And yet a calculation indicates clearly that under all these circumstances the protein input is always as high as the figure determined essential-a gram per day per kilo of body weight-though often higher; and that though the relations of fat to carbohydrate fluctuate greatly, the caloric needs of the body are covered in accordance with the figures of our closely determined standards. Man is truly an omnivorous animal, and this is indeed well for him. The powers of adaptation and compensation are nowhere better illustrated than in the function of digestion and nutrition. It is this broad experimental truth that places the ban of scientific disapproval upon every exclusive school of diet, if the popular fads of diet may be so designated. Of course, digestion and nutrition can be maintained with adherence to all sorts of fad diets-not as an illustration that the fad diet is correct, but simply as an illustration of the powers of adaptation-just as in the instances of the different dietaries mentioned above. The term "normal diet" must, therefore, be given a very wide scope, and for further information we must depend upon carefully planned and executed investigations of natural classes rather than upon isolated laboratory experiments.

Masses of Normal Diets.-Let us now consider the masses in various normal diets. Included in the mass of a diet is the input of water, whether consumed with meals or between meals. Normally, water is consumed with meals. Under the influence of a recent fad, numbers of individuals now take no fluid with meals; of course, they get along as well, and no better, than their unfadded fellowmen. Since the water ingested as beverage is added to that of the diet, it ought to be originally included in the calculations. The water taken daily by different individuals varies widely; usually it will run from half a liter to a liter and a half; it may fall materially below a half liter, and may be as high as two or three liters. It will be assumed in these calculations that the water taken as beverage is one liter per day. For the determination of the masses of the diet, the protein input in mixed diets will be fixed at 70 grams per day; the distribution of fats and carbohydrates will express largely the economic basis of the diet.

A milk diet for an adult would consist of 5 liters per day, if the input of 3000 Calories is to be attained. Such a ration is not only rather 
large in bulk (water as beverage would not be necessary), but it is needlessly heavy in protein, 150 grams per day. If to 3 liters of milk 300 grams of sugar were added, the bulk of the diet would be more agreeable, the protein input reduced to nearer the requirements and the caloric value maintained. This could also be attained by adding cream, much more expensive and less digestible than sugar. The solids of a milk diet of 5 liters are about 600 grams; of 3 liters plus 300 grams sugar, 660 grams. 'The total sum of these solids is digestible; it will not be all resorbed, but it is all digestible.

A pure protein diet would consist of 750 grams of casein per day. Such a diet can be borne in the dog, it is questionable whether a man could swallow it or retain it. A meat diet can, however, be taken by men living in the open, and especially in cold weather, without difficulty, though the ration becomes tiresome. If fresh meat were consumed and choice were available, it would be best to have the ration consist of about equal parts of lean meat and fat. For a moderately active life such a ration would consist of about 1500 to 1800 grams per day, depending upon the fatty tissues or fat utilized. For food under circumstances where a pure meat diet is necessary, desiccated meats are now available. On the assumption of complete desiccation, such a ration would consist of 250 grams each of protein and fat. The body could, of course, handle more protein and less fat, or more fat and less protein-but equal parts of each would suit the average digestion best. Adding the water to the weight of the natural meat and fat first given, the total mass of the day's ration would be 3000 grams. In the use of the desiccated meat, one liter of water would not be enough; a man would need to drink enough water to bring up the weight to 3000 grams. All the solids of such a diet would be classed as digestible; it would not be all resorbed, but it would be digested.

Bread represents a complete diet. Campers who have tried both bread diet and meat diet are a unit in preferring bread diet to meatfat diet, except under conditions of extreme cold. A satisfying ration of bread would weigh, with a liter of water, 2500 grams. Camp biscuit, which differs from bread in an added content of fat, is rather better, as the weight can be reduced to 1200 grams, and the lesser input of starch is an advantage. The solids of such a diet would be about 900 grams for both bread and biscuit. The indigestible residue would be less than 40 grams per day.

A diet of buttermilk and potatoes, commonly used in the treatment of obesity, would weigh 5 kilos (2 liters buttermilk and 3 kilos cooked potatoes) with a caloric content of $3000 \mathrm{Cal}$. Since few could, or would, ingest 5 kilos of food per day, the rationale of the use of this regimen for the reduction of obesity is obvious; an amount of the diet that satiates physically, does not contain enough heat to maintain the body temperature, which is then maintained by combustion of the body fat.

The usual mixed diet of civilized communities contains from 75 grams to 150 grams of protein ingested as meat, eggs, milk, cheese, 
bread, cereals, and legumens, the protein of meat comprising usually over half of the total protein; from 50 to 150 grams of fat, consumed as cream, milk, yolk of egg, butter, olive oil, fat of meat, and animal and vegetable fats used in cooking; and from 300 to 600 grams of carbohydrates, in the form of cereals, starches, breads, milk, and sugar. Tastes favor the use of fats; economy is against them. The universal tendency of the past, to vary the diet with the season, is being gradually eliminated by the development of transportation and storage facilities and the art of packing fruits and vegetables, these all operating to increase the price of raw produce during the summer and to reduce the price during the winter. In a number of series of metabolic investigations, the writer has had occasion to study the composition of the mixed diets of free choice of several groups of adult normal men. The weights of these diets, including one liter of water as a beverage, varied from 2500 to 3500 grams per day. The solids of these mixed diets varied from 500 to .800 grams per day. The indigestible solids varied from 20 to 40 grams per day. In a few instances the diet weighed as low as 2000 grams per day, in a few as high as 4000 per day, without in any instance being especially individualistic. These figures are fairly representative of mixed diets of adult men not engaged in hard physical work.

Strictly vegetarian diets must naturally present large bulk. The more intelligent vegetarians, who consume bread and the cereals largely, have less bulk in the diet than have those who incline to cover their nitrogen needs with legumens. With a heavy ration of dried beans and peas in the diet, the total bulk can be held lower, but such a diet is apt to be indigestible. For the strict vegetarian more than for all others, bread is the staff of life. A properly selected vegetarian diet need not weigh, with the liter of water, over 3 kilos, or at the most 4 kilos. The fruitarian diet, an uncommon and recent expression of the food fad, must be a diet of still greater bulk, unless the individual is able to digest regularly large amounts of nuts. The food in such a diet will weigh from 3 to even 5 kilos per day. The solids of these diets vary from 500 to 900 grams per day, since for the most part starch is the main fuel and fat is present in small amount. The amount of indigestible solids need not be over 40 to 50 grams per day, but may be as much as 100 grams. Without any discussion of the desirability of such large indigestible residues, either in health or in the treatment of constipation, it may be pointed out that an intelligent use of fruits and vegetables will enable an individual to secure a large fecal residue without the diet being either indigestible or too prone to bacterial decomposition. What is wanted is indigestible residue, not a large residue composed of material in itself digestible but consumed in excess of the capacity of the individual digestive system.

The diets of laboring classes furnish striking instances of adaptation. The fish and rice diet of the Chinese coolie is equivalent to the potato and herring diet of the North German peasant. The idea that the 
coolie maintains his nutrition upon an amount of food, protein, and starch, that would not be found sufficient for a Caucasian, is erroneous. When the body weight of the coolie is taken into consideration, the ration is within modern figures. During the cane season, the negro workers in the fields live principally upon the cane juice; and the saving power of sugar for protein cannot be better illustrated than in the diets of these laborers. The protein input during this brief season is low, probably not over 40 grams per day; but the great excess of sugar makes it enough, for the time at least.

The element of bulk in the diet is one too frequently left out of account. Satiation is due more to bulk than to ingredients, though certain articles of food notoriously satiate. It is possible to so arrange a diet as to fill the stomach thrice daily and yet not maintain nutrition. It is also possible to maintain nutrition on a diet whose bulk is so low that it does not furnish over one good meal. The tendency to fewer meals is based upon the use of concentrated foods quite as much as upon the idea of taking less food. The use of a diet of large bulk has in general the tendency to lower the percentage of resorption, and to increase the activity of bacterial processes, though in many instances no such effects are to be noted. The now popular notion that the husks and shells and outer coverings of foods furnish elements indispensable to good nutrition was born in the imagination of the writer of advertisements of patent foods. Whatever the relation of polished rice to beri-beri, the adoration of bran is worship at an empty shrine. On the other hand, there is no doubt that the use of a too concentrated diet, devoid of indigestible residue, may lead to impaction of feces, or at the least to their retention in the body for prolonged lengths of time.

\section{RESORPTION OF THE PRODUCTS OF DIGESTION}

There is no resorption of the products of digestion by the mucous membrane of the mouth or esophagus. Only sugar could be concerned, since the proteins and fats undergo no change in the mouth. The digestion of carbohydrates in the mouth, during even the most prolonged mastication, is very slight. There is in any event no evidence that the mucous membrane of the mouth and esophagus possess the power of resorbing sugar.

Resorption in the Stomach.-In the consideration of the resorptive function of the stomach, it is necessary to realize the difference between the natural conditions in digestion and the forced conditions of experimentation. The question of the resorption of the products of digestion in the stomach (except in the case of the administration of predigested foods), concerns sugars alone, since the fats are not digested in the stomach at all, and the digestion of the proteins does not reach the stage of peptone. It may be positively stated that no undigested fat 
and no protein in the states in which protein is found in gastric digestion, are there resorbed. With the carbohydrates, there is some degree of digestion, from 10 to possibly 40 per cent. of the starches may be converted into sugar in the stomach before the acidity of the gastric secretion checks the action of the salivary ferments. It has been usually assumed that this sugar was resorbed from the stomach. This assumption is probably incorrect. It is true that if a stomach be filled with a sugar solution and the pylorus ligated, sugar will be resorbed. It is, on the other hand, very probable that in normal digestion, where water is being continually secreted into the stomach, and the contents gradually passed into the duodenum, no sugar is resorbed. It is, of course, difficult to prove this. Possibly the non-absorption of sugar in the stomach may be due to the layer of mucin-HCl that covers the mucous membrane, through which salts might diffuse, but sugar only with difficulty. In the intestine mucin is much less in evidence. But if the sugar be resorbed in normal digestion, the amount resorbed must be small. This physiological opinion is supported by pathological observations. Instances of pyloric obstruction are unfortunately frequent enough. Observation of the processes of digestion in these cases does not lead to the view that there is any material resorption of sugar from the stomach. Our present knowledge may, therefore, be stated as follows: experimentally, the resorption of sugar from the stomach can be forced; in normal digestion it does not occur at all, or at the most in but small amount.

It is also clear that normally no water is resorbed from the stomach. On the contrary, the stomach is an active secreter of water. In this regard, the stomach in the carnivora operates conversely to the psalterium of the ruminants. The stomach in the horse, and in the other non-ruminating herbivora, secretes large amounts of water. In the psalterium, water is resorbed, so that the contents when passed into the lab-stomach resemble little cakes from which the water has been pressed. Normally, the human stomach does not secrete water as freely in the event of copious ingestion of water with the meals as with the ingestion of food without water. For the pathologically dilated stomach, with or without pyloric obstruction, it is known that the water secretion of the stomach may continue in spite of the presence of large accumulations of water there and to the great detriment of the patient.

Resorption from the Small Intestine.- It will be of advantage to subdivide this subject into three parts: The qualitative resorption of the products of digestion; the quantitative relations of the same, and the action of the resorption membrane upon the products of digestion during their passage through the mucosa.

The resorption of the products of digestion occurs through the lining epithelial cells, not between them; it is intracellular, not intercellular. The resorptive function of this lining epithelium is not to be defined in terms of a simple transfusion membrane; nor is it to be fully defined in terms of a semipermeable membrane. Direct experiment will 
illustrate this. If a living detached intestinal lining, or a detached peritoneal membrane, be studied for its resorptive properties, such and such results will be noted. If now this membrane be killed with chloroform, different results will be observed. If finally the membrane be killed with formaldehyd, still different results will be obtained. The differences obtained under the three sets of conditions may be interpreted as follows: The fullest properties are to be observed with the unaltered detached membrane. Following the action of chloroform some of the reactions disappear, but many are retained. Following the action of formaldehyd, which alters greatly the state of the tissue, all the distinctive reactions disappear, the membrane retains only the properties of a parchment membrane. After the action of the chloroform, the membrane has the quality of semipermeability, but has lost the selective properties of the native membrane. These selective properties are vested in the chemical and physico-chemical attributes of the living cells. Chloroform kills the cells, but does not alter their physico-chemical properties so markedly but that a high degree of semipermeability is retained. Formaldehyd so completely alters the chemical and physico-chemical states of the cells as to destroy all but simple transfusion, such as is displayed by a parchment membrane. The explanation of the selective and synthetic properties of the living epithelial cells is not to be sought in any mystical or philosophical interpretation of the term "vital." It is to be sought in a rational understanding of the chemical and physico-chemical attributes of living cells, attributes as yet only in part open to our crude methods of investigation and analysis.

An histological illustration of the living intestinal membrane will aid the visualization of the concept that is to follow.

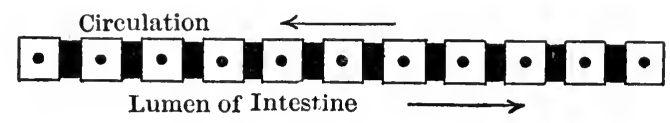

The material to be resorbed must first pass through the proximal lining wall of the epithelial cells. It then becomes incorporated with the protoplasm of the cells. Later it must pass through the distal lining wall of the cells. Upon reaching the postcellular space, it will be transferred to the streams of circulation. The products of the digestion of carbohydrates and protein pass into the venous, $i$. e., portal circulation; the products of digestion of fat pass largely into the lymphatic, $i$. e., retroperitoneal lymph circulation. We do not know what determines this separation of the routes of transportation. For the present, the question does not concern us. Of the utmost importance here is the understanding of the fact that during the act of passage through the cellular membrane, the materials are for a time incorporated with the protoplasm of the cells. And within the very limited spacial dimensions of this incorporation are encompassed some of the most important 
synthetic reactions of the body. The lining membrane of epithelium is not only a membrane of resorption, it is also a tissue of synthesis. Possibly it would be correct to say that the proximal lining wall of the cells - the lining facing the lumen of the intestine-serves as the direct transfusion membrane; the synthetic functions devolve upon the protoplasm, the rear lining wall being permeable to the synthesized products. It is clear, however, that under such an interpretation the rear lining wall must have different physical properties than the front lining wall, for some of the synthesized products are colloidal, and colloids do not pass through the anterior lining wall. Let us now apply this conception to the facts for the resorption of the different foodstuffs, from the data of which this theory was originally determined.

Resorption of Carbohydrates.-The facts are as follows: Maltose can pass through the intestinal wall, the contents containing no maltase. Cane sugar can pass through the intestinal wall, the contents containing no invertase. Milk sugar can pass through the intestinal wall, the contents containing no lactase. Dextrose passes through the intestinal wall. Dextrins do not pass through the intestinal wall, in the absence of amylase in the contents. The velocity of resorption of monosaccharids is much greater than of disaccharids. In the blood of the portal system after all these experiments, usually only one form of sugar is to be found, namely, d-glucose.

The explanations must be as follows: Since maltose can be resorbed from intestinal contents containing no maltase and reappears in the blood on the other side in the form of d-glucose, the protoplasm of the lining cells must contain a maltase, and a reaction of cleavage must occur within this protoplasm. Since cane sugar can be resorbed from intestinal contents containing no invertase and reappears in the blood on the other side largely in the form of d-glucose, the protoplasm of the lining cells must contain an invertase, and a reaction of cleavage must occur within the protoplasm. Since milk sugar can be resorbed from intestinal contents containing no lactase and reappears on the other side in the form of d-glucose, the protoplasm of the lining cells must contain a lactase, and a reaction of cleavage must occur within the protoplasm. Since dextrins and starches are not resorbable, the protoplasm of the lining cells obviously does not contain amylase. It is thus very clear that within the protoplasm of the lining cells important digestive functions are vested-the power of splitting disaccharids into the component monosaccharids. So far as we can judge, the body could digest these disaccharids in the entire absence of maltase, invertase, and lactase from the secretions of the salivary glands, pancreas, and succus entericus. It might be natural to infer that the resorbing cells secrete these ferments into the lumen with the succus entericus, retaining some ferment within their protoplasm to act upon such molecules of disaccharid as might escape cleavage within the lumen. This is doubtful. Firstly, because, so far as we can judge, the secretion of the 
ferments of the succus entericus are not the result of the activity of the simple lining epithelium (unquestionably the resorption membrane), but of special glands. Secondly, it is possible directly to show that the lining of an intestine is able to split milk sugar when the succus entericus is unable to accomplish this reaction. The point in interpretation is not important, but it seems most likely that these intracellular ferments are not eliminated into the lumen of the intestine with the succus entericus, but are utilized solely for intracellular digestion. The blood and the other tissues do not possess the power to hydrolyse cane sugar and milk sugar. It is upon the intracellular lactase and invertase of the intestinal epithelium that the body must depend for protection from the presence of the unaltered disaccharids; whatever cane sugar and milk sugar may escape intra-intestinal cleavage is subject to intracellular cleavage in the act of resorption and passage through the lining epithelium. It might even be suggested that this is, in the adult, the sole or chief method of digestion of milk sugar, the demonstration of lactase in the pancreatic and intestinal juices being so difficult of accomplishment.

Since one of the component primary sugars of cane sugar and milk sugar respectively are d-levulose and d-galactose, and since d-glucose only is to be found in the blood, it is clear that within the protoplasm of the lining epithelial cells, or liver, d-levulose and d-galactose are converted into d-glucose. These may be termed acts of synthesis, using the term in its ordinary sense. The conversion of d-levulose and $d$-galactose into $d$-glucose means the conversion of one stereoisomeric configuration of the hexose into another. It can be accomplished in the laboratory without difficulty, especially in the case of d-levulose, which on standing in sterile alkaline solution passes into d-glucose. As an hypothesis of physiological chemistry, d-glucose may be regarded as the most stable stereoisomeric state of the hexoses, toward which the d-levulose and d-galactose naturally tend. These conversions do not occur within the lumen of the alimentary tract; the digestive juices are devoid of such power. The great importance of this function of the intestinal epithelium is, therefore, apparent. This faculty of the protoplasm of the lining epithelium rests obviously in a ferment, a transformation ferment for which no special name has been devised. It may be here recalled that d-galactose occurs not only in the milk of the active mammary gland, but also in the central nervous system; since only d-glucose is to be found in the blood, it is clear that in the breast gland and in the central nervous system this reaction can be reversed and d-galactose formed from $\mathrm{d}$-glucose. This is not true for d-levulose; the animal body does not normally form it from d-glucose, though the intestinal epithelium and the liver can form d-glucose from it.

To summarize, the following reactions of digestion occur within the protoplasm of the lining epithelium of the intestinal tract: 
Maltose + water $=d$-glucose $+d$-glucose
Cane sugar + water $=d$-glucose $+d$-levulose.
Milk sugar + water $=d$-glucose $+d$-galactose.

The following reactions of transformation occur there:

D-levulose $\rightarrow$ d-glucose.

D-galactose $\rightarrow$ d-glucose.

'To what extent the hydrolytic functions of these cells are exercised in normal digestion we do not know. Probably by far the larger part of the cane sugar of the diet is split in the intestinal tract, and only a small part resorbed unsplit is hydrolyzed within the protoplașm of the lining epithelium. For milk sugar this is not so certain. The demonstration of lactase in the pancreatic juice fails in experiment; in the succus entericus it is difficult of demonstration, and when present is of low activity. Yet large amounts of milk sugar are easily digested. We may, therefore, fairly ascribe to the lactase of the epithelial protoplasm an important range of function in the digestion of milk sugar.

The transformation of $\mathrm{d}$-galactose into $\mathrm{d}$-glucose is apparently not confined to the intestinal epithelium. The conversion of levulose into glucose is shared with the liver. And if a goodly amount of levulose be ingested on the empty stomach, its resorption occurs so rapidly that much reaches the liver unchanged. Normally the liver converts this levulose into glucose. But in many organic diseases of the liver this power is reduced or lost, the levulose passes through the liver unchanged, and is eliminated in the urine.

There is a limit to the action of the invertase and lactase of the epithelial protoplasm. This is showed in the fact that following the ingestion of very large quantities of cane sugar or milk sugar, most often in the case of cane sugar, traces of saccharose or lactose are to be found in the urine. This means simply that with the great velocity in resorption from large amounts of concentrated solutions of these sugars, the epithelium is so to speak overwhelmed, and small amounts pass unhydrolyzed into the retro-epithelial spaces, to be passed into the circulation, thence to be carried through the liver unchanged to the kidneys for elimination. There is no limit to the reaction of transformation with reasonable amounts ingested, and in the absence of hepatic disease, $i$. $e$., following the ingestion of even large amounts of cane sugar or milk sugar, d-levulose or d-galactose is not to be found in the urine. It is possible, however, that proper attention has not been given to this subject, since to ordinary examination these primary sugars present in the urine would pass as d-glucose.

There is nowhere in the digestive tract, either in the juices of digestion or in the resorption membrane, any known power to convert pentoses into hexoses. There is evidence that pentoses are utilized in the body, and it is believed that their utilization follows their transformation into d-glucose. But this transformation, so far as we know, 
does not occur in the acts of digestion or resorption. Direct and comprehensive investigations might possibly indicate the contrary; there is little data from which conclusions may be drawn. It is known that within the body pentoses can be formed from d-glucose and conversely d-glucose from pentoses.

The Resorption of Protein.-Extensive as is our knowledge of the digestion of protein, by ferments and by acids, within the body and without, we are not yet fully informed as to the facts for the resorption of the products of protein digestion. It is not a question of how far down the scale of the products of protein digestion resorption occurs; it is a question how far up the scale it may occur. The data are conflicting, but it is clear that the latest data, obtained with better methods of chemical analysis and more careful experimentation, indicate that much of the earlier data were not trustworthy. The amount of aminoacid that is to be found in the intestine in any one moment is no evidence of the extent of cleavage, since the amino-acids are absorbed very rapidly. With increasing knowledge, the tendency is more and more to limit resorption to products low in the scale. Realizing that possibly this tendency may be put somewhat strongly, it is probably a fair statement that a judicious interpretation of the best data indicates that the resorption of the products of the digestion of protein occurs in the states of polypeptid and below; that it does not occur with or above the stage of peptone. The digestion of protein may not proceed to the state of complete cleavage to monamino-acids and di-aminoacids. Bi-, tri-, tetrapeptids and polypeptids may be included in the normal end products of the natural digestion of protein. These, with mono- and di-amino-acids are to be regarded as the states of resorption of the products of the digestion of protein. This conclusion is based partly upon the results of experimental work, and partly upon induction. Since proteins are formed of amino-acids as building stones, our present chemical conceptions of their composition and constitution do not permit us to believe that the body can build them from the peptones of another animal or plant-the reconstruction must be more extensive than that. Just as one cannot rebuild a house by merely taking off and replacing the roof, so one cannot form new protein in the biological sense by enlarging a foreign peptone. The proteoses still bear the biological stamp; we cannot, therefore, conceive them as the nuclei for the formation of protein. The peptones of different proteins are very different bodies, and when one considers that serum albumin and serum globulin are formed from what-not mixtures of protein in the diet, it does not seem possible to regard peptones as building stones. The resistant polypeptids also are very different bodies in different peptones - contain different amino-acids. Obviously, the more extreme the cleavage, the greater the freedom of choice afforded the synthetic functions in the building of protein. This induction is supported by a great deal of experimental and chemical investigation, and we are thus led to the proposition as stated; the resorption of 
the products of the digestion of protein by the intestine is in the states of peptids and di- and monamino-amino acids. These and these alone are able to afford to the receiving body full opportunity for the biological synthesis of protein. This conclusion is supported further by our knowledge of the phenomenon of anaphylaxis.

In another place, it will be pointed out that when protein is burned, the process consists first of hydrolytic cleavage, following which the amino-acids are deaminated. The oxy-fatty acids resulting from this process may be burned directly. But there is good evidence that to some extent, probably to a large extent, sugars are formed from them, to be added to the general sugar content of the body. It will be shown in another place, that the body resists the storage of protein; that whenever protein is ingested in excess of the body needs it is promptly burned and the nitrogenous products eliminated in the urine. Do these processes occur within the alimentary tract? Are the aminoacids subject to deaminization within the intestine or in the intestinal wall, and the fatty acids resorbed as such, to be transformed into sugar in the liver? The data today are decidedly against this hypothesis. The digestive juices of the alimentary tract contain no deaminization ferment. The bacteria of the intestine elaborate deaminization ferments that operate largely in the colon upon the amino-acids that have escaped resorption. There is no evidence that they operate to any material extent high in the tract where the end products of the protein digestion are resorbed. Our best evidence is that the aminoacids are resorbed as such. Their cleavage in the catabolism of protein is not a function of the digestive tract, but of the tissues. The writer believes that all protein is resorbed in the state of peptids and aminoacids and recondensed to protein, in which state it becomes the substrate of the protein metabolism. In the event of ingestion of amounts beyond the needs of the body for repair and regeneration of the cells, beyond the maintenance of the status quo of the body protoplasm, the excess of protein is catabolized. In a word, the digestive tract is not concerned in the catabolism of protein at all. This is the exclusive function of the tissues.

The fact that the blood of the portal vein is relatively rich in ammonia has been urged in favor of the deaminization of amino-acids in the intestine. When the blood proteins are formed in the intestinal wall, in accordance with the interpretation here adopted, superfluous aminoacids will be left over. 'These might possibly be deaminated in situ, and from them the ammonia of the portal blood derived. Ammonia is also evolved by bacterial action within the intestine.

It having been made very probable that the products of the digestion of protein, whatever the type, are resorbed in the form of peptids and amino-acids, once these have passed through the anterior or front wall of the epithelial cells of the intestinal mucosa and have been incorporated with the protoplasm of these cells, what occurs? The older view was that they were transported in this state to the liver, 
there to be converted in large part into protein, in part to be burned. It was, of course, recognized that these end products might pass through the liver and be utilized directly in other tissues of the body; but the larger utilization was localized in the liver. For this view, held generally for many years, there was no direct evidence; it simply appeared to be the natural inference. When amino-acids were sought for in the blood of the portal vein, only doubtful traces were to be found. This, however, did not discredit the view, since if one were to calculate the volume of portal blood, the number of times it passes through the intestine and liver in the time consumed in a digestion and divide this figure into the amount of amino-acids furnished by the digestion of the protein of a meal, it is clear that only traces of amino-acids could be expected to be present in a particular sample of portal blood, representing merely a momentary phase of the act of digestion. The toxicity of the portal blood, as reported in dogs and geese with the Eck fistula and its apparent dependence upon the amount of protein in the diet, was also taken as evidence of the resorption of the products of protein digestion in the form of amino-acids. It is not now possible, on the basis of the analytical evidence at hand, to consider the toxic symptoms that may develop in connection with the Eck fistula as the results of amino-acids. The positive chemical demonstration of aminoacids in the portal blood or in the general circulation of animals with the Eck fistula (or in the normal) has never been accomplished. As explained above, with the great dilution of the products of digestion by the enormous volume and velocity of the circulation, such could hardly have been expected. Within recent years, moreover, the investigations with the Eck fistula have shown that the results of this operation, properly performed, have been greatly exaggerated; and the dependence of the symptoms upon the amount of protein in the diet greatly overrated. It were a wise man who could state today in what manner the Eck fistula modifies the protein metabolism of the animal possessing it, or the cause of the symptoms that may attend it.

The more prevalent view now runs to the effect that these end products of the digestion of protein, following their passage through the front wall of the epithelial cells, are in the protoplasm of these cells recombined to form protein. In a word, the cleavage reaction of digestion is reversed, and the building stones are again united to form protein, which is then passed into the portal circulation. This view is not capable, at present at least, of direct demonstration. It is, however, strongly supported by indirect evidence and sound fundamental considerations. There is now an enormous literature on the biological specificity of the proteins of the body. Under all conditions, the tissues of the body form higher proteins that are peculiar to itself. 'To accomplish this, as has been pointed out, it is necessary that the digestion of protein proceed to an advanced point in order that the units of disintegration, the building stones, may present the widest variety for selection. There is strong indirect evidence that the construction of 
a protein by the body is preceded by the almost complete tearing down of the protein material - either in digestion or in tissue metabolism. The disintegration of a protein into its building stones and the reconstruction of the new protein from these building stones seems to be the universal method of the protein metabolism. In the case of the digestion of sugars and of fats, direct demonstration can be accomplished to the effect that the states in which these substances exist in the portal and lymphatic circulations are the states in which they are to operate as the substrates of their respective metabolism. There are no d-levulose or d-galactose in the circulation, only d-glucose, the regular sugar of the metabolism. Now, entirely apart from the question of toxicity of the products of the tryptic digestion of protein, reasoning by analogy we should expect to have the end products of the digestion of protein recombined in the intestinal epithelium to form protein. It is not to be denied that the tissue cells could accomplish this. Evidently all cells and especially muscle cells have some anabolic faculty for protein. Direct experiments with the liver, however, show that when amino-acids are passed through it, these are deaminated and sugar formed from the fatty acid fraction, they are not apparently utilized for the formation of protein. That this result must necessarily speak against the formation of protein from amino-acids in the liver, is not here contended.

Indirect evidence and general considerations which will be presented in another section lead us then to the view that the synthesis of protein from the end products of digestion occurs in the protoplasm of the epithelial cells of the intestine. The form of protein may be safely stated to be the serum albumin and serum globulin of the blood serum. That serum albumin should be regarded as the primary protein in the anabolic series and the globulins placed higher in the scale is due to the greater biological specificity of the globulins as contrasted with serum albumin. For us at this time we rest with the proposition that serum albumin and serum globulin are formed in the protoplasm of the epithelial cells of the intestines from the end products of the digestion of protein; and that these proteins are the substrate upon which all the later processes of metabolism of protein in the body are based. Serum albumin and serum globulin are, in a word, the raw materials of the protein metabolism.

It will be recalled that all the common forms of protein are composed of the same amino-acids, but in widely different proportions. When now the protoplasm of the intestinal epithelial cells forms serum albumin from them, there is selection and some of the amino-acids will not be utilized, they are superfluous. What becomes of these? There are two possibilities. Either they are deaminated in the intestinal epithelium, and the ammonia and fatty acids carried to the liver to be converted there into urea and sugar; or they are carried unchanged to the liver, there to be deaminated and converted into ammonia and sugar. The latter may be regarded as the more likely occurrence. In either 
event, this could account for the amino-nitrogen supposed to exist in the portal blood.

The Resorption of Fats.-As old as modern physiology is the question of the state of resorption of fat. Decades ago the question was approached by histological methods, and from the appearances thus obtained the inference was drawn that fat could be absorbed unchanged, as neutral fat, in a state of fine globular subdivision, without having been acted upon by the juices of the digestive tract. That fat was split in the intestine was known from the first; the question was whether all the fat was split, or whether some of the fat might not be resorbed in the unchanged state. It has now become clear that the use of the histological method is fraught with danger. Within recent years, however, even the results obtained with this method have spoken against the idea of the resorption of neutral fats. It can only be shown that fat is present within the cells; it cannot indicate whether it was resorbed as such or formed within the cells. If the problem were to be defined as follows: are the fats absorbed as neutral fats, or are they absorbed as fatty acids and glycerol and carried as such into the circulation? the demonstration of stained neutral fat within the epithelial cells would bear upon the question. This is not at all the problem. The neutral fats are to be found as such in the epithelial cells. The question stands: is the fat absorbed into these cells as such, or is it formed there after resorption of the fatty acids and glycerol? Within the last ten years the accumulated evidence points more and more strongly in the direction of the statement that fats are resorbed only in the state of cleavage. In other words, undigested neutral fat is not capable of resorption; fatty acids and glycerol, the products of hydrolysis of fats, are alone capable of resorption. Resorption of fats, as of protein and starches, must be preceded by digestion.

The hypothesis of the resorption of neutral fat was based upon the idea that if it were in a state of extremely fine subdivision, in a state of minute emulsion, the globules could be carried mechanically through the lining of the intestine. Whether between the cells or into the cells and by what forces carried through, was not stated. Modern investigations in physical chemistry have taught us the meaning of the state of emulsion for the lipolytic processes of the intestinal juices. But there are no data to indicate that the state of emulsion accelerates the velocity of a process of diffusion. The bile and the alkaline pancreatic juice bring about a noteworthy solution of the fatty acids. And it is the soluble fraction alone that is available for diffusion. That cells can take up particles of solids has been shown, best for plant cells. But there is no direct evidence that the epithelial cells of the intestine take up the solid particles of neutral fat. All the indirect evidence is against this view.

If soaps are given to a dog, fat will be formed from them. Since there is no evidence of a synthesis of glycerol within the lumen of the intestine, the conclusion is obvious that the glycerol is synthesized 
in the protoplasm of the epithelial cells and there joined to the fatty acid of the ingested soap to form neutral fat. The same result will be obtained if free fatty acids are fed to a dog. If fatty acid and glycerol are fed to a $\mathrm{dog}$, microscopic examination of the intestinal wall will show fat in the protoplasm of the epithelial cells. In some instances it has been possible to effect this demonstration with excised sections of the intestine.

If the state of emulsion alone could be held responsible for the resorption of fat, this ought not to be limited to neutral fats, but ought to be demonstrable for other substances that can be gotten into a state of fine emulsification. The experiments, however, indicate the contrary. Many colloids can be brought into the most fine subdivision, but are not resorbable. And for the hydrocarbons in particular, as paraffin, it can be shown that even when administered in the most highly emulsified state, where the particles may be shown to be smaller than those in a fat emulsion, no resorption occurs. Any fat that can be split can be resorbed; nothing is resorbed that cannot be split.

As will be later pointed out, the resorption membrane does not effect any qualitative alteration in the fatty acids; that is, oleic, palmitic, and stearic acids are to be recovered in the fat of the lymphatic duct in the same proportions they held in the fat of the diet. As a rule, also, the resorption membrane does not alter the alcoholic component. Sometimes, however, this can be done. If the fat of the walrus (in which the glycerol is replaced by cetyl alcohol) be given to a human being, as was once done in the case of a fistula of the thoracic duct, the fat recovered after its digestion was largely in the state of tripalmatin. The only explanation of this finding lies in the assumption of cleavage within the lumen of the intestine, resorption of the products and the substitution of glycerol for cetyl alcohol in the recombination within the epithelial cells of the intestinal lining.

The sum total of the data of today justifies, therefore, the belief that fats are never resorbed undigested, but only in the state of fatty acids and glycerol. There is no evidence that the fatty acids are ever synthesized or converted into each other in the intestinal wall. Glycerol can be formed there (or obtained from the circulating blood stream) and abnormal fats can thus be converted into normal fats or glycerids. Some abnormal fats, however, are split, recombined unchanged and thus added to the fat content of the body.

On reaching the retroperitoneal lymph circulation, ending in the thoracic duct, the fat is held largely in an insoluble state. On reaching the blood, for a time this persists; before long, however, the blood presents no signs of lipemia. This is due in part, no doubt, to the deposition of the fat in the different tissues. In part, it is due to the fact that the blood holds a certain amount of fat in a water-soluble, diffusible state. Whether this fat is in combination with protein or how else combined is not known; but combined it is in a state soluble in water and diffusible through a common parchment membrane. 
It is tempting to assume that this state represents the active state of the fat for the reactions of metabolism; and that neutral fat, wherever and however found, is only storage fat. A parallel might be drawn from the carbohydrate metabolism. Glycogen is the storage state of carbohydrate; the soluble, diffusible glucose is the active chemical state for the purposes of metabolism, $i$. e., chemical reaction.

While it is known that the chief resorption of fat occurs through the lacteals, still resorption into the portal system has not been definitely excluded. All the fat ingested at a feeding has never been recovered from the thoracic duct, but this experiment is not to be given an exclusive interpretation. That fatty infiltration of the liver follows the ingestion of a meal rich in fat proves nothing directly, since the fat might have been deposited from the arterial circulation. In the cat, in which such a fatty infiltration of the liver can be provoked by a heavy feeding with cream, fatty infiltration of the kidneys is still more marked, an observation that robs the fact of the occurrence of fatty infiltration of the liver of any special significance.

The Synthetic Functions of the Intestinal Epithelium.-These may be summarized as follows: The conversion of d-levulose and d-galactose into d-glucose; the formation of serum albumin and serum globulin from the products of the digestion of protein; and the formation of neutral fats from glycerol and the fatty acids. These all we ascribe to ferment action. The formation of protein and fat fall under the concept "reversed action" of ferment. It will be recalled that a ferment simply accelerates the velocity of a reaction in whatever direction it may be proceeding. Theoretically, when the experimental conditions can be obtained each ferment or catalyzer will accelerate the velocity of its reaction in either direction-breaking down or upbuilding. For the purposes of this discussion it will be assumed outright that these reactions of synthesis in the intestinal wall are enzymic in nature. Whosoever wishes to regard them as vital and not enzymic may be permitted to hold that opinion without discussion. Under the assumption that these syntheses are enzymic, two interpretations are possible. One is that the syntheses are due to proteolytic and lipolytic ferments identical with those that accomplish the cleavages within the lumen of the tract. The second is that special synthetic ferments are concerned therein. It is again the question of dualism in ferment action. Experiments with reversions are difficult; the intricacies are to a large extent inherent in the problem, and are present, therefore, in experiments with inorganic substances. These are increased by the lability of organic substances involved in experimentation with the biological aspects of the question. Under these circumstances, the experimental data do not speak decisively for either point of view. But the weight of physico-chemical theory and analogy is decidedly on the side of the view that such reversions (especially in the case of fat), are due to the action of the same ferments that produce the cleavage in digestion. In the case of the proteins, the biological 
specificity of the serum albumin and serum globulin formed from the diversified proteins of the diet pleads against the interpretation of synthesis by the same ferment that accelerates hydrolysis.

Time Relations of Resorption.--There is no direct experimental way of measuring the rate of resorption from the intestine. Indirect evidence, the known data of the heat curve following the ingestion of varying amounts of protein, and the curve of the output of nitrogen, enable us, however, to make an approximate computation. The resorption is very rapid early in the course of intestinal digestion. Probably within two hours after the stomach has begun to discharge its contents regularly into the duodenum, the maximum of the curve of resorption is reached. Within four or at least six hours after the stomach begins to discharge its contents into the duodenum, the resorption is practically completed. In fact, it might be said that intestinal digestion and resorption are completed almost as soon as the stomach is emptied of its contents. Digestion is very rapid in the intestine, most rapid for the carbohydrates, less so for the protein, least so for the fats. Resorption is also very rapid, and the order remains the same; sugar, protein, and fat.

Space Relations of Resorption.--The most active resorption occurs in the lower duodenum and upper jejunum. The curve of resorption plotted for the whole small intestine would rise sharply to the beginning of the jejunum, and then fall away gradually to the lower part of the ileum. It is, however, very doubtful if this expresses in any way the powers of resorption of the different sections of the intestine. For it must be remembered that the lower duodenum and upper jejunum have presented for their action the most concentrated solutions of products to be resorbed, the greatest absolute amounts of the end products of digestion. If a cut-off be established between the lower end of the duodenum and the upper end of the ileum, so that the jejunum is excluded from resorption, it will be found that the velocity of resorption is in nowise lowered thereby. In other words, the upper ileum absorbs as well as the jejunum, when it has the same opportunity. This would not hold for the lower ileum. Practically no resorption occurs from the colon.

Relation of Velocity of Resorption to Mass of Content.-Other things being equal-digestibility of contents, total mass, etc.- the time of resorption is not materially lengthened by moderate increase in the amounts to be resorbed. Though this cannot be shown directly, it may be inferred from indirect evidence. When the resorption is studied in a localized portion of the intestine, it seems that with a constant volume, the velocity of resorption is proportional to the concentration of the substance to be resorbed; when the concentration is held constant, the velocity of resorption is proportional to the volume placed in the intestine. From this we are permitted to infer that the prolongation in the time of digestion that is observed after gorging is due to retardation of the chemical processes of digestion more than 
to retardation in resorption. This may not be strictly true; but it is quite certain that within wide limits, the fats being least resorbable, the intestinal wall absorbs the products of digestion about as fast as they are furnished by the chemical processes of digestion.

Relation of Bulk in the Small Intestine.-The total bulk presented to the intestine in the day is large. Let us say that a mixed diet contains with the water taken as beverage, 3 kilos. To this must be added a kilo of saliva, half a kilo of gastric juice, a kilo of bile, and possibly half a kilo of pancreatic and intestinal juice. The total is 6 or 7 kilos. The average stool from such a diet will weigh not over 100 or 200 grams. Of the rest, 600 or 700 grams of solids and in the neighborhood of 6 liters of water have been resorbed in the intestine. This is divided into three meals, extending over a time of some sixteen or eighteen hours, making 2 kilos per meal. Now the earlier idea was that the stomach delivered its contents to the duodenum in rather large consignments. This we know not to be true. When the pylorus relaxes, a very small amount is passed into the intestine. When the acidity of this portion of contents in the duodenum is neutralized, the pylorus again relaxes and another small portion is admitted into the duodenum. It is the duodenum that holds the pyloric string, the stomach is willing to empty en bloc. The neutralization of the gastric acidity is not, however, the sole method of regulation, since this mechanism is followed even in the absence of gastric acidity.

Now it is not to be inferred that as the successive consignments from the stomach are neutralized they accumulate in the intestine, so that we would within a few hours have the total mass within the intestine increased in proportion to the amount of the gastric contents. By no means. The resorption of water proceeds so rapidly in the upper small intestine that it is scarcely an exaggeration to say that it is absorbed as fast as it enters from the stomach. While it is true that desiccation of the feces occurs in the colon, a stool of 100 grams weight in an individual with the habit of daily defecation probably did not weigh over 200 grams when it entered the head of the colon. The upper intestine acts in the resorption of water as actively as the psalterium of eattle. So that, as a matter of fact, the bulk of the contents of the small intestine is not greatly increased during the time of intestinal digestion. With 2 liters of fluid to resorb with each meal, it is doubtful if the bulk of the contents of the small intestine is increased over half a liter at any time. From four to twelve hours are required for the passage of a marked content from the duodenum to the colon.

The Modus Operandi of Intestinal Resorption.-This is a difficult subject to discuss. On the one hand stand the failures of those who have attempted to force an interpretation of the process of resorption to fit gross physical concepts. On the other hand stand the vitalists, holding that no physico-chemical explanation is possible. For those of us who believe that in the ultimate all manifestations of life rest 
upon chemical and physical properties, the position of the vitalist is as irrational as the traditional practice of the ostrich. On the other hand, our knowledge of chemistry and physics and our ability to reproduce essential conditions in experimentation directed to the elucidation of such a complex problem as resorption, are limited; and we must expect to fail today where we may succeed tomorrow. If the present physical theories cannot be conclusively applied to the data, under the present conditions of experimentation, this furnishes no basis for an ultimate conclusion. A number of physical and physicochemical factors can be shown by direct experiment to play a rôle in resorption from the intestine. No one of these factors can be held to satisfactorily explain all the known facts. Nor is it now possible to combine these several factors, in an experimental way, so as to reproduce the phenomena. It must be realized at the outset that the problems of the resorption of the products of digestion and of metallic salts are not identical. There is evidence that the resorption of the products of digestion is entirely intracellular. On the other hand, there is good evidence that the resorption of the metallic salts is largely if not entirely intercellular. Thus the results of experiments on the reactions of the intestine, within the body and without, to different solutions of metallic salts cannot be directly applied to the problem of the reaction of the intestine to the products of digestion.

It will suffice for our purpose to name and define the several factors that are of known influence in the resorption of the products of digestion from the intestine.

Diffusion; Osmotic Pressure.-This is a factor of great importance in the resorption of salts, but of lesser importance in the resorption of the products of digestion. The osmotic pressure of sugar is high, of amino-acids low, of fatty acids very small. The resorption of sugar is more rapid than that of amino-acids, and much more rapid than that of the fatty acids; but the velocities of these resorptions are not related to each other as are the osmotic pressures of the solutions of these substances. For metallic salts the rates of resorption run parallel to the diffusion velocities, the latter being the expression of the coefficients of dissociation of the molecule and the migration velocity of the ions. This has clearly no determining application to the resorption of sugar, amino-acids, and fatty acids. Sugar is resorbed more rapidly than proportional to its diffusion velocity; for the amino-acids no data are available; the fatty acids are resorbed far more rapidly than in proportion to the diffusion velocity. The same fact holds true for urea and for many other organic substances. The factor of osmotic pressure per se could, under the theory of resorption of the products of digestion through the protoplasm of the epithelial cells of the intestinal lining, apply at the most only to the passage of the substances through the anterior cell wall. The known facts for the relationship of resorption to osmotic pressure hold, with quantitative differences, 
for the dead intestinal wall; and hold also to some extent for artificial gelatinous membranes.

Molecular Imbibition and Capillary Imbibition.-These have been invoked in explanation of the resorption of the products of digestion. Their importance at the most must be small, since the capacity for imbibition of a tissue like the intestine is low. For the intercellular resorption of salts, imbibition may have an importance; for the intracellular resorption of the products of digestion, it can have none of moment.

Intra-intestinal Pressure.-Of great importance, as illustrated in direct experiments with the living intestine, is the intra-intestinal pressure. In this intra-intestinal pressure are several factors: the tone of the intestinal wall; the weight of the intestinal tract; the contracture of the intestinal muscilaris; the pressure of the abdominal wall; and the pressure of the diaphragm. During inspiration the diaphragm exerts pressure; during expiration the abdominal wall exerts pressure. By opening the abdominal wall, by removing the pressure of the diaphragm, by placing within the peritoneal cavity varying compressions through the presence of fluids, by releasing the gases from within the intestine and by obliterating peristalsis, it is possible in direct experiment to create many different modifications in the intra-intestinal pressure and to study the conditions of resorption under these varying circumstances. Such tests have demonstrated the great influence of variations in intra-intestinal pressure upon the velocity of resorption. This pressure does not, of course, create more than a physical tendency to the passage of a substance through the intestinal wall; it simply aids the passage by direct pressure from behind, just as increased atmospheric pressure will accelerate the filtration of a solution through a filter. It is a quantitative force merely, but undeniably one of great importance.

Blood Pressure.-The blood pressure in the arterial capillaries aids in resorption by driving the resorbed fluids into the capillaries leading to the portal system. The lower the concentrations of resorption material behind the separating membrane, the greater the velocity of the purely physical stream of diffusion. This is a factor usually neglected in experiments.

Surface Tension and Semipermeability. - Two further physical factors, of undoubted importance, but as yet outside of experimental measurement, are the surface tension of the emulsified intestinal contents and the semipermeability of the intestinal wall. That the membrane of lining epithelium is semipermeable, in the same sense as artificial semipermeable membranes though with different qualitative manifestations, is well known. These two factors must certainly influence the processes of resorption in both qualitative and quantitative directions.

Physico-chemical Constitution.-Are we warranted in the assumption that the resorption of the products of the digestion of carbohydrates, 
proteins, and fats are accomplished solely through the exhibition of the above-named factors of physical properties? In my opinion, we are not warranted, from the available data, so to judge. This does not mean flight to vitalism. It simply means that there is among others one or more factors as yet unmeasurable though of undoubtedly great importance, that have not been introduced into the accounting. One factor is the physico-chemical constitution of the protoplasm of the epithelial cells. This protoplasm may be defined as a fluid lipo-proteinsaline complex. While from one point of view it is to be regarded as a two-phase system, a system of coexisting phases, from another point of view it is a fluid. It is a structure with very high surface tension, with marked capacity for adsorption reactions, with a pronounced tendency to chemical reactions, a system in which variations in the equilibrium are easily produced. The physico-chemical structure of such protoplasm is dependent upon its life. When the circulation ceases, when the processes of oxidation are suspended, when the reactions essential to its function are suppressed, its state of equilibrium must suffer alteration. From the purely physico-chemical point of view, the constitution of the protoplasm must change with death, because when withdrawn from the conditions of metabolism and function, the chemical reactions within the protoplasm are changed, and consequent upon this the physico-chemical state of the protoplasm must be altered. Therefore, one ought never to force upon living tissue the negative conclusion of experiments done on dead tissue. This influence of the physico-chemical constitution of the epithelial cells upon the processes of resorption in the qualitative sense must not be overlooked. It is possible that herein lie largely the qualitative differences that have been noted between purely physical resorption under the conditions of experimentation and the phenomena observed during life. Most of the differences have been quantitative to be sure, and these we may infer lie largely in the incomplete conditions of our methods of experimentation. But some of the differences are qualitative. For the elucidation of the qualitative differences it is not enough to study the qualitative properties of the substances to be resorbed; valuable as these data are, that is only one side of the matter. Equally important is the physico-chemical state of the resorption membrane. For certain cell membranes it is known that they absolutely bar the passage of otherwise easily diffusible ions. This exclusion is due to a physico-chemical property of the cell wall. Protoplasmic membranes act not merely as physical membranes but also as reaction surfaces. When a solution is placed upon a layer of protoplasm, not only is it placed upon a diffusion membrane, but we have in effect two chemical systems placed in contact. And the chemical and physico-chemical reactions between the two systems may modify greatly the phenomena of physical diffusion, in both qualitative and quantitative senses. One can obtain a certain experimental insight into this matter by watching the processes of diffusion through a detached 
serous membrane. As the membrane dies, its properties as a diffusion membrane change. These changes are the result of alterations in the chemical and physico-chemical constitution of the protoplasm of the cells, due to the withdrawal of circulation, the cessation of oxidation and other metabolic reactions and to the fall in temperature. It is this factor, the importance of which we are just beginning to appreciate and the experimental definition of which is not yet possible, that has been overlooked in past endeavors to explain intestinal resorption upon the purely physical basis. It is this fact, misinterpreted and glorified with the title of "living," that has led the vitalist to reject the conception of intestinal resorption as a physical and physico-chemical phenomenon. There is no known fact of intestinal resorption that cannot be reasonably explained upon the basis of the conception of purely physical forces operating in connection with a membrane of the physico-chemical properties of protoplasm; the complete experimental reproduction of the total phenomenon has, however, not yet been achieved. But to consider a thing in experimental science as unachievable because in the incomplete state of our technique not yet achieved, is so unscientific as to border on absurdity.

\section{SUMMARY OF PATHOLOGICAL VARIATIONS IN THE PROCESSES OF DIGESTION}

A systematic classification of the disturbances of the functions of the alimentary tract permits of the possible pathological variations being grouped under several headings.

Disturbances due to improper methods of mastication.

Disturbances due to toxic or otherwise injurious ingesta.

Disturbances due to anomalies in secretion of digestive juices.

Disturbances due to abnormalities in motor functions.

Disturbances due to qualitative deviations in the chemical reactions of the processes of digestion.

Disturbances due to quantitative variations in the chemical reactions of the digestive processes.

Disturbances due to defects in the processes of resorption.

Disturbances due to bacterial infections and reactions within the tract.

While this treatise is not designed to discuss the diseases of the alimentary tract, the physiological knowledge now at our command enables us to make a survey of the field of pathological variations. The ill results of deficient mastication and ensalivation of the food are fourfold. The trituration is not adequate, leaving large pieces of food to be passed through the stomach and intestine. This is of little consequence to carnivora, but is liable to be followed by signs of alimentary distress in man. The lack of proper ensalivation makes more difficult the attainment of an adequately emulsified chyme in the stomach. 'This state of chyme is of great importance to intestinal 
digestion. Mastication tends reflexly to increase the secretion of gastric juice, a fact that seems to hold even for the act of sucking in the infant. Lastly, lack of ensalivation means absence or deficiency in the salivary enzymes. This is probably the least important result, as this defect is easily made up in the small intestine. 'The stress laid upon the importance of the salivary enzymes in the modern fad of supermastication is not justified by our knowledge of their scope in the total processes of digestion. But trituration and ensalivation are of great importance to the chyme-forming function of the stomach.

Disturbances due to toxic or other injurious effects of the ingesta, exogenous intoxications due to decomposition of foods, lie outside the scope of this treatise.

The variations in the secretion of the various alimentary juices have been considered in the appropriate sections. Disturbances due to alterations in the motor functions have also been considered. Apart from neoplasms, parasites, and bacterial infections, these include the majority of clinical instances of alimentary disturbances.

Disturbances due to qualitative deviations in the chemical reactions of the processes of digestion are not known. So far as we know, the chemical reactions are accomplished or left in part uncompleted; there is no qualitative deviation with the evolution of an abnormal product of digestion.

Disturbances due to quantitative variations in the chemical reactions of the processes of digestion are frequent, and have been considered in appropriate sections. The variations are all in the direction of deficiency. Conditions outside of the absence of digestive juices might, however, conceivably operate to frustrate the chemical processes of digestion. This in itself would have little consequence were it not for the favorable conditions for the development of bacteria thus produced. The usual causes of deficiency in digestion are absence of one or more of the alimentary secretions, or abnormal bacterial infections.

Disturbances due to defects in the processes of resorption are not of frequent occurrence. Strictly speaking we mean the non-resorption of the normal products of digestion. The seat of the defect may lie in the intestinal mucosa or in the retroperitoneal lymphatic system in the case of the fats; in the intestinal mucosa alone in the case of protein and sugar. It is not to be assumed that defects in resorption need be accompanied by microscopic alterations in the intestinal mucosa demonstrable by present methods. On the other hand, resorption may be normal through an intestinal mucosa the seat of extensive lesions. Ulcerations do not apparently modify to any extent the resorption processes of the intestine. When one considers the phenomenon of anaphylaxis, one is led to wonder why sensitization to foreign protein is not developed in the subjects of gastric or intestinal ulcer by resorption of unchanged protein through the ulcerated area. Possibly such have occurred, the source of sensitization having escaped detection. Man is apparently not very prone to anaphylaxis. Nevertheless, 
instances of anaphylaxis have been reported in children following the ingestion of protein, especially egg albumin, that lead one to infer that sensitization had previously occurred through alimentary resorption of unaltered protein. How this resorption was accomplished is not known. The mucosa of the alimentary tract of newborn animals has been experimentally shown to be permeable to foreign proteins.

Disturbances due to bacterial processes in the alimentary tract are very common, and deserve a detailed consideration that lies outside the scope of this work. Bacteria may operate in three directions: by the abstraction of nutrients designed for the organism, a factor that may be disregarded; by the formation of toxic substances from the products of the digestion of the foodstuffs; and by the elaboration of specific poisons. In reality the bacterial infections of the alimentary tract lead to specific diseases, only a few of which are yet individually recognized and delineated. What is commonly, though erroneously, termed gastro-intestinal auto-intoxication (exogenous intoxication being excluded), is in reality bacterial infection and intoxication, not auto-intoxication. It is as incorrect to speak of an intestinal bacterial infection as an auto-intoxication as to term hookworm disease an autointoxication. Further investigations into the flora of the intestine will certainly lead to the definition of specific disease types, due to the presence of specific varieties of microörganisms. Asiatic cholera is an instance of an exaggerated intestinal infection. What is now needed is less verbiage concerning symptomatology and more concrete work directed to the study of the bacterial flora of the intestinal tract. No instance of auto-intoxication due to qualitative perversion or quantitative variation in the chemical processes of digestion has ever been reported, unless we wish to term hyperchlorhydria an auto-intoxication. The so-called gastro-intestinal auto-intoxications are bacterial infections and intoxications.

\section{GENERAL RELATIONS OF HEAT AND ENERGY IN DIGESTION}

The reaction of the hydrolytic cleavage of the proteins, fats, and carbohydrates, under which term the digestion of these substances is described, is practically isothermic. By this we mean that no heat is lost or gained in the reaction. Oxidations are exothermic, that is, heat is set free; reductions are endothermic, heat is combined. Applied concretely to the substances of digestion and their products, we understand that when a starch is converted into sugar, the sugar has about the same caloric value as had the starch; when a fat is split, the fatty acids and glycerol have about the same heat value as had the fat; when a protein is hydrolyzed, the amino-acids have about the same caloric value as had the protein. 'This is not strictly true, but the differences are slight. The invert sugar derived from the hydrolysis of a unit of cane sugar yields 3 per cent. less heat than does the cane 
sugar. The loss for starch is difficult of measurement, but is less than 5 per cent. The loss for fat is 5 to 7 per cent. The loss for protein is somewhat greater, though not accurately known, probably under 10 per cent. From the standpoint of the chemical reactions of digestion, therefore, little energy is lost in the processes of alimentary digestion. In any event, the total process would be an absolutely isothermic one, since the end products of digestion (with the exception of glucose) are in the processes of resorption recombined. Exactly as much heat as might be set free in the reactions of digestion is again combined in the reactions of reconstruction. The end products of the digestion of protein and fat are recombined in the wall of the intestine to form the states in which they were ingested, protein and fat; therefore, the total reaction must be isothermic, even though the cleavage reactions themselves were exothermic.

These facts of the heat relations of the chemical processes of digestion are diametrically at variance with the widely diffused idea that enzymic action involves an expenditure of energy. It cannot be too repeatedly stated that the reaction of fermentation rests upon a reduction in the internal chemical resistance, and not upon an increased outlay of energy. It is not more work done; but simply the same work done in a shorter period of time.

Because no heat is lost in the chemical reactions of cleavage and reconstruction in the processes of digestion, it would, however, be incorrect to conclude that no work is done in the act of digestion considered as a whole. The secretion of the digestive juices entails a certain heat production. The processes of resorption are associated with a small loss of heat. The largest factor lies unquestionably in the muscular movements of the alimentary tract. When one considers the masses of food, observes the churning movements of the stomach and the peristalsis of the intestinal coils, it is clear that quite a little work is expended. The amount of heat lost in these processes cannot be measured. It may, perhaps, be judged to lie within 100 Calories per day.

This loss of heat is not to be confused with the larger production of heat associated with the metabolism following the ingestion of food. If we determine the caloric needs of a starving individual, and then administer in food (absorbed) exactly that amount of calories, it will be found that these are not sufficient to support the heat production; this rises following the ingestion of food, and the individual will not be in caloric equilibrium. This loss of heat following the ingestion of food is greatest for protein, less for fat, and least for sugar. This heat production is due to what is termed the specific dynamic action of the foodstuffs, an action greatest for protein and least for sugar. The results of the operation of this specific dynamic action of the foodstuffs (which subject will be detailed in another chapter) is a loss of heat in the processes of metabolism following digestion; there is a diflerence between the plane of maintenance in starvation and the plane of main- 
tenance in feeding, there is a heat production associated with the latter that is a loss to the body. This heat production varies under different conditions of external temperature and with different diets; it may be judged to vary from 150 to 300 Calories per day for average mixed diets. This is a much greater loss than that which occurs in association with the processes of alimentary digestion. It should be clearly understood that although this larger heat production follows the ingestion of food, the loss does not occur within the alimentary tract, but within the metabolism following the entrance into the circulation of the products of digestion. There is a small primary loss of heat in the processes of digestion; there is a larger loss of heat in the post-digestive metabolism.

\section{THE FECES}

The Mass of the Feces.- The mass of the feces depends upon three factors: the character of the diet, the bacterial processes in the intestine, and peristalsis. When all three operate in the one or other direction the smallest and the largest bulks of feces are to be observed. As a rule, the content of resorbable material in the stools is inversely proportional to the period of retention in the small intestine. Colonic diarrhea has little influence on resorption. Under pathological conditions a fourth factor may be added, the excessive secretion of water by the intestinal tract. As described under the consideration of resorption in the intestine, the normal function is the resorption of water. A small amount of water is, of course, contained in the succus entericus, but a great deal more water is resorbed. Under certain conditions of bacterial infection of the tract, this stream may be reversed, and large amounts of water eliminated; and to the withdrawal of water from the tissues, many of the most prominent symptoms of these intestinal infections are to be attributed.

'The weight of the daily milk stool of an adult will rarely run over 50 grams. The weight of the daily stool of an ordinary mixed diet may vary from 50 to 300 grams; if the diet consist of meat, fats, bread, and dairy products, with few fresh vegetables or fruits, the weight will often be under 100 grams; the higher the proportion of fruits and vegetables, the higher the weight of the stools. The daily stool of a vegetarian or a fruitarian may weigh a half kilo or even more. The increased weight of the stool in the vegetarian and fruitarian is due partly to the greater amounts of indigestible solids; but it is due in part to the greater amount of unresorbed digestible solids, and to the greater content of bacteria and water. The more frequent is defecation, upon a mixed diet, the greater the weight of the stool, the difference being largely water.

The water of the feces varies with the diet, the bacterial condition of the tract and the frequency of defecation. It is especially high in the normal milk stools of children, where it runs from 80 to 90 per cent. 
The milk stool of the adult is much more solid. Milk and meat stools may contain no more than 50 per cent. of water; they may contain 75 per cent. In the large stools of the vegetarian or fruitarian, the water content is often high, three-fourths; but one may see the stool of the same diet containing less than one-half water. From this data it is clear that in the case of the small stools of a milk or meat-fat-cereal diet, the water output of the intestinal tract may be as low as 25 grams; whereas in the case of a vegetarian or fruitarian diet, the water output of the tract may be as high as 500 grams. The meaning of such variations for the function of the kidneys is obvious. In the use of vegetables and fruits in the treatment of constipation, one secures larger stools, not only because of the greater mass of indigestible material and unresorbed digestible residue, but also because of the greater water content of the stools. This larger water content is doubtless due in part to the greater bacterial activity prevailing in large mixed stools. The water content of pathological stools may be very high; it is not unusual to find watery stools that contain less than 50 grams of solids to the liter.

The average dried weight of the daily stool varies from 15 to 50 grams; in the case of vegetarians it may rise to over 100 grams per day. The lowest values are to be seen in meat-rice-fat stools. Ordinary average dried weights present less fluctuation, varying from 20 to 40 grams. The heat value of dried feces varies from 5 to 6.5 Cal. per gram, the highest values being seen in the stools of milk or meat diets.

Peristalsis.-Under the term peristalsis we group the muscular movements of the intestine. Though not usually classed together, the movements of the stomach are identical in physiological meaning. Two kinds of movements are to be observed: (a) Mixing movements comprising rotary contractions in circumscribed sections of the intestines, combined with propulsive and reversed movements. For both the stomach and intestine, localized reversed peristalsis has been observed and evidently forms part of the normal mechanism. (b) Propulsive movements, by which the contents are gradually propelled toward the colon. In the colon only propulsive movements occur, to any extent at least. The time normally required to propel the contents of the duodenum to the upper end of the colon varies in different individuals from four to twelve hours, or even more.

The obvious functions of intestinal peristalsis are four: the emulsification of the contents, this being greatly aided by the mucin secreted by the intestine; the admixture of the ferments with the emulsified content of foodstuffs, which state of homogeneity (using the term in its ordinary meaning) affords to the ferments the maximum surface contact with the particles of foods; shifting of the contents, whereby fresh portions are successively brought into contact with the resorption membrane; and the removal of the gases of bacterial decomposition, the accumulation of which would soon lead to dilatation and paralysis of the muscular coats and inhibition of resorption. It is difficult to 
overestimate the importance of the functions of gastric and intestinal peristalsis; there is far more disease of the alimentary tract due to disturbances of motility than to disorder of chemical action in digestion; while there are several ways of chemical action, there is but one motility. The crucial importance of peristalsis for the processes of resorption may be shown in a simple experiment. To ordinary gastric contents, or to the contents of the small intestine, properly emulsified, a known amount of an easily diffusible substance like glucose is added, and the mass placed in a dialyzer. For the first few minutes, the diffusion of the sugar will be rapid, then it will fall quickly to a minimum. In such a thick viscid mass, convection streams do not occur. And thus after the first few moments, the velocity of dialysis will be reduced to the velocity of the diffusion of the sugar through the mass of the contents to the line of membrane, a slow process. If, however, the mass of contents be regularly and vigorously stirred, diffusion will remain rapid, up to a certain point proportional to the vigor of stirring. In each moment the peristalsis of the intestine removes from the epithelial layer the contents whose products of digestion have been resorbed, and places thereon a fresh layer of maximum concentration, thus affording to the resorptive surface the fullest opportunity for functionation.

Normal intestinal peristalsis is probably a function which when once set into operation by the passage of food into the duodenum is automatic and self-regulatory within wide limits, decreasing gradually as the mass of the contents passing down the tract becomes reduced in volume. Diet has, of course, some influence upon it. A large amount of fat in the diet tends to stimulate peristalsis. And the presence of large masses of indigestible residue tends to accelerate peristalsis. Many chemical substances also, such as the organic acids in fruits and vegetables, act as stimulants or irritants. There can be no question that bacterial processes are of importance, and that with certain flora peristalsis may be greatly stimulated. But on the whole, with normal diets and in health, it is an automatic function, quite independent of the momentary contents of the tract.

With the colon it is clearly different. The study of the intestinal contents and habits of defecation of animals illustrates clearly that colonic peristalsis is proportional to the mass of colonic contents. The physical irritation of the indigestible residue (upon which, for example, the laxative action of figs and of coarse oatmeal depends), and the chemical irritation of the products of bacterial action (prominent in the herbivora) are factors to be sure; but on the whole, the mass of colonic contents determines frequency of defecation. The system of daily defecation in man represents the attempt to establish a different rule for the colon. The carnivora do not defecate daily. Nor would man defecate daily on a concentrated diet of meat, fat, bread, milk, and cheese. When one considers the size of a stool of 50 grams weight and contrasts it with the capacity of the colon, it is clear that if the 
peristalsis of the colon were related only to the mass of the feces, daily defecation would not occur; such a small stool is almost lost in the colon. If defecation be desired daily, the mass of the stools must be increased or the colon must be given a different rule of action, the habit of periodicity. Without distention, the normal colon holds 6 kilos of water, the sigmoid flexure alone more than 1 kilo. The amount of feces found in the colon in cases of sudden death from accident vary from half a kilo up to 2 kilos. An impacted colon may contain 5 kilos of feces; in the extreme cases of colonic dilatation, which is what the "balloon men" of the circus are, the capacity is almost beyond credence.

The time relation between the ingestion of a food and the discharge of the stool representing it, is quite variable. The marking of the stools in metabolic investigations has given good data on this point. If charcoal be given with a breakfast, it may rarely be recovered on the same evening. In a goodly number of individuals, it will be recovered on the next morning, after twenty-four hours. In the majority of individuals it will be recovered on the second morning, after fortyeight hours. Sometimes it will not be recovered until the third or even fourth day, in individuals in perfect health and with daily evacuation of the bowels. Whether an individual will pass the charcoal stool in twenty-four, forty-eight, or seventy-two hours, depends not upon the retention of the food in the stomach or small intestine, but upon the duration of retention in the colon. Some colons keep quite empty; others tolerate an amount of feces representing the diet of several days. Within reasonable limits, the one state of affairs cannot be termed more normal than the other.

Reaction of the Feces.- The reaction of normal feces may be neutral, faintly alkaline or faintly acid. The milk stools of infants are usually acid. The degrees of acidity or alkalinity involved in normal feces is very slight. Abnormally, excesses of acidity are common, both in children and in adults, due to acids formed through bacterial action.

Odor of the Feces.-The substances to which feces owe their odor under different circumstances are only known in part. They are probably all of bacterial origin. Hydrogen sulphid, mercaptans, methane, indol, skatol, and volatile fatty acids are the substances normally most concerned in the fecal odor. Their derivation will be discussed under bacterial processes in the digestive tract.

The gases of the intestinal tract, apart from carbon dioxid and nitrogen that remain unabsorbed when swallowed, are also derived from the bacterial reactions. They include hydrogen, hydrogen disulphid, methane, mercaptans, phosphin, and traces of primary alcohols. The constitution of these gases testifies directly to the very slight occurrence of oxidations in the intestinal tract. The carbon dioxid is derived from the blood and from alcoholic fermentation.

The feces contain many substances of bacterial derivation, which will be considered in the section devoted to that subject. They include 
many members of the series of fatty acids, unsaturated acids, alcohols, amino-acids, derivations of the benzene ring, and mixed compounds.

Solids of the Feces.-Indigestible Residue of the Diet.-This will vary from nothing, in the case of milk diet to as high as 60 or 70 , grams per day in the case of the diet of vegetarians or fruitarians. Under this heading are included cellulose, connective tissue, skins and coverings, seeds, gums, and resins, pectins, chlorophyl, chitinsvarying obviously with the diet. The indigestible residue of an average mixed diet will not usually exceed 10 grams per day.

BACTERIA.-By means of centrifugation it is possible in an approximate manner to separate the bacteria from the other solids of the stools. The results of such segregations have taught us the surprising fact that from one-fourth to one-half of the dried residue of feces consists of the bodies of bacteria. In other words, in the stools of a day, from 5 to 20 or even more grams (dried weight) of bacteria will be discharged. High figures are obtained for the milk stools of children, due to the luxuriant proliferation of the lactic-acid group. High figures are also contained in the large stools of vegetarian diet, rather low counts being often noted in the small stools of a concentrated meatfat-bread diet. Since these bacteria are in large part purely saprophytic germs, there is no necessary relationship between the counts and fermentative activity within the intestinal cavity. The larger proliferation occurs undoubtedly within the colon.

Residue of Digestible Food.-This exists in two forms: food that has escaped the chemical action of the digestive juices, and food that has been digested but not resorbed.

For the carbohydrates, the first alone is normally found, if at all. The digestibility of carbohydrates depends largely on proper cooking. If starchy foods be imperfectly cooked, or if unmasticated though properly cooked (as in the swallowing of whole rice, beans, or oats), they may pass undigested into the feces. The same holds good of improperly made bread. When starches and grains are properly cooked and masticated, it is not usual to find undigested remnants in the stools. All sugar formed from starch is either resorbed or falls a prey to bacterial fermentation, none is found in the stools. For the proteins, both forms are to be found; undigested fiber of meats, and traces of peptone, peptids, and amino-acids. With proper cooking and mastication, few meat fibers are to be found in the feces, except in the absence of pancreatic juice. The amount of peptid and aminoacids, determined by methods that have not much certainty or accuracy, usually falls below one gram, calculated from the nitrogen content. Such amino-acids as are not resorbed are subject to deaminization by the intestinal bacteria.

Nitrogen.-The total nitrogen of feces may vary from 0.5 to 1.5 gram per day, on a ration of 75 to 100 grams of protein. The percentage of nitrogen in the moist stool or dried feces has little meaning; it is the actual amount per day that is important. This nitrogen is divided 
into several fractions: the undigested proteins of the diet; the unresorbed amino-acids of the diet; the unresorbed amino-acids of the diet transformed by bacteria; the nitrogen of the mucin and nucleoproteid of the alimentary secretions; the nitrogen of the biliary constituents. It is not possible to even attempt to divide into these several fractions the figures for nitrogen obtained for stools by the Kjeldahl method. It may be fairly stated that with mixed diets the nitrogen of the mucin, the nucleoproteid, the bile, and the bacteria exceeds the nitrogen of the undigested and unresorbed protein. This fact is too often overlooked in the interpretation of the nitrogen data for input and output in metabolic experiments. If any material portion of the mucin secreted by the intestinal tract, estimated at some 20 grams per day, were discharged with the feces, this ratio would be much higher; but the larger part of this mucin is digested and resorbed. Normally coagulable protein and proteoses are not present in the stool. A goodly part of the nitrogenous constituents of the bile are resorbed, the amounts respectively resorbed and eliminated in the stools being, however, not capable of estimation. If the diet consists of meat, carbohydrate, and fat, the nitrogen of the stools will be so little as to suggest that it represents solely the residues of the alimentary secretions, apart from bacterial bodies. Such feces may contain no more than half a gram of nitrogen per day. The feces of a man in starvation contain nearly as much, usually about a third of a gram. The stool in starvation may contain a higher percentage of nitrogen than the stool of a diet. The stools contain on a sugar diet nearly as much nitrogen as on a meat diet if the latter is properly digested. It is clear that under the most ideal conditions, the nitrogen of the stools is little more than the nitrogen of the unresorbed alimentary secretions. A meat diet stimulates the alimentary secretions to a greater extent than does a sugar diet, and this will explain the slightly greater nitrogen content seen in the stools of meat diet. It is with mixed diets that we observe much larger figures for the nitrogen of the feces. So soon as vegetables and fruit enter into the diet in considerable amounts, the resorption of the products of digestion of protein falls. This is in all probability largely a physical condition; the undigestible residue of cellulose, etc., hinders the functions of peristalsis, so that the entire contents of the intestine are not brought into contact with the mucous membrane and as a consequence resorption is not complete. Another reason may lie in the fact that, as a rule, the stools of a mixed diet lie in the small intestine a shorter time than do the stools of a meat and starch diet. The milk stool of adults contains more nitrogen than the meat stool. Infants utilize the casein in milk better than do adults. But adults utilize the fat better than do infants. In the case of infants, the fat is largely in the state of neutral fat, suggesting that the lipase secretion of the pancreas is not as developed in infants as is the trypsin secretion.

From these data it is evident that a normal subject should resorb at least 85 per cent. of the protein of a normal diet. This figure may 
rise to 90 or even 95 per cent., and may exceptionally fall in subjects in full health to 80 per cent. It must be kept clearly in mind that these figures hold only for normal rations of protein. There is no question that with very heavy rations of protein, lower resorption may be observed. It varies also with the form of protein. It has been often noted that resorption on a milk diet is less than in the case of meat. And in the vegetarian, resorption may be notably lower.

The normal stools do not usually contain any protein precipitable by salts. Polypeptids and amino-acids are, of course, always present in the amounts previously stated.

Pathologically, much larger amounts of unresorbed protein or nitrogen are often to be encountered. The ways in which different pathological conditions operate to bring about this result may be grouped under three heads: diarrhea; lack of digestion, due to absence of ferment; and disease of the resorptive membrane. Diarrhea acts in itself by so reducing the stay of the food in the intestine as to give the intestinal juices little time to accomplish the reactions of cleavage and to permit of resorption. In proportion to the violence of the diarrhea, the food reappears more or less unchanged. It is not uncommon to find food discharged within an hour of the time of ingestion, of course, quite unaltered. Apart from this, however, diarrhea acts by disturbing the quality of the succus entericus and also, apparently, by modifying the normal flow of pancreatic secretion.

The absence of the gastric secretion has usually no effect upon the fecal nitrogen, the digestion of protein is fully accomplished by the intestinal and pancreatic juices. The absence of pancreatic juice may result in no disturbance of normal digestion, if the diet be well arranged and the ration of protein be not excessive. It may result in a marked increase in the undigested and unresorbed protein. The defect lies in chemical digestion, not in resorption; such protein as is digested is resorbed.

In diseases of the intestinal mucosa, pronounced reduction in resorption may result. In some cases of pernicious anemia, in enterocolitis of infants, in tubercular enteritis, one may find in the stools large amounts of amino-acids, the protein of the diet having been digested by the gastric and pancreatic juices far in excess of the faculty of resorption. In other cases, however, where on later autopsy the intestinal mucosa has presented widespread signs of advanced degeneration and atrophy, the resorption was entirely normal. It is never possible by a histological examination to determine to what extent the disease of the mucous membrane of the intestine might have disturbed its powers of resorption. That the total intestinal lining is in excess of the normal needs of the body, thus permitting a leeway and compensation, is shown in the results of operative experiments on the intestine, by means of which portions of the gut are switched out of function. If one-third of the intestine be circuited out of function, there is no resultant disturbance in the utilization of foodstuffs. When, however, 
two-thirds of the length of the intestine are switched out of function, there is a moderate reduction in the utilization of protein, a marked reduction in the utilization of fat, with no disturbance in the digestion of carbohydrate. Half the length of the intestine scems capable of permanently sustaining life. This is made all the more sure because the villi hypertrophy after resection of a large portion of the intestine.

Surveying the whole field of diseases of the digestive tract and its accessory glands, one must be struck with the rarity of reduction in the digestion and resorption of protein so marked as to threaten the maintenance of the nitrogenous balance of the body. Such a state of affairs is almost unknown, if indeed it has ever been shown in an individual properly dieted, and not the subject of uncontrollable diarrhea. The man with cholera, of course, does not digest enough protein to maintain nitrogenous balance; the man with pyloric obstruction often does not digest enough protein to maintain nitrogen balance. But in such instances, if food can be gotten into the intestine and retained there, a sufficient digestion would occur. Apart from such very exceptional conditions, protein starvation through non-digestion is practically unknown.

Fat.-In the normal stool, fat exists in two forms: digested, or split; and undigested, as neutral fat. On a fat ration of 100 grams, from 2 to 10 grams may be found in the stools. With larger rations, the amounts tend to rise, to maintain the same ratio. Just how much of this fat is split and how much is undigested, varies with different individuals. We have not much data; until recently the methods of analysis have been unsatisfactory. Frequently one finds the split fat exceeding the neutral fat; that is, the fault is more one of resorption than of digestion. The fats of high melting points are less well digested than fats of low melting point. A larger amount of fat is often to be found with a milk diet than with a mixed diet. Occasionally, one meets with normal individuals who on a ration of 100 grams of fat will discharge as much as 20 grams in the stools; such cases are, however, rare, and the figure stated may be taken as the highest normal limit. Some of the fatty bodies of the feces are derived from the alimentary secretions, from the bile and succus entericus; the amount is not determinable, but is certainly not small. The stools in starvation are notably rich in fat, and the stools on a rice-meat diet may contain more fat than the diet. These statements hold for true fat and are not a fictitious result due to the presence of numerous other ether-soluble substances in the stools. The fat of the stools has a higher melting point than the fat of the diet, due apparently to relatively low resorption of tristearin.

The separation of fatty acids and soaps has little meaning, resting upon a chemical misconception. What we need to know may be stated under two questions: How much of the known fat of a diet was split in digestion? How much of the digested fat was resorbed? The figures for neutral fat and total fatty acid of higher fats, answer these questions. How much of these fatty acids appear in the form of soaps, has 
nothing to do with the functions of digestion or resorption, but is quite accidental. If the diet contains cations in scarcity or in plenty, if the calcium and phosphoric acid elimination of the small intestine are so and so, more or less of the fatty acids will be discharged as soaps. The variables that determine the amount of soap do not lie at all in the function of digestion.

If the bile be absent, the fats may be normally digested and resorbed. If any defect appear, which is not the rule with a normal and selected diet, the defect will usually lie in resorption, $i . e$., the stools will contain the normal amount of neutral fat but an excess of fatty acids. Rarely, the neutral fat may be increased also.

If the pancreatic juice be absent, disturbance in the digestion of fat is likely to appear with a normal and selected diet. The amount of neutral fat will be increased, often greatly; the amount of fatty acid will usually be normal. With a diet poor in fat, the digestion may be normal; an excess of neutral fat will usually appear on increasing the fat of the diet. Some cases digest normally large rations of fat.

If both the bile and pancreatic juice be absent, as is sometimes seen in lesions affecting the papilla of the duodenum or the common opening of the ducts, digestion and resorption will usually be greatly reduced; often, however, no more than the usual amount of fatty acid will be present in the stool that is loaded with neutral fat. One must not be surprised, however, to find a normal digestion of say 50 grams of fat per day in the complete absence of bile and pancreatic juice.

Ten grams of fat or fatty acid, present in a stool of 50 or 75 grams in weight, lends to the stool a macroscopic appearance of fat, especially when the stool is cold. It is, however, not possible to judge of the fat content of a stool by inspection. It must be furthermore insisted upon that to form a reliable judgment, the fat in the diet must be known and controlled, and the test should extend over a number of days.

Biliary and Intestinal Secretion.-The feces contain the remains of the biliary and intestinal secretions. Unknown amounts of cholesterol, biliary acids, pigments, and lipoids, and of mucin are normally present. The cholesterol and lipoids are usually weighed with the total neutral fat, which, unless corrected by a determination of the acid-number, will lead to a faulty result, though no great error would be possible. The bile pigment is usually present in the form of urobilin or in other reduced state. Bilirubin or biliverdin may, however, be rarely present in normal feces. In cases of diarrhea the unaltered pigment is very likely to be present.

The Color of the Feces.- The color of the stools is due to three different factors: the diet, bacterial processes, and the biliary pigments. The bile does no usually determine the color. Milk gives a pale-yellow stool in infants, but in adults the milk stools are usually more deeply colored. Meats lend a dark or blackish color, due to reduced hemoglobin pigments and sulphid of iron. Many vegetables turn the stools 
black. With a mixed diet the colors vary from deep yellow to dark brown. Green stools are sometimes to be seen in adults or children in health on a mixed diet. White stools are sometimes to be seen in health, due to the reduction of bilirubin to a leukourobilin. Many variations in the color of stools can be shown to be independent of the bile or any article in the diet, and for these we hold bacterial processes of unknown nature responsible.

\section{PRODUCTS OF BACTERIAL ACTION ON FOODSTUFFS}

Bacteria acts upon the food in the stomach to no demonstrable extent normally; bacterial action in the small intestine is normally demonstrable. It is in the colon, however, that bacteria displays their most striking activity. The immunity to bacterial decomposition enjoyed normally by food in the stomach is due in part to the brief stay there, to the fact that the foods are but slightly digested in the stomach (bacteria operate actively upon the products of digestion rather than upon native foods), and to the action of hydrochloric acid. Whatever action on foods is to be noted in the stomach, even under pathological conditions, is usually confined to fermentation of sugars. Putrefaction is rare, even in diseases of the stomach, unless the foods are very unduly retained there by pyloric stenosis or dilatation of the viscus, and in particular in connection with ulcerative carcinoma. In the small intestine, the processes of digestion and resorption proceed with such rapidity that the bacteria have little opportunity to act upon the larger fraction of the foods. Upon the unresorbed residues, however, and upon the alimentary secretions themselves, bacteria operate in a pronounced manner. Neither the bile nor any other intestinal secretion is today regarded as endowed with notable antiseptic properties. The hydrochloric acid is indirectly more effective in the repression of intestinal bacterial action than are the bile and intestinal secretions directly. The normal chemical action of the hydrochloric acid (and pepsin) in the gastric digestion so accelerates the later proteolytic action of the trypsin and erepsin as to depress bacterial action by removing the media of their development. In a certain sense, the alimentary secretions may be said to furnish more nutriment to bacteria than do the unresorbed residues of the products of the digestion of the foods. The mucin secreted by the tract amounts to probably 20 grams per day. The pancreatic juice is rich in protein and very fermentable. The bile is easily decomposed, and as elsewhere stated, most of the constituents of the bile are greatly modified by bacterial action in the tract. The results of the activities of bacteria on these alimentary secretions are well seen in starvation. Intestinal putrefaction does not cease in starvation. On the contrary, after the first few days the signs of intestinal putrefaction in the urine and feces indicate that the bacteria in the intestine are very active. The substrate of their operations is 
the protein of the alimentary secretions. These proteins are apparently rich in phenyl-amino-acids and tryptophan, as phenol and indol compounds are prominent in the urine and feces of the starving individual. The action of bacteria under these conditions is heightened by the presence of constipation. And though we must believe that the mass of alimentary secretions is much reduced in the state of starvation, their prolonged retention in the lower intestinal tract affords to bacteria full opportunity for action. Anaërobic bacteria are now regarded as chiefly responsible for putrefactive processes in the intestine.

The fermentation of carbohydrates in the intestine leads to the formation of lower fatty acids and alcohols, lactic and succinic acids; and to the evolution of carbon dioxid, methane, hydrogen, and other hydrocarbon gases. Raw starch that has escaped digestion usually escapes bacterial decomposition. If cooked starch escapes digestion, as is often the case in improperly prepared foods, it will often be found in the stools. In the human intestine the fermentation of starch and cellulose is normally very restricted. On the other hand, it may be regarded as certain that all sugar that is unresorbed is completely fermented in the intestine. There is evidence that in different diseases of the alimentary tract, especially in children, abnormal flora may predominate, with the production of abnormal types of fermentation of carbohydrates.

The fats are very resistant to fermentation in the intestine, and even under pathological conditions it is rare to find an active fermentation of fat. There is no foundation for the fear that an acidosis may be caused by the formation of the ketonic acids from fatty acid in the intestine. It is true that in many cases of acidosis there are marked signs of intestinal irritation, and vomiting is often a prominent symptom. But these facts furnish no ground for the assumption that the acetone bodies were formed in the alimentary tract; the correct interpretation must regard the vomiting as a symptom of an internal auto-intoxication, just as may be seen, for example, in uremia.

The native proteins are resistant to putrefaction in the intestine. It is the products of the digestion of proteins, the amino-acids, and the products of the digestion of the alimentary secretions, also aminoacids, of course, that constitute the main substrate for bacterial action. This occurs to some extent in the entire intestinal tract, it is, however, more marked as the contents descend the tract, being most pronounced in the colon. The chief interest in the processes of putrefaction centres in the derivatives of tyrosin, phenyl-alanin, tryptophan, and histidin.

Putrefaction of Tyrosin and Phenylalanin.-The putrefaction of these two phenyl-amino-acids follows a course very different from the tissue catabolism of these two substances. In the processes of putrefaction the tendency is to retain the aromatic ring and to build down the fatty acid following deaminization; thereupon the aromatic derivative is usually conjugated. In the catabolism in the tissues, the aromatic ring is ruptured, and the entire molecule burned. It may perhaps be 
going too far to state that none of the aromatic derivatives of protein catabolism appear in the urine; it will be pointed out that possibly a trace of benzoic acid is thus derived. But for practical purposes, all the aromatic bodies of the urine are derived from the bacterial decomposition of the aromatic amino-acids in the intestine. Tyrosin is first deaminated, then the resulting p-oxy-phenyl-propionic acid is oxidized to p-oxy-phenyl-acetic acid; this is then reduced to phenol or cresol.

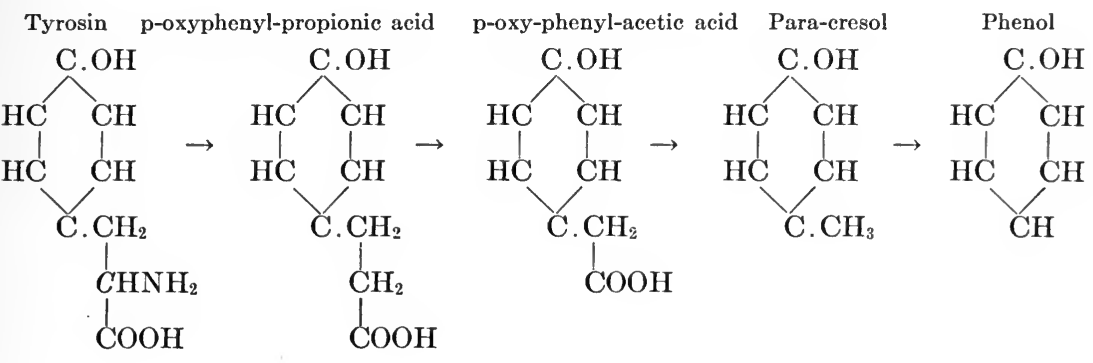

Some of the phenol is oxidized to hydrochinon, a part to orthodioxybenzene. These all are eliminated in part as simple salts, in larger part conjugated with sulphuric acid as the aromatic or ethereal sulphates, and in traces uncombined. The conjugations do not occur in the intestine; the substances are absorbed directly and the conjugation occurs in the liver. If the amounts be large or the available sulphuric acid be scanty, conjugation will occur with d-glucuronic acid, beyond the small fraction always thus combined. It is possible that a fraction of the tyrosin or phenylalanin is converted into benzoic acid, and that the trace of hippuric acid present in the urine of a subject on a meat diet may have been thus derived.

The putrefaction of phenylalanin is not well understood. In the horse phenylalanin is converted first into phenylpropionic acid, in a manner analogous to the building down of tyrosin, then into phenylacetic acid, which is eliminated bound with glycocoll as phenaceturic acid. But this last-named substance is not found in human urine. It has been suggested that the phenylalanin might be converted into benzoic acid, but this is unlikely in any quantity. However accomplished, it is certain that it is putrefied to paracresol or phenol, just as is tyrosin. As stated, a small fraction of these aromatic bodies is eliminated in the urine free, a variable amount as simple salts, the larger amount normally conjugated with sulphuric acid. It must, however, not be assumed that all the aromatic bodies resorbed from the intestine reappear in the urine; quite the contrary, a certain undetermined fraction is burned. This is made certain by the familiar observation that if a dose of phenol be ingested, it can never be recovered quantitatively in the urine, and it is believed that the missing fraction is burned. Phenol and cresol are never in the body converted into indol or skatol. Outside of the body histidin yields imidazolethylamin. 
Tryptophan.-The parent substance of indol and skatol is tryptophan. Tryptophan is indol- $\alpha$-amino-propionic acid. This is deaminated, the propionic acid oxidized to acetic acid, this then splits off or is burned, and the indol oxidized to indoxyl, the later reactions occurring in the tissues after resorption from the intestine.
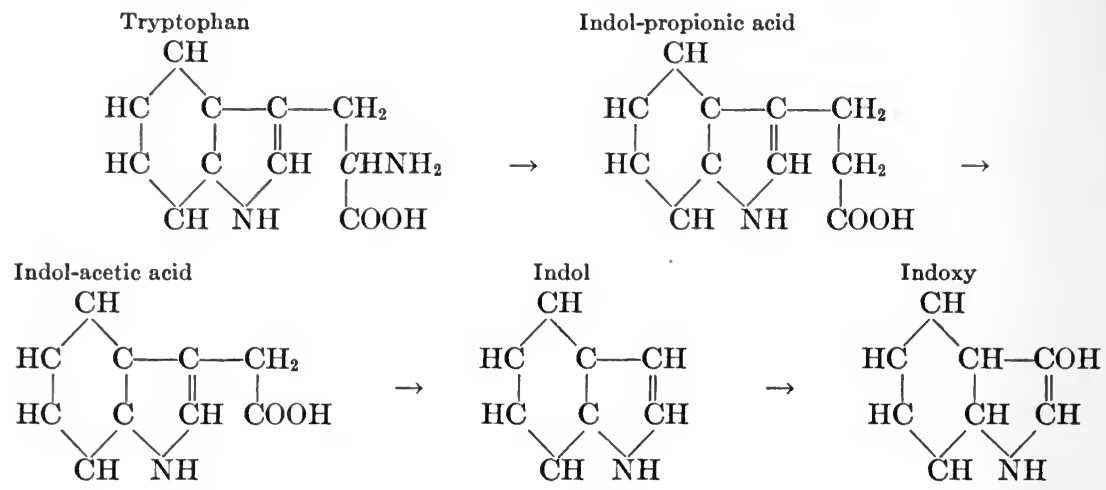

The indoxyl is conjugated with sulphuric acid, sometimes with d-glucuronic acid. The indol-acetic acid is probably the urorosein of the urine. From indol-acetic acid, by splitting off $\mathrm{CO}_{2}$, methylindol or skatol is derived.
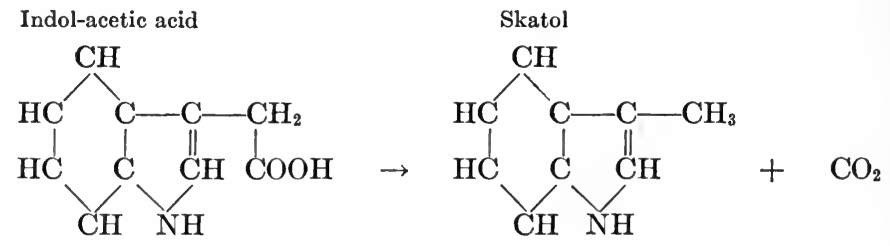

So far as known indol or its related substances are never converted in the body into phenol. The two series are entirely independent. It is possible that tryptophan in the intestinal tract has also another source. According to present conceptions hematin, the non-protein component of hemaglobin, is a tetrapyrrol, linked by iron.

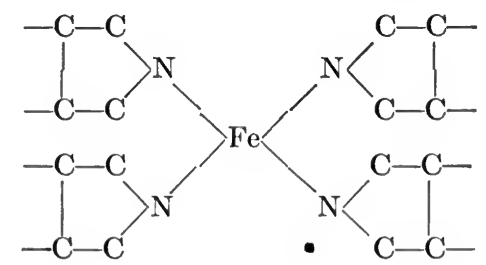

A glance at the constitution of tryptophan suggests that this body may be formed by the combination of one of the pyrrol groups with a benzene 
ring. The point is of interest, for it may indicate the synthesis of the indol ring in plants. For us here the suggestion has a different meaning; if this be the constitution of hematin, might we expect to find tryptophan (or prolin) in the bile or intestine as the result of its catabolism?

This is not the only direction in which bacteria decompose tyrosin, phenylalanin, and tryptophan. The tyrosin and phenylalanin may be converted into amins instead of being deaminated, $\mathrm{CO}_{2}$ being split off.

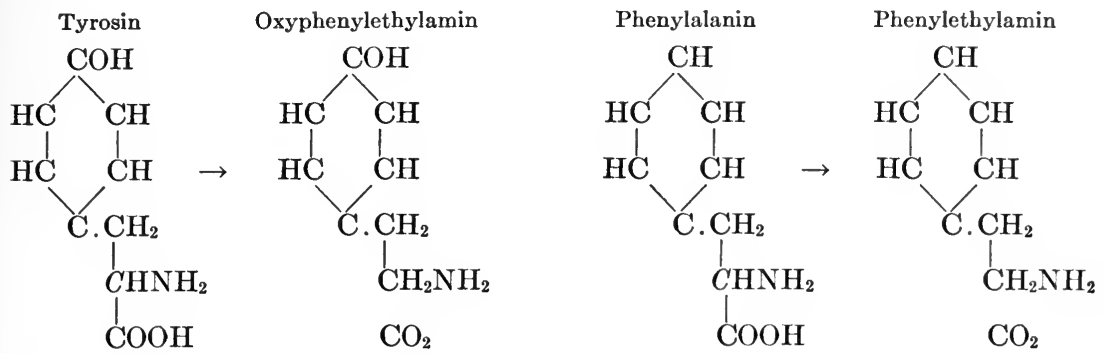

These are not regular constituents of the urine, but appear usually in the decomposition of protein.

The amount of sulphuric acid combined with these aromatic bodies varies from 0.1 to 0.7 gram per day, a striking enough variation. It is not possible with any degree of accuracy to estimate these bodies directly, we can only measure the acid with which they are conjugated. Current in the medical literature during the last twenty years are four erroneous statements bearing upon this subject. Diagnosis, physiology, and pharmacology have been erected on these four propositions, not one of which is sound. These are as follows:

(a) The aromatic bodies combined with sulphuric acid in the urine are the only aromatic bodies in the urine, and the estimation of the ethereal sulphates may be used to measure the total aromatic bodies in the urine.

(b) The conjugated sulphates of the urine represent the quantity of aromatic bodies formed by bacteria in the intestine.

(c) The quantity of indoxyl is representative of the aromatic bodies of the intestine, and of the quantity of aromatic bodies formed in the intestine by bacteria.

(d) The aromatic bodies of the urine are representative of the intensity of bacterial processes in the intestine.

It is easy to demonstrate that each one of these propositions is unfounded; but more, each may be shown to be untrue.

(a) The urine contains (apart from hippuric acid which is not here concerned) aromatic bodies not conjugated with sulphuric acid. For example, both p-oxy-phenyl propionic and p-oxy-phenyl acetic acid are present as simple salts in the urine, in notable but undetermined amounts. Furthermore, waving aside the possibility of free volatile aromatic bodies being present, for which there is some evidence, aro- 
matic bodies are always present in conjugation with d-glucuronic acid. The methods for the estimation of the aromatic bodies that exist in the urine apart from the ethereal sulphates are so difficult and so unreliable, that we are not in a position to state how large the fraction is. One sees, however, urines of vegetarians on a low protein input, in which it is certain that a large fraction, possibly the larger fraction, of the aromatic bodies in the urine exists outside of the ethereal sulphates.

(b) The conjugated sulphates are not representative of the quantity of aromatic bodies formed in the intestine, unless all exogenous aromatic bodies are excluded. 'This, however, can be done only on a meat diet, as all fruits and vegetables contain aromatic bodies, that in part pass into phenols or cresols, in part into benzoic acid. It is a matter of experience that the ethereal sulphates of the urine rise when a subject is taken from a meat-fat-starch diet and placed upon a vegetable diet. It is possible, of course, that the reactions of putrefaction of protein are increased with a vegetable diet; certain vegetable proteins in particular (as the legumens) seem very prone to putrefaction. But on the other hand, it is certain that phenol-bearing bodies in fruits and vegetables augment the phenols of the urine. It is clear, therefore, that if one wishes to use the ethereal sulphates (or total urinary aromatic bodies) of the urine as an index of the bacterial formation of phenols in the intestine, the exogenous phenols would at least have to be excluded from the diet-something that the proponents of the proposition have not done.

Furthermore, if the aromatic bodies of the urine are to be regarded as an index of the aromatic bodies of the intestine in a quantitative way, it will need to be established that none of these aromatic bodies that are resorbed from the intestine are destroyed in the body. Now it is certain that resorbed aromatic bodies of the intestine are to some extent destroyed in the body. The degree of destruction is not known and is not now measurable. It is possible that the variations in urinary aromatic bodies in different individuals might be due to differences in the powers of combustion of aromatic bodies, and we have no way of excluding this. The fact that the partition of the aromatic bodies is known to vary from individual to individual supports this possibility.

More important still is the tacit assumption that either all the aromatic bodies formed in the urine are resorbed or that the degree of resorption is more or less constant, from time to time in the same subject and also in different individuals. Now this is unfounded. Often the resorption of the aromatic bodies from the intestine, especially in connection with a simple meat and starch diet, is nearly complete; the stools yield little. But on the other hand, it is common to find a stool, in the entire absence of diarrhea, that gives an intense reaction for aromatic bodies. One sees formed stools that contain more aromatic bodies than does the urine. As a rule, constipation affords greater opportunity for resorption, while diarrhea lessens resorption. In many diseases with loose and frequent stools, the feces are heavily charged 
with aromatic bodies, while the urine is nearly free. It is entirely possible that apart from the question of diarrhea, variations in resorption may occur, due to conditions in the mucosa. This is suggested by the facts in starvation. In starvation the bacteria form large amounts of aromatic bodies from the proteins of the alimentary secretions. It is usual to find a moderate reaction for aromatic bodies in the urine and a heavy reaction in the stools, and this even though the stool be passed daily. Now with all the possible variations in the degree of resorption of aromatic bodies from the intestine (to say nothing of the differences in the amounts actually formed there) how can the quantity in the urine, even if completely and properly estimated, be taken as an index of the amount formed in the intestine?

(c) Today, when the direct source of indoxyl is definitely understood, to regard the quantity of indican in the urine as an index of the total aromatic bodies formed in the intestine, either by bacteria or in any way there derived, is nothing less than an egregious blunder. Indoxyl is derived from tryptophan; the phenols are derived from tyrosin and phenylalanin; these are independent processes, and the products depend upon the substrate concentrations. If a diet be poor in tryptophan, the stools and the urine must be poor in indoxyl, it makes no difference how much phenol derivatives it contains. If a diet be rich in tyrosin and phenylalanin, the feces and urine may be rich in the different phenol derivatives, it makes no difference how much indoxyl is present. The presence of indoxyl is a reaction only for the putrefaction of tryptophan. If the proteins of the diet be poor in tryptophan, it makes no difference how many or what bacteria are in the intestine, they cannot form indoxyl from the products of digestion. If an individual be given a diet containing fat and starch with 25 grams of egg albumin and the same amount of gelatin, the reaction for indoxyl in the urine becomes very faint. Gelatin is devoid of tryptophan, egg albumin is not rich in it. It is not possible, however, to exclude tryptophan from the alimentary tract. A diet free of tryptophan will not support nitrogenous balance, as the tryptophan is absolutely essential to the anabolism of protein. This deprivation, however, the body could tolerate for the purposes of a test. But the test could not be relied upon because the intestinal mucin contains tryptophan and the indoxyl reaction persists during starvation, being then less prominent in the urine, but intense in the feces.

With a fixed diet, constant in tryptophan, would it be possible to assume that the indoxyl of the urine is an approximate index of the putrefaction of tryptophan in the intestine? Only if all the indol were resorbed or if the degree of resorption were constant. These conditions cannot, however, be experimentally attained. A strong indoxyl reaction in the urine might be due to a good resorption and a faint reaction to a poor resorption, the intestinal content of indol being the same in the two cases. Nor are diarrhea or constipation required to insure respectively low and high resorption. The color 
reactions of indoxyl are so pronounced and the amount required to give a test so minimal, it is clear that variations that could have no other than an incidental meaning might lead to striking differences in the color tests in the urine. It is, therefore, certain that the reaction for indican is much less qualified to be used as an index of the aromatic bodies in the intestine than is the estimation of the total ethereal sulphates, in which the indican is largely included. Indicanemia as a sign of uremia, as recently suggested, has not been established, though this present verdict is still insecure on account of difficulties in the methods of analysis. If proved, it would amount to a specific uremic threshold value for indican, though in nowise suggesting the substance as the toxic agent in the condition.

(d) It may be true, but need not be true, that the total aromatic bodies of the urine are an index of the intensity of bacterial processes in the intestine. There are three factors that may modify the results: the factor of resorption, the flora of the intestine, and the nature of the diet. The factor of resorption, and the marked variations to which it is subject, has been discussed and needs no further reference. But it is clear that in the sense in which proposition $(d)$ is made, the total amount of aromatic bodies formed in the intestine (fecal plus urinary aromatic bodies) cannot be assumed to be an index of the intensity of the bacterial processes in the intestine. At the most, the total production of aromatic bodies must depend on the nature of the diet, and on the flora of the intestine. There are saprophytes that are active in the formation of aromatic bodies; there are pathogenic germs, like the cholera bacillus, that are active. There are saprophytes like the bacillus of lactic-acid fermentation, that are not active in the formation of aromatic bodies; there are disease-producing germs, like the typhoid bacillus, that are but slightly active. It is, therefore, not only a question of the intensity of the bacterial processes of the intestine, it is a question also of the flora. One flora may be very active, but not operate especially in this direction. Another flora may be inactive, but may operate especially in this direction. Thus in some of the gastro-enteric diseases of children, the aromatic bodies are present in the urine and stools in very small amounts, even without diarrhea. In starvation, on the other hand, the intensity of the bacterial processes in the intestine cannot be regarded as abnormal, but there will be a goodly production of aromatic bodies from the intestinal mucin.

Not only is the flora of importance, the type of proteins in the diet must be of some importance. As a rule, the plant proteins are somewhat richer in tyrosin and phenylalanin than the animal proteins that are used as food. All circumstances of resorption and flora regarded as fixed, it seems clear that more aromatic bodies are formed on a mixed diet than on a meat and starch diet, though the occurrence of preformed phenols in plants leads one to make the statement less equivocal than the figures would indicate. In actual life, the factors of resorption and intestinal flora may be regarded as of much greater importance than the question of the aromatic content of the proteins of the diet. 
In times gone by, it was usual to draw conclusions from the ratio of ethereal sulphates to total sulphates. These two magnitudes are now recognized to represent different variables, and the ratio has no meaning that bears on the interpretation of the ethereal sulphates as an index of intestinal processes. The estimation of the total ethereal sulphates remains the sole method upon which any conclusion as to the formation of aromatic bodies in the intestine may be based. But such conclusion, for the reasons and facts above stated, is very likely to be incorrect; so liable to be wrong in fact as to make this estimation an unreliable guide for diagnosis or treatment.

The conjugation of the aromatic bodies with sulphuric and glucuronic acids in the liver is in a certain sense an act of distoxication. The phenols are quite innocuous when conjugated with sulphuric acid. This state of conjugation is in nowise peculiar to the phenols, many substances are thus distoxicated, for example, orcin, thymol, pyrogallol, and resorcin. The reaction occurs in the liver, and the sulphuric acid is withdrawn from the common sulphate derived from the oxidation of cystin. The normal catabolism of protein yields more sulphuric acid than is required for conjugation with aromatic bodies formed in the intestinal tract. That traces of glucuronic acid exist in normal urine combined with aromatic bodies is not the result of lack of sulphuric acid; it is to be regarded as a partition. The conjugation in the liver is probably a purely chemical reaction, requiring no action on the part of the liver cells and constituting, therefore, no task in the metabolic sense. In this respect it resembles the combination of the ketonic acids with ammonia, although the reactions are not exactly of the same order. In any event, there can be no idea that the conjugation of the aromatic bodies in the liver is a burden to the liver that one should attempt to minimize as much as possible by restriction of the formation of the aromatic substances in the intestine. There is, furthermore, no foundation for the idea that the elimination of these bodies constitutes a burden to the kidneys, since in the conjugated state the aromatic bodies are practically non-irritating.

Normal urine contains traces of a base, methyl-pyridyl-ammonium.

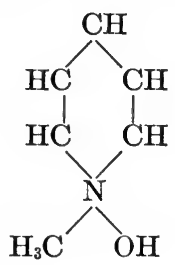

This must in some way be derived from the benzene nucleus of one of the aromatic amino-acids, probably by bacterial action within the intestine, rather than in the catabolism of protein-unless indeed it is derived from coffee, tea, or tobacco.

Hippuric Acid.-Hippuric acid is a combination of benzoic acid and glycocoll. The reaction is as follows: 
Benzoic acid + glycocoll $=$ hippuric acid + water

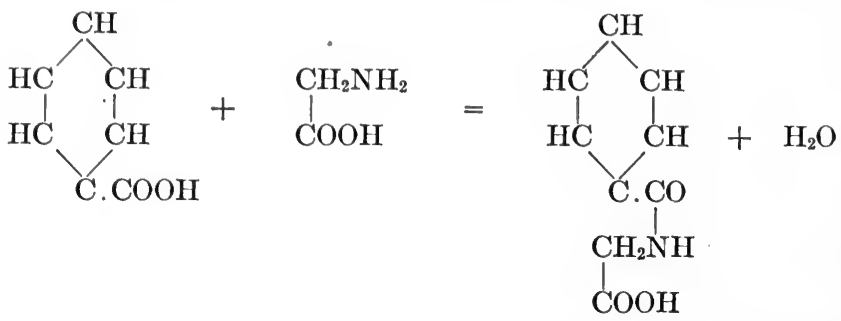

This is again a reaction of distoxication. The benzoic acid withdraws glycocoll from the urea metabolism, quite as the ketonic acids withdraw ammonia from the urea metabolism. This reaction is an illustration of a common type in the pharmacological field, many other substances being thus distoxicated by combination, among them salicylic acid, toluylic acid, phenyl-acetic acid, nitrobenzoic acid, thiophen aldehyd, cinnamic and quinic acids.

There are apparently four sources for the benzoic acid that is recovered from the urine as hippuric acid. (a) From the aromatic aminoacids of the digested proteins of the alimentary tract. (b) Preformed benzoic acid in fruits and vegetables. (c) Benzoic acid formed from aromatic compounds of various linds, present in fruits and vegetables and set free by hydrolysis in the alimentary tract. (d) Benzoic acid formed from aromatic bodies in fruits and vegetables by bacterial action within the alimentary tract.

Traces of hippuric acid, up to 0.1 gram per day, are to be found in the urine in starvation, on milk diet and on meat diet. The sole source of this benzoic acid must be the phenyl-amino-acids, either within the alimentary tract or in the protein catabolism. It seems quite certain that the former is the single source. When phenyl-propionic acid is introduced into animals, it is oxidized to benzoic acid in part; in part it remains as phenyl-acetic acid. The phenyl-propionic acid would be most naturally derived from phenylalanin.

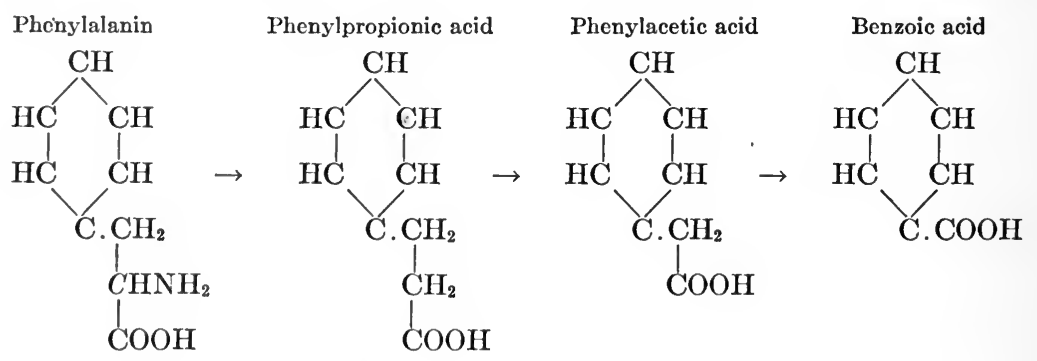

From the phenyl-acetic acid phenol is also formed by bacteria.

It is possible that tyrosin might also be converted into benzoic acid, although it is a little difficult to understand how the tyrosin is to be reduced to phenylalanin. 
On mixed diets the hippuric output varies from 0.3 to 1 gram per day. The urine of vegetarians and fruitarians may contain up to or over 2 grams per day. Hippuric acid in the urine is characteristic of the herbivorous diet. In man the largest portion of the hippuric acid is formed from benzoic acid derived from the components of the diet under fractions $b, c$, and $d$. Many fruits, like cranberries, contain large amounts of benzoic acid, and all fruits and vegetables contain goodly amounts of different compounds that on hydrolysis or oxidation yield benzoic acid.

The site of the conjugation of benzoic acid and glycocoll to hippuric acid is the kidney. By this we do not mean that in the nephrectomized animal hippuric acid cannot be formed. But for practical purposes the reaction is held to occur in the kidney. The conjugation is a ferment reaction, and is accomplished under favorable conditions by fresh kidney pulp and in the perfused kidney.

Benzoic acid is also conjugated with d-glucuronic acid and a trace of this compound is believed to be present in normal urine.

The amount of glycocoll available in the normal protein catabolism is far more than sufficient to combine with any amount of benzoic acid that may be ingested in the natural state in foods. When benzoic acid is ingested directly it is found that glycocoll is available in amounts far in excess of the quantity of glycocoll to be derived from the day's catabolized protein. This glycocoll must be synthesized in the body or else derived from the body protein. Experimental investigations have indicated that the latter course is the method adopted to secure the glycocoll. The body builds down protein from its tissues and thus secures glycocoll. When large doses of benzoic acid are administered to an animal, there will be an excess of nitrogen in the urine; this corresponds to the superfluous fraction of the molecule of protein catabolized to secure the glycocoll. This increase in nitrogen, curiously enough, is not in the state of urea but is included in the rest nitrogen, and probably is in the form of a polypeptid body like the oxy-proteinic acid. It is likely from this that the catabolism of protein under these circumstances is different from the normal. Whether the glycocoll is secured merely in proportion to its occurrence in the protein catabolized, or whether it is also formed from other amino-acids is not known. The body proteins drawn upon are supposedly the proteins of the connectivetissue group, some of which are very rich in glycocoll.

Whether the conjugation of benzoic acid with glycocoll is to be regarded as a burden to the kidneys is difficult of determination. The kidney in nephritis is sometimes defective in this function; but it has not been shown that the elimination of the hippuric acids is, like sodium chlorid in excess, a burden to the organ. The carnivorous diet is commonly regarded as being most onorous to the kidneys, while the largest amounts of hippuric acid are to be found in the urine of vegetarians and fruitarians. 


\section{CH A P T ER I V}

\section{METABOLISM}

\section{GENERAL CONSIDERATIONS OF METABOLISM}

UNDER the term metabolism we understand the series of chemical processes through which the foodstuffs are carried $(a)$ in the conservation of the tissues of the body and $(b)$ in the maintenance of body temperature and of physical work. The upkeep of a machine comprises the replacement of worn parts, lubrication, and fuel. The upkeep of the body comprises the replacement of worn parts and fuel. What do we mean by the replacement of worn parts? Cells have a limited life, and the status quo must be maintained by the regeneration of new cells to replace those that die. These new cells are formed from the foodstuffs; not normally from the materials of the dead cells. This is a point of fundamental importance. When a cell dies, its material is disintegrated and cast out; it has normally no further utilization in the body. The new cell, formed by process of reproduction from a preëxisting cell of its type, is maintained in its growth by new material derived from the foodstuffs. In the case of a machine, the worn parts may be recast into new parts; in the case of an animal organism, the worn parts are normally discarded. All cell formations during normal life are, therefore, maintained through the utilization of new raw material, from the daily food in large part, in small part from stored food if need be. For the most part, however, the formation of new cells is dependent upon daily food. As will be later described, in starvation the body may exercise a faculty of adaptation in this respect, in that the formation of the important cells of the body is maintained at the expense of the degeneration of less important cells, i.e., the nervous system and glands are maintained at the expense of connective tissue and skeletal muscles. With the maintenance of cellular regeneration in adult life is to be classed the maintenance of growth in the young. It means, however, more than this. Living cells must suffer wear and tear in the course of chemical functionation. The relative values of these two fractions of regeneration of new cells and wear and tear of functionating cells vary with different tissues. Together the two comprise the total upkeep of the cells. Body heat and body work may be classed together. For these the foodstuff's act as fuel. The upkeep of the body means then the maintenance of the working parts and the supply of fucl.

These two phases of metabolism are shared by each of the three main foodstuff's - the proteins, the carbohydrates, and the fats. In 
the quantitative sense, it is true the proteins have the more important share in the upkeep of the tissues, and the fats and sugar the most important share in the production of heat and support of work. But heat is always evolved in the protein catabolism and the entire fuel needs of the body can be maintained by the combustion of protein. On the other hand, lipoids and sugars are indispensable constituents of protoplasm.

In miniature, both in time and space, the processes of the growth and maintenance of tissues are illustrated in the hatching of the chick. The egg contains fat, a little carbohydrate, and much protein. On account of lack of oxygen, there is no heat metabolism in the hatching chick, the heat necessary for the preservation of cell life must be furnished from the outside. From the materials in the egg, all the structures of the chick, all the cells of the glands, nervous system, connective tissues, skeleton, skin, nails, feathers, digestive tract, etc., are formed. Once the anlage is established, growth and upkeep are identical in terms of metabolism, the difference is in quantity. Illustrated in the hatching of the chick are the processes of upbuilding mostly, and but few of disintegration, on account of the lack of oxygen and the short period of time involved. When the chick is hatched, the heat metabolism is added to the tissue metabolism, and the processes of dissimilation are inaugurated. During the period of growth, the building-up processes are relatively disproportionate to the processes of dismantlement; during mature life they are balanced; with the advance of age the disproportion of youth is reversed. The unit of metabolism per unit of tissue is greatest in youth and least in old age; this applying to both the building-up and the building-down processes. The building-up processes of metabolism we term anabolism; the building-down process we term catabolism. In the series of processes termed anabolism the foodstuffs are transformed and organized into the cells and tissues of the body. In the series of processes termed catabolism, worn protoplasm and the constituents of dead cells are disintegrated and cast out of the body. And side by side with these processes are the reactions of combustion, whereby the temperature necessary for the life of the cells is maintained and the energy needed for external work furnished. Life represents, therefore, a state of equilibrium.

For each of the three main foodstuff's we may divide the subject matter as follows:

The chemical state of the foodstuff as the basis of utilization.

The state in which it exists in the cells and other tissues.

The chemical processes in the anabolic transformations.

The chemical processes involved in the disintegration of the cells and tissues.

The chemical states of the end products of catabolism.

The elimination of the end products of catabolism from the body.

Schematically, the processes of metabolism may be sketched as follows: 


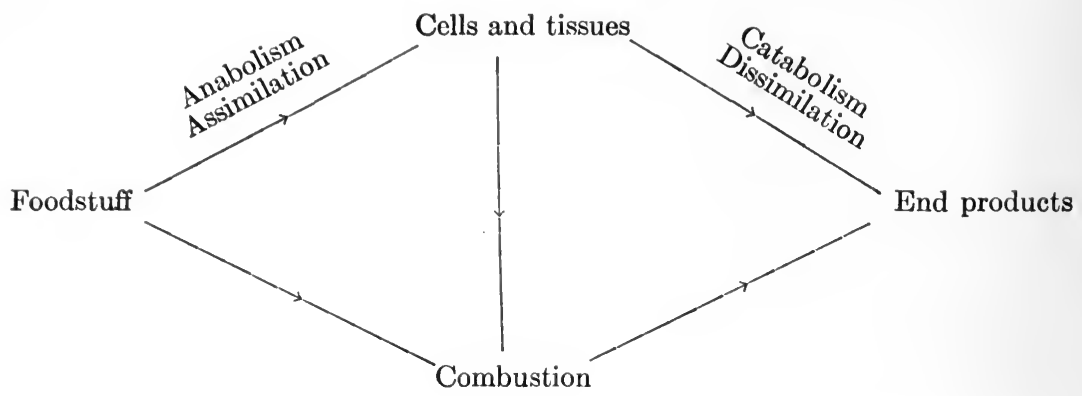

This scheme applies to protein, fat, and sugar, with quantitative variations only. Because not yet clearly understood, the anabolic functions of sugar and fat have been long neglected; modern chemical and physiochemical investigations, however, have taught us their great importance. The lesser importance of the fuel relations of protein have also been realized within recent years.

A subdivision of the consideration of metabolism into chapters devoted to carbohydrates, fats, and proteins serves well if we devote a separate chapter to the discussion of the purin metabolism, that cannot be successfully placed under either protein or carbohydrate.

\section{CARBOHYDRATE METABOLISM}

In the discussion of the digestion and resorption of the carbohydrates we learned that the end product of these processes was the aldohexose, d-glucose. This is the form of sugar delivered to the blood of the portal vein; it is the form of sugar delivered from the liver to the blood of the hepatic vein; it is the only form of sugar known to exist normally in the blood and circulating fluids. There are other sugars fixed in the tissues; these must, therefore, be derived from d-glucose. In the tissues is to be found a polysaccharid, the animal starch, glycogen; this must be derived from d-glucose. The body forms fat from d-glucose. And lastly, the body burns d-glucose directly. The metabolism of d-glucose may thus be represented as follows:

$$
\begin{gathered}
\text { sugars of tissues, combined and free } \\
\text { d-Glucose } \\
\begin{array}{c}
\text { glycogen, proximate form of storage } \\
\text { fat, ultimate form of storage } \\
\text { fuel }
\end{array}
\end{gathered}-\left\{\begin{array}{l}
\text { pentose } \\
\text { galactose } \\
\text { glucose } \\
\text { glucosamin } \\
\text { galatosamin } \\
\text { chondroitin }
\end{array}\right.
$$

\section{COMBINED SUGARS OF THE TISSUES}

That a moiety of carbohydrate could be obtained in the chemical disintegration of the proteins has long been known. Whether this carbohydrate was an admixture or present in combination; and if 
combined, whether loosely or firmly combined, was not always easy of determination. In some instances glycogen was undoubtedly involved. For the most part, however, it is now clear that we have to deal with special forms of sugars. The demonstration that these are all derived from $\mathrm{d}$-glucose is contained in the fact that they are all formed on a milk diet, the sugar of which is in digestion converted into d-glucose. In the plant world sugars exist in fixed combinations with many other substances, and of these the glucosids are perhaps best known. It is possible to compare the fixed combinations of sugar in the animal body with the glucosids of plants, since both are constituents of metabolism.

The Pentoses.-The pentoses are sugars containing five atoms of carbon, three of which are asymmetric, and possessing the common formula: $\mathrm{C}_{5} \mathrm{H}_{10} \mathrm{O}_{5}$. While in animals they exist only in the form of the sugars, in plants they exist in the form of polysaccharids termed pentosans, related to pentoses as starch is related to dextrose. So far as present information goes, pentoses exist in the body only in one localization, chemical and morphological, namely, in nucleic acid, in the nuclei of cells. No pentose is to be found in the blood, in the circulating fluids, in protoplasm or in connective tissues outside of the nuclei. In the nucleic acids isolated from the liver, pancreas, thymus, lymphatic glands, kidneys, muscle, and brain, one form of pentose has been found, d-ribose. The stereoisomeric equations of d-ribrose, $d$ - and l-arabinose, and d-glucose are reproduced for purposes of comparison.
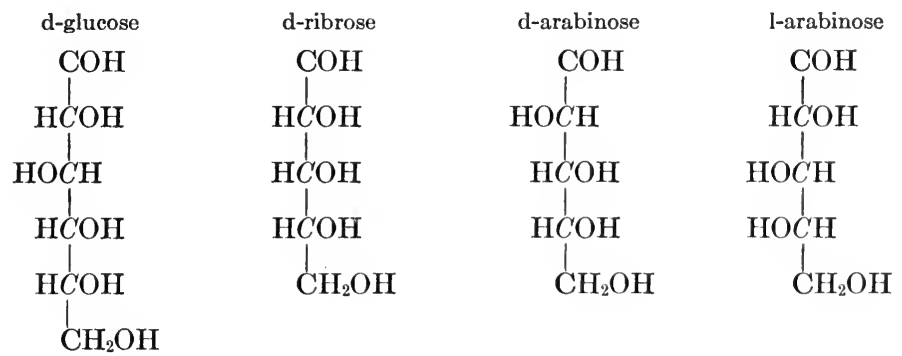

As will be elucidated later, d-ribrose is combined with purins and pyrimidins to form ribosids, which with phosphoric acid form nucleotids that in simple or polymolecular states constitute the nucleic acids of the nuclei of cells. Thus far pentose has not been shown to exist in the body in any other combination. 'The pentose of the glands was formerly supposed to be l-xylose, but the sugar is now known to be d-ribrose. The amount of pentose in the nuclei-rich glands is quite large; fresh liver, kidneys, lymph glands and thymus contain as much as a half of 1 per cent., while pancreas contains 2 per cent.; brain and muscle contain 0.1 per cent. The total pentose content of the adult body may be judged at 20 grams. Since no pentose is to be found in the blood, it would follow that in the catabolism of nucleic acid the ribrose is either burned directly or else converted into d-glucose. Since the formation of nucleic acid is independent of any pentose of the diet, 
it is obvious that it must be derived from d-glucose. A glance at the two equations, however, does not indicate how this is attained.

Pentose is rarely found in the urine, supposedly in combination with urea. Pentosuria is usually an idiopathic condition, without symptoms and inclines to run in families. Pentose has, however, been found in the urine in typical diabetes, side by side with d-glucose, though in much smaller amount. The variety of pentose seen in idiopathic pentosuria is not d-ribrose, but d- and l-arabinose in equal amounts. In one case l-arabinose was eliminated alone.

There are three possible explanations of pentosuria: the sugar might be of alimentary, of glucolytic or of nucleic origin. It seems certain that the pentose is not derived from the diet. The condition persists despite pentose-, pentosan-, or carbohydrate-free diet. It is not exaggerated by the administration of pentoses; the subjects of this curious anomaly burn pentoses in a normal manner; even ingested, arabinose is burned and not added to the arabinose of the urine. As elsewhere stated, the pentoses of the diet are probably converted into $\mathrm{d}$-glucose in the intestinal wall, which explains this finding. It is clearly not a form of alimentary melituria.

If the pentosuria were of nucleic origin, this would mean that somewhere in the body d-ribrose is converted into d-and l-arabinose, instead of being burned or converted into d-glucose. This, of course, is possible, as we know experimentally that the various hexoses are convertible into each other, and sometimes with ease. Nevertheless the stereoisomeric equations for these pentoses are far apart.

If, lastly, the pentoses originate in d-glucose, the action is indeed an abnormal one. The occurrence of pentosuria in diabetes (the exact. pentose concerned has not been determined) furnishes us with an illustration, however, since here we must regard the pentose as derived from d-glucose. This deflection only affords another illustration of the perversion of the carbohydrate metabolism in diabetes.

Of the origin of the pentose in the body as utilized in the synthesis of nucleic acid, we have no experimental or chemical evidence. It is not possible to believe that as in the plant it can be built up from formic acid. It is difficult to believe that it could be formed from glycerol. It must be assumed that d-glucose is built down to the pentose. Where and how this is accomplished is not known. Certain bacteria are able to form l-xylose from d-glucuronic acid.
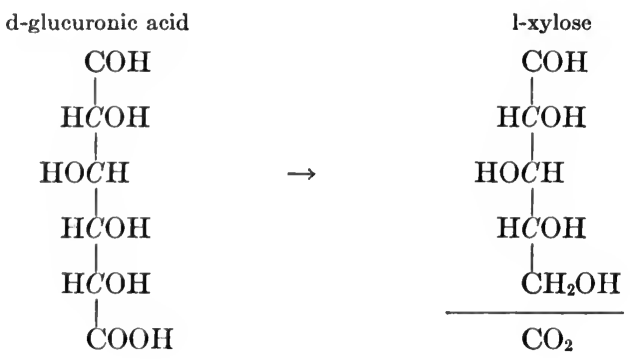
It does not seem possible to regard this conversion as illustrating a type of reaction to which we could appeal in explanation of the formation of $\mathrm{d}$-ribrose from d-glucose. The synthesis of nucleic acid is in this, as in the derivation of purin and pyrimidin from protein, a most original and self-sufficient process.

Galactose.-Under the term galactose we understand d-galactose. Galactose exists to a limited extent in plants, in the forms of galactosids, in gums of a starch-like nature termed galactans and in plant amyloid. In animals galactose exists in milk sugar, in the central nervous system and in the peripheral nerves, in true mucins (of the higher animals as well as in such mucins as the covering of frog's eggs) and apparently in some nuclei, though it is clear that galactose plays no such rôle in the nuclei as does pentose. It is not present in the blood or circulating fluids or in the urine. When galactose is injected into the circulation, it will be eliminated only in part in the urine, if at all. From this it is apparent that the body can burn it directly or convert it into d-glucose. In the digestion of milk sugar or of the galactoseyielding carbohydrates of plants, the galactose is in the intestinal wall (or eventually in the liver) fully converted into glucose. And so far as we are aware, this reaction is never reversed in the metabolism, though it is reversed in special tissues-in the mammæ and in the central nervous system. Idiopathic galactosuria is unknown; alimentary galactosuria has been reported in a few cases of hepatic disease. It is, of course, possible that on a diet of milk, the intestinal wall might be overflooded with galactose and some pass into the portal circulation, later to pass through the diseased liver into the general circulation, carried to the kidney and there eliminated. But the reported cases of galactosuria should be viewed with suspicion, because of the methods of analysis used. For the positive separation of galactose from glucose and its identification, several methods must be employed: the rotation of the plane of polarized light, the refractive index, the formation of mucic acid, the formation of galactose- $\alpha$-methyl phenylhydrazone, and the fermentation test with a culture of Saccharomyces Ludwigii that has been recently tested on galactose and glucose.

The galactose fraction of the milk sugar of the lactating animal is formed from the blood glucose in the cells of the mammary gland. It is the reversal of the reaction whereby in digestion milk sugar is split into glucose and galactose, and the galactose then converted into glucose. The cells of the mammary gland convert glucose obtained from the blood into galactose, and then combine with this other glucose from the blood to form milk sugar. It is a purely localized function; and so far as known, galactose never escapes into the circulation, though as will be soon elucidated, the finished milk sugar often does. Extirpation of the mammæ of an actively lactating animal is followed by a transcent hyperglucemia, with glucosuria; glucose alone appears in excess in the blood and in the urine. It is as though the sugar-forming functions of the body had established a certain high plane of produc- 
tion of glucose, to supply the usual needs of fuel and the extraordinary demands of the breast glands; and on the latter being suddenly removed, the overproduction of glucose could not be promptly shut down. The formation of milk sugar is independent of the galactose of the diet; nor is galactose in the diet utilized directly in milk formation. In other words, so far as the sugar is concerned, milk is no better a food for the nursing mother than any food that contains the available carbohydrates; in whatever state ingested, carbohydrate must be converted into glucose and the galactose formed in the mammæ from the glucose.

Galactose exists in the central nervous system and in the peripheral nerves in stable combination with the lipoids. The amounts are not striking, but the combination is fundamentally essential. So little is known, despite extensive and intelligent investigation of these lipoids of the nervous system, that we are not in a position to indicate with what manner of combination we are here concerned. An illustration will make this plain. On analysis of the white matter of the brain, after a series of procedures a substance is obtained that is termed cerebron. On hydrolysis this splits into a substance termed cerebronic acid, sphingosis and galactose. Thus supposedly:

$$
\mathrm{C}_{48} \mathrm{H}_{93} \mathrm{NO}_{9}+\mathrm{H}_{2} \mathrm{O}=\mathrm{C}_{22} \mathrm{H}_{50} \mathrm{O}_{3}+\mathrm{C}_{17} \mathrm{H}_{35} \mathrm{NO}_{2}+\mathrm{C}_{6} \mathrm{H}_{12} \mathrm{O}_{6}
$$

Neither the constitution, the molecular weight nor even the elementary analysis of the cerebron, cerebronic acid or the sphingosis is understood. Galactose may be also obtained from other fractions of the material than the cerebron. But aside from all this, which must be clarified by future research, it is certain that we deal here with a combination of fundamental importance. Galactose exists also in the nervous cells. In the catabolism of the nervous tissues the galactose must be set free, and so far as we can judge is not eliminated in the urine unchanged, since we have evidence that the body tissues and fluids can burn it or convert it into glucose, most probably the latter.

The galactose in mucin and in the nuclei is in stable combination, the mucin being one of the most typical of the so-called glycoproteids. The amount of galactose present is, however, very small.

The formation of galactose from glucose for the purpose of combination with lipoids, mucin and nuclei constitutes the best-known anabolic processes of the sugar metabolism. It will be later pointed out that glycogen seems to bear analogous relations to protein, though as a whole, it is to be viewed as a storage form of sugar. But in the case of galactose, we deal with an anabolism that has no relations to the combustions of the body but is concerned solely with the formation of chemical complexes of the highest cellular importance. The mechanism of the transformation of glucose into galactose is not known. It is easy in the laboratory to form glucose from galactose and possible also to form galactose from glucose. The localization of the formation of glucose from galactose in the intestinal wall, and of the formation of 
galactose from glucose in the central nervous system, mammary glands, mucin-forming cells, and in nuclei indicates that special conditions are requisite.

Fructose (Levulose).- Although fructose is not an anabolic state of glucose it may be appropriately considered here. Under the term fructose we understand d-fructose. Fructose is present in nature in the form of cane sugar, and in all fruits and in complex polysaccharids that are termed fructans, of which inulin is an illustration. In digestion, the fructose derived from fruits and from cane sugar is converted into glucose in the intestinal wall and in the liver. When fructose is introduced into the blood, it is eliminated in part only by the kidneys. The tissues apparently have power to convert fructose into glucose. There is evidence that the body does not burn fructose directly, but only after conversion into glucose. Unlike galactose, fructose has no metabolic relations and is not to be found in the tissues of the body except in circulation.

Fructose sometimes appears in the urine. Three types of fructosuria are to be distinguished. Alimentary fructosuria is seen in not a few cases of diabetes, in some diseases of the liver, and sometimes unassociated with any disease. It may occur in uncomplicated pregnancy. It is due simply to the fact that following the ingestion of a large amount of cane sugar, the intestinal wall is overwhelmed and the liver does not complete the conversion of levulose into glucose. It can be provoked, experimentally, by ablation of the larger portion of the liver; by ligation of the portal vein; is noted in some cases of Eck's fistula; and clinically is so often present in chronic degenerative diseases of the liver, as cirrhosis, as to constitute a diagnostic test. It is not seen in the acute degenerative diseases of the liver, such as acute yellow atrophy and eclampsia. Undoubtedly most of the instances of fructosuria seen in diabetes are alimentary fructosuria associated with hepatic diseases. Rarely, however, an alimentary fructosuria is seen in which there are no indications of hepatic disease.

Mixed melituria is common in pronounced diabetes, fructose and glucose being eliminated side by side, the former always in the lesser amounts. The effect of restriction of the carbohydrate input is more markedly evinced upon the fructosuria than upon the glucosuria. There is no doubt that mixed melituria is an unfavorable sign in diabetes. The meaning is probably defective hepatic function. The usual case of diabetes converts fructose into glucose quite as in the normal; the inability to do so constitutes only an added defect.

Finally, cases of supposedly idiopathic fructosuria have been reported, in which the condition is stated to have persisted in the absence of fructose from the diet. These cases have not been properly studied. Not only is it necessary to exclude all fructose-carrying foods, it is also necessary to test the influence of different sugars with a carbohydrate-free diet. There is one further explanation that has been overlooked. The fructans, like inulin, are supposed to be resistant to the 
ferments of the alimentary tract, though easily hydrolysed by bacteria. This might serve as the source of the fructose. The tests commonly applied for the identification of fructose are not reliable. The color tests and the reactions with the different salts of copper are not reliable criteria. One must determine the rotation of the plane of polarized light, ascertain the refractive index, demonstrate the presence of the keton group, form the characteristic fructose-methyl-phenyl-hydrazone and apply the fermentation test with a proved culture of Sacch. membranefæcans. If a fructosuria were determined to exist under controlled conditions in the diet (on a diet of glucose and casein for example), it would be explicable only as a reversion of the reaction whereby normally glucose is formed from fructose. This reversion is easily accomplished in the laboratory. But so long as we possess in the body no demonstration of such a conversion normally, one must be rigid in the application of the analytical criteria. No idiopathic fructosuria has thus far been demonstrated to be of metabolic origin. Fructose has, however, been isolated from the blood of the non-diabetic subject.

Chondroitin.-In cartilage, bone, tendons, connective tissues of the type of fasciæ, reticulin, hyalin layer of bloodvessels and especially in the pathological material termed amyloid is an ethereal sulphuric acid termed chondroitin-sulphuric acid. Traces seem to occur also in the urine. When this conjugated sulphuric acid is split by heating, an amino-polysaccharid is recovered that is evidently a highly complex anabolized form of carbohydrate. The substance is so different from glycogen that it is better to regard it as a substance anabolized from glucose than as a modified glycogen. Of its internal constitution nothing is known; of its derivation nothing is known.

An apparently somewhat similar substance has been obtained from the liver, spleen, kidneys, pancreas, and mammary glands, termed glucothionic acid. It is also an ethereal conjugation of a carbohydrate with sulphuric acid. In the case of glucothionic acid, the carbohydrate does not seem to be a polysaccharid. On the other hand, it has no relation to the pentose of nuclein.

The last of the anabolic states of glucose is d-glucosamin. This is an amino-sugar, a combination of an amino-group with glucose.


It exists in complex combination in the chitins of the shells of many crustaceans and in the wings of insects. Glucosamin exists in most 
proteins. The glucoproteins contain 20 to 30 per cent., ovalbumin 10 per cent., the serum albumin, globulins, and myosins contain traces, while casein is free of it, as is codfish flesh. In its native state it exists probably as a polysaccharid. Galactosamin usually coexists with glucosamin in the true mucins. It is also to be obtained in the hydrolysis of the bodies of bacteria. It is sometimes found in the contents of cystic tumors of the broad ligament and in other mucinaginous neoplasms. Feeding experiments designed to produce such conditions have not been successful. It seems clear that the amino-sugar is formed in situ in the tissues where it occurs, and is thus a typical anabolic product of glucose.

The Catabolism of the Special Tissue Sugars.-Pentose, galactose, galactosamin, and glucosamin we have named as the anabolic products of glucose that exist in the state of primary sugars. They are constituents of tissues and cells. What becomes of them when the cells and tissues of which they are a part undergo catabolism? We do not know. They might be converted into glucose by the reversions of the reactions through which they were formed; they might be eliminated unchanged as end products. That they could be burned directly is hardly to be regarded as probable. The amounts involved are so small that one could not hope to detect them in the urine. The products of catabolism are so rarely utilized in the organism that from the general point of view one would be inclined to the opinion that these sugars (except the glucosamin) are eliminated unchanged.

\section{THE DERIVATION OF THE SUGAR OF THE BLOOD}

Glucose is the substrate of the sugar metabolism of the body; all the carbohydrates of the diet are converted into it, and from it all the forms of carbohydrate of the tissues are formed. Is the carbohydrate of the diet the sole source from which the glucose of the blood is derived? This is a fundamental question, and one that for years presented seemingly interminable experimental difficulties to the investigator. We are now on clear ground in part; and in any event the facts known are of such nature as to enable us to consider in a provisional manner the entire problem.

Derivation of Sugar from Proteins.-Obviously, there are two further sources for the derivation of glucose than the carbohydrates of the diet; the proteins and the fats. When we recall that a half century ago it was the ruling view that the fattening of animals was accomplished through the protein metabolism, it is all the more interesting to realize how difficult it has been within recent years to prove that sugar can be formed from protein. In fact, it has been only since our conception of the internal constitution of the molecule of protein became definable, that it has been possible to so arrange the experiments and control the variables as to demonstrate the formation of sugar from protein. 
Proteins are composed of linkings of amino-acids. And it is from the fatty acids of these amino-acids that glucose is formed. When a protein is broken down in catabolism, it is split by hydrolysis into the component amino-acids. From this point on there are two chemical possibilities: the amino-acids may be split by deaminization, the nitrogenous fraction converted into urea and the oxy-fatty acids burned directly; or sugar may be formed from the oxy-fatty acids or from the amino-acids, and this sugar then burned. The body can, of course, burn all the fatty acids that are encountered in the molecule of protein; the body can burn all the amino-acids that are to be obtained from the molecule of protein. Direct experiments also prove that while the body does not form sugar from the simple lower fatty acids, it does form sugar from some amino-acids introduced from without. From the standpoint of energy, there is no gain to the body in first converting the aminoacids into sugar and burning them in that state; but it is a question of physiological fact and not of teleology. On the other hand, it is clear that all the carbon of the molecule of protein is not converted into sugar; some of the oxy-fatty acids are burned directly. In other words, all of the amino-acids derived in the hydrolysis of protein are not sugar builders. From the standpoint of general theory, it would be very simple if it could be assumed that all the carbon of the molecule of protein (and of the fats) were converted into sugar. This would make sugar the sole fuel of the metabolism. We are not, however, warranted in assuming this position, though there are general considerations and qualitative arguments that make the view very attractive.

What is the crucial experiment that proves that sugar is formed from protein? A dog is first fasted until poor in glycogen. It is then made diabetic by ablation or destruction of the pancreas. If now the dog be maintained upon a carbohydrate-free diet, containing only protein free of preformed carbohydrate (as casein), and the sugar of the urine estimated day after day, in favorable cases where it is possible to keep the animal alive for a long time, it will be found that the amount of sugar eliminated in the urine exceeds greatly the sugar that could have been derived from all forms of storage of carbohydrate in the body of the dog. The sugar eliminated in the urine of such a dog must have come from protein or from fat. By direct experiment, it is possible to follow the formation of sugar from certain amino-acids. And in the dog with phloridzin intoxication, it is also possible to show in toto the formation of sugar from protein and directly also the conversion of certain amino-acids into glucose. The question that concerns us here relates to the extent that this occurs in normal metabolism. Do the experimental facts hold for the normal, or do they merely illustrate adaptations to extraordinary conditions? If they are phenomena of the normal metabolism, what are the quantitative relations, what amounts of sugar may be normally derived from the protein metabolism?

Today it must be regarded as certain that the formation of sugar from amino-acids is a fact of normal metabolism, and not an adapta- 
tion to meet an experimental or pathological condition. The ultimate cause of glucosuria in the diabetic is the inability to burn sugar. The cause of glucosuria in phloridzin intoxication is abolition of renal retention of the glucose in the blood. The conversion of certain aminoacids into sugar holds for the postmortem experiment of perfusion through the liver as well as for the two conditions of diabetes and phloridzin intoxication. Since it is inconceivable that the formation of sugar from amino-acid should be an adaptation in three such different conditions of metabolism and experimentation, we are forced to regard it in all three as a physiological procedure, and to assume that under all known conditions, physiological and pathological, sugar is formed from the amino-acids derived from the catabolism of protein.

What amounts of sugar are thus derived? Is the total carbon of the protein molecule converted into the state of sugar? No positive answer can be given; to date, however, it seems most probable that only a fraction of the amino-acids is converted into sugar. A fraction is burned directly. Whether this means that certain amino-acids are not sugar builders; or whether it means that some condition limits the conversion of these amino-acids into sugar, is not known. The experimental solution of the question has been approached in two ways: one by the determination of the quantity of sugar that can be gotten out of a unit of protein, under constant conditions of experimentation; and the other by testing the individual amino-acids for their chemical qualifications as sugar builders. The molecule of common protein contains about 50 per cent. of carbon. If this were all converted into sugar, that would equal the formation of 1.25 grams of sugar from 1 gram of protein. Or stated in the form of the nitrogen: glucose ratio, it would mean that 1 gram of nitrogen in protein would correspond to 8 grams of sugar. Now this is far more than is ever seen in experiment (though it has been reported in human diabetes), in which the formation of four parts of glucose to one part of nitrogen in the protein (nitrogen: glucose ratio $1: 4$ ) seems to be the limit. It is, however, unfair to assume that all the protein, under the conditions of the experiment, underwent catabolism; how much may have been retained in the body for purposes of the protein metabolism cannot be accurately determined. The quantitative experiment, therefore, indicates that at least half of the carbon of the protein molecule may be converted into sugar; it does not inform us whether more, or how much more, might be converted.

The qualitative method of experimentation (to be later discussed in detail) consists in testing the several amino-acids for their convertibility into glucose. There are in the protein molecule nearly twenty different amino-acids. Many of these have not been tested. Some of the most prominent have been tested with positive results. Others are known not to yield sugar. From these results it is clear, for the present at least, that no decision may be reached as to the amount of sugar that is actually derived from the protein of the daily metabolism. 
If the total carbon of the protein were converted into sugar, a man of 70 kilos weight on a daily ration of 70 grams of protein, would derive from this source about 100 grams of sugar daily. If the amount of sugar were calculated according to the highest nitrogen-glucose ratio that is to be observed in experimental diabetes, about 50 grams would be daily so derived. These amounts of sugar would yield correspondingly 400 and 200 Calories of heat-but a small fraction of the total heat requirements of the body. The average diet of a man of 70 kilos, on a ration of 70 grams of protein, will contain 400 to 500 grams of carbohydrate.

As will be pointed out in the discussion of diabetes, the quantitative relations are there different and of vastly greater importance. Since the diabetic is thrown upon the combustion of protein to a much greater extent than is the normal body, the amount of sugar derived therefrom may be two or three times the amount thus derived in the normal body. It is this sugar, which the diabetic body cannot burn, that accounts for the persistence of glucosuria on a carbohydrate-free diet; and it is the loss of this sugar that reduces so greatly the efficiency of the combustion of protein in the diabetic organism.

Derivation of Sugar from Fats.-Is sugar derived from fat in the animal body? This is a fundamental question, but one for the future. That fat is formed from sugar both in plants and animals is not to be questioned. That in plants the reversion of this reaction, the formation of sugar from fat, occurs, is also not to be questioned. This process is easily to be observed in the germination of seeds of all kinds. Starch is in seeds the proximate form of storage; fat is the ultimate form of storage, in some seeds more than in others. When the seed undergoes germination, the starch is first converted into sugar; thereafter the fat is converted into sugar, attended with the evolution of carbon dioxid. The carbon which the growing plant obtains from the carbon dioxid of the air, the germinating seed obtains in part from its fat. The entire process, the reactions in each direction, can be well observed in the ripening and germination of the nuts.

Does such a reaction occur in the animal body? We do not know. As will be pointed out in the discussion of the fat metabolism, we do not know positively the reactions through which fat is burned in the body. Obviously, it might be burned directly as commonly believed; or it might be first converted into sugar. Could this last eventuality be demonstrated, it would simplify enormously our conception of the fuel combustion of the body. Up to the present, there is no way of showing that sugar is formed in the body from fat. There is confessedly also no way of proving that it is not so formed. When the diabetic dog eliminates more sugar than can be accounted for by the stored carbohydrates in the body, the assumption that this sugar is all formed from protein and none from fat is simply an inference along the line of least resistance. There is no possibility for the conversion of fat into sugar in the processes of digestion; the fat is resorbed as such. 
There is also no likelihood of the formation of sugar from protein in the processes of digestion. It is in both instances a derivation in metabolism only. If sugar were formed from fat, in a relation of simple reversion of the reaction whereby fat is formed from sugar, 1 gram of fat would yield nearly 2 grams of sugar.

In a body whose metabolic processes are being normally maintained by the foodstuffs, the sugar of the blood is derived in large part from the carbohydrates of the diet, not over 50 or at the most 100 grams being derivable from the protein that is daily undergoing catabolism. When for any reason the metabolism of the body is not being fully supported by the foodstuffs, we are justified in the inference that more sugar is derived from protein in proportion to the greater catabolism of tissue protein; whether when the fats of the body are burned sugar is formed, can be neither affirmed nor denied.

The Disaccharids in Metabolism.-Cane Sugar or Saccharose.-Speculation has long been rife concerning the possibility of the entrance of unchanged foodstuffs into the circulation through breaks in the surface of continuity of the mucosa of the alimentary tract, as in the case of ulcerations. For the proteins, the matter may have an important bearing, as will be elsewhere elucidated, since the entrance of native foreign protein might lead to the establishment of anaphylaxis. So far as known, starch under no circumstances enters the circulation. There is likewise no evidence that the disaccharids ever enter the circulation through ulcerations. They do, however, enter the circulation when an excessive overload is placed upon the resorption membrane. It is possible by the ingestion of large amounts of disaccharids, to intensify the concentration of the sugars in the contents of the intestine, leading to such high velocity in resorption as to outrun, so to speak, the cleavage reactions that normally occur within the intestinal wall. In a word, it is possible to absorb a disaccharid more rapidly than it can be split into its component primary sugars. This does not seem true of all individuals, but it is certainly true of many normal subjects. Such a thing never occurs in connection with the digestion of starch. No matter how massive the meal of starch, the rate of conversion into maltose is so slow that the maltose in resorption is always converted into glucose.

Cane sugar or saccharose is normally not a constituent of metabolism. By this we understand that when ingested as such, in the form of the sugar of the table or in the fruits in which it is contained, it is always completely split in the processes of digestion, the fructose fraction is converted into glucose, and thus the entire product in the circulation is glucose. When cane sugar is introduced into the circulation, even in small amounts, it is quantitatively eliminated by the kidneys. In other words, the body cannot hydrolyze it and cannot burn it directly; it is, therefore, eliminated like a foreign body. Under abnormal conditions of diet, and possibly in abnormal conditions of the alimentary tract, the ingestion of large or even moderate amounts 
of saccharose will be followed by melituria. There is probably a point with every healthy body beyond which the ingestion of cane sugar on the empty stomach will be followed by melituria. Sometimes this limit of assimilation may be as low as 100 grams; usually 200 or 300 grams can be ingested without more than a transient trace of melituria. The sugar that appears in the urine may be glucose, fructose, or cane sugar itself, or these may be combined. The more normal type, if such an expression may be used, would be saccharosuria, though combined melituria is seen in entirely healthy persons. The presence of cane sugar in the urine is easily established, especially if it be there alone, as the special yeasts will not ferment it. If glucose or fructose be present also, the polariscope will give definite information. The rotation of the plane of polarized light should be noted in direction and degree, and the urine then acidulated with hydrochloric acid to about tenth normal strength and boiled under a reflux condensor for a half hour, following which the rotation is again observed after the exclusion of birotation by the addition of a little alkali. A shifting of the rotation to the left will indicate the presence of saccharose. Fehling's solution is also reduced by the inverted sugar.

The commonly accepted explanation of alimentary saccharosuria is that when ingested in large amount, the velocity of absorption exceeds the velocity of cleavage, and some unsplit sugar reaches the portal circulation; and as the liver is not believed to have the power of splitting cane sugar, saccharosuria results. It has, however, been shown by experiment that if the thoracic duct be ligated in a dog, it is very difficult to produce alimentary melituria. From this experiment one might infer that under the conditions of excessive ingestion of sugar, a portion is resorbed through the lacteal system, instead of through the portal system as normally. When one considers the minimal amounts of cane sugar that are to be recovered in the urine after the ingestion of half a pound, this explanation does not appear improbable. We know, however, that cane sugar injected into the portal vein is not hydrolyzed in the liver, but is eliminated by the urine.

Maltose.-Maltose does not exist in plants except in traces. Its occurrence in the diet is referable to the maltose of the factory, this sugar being largely used in confections. Although it is a stage in the conversion of starch into sugar, this stage is transient; and so far as known, such a thing as the accumulation of maltose in the act of digestion or in metabolism does not oceur. Normally tissues contain traces of maltose, most easily demonstrated in the muscles. Injected into the circulation, it is split into glucose, and the procedure is tantamount to the injection of so much glucose. Apparently all the glands of the body, as well as the muscles, are able to effect this hydrolysis of maltose to glucose. An alimentary maltosuria occurs under the conditions of excessive ingestion described for cane sugar, and in about the same quantitative relations. The sugar eliminated is always both maltose 
and glucose if the ingestion be excessive; maltose may appear alone if the ingestion be smaller.

There is a rare form of maltosuria that is not alimentary. In a few cases of diabetes maltose has been found in the urine. It has been also noted in the urine following ablation of the pancreas in the dog, and has been reported in acute pancreatic disease in man. Its presence in diabetes might be simply explained as the result of the law of mass action. The failure to remove glucose from the system, in a chemical hydrolysis of maltose, will result in the cessation of the reaction before all the maltose is split; and thus in the hyperglucemia of diabetes, we might expect maltose to be present in more than the normal traces, and to be eliminated in the urine.

Lactose.-The relations of the metabolism to lactose are quite identical with those of cane sugar. When injected into the circulation it is quantitatively eliminated in the urine. When injected into the portal vein, the same result is attained. In other words, the tissues cannot burn it and cannot hydrolyze it. It is frequently present in the urine; and this lactosuria is of two types-alimentary and lactational.

Alimentary lactosuria, following the ingestion of even excessive amounts of milk, may be said to occur rarely in the normal individual. In invalids with reduced powers of digestion, in whom forced feeding with milk is attempted, traces of lactose may appear in the urine. It is common, however, in different forms of gastro-enteritis in children. While it does not occur easily in the normal adult on ingestion of milk, it is easily provoked by the administration of milk sugar in concentrated solution on the empty stomach; as a matter of fact lactosuria is more easily provoked than is saccharosuria. In this case the sugar in the urine is nearly always lactose alone; galactose does not occur, and glucose is rare. The condition is due to the velocity of resorption being greater than the velocity of cleavage, or to the fact that a fraction that had escaped cleavage escapes the liver also by being absorbed into the lacteal system. As stated, however, in direct experiment, the liver is not found capable of splitting milk sugar.

Lactational lactosuria is very common. It may appear during the last days of gestation or during the early days of the puerperium. It is made more prominent by anything that hinders the normal formation and discharge of the milk, and usually disappears when lactation is fully established. It may reappear, or appear, when the child is weaned, or if for any reason lactation be suddenly suspended. It may be provoked sometimes in the pregnant woman by the ingestion of a large amount of glucose or cane sugar, which serves to act as a stimulant to the function of the mammary gland. If the mammary glands of an animal be removed, lactosuria cannot occur.

The sugar is usually not present in large amounts, but this statement has many exceptions. The explanation of its occurrence is very simple and obvious. The intra-acinal pressure becomes excessive, and the milk sugar transudes into the paraductal spaces, to be taken 
up by the circulation and eliminated because the body cannot utilize the sugar. The occurrence of lactosuria in the pregnant or nursing woman is made quite certain if the urine reduces Fehling's solution but will not ferment with Saccharomyces cerevisiae.

\section{THE STATE OF GLUCOSE IN THE CIRCULATING FLUIDS AND TISSUES OF THE BODY}

'The glucose content of the body is largely in the circulating fluids, en route to the organs of combustion. According to our best information, this glucose is in the free chemical state, uncombined with any other substance. It is true that combinations of glucose have been described; it cannot be denied that such exist and they may have a great importance. These descriptions refer to glucose-protein and glucose-lipoid complexes. Upon the basis of this, it has been further assumed that the sugar in colloidal state was not subject to combustion, which involved only the free sugar. The limit of colloidal sugar, according to this view, would be the limit of the colloids with which it is combined; and for diabetes in which a hyperglucemia with glucosuria exists, it has been assumed that the amount of sugar present exceeds the colloids, or at least the combining power of the blood. Continuing, the further assumption was made that the kidney retains the colloidal sugar, and permits the free sugar to diffuse into the urine. For the glucose-protein complex, the data are meagre in amount and of very doubtful quality. For the two lipoid combinations, the so-called jecorin and glucose phosphatid, something more can be said, though even here from the purely chemical point of view the methods have been untrustworthy. Lipoids are very prone to carry into an ethereal extract small amounts of crystalloids in the watery media from which they are isolated. These adsorption inclusions are often very tenacious, and simulate chemical combinations to a surprising degree. For the jecorin and glucose phosphatid we have no such data on the chemical state of composition that we possess, for example, for the galactose lipoid or for the pentose combination in nuclein. While granting the probable occurrence of such combinations and without restricting in any way the scope of their possible importance, it is necessary to leave the question in this unsettled state. The amounts of glucose involved are in any event small, a mere fraction of the total glucose of the blood.

The glucose in the cells is in all probability also largely in the free chemical state. Since all cells contain glycogen, and since the methods for the demonstration of glucose in tissues are very likely to convert glyeogen into sugar, the data bearing on the amounts of glucose contained within cells are very untrustworthy. Certainly, fixed cells and the blood cells contain far more glycogen than glucose. There is no evidence that all the cells that contain glyeogen utilize it; it would be there as the result of mere diffusion of sugar from the circulation 
fluids. The anabolic process whereby glucose is converted into pentose in the regeneration and multiplication of the nuclei, is quite certainly accomplished in situ within the cells; the amount involved is, however, extremely minute.

\section{THE QUANTITY OF GLUCOSE IN THE CIRCULATION FLUIDS AND TISSUES OF THE BODY}

Accurate quantitative analyses of glucose in a fluid like the blood are not easy or especially accurate. The glucose content of the blood of different animals runs from 0.05 to 0.1 per cent. In man the content seems to lie close to 0.08 per cent. The figure is apparently quite constant for each species. To what fluctuations it may be subject under different conditions of nutrition, diet, metabolism, climate, and in different diseases has not been properly studied. The blood contains more sugar during infancy than during adult life. The glucose content of cells is unquestionably much lower than that of the blood plasma.

When one contemplates the fluctuations in the combustion of sugar under the different conditions of adaptation in the heat metabolism and under varying stresses of work, the constancy of the sugar concentration of the blood is all the more striking. It is regulated by the coadaptation of three factors: combustion, formation from glycogen, and elimination by the kidneys. The last-named variable is seldom in operation under ordinary conditions. The relations of the kidneys to the glucose concentration of the blood may be compared to the relation of a dam to the waters of a mill pond. When the level of the impounded water is below the top of the dam, none overflows; when the water rises above that level, overflow occurs until the level is reduced to that of the dam. In the kidney, so long as the glucose concentration does not, in man, exceed about 0.1 of 1 per cent., the kidney is impermeable to it. But when the glucose concentration rises above this level, sugar is eliminated by the kidney until the content in the blood is lowered to the normal figure. For this extremely interesting mechanism we have as yet no explanation in the theory of secretion. How a fixed level exists in the kidney, and to what reductions it may be subject, is not well known. For one substance in particular, phloridzin, it is established that it causes glucosuria by lowering the level of retention; or in the case of profound intoxication, by the complete opening of the floodgates, so that practically all the sugar of the blood is eliminated, the renal power of retention of glucose being apparently lost. This matter will be discussed in detail later. Whether in any of the incidental, so-called non-diabetic forms of glucosuria, that will be later described, a similar mechanism is operative; or whether all of these naturally occurring forms of glucosuria are, like the diabetic glucosuria, due to hyperglucemia, is not known. This is a point that from the standpoint of theory, as well as for practical reasons, is worthy 
of investigation. In phosphorus poisoning the sugar content of the blood sinks to almost nothing, due to the abolition of glycolysis.

The comprehension of the constant concentration of glucose in the blood, below the level of renal elimination, is made clear in outline, if not in detail, by terming it an equilibrium in a reaction system. The combustion of sugar is of two types, that may be termed induced and forced. Under induced combustion we understand the combustion in the resting state, the basal production of heat required to maintain the body temperature under the existing conditions of heat radiation and dissipation. By forced combustion we understand the production of heat in excess of this, due to voluntary muscular exertion; and followed, of course, since the body temperature remain normal, by an adaptation for proportionately increased heat radiation and dissipation. Whether the combustion be induced or forced in type, the seat of the combustion is certainly in large part in the muscular system. The concentration of glucose in the blood is. maintained by the conversion of glycogen into sugar. When the momentary rate of combustion is increased, proportionately more glycogen is converted into sugar and the concentration thus maintained; when the rate of combustion is reduced, less glycogen is converted into sugar and hyperglucemia thus avoided. Just as in a chemical system operating under the law of mass action, we can accelerate the velocity of the reaction from substrate to product by the removal of the product; so when the product sugar is removed by combustion, we observe the conversion of glycogen to sugar accelerated. If the products are not removed, or in the case of the glucose not used and accumulate, then the reaction of substrate to product becomes retarded. The actual point of equilibrium, in a system in catalytic reaction, depends upon the relations of substrate concentration, product concentration, and ferment concentration. In other words, the velocity of formation of glycogen into sugar in the cells is a function of the amount of glycogen (which in the reaction sense may be termed constant), of the ferment concentration (which in the normal case is also probably constant), and of the concentration of glucose within the cells. Now the concentration of glucose within the cell will depend upon the concentration of glucose in the circulation fluids. And if the sugar there is rapidly removed by combustion, then the diffusion of glucose from the cell into the circulation will be rapid, and the consequent lowering of the glucose concentration within the cell would lead to acceleration of the reaction of conversion of glycogen into glucose. One additional fäctor in further increasing the reaction would lie in an increase in the glycolytic ferment in the cells in which the conversion of glycogen into sugar occurs. From the standpoint of the theory of the law of mass action applied to the catalytically accelerated reaction, the constant concentration of glucose in the circulating fluids of the body presents no difficulties. That the process has not been experimentally reproduced, is a question of chemical and experimental difficulties. The quantitative relations 
of the conversion of glycogen to sugar are easily stated. A body of 150 pounds, on a fixed protein ration of 1 gram of protein per kilo, and being supported by the combustion of sugar alone and not of fat, would burn in the course of a day of leisurely activity some 500 grams of sugar. This corresponds to the burning of about 6 milligrams per second. While the molecular weight of glycogen is not known, analytical results indicate that about 9 parts of glycogen will yield 10 parts of sugar. While on the one hand, it is clear that the formation of 6 milligrams of sugar from glycogen per second in a reaction system as large as the human body is chemically a trifling accomplishment, it is equally clear that the maintenance of the station of equilibrium is an operation of great delicacy and striking mutability. In the opinion of the writer, the retention of glucose in the plasma of the blood by the kidneys is an expression of semipermeability resting upon a physico-chemical basis, and is not due to an assumed colloidal combination of glucose. The idea that diffusible sugar would pass through the kidney and only colloidally combined sugar could be retained is too obvious, and in addition is devoid of a proper foundation. As stated, it is quite certain that the larger fraction of glucose in the plasma of the blood is not combined but free.

Before discussing the chemical reaction and the physiological relations of the combustion of glucose, it will be advantageous to consider first the formation of glycogen and fat from glucose, the relations of glycogen in metabolism, its localization in the tissues and to discuss the relations of the combustion of sugar to the glycogen of the liver and to the glycogen of the extrahepatic tissues. Just as we have termed glycogen the proximate storage state of glucose, and fat the ultimate state of storage; so in a limited sense, we may term the hepatic glycogen the proximate depot of glycogen, and the muscular and other extrahepatic glycogen the ultimate depot of glycogen. And just as, in a certain sense, fat is not burned in the body until the glycogen is reduced to a certain level, so the ultimate glycogen is not converted into sugar until the hepatic glycogen is reduced to a certain minimum.

\section{THE FORMATION OF GLYCOGEN AND THE RÔLE OF GLYCOGEN IN THE CARBOHYDRATE METABOLISM}

Glycogen is animal starch, a polysaccharid of the elementary composition $\mathrm{C}_{6} \mathrm{H}_{10} \mathrm{O}_{5}$, of large but unknown molecular weight. In water it forms a colloidal suspension, not a true solution; the glycogen will not diffuse through a membrane, offers no osmotic pressure of measurable degree, does not conduct the electrical current, but when placed in the field of a galvanic current migrates to the anode. Suspensions of glycogen rotate the plane of polarized light to the right, but do not reduce metals in alkaline solution. So far as known, the glycogens of the different parts of the body are identical; it is not certain that the glycogens of different species are identical. Glycogen exists in the 
body only within cells and tissues, never suspended in the circulating fluids. The blood does contain glycogen, but only within the leukocytes, not in the plasma. Within cells, the gly cogen exists in the protoplasm in amorphous or granular state as free glycogen; and secondly, in combination with other protoplasmic constituents. These two states we may denominate free (storage) and combined glycogen. The free glycogen may be recovered, after the coagulation of the proteins of a tissue, by prolonged extraction with hot water. The combined glycogen can be recovered only after digestion of the protein by a ferment like trypsin. The free glycogen can be removed from an organ or animal by starvation, work, and refrigeration. The combined glycogen cannot be so removed. The amount of free glycogen present under ordinary conditions greatly exceeds the amount of combined glycogen. On the basis of calculations of analyses of the tissues of the dog, the amount of combined glycogen in an average human body may be estimated at not to exceed 100 grams. When we speak of a glycogenfree liver or other organ, this refers to the free glycogen, not to the combined glycogen. This combined glycogen is a constituent of protoplasm, combined with the protein-lipoid complex of which protoplasm is composed. The amounts of storage glycogen that may be found in the body are varied with different conditions of nutrition. The liver may contain from a few grams up to 150 or even 200 grams. Muscle well stocked with glycogen may contain as high as 4 per cent., the other glands of the body quite as much. Small animals have been analyzed to contain as high as over 3 per cent. for the entire body. For a man of 70 kilos, this would correspond to over 2 kilos of glycogen, or $2 \frac{1}{2}$ kilos of glucose, adequate fuel for the body for five days.

The formation of glycogen from glucose, as a chemical reaction, is the reversion of the hydrolysis whereby glucose is formed from glycogen (glucose + water $=$ glycogen), it is the reversion to the state of polysaccharid from the primary sugar. An intermediary stage must be maltose. So far as we can observe, the formation of starch in the plant and of glycogen in the animal are quite parallel processes in chemical procedure and in metabolic meaning. Upon the basis of theory, we refer this reaction to the activity of a glycolytic enzyme.

The glycogen of the liver is derived directly, and we believe under normal conditions solely, from the diet. This statement is not an actual contradiction of the fact that the fetal liver contains glycogen. The direction of the stream of migration of glycogen seems always to be centrifugal from the liver. The liver can be freed of its glycogen (except, of course, that combined in the protoplasm, which is always excepted from this discussion) by starvation, especially if aided by exercise. Under such conditions, the body contains still quite large amounts of glycogen. But no tendency is to be observed for this to return to the liver. Thus the liver which, as we shall later describe, is a veritable dumping ground for fat, receives no return glycogen from the tissues that it has itself enriched. 
The liver withdraws the excess of sugar from the hyperglucemic blood of the portal vein during the course of digestion of carbohydrate, converts it into glycogen, and stores it in the protoplasm of the hepatic cells. Normally, the blood of the hepatic vein contains no more sugar than 0.1 per cent. So far as our analysis goes, the blood of the hepatic vein contains no more blood than does the venous blood that it meets in the inferior cava. Under many circumstances, however, there must be a total quantitative difference in time, since under conditions of liberal ingestion of carbohydrates, the blood of the hepatic vein must contain an excess of sugar, being carried to the muscles for conversion into glycogen. In any moment of analysis, however, this is too small to be detected.

The Limit of Assimilation of Carbohydrates.-By the term limit of assimilation of carbohydrates we mean the amount of carbohydrate that can be ingested and converted into glycogen in the liver without the content of glucose in the hepatic vein (that is, in the circulation), rising materially above the normal, in short without hyperglucemia. In other words, the limit of assimilation of carbohydrate corresponds to the limit in time of the glycogen-forming function of the liver. If more glucose enters the liver through the portal vein, in the unit of time, than can be converted into glycogen and stored in the liver, then hyperglucemia will result, and, of course, glucosuria. There is no limit for starch normally. The liver can always handle in the unit of time all the glucose that can be formed in the process of digestion from any ingestion of starch. For the disaccharids and for the three monosaccharids (glucose, fructose, and galactose), the limit of assimilation lies, for administration in simple solution on the empty stomach, from 100 to 250 grams, varying with different individuals. In the difference between individuals, 100 to 250 grams, are included two variablesvariation in powers of digestion, and variation in hepatic function. The difference between starch and sugar is merely a question of velocity of absorption. Two hundred grams of sugar placed in the duodenum, would probably be resorbed within an hour. Two hundred grams of starch would probably not be digested and resorbed within five or six hours. The velocity of digestion is always slower than the normal velocity of glycogen formation; were this not true, alimentary glucosuria would be a daily occurrence.

Formation of Glycogen in the Liver.-From what constituents of the products of digestion is glycogen formed in the liver? Only from glucose, and therefore, only such substances as are capable of transformation into glucose can be regarded as builders of glycogen. Perfusion experiments on the liver, best done on certain toads, have indicated that glycogen can be formed from glucose, fructose, galactose, maltose, glycerol, and formaldehyd; from cane sugar, milk sugar, and pentoses no glycogen can be formed, also none from protein (?). The meaning of these experimental results is that the liver is able to split maltose into glucose; convert fructose and galactose into glucose; 
transform glycerol into glucose (through the stage of glycerose); and like plants can reduce formaldehyd to glucose. From the glucose thus derived, glycogen is formed. Glycogen is not formed from cane sugar, milk sugar, and pentose because the liver cannot split the disaccharids and cannot convert the pentose into hexose. The experimental results in the case of amino-acids are contradictory. For though the liver does not show an increase of glycogen, it can be shown to display an increase of sugar when certain amino-acids are perfused through it. If an animal made glycogen-free by starvation be fed on a pure protein (like casein) in excess of the immediate needs of the body, the liver will store glycogen, which can have been formed only from the amino-acids of the digested casein, via glucose.

Storage Capacity of Liver for Glycogen.- - To what variations this assimilative function of the liver is subject, is not well known. Like all functions, it may be assumed to be able to stand an overload; like all functions it must be susceptible of deterioration. Experimentally it can be reduced or abolished by the medullary puncture of Bernard. Apparently there is in the medulla oblongata a glycolytic centre for the liver, the two branches of the arc being the pneumogastric and the splanchnic nerves. The splanchnic nerves must be the path of innervation, since the puncture does not produce glucosuria if they are severed; but it does succeed if the vagi are cut, and, best of all, if the cêntral cut ends of the vagi are stimulated. The Bernard puncture succeeds in small animals, birds, and reptiles; and the facts hold inferentially for man. The operation is followed by two results; the liver is made free of glycogen by reason of the conversion of its stored glycogen into sugar; and hyperglucemia results from this excessive transfer of glucose into the circulation and glucosuria follows. The glucosuria endures only until the excess of sugar is removed from the blood. If the liver were free of glycogen before the puncture was done, no hyperglucemia or glucosuria result. If now sugar be administered, it will pass without hindrance through the liver, into the circulation and out of the kidneys. The same result will be obtained if sugar be injected into a branch of the mesenteric vein. In a word, the only point of operation is the stored glycogen of the liver; if there be no stored glycogen, there is no result. 'The storage capacity for, or content of, glycogen in the muscles or the other tissues is in nowise disturbed. Even if the liver of the animal receiving the puncture be filled with glycogen, no hyperglucemia or glucosuria will occur if the vessels of the liver be ligated. The operation obliterates the storage capacity of the liver for glycogen; it wipes out any existing deposition and prevents any new deposition.

The explanation of this peculiar phenomenon, which is of the greatest interest to the physiologist but lacks entirely the importance for the understanding of diabetes that was previously ascribed to it, is not easy to give. If the glycogen stored in the liver were assumed to lie there in some state of combination, the simple freeing of which would 
set it loose in the circulation, an explanation might be sought in that direction. But that is not to be considered. 'The explanation most probably lies in a disturbance of the relations of equilibrium and ferment action. The conversion of the glycogen into glucose is an act of fermentation. A shifting of the station of equilibrium alone it cannot be, since with the hyperglucemia, the direction of the reaction ought to be shifted toward the glycogen, not away from it. The assumption of the formation of an excess of the glycolytic ferment might, without serious contradiction, explain the result. From the standpoint of the modern hypothesis of hormones, an explanation might also be sought, though it is difficult to adjust this hypothesis to the purely nervous impulse. This could, however, account for the formation of a large excess of glycolytic ferment, through whose activity the glycogen would be hydrolyzed to glucose. The really most difficult feature of the situation lies not in the fact that the glycogen is converted into sugar to the full extent of the contained glycogen; but when the liver contains little glycogen and glucose is presented, the liver is prevented from forming glycogen under conditions that normally would be attended by a rapid formation of glycogen. The reaction of hydration is accelerated and the reaction of anhydration is prevented. Of course, if one were to assume one ferment for the transfer of glucose to glycogen and another ferment for the hydrolysis of glycogen to glucose, it were easy to say that the Bernard puncture stimulates the formation of the hydrolyzing ferment and inhibits the formation of the synthetic ferment. But in such an explanation nothing would be gained.

In some cases of extreme jaundice the storage capacity of the liver for glycogen is lessened or destroyed. In many instances of advanced organic disease of the liver an alimentary fructosuria is present, due to the inability of the liver to convert the fructose into glucose. In the same class of cases, however, the liver does not exhibit any loss of the power of converting glucose into glycogen. To the chemical point of view this is simple. The two are totally different reactions, and it is natural that the functions, though accomplished by the same organ, should be separate. It simply means that the function of the conversion of fructose into glucose and the function of the conversion of glucose into glycogen are not interdependent. Another illustration of this fact is contained in the experimental observation that following extirpation of the pancreas, the liver cannot form glycogen from glucose but can convert fructose into glucose.

In carbon monoxid intoxication we have another illustration of the disturbance of the glycogen-storing function of the liver. Just as is the case of the Bernard puncture, the glycogen of the liver is hydrolyzed to sugar and the circulation flooded, with resulting glucosuria.

There is under all conditions a certain antagonism between the storage of glycogen and fat in the liver, as though there were not room for both. Whether this may be the explanation of the frequent occurrence of glucosuria in fatty degenerations of the liver is only conjectural. 
The maximum storage capacity of the liver may be set at from 150 to 200 grams. Now since the total sugar needs (as fuel) of the body not engaged in hard work are not over 400 to 500 grams per day, it is clear that the liver could handle the entire input and from its store supply the fuel glucose of the whole body. Since the absorption of food is, however, not usually evenly distributed through the twentyfour hours of the day, it is probable that such is not commonly the case. Nevertheless, individuals who consume only moderate amounts of sugar per day, say 300 grams, and with it considerable fat, might easily hold in the liver all the sugar ingested, pending the needs of the body for fuel. In other words, the provision for proximate storage would be sufficient for the entire metabolism if the life and diet of the individual were arranged to that end. As a rule, this is not so, and the liver is unable to store all the carbohydrate, it must be stored in the depots of ultimate storage of carbohydrate, in the muscular system. How is this transfer accomplished?

Transference of Glycogen.-The blood contains no glycogen except in the leukocytes. It is beyond possibility to suppose that the leukocytes transport the glycogen of the liver to the other tissues of the body. The glycogen must be carried in soluble form. There is but one way to accomplish this-by the reconversion of the glycogen into glucose. It is apparent from this that we may assume that there are four levels in the sugar concentration of the circulating fluids. The lowest level is the level of combustion; the second level is that of the formation of glycogen by the muscular system; the third level is the level of the formation of fat from sugar; the highest level is the level of renal retention. To present this hypothesis in concrete form, let us assume arbitrary figures for these four levels of glucose in the blood, namely, 0.07, 0.08, 0.09, and 0.1 per cent. When the body is upon a minimal diet, with just enough carbohydrate absorbed evenly through the day to maintain the body heat, the glucose concentration in the blood would stand at the lowest level, 0.07 per cent. If the carbohydrate be absorbed unevenly, so as to overtax the liver at times, or if an excess over fuel needs be ingested, the concentration in the blood would rise to the second level, 0.08 per cent. Thereupon the glycogen-forming function of the muscles would become operative. These would store up in glycogen the glucose that passed out of the liver in excess of the concentration on the lowest level, to yield it for fuel as needed. If the ingestion of carbohydrate in excess of the fuel needs of the body were long continued and large enough, the blood concentration of the glucose being kept up to 0.08 per cent., glycogen would be formed and stored in the muscles and other tissues until their full storage capacity was reached. Then the blood concentration would per force rise higher, to the next level 0.09 per cent. At this point the function for the formation of fat from sugar would become operative, and the concentration of the sugar in the blood would be kept down below the renal level of 0.1 per cent. by the formation of fat from the excessive sugar. This 
is a function of wide adaptation, and large ingestions of carbohydrates may be tolerated without the renal level being attained. It is possible to inject considerable amounts of glucose and demonstrate by repeated analyses of the blood that the sugar content of the blood is high, without the production of glucosuria. This fact is not an argument for the binding of sugar in the blood; it merely indicates that the conception of threshold value must not be interpreted in too rigid a manner. An enormous single ingestion of sugar will send the blood concentration up beyond the renal level. It is naturally not to be assumed that the hypothesis predicates the application of this scheme rigidly, it is, of course, adaptive and fluctuating and there is overlapping; but the dynamic point of view is illustrated. The thoughtful reader will ask at once if the fact that the diabetic, who has hyperglucemia, does not build glycogen and fat, is not a contradiction of this scheme. It is not, for the simple fact that among the defects of the diabetic metabolism are the more or less marked loss of the functions of formation of glycogen and fat from glucose. One occasionally meets with a diabetic who exhibits a pathological obesity, an illustration of the result when the power of forming fat is retained in the diabetic body with hyperglucemia. The hypothesis as outlined must in the end rest upon the broad chemical generalization that the phenomena under discussion are to be interpreted as expressions of mass concentrations and reaction velocities.

Glycogen Content in Muscle.- The glycogen content of muscle varies, of course, with the state of nutrition. While under conditions of forced feeding it may be as high as 4 per cent., it is usually not over half that figure. In other words, the muscular system can contain enough glycogen to support the heat of the body for five days; but as a rule it contains not more than enough to support the heat of the body for two days. This fact of analysis is confirmed by observations of the respiratory quotient, which for a few days in starvation displays the value noted in the combustion of sugar, falling thereafter to that related to the combustion of fat and protein. The situation is, of course, not rigid; there is some overlapping, by which we mean that some fat is burned before the glycogen of the body is depleted. Glycogen is stored in both the voluntary and involuntary muscles, most in the former, whose much greater bulk in any event would make them outweigh the involuntary muscles in importance. When the liver content of glycogen is high, glucose passes into the blood of the hepatic vein and thence into the general circulation in excess of the heat requirements of the body, there occurs a slight rise in the glucose concentration of the blood, a rise to the second plane of the scheme enunciated. Consequent, thereupon, a slight rise in the glucose concentration occurs within the muscle cell, and this we must regard as the moment determining the formation of glycogen there. The act of formation of glycogen from glucose we regard here, as in the liver, as the reversion of the reaction of hydrolysis whereby glucose is formed 
from glycogen. The glycogen formed within the muscle cell is stored in amorphous or granular form. There must, of course, be variables in this function. Lately, we have learned that certain dystrophic diseases of the voluntary muscles are associated with reductions in the output of creatinin, which we regard as the specific end product of the muscular metabolism. Whether such diseases are associated also with reduction in the glycogenous function of the muscles has not been determined, though it is clear that the powers of combustion are in nowise reduced. Once deposited in the muscle cell, so far as we know, the glycogen is not disturbed unless a fall in the concentration of glucose within the cell provokes its hydrolysis. The one exception to this statement, noted in diabetes, will be discussed under that heading. It will suffice here to say that while normally hyperglucemia is followed by the maximum storage of glycogen in the muscle cell, this does not hold in diabetes. But of other known outside influences the glycogen of the muscle is free. Thus the puncture of Bernard has no effect upon it, and no chemical substances exert any influence upon it, so far as we know.

Conversion of Glycogen into Glucose.-The conversion of glycogen into glucose, both in the liver and in the muscles, is dependent upon the accelerating action of a ferment, termed glycogenase. This ferment is formed in the hepatic and muscular cells, and is not derived from the circulation. The blood plasma contains, it is true, a glycolytic ferment; but the glycolytic functions of the muscle and liver are not dependent upon their blood supply. In truth, the enzyme of the blood is probably derived by diffusion from the liver and muscles. It is a so-called soluble ferment, in that it can be extracted from the parent cells. When brought into contact with glycogen, under proper conditions of concentration, reaction, and temperature and free of bacterial infection, glucose is formed, through maltose as the intermediary state. Traces of dextrins also can often be detected, not only in experiments with the isolated ferment-extract acting upon glycogen, but also in experiments with aseptic postmortem digestion of the liver and muscles. If a well-nourished liver or muscle, free of bacterial contamination, be allowed to lie, it undergoes a postmortem digestion involving the protein, fats, and glycogen, due to the action of its own intracellular ferments. Following such a postmortem digestion, glucose, with traces of maltose and dextrins, can be isolated, and the glycogen content demonstrated to have fallen proportionately. Such experiments may be conveniently carried out, if glycolysis is the object of the test, with the liver and muscle of the mollusca.

Combustion of Glycogen.-The glycogen of the muscle is available for both the induced and the forced combustion, the autogenous combustion of basal heat production and the combustion of muscular work. It disappears in the resting, starving body; it disappears in the work of muscular contractions. The glycogen content of an isolated muscle can be shown to diminish proportionately to the intensity and 
duration of the contractions caused by stimulation of its nerve by an electrical-current. The glycogen content of the two hind legs of a frog may be contrasted, the one being made to contract by electrical stimulation while the other lies quiet; the glycogen content of the resting leg will be found to be greater than that of the exercised leg. When a muscle is paralyzed, by section of its motor nerve, glycogen accumulates in it. If the peripheral end of the cut nerve be stimulated, the muscular contractions will be associated with the disappearance of this glycogen. Finally, the entire body of an animal may be stocked with glycogen by forced feeding. If now the animal be worked hard for a number of hours, and then killed, it will be found that the muscles are very poor in glycogen, though control animals demonstrate that before the work was undertaken, the muscular system was rich in glycogen. That the work was accomplished on the combustion of glycogen (via glucose, of course) is proved by the fact that the respiratory quotient during such an experiment is found to be high, that is, to have the value known to be associated with the combustion of sugar.

It is possible also to make the experiment on the isolated muscle by contrasting the oxygen and carbon dioxid contents of the arterial and venous blood of the muscle. The resting muscle absorbs some oxygen and gives off some carbon dioxid. This is the simple heat production of the muscle acting as the fire box of the body. 'The oxygen that is absorbed is often in excess of the amount of carbon dioxid discharged by the resting muscle. If the muscle be stimulated to contractions, both the absorption of oxygen and the discharge of carbon dioxid will rise rapidly, the later often disproportionately. This disproportionate rise in the $\mathrm{CO}_{2}$ output during muscular work may in a certain sense be regarded as reciprocal to the disproportionate absorption of oxygen during rest, and indicates that in some way the muscle has a capacity to bind or store oxygen. That the muscle, on contraction, can give off carbon dioxid when operating without circulation in a system devoid of oxygen, is well known; and possibly this carbon dioxid represents in part the oxygen that was stored during the period of rest. On the other hand, as will be elsewhere noted, carbon dioxid can be derived from glucose without the addition of oxygen.

The muscle cells possess two distinct functions of combustion; combustion in the state of rest; and combustion for the support of contraction, an instance of the transformation of latent energy into work. One can make steam for heating purposes, or one can utilize it in an engine for the purposes of mechanical work. So does the body burn sugar, and both functions are localized in the muscular system, though the two processes are quite as distinct as a steam-heating plant and a steam engine. The combustion for the maintenance of the body heat occurs, however, not wholly in the muscles. There are active combustions in other tissues, notably in the liver, and particularly in connection with the protein catabolism. But the burning of carbohydrates, including the sugar derived from protein (and of fats possibly), occurs unquestionably 
in largest part in the muscular system. As fuel for this resting combustion for the maintenance of body heat, the muscles use the glucose of the cireulating blood, not their stored glycogen, so long as glucose is available in the blood. The loss in the glucose of the blood is replenished by the liver through the conversion of its stored glycogen into glucose. Only when the hepatic glycogen is reduced and the glucose concentration of the blood lowered to a minimum, does the muscle cell convert its own glycogen into glucose and burn it. The muscle does not return glucose to the circulation; it retains it and burns it. When the liver is ablated, the blood content of glucose sinks to almost nothing.

When, now, with the liver and muscles well stocked with glycogen we perform muscular work, whence is the sugar for the work derived, from the blood or from the contents of the muscle cells? It might be supposed that the modus operandi were different for the autogenous combustion of sugar and for that associated with muscular contractions. This is, however, not the case; the combustion associated with muscular contraction are also supported by abstraction of glucose from the circulating blood. The loss in the blood is made good by the conversion of glycogen into glucose in the liver. This proceeds, in the course of prolonged muscular exertion, until the hepatic stock of glycogen is reduced to traces, then the muscle cells convert their own glycogen into glucose and utilize it. In other words, the muscle cells respect their own stored glyeogen and draw upon the glycogen of the liver for fuel so long as this lasts. While the glycogen of the muscle is proximate to the muscle cells in the spatial sense, it is ultimate to them in the physiological sense. This seems to be especially true of the heart muscle; it is possible in direct experiment with the isolated heart to demonstrate the dependence of its contractions upon the glucose content of its medium.

That the body can burn sugar directly from the act of digestion, in other words, that the liver allows the glucose when needed to pass into the general circulation, can be shown in experiment. If an animal be worked and starved until free of glycogen in the practical sense, it will maintain its heat production upon the combustion of protein and fat, and this will be indicated in the respiratory quotient, which will be between 0.75 and 0.8 . Very soon after the ingestion of carbohydrate the respiratory quotient will rise to about 0.9 , indicating that glucose is at the time the chief fuel of the body. If the animal be not fed again, the respiratory quotient will fall, in from five to ten hours, depending upon the amount of carbohydrate ingested, to the starvation quotient.

If an animal, glycogen-free in the practical sense, be fed a protein free of carbohydrate (as casein or codfish) in excess of the needs of the body, glycogen will be stored in the muscles as well as in the liver. It has been inferred that the liver forms the glucose from the amino-acids and that the muscles derive it from the liver. But 
as the storage in the muscles appears early and is not preceded by a noteworthy storage in the liver, it may be fair to question whether the muscles as well as the liver may not have the power of forming glucose from the amino-acids. The respiratory quotient of such an animal, temporarily taken from the diet, will be low. If now the animal be exercised, the quotient will rise, showing that the muscular contractions were associated with the combustion of sugar that in the state of glycogen was lying in the resting muscles, while the heat of the resting animal was being maintained by the combustion of protein and fat.

When as the result of starvation, exercise, or refrigeration the glycogen of a body is exhausted, upon what is the production of heat and the maintenance of muscular work supported? By the combustion of protein or fat? In what chemical state does this combustion occur?

Muscular Work and Protein Diet.-It is certain that the most extensive work can be preformed on a pure protein diet, a diet of protein like casein that contains no preformed moiety of carbohydrate. But it seems now equally certain that it is only the carbonous fraction of the protein molecule which supports muscular work. It is immaterial here whether all this carbon be converted into sugar or whether some of the fatty acids be burned direct, this fraction alone supports the muscular work. The oxidation of the nitrogenous fraction does not occur in the muscles, but in the liver in large part, and is not available for muscular work. 'Muscular work on a protein diet is still, therefore, work performed on the basis of sugar combustion. Protein and glucose both yield about 4 Calories of heat in the body; but while all the 4 Calories in glucose are available for the support of muscular work, not all of the 4 Calories in protein are so available. This will be elucidated in a more appropriate place later. It is here only important to have it understood that when the muscle works on a protein fuel, it actually works on the combustion of sugar derived from the protein.

Muscular Work and Fat Diet.-Finally, it may be easily shown that muscular work can be supported solely by the combustion of fat, and that the fat thus burned yields its full heat value, 9.4 Calories per gram, to muscular work. In what chemical state is this combustion of fat accomplished for the maintenance of muscular work? It would be very simple if we could assume that fat were burned as sugar. If we could assume that fat, like glycogen, is simply an insoluble state of storage, and that like glycogen it has first to be returned to the state of glucose to be burned, it would make the mechanism of the body combustions very simple. But we have no experimental evidence that this is true. For the present at least we must believe that fat is burned in some way directly; and this form of combustion, whatever it may be, occurs in the muscle cell when it supports the work of contraction. 
What has been said of the full caloric value of protein not being available for muscular work, does not conflict with the law of the isodynamic heat value of the foodstuffs. For heat production, protein, sugar, and fat are isodynamic; for muscular work, however, while fat and sugar are isodynamic, the full caloric value of protein is not available. It will be well to recall here that of the combustion that occurs during muscular exercise in excess of the combustion during rest, only from 20 to at most 35 per cent. is converted into mechanical work, the balance is simply dissipated as body heat.

\section{THE FORMATION OF FAT FROM GLUCOSE}

Fat we have designated as the ultimate state of fuel storage. When the deposition of glycogen has reached a high plane, the intensity of the formation of glycogen would obviously suffer inhibition. If now the input of carbohydrate be maintained, it is clear that the plane of glucose concentration in the blood must rise. The plane of glucose concentration in the blood upon which the formation of fat is based, is the highest plane normally attained. It is just below that of the limit of renal retention, and if the ingestion of carbohydrates be maintained, fat will be continuously deposited and the body thus protected against hyperglucemia and glucosuria. There is, of course, a limit even to fat formation, though it is often very distant. Nevertheless, the occurrence of glucosuria so frequently seen in the very obese indicates that they are living at this limit; and a slight excess of carbohydrate input is followed by glucosuria because the storage capacity for glycogen being fully utilized and the storage capacity for fat being nearly completely utilized, hyperglucemia is easily produced. This is all the more true since the obese person usually has a very fatty liver and the presence of much fat, even in the healthy liver, is inimical to the glycogenetic function of that organ. It must not be understood that the formation of fat does not set in until the storage of glycogen is completed. The situation is not so literal as that, there is some overlapping, some fat is formed before the tissues are surcharged with glycogen. But, as a whole, the state of affairs is made clear in the statement that the ultimate depots of storage are not utilized until the proximate depots of storage are filled.

It is only by exclusion that we can arrive at any idea of the site of the formation of fat from glucose. It is not formed in the muscles. When the muscle cells have their fill of glycogen, they refuse glucose from the blood stream; they do not accept it and form fat from it. This excess of glucose then circulates. For a long time it was believed that in the liver occurred the formation of fat from glucose. This may be true to a limited and localized extent; but the fat of the general tissues is not formed in the liver. It is quite inconceivable 
that this fat should be formed in the liver and then transported all over the body. The experimental evidence is against it; whenever fat wanders it seems to go always toward the liver, and not from the liver. The situation is the converse for fat and sugar. The liver sends sugar out, and does not receive it from the circulation; fat the liver does not send out, but fat the liver does receive from the circulation. The site of the formation of fat is probably in the areolar and connective tissues-in the bone marrow, subcutaneous areolar tissue, in the connective tissues about the muscles, under the serous membranes, etc. The excess of the sugar is carried to these tissues, and here by cellular action converted into fat. Apparently it is merely a question of glucose concentration; this is the moment upon which the formation of fat from sugar must be based. The function, of course, is variable in different individuals.

To this rule of action of this function there are exceptions. Pancreatic diabetes, typical diabetes in man, is one. The diabetic organism has lost in part the power of forming fat from sugar. 'This is all the more striking when we recall that on the basis of the law of mass action, the hyperglucemia should lead to the excessive formation of fat. Now and then a case of diabetes is seen, in which, with loss of the power of burning sugar and the consequent presence of hyperglucemia, the function of the formation of fat from glucose is retained; such diabetics fatten inordinately. Later they may lose the function, and will then lose the fat. If the diabetic organism could retain the power of forming fat from sugar, it would, in this act, after a fashion, circumvent the defect in the combustion of sugar. On the other hand, one may meet with occasional individuals who have normally almost no power of forming fat, or what is equivalent in result, an unlimited power of burning glucose. The combustion of glucose is normally restricted to the heat needed to maintain body temperature under the conditions of the existing dissipation of heat. But in these occasional individuals, the more carbohydrate they eat the more they burn, the mechanism of heat dissipation being operated to the maximum. The ingested sugar must in these cases be burned, if resorbed, since there is no glucosuria and, therefore, no hyperglucemia. Typical instances of acute exophthalmic goitre seem to behave in the same way. The powers of digestion do not seem to be equal to the capacities of combustion, the more eaten, up to the limit of digestion, the more burned. It is not the place here to discuss whether this be cause or effect. It is simply necessary to understand that on the one hand the power of forming fat from glucose may be lost even with hyperglucemia; and on the other hand, it may be impossible with the maximum ingestion of carbohydrates to achieve the formation of fat, evidently because the slight hyperglucemia necessary for the formation of fat is not attained. 
Chemical Process.-Through what chemical process is the formation of fat from glucose accomplished? The question is related solely to the derivation of the higher fatty acids from glucose; the glycerol is another matter, since the body can and does produce it independently. It is known in plants that when fat is formed from sugar and sugar from fats the free fatty acids are transiently present. If an animal be fed a known constant diet, rich in carbohydrate, and the nitrogen in the stools and urine, and the carbon in the stools, urine and expired air be estimated, the exact amount of carbon left in the body may be calculated. And although it is known that this carbon is left in the body in the form of fat (the experiment deals with an animal stocked with glycogen) no trace is left of the mechanism of the transformation. To form one molecule of stearic acid, three molecules of glucose or six molecules of glycerose may be conceived to unite, after the fashion of a chain, connected by the aldehyd groups. Reduction, or successive oxidation and reduction, would permit the stearic acid to be attained. Any attempt to write equations by which we might be led from the molecule of glucose through lower fatty acid to higher fatty acid, must reckon with the certain fact that the reaction in all its physiological reactions is known to be an isothermic one, the heat value of the fat formed is the same as the heat value of the glucose. Just so in the combustion of fat; the full heat value is obtained, which indicates that if we are to assume that the fat is burned via sugar any equation that we may write must check with this observation. If all the carbon in sugar were stated in terms of fat, 1 gram of glucose would form 0.53 part of fat, with a heat value of 4.8 Calories, much higher than the 3.8 Calories observed for the sugar. It is clear that if the fat formed from a unit of glucose is to have the same heat value, some carbon must be extruded in the reaction. This was taken into account in one of the oldest formulations, as follows:

$$
\begin{array}{ccc}
\text { Glucose } & \text { Lactic acid } & \text { Stearic acid } \\
9 \mathrm{C}_{6} \mathrm{H}_{12} \mathrm{O}_{6}=18 \mathrm{C}_{3} \mathrm{H}_{6} \mathrm{O}_{3}=2 \mathrm{C}_{18} \mathrm{H}_{36} \mathrm{O}_{2} & +18 \mathrm{CO}_{2} .
\end{array}
$$

In this equation, however, the heat value of the fat formed from 1 gram of sugar would be only 3 Calories. If we were to write the reaction:

$$
\begin{array}{ccc}
\text { Glucose } & \text { Lactic acid } & \text { Stearic acid } \\
18 \mathrm{C}_{6} \mathrm{H}_{12} \mathrm{O}_{6}= & 36 \mathrm{C}_{3} \mathrm{H}_{6} \mathrm{O}_{3}= & 5 \mathrm{C}_{18} \mathrm{H}_{36} \mathrm{O}_{2} \times 18 \mathrm{CO}_{2}
\end{array}
$$

the heat value of the fat obtained from 1 gram of glucose would be the same as that of the sugar. But what is the use of such equations? They teach us absolutely nothing of the actual state of affairs. Comprehensible qualitative equations will be advanced under the heading of the Fat Metabolism. 


\section{THE COMBUSTION OF GLUCOSE}

When 1 gram of starch is burned in the calorimeter it yields 4.2 Calories of heat, the disaccharids yield 3.95 and glucose 3.75 Calories per gram. While the body burns the carbohydrates completely, and the values for the calculation of heat production should be the same in theory, in practice it is necessary to have a rounded figure, all the more because it can be shown that our analyses in the body obtain nearer the theoretical values for the sugars than for starch, because the sugars are more completely absorbed than starch and because the usual diet contains all the forms of carbohydrate. The figure 4 Calories per gram has been found to form a satisfactory basis of calculation. Glucose is burned completely to water and carbon dioxid, and all carbohydrates are converted into glucose before being burned. The equation:

$$
\mathrm{C}_{6} \mathrm{H}_{12} \mathrm{O}_{6}+6 \mathrm{O}_{2}=6 \mathrm{H}_{2} \mathrm{O}+6 \mathrm{CO}_{2}
$$

illustrates that the molecule of glucose contains enough oxygen to form the water; needed are the six molecules of oxygen to combine with the carbon to form the six molecules of carbon dioxid. Placed in terms of weights, 1 gram of glucose combines with 1.066 grams of oxygen to form 0.6 gram of water and 1.066 grams of carbon dioxid, with the liberation of 3.75 Calories of heat. Assuming that the carbon dioxid is eliminated as rapidly as formed, 0.29 gram of carbon dioxid eliminated corresponds to 1 Calorie of heat. This reaction occurs in large part in the muscular system. It certainly does not occur to a demonstrable extent in the circulating fluids. To some extent it does occur in the large glands, especially the liver. But for practical purposes, the muscles are the fire box of the body. In the reaction as written stand only the original substance and the final end products. What are the intermediary stages in the reaction; through what chemical processes is the molecule of glucose burned? While it is important to realize that this question cannot be answered now with definiteness, it is equally important that we make attempt to answer it, and to define our knowledge relating to it. The glucose may be burned directly or indirectly, though in either event the reaction must be one in many stages.

Direct Oxidation of Glucose.-The direct combustion of glucose, so far as our present chemical conceptions go, could only occur through stages that would involve d-gluconic acid, d-glucuronic acid, d-saccharic acid or oxalic acid. Without further discussion oxalic acid may be ruled out of consideration. The formulæ of $d$-glucose and of the other three-named substances is here given: 


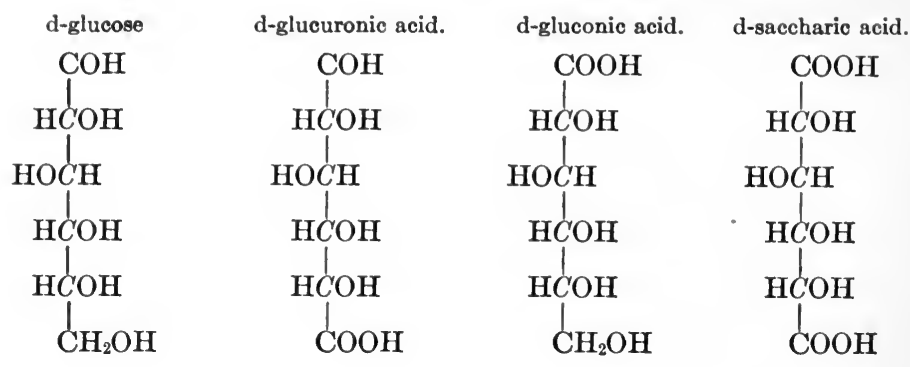

From these equations it is clear that even these could be but the very beginnings of an oxidation.

D-glucuronic acid exists in traces in normal urine, and there can be no doubt that it is derived from glucose. It appears in the urine only in the paired state; it has never been found in the circulating fluids or tissues in the free state. Substances such as camphor, chloral, and thymotin-piperidid are eliminated conjugated with d-glucuronic acid. In an individual with normal carbohydrate deposits, the ingestion of such substances is followed by the elimination of a correspondingly increased amount of d-glucuronic acid; if administered to the starving animal this is not the case. A dose of thymotin-piperidid that would be lethal to a starving animal is not so when administered to an animal in normal nutrition, because it is distoxication by conjugation with d-glucuronic acid. The question really at issue is whether the d-glucuronic acid is formed naturally and simply seized upon by the substances with which it conjugates; or whether these substances, so to speak, provoke the formation of d-glucuronic acid. In the first instance, it would be termed a normal intermediary product of metabolism; in the latter event a pathological product. The occurrence of d-glucuronic acid in the body will be discussed in another content. There is, however, no warrant for the idea that the total combustion of glucose could possibly pass through the stage of d-glucuronic acid.

D-gluconic acid is easily formed from glucose by oxidation in the laboratory, and bacteria accomplish the addition of oxygen also. The body burns it with ease, and in the diabetic it acts antagonistic to the acetone complex. It has, however, never been demonstrated to occur in the animal body. d-saccharic acid, which is closely related to both d-glucuronic acid and d-gluconic acid, is not known to occur in the body. When administered, it is burned, like the other two bodies under consideration. When a rather large dose of d-gluconic acid is given to an animal, a small amount of d-saccharic acid may be eliminated in the urine. The fact that all these three substances are burned in the diabetic organism as well as in the normal need not, as has been supposed, throw doubt upon the theory that glucose is burned normally through such stages; the defect in the diabetic might be precisely in the first stage. What 
does throw grave doubt upon the hypothesis is the fact that these substances, one and all, are not revealed and do not behave in metabolism in such a way as to warrant the assumption that the normal combustion of sugar proceeds through them. Yet the question must be left open.

Indirect Oxidation of Glucose.-The most likely possibility of an indirect oxidation of glucose is to be sought in the reaction of alcoholic fermentation. According to this hypothesis, glucose is first converted into lactic acid, this then splits into ethyl alcohol, precisely as in the fermentation with zymase, and ethyl alcohol then oxidized through acetic and formic acids to water and carbon dioxid. Thus:

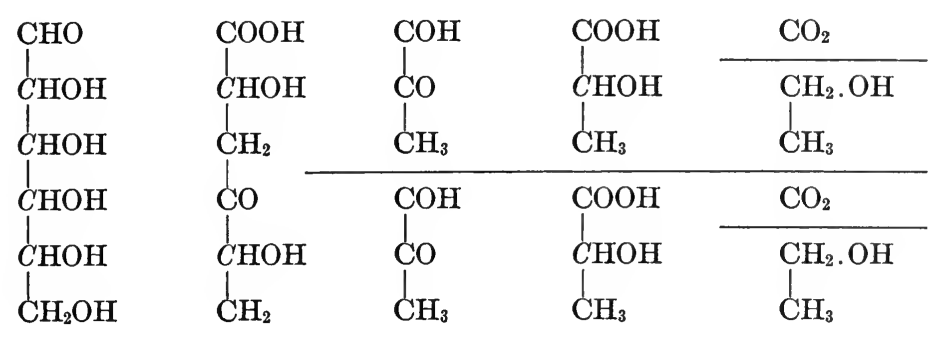

The intermediary equation following that of glucose is purely hypothetical, the next is methylglyoxal, following this lactic acid appears and from this ethyl alcohol and carbon dioxid. The intramolecular alterations in the hypothetical substages are all expressions of successive incorporations and extrusions of hydroxyl and hydrogen groups to accomplish the intramolecular transformations. Lactic acid may be derived from acetol through methylglyoxal, so that this step is not hypothetical. This scheme has been worked out to follow the line of least resistance in the transformation in alcoholic fermentation, in which there is no doubt of the intermediary occurrence of lactic acid and probably of methylglyoxal.

What have we for data bearing upon this scheme? That bacteria and plants act thus upon glucose would not, of course, prove that animals do so burn it. Traces of ethyl alcohol are always to be found in freshly analyzed muscles. This could have come only from carbohydrate. Lactic acid also is to be found in fresh muscle, especially following heavy contractions; and it occurs also in the autolysis of muscle in such large amounts as to make the derivation there only possible from sugar. Lactic acid can, of course, be also derived from protein (amino-acids). But the more the matter is investigated, the more likely it appears that it is derived in the muscle from glucose. When the muscle works with little or no oxygen, lactic acid appears in large amounts, an observation that is hardly to be harmonized with any other view than the derivation of the lactic acid from glucose. Muscular contraction, under normal conditions of glucose concentration, does not lead to any exaggeration in the nitrogenous 
catabolism of the contracting tissues, which is difficult to harmonize with the appearance of lactic acid when the muscle works without full supply of oxygen, if that lactic acid be held derivable from the protein. The cleavage of glucose to lactic acid and carbon dioxid occurs without oxygen. If a muscle be pumped free of oxygen, placed in a chamber filled with nitrogen and stimulated to contractions, carbon dioxid will be given off and the muscle will turn acid from lactic acid. This is perfectly comprehensible, according to the scheme outlined above. The demonstration would be complete if the same muscle could be shown to contain ethyl alcohol, but unfortunately we have no delicate tests for traces of ethyl alcohol.

Direct experiments are difficult and have as yet not given unequivocal results. Sterile extracts and precipitated extracts of muscle and other animal tissues seem able to form a certain amount of alcohol and carbon dioxid from glucose. In other words, they contain zymase. But the results quantitatively are not satisfactory. If an extract of muscle be mixed with a glucose solution of known strength, there is little result. If now a pancreatic extract, which is thermolabile, be added, the glucose can be shown rapidly to diminish. Expressing such an experiment in the language of the theory of catalysis, we would say that the muscle contains the enzyme, the pancreas the zymo-excitor. The relations are quite the same for zymase. The expressed ferment of the yeast is able to ferment glucose but little except after the addition of a solution of phosphate. Here the yeast extract contains the ferment, the phosphate is the zymo-excitor. Under the conditions of the tests, it has not been possible to demonstrate the presence of either ethyl alcohol or carbon dioxid. Indeed, recent work has purported to show that the glucose that is removed has been simply converted into isomaltose. While in work of this kind the success of an experiment is of the most convincing influence, failure does not negate the hypothesis for the testing of which the experiment was undertaken. The correlation of muscle extract and pancreas extract is so harmonious with our experimental knowledge of diabetes, that the apparent failure of direct fermentation tests should not be taken as conclusive. We know that when the pancreas is removed, the muscles cannot burn glucose properly. This positive fact is far more fundamental than the negative result of test-tube experiments with extracts of muscle and pancreas. The action of the internal secretion of the pancreas to sugar combustion has also been assumed to lie in a relation to the passage of the glucose from cells to blood stream, an hypothesis intangible and far-fetched.

Relationship of Pancreas.-The relationship of the pancreas to the metabolism of glucose is established in an unequivocal manner. There exist also data tending to show that the thyroid and the adrenal bodies have some relation to the sugar metabolism. While the ablation of the thyroid body does not cause any reduction in 
the combustions of the body, the hyperactivity of this ductless gland certainly has a stimulating action. This is seen markedly in acute exophthalmic goitre, in which the combustions of the body (protein, fat, and sugar) are greatly increased, the heat production of the body being sometimes doubled. This result can also be produced by the administration of desiccated thyroid glands of animals, and upon this exaggeration of combustions the treatment of obesity by thyroid substance, now recognized as dangerous, is based. This is simply a clinical fact, for it we have no explanation that rests upon an experimental basis, and a hypothetical explanation would scarcely be of service. Curiously, direct experiments seem to show that increased thyroid activity operates against the pancreatic stimulation of the muscular combustion of sugar. In such direct experiments, however, the relations are so complicated that one must exercise the greatest care in the interpretation of the results. Far more important is the clinical fact that hypertrophy of the thyroid body is associated with exaggeration of combustions, and following partial extirpation of the hypertrophied thyroid gland, the previous exaggeration in combustion is diminished.

Relationship of Adrenal Bodies. - That the adrenal bodies have some relation to the intestinal secretion of the pancreas, is certain. How this is accomplished is not understood. That it has any relation to the so-called internal secretion of the pancreas is not clear. Excision of the adrenal bodies nullifies the effects of the Bernard puncture. But such extirpation must effect a more or less complete section of the splanchnic nerves, which are the efferent paths of the nervous impulses concerned in the effects of the Bernard puncture. Chronic diseases of the adrenal bodies may be associated with glucosuria. But this is not the rule, rather the exception, certainly for tuberculosis of the adrenal bodies. At present there is too much confusion and too little definiteness for inferences to be reasonably drawn. The relation of the internal function of the pancreas to the mydriatic action of epinephrin has presented a new point of view in the problem of interorganic function; but an interpretation of these relations is not yet at hand.

Combustion without Oxygen.-The matter of combustion without oxygen merits a further consideration. The phenomenon holds for wider relations of life than the isolated muscle. Many worms can be kept in an atmosphere without oxygen for days, with noteworthy movements. A frog at low temperature will tolerate an oxygenfree atmosphere for a day. In such instances the atmosphere of the experimental chamber contains quite a little carbon dioxid. This may have been derived in part from oxygen, which as we have seen, the muscles can store. In addition to this, the cleavage of glucose into ethyl alcohol and carbon dioxid occurs without oxygen. It is possible that there are other reactions of cleavage that either yield oxygen for the combustion or yield $\mathrm{CO}_{2}$ directly. We shall 
learn later that the reduction of diacetic acid yields carbon dioxid and acetone, and $\mathrm{CO}_{2}$ is thrown off when taurin is formed from cystin. Not much heat is liberated in the cleavage of glucose to ethyl alcohol and carbon dioxid; 1 gram of glucose splits to form 0.511 gram of ethyl alcohol and 0.489 gram of carbon dioxid with the liberation of only 0.3 Calorie of heat; in the 0.511 gram of ethyl alcohol lies the remaining 3.5 Calories of heat.

It is to be granted that the proposition that all the sugar of the body is burned through ethyl alcohol is striking, and rendered none the less striking when the physiological action of alcohol is recalled. When the mass relations are considered, however, particularly in relation to the fact that the stage of alcohol is only intermediate and transitory, the wonder disappears. We have seen that the resting man burns about 6 milligrams of glucose per second, at hard work possibly 15 to 20 . This would correspond to from 3 to 10 milligrams of alcohol per second, or from 250 to 800 grams per day. Such huge doses of alcohol could not be burned if ingested by the mouth. But this is the result of concentration. Generated in each second and distributed throughout the entire muscular system, appearing only for the instant as an intermediary product, it is clear that our toxicological conceptions of alcohol cannot be applied to this consideration. If a solution of glucose be infected with an alcoholic yeast and a vinegar yeast, and permitted a free supply of oxygen, alcohol will appear only as a transient stage, to be fermented to acetic acid as fast as it is formed from the sugar. So in the body, with the free supply of oxygen, the alcohol would be burned as rapidly as formed. In the absence of oxygen it would accumulate, together with the lactic acid, and doubtless act as a toxic agent, or at least tend to inhibit the reaction to which it was due. The combustion being regarded as localized within the muscle cells, the alcohol would never reach the circulation or the nervous system, upon which its toxic actions are displayed. Since the combustions proceed evenly, and since the amount of alcohol to be recovered from fresh muscles is the merest trace, it is evident that it would be permissible to assume that the alcohol would be burned as rapidly as formed. Thus the total concentration in any moment could never be over 20 milligrams distributed throughout the entire muscular system-a concentration that could not in the wildest stretch of imagination be regarded as serving to cast a reflection upon the credibility or admissibility of the theory that glucose is burned in the body through the stage of ethyl alcohol.

Formation of Lactic Acid.-Two points remain to be considered in this connection. The formation of lactic acid from glucose is a reversible reaction. In some severe cases of diabetes, the administration of lactates is followed by increase in the glucosuria, the sugar having been obviously derived from the ingested lactic acid by reversed reaction. Experimentally, this holds for the depancreatized dog. 
It holds also in the animal with profound phloridzin intoxication, in whom ingested lactic acid becomes converted into glucose. The two experiments are, however, absolutely different in the matter of the glucose concentration of the blood. This is abnormally high in the depancreatized dog, and almost nothing in the phloridzinized dog; yet in each instance glucose is formed from the ingested lactic acid. We have said that when ingested in the normal body, lactic acid is burned; probably not directly, but only after conversion into sugar. Lactic acid is an intermediary stage in the cleavage of sugar to ethyl alcohol, according to that theory of combustion. An excess of lactic acid (such as would occur in the liver following ingestion by mouth) would according to the laws of general chemistry be expected to result in a reversion of the reaction (formation of glucose), which is the experimental result. Since it is difficult to imagine in what other way than through ethyl alcohol lactic acid is burned, these observations serve to strengthen belief in the correctness of this theory of combustion of glucose.

The cleavage of glucose through lactic acid into ethyl alcohol and carbon dioxid is, of course, only a cleavage, exothermic it is true. But the real combustion is yet to occur. This we believe is accomplished to the completed stage largely in the muscles. Oxidation ferments, known to be present, carry the ethyl alcohol through acetic acid and formic acid, in fractional stages, to the end products of water and carbon dioxid. These pass into the venous capillaries, and this conception agrees fully with the fact known from another quarter, that the highest concentrations of carbon dioxid known in the body are to be observed in the muscle tissue.

\section{Lactic Acid.}

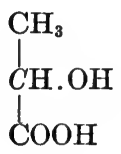

Lactic acid occurs in the body under many different circumstances and of different derivations. There are, however, three sources that may be definitely determined: derivation from glucose, from aminoacids, and in the alimentary tract through bacterial action. The lactic acid formed in the alimentary tract is held to have been derived from carbohydrate.

Lactic acid is very easily burned in the body. The lactic acid that is formed in the gastric and intestinal fermentation is inactive. The lactic acid that is formed in the body is dextrorotary. It is doubtful if the lactic acid of normal intestinal fermentation ever appears beyond the hepatic metabolism, being burned instead. It may also be assumed that any lactic acid normally produced in metabolism is burned. Whenever we encounter it as a metabolic substance, it has either escaped combustion, or, in fact, its very formation was an 
abnormality. For all practical purposes, whenever it appears in the blood in considerable amounts it is eliminated in the urine. When the body is prevented from burning it the kidneys present the only avenue of elimination, largely as ammonium salt.

Lactic acid is present in the intestinal contents of normal breastfed infants. It is contained performed in mixed diets. It is to some extent formed in the intestine in normal digestion, though probably not in the stomach. Under many conditions of increased bacterial activity, large amounts are formed in the intestine. In the stomach, also, in connection with chronic gastritis, atrophy of the gastric mucosa and carcinoma, especially if associated with pyloric obstruction, lactic acid may appear and in very large amounts, due, of course, to the action of bacteria upon carbohydrates. While lactic acid is very often present in the gastric contents of cases of gastric carcinoma, it is in nowise pathognomonic of this lesion. The lactic acid so formed is usually entirely burned on resorption. But in a few reported cases the urine has contained the dextrorotatory acid. On resorption the levorotatory form is apparently converted into the dextrorotatory form. It is possible that the presence of lactic acid in the urine of a case of gastric carcinoma might be interpreted as a sign of organic hepatic disease, either involvement by the neoplasm or an inflammatory or degenerative reaction.

Lactic acid originating within the metabolism could be derived from either glucose or from amino-acids. In birds the ingestion of derivatives of lactic acid is followed by the formation of uric acid. It is, however, not possible to assume any formation of lactic acid from purin in the higher organism. In theory it is easy to derive lactic acid from certain amino-acids; in experiment it is difficult. The equations illustrate the possibilities in the chemical sense.
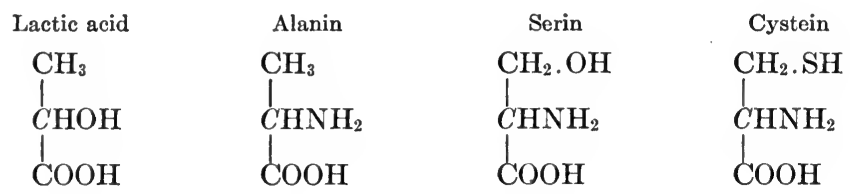

Other possibilities are known, such as the formation of p-oxyphenyl lactic acid from tyrosin. But when these possibilities are all weighed, in the light of the amounts of lactic acid formed and the conditions under which it occurs; and above all when the experimental investigations directed to the elucidation of the derivation of lactic acid under these conditions are reviewed, we are led to the conclusion that as noted physiologically and pathologically, lactic acid is usually derived from glucose.

The normal blood, muscles, and liver contain traces of it, the urine none. In the presence of a free supply of oxygen in the muscles it is quickly oxidized; in the absence of oxygen it accumulates. It is present 
in notable amounts in rigor mortis, and is developed in large amounts in autolysis. It is possible to infer that it passes into the circulation only when the conditions of combustion in the muscle are unfavorable.

Asphyxia leads to the accumulation in the blood and tissues of large amounts of lactic acid. Dyspnea has the same result if long prolonged. Intoxication with carbon monoxid achieves the same result, in an obvious indirect manner. It is also seen in other intoxications, in which interference with the processes of oxidation is more or less directly observed, thus: hydrocyanic acid, amyl nitrite, cocain, arsenic, phosphorus, and the narcotic anesthetics. It may be seen in severe essential anemia.

Diseases of the liver frequently present an excess of lactic acid in the blood and urine, possibly in part of intestinal derivation. Thus cirrhosis, fatty degeneration, acute yellow atrophy of the liver. It is also seen in eclampsia, which may be referred to the liver. In severe instances of diseases of the liver the elimination of large amounts of lactic acid, up to or exceeding 20 grams per day, amounts to a veritable acidosis.

There is no excess of lactic acid in diabetes. On the contrary, in some cases of diabetes, the administration of lactic acid will be followed by an increase in the glucosuria, due to the formation of glucose from lactic acid. This is an illustration of reversed reaction, lactic acid being normally formed from glucose in the cleavage that precedes its direct oxydation. There is no excess of lactic acid in rachitis and osteomalacia, and any attempt to place upon lactic acid, directly or indirectly, the responsibility for these diseases is futile.

Glucuronic Acid.- In the discussion of the modus operandi of the combustion of sugar, the possibility of the molecule of glucose being burned through d-glucuronic acid was stated, but the conclusion was reached that the existing data do not tend to maintain the proposition. The substance, however, is a constant concomitant, at least, of the carbohydrate metabolism; and its qualities in certain directions are unquestionably of importance to the organism.

Glucuronic acid exists in the body in the state of conjugation, in combinations of the type of glucosids. When the isolated facts are collected, it appears that the number of substances with which it may be conjugated is almost unlimited. On analysis, however, it will be seen that they are all either alcohols or phenols. Not that the substances entering into the conjugations were either alcohols or phenols originally, but in the body they undergo conversion into alcohols or phenols, since it is with the alcoholic hydroxyl group of these substances that the combination occurs. To accomplish this, hydrocarbons must be hydroxylated, as must be also members of the heterocyclic and the hydro-aromatic series. Ketones must be converted into secondary alcohols and aldehyds into primary alcohols. As they issue from the body, therefore, the numerous substances that conjugate with glucuronic acid exist in the state of alcohols or phenols. Among the most important 
of these substances are carbolic acid, camphor, chloral, phenacetin, acetanilin, resorcin, acetone, menthol, morphin, and pyramidon. Important in pathology rather than in toxicology are the conjugations with acetone and phenols.

The conjugations of glucuronic acid rotate the plane of polarized light to the left; the free acid, however, to the right. The substance has marked powers of reduction, but does not ferment with the common yeast germs. It can, however, be fermented to l-xylose, and this was previously pointed out as a possible source of derivation of pentose in the body. It is present in the blood, in tissues, in the bile, and in the urine.

When ingested some will appear as conjugated phenols (the ethereal sulphates will be reduced), some may appear in the free state if the ingestion be massive, there is usually much oxalic acid formed, and traces of gluconic and saccharic acids may be observed. Glucosuria may also occur. There seems under all circumstances, physiological and pathological, to be a mass relation between glucuronic and sulphuric acid for the combination with the aromatic substances resorbed from the intestine, they being, so to speak, contenders for these aromatic bodies. If either one be held constant and the other increased or diminished, the division of the aromatic bodies between them will be varied in the direction of highest concentration. This is one reason why the estimation of the ethereal sulphates in the urine cannot be employed as a measurement of the amount of aromatic bodies in the urine.

The localization of the conjugation in the body is not well understood. Though commonly stated to occur largely in the liver, there is no evidence of this, unless the presence of glucuronates in the bile is to be accepted as such. Since different extreme organic diseases of the liver and specific intoxications affecting the liver do not result in any reduction of the function, a localization restricting the conjugation to the liver is very improbable. General considerations would suggest the muscles.

Since glucuronic acid, independent of the diet, is always to be found in the body available for conjugation, it has been usually inferred that it constitutes a normal constituent of the carbohydrate metabolism, the product of a side-reaction. There are, however, serious chemical qualifications to this view. The direct oxidation of d-glucose to d-glucuronic acid is very difficult of comprehension, since from all we know of the molecule of glucose the aldehyd group $(\mathrm{COH})$ is much more easily oxidated than is the primary alcoholic group $\left(\mathrm{CH}_{2} \mathrm{OH}\right)$. But in glucuronic acid it is the primary alcoholic group and not the aldehyd that has been oxidized. Under these circumstances it is much more logical, chemically, to assume that the glucose combines with the alcohols or phenols by means of the aldehyd group; this being thus protected from oxidation, the primary alcoholic group undergoes oxidation, with the production of the conjugated glucuronates. 
In other words, the conjugations occur with the glucose, not with the glucuronic acid preformed; and the conjugated glucose is then oxidized to a conjugated glucuronate. Thus the amount of conjugated glucuronic acid is really a function of the amount of pairing alcohols and phenols, if the glucose concentration be normal. Otherwise we are to suppose that glucuronic acid is formed from glucose, and if the body does not happen to contain much of the pairing substances, the excess is subject to further oxidation. This is contrary to observed facts.

This conception of the situation makes far more intelligible the distoxication function of glucuronic acid. The toxicity of the alcohols and phenols when conjugated with glucuronic acid is practically nil. A certain amount of the aromatic substances formed by bacterial action upon the end products of protein digestion in the intestine are regularly conjugated in this manner, the precise amount depending upon the mass relations with sulphuric acid in the body. When any of the denominated substances are ingested, they are more or less completely conjugated and thus eliminated in an innocuous form.

Glucuronic acid is also increased in certain diseases. Thus in profound jaundice; in some cases of diabetes; in conditions of highly exaggerated dyspnea as may be seen in diseases of the lungs and heart; associated with multiple fractures of the bones and with widespread traumatism to muscles; in starvation and sometimes in apparently normal health, excesses of glucuronic acid appear in the urine. Under these circumstances it is assumed that the excess is not provoked by the presence of any pairing body, but is primary. The association with muscular traumatism is very suggestive. Such primary increase in the formation of glucuronic acid would indicate that under certain conditions the side-reaction may be exaggerated. Its occurrence in conditions of dyspnea suggest that in oxygen hunger such exaggeration of the side-reaction occurs. The increase in jaundice may be simply a resorption with the bile. The occurrence in diabetes and acidosis has been frequently misinterpreted. It ought to be regarded here as a provoked glucuronuria, due to the presence of acetone in the blood, acting as a pairing substance. When one regards the chemical difficulties attending the isolation of glucuronic acid, one is led to view the reported instances of primary excess of the substance with reserve. But even were such to be established, it would give no warrant for the hypothesis that it is a fundamental stage in the combustion of glucose, or that the defect of diabetes lies in the failure to effect oxidation at this point. The known facts do not warrant any definition of glucuronuria as a precursor of diabetes.

In practical urinalysis the occurrence of glucuronic acid may cause confusion since by its reactions of reduction it stimulates glucose. But it is easily differentiated by examination of its rotation of the plane of polarized light, its resistance to fermentation and its relations of phenylhydrazin. 
Oxalic Acid.

\section{$\mathrm{COOH}$ \\ $\mathrm{COOH}$}

Oxalic acid is present in traces in the normal tissues, circulating fluids and the urine, and may be present in the urine in considerable amounts. The term oxaluria, as usually employed by the physician, does not refer to the amount of oxalic acid in the urine, but to the amount of oxalate crystals that the urinary sediment contains. There is no relation between the content in oxalic acid and the sediment of oxalates; there may be much sediment with a low oxalic acid content; there may be a high content with no sediment of crystalline oxalates. What it is that leads to the formation of the crystals in the one case and the solution of the salts in the other case, is not known. The calcium salt is the most insoluble, the magnesium salt is quite insoluble, the potassium and sodium salts are soluble. Acidity seems to increase solubility. In the crystals of calcium oxalate, calcium carbonate is very apt to be occluded.

Fruits and vegetables contain varying amounts of oxalates. These seem to be resorbed from the acid stomach and the upper duodenum; from the neutral or alkaline lower intestine there is little absorption, as the salts are precipitated. Thus the administration of acid increases alimentary oxaluria, the administration of alkali reduces it. Of the total percentage absorbed from the food under even known conditions of diet, little is known. This inability to demarcate the exogenous oxalic acid makes very difficult the determination of endogenous oxalic acid.

Not only do the fruits and vegetables contain oxalic acid, but it is formed by fermentation in the intestinal tract. The amounts so formed are not known, nor have pathological variations been determined. It is probable that bacteria form it from amino-acids as well as from carbohydrate; it does not seem possible to restrict it by control of diet.

For the endogenous oxalic acid there are several sources. Reference has already been made to its derivation from glucuronic acid. Organ pulp digested with glucuronic acid forms oxalic acid. Traces of glyoxylic acid may be found in the urine, which might have been derived from the cleavage of the glucose molecule (methyl glyoxal), and this could, as a side-reaction, give rise to oxalic acid.

When an animal is placed on a pure protein or milk diet, oxaluria persists. It can be shown that the administration of glycocoll or creatin will result in increased oxaluria. This is the explanation of the large amounts of oxalic acid that are formed following the ingestion of gelatin with the heavy content of glycocoll. Organ pulp digested with glycocoll yields oxalic acid. In a way, oxalic acid may be regarded as the end product of the oxidation of glycocoll in the direct sense, 
though it is certain that for the most part the catabolism of glycocoll does not follow this route.

Oxalic acid may also be formed in the test-tube from pyrimidin, purin, ethylenglycol, and alloxan. Digestion of purin and pyrimidin with organ pulp also yields oxalic acid. But it is very doubtful if the purins and pyrimidins in the body are to be regarded as sources of oxalic acid.

The fact is that on a diet free of oxalates, the oxalic acid of the urine is but the merest trace. Thus, whatever the chemical or experimental results, it is clear that normally but little oxalic acid is formed from amino-acids, glucose or purins. The difficulty in the interpretation of increased oxaluria is due to the fact on the one side that we cannot rule out the formation by bacteria within the alimentary tract, even though we could control the input; and on the other hand, we do not know just to what extent the body can burn absorbed oxalic acid. When a given amount is ingested, most of it reappears in the urine; but since the unresorbed fraction cannot be measured, we do not know how much was burned. Of injected sodium oxalate, nearly all is promptly eliminated in the urine. In a word, we lack in the investigation of provoked or spontaneous oxaluria the controls that alone would enable us to make a reliable interpretation.

Excessive oxaluria is common (whether of alimentary or endogenous origin is unknown) and of vague meaning. Oxaluria has played an active though supposititious rôle in practical medicine. It has been widely employed as a convenient receptacle for the deposition of undiagnosticated cases of illness of all kinds. There is probably an idiopathic oxaluria, of unknown derivation, a metabolic curiosity at present. There is no known relation of oxaluria to any pathological entity, within the intestinal tract or in the metabolism.

Oxaluric acid, a combination of urea and oxalic acid, may be found in traces in the urine. This conjugation occurs so easily in the testtube, one wonders that more of the combination does not occur normally in the urine. It has no known meaning.

\section{Glycerol.}

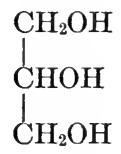

When the body forms the higher fatty acids from glucose, it must also synthesize glycerol to combine with them for the formation of fat. When higher fatty acids are ingested, they are resorbed and at once combined with glycerol to form fats, and this glycerol likewise must have been synthesized in the body.

This glycerol may have been derived from two different sources: from glucose and from amino-acids. The derivation from glucose must occur through the stage of lactic acid, according to present conceptions. The following equations illustrate the two lines of possibilities. 


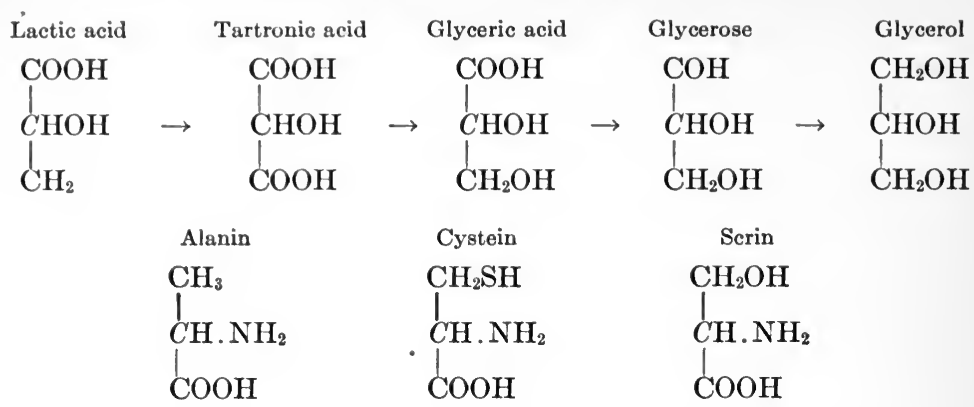

Cystein after deaminization and oxidation would yield glyceric acid; serin on deaminization would yield glyceric acid; alanin would yield lactic acid.

The line of reaction is clear in the series of carbohydrates. For the amino-acids, the direct line of reaction for the three stated aminoacids is not clear, but the general configuration is such as to make it very likely that glycerol might come from them directly as well as obviously via lactic acid. When, however, we come to the consideration of the amounts of glycerol that the body can form and contrast these figures with the amounts of the three amino-acids that could be formed in the nitrogen metabolism, the content of common protein in these three amino-acids being known, it is clear that the glycerol could have been originated only from glucose. The experimental investigations have led to the same result. And thus we feel certain that glycerol in the body is a derivative of glucose, and a constituent of the carbohydrate metabolism.

When glycerol is ingested it is burned (via glucose), unless the dosage is excessive. Then some may appear in the urine. The blood contains normally traces of glycerol, which are tolerated and retained by the kidneys. The urine, however, occasionally contains traces of glycerol under conditions of normal metabolism. It occurs as a byproduct in alcoholic fermentation (via lactic acid), and as such may be present in the normal intestinal contents. When ingested in huge doses it has been reported as increasing the output of uric acid; this might be taken to indicate the formation of tartronic acid, through lactic acid; from this dialuric acid could be derived, and from this uric acid, as in birds. The experiments, however, are not convincing.

The intimate relations of glycerol to the glucose metabolism are indicated in several ways. When glycerol is fed to the depancreatized dog, the glucosuria is increased. When it is fed to the phloridzinized dog, the glucosuria is increased. When it is perfused through the liver, the liver content of glucose and glycogen is increased. When glycerol is fed to the diabetic it acts just as sugar does; it tends to increase the glucosuria, and it acts antagonistically to the acidosis. There is but one interpretation of these several results: the glycerol is converted into glucose via lactic acid. And conversely to this, from 
glucose through lactic acid glycerol is formed. It is important that this relationship be fully realized. When glycerol is ingested and burned, it is in reality converted into glucose. In the catabolism of fat the glycerol is converted into glucose. Possibly one of the reasons why the diabetic organism cannot form fat properly may lie in the inability of the body to form glycerol from glucose.

When one administers higher fatty acids they are converted into neutral fats with such facility that one is almost made to feel as though the body contained stores of glycerol ready for use. The facility with which glycerol is formed when needed indicates that, like ammonia for protein, it is a normal intermediary stage in the carbohydrate metabolism. It may be assumed that wherever there is lactic acid there is also glycerol as a side reaction. When there are fatty acids to combine with it, it is thus abstracted from the carbohydrate metabolism. Otherwise it exists simply as a side reaction within the main stream of the glucose metabolism. When fats are burned, it is certain that the glycerol derived from the hydrolysis of the neutral fats is converted into glucose. The discussion as to the convertibility of fats into sugar concerns only the fatty acid. That the glycerol fraction is converted into glucose has long been known and recognized. The amounts involved are not small. From the glycerol derived from the cleavage of 1 gram of fat not less than one-eighth gram of glucose would be formed.

The following chart indicates the metabolic relations of glucose:

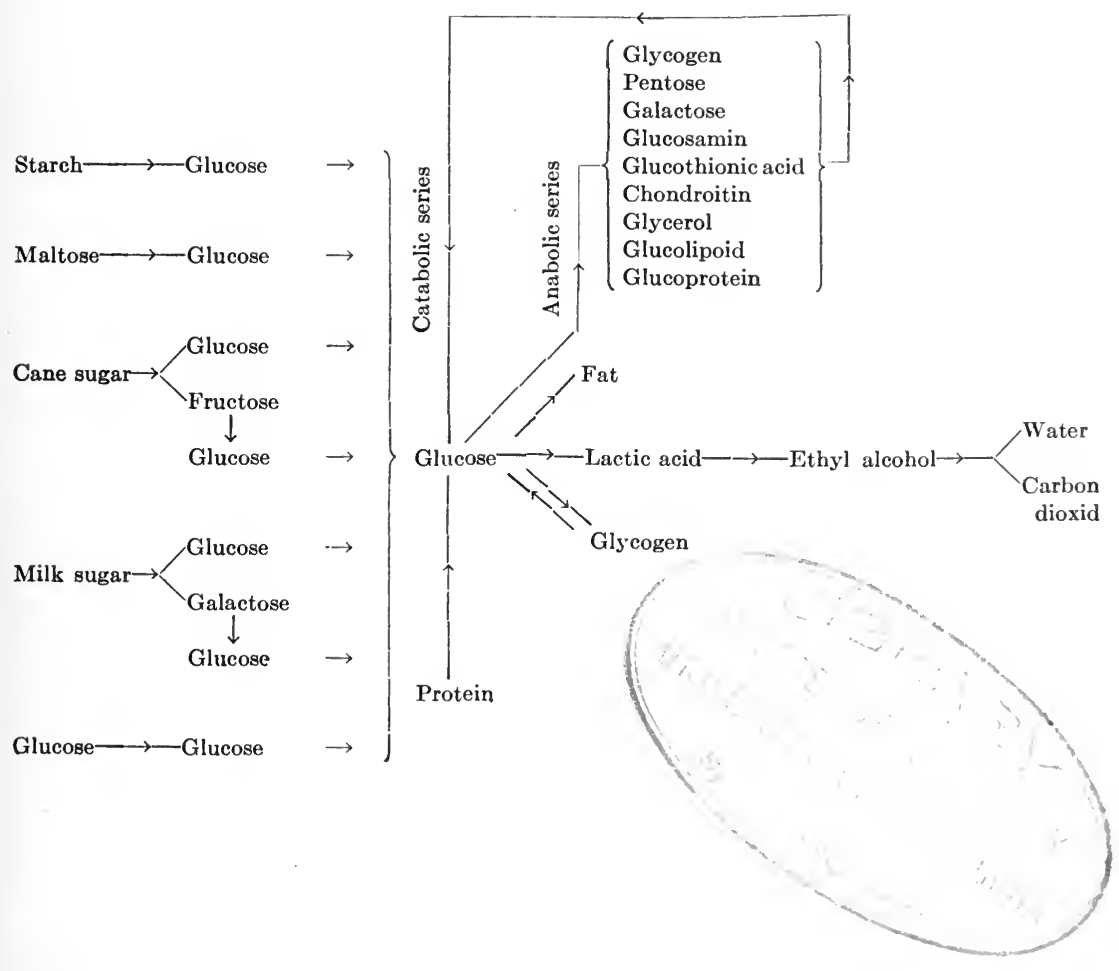




\section{GLUCOSURIA}

Under the term melituria are included all the different conditions in which a sugar is present in the urine. The specifications of the chemical nature of the sugar are contained in the term maltosuria, saccharosuria, lactosuria, pentosuria, fructosuria, and glucosuria. With the exception of glucosuria all these forms of melituria have been described under the sugars concerned. They are of subordinate interest, their chief importance being, in fact, as well as in theory, their relations to glucosuria and the light they may throw upon it. Glucosuria is a condition whose meaning is so fundamental to the understanding of the carbohydrate metabolism as to warrant an extended discussion of the facts and of their theoretical interpretation.

According to our knowledge of the relations of glucose in the body, there are two types of glucosuria: hemic and renal glucosuria. By hemic glucosuria we understand the elimination of glucose in the urine because of excessive concentration of glucose in the blood; by renal glucosuria we understand the elimination of glucose in the urine because of lowering of the level of renal retention. In hemic glucosuria, the height of the impounded waters rises above the floodgate; in renal glucosuria, the level of the floodgate is lowered. We are justified in the assumption that the concentration of glucose in the blood and the level of renal retention may be variable in time in the same individual in health and in disease, and from individual to individual.

Founded upon our knowledge of the relations of glucose in the body, we may postulate seven ways in which hyperglucemia could be attained: (a) By excessive ingestion of sugar, ingestion exceeding in time the normal function of glycogenesis of the liver; $(b)$ by reduction in the function of glycogenesis of the liver, so that a normal input cannot be handled; $(c)$ by exaggeration of the glycolytic function of the liver, glycogen being converted into glucose in excess of the utilization of it; $(d)$ by reduction in the glycogenetic functions of the muscles, or reduction in the storage capacity for glycogen in the muscles; $(e)$ by exaggeration of the glycolytic function of the muscles, glycogen being converted into glucose in excess of combustion and returned to the circulation; $(f)$ by reduction in the formation of fat from glucose; and $(g)$ by reduction in the combustion of glucose in the muscles. As we shall see, we are not yet certain that all of these possibilities are represented in experimental or clinical instances of glucosuria. Nor are we able to assign every case of glucosuria; a great many in fact, we are unable to assign to any definite place in this classification.

The lowering of the level of renal retention is a process that is localized in the kidney. The attempt has been made to localize this in the blood. It has been stated, in hypothesis, that the kidney retains combined glucose (glucose-lipoid, glucose-protein, colloidal glucose in short), but does not retain free glucose; that normally the retention 
of sugar is simply an expression of the fact that all the sugar of the blood is combined and none is free. When, therefore, glucose appears in the urine this is accepted as a sign that there is free sugar in the blood. And the instances of glucosuria due to the lowering of the level of renal retention and not to hyperglucemia are held to be due to the abnormality of free glucose in the blood, not to any alteration in the renal function. Apart from the fact that this hypothesis is entirely unable to explain glucosuria with hyperglucemia, it has for its support no conclusive chemical or experimental data, and has against it the positive physico-chemical fact that by far the larger portion of the sugar of the blood is in the free state. The lowering of the level of renal retention is, therefore, to be regarded as a process, reaction, or state in the renal structure. When we recall that the kidney that normally retains colloids entirely, under certain conditions of disease eliminates protein in such a way as to exclude physical leakage, we have no ground for wonder that under conditions of disease the power of retention of glucose should be lowered or abolished. Nor is it to be regarded as remarkable that the kidney should be able to retain the glucose below a certain concentration and permit it to pass out when above that concentration, since this may be solely a question of the relation of osmotic pressure to a state of semipermeability. We come here into contact with the difficult question of the modus operandi of secretion. But the problem does not bear heavily upon our conception of glucosuria. When we know why and how the kidney secretes sodium chlorid and urea, it will be time to take up the question why the kidney retains glucose below a certain blood concentration and eliminates it above that concentration.

Hemic Glucosuria.-The first matter to be determined is the level of renal retention of glucose. What is the normal glucose content of the blood, and to what fluctuations is it subject? The data are not extensive and trustworthy enough to enable us to give a precise answer. It is not easy to procure the amounts of blood needed for careful quantitative estimations; and these are difficult of accurate execution. In the past the tendency of the figures was too high. The figures given in the arbitrary scheme-varying from 0.6 to 1 per thousand-are probably not far from the truth. The adjustment is usually a very fine one; but this need not be true. By this we mean that usually a slight increase will cause glucosuria. It is doubtful if glucose in concentration of over one part in the thousand should be termed normal; the majority of the best analyses do not run over 0.8 part per mille. What we need are not haphazard comparisons from individual to individual, but repeated analyses on one individual or animal to determine to what extent fluctuations occur in the glucose concentrations of the blood under different conditions of diet, nutrition, and work.

When now we come to the analyses of the blood of individuals with glucosuria or diabetes or of depancreatized $\operatorname{dogs}$, we find striking contradictions, or at least what at first glance strike one as contradictions. 
While one usually finds the highest glucosuria with the highest hyperglucemia, one may find pronounced glucosuria with low hyperglucemia, or with none; and one may find striking hyperglucemia with low or no glucosuria. It is only in typical rapid diabetes in man and in the diabetes following ablation of the pancreas in animals that the consistent concordance of high hyperglucemia and high glucosuria is to be encountered.

Reflection, however, makes this lack of apparent concordance clear. If the dam impounding the waters of a basin be a broad one, the water will not rise much above the level of the dam, because the wide overflow keeps it down. If the kidneys eliminate the excess of glucose, we need not find any rise in the concentration of glucose in the blood. The ingestion of a large amount of sodium chlorid will be followed by the rapid elimination of the salt in the urine; absorption and elimination are both rapid. But one would not find the conductivity or the depression of the freezing point of the blood increased during the time of the experiment, the blood would not contain any analytical increase in sodium chlorid. The blood passes so rapidly through the cycle of circulation that with normal action on the part of the kidneys there is no retention, no rise in the salt level of the blood. The same is true of urea, of which the blood contains normally the merest traces; it may be ingested in large amount, without the urea concentration of the blood being analytically raised. This ought theoretically to be the normal procedure. So soon as the glucose concentration of the blood rises to the level of renal elimination, this elimination should be effective enough to keep the blood concentration down to that level. In the case of very excessive amounts of sugar this could not be expected to hold true; but for all ordinary cases it can be shown that the velocity of elimination displayed by the kidneys ought to be sufficient in itself to prevent any material rise in the glucose concentration of the blood. Short as the list of reliable analyses is, they are enough to teach us that we cannot from high glucosuria always reason to high hyperglucemia.

From this it is clear that the relation of the kidney to the concentration of glucose in the blood need not be constant in different individuals and in the same individual from time to time. The variation seems to occur always to the side of lesser elimination. In a word, the kidney seems to get accustomed to the increased concentration of glucose, the elimination is restrained, and the level of glucose in the blood rises more and more. We have a parallel situation in the case of uric acid. The elimination of uric acid in the normal and in the subject of chronic lead poisoning will be the same; but the blood of the man with lead poisoning will contain ten times as much uric acid as is to be found in the blood normally. The attempt to raise the concentration of uric acid in the blood of the normal man will fail, because the kidneys will eliminate the ingested uric acid promptly, which they will not do in the case of the subject of lead poisoning. In a word, the kidney in 
lead poisoning is tolerant, insensitive to uric acid, the level of elimination has been raised - the dam has been built up.

From this it must be borne in mind that what we really mean by hemic glucosuria is not glucosuria with analytical hyperglucemia, but glucosuria associated with physiological hyperglucemia that would become analytical hyperglucemia if the renal elimination were not effective. The adjustment for the renal elimination is usually far more fine than are our methods of analysis; the renal elimination may keep the glucose content down to within the normal analytical limits. In alimentary glucosuria, so far as we know, the elimination is always effective, the blood concentration does not rise materially. But in many cases of diabetes this fine adjustment is cut out of operation in some way unknown to us yet, the renal elimination is not effective, and the concentration of glucose in the blood increases, often to great height. When one contemplates the figures for glucose in the blood -7 to 10 per mille-and contrasts these with the amounts of sugar in the blood in any moment of time, and, finally, with the amounts of sugar eliminated in the urine during the day, one is indeed led to realize how poorly sometimes the kidney eliminates the sugar. If the input of carbohydrate be reduced in such a case, the amount of glucose eliminated will be reduced, but the concentration in the blood will remain the same. Indeed, it may be possible to render the urine free of sugar, while the blood content remains analytically much above the normal. This toleration for glucose may develop very gradually, as has been observed in cases of simple diabetes that were getting worse; but in the case of the depancreatized dog, the condition develops simultaneously with the glucosuria.

Of the nature of this modification of function we are ignorant. It is not possible to believe that it rests upon any anatomical lesion of the kind at least that we are acquainted with in the kidney. It is true that many advanced cases of diabetes have renal lesions; but early in pancreatic diabetes in dogs there are no renal lesions so far as we know. It may be objected that possibly there are renal lesions beyond the horizon of present histological methods; this, even in the face of the thousands of pages that have been written in description of the hundreds of thousands of sections of kidneys that have been cut, will be cheerfully granted. In the essential sense, structure and function may be assumed to go together in these matters. It is possible that exposure to high concentration of glucose may so affect the physicochemical constitution of the protoplasm as to render the semipermeability to glucose less effective. And, finally, it is important to realize that in this proposition we deal with a hypothesis directly opposed to that upon which the conception of renal glucosuria is founded.

If a discussion of the clinical and experimental occurrences of glucosuria is to be profitable, we must make the attempt to determine the type to which the particular instance belongs. Otherwise the list becomes simply a series of empirical findings of glucose in the urine. 
But the attempt to assign the clinical and experimental instances of glucosuria to groups is difficult always, and often impossible, owing to the fact that we have so few analyses of the glucose content of the blood, and so few controls of or experiments with the diet. The interpretation of much of the clinical data is, therefore, for the present, clearly provisional.

(a) Glucosuria Due to Excessive Ingestion of Carbohydrates, Ingestion Exceeding the Normal Glycogenetic Function of the Liver.-This form of glucosuria must be clearly separated from the melituria due to the excessive ingestion of diffusible sugars in excess of the digestive capacity of the alimentary tract. In true alimentary glucosuria an amount of glucose is poured into the portal blood in excess of the normal power of the liver to convert it into glycogen. In the alimentary melituria, sugar, not glucose, is absorbed into the portal circulation, which sugar must be in the liver transformed into glucose; if the liver cannot do this at all or if the amounts to be so transformed are in excess of the powers of the liver, the particular sugar will appear in the urine.

When cane sugar is ingested, especially in large amounts and upon the empty stomach (from 100 to 250 grams in different individuals) it may be resorbed in small part unchanged. This resorbed cane sugar passes through the liver, which has no power to split it, and is eliminated in the urine. Sometimes traces of fructose and glucose also appear in the urine, due to the fact that the quantity of glucose and fructose passed into the portal circulation as the result of the digestive cleavage of the cane sugar, was in excess of the glycogenetic power of the liver.

When milk sugar is ingested in large amounts (from 50 to 200 grams in different individuals), especially on the empty stomach, it may be resorbed in small part unchanged, and since the liver has no power to split it, it will be eliminated in the urine. Glucose will very rarely be present in the urine, due to the fact that the digestion of milk sugar is relatively slow (slower than cane sugar) and the limit of the glycogenetic function of the liver is not so easily reached. Theoretically, the use of test meals of cane and milk sugar for the purpose of determining the digestive function, the power of splitting the sugars, ought to be applicable; but practically, there is no data bearing upon the question. As a test for the glycogenetic function of the liver, however, cane sugar and milk sugar ought not to be used, glucose itself should be employed.

When fructose is ingested in large amounts (in from 100 to 200 grams) unaltered fructose may appear in the urine in traces; if the hepatic function of conversion of fructose into glucose be reduced, fructose will appear in the urine in large amounts and over a prolonged period of time. This alimentary fructosuria is a test of great value in organic diseases of the liver, as elsewhere detailed. If the hepatic function of conversion of fructose into glucose be normal and the ingestion be very heavy, glucose may appear in the urine, with possibly 
traces of fructose. The fructose reaction is a test for a specialized hepatic function, and has so far no known application outside of that.

The test meal of glucose is the correct test for true alimentary glucosuria, as the term is commonly applied. It is a test to determine the hepatic function of glycogenesis. Normal individuals will tolerate from 100 to 200 grams of glucose, taken upon the empty stomach, without glucosuria.

Melituria, due to the ingestion of excessive amounts of sugars, amounts in excess of the normal powers of digestion and of the normal glycogenetic function of the liver, is relatively common. The limit of normal hepatic function in this direction seems to be rather low in certain individuals, and during the years of adolescence and with senselessness in diet, such melituriæ may be frequently produced. They are, of course, easily detected and excluded by supervision of the diet. There is no such thing, apparently, as glucosuria following the ingestion of no matter how much starch in health; the normal glycogenetic function of the liver is superior to the digestive capacity of the intestine for starches. Such a glucosuria (glucosuria ex amylo) is always abnormal, but is not always, as was long believed, diabetic. In theory as well as in fact, there is no reason why a reduction in the glycogenetic function of the liver below the plane of the digestion velocity of starches should be peculiar to diabetes.

In some cases of alimentary glucosuria, this occurs only when the ingestion of carbohydrate is done on the empty stomach; in other cases it will occur when the carbohydrate is ingested with a meal. In some cases, the elimination of glucose continues only during the period of time occupied in digestion (a half dozen hours after the meal); in others it is much more prolonged. In some cases work during the time of digestion will prevent it; in others not.

An easily aroused alimentary glucosuria, one in particular that lasts into the next day following the experiment and that is not prevented by work, should arouse suspicions of a masked diabetes. This rule holds of course, for any easily aroused glucosuria, such as that of fright or excitement. In such individuals the limit of assimilation of sugar should be determined, and the test repeated at regular intervals.

(b) Glucosuria Due to Reduction in the Normal Glycogenetic Function of the Liver.- Whenever, in a body whose liver is poor in glycogen, the ingestion of a normal ration of carbohydrate or specifically glucose, is followed by a glucosuria, we may reasonably assume that the glycogenetic function of the liver is reduced. Such a state of affairs need not lead to more than a transient glucosuria, if the combustion of sugar and the glycogenetic functions of the muscles be normal. Otherwise glucosuria would follow every meal in the dog with an Eck fistula, which is not the case. A strictly analogous condition is the fructosuria of hepatic disease, in which the hepatic function of the formation of glucose from fructose is reduced. Here the formation of glycogen from glucose is reduced. In the one case the fructose passes into the general 
circulation; in the other, glucose. These conditions may be classed under the term, in use especially in France, heptargia. The alleged reduction in the toleration of sugar in pregnancy requires confirmation.

Reduction in the power of forming glycogen from glucose is present in all severe cases of diabetes. Occasionally, in the febrile infections, in pneumonia and influenza, especially in exophthalmic goitre, in alcoholism of acute type and in lead poisoning, we observe instances of glucosuria following the administration of very moderate rations of sugar. Since it is quite certain that the liver in these conditions is poor in glycogen, it seems reasonable to regard these instances of glucosuria (which may even be glucosuria ex amylo) as due to reduction in the glycogenetic functions of the liver.

In another group of cases, chronic in nature, the same conditions are noted; in exophthalmic goitre, in gout, obesity, hypertrophic cirrhosis of the liver, in fatty liver, chronic alcoholism, and chronic fever. It is clear from an analysis of these conditions, that it is not always possible to rule out defective glycolysis, and that in any event, the state of affairs in the liver is different in the different conditions. In some it is possible that the liver, instead of being poor in glycogen, is overstocked and can hold no more. In others, the liver is infiltrated with fat and affected with fatty degeneration, conditions in which the formation of glycogen from glucose is antagonized. In some neither of these conditions hold, and we have apparently simply a reduction in this function of the liver. In most cases of profound organic disease of the liver, the function of glycogenesis is retained in a most remarkable manner. In fact in the most advanced states of fatty degeneration of the liver, as may be seen in pernicious anemia sometimes, the function is normal. It is usually normal in jaundice. In organic diseases of the liver as a class, this function of the liver is usually retained while the function of conversion of fructose into glucose is usually lost. So far as gout is concerned, it is most often in gout with obesity that this glucosuria is met with. And in these instances, as well as in the apparently simple cases of obesity in which it is encountered, time will not infrequently reveal that the cases were masked incipient diabetes.

There is supposed to be a certain parallelism between glycogenesis and the distoxication function of the liver. If the reactions of distoxication were all, as so often, reactions with the formation of complex combinations of the general type of glucosids, the relation to glycogenesis would be intelligible. But it holds also for distoxication of the metals, which are combined with fractions from the protein and not from the carbohydrate group. Diseased liver cells that have lost in part their power of converting glucose into glycogen are often the subject of more or less extensive fatty degenerations, and these may possibly operate against the protein reactions with the heavy metals.

Assuming for the purpose of argument, that the actual state of affairs in this abnormality consists in a reduction in the glycogenetic ferment, the above presentation assumes further that this is due to 
disease of the liver cells that produce this ferment-just as hepatic fructosuria is supposedly due to the non-formation of the transformation ferment on account of disease of the liver cells. But it is possible that the modus operandi is not direct, but indirect. Ablation of the pancreas is followed promptly by reduction in the glycogenetic function of the liver. In many of the instances cited as illustrations of this type of glucosuria, it is certainly an open question if we are not dealing with acute or chronic disease of the pancreas, recoverable in nature, leading to a certain degree and for a certain time to reduction in the glycogenesis of the liver. How the pancreas brings about this result is not known; whether by repression of the formation of the glycogenetic ferment, by the removal of some condition essential to the action of a zymo-excitor, or by excessive glycolysis, we have as yet no idea.

(c) Glucosuria Due to Exaggeration of the Glycolytic Function of the Liver.-This is a very common type of glucosuria. It consists in the rapid conversion of glycogen into glucose, in excess of the combustion needs of the body and in excess of the glycogenetic function of the muscular system. There is no doubt that when the liver is rapidly emptied of glycogen, the normal glycogenetic function of the muscular system is easily exceeded, and a temporary glucosuria is the result. The criterion of this type of glucosuria is that it does not occur following starvation, hard work or refrigeration; in other words, it cannot occur when the liver is poor in glycogen. This and the type just described are opposites in this. When a procedure that causes glucosuria by exaggeration of hepatic glycolysis is tried in an animal whose liver is poor in glycogen, it will fail, nor will glucosuria occur if the animal be given a moderate amount of sugar. The procedure that causes glucosuria through defective hepatic glycogenesis will not cause glucosuria in a temporarily starving animal whose liver is full of glycogen, but will do so in an animal whose liver is poor in glycogen, following the ingestion of a normal ration of sugar. In practice, however, it is frequently not possible to classify the results, as glucosuria occurs either way, $i$. e., the two types exist in the same individual.

The classical illustration of this type of glucosuria is to be found in the medullary puncture of Bernard. Many forms of traumatism of the nervous system may produce this glucosuria, though not so uniformly. If the shock or stimulation of any part of the central nervous system, even of the cervical or thoracic sympathetic ganglia, be pronounced enough, it acts upon the glycogenetic centre just as the actual puncture would. Of disease lesions that may so act, we may mention tumors, apoplexy, concussion, and sclerosis. In some of the chronic organic diseases of the nervous system intermittent glucosuria occurs, and it is usually to be classed here. In some of these instances, however, the condition is dependent upon the ingestion of carbohydrates, so that they may belong to the type just previously considered. Functional conditions of the nervous system may also bring about this glucosuria. Thus fright or extreme excitement of any kind may be 
associated with glucosuria; of this the glucosuria seen in chained or caged wild animals is an illustration. These glucosuriæ are all transitory or at least intermittent. They are due to the sudden unloading of the hepatic glycogen in the form of glucose, brought about we believe by a sudden excessive formation, or at least action, of the glycolytic ferment of the liver.

A great many poisons, that in large doses produce glucosuria, seem to operate by action upon the glycolytic centre in the medulla oblongata. Strychnin, which produces glucosuria when given in huge doses, does not so act if the spinal cord be cut or if the liver be removed or made free of glycogen. Arsenic apparently tends to produce glucosuria in both ways. By a direct action upon the liver cells, it depresses the function of glycogenesis; by its action upon the glycolytic centre, it tends to exaggerate glycolysis in the liver. Morphin, chloral hydrate, amyl nitrite, cyanids, nitrobenzol, anilin, antipyrin, and many other drugs in large doses act in the same manner, by stimulation of the glycolytic centre. For some of these, the question of an internal asphyxia may be properly raised, though the present data justify their provisional classification here. In any event, the matter is of lesser importance, since it has been shown that asphyxia is not associated with glucosuria in an animal whose liver is free of glycogen.

The action of some poisons is very difficult of explanation. In phosphorus poisoning the liver is freed of its glycogen, without, however, either hyperglucemia or glucosuria occurring. It is not known what becomes of the glucose. It might have been burned in excess. It might have been converted into fat or it might have been converted into lactic acid; in any event its storage in the muscles in the form of glycogen is excluded.

Caffein and the kindred diuretics may produce a glucosuria that is related to the unloading of glycogen in the liver. If caffein be given as a diuretic to an animal poor in hepatic glycogen, no glucosuria will occur. But if with the caffein be given a moderate ration of sugar, glucosuria may occur. Whether this can be due entirely to disturbance in the glycogenetic function of the liver is questionable, since the glucosuria is proportional more to the diuresis than to the input of sugar. It is possible that we have here an illustration of a slight, transitory lowering of the renal level of the blood sugar, an illustration of a toxic renal glucosuria, in part at least.

When solutions of common salt of certain concentration are injected intravenously, glucosuria follows. Since glucosuria does not occur after section of the splanchnic nerves, it is inferred that sodium chlorid, like the poisons mentioned, acts on the glycolytic centre. 'The glucosuria is prevented by calcium solutions, and this fact strengthens the interpretation given, since calcium is antitoxic to sodium under these circumstances of experimentation. Some of the investigations have yielded results that suggest that possibly the renal permeability to glucose is reduced by the action of strong saline solutions. In the 
absence of adequate blood analyses, however, this suggestion cannot be properly evaluated, though it appears to be reasonable.

Exceeding important, in fact and in theory, are the relations of the adrenal bodies to the sugar metabolism. 'There are great experimental difficulties, and the data are in part very conflicting, but a number of certain and striking facts stand out: (a) The injection of epinephrin causes in the normal animal glucosuria and hyperglucemia; $(b)$ the ablation of the adrenal bodies causes hypoglucemia; and $(c)$ the ablation of the adrenal bodies prevents the glucosuria of medullary puncture.

The injection of large doses of epinephrin causes glucosuria not only in the normal animal, in the animal with liver filled with glycogen; it causes glucosuria in the starving animal as well, not so pronounced in amount but still present. It does not cause glucosuria in the starving animal following the action of phloridzin. The best interpretation is to assume that epinephrin stimulates the glycolytic function of the liver and of the muscles as well. When the body has been well drained of glucose through the combined actions of the combustions and phloridzin elimination, epinephrin loses its effect. This interpretation is supported by the observance of the disappearance of muscle glycogen following the use of epinephrin. It must, however, be shown that the period of muscle loss of glycogen is coincident with the period of hyperglucemia, for this interpretation to stand. The idea that epinephrin acts as a depressor to the internal secretion of the pancreas, and thus produces glucosuria in an indirect way, has not received support in the latest experimental studies. Epinephrin is probably a direct stimulant to glycolysis. In how far glycolysis in the daily functions of the organism is regulated by the adrenal bodies is entirely unknown. The glucosuria of epinephrin is in no way an expression of its vasomotor properties.

The ablation of the adrenal bodies causes hypoglucemia. This is very difficult of explanation. It cannot with our present ideas be conceived as operating through the pancreas, since no known exaggeration of pancreatic action can cause a hypoglucemia. There is no excess of combustion, there is no depletion of body glucose through renal elimination. All that seems to be left is to assume that the station of equilibrium between glucose and glycogen (or between glucose and fat) has been shifted away from glucose and toward glycogen. In other words, the storage of glycogen in liver and muscle is increased at the expense of the blood concentration of glucose. The emotional glucosuria of animals does not develop after adrenalectomy.

Ablation of the adrenal bodies prevents glucosuria after the Bernard puncture. This has been interpreted to mean that the fibers from the glycolytic centre to the liver run via the sympathetic nerves through the adrenal bodies, or that the centre operates in some way through the mediation of the adrenal bodies. No interpretation of the fact is possible with the present data.

Recent experimental researches indicate that either hypercapnia 
or acapnia may be followed by hyperglucemia and glucosuria. Though the mechanism of these relations is not yet clear, it seems probable that some of the diverse clinical forms of glucosuria of acute type belong to the one or other of these conditions.

Lastly, the glucosuria of diabetes is in part founded upon an exaggeration of hepatic glycolysis and constitutes one of the metabolic defects of diabetes, clinical and experimental.

(d) Glucosuria Due to the Reduction of the Glycogenetic Function of the Muscles, or Reduction in Their Storage Capacity for Glycogen.Such a state of affairs would lead to hyperglucemia, under certain conditions of diet and hepatic function at least, and thus to glucosuria. Such a state of affairs exists in diabetes. Beyond this, we know little. Some of the facts known for phosphorus poisoning could be reasonably interpreted as due to defective muscular glycogenesis. It is possible that some such thing might occur in the muscular dystrophies. When the motor nerve of a muscle is cut, the muscles become rich in glycogen. How long this state of affairs lasts, whether it continues during the later atrophic degeneration of the muscle fiber, is not known. Muscle cells in chronic neuromuscular diseases may be found poor in glycogen; but there are no control studies to indicate that this may not have had a general nutritional foundation.

(e) Glucosuria by Exaggeration of the Glycolytic Functions of the Muscles.-This would result in sugar being returned to the circulation, something that we do not believe ever occurs normally. It is quite certain that it occurs in diabetes, else how can we understand the disappearance of glycogen from the muscles in acute pancreatic diabetes, when the body burns almost no sugar and there is pronounced hyperglucemia. It means a shifting in the equilibrium glucose $\longleftrightarrow$ glycogen. Carbon monoxid, ether and chloroform produce glucosuria in the animal whose liver is free of glycogen and without the ingestion of carbohydrate. Since in these instances the glucosuria is apparently due to hyperglucemia and not to any lowering of the renal level, unless the sugar can be derived from an exaggeration of the protein catabolism (which does not appear to exist) there seems to be no way of deriving the sugar except by abstraction from the stock of muscle glycogen. It is not to be inferred that the action of these intoxications is confined to the direction here under consideration. It can be shown that ether and chloroform reduce the glycogenetic function of the liver. And carbon monoxid produces a larger glucosuria, apparently, with the liver stocked with glycogen than empty. Carbon monoxid does not act through simple oxygen hunger, since this does not in itself lead to glucosuria.

(f) Glucosuria Due to Reduction in the Formation of Fat from Sugar. - The result of a reduction of this function would under certain conditions of diet lead to hyperglucemia. It occurs in diabetes as one of the defects of that disease. 
(g) Glucosuria Due to Reduction in the Combustion of Glucose in the Muscles.-This is the cardinal defect of diabetes. It is likely that it occurs, to some extent and as a transient condition, outside of diabetes. It is possible that such a defect is produced in one of two ways; either the muscle ferment is defective or the pancreatic zymoexcitor is defective. We know nothing of the relations of the muscle ferment. We know that ablation of the pancreas or loss of its function through disease leads to loss of the power of burning glucose in the muscles. It is possible that acute febrile infections, or local lesions or infections in the duodenum or bile ducts, might lead to mild inflammatory or infectious lesions of the pancreas, attended with partial loss of the internal function, followed by recovery. Possibly some of the glucosuria of febrile diseases have this etiology. It is clear that this hypothesis might also explain the action of some poisons that up to the present have been considered to act upon the liver for the production of glucosuria. This is a matter for controlled future investigation.

Glucosuria of Unknown Cause.-Leaving aside the many occasional findings of sugar in the urine without determinable causation, there are certain quite regular types that must be mentioned. One of these is the glucosuria of acromegaly, due probably to lesions in the hypophysis cerebri. Another is the singular glucosuria of starvation seen in some animals. There is no such thing as a starvation diabetes in man. The vagabond glucosuria that was once regarded as such, is probably usually due to chronic alcoholism. The glucosuria associated with certain diseases of the skin is in a large percentage of cases a presenting symptom of insidious, so-called latent, diabetes. The glucosuria of acid intoxication is wholly unclear. The glucosuria of adolescence, an uncommon but undoubted condition, is also unclear, though its relations to vasomotor variations suggest that it may be of renal type. Glucosuria may occur in connection with refrigeration, and may here be accompanied by lactic acid in the urine. Refrigeration is, of course, associated with excessive combustion of sugar and the consequent disappearance of glycogen. Unless the cold, like shock, acts upon the glycolytic centre, the phenomenon is very singular.

Glucosuria Due to Renal Permeability.-In all the instances above considered, glucosuria is due to hyperglucemia; the excess of blood glucose simply flows over the renal gate. Under true renal glucosuria we understand the elimination of glucose in the urine in the absence of hyperglucemia, due to the lowering of the level of renal retention of glucose. This level may not only be lowered, it may be practically abolished, so that the concentration of glucose in the blood becomes very low. The classical illustration is furnished in phloridzin intoxication.

Phloridzin is a glucosid which on cleavage yields phloretin and glucose. When injected once into the circulation, glucosuria appears within a short time, persists for a number of hours and then disappears. With a moderate dosage, the glucose concentration of the blood is 
not lowered, being maintained by the glycolytic functions of the liver and muscles. When the animal is starved and the administration of phloridzin is properly proportioned and long continued, the glucose concentration of the blood is lowered, sometimes markedly.

The action lies in the kidney. If the kidneys be extirpated and the animal then poisoned with phloridzin, there is no increase in the blood concentration of glucose above that of the control animal. In birds, in whom the kidney is very resistant to hyperglucemia, the administration of phloridzin is promptly followed by glucosuria. Perfusion of the kidney with a phloridzin-containing blood is followed by the elimination of glucose in the secreted urine. When phloridzin is injected into one renal artery, glucosuria appears first on that side and on the other side only after time has passed for the glucosid to have been carried to the other kidney by the circulation. 'The action of phloridzin is not prevented by extirpation of the liver, while it lowers the hyperglucemia produced by extirpation of the pancreas.

An early hypothesis for the action of phloridzin lay in its cleavage. It was supposed that the kidney split the glucosid into the two component parts and eliminated the glucose fraction. The phloretin then continuing in the circulation, combined with another molecule of glucose to regenerate the molecule of phloridzin, which in turn was again split in the kidney and the glucose eliminated, etc. This hypothesis failed because it could not be harmonized with the facts. It was in the first place entirely unclear why the kidney should eliminate the molecule of glucose derived from the postulated cleavage of the phloridzin; to assume a secretory defect in the kidney means to yield the entire argument. Furthermore, under the terms of this hypothesis, a molecule of phloretin should have the same action as a molecule of phloridzin, which is not the fact. The assumption that the kidney splits phloridzin into glucose and phloretin is not supported by experiments with renal pulp. The curves of elimination of sugar under controlled and varied conditions of diet are entirely against this hypothesis.

A widely supported theory attributes to the phloridzin the power of splitting sugar combinations of the blood, with elimination of the freed sugar. The hypothesis runs to the effect that the kidney retains only bound sugar, not free sugar; that the sugar of the normal serum is bound and therefore none is susceptible to renal elimination. In phloridzin intoxication, the kidney acquires the power of splitting the sugar combination, setting the sugar free, when the normal renal action eliminates it. The data upon the basis of which it is assumed that the larger part of the glucose of the blood is bound in complex form, is not chemically trustworthy, as already stated; on the contrary, the best physico-chemical investigations lead to the conviction that the larger portion of the sugar of the blood is free. There is something rather mystical in the hypothesis that a poison bestows upon a tissue a specialized function that it does not normally possess. The 
curves of glucose elimination under the influence of phloridzin, with known and controlled conditions of diet, are not to be harmonized with this hypothesis. The fact also that in nephritis and in the sclerotic kidney of old age, phloridzin has little power to produce glucosuria, is opposed to this hypothesis.

The best explanation is simply to say that the phloridzin abolishes or reduced the property of the kidney to restrain glucose from elimination, just as renal lesions reduce the power of the kidney to effect elimination of certain substances. In each instance a function is lowered; in the one instance a retention function of a semipermeable membrane is lowered; in the other instance an elimination function of a semipermeable membrane is lowered. That the phloridzin glucosuria cannot be exaggerated by diuretics does not speak against this interpretation. We form no picture of this defect of the kidney; future investigation must determine that. An anatomical defect is not known; nephritis and albuminuria are not produced in ordinary phloridzin intoxication, except after long-continued and excessive administration.

The march of events in a long-continued intoxication with phloridzin is very instructive. The continued elimination of the glucose in the urine, together with the normal combustion of glucose, serve to reduce the glucose concentration of the blood. To replace the loss, the liver converts its glycogen into glucose, and soon becomes free of glycogen. Thereupon the glycogen of the muscles maintains the combustions of the body. The elimination of sugar continues. Whether this sugar is now in part derived from the glycogen of the muscles, returned into the circulation, is not known, though it has been usually assumed that this is the case. When the carbohydrates of the body are reduced to a low point, the body falls back upon the combustion of fat and protein. From the amino-acids of the protein catabolism glucose is formed; and this, too, of course, falls prey to elimination. It is probable that from the moment the hepatic glycogen is exhausted the body maintains its combustion upon the glycogen of the muscles so long as it lasts, and the sugar of the urine represents, therefore, sugar formed from protein solely. It is difficult to prove this; but if this interpretation were to be adopted, we would be spared the assumption that the glycogen of the muscles after conversion into sugar, instead of being entirely burned there, is in part returned to the circulation. When the glycogen of the muscles is low or exhausted, then the body is entirely dependent upon fat and protein. Both are burned, in different proportions in different animals. In different specimens of urine from different dogs, the glucose : nitrogen ratio may vary from 4.4 to $2.8: 1$. These represent partly the ratios of utilization of fat and protein in different animals, in part the different ratios of sugar formation from the unit of protein in different animals. That these eventualities explain entirely variations in glucose : nitrogen ratios, is not, however, asserted. The higher ratios are seen in the earlier days, and depend on the conversion of hepatic glycogen into sugar. Later the ratio is usually close to $3.6: 1$. 
When to such a starving animal, fully intoxicated with phloridzin, sugar is administered, it will not be burned, it will be eliminated, the variations being slight and irregular. If lactic acid be administered, the glucosuria will be increased. Glycerol has the same action. And these two experiments constitute beautiful illustrations of the reversibility of reactions.

When fats are ingested, the glucosuria is not increased. It has been stated, on experimental grounds that do not afford a basis for positive confidence, that the administration of fat in excess to a phloridzinized animal is followed by the deposition of glycogen in the muscles, though not in the liver. The amounts reported are not enough to exclude the glycerol of the fats as the responsible source.

When protein (protein free of preformed carbohydrate) is administered, the sugar in the urine rises and maintains a relation in each animal, quite parallel to the curve of nitrogen elimination. Many amino-acids have the same result upon the glucosuria. Glucose is formed from some of the amino-acids of the catabolized protein, and some may be burned, though largely eliminated. In such phloridzinized dogs many of the most conclusive demonstrations of the formation of sugar from protein have been accomplished. Phloridzin does not in itself exaggerate the protein catabolism. On account of the losses of glucose in the urine, the body exaggerates the catabolism of protein to cover its needs. Since the burning of protein is an expensive way of supporting the body heat, and especially in connection with the constant loss of sugar, the heat metabolism is greatly exaggerated. Much fat will be burned, if available, and this leads to the acetone complex, to acidosis.

Marked fatty infiltration of the liver occurs in connection with phloridzin intoxication. This is another illustration of the antagonism between fat and glycogen in the liver. There being no glycogen, fat wanders in, the liver cells being normal. If a heavy sugar input be accomplished, or if the administration of phloridzin be discontinued, the glycogen will return to the liver and the fat will emigrate to the peripheral fatty depots.

Natural Renal Glucosuria.-Does renal glucosuria occur as a state of disease? Mention was made of the possibility of the glucosuria of salt injections and of caffein being in part at least the expressions of a lowering of the renal retention of glucose. In some diseases of the kidney, notably in the arteriosclerotic type of gout and in the arteriosclerotic form of chronic nephritis, glucosuria is common. It is in just these cases that the renal level for uric acid is known to be raised. It is possible that here the renal level for sugar may be lowered. Careful investigations, with controlled and varied relations of diet and with frequent analyses of the glucose of the blood, would probably afford a definite decision. Since these individuals are helped rather than harmed by venesection, little difficulty need be expected from this direction. A complication, however, would lie in the fact that follow- 
ing venesection, a rise occurs in the sugar concentration of the blood. Nevertheless, in view of the great difficulties attending the production of chronic nephritis in animals, such investigations offer promise of results.

\section{THE CARBOHYDRATE METABOLISM IN DIABETES}

The disturbances of metabolism in diabetes are of such importance that an understanding of them affords conceptions of metabolism at present of greater value to the physician than any derived from the study of any other disease. Many of the cardinal facts of metabolism have been determined through the study of diabetes and allied conditions. In diabetes, clinical as well as experimental, we are able to view and to follow definite alterations in the chemical procedures in the body. Of all diseases, outside of the microörganismal infections, diabetes permits of experimental production in the most classical manner; and the inter-interpretation of the facts of clinical and experimental diabetes has afforded striking illumination of the clinical disease. Finally, we have in diabetes the best-known instance of an autointoxication. It will be advantageous to discuss seriatim the disturbances in metabolism that are known to occur in this disease.

Definition of Diabetes.-It will be necessary in the first place to define the use of the term diabetes. The facts determined for diabetes due to removal of the pancreas in the dog, may in many instances be utilized as a control in our definition of the clinical term diabetes. From the standpoint of the student of metabolism, diabetes in the human subject may be more accurately defined than has been possible according to usual clinical standards. For the definition of the momentary status of an instance of glucosuria, it is a clinical rule that if the condition subsides promptly on the withdrawal of carbohydrate from the diet, true diabetes does not exist. Occasionally a typical case of diabetes develops in an individual who for a time previous has exhibited nothing but an alimentary glucosuria, a lowered limit of assimilation for carbohydrates. In the instances of glucosuria that persist after the withdrawal of carbohydrate from the diet, the younger the subjects the larger will be the proportion of cases of typical diabetes. We may group these cases of extra-alimentary glucosuria under three headings.

(a) Classical diabetes, most often seen in those under middle life, of acute or relatively rapid onset; causeless so far as the subject is able to determine; not preceded by disease of the liver, gout, obesity or other known condition; of rapid course, and exhibiting all the metabolic abnormalities to be later detailed; paralleling pancreatic diabetes in the $\operatorname{dog}$ and proceeding relentlessly to a fatal termination. Lesions of the pancreas-rarely gross, sometimes leading to a more or less complete distruction of the gland, involving specifically or solely the 
islands of Langerhans and resulting in their atrophy and disappearance - are found in practically all bodies dead of such diabetes.

(b) Instances of diabetes that begin in simple alimentary glucosuria, and pass slowly and insensibly into typical forms of diabetes, differing from those under subheading (a) only in much greater chronicity. The majority of these cases of diabetes are to be seen in after-middle life. Some of these present every metabolic sign of diabetes; many, however, do not; in particular the power of forming and of burning fat is often retained.

(c) Instances of diabetes that are apparently founded upon previous disease, as gout, arteriosclerosis, obesity, or cirrhosis of the liver. While a small percentage of the cases thus determined to possess glucosuria on a diet free of carbohydrate do develop into typical diabetes with all the metabolic signs of the disease, the larger proportion of them do not present all the metabolic signs, the power of forming and burning fat being most often retained. One meets with instances in which through years glucosuria persists despite all regimen, without emaciation, free of the acetone complex, with few of the symptoms of diabetes. It is in the bodies of those who have suffered from diabetes of the forms under $(b)$ and $(c)$ that lesions of the pancreas may be missed, and specifically the islands of Langerhans are to be observed in normal or reduced numbers.

Etiology of Diabetes.-So far as we have experimental knowledge, the pancreas is the only organ the loss of whose function is followed by diabetes. There is experimental evidence that the internal secretions of the thyroid and adrenal bodies are antagonistic to that of the pancreas; and that exaggeration of the function of these bodies tends to the depression of the internal function of the pancreas and of the combustion of glucose. But there is no evidence, clinical or experimental, that such exaggerations of the functions of the thyroid or adrenal bodies ever so far depresses the internal function of the pancreas as to cause diabetes, though experimentally they may intensify glucosuria. So long as we possess no further knowledge it will be safe to refer to the pancreas, directly or indirectly, the etiology of all cases of typical diabetes, whether the histological examination with present methods reveals alterations or not.

The clinician, on the other hand, must realize that glucosuria merely, even ex amylo or persisting after the complete withdrawal of all carbohydrate from the diet, will probably not be found in the future to be an absolute criterion of diabetes. The fundamental defects of the diabetic metabolism are inability to burn sugar and inability to burn fat as in the normal. There is evidence that hyperglucemia and glucosuria may persist in a body that burns fat perfectly and sugar fairly well; in whom, in short, there is but a partial defect. This partial defect may indeed become complete. But it may remain stationary and the subject live indefinitely in that condition. The normal man has an almost unlimited power of burning sugar. This may be greatly reduced 
without the man becoming a typical diabetic, and without the other metabolic disturbances of classical diabetes becoming established.

It has been long assumed, in a half-conscious manner that has rarely found definite expression inside of the physiological laboratory but which has nevertheless moulded past conceptions of the disease, that there is a total suboxidation in diabetes. By this we mean that the total combustions in the diabetic body are less than in the normal. By fuller utilization of the mechanism of heat dissipation (such as occurs in myxodema) it would be possible to maintain the body temperature on a plane of total oxidation considerably lower than the normal, though with a corresponding restriction of the function of adaptation of the organism. But the direct experiment, the measurement of heat production in the diabetic by the methods of direct and indirect calorimetry, has demonstrated the incorrectness of this assumption. In fact, the total heat production of the diabetic body is found to be a little above the normal, 6 to 7 per cent. A calculation of the data obtained from dogs with experimental diabetes leads to the same conclusion. This is exactly what should have been expected on mechanical as well as thermodynamic grounds. It takes more fuel to get ten horse-power out of a worn ten horse-power engine than out of one in perfect condition; and it requires more fuel to yield 2000 Calories of heat available in terms of cellular energy in the disturbed metabolism of the diabetic body than in the normal individual. While it is true that the diabetic has lost in whole or in part the power of burning glucose, the power of burning most other substances is retained. Since the total combustions are normal or excessive and the combustion of glucose low, it must follow that the combustion of fat and protein though qualitatively abnormal is still excessive; and this is the fact, determined by direct test. In the terminal stages of diabetes, as in diabetic coma, the total combustions of the body are probably reduced below the normal.

A mechanical illustration will make the matter clear. Modern marine boilers are installed for heating by the burning of crude oil or of coal, the fire-box having a double installation. Now the installation for burning oil may become disabled without the installation for the burning of coal being in the least disturbed; under such circumstances steam would be kept up by the burning of coal. So in the diabetic body, the installation for the burning of glucose breaks down, that for the burning of protein and fat is retained, and the body produces heat by the combustion of these substances. As will be later pointed out, frequently the installation for the combustion of fat breaks down also, leaving the poor diabetic body with very defective means to maintain heat production. Even under these extreme circumstances, the power of the body to burn many extraneous chemical substances is not reduced, and the total heat production is at least normal.

It is important in this connection to make one further point clear. 
While the total combustions of the resting body in diabetes are normal or excessive, the facultative increase of combustion in the sense of the normal power of work is always lowered. 'To the point of absolute muscular exhaustion, the normal individual can increase the combustions of the body for the support of work; the limit of muscular work lies in the fatigue of heart and neuromuscular apparatus, not in any limit of combustion. With the diabetic, however, this facultative increase of combustion for the support of muscular work is limited; in marked degree, it is true, only in severe diabetes.

Experimental Diabetes.-It will be of advantage at the outset to sketch the known facts of experimental diabetes. This is provoked by the abolition of the internal function of the pancreas, either by extirpation or atrophy of the organ. Complete extirpation of the pancreas in the dog is followed within a few hours by glucosuria, the to-be-enumerated metabolic defects develop rapidly, the animal wastes, the wound of operation usually refuses to heal and the animal dies within a few weeks of septic infection or diabetes. The behavior of the wound of operation in the dog is in striking similarity to the behavior of human diabetic tissues under operations, long known through unfortunate experience to exist in the human subject with diabetes, in whom sepsis and gangrene occur upon slight provocation. In older dogs, the reaction of the animal is more favorable, the wound heals, the duration of life is prolonged, and polyuria, polydipsia, and polyphagia develop in characteristic manner. In the rapid decline to be often observed, especially in younger dogs, in whom sepsis is the chief cause of death (though the intensity of this sepsis rests upon the reduced tissue resistance of diabetes), the symptoms of polyuria, polydipsia, and polyphagia may not occur at all. If a small part of the pancreas be left, with proper arterial and venous attachments, diabetes will not develop at the time. If later this piece be extirpated at a second operation, diabetes will develop at once. In some instances, diabetes develops gradually, due to the atrophy of the piece of pancreas left within the body; in other instances, for a year at least, diabetes has not developed. If the common pancreatic duct, or ducts, be ligated, the gland will undergo a gradual atrophy, and later diabetes will supervene. In some instances, however, in which the operation of ligation has been done with great care, diabetes has not developed within a year, the only symptom related to the metabolism being a greatly reduced limit of assimilation of sugar. On section of such an animal, a mass of scar tissue is found to occupy the site of the pancreas; and in this scar tissue is no trace of acinal pancreatic tissue, but islands of Langerhans are to be seen. It may be confidently believed that after the lapse of still longer time, when the tissue has had opportunity to undergo complete atrophy, which may be greatly prolonged, diabetes would develop. Should the experiment not justify this prediction, the best explanation would be to assume that the islands of Langerhans are not derived from the acini of the pancreas, and that complete 
atrophy of the secretory portions of the gland may occur with retention of the internal function of the pancreas, due to retention of the islands of Langerhans in state and number sufficient to maintain functions. It may also be confidently predicted that if the atrophied scar tissue of the site of the pancreas be removed, diabetes would develop.

The best method of establishing the disease experimentally is to do the operation in two stages, allowing a portion of the gland to remain, either to undergo atrophy or to be removed by a later operation. The operation succeeds, $i$. e., produces glucosuria and inferentially all the rest, in birds, frogs, and lizards.

Hyperglucemia is promptly produced, and is the cause of the glucosuria. The limit of assimilation of carbohydrates is reduced to the lowest level. If the liver be removed with the pancreas, which is possible in the frog, hyperglucemia and glucosuria do not occur. Starvation lowers but does not remove the glucosuria. The explanation of this fact is as follows: The glucose eliminated by the diabetic comes from carbohydrates of the diet and from protein, either of the diet or from the tissues of the animal. The sugar derived from protein is formed in the liver. With the liver removed, therefore, there is no formation of glucose from protein and consequently no glucosuria.

At autopsy, the liver and muscles are found to be very poor in, or totally free of, glycogen. The liver, muscles and blood are, however, rich in fat. These results remind one of the findings in starvation. The blood will not produce glucosuria, or any other result, on injection in large amounts into a healthy animal. Nor will the injection of the blood of a healthy dog have the slightest effect on the glucosuria of the depancreatized animal. The blood of the diabetic dog does not display any alteration in the normal slight glycolytic activity. The administration by mouth or hypodermic injection, of pancreatic extracts has no influence upon the course of the metabolism.

If the $d o g$ be reduced to the lowest level of starvation before the operation, glucosuria may not occur, only a moderate hyperglucemia. Though little sugar can be burned by such an animal, still less is formed in the atrophied state of the tissues. This fact does not speak for or against the possible origin of sugar from fat. The administration of epinephrin exaggerates the glucosuria in early cases. This is due to the fact that this substance stimulates the glycolytic function of the liver to even more than the already abnormal extent present in diabetes. When the liver has been cleared out, so to speak, further injections of epinephrin have no further action in this direction.

It has been stated that ablation of the pancreas in the pregnant dog does not result in diabetes, the interpretation being obviously that the internal secretions of the pancreas of the young in the uterus pass into the maternal circulation and carry on the function. In the human subject, pregnancy does not act as a positive influence upon diabetes.

All the metabolic defects in the diabetic, to be detailed seriatim, hold in the diabetes of the depancreatized dog. It is not common, 
except in the late stages, for the function of combustion of sugar to be entirely lost, though it is always very low. The glucose: nitrogen ratio runs about 2.7 to $3: 1$, it may be as low as $2: 1$, or as high as $5: 1$. When it is recalled that it was with the dog that the so-called normal ratio, supposed to represent the relation of the formation of sugar from protein $(4.4: 1)$ was established, one is led to infer that the diabetic dogs still burn some glucose.

The sum total of our experience with pancreatic diabetes runs to the effect that the pancreas forms an internal secretion that in some way is essential in the muscles to the combustion of sugar, carrying what in a general way may be termed the function of a co-ferment or zymo-excitor. The pancreas does not itself form a glucolytic ferment, but it does form something that is in some way necessary for the combustion of glucose by the muscle cells. Whether all the other defects in the metabolism of the diabetic are secondary to this or whether the removal of the pancreas affects directly other acts of metabolism, notably the formation and combustion of fat, will be discussed later. When an extract of pancreas is mixed with an extract of muscle and a solution of glucose, the glucose disappears in part. Properly executed, there is no question of the fact of this result. Recent experiments, however, indicate that the glucose, instead of being burned (lactic acid or alcohol have never been demonstrated in the mixture), has been converted into a maltose. In other words, the experiment yields the condensation or polymerization function of the muscle, (of which the physiological expressions are the formation of maltose and glycogen from glucose in the muscle cell) instead of the combustion of sugar. It is not now possible to make any definite statement of the relation of the pancreas to the muscle. Certain only is the fact that in some chemical way the pancreas coöperates with the muscle in the combustion of sugar. It is the conviction of most workers that this internal function of the pancreas is localized in the islands of Langerhans.

Physiologists who endeavor to locate the etiology of diabetes in the central nervous system, have for two reasons objected to the interpretation of the results of extirpation of the pancreas here given; firstly, that extirpation of the duodenum produces glucosuria; and secondly, that the results of extirpation of the pancreas persist when pancreatic extracts are injected into the animals. That extirpation of the duodenum causes a glucosuria is true; but that extirpation of the duodenum, without injury to the pancreas, causes a diabetes with the metabolic disturbances known to exist in pancreatic diabetes, has not been shown at all. The second objection carries more weight, though it is not now tenable. The results of extirpation of the thyroid body are completely nullified by injection with thyroid extracts, and it is assumed that the same ought to hold for the pancreas. But for this line of argument to hold, it would need to be assumed that the metabolic products of all tissues are stable, as thyroid substance is - a conten- 
tion that no physiological chemist could concede. Not only may the substance supposedly formed in the pancreas be unstable, it is also probable that it is continuously secreted, and that the gland contains at the one moment of preparation of the extract only the merest trace of the substance. The experiment contains so many possibilities of error that the present failure, or even the definite failure, of injections of pancreatic extracts is not a valid objection to the interpretation of the facts given - that the pancreas furnishes to the body a chemical substance essential in the muscles to the normal combustion of sugar.

The fact that acromegaly is sometimes associated with diabetes is not out of harmony with the statement that the pancreas is the only tissue of the body whose removal will provoke diabetes. The relations of acromegaly to diabetes are certainly unclear. But lesions of the hypophysis or the removal of that body does not lead to the establishment of diabetes, as in the case of removal of the pancreas, though the limit of assimilation of carbohydrates is disturbed. The experimental data are not clear-cut at present, and in part even contradictory. But in a tentative way we may define the relations of the hypophysis to the metabolism of carbohydrate as follows: Overfunctionation of the body results in lowering of the toleration for carbohydrates; upon what this is founded, is not known. Deficient functionation, as ablation, increases the toleration of the body for carbohydrates, the animals will tolerate without glucosuria large injections of glucose. The mechanism of this increased toleration is not clear; in particular, we do not know whether it is due to hyperglucemia with increased impermeability of the kidneys or to increased glycogenesis. The dogs who have undergone this operation tend to fatten rapidly, which is what would be expected in accordance with the law of mass action. The relations of these functions to the pancreas are unclear.

In all discussions of the relations of disease of the pancreas to diabetes, it must be understood that disease of the pancreas in toto is not present. It is rare even in the typical rapid diabetes of youth, for the intestinal functions of the pancreas to be in the least disturbed. On the other hand, obstruction of the common pancreatic duct or tumors of the pancreas do not lead to diabetes unless atrophy of the whole tissue follows. The secretory function of the pancreas as a digestive gland and the internal function of the pancreas as related to the metabolism of carbohydrate, are separate and independent functions. We have only to review the work of the liver to realize how many chemical functions may devolve upon one organ. This conception is made more easy in the case of the pancreas by the fact that whatever their origin, in their adult state the acini and the islands of Langerhans are different structures.

The Combustion of Glucose.-Reduction in the power of burning glucose is the fundamental defect of the disturbed metabolism of diabetes. No mystical appeal to the central nervous system as the seat of diabetes can alter this established fact. We have here another 
illustration of an old theory coming into its own. Just as we today accept the theory of fermentation enunciated a half century ago by a long-visioned chemist, so we have through the experimental investigations into diabetes established the truth of the teaching of the physiologically trained clinicians of nearly a half century ago, that the defect in diabetes lies in the inability to split the molecule of glucose preliminary to oxidation. It is now an established fact that fat, protein, and sugar must all be split before they are available for oxidation in the body. The investigations into the fermentation of sugar indicate that, disregarding earlier intermediary stages, the molecule of glucose must be split into two molecules of lactic acid before it can be converted into alcohol. In the animal body, according to our present evidence, the molecule of glucose must be split into two molecules of lactic acid before it can be burned. The cleavage into lactic acid is probably not direct, as indicated in another connection. Intermediary stages, as yet not clearly defined in the chemical sense, undoubtedly appear earlier than lactic acid. Methylglyoxal, in one form or other, seems a likely intermediary stage. But for the present we may, in the practical application of this teaching to diabetes, rest with the statement that the diabetic cannot split glucose into lactic acid. From the stage of lactic acid on, through ethyl alcohol, assuming that such is the direction of the oxidation in the animal body, the powers of the diabetic organism are normal; but the formation of lactic acid from glucose the diabetic body cannot accomplish. The formation of glucose from lactic acid the diabetic body can accomplish as in the normal; but the cleavage of glucose into lactic acid, the diabetic cannot accomplish. .

Relations between Glucose and Lactic Acid.-It is necessary at this point to expound a little more in detail the reciprocal relations of glucose and lactic acid. The facts are capable of a simple interpretation according to the law of mass action. Whenever the concentration of lactic acid is low, the reaction runs in the direction of the formation of lactic acid; when the concentration of lactic acid is high, the reaction runs in the direction of the formation of glucose. In the muscles, where the combustion of sugar occurs, the intermediary lactic acid appears but in traces, and is there quickly oxidized. When, however, a large amount of lactic acid is ingested and absorbed, the concentration of lactic acid in the liver and probably also in the blood, is so great that the direction of the reaction is reversed, and glucose is formed. Small amounts of lactic acid are burned in the normal body, in the dog with phloridzin intoxication and in the diabetic; larger amounts result in the formation of glucose under all three conditions. It is clear, therefore, especially in consideration of the existence of hyperglucemia, that the diabetic would be able to complete the oxidation of glucose in the normal manner if once glucose could be converted into lactic acid.

Contrary to earlier opinions, the combustion of glucose occurs largely in the muscles, not in the liver. The muscles we may regard as the 
fire-box of the body. The pancreas contributes some chemical factor to this muscular metabolism, and when this contribution of the pancreas is lacking, the power of the muscle to split glucose into lactic acid is impaired or lost. This in a nutshell is the present conception of the non-combustion of sugar in diabetes.

Demonstration of Non-combustion of Glucose.-The fact of the noncombustion of glucose, or at least its greatly reduced combustion, in diabetes can be demonstrated in several ways. In one of the crucial animal experiments in diabetes, a dog weighing 5.8 kilos, whose body under the conditions of the experiment could not have contained over 250 grams of stored glycogen and sugar, was made diabetic by ablation of the pancreas. He was fed on nutrose (a casein compound free of preformed carbohydrate) and during twenty-five days eliminated nearly 1200 grams of sugar. A kilo of sugar was eliminated from a non-carbohydrate source by an animal of but little over five times that weight, in less than a month. This experiment, which first demonstrated indisputably the formation of sugar from protein or fat, demonstrates at the same time the lack of combustion of sugar in the animal, since obviously such an amount of sugar could not have been eliminated under these circumstances if the body were utilizing it in its heat metabolism. The only intelligible interpretation of the result is that the body was forming sugar (from protein certainly and possibly from fat), and eliminating it because it could not be burned. This same fact is seen in clinical diabetes. The blood and tissues are flooded with glucose (hyperglucemia); if the carbohydrate of the diet be increased, the increase appears almost quantitatively in the urine; if carbohydrate be stricken from the diet the glucosuria falls; if the meat of the diet be raised, the glucosuria will rise; if the meat be reduced, the glucosuria falls. However derived-from carbohydrate of the diet, from meat of the diet or from the body flesh-the sugar is largely eliminated and only burned to a limited extent.

The respiratory quotient indicates the same fact of the non-combustion of glucose. The respiratory quotient on the maintenance of the body heat by the combustion of glucose is nearly 1 ; by the combustion of fat it is something over 0.7 ; by the combustion of protein it is about 0.8. Now in diabetes the respiratory quotient, coexisting with hyperglucemia and glucosuria, varies from 0.6 to 0.8 (below 0.7 , for reasons that will be detailed later); in other words, the diabetic presents the respiratory quotient of an organism supporting its heat mechanism largely or wholly by the combustion of fat and protein. If a dog be freed of glycogen by work, starvation or refrigeration, and then placed at work, it will be found to have a respiratory quotient of from 0.7 to 0.8 , since it is supporting its heat mechanism on the combustion of fat and protein. If to such a dog carbohydrate be given, the respiratory quotient will rise in a short time. When, however, carbohydrate is given to a diabetic the respiratory quotient will not rise, but instead the carbohydrate will be eliminated in the urine in the form of sugar. 
The failure of the combustion of sugar can also be shown by another calculation. The heat production being known, the nitrogen input and output, and the sugar input and output, it can be made certain (barring the formation of sugar from fat) that the body heat is supported by the combustion of protein and fat, and that the sugar formed from protein or ingested in the state of carbohydrate is eliminated in the urine. Such calculation, made roughly in clinical diabetes, gives a very fair indication of the degree of utilization of carbohydrate in the heat metabolism. Such a calculation has the advantage over the use of the glucose: nitrogen ratio, to be mentioned later, in that the fluctuations in the nitrogen factor are controlled. The heat production cannot, of course, be usually measured; but it may be approximated by calculation of 30 to 35 Calories per kilo body weight per day. The mere measurement of the carbohydrate input and sugar output may lead to valuable results in mild cases; but in severe cases, where the sugar output exceeds the carbohydrate input, the nitrogen input and output must also be measured.

The fact that the combustions of the body in diabetes are largely confined to protein and fat, is clearly shown in the caloric equivalents that have been determined in the disease. The following table contains the equivalents for the different foodstuffs, and in the lower space those that have been observed in diabetes:

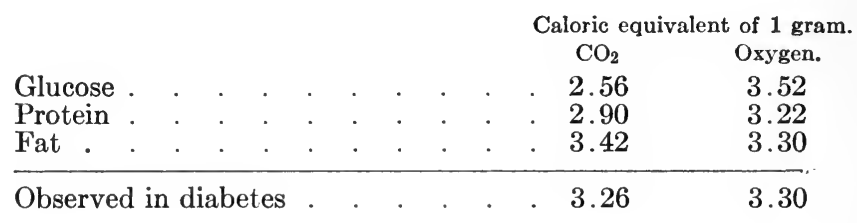

It is clear that the observed equivalents in diabetes have been attained largely through the combustion of fat and protein, since they are far removed from the figures for glucose. The $\mathrm{CO}_{2}$ elimination in diabetes indicates directly the lack of combustion (or toleration) of glucose. In severe diabetes, the $\mathrm{CO}_{2}$ elimination is quite constant from day to day, irrespective of the variations in the carbohydrate of the diet. Variation in the input of carbohydrate is followed by fluctuation in the output of $\mathrm{CO}_{2}$ in a body being heated by the combustion of sugar.

From the dynamic point of view the failure to burn sugar in the diabetic is all the more striking when viewed in connection with the excess of sugar in the blood. 'This should, unless otherwise removed, lead to increase in the rate of combustion. But the ferment is inactive.

Amount of Combustion in Diabetes.-It is not usual, so far as can be learned, for the power of burning glucose to be entirely lost, even in severe diabetes. The difficulties in the way of the accurate determination of this point are at present not surmountable. In the last stages of clinical diabetes, it seems likely that no sugar is burned, just as in the last stages of pancreatic diabetes in the dog. It may probably 
be stated with approximate correctness, that the average diabetic can burn 25 to 50 grams of glucose per day. The normal individual, with work or refrigeration, can burn a kilo or more of glucose per day. According to degree of severity, diabetics may be graded. There are mild cases of true diabetes who can burn 100 to 150 grams of sugar per day plus the amount derived from the protein of the diet, say 50 grams. As the severity of the disease rises, these figures fall, until we come to a large group of cases who are able to burn only the glucose formed from the protein of the diet-50 to 75 grams per day-added sugar in the diet being eliminated. The ingestion of an excess of protein will usually be followed by the reappearance of glucosuria in these cases. In still more severe instances the sugar formed from the protein of the diet cannot be burned, and glucosuria appears on a protein-fat ration. If the protein be cut to a low figure and the fat be held high, many of these cases will lose their glucosuria. In others, however, it will persist under all circumstances. And since, with increasing severity of the disease, the combustion of fat becomes imperfect and greater resource must be had to the protein metabolism, such cases will present heavy glucosuria on the best protein-fat regimen that can be maintained. It is not usual to recover in the urine all the sugar that the protein can be calculated to yield. There are, however, unusual cases in which not only is all the sugar to be recovered from the urine that can be derived from the catabolism of the protein, but apparently more; and in some extreme cases so much more that we are almost compelled to assume a final derivation of this sugar from the fats, even under the assumption that absolutely no sugar is being burned.

If to a diabetic $\operatorname{dog}$ on a fixed protein-fat regimen a unit of sugar be given, this will reappear quantitatively in the urine. In experiences with established diabetes in the human subject, this, too, is the rule. There are, however, cases in which, after a time, less than the added unit of sugar appears in the urine; the combustion of sugar improves and we speak of the function being improved by use. In other cases, however, the contrary result is attained; more than the unit of sugar administered is recovered in the urine, the patient has lost even of his already reduced powers of burning sugar, and we regard this as an expression of the injury that the increased concentration of glucose has done to the metabolism. Valuable as it is to the diabetic to use his powers of burning sugar to the fullest, even so injurious is it to him to have his system flooded with an excess of ingested glucose that he cannot burn.

The whole question, of utmost importance in the treatment of diabetes, cannot be correctly settled until the purely physiological question of the amount of glucose that a body forms from a unit of a fixed protein is known. This will be discussed under another heading. For the present, however, there is no warrant for the idea that the body in diabetes can burn no sugar. It is certain that the amount that can be burned in typical cases is very small. The state of the 
glycogen depots of the body postmortem cannot be used as a basis for a judgment of the sugar-burning powers of the body before death. These are in all cases highly depleted and may be exhausted. But this could be the result of defective glycogenesis as well as of excessive glycolysis. So far as the direct relations are concerned, there is no theoretical reason why the combustion of sugar should not cease without disturbing the depots of glycogen. But diabetes represents no such single defect in the carbohydrate metabolism.

Work and the Combustion of Glucose.-Work increases to some extent the combustion of glucose in the body of the diabetic, in inverse proportion usually to the severity of the disease. There can be no question that in the cases where graduated exercises are associated with progressive reduction in the elimination of sugar on a fixed diet, the power of burning sugar is thereby progressively improved. In severe cases no amount of exercise will be followed by a reduction in the elimination of sugar. On the contrary, one of two untoward effects may occur. The sugar in the urine may be increased; this will indicate that the work is being done on the combustion of protein (of the diet or of tissue), and the increment of sugar in the urine is derived therefrom. Or the acetone bodies may be increased; this indicates that the work is being done on the combustion of fat and the acetone bodies serve to illustrate the added defect in this metabolism. If the acidosis be increased, the $\mathrm{CO}_{2}$ elimination will be augmented through expulsion of carbonic acid from the blood, due to the reduced capacity of the blood for carbonic acid. This is an illustration of the caution that must be employed in the interpretation of the respiratory quotient, since this increase in $\mathrm{CO}_{2}$ might have been erroneously regarded as due to an increased combustion of sugar. In depancreatized dogs, the power of burning sugar for the support of work is directly proportional to the power of burning sugar at rest. That work in the dog is done on protein and fat has been directly shown by the determination of the respiratory quotient. But in human diabetes there are striking exceptions. One meets with cases of severe diabetes with heavy glucosuria and pronounced acidosis, in whom obviously there is little power of burning sugar at rest, who nevertheless under proper exercises display striking reductions in both glucosuria and acidosis. On the other hand, in mild cases work may be badly tolerated. The determination of the metabolism on which a diabetic subject accomplishes work is of great therapeutic importance, since the work that is a benefit to one will be an injury to another.

In many cases of diabetes, intercurrent fever tends to lower the glucosuria, by increasing the combustion of glucose just as in the case of work. In other cases, especially in severe diabetes, the converse is true; the glucosuria is increased, obviously at the expense of the protein metabolism; and the acetone bodies may be increased, indicating excessive combustion of fat. Any further speculations, based upon the hypothesis that diabetes is a disease of the central 
nervous system and localized in an area related to that of the heat centres, are without foundation.

Toleration of Other Sugars.-Levulose and lactose (through its content of galactose) are sometimes tolerated and utilized better by the diabetic than is glucose. This is a fact whose meaning is not at all clear. The experiment is easily made either by giving fructose or galactose alone, or by giving cane sugar or milk sugar. In the event of a positive result, the glucose in the urine will not be increased to the extent noted in the case of the ingestion of a similar amount of glucose. According to our physiological information, fructose and galactose are in the mucosa of the intestine and in the liver converted into glucose. Since the combustions occur in the muscles and not in the liver, and since, furthermore, the body is believed to burn fructose and galactose only after conversion into glucose, the situation is entirely unclear. The facts hold for diabetes in the depancreatized dog. It has been' suggested that possibly the liver can form glycogen from fructose and galactose directly, without prior conversion into glucose. This explanation is not improbable. There is nothing to indicate that fructose and galactose cannot be polymerized to a polysaccharid. Indeed, we have galactosans in nature, though they do not much resemble starch or glycogen. All the natural starches yield on hydrolysis only glucose, not fructose or galactose, though possibly these were contained in the polysaccharids and converted into glucose in the chemical operations of hydrolysis. This explanation would account for the fact that following the ingestion of fructose the sugar of the urine is not increased. It would also account for the unfortunate fact, observed in the treatment of diabetes, that after a few days or a week, the regular administration of fructose is followed by rise in the glucosuria until the unit of input is recovered in the urine. This, under the explanation suggested, would indicate that the toleration for fructose lasts only so long as the body can convert it into glycogen and store it in the liver; so soon as the storage capacity for glycogen is exceeded, under the conditions in the liver that prevail in diabetes, the total ingestion of fructose reappears in the urine in the form of glucose. If when this point is reached, the glucosuria falls coincidently with the cessation of the administration of fructose, it is clear that the stored glycogen is being retained, and this is of undoubted advantage to the diabetic. Whether the missing fructose is stored as glycogen or burned at the time could be determined by estimations of the respiratory quotient. Variations in the toleration, $i$. e., utilization, of starches of different derivation are also known. Thus the starch of the potato is supposed to burn better than that of maize. There is no doubt of one fact, diabetics tolerate oatmeal better than any other carbohydrate. It is common to see a diabetic with little glucosuria and low acidosis on 100 grams of starch per day in the form of oatmeal, when 50 grams of glucose will pass almost quantitatively into the urine. This toleration is not invariable, and it is often lost. For this fact, striking as it is, we have no explanation. 
Pentoses do not increase the glucosuria, though they are burned in the body. This, again, is not now comprehensible, so long at least as we hold to the general notion that pentose is only burned after conversion into hexoses. For one pentose in particular, rhamnose, it has been shown that the diabetic can burn up to 100 grams per day. Curiously enough, however, the utilization of this substance seems to provoke an exaggeration of the protein catabolism, as evidenced by increase in the glucose and nitrogen in the urine.

Glycogen Theory.-Against the interpretation of the defect in the combustion of glucose in diabetes as given above, stands another hypothesis, which, since its promulgation by one of the most distinguished students of diabetes, deserves adequate statement and consideration. This hypothesis runs to the effect that only such sugar can be burned in the body as has been converted into glycogen in the body; in short, it is the glycogen that is burned. According to this view, glycogen must be incorporated into the muscle cell, anchored in its protoplasm, before it can be burned. And the diabetic is held to have lost the power of anchoring glycogen in its muscle cells. In favor of this hypothesis are the facts that fructose and galactose are, for a time at least, better tolerated than is glucose; and the unquestioned fact, known to all students of diabetes, that the ability to store and hold glycogen is a faculty of crucial importance. Reference will be made later to this importance of stored glycogen to the diabetic. The question is not as to these facts; it is as to whether they necessarily, or even most naturally, favor the hypothesis stated. There is in physiology no adequate ground for the assumption that the muscles cannot burn sugar directly from the blood stream without first converting it into glycogen. To be burned it must be first returned to the state of glucose. Taken directly from the blood stream, it might be urged, it is in the free state. In this hypothesis, on the other hand, it is bound to protoplasm, the anchorage of the glycogen being assumed to hold after its cleavage into glucose. But why should not the protoplasm bind the glucose directly, if such anchorage be held necessary? Can only that glucose be bound that was bound as glycogen? Such a proposition is most unusual. If an animal be freed of glycogen by starvation, work or refrigeration, it maintains its body heat by combustion of fat and protein and has the corresponding respiratory quotient. If, now, to such an animal sugar be given, in a very short time the respiratory quotient rises, indicating that this sugar is being burned. Is it to be assumed that this sugar carried by the blood to the muscles must be first polymerized to glycogen and then returned to glucose before it can be burned? There are no physiological or chemical data to warrant this assumption; and until such are forthcoming, no hypothesis of diabetes can be founded upon it. The mere fact that the storage of glycogen is of great benefit to the diabetic organism is not enough to warrant the physiological dictum that sugar cannot be burned directly in the muscles, but only after conversion 
into glycogen. As a matter of fact, defective glycogenesis and defective combustion of glucose do not always run parallel in diabetes.

Lactic Acid Theory.-Quite another explanation for the defective combustion of glucose in diabetes centres about lactic acid. In this it is assumed that in the muscles glucose is split into lactic acid, which is then carried to the liver to be reconverted into glucose, thus maintaining a vicious circle. In the interpretation given above, it was held that the cleavage to lactic acid in the muscle does not occur; did it occur as in the normal, the lactic acid would be burned. The proposition here being considered is just the converse of this; it is assumed that the glucose is split to lactic acid, but instead of this being burned in the muscles, it is returned to the liver, to be there converted into glucose and returned to the circulation and muscles. This would naturally result in hyperglucemia. But is it true that the diabetic cannot burn lactic acid? No, the diabetic can burn lactic acid. The hypothesis is surely based upon a misunderstanding of the mass relations. When 100 grams of a lactate are given to a severe case of diabetes, it reappears in part as sugar in the urine. When it is given to the normal individual, we may be sure that the formation of sugar occurs also, but it does not become visible as in the diabetic. A heavy ingestion of lactic acid so increases the concentration of lactic acid as against sugar in the liver, that the reversal of the normal reaction is accomplished, the lactic acid is converted into glucose. This occurs in phloridzin intoxication, just as we may be sure it occurs in the normal. But from this fact to assume that the traces of lactic acid that result in each moment from cleavage of glucose in metabolism are converted back into sugar, is entirely arbitrary. As a matter of fact, less lactic acid is present in the muscles in diabetes than in the normal, in strict agreement with the theory that the defect in the diabetic consists in inability to split glucose into lactic acid. It is present in the muscle only as a transient intermediary stage, and from this lactic acid, in the normal and in the diabetic, we may be sure no sugar is formed. For such a postulated inability to burn lactic acid and its consequent conversion into glucose, no foundation exists. So far as the writer is able to see; there is but one condition in the body in which ingested lactic acid, under proper conditions of concentration, cannot be utilized as in the normal-in hepatic degeneration of the type seen in phosphorus poisoning and acute yellow atrophy.

For the present, therefore, the best interpretation of the failure to burn glucose in diabetes rests upon the proposition that on account of the absence of the internal pancreatic secretion the muscle cell has lost the power of cleavage of the molecule of glucose into lactic acid, or some previous intermediary stage, whereby the oxidation ferments are denied opportunity of action.

Combustion of Substances Other than Glucose.-The faculty of combustion in the diabetic has been tested in many directions. Citric acid, tartaric acid, lactic acid, acetic acid, inosite, mannite, benzol, 
d-gluconic acid, d-glucuronic acid, and d-saccharic acid are all burned, in every way as in the normal. Benzol is a substance of difficult oxidation, but the diabetic burns it as well as the normal. When one contemplates the equation for d-gluconic, d-glucuronic, d-mucic, and d-saccharic acids, all combustible, and notes that one contains the aldehyd group and another the terminal alcoholic group intact, it is clear that the defect in the diabetic cannot lie in the direction of these groups. Beta-oxy-butyric acid the diabetic cannot burn, of which more later. Some diabetics are not able to oxidize dioxyphenylacetic acid. The abnormalities in the combustion of fat and protein will be discussed under the appropriate headings.

Hyperglucemia.- In all cases of diabetes, experimental and natural, varying from case to case, an excess of glucose exists in the blood. This might be due to one or more of three conditions:

Non-combustion of glucose.

Elevation of level of renal retention.

Overproduction of sugar.

Overproduction of Sugar.- The last may be discussed first and dismissed. There is no evidence of an overproduction of sugar in diabetes. All the sugar can be accounted for without assuming overproduction. From carbohydrates there can be no overproduction. The diabetic simply eliminates the sugar that the normal body burns. Sugar is formed from protein in the diabetic just the same as in the normal body. Whether this function is facultatively increased in diabetes, $i$. $e$., whether from a unit of protein more sugar is formed than under normal conditions, will be discussed under another heading, as will also the question whether this may be a pathological facultative function, operative in diabetes but not in health. The total amount of sugar at the disposal of the functions of the body is surely no greater in the diabetic than in health. But in health the body burns it, stores it as glycogen, stores it as fat and uses it to save the protein metabolism; the diabetic does none of these in the normal manner, but instead carries it in the blood and tissues and eliminates it in the urine. There may be a slight excess of sugar production from protein, though this is doubtful. But in the sense of an overproduction of sugar beyond the needs of or utilization in the body, so that after using all it needs the body is flooded with the excess of sugar-no such thing exists. The normal body can and regularly does burn far more sugar than any diabetic organism possesses. The hyperglucemia is due to deficient utilization of glucose, possibly to elevation in the level of renal retention.

The glucose concentration of normal blood seems to vary from 6 to 9 parts in the ten thousand. In the lowest values seen in diabetes, 15 to 25 parts in the ten thousand, we have already a goodly increase. From this up values are recorded to over 100 parts in the ten thousand. It is greatly to be desired that quantitative analyses of the blood of diabetics under controlled conditions of diet be done; the present 
data are too fragmentary, and all considerations based thereon must be subject to reservation.

In many cases there is a proportional relation between the hyperglucemia and the glucosuria. In some cases the proportionality is better noted if we compare with the blood concentration the weight of sugar eliminated rather than the percentage. In many cases, however, no such proportionality is to be observed; we see high blood concentrations with low glucosuria, relatively low excess in the blood with high glucosuria. The most striking exceptions are in the direction of hyperglucemia with slight glucosuria. Early in diabetes we may find little or no excess in the blood, with active elimination by the kidneys. In theory, obviously, this ought to hold if the kidneys do their work; when one considers how much sugar the kidneys can eliminate, one is led to wonder why in many cases of diabetes the kidneys do not succeed in keeping the glucose of the blood to the normal level.

In some cases of diabetes, especially in early stages, the glucosuria is quite independent of the diet, remaining about the same on any reasonable diet, even if quite free of carbohydrate. Usually, however, as carbohydrate is added to a protein-fat diet, the glucose in the urine rises in proportion; and as the ingested amount is increased absolutely more and more of the amount ingested is recovered, until finally, all is recovered in the urine.

When a diabetic has been made aglucosuric by diet, as often succeeds, it will be found that the sugar content of the blood is lower than in the same case on carbohydrate diet with glucosuria, but above the normal concentration it still remains. This will in the future probably prove to be a prognostic test of value, as in this way the disease may be unmasked.

Elevation of Level of Renal Retention.-When one contemplates these relations, one cannot but feel impressed with the conviction that the kidney ought to be able with the degree of hyperglucemia present in most cases of diabetes, to keep the glucose concentration in the blood down to the normal level. Thus the question arises, Is not the renal level of retention of glucose raised in diabetes, conversely to phloridzin intoxication in which it is lowered? We have an analogy in nephritis, in which the level of retention of uric acid is raised much above the normal. In typical advanced diabetes, it may be stated as the rule that under strict conditions of diet the glucose in the blood is higher than it should be in consideration of the degree of sugar elimination in the urine and in contrast with our knowledge of what the kidneys can accomplish in this direction under other conditions. One sees high hyperglucemia with low glucosuria. What else does this mean than a renal retention, a raised threshold value? By this we do not mean nephritis, we mean simply an alteration in a specific function of the kidney, an increased impermeability. If we are asked to explain what is meant by increased renal impermeability, we reply by asking for a definition of the normal permeability, whereby a low concentra- 
tion of glucose in the blood is effectually retained. To regard the hyperglucemia as due to an increase in the sugar-binding colloids of the blood is as unfounded as to regard the glucosuria of phloridzin intoxication as due to a reduction in the sugar-binding colloids of the blood. There is little experimental literature on the subject, though the observations stated hold for pancreatic diabetes in the dog. If a depancreatized dog be given a chromic acid nephritis, the glucosuria is lowered. It is also a common experience that supervening nephritis in the diabetic is often associated with lowering of the glucosuria. All this, however, need mean nothing directly to the point at issue. The question must be settled by future investigation. The matter is of practical importance. For if such a thing occurs as increase in the impermeability of the kidney to glucose, the glucosuria loses in part its indicative value in diabetes; if one could reason from glucosuria to glucemia, our conceptions would be much simpler than if no such relationship is to be postulated. And it is already apparent that in many cases of advanced diabetes, no such relationship exists. There is in the blood of the diabetic, as in the normal, a small fraction of the blood sugar that resists fermentation; and this fraction is absolutely increased to some extent in diabetes. When the blood is boiled with weak hydrochloric acid, the total blood sugar becomes susceptible to fermentation. But the fraction thus bound is small, and no argument can be based upon it that is to apply to the whole question of the foundation of hyperglucemia.

Mixed Melituria.-Pentose, fructose, maltose, and traces of dextrin and even of glycogen have been reported in the urine of diabetics. While there can be no doubt of the reliability of some of the observations, it is certain that had stricter methods of identification been employed the number of instances would be much less. The color methods alone ought not to be relied upon to serve as criteria of fructose and pentose. Maltose is a normal sugar in the blood in the merest traces, in theory at least, since it is an intermediary stage in the conversion of glucose into glycogen, and vice versa. In the diabetic its presence may be reasonably regarded as evidence of defective glycogenesis. That traces of glycogen occur in the urine is made intelligible when we consider the fact that in diabetes the renal epithelium is often stuffed with glycogen. Dextrin, if it really occurs, would have the same meaning as maltose.

In many cases of the reported instances of fructosuria in diabetes, alimentary or hepatic fructosuria have not been excluded. In some of the reported cases, however, such exclusion has been rigidly accomplished. We face here a reversion of a normal reaction, the conversion of glucose into fructose instear of fructose into glucose. Careful analyses have tended to indicate that fructose may occasionally be present in the blood normally, and it is, therefore, possible that in its appearance in diabetes we are dealing simply with a pathological exaggeration of a normal reaction. It is in the severe cases of the disease in 
particular that fructose is found in the urine. It is possible to assume that a mass relation between glucose and fructose holds in the blood, with the station of equilibrium very much in favor of glucose. At normal concentration of glucose, the amount of fructose would be very low, below the level of analyses. With the marked hyperglucemia of severe diabetes, the operation of the law of mass action would result in the formation of larger amounts of fructose, so that its presence might become evident. Such a consideration, based upon the chemically well-known ease of conversion of glucose into fructose and fructose into glucose, becomes a reasonable interpretation of the fructosuria of diabetes. In a certain sense the sign may become one of unfavorable prognostic meaning. Viewed in this manner, there is nothing contradictory in the toleration of fructose by the diabetic and the occurrence of fructosuria in diabetes.

With regard to lactose no abnormal relation exists in diabetes. The diabetic mother forms milk sugar from glucose in a normal manner. Nor is lactosuria any more common than in the normal.

Pentosuria occurs in diabetes so rarely that it is a question whether it has been more than a coincidence. For the identification of pentose sugars in the urine, more rigid methods are in place than have been usually employed in the past. If future investigations should indicate that pentosuria does occur as a complication of diabetes, we will have before us simply another disturbance of the carbohydrate metabolism; and its occurrence in the diabetic organism will be no more strange than is its occurrence under other conditions, of which we know so little. When the chemical meaning of pentosuria of idiopathic type is understood, that of diabetes will probably present no difficulties.

Hepatic Glycogenesis.- It is difficult to measure the hepatic function of conversion of sugar into glycogen and the storage of the latter, because of our inability to control hepatic glycolysis. We can always study hepatic glycolysis, because we can control glycogenesis by fixing the input. Normal for each individual is a physiological figure for the storage of glycogen in the liver, and as a rule, with usual diets the body operates within this limit. When to a diabetic dog whose liver (already poor in glycogen) has been freed of glycogen by starvation, glucose is given, it will appear in the urine in a very short time. This indicates that the formation of glycogen in the liver is defective, unless the function of glycolysis operates so rapidly that the glycogen is built down to sugar as fast as it is built up to glycogen. If in such an experiment the respiratory quotient were to remain constant and not all of the ingested sugar were recovered in the urine, we would know that some of the sugar was stored as glycogen; but we would not be in a position to know whether deposition occurred in the liver or in the muscles. The toleration of starch when contained in oatmeal as contrasted with the toleration of starch or sugar in other forms, has been usually interpreted to rest upon variations in hepatic glycogenesis. The same explanation is suggested for the toleration for fructose as 
against glucose. Since the glucose formed in the liver from glycogen, howsoever derived, is always the same, it is natural to localize the site of toleration of oatmeal in the liver. While the data are largely qualitative and not satisfactorily conclusive, we may provisionally conclude that insufficient formation of glycogen from glucose occurs in the liver as a defect in diabetes. This opinion is solidified by indirect evidence. The liver of the diabetic is, as previously stated, very poor in glycogen though the blood that drips from it is rich in sugar. In the depancreatized dog, the liver is no poorer in glycogen during starvation than after a carbohydrate meal. At the same time it must be confessed that these results could be quite as well explained by an exaggeration of glycolysis associated with even normal glycogenesis. The equilibrium is obviously disturbed, and this is all the more striking when the hyperglucemia is considered. But such a disturbance in the equilibrium could be as easily the result alone of excessive glycolysis (which can be proved) as of defective glycogenesis.

An excess of glycogen may be found in the leukocytes and in the renal epithelium, though the demonstration has been usually morphological and not chemical. If this be true, it suggests that the leukocytes and the renal cells form glycogen from the hyperglucemic blood, an illustration of the law of mass action.

Hepatic Glycolysis.-The formation of glucose from glycogen is unquestionably excessive in diabetes. Normally, the hepatic regulation of glycolysis is very accurate, no hyperglucemia occurs no matter how little or how great the combustion of sugar, no matter how much carbohydrate the diet contains up to the storage capacity of the liver for glycogen. In diabetes the conversion of glycogen into sugar operates excessively, so that the sugar-holding, or more properly speaking glycogen-holding power of the liver is reduced to the minimum. The removal of the pancreas acts as directly in this direction as does the puncture of Bernard and almost as quickly. Within a few hours after the removal of the pancreas in a fasting dog, the hyperglucemia and glucosuria appear, and in a surprisingly short time the liver will be found poor in glycogen. The same result will be attained, though in less time, in a dog in full carbohydrate nutrition; the hyperglucemia and glucosuria appear promptly, but more time will be required to free the liver of glycogen. This defect has no direct relation to the inability to burn sugar, since it may be produced by the puncture of the medulla and through the action of many drugs, without the power of burning sugar being in the least disturbed. The pancreas, moreover, has a direct relation to the hepatic function of glycolysis, and the writer does not believe that this can be explained as the result of any interference, through the removal of nerve fibers in the extirpation of the pancreas, with the action of the glycolytic centre in the central nervous system. How the removal of the pancreas results in stimulation of the glycolytic centre or function is too distant a conjecture to be attempted. One might obviously assume that the pancreas 
furnishes a substance that physiologically represses the glycolytic centre (in somewhat the same general sense as the vagi repress the heart action), but that is an explanation after the fact. Throughout the course of diabetes this excessive activity of the glycolytic function of the liver is in evidence; and so far as its normal rôle in the regulation of the carbohydrate metabolism is concerned, the liver might as well be absent. It is certainly of little use to the diabetic.

Glycogenesis and Glycolysis in the Muscle.-The muscles of the diabetic are poor in glycogen, despite the fact that the blood is excessively rich in glucose, which under normal conditions would result in the formation and storage of large amounts of glycogen. The renal cells that normally contain little glycogen are in diabetes filled with it; but the muscle cells, whose function it is to form and store glycogen, are empty. So far as we are able to determine, the fault lies rather in defective glycogenesis than in excessive glycolysis. That the latter exists, however, is made evident by the rapidity with which the muscles become poor in glycogen after extirpation of the pancreas. This defect in the carbohydrate metabolism of the muscles, all the more striking when the hyperglucemia is recalled, is a clear-cut illustration of a pathological alteration in a station of equilibrium. From a concentration of glucose as much as tenfold the normal, little or no glycogen is formed. This may be reasonably interpreted as an expression of defective ferment action, since the formation of glycogen from glucose we regard as an enzyme reaction. It is a defect of grave misfortune to the diabetic organism. Clinical experience leaves little doubt of the truth of the dictum that the lower the reserve of glycogen in the diabetic, the nearer to the danger line is he. The glycogen seems to spare the fat metabolism and acidosis is liable to arise with almost explosive violence when the depletion of the store of glycogen has reduced the reserve to a minimum. Many an unfortunate diabetic has, by excessive carbohydrate starvation, been brought to the danger line of such an acidosis, not infrequently to succumb. To fully appreciate the value of the glycogen stores to the diabetic, one does not need, however, to subscribe to the hypothesis that fat can only be burned with sugar, or believe that glucose is only burned after conversion into glycogen. Future investigations must show the exact relations.

The Formation of Fat from Glucose.-Not only is the formation of glycogen, the proximate state of storage of sugar, defective in diabetes; the formation of fat, the ultimate state of storage, is also defective. In the normal body, hyperglucemia leads first to formation of glycogen, then to formation of fat; in the typical instance of diabetes, hyperglucemia is followed by neither. This again is a disturbance of the normal state of equilibrium, since with such a hyperglucemia as the diabetic possesses, the formation of fat from sugar ought to be greatly increased, instead of which it is decreased. Were this function in a normal state (even though no sugar were burned), the body would be able to convert into fat all the sugar formed from protein, and this 
would thus be saved to the organism. Under such circumstances, even though sugar could not be burned directly, it would be burned via fat and the hyperglucemia and glucosuria of the diabetic would not exist. So far as can be seen, this is a direct and fundamental defect in the function of lipogenesis, and cannot be grounded upon any known defect in the sugar catabolism. Nor can it be attributed to the lack of glycogen, since the fat is surely formed from glucose and not from glycogen. Not all cases of diabetes, even severe ones, display this defect. Occasionally, one sees severe diabetes with obesity-just what would be expected with cessation of the function of sugar combustion and retention of the power of lipogenesis. Even such cases, however, are apt, after a time, to become emaciated and to lose their power of forming fat.

A possible chemical explanation of the inability of the diabetic to form fat from sugar lies in the behavior of butyric acid in the diabetic metabolism. As will be stated in the chapter devoted to the metabolism of fat, according to our present conceptions when a fatty acid is burned it is built down in regular stages to butyric acid, and through this to final oxidation. Now in the diabetic this butyric acid is oxidized to beta-oxy-butyric acid, from which proceed diacetic acid and acetone. In the formation of fat from sugar, it would seem possible that butyric acid might be the stage to which the transfer is made from the sugar to the fatty acid. This we may provisionally sketch as follows: glucose $\rightarrow$ lactic acid $\rightarrow$ acetic aldehyd $\rightarrow$ beta-oxy-butyric aldehyd $\rightarrow$ butyric acid $\rightarrow$ higher fatty acid plus glycerin $\rightarrow$ fat. Since now in diabetes the butyric acid derived from catabolized fat is not treated in the normal manner, there is no reason why the butyric acid derived from glucose should have any different fate. We might, therefore, assume that it is simply joined to the acetone group, instead of being converted into fat as in the normal. Not only would this serve to explain the inability of the diabetic organism to form fat from sugar, it would also constitute an additional source for the acetone bodies. This is merely an hypothesis, to be tested in the future. The fact that the administration of sugar is usually followed by reduction in the acidosis is unfavorable to the hypothesis, since with the concentration of glucose in the circulation increased, the formation of butyric acid ought in accordance with the law of mass action to be increased. Favorable to the hypothesis is the fact that it is precisely in the diabetic with acidosis that we see most marked the failure of the function of the formation of fat from sugar. Directly unfavorable to the hypothesis is the fact that the scheme demands the cleavage of glucose into lactic acid, whereas precisely in the inability of the diabetic body to form lactic acid from glucose lies apparently the fundamental defect of the metabolism in diabetes.

It is possible that some cases of pathological obesity are due to defective combustion of sugar with retention of the function of lipogenesis, $i$. e., are masked diabetes. Some clinical cases of diabetes supervene on sudden, unnatural obesity, and this might be the 
unmasking of the disease. It is, however, conceivable that such cases in later life might remain stationary, the obesity masking the mild diabetes completely. A determination of the respiratory quotient would, however, serve to uncover such a case, since it would yield a low quotient, indicating the lack of combustion of sugar.

The Origin of Glucose in Diabetes.-When the normal body fasts or is placed upon a protein-fat diet, the sugar metabolism becomes, so to speak, quiescent; under identical conditions in the diabetic, glucose continues to be eliminated, and often in large amounts. Whence is this glucose derived? This cardinal problem in diabetes has been brought much nearer solution in the last half dozen years. This brings us to the consideration of the protein metabolism in diabetes.

The Protein Metabolism in Diabetes.-The protein metabolism may be said to be always exaggerated in diabetes. This is strikingly shown in the fact that the nitrogen in the urine of the starving diabetic is from two to five times as much as in the starving normal individual. The nitrogen balance is usually maintained, but only by the ingestion of masses of protein in excess of those normally required to maintain such an equilibrium. The mere fact that the diabetic loses in large part the carbon of the protein molecule, as will be later discussed, would serve to explain this. If, however, the average diabetic be placed upon a fat-protein diet, it will be found that the nitrogen balance will be reached on the same input of protein needed by the normal individual. But the adaptation is much lessened. If the fat be held high and the protein reduced, the reduction in protein input will be followed by a nitrogen defect more quickly in the diabetic than in the normal. In the normal body under such circumstances, the glucose that is formed from the catabolized protein spares protein, as does also the fat; in the diabetic, this sugar is largely lost, and the sparing power of fat for protein is reduced also. Many cases of severe diabetes, with the free use of fat will maintain a nitrogenous equilibrium on a relatively low protein input, no more than 10 grams of nitrogen per day. But it is inclined to be precarious, and often cannot be maintained. This fact is of importance, since in periods it is desirable to reduce the glucosuria by reduction of the protein (source of the sugar), while at the same time the nitrogen equilibrium ought not to be disturbed. It is indeed possible, for periods at least, to retain nitrogen in the diabetic body, though this is usually not a true retention in the sense of tissue building, but a spurious retention, to be lost again when the diet is reduced. Apparently a body cannot build flesh unless it can store glycogen. The average diabetic will ingest with 200 to 250 grams of fat (if it can be ingested), 125 to 150 grams of protein per day; and the metabolism apart from the loss of the sugar derived from the protein is not then greatly different from the normal.

The exaggeration of the protein catabolism seems more marked in the pancreatic diabetes of the dog than in man, for which reason, as well as for others, we term the experimental disease in the dog a com- 
plete diabetes. The average nitrogen output of normal individuals in fasting runs from 4 to 6 milligrams per kilo per hour. In diabetes the fluctuations are much greater than in the normal, and the values run up to 10 milligrams per kilo per hour. 'The fluctuations in time observed in diabetes are of especial interest. The curve of nitrogen output always follows sometime behind that of the sugar, as in the phloridzinized dog. But the irregularities in the flow of the nitrogen output suggest at least that the inner mechanism of the disintegration of protein is disturbed. Extreme cases of diabetes will give much larger eliminations of nitrogen than the figures just given, approaching those of the depancreatized diabetic dog. The administration of glucose has no sparing power for protein in severe diabetes, even when some of the administered sugar is not recovered in the urine. Normally, the combustion of glucose spares both protein and fat and the saving power for fat is total in the sense that the body will burn no fat if a sufficient amount of sugar be available. In diabetes the saving power of sugar for fat is still shown in the action of sugar upon the acidosis. This is retained much longer than is the saving power for protein. So long as the body cannot burn the sugar formed from protein, the ingestion of further sugar would be expected to have no saving power for protein.

In diabetic coma amino-acids appear in the urine, indicating that the catabolism of protein in part is not completed to the normal end products.

When in severe diabetes, the power of burning sugar is reduced to the lowest level, and when also the power of burning fat is markedly disturbed, the maintenance of nitrogenous equilibrium is only possible through the ingestion of greatly increased amounts of protein. It may require protein representing 25 or 30 grams of nitrogen, or even more, to maintain the nitrogen balance. Under such circumstances, and especially in the absence of the protein-saving power of sugar, it may not be possible, even with the most maximum ingestions of protein, to retain nitrogen at all, $i$. e., under no circumstances can flesh be restored. In a certain sense, the plane upon which a diabetic can maintain a nitrogenous equilibrium is an indication of the severity of the disease - though many exceptions to the rule occur. Not only is the demand for a large protein ingestion to maintain the nitrogenous equilibrium an indication of the severity of the metabolic abnormality, the large amounts of glucose derived therefrom exaggerate the hyperglucemia and glucosuria. And this hyperglucemia is directly an injury to the tissues.

In this presentation of the facts, it has been obviously assumed that the exaggeration of the protein catabolism, no matter how pronounced, is merely secondary to the states of the catabolism of glucose and fat and not primary. That the diabetic is hard put to for fuel to maintain the body temperature is obvious. But is this dire necessity the sole causation of the exaggerated catabolism of protein? Or is 
there, as in phosphorus poisoning, a primary autolytic exaggeration of the protein catabolism? When one contemplates the muscular wasting of a severe case of diabetes, the thought of a primary toxic proteolysis comes strikingly to mind. The hyperglucemia is toxic to the tissues; experiments on the action of sugar solutions in the biological laboratory leave little doubt of this. The cessation, or rather reduction, in the muscles of the function of combustion of glucose would produce conditions tending to atrophy, entirely independent of the carbohydrate and fat metabolism. When the diabetic suffers from gangrene or septic infection, then surely a toxic cytolysis is superadded to the metabolic exaggeration of protein catabolism. But direct experiments with the nitrogen equilibrium under controlled conditions have never given figures that warrant the interpretation that in uncomplicated diabetes a toxic exaggeration of the protein catabolism exists, primary in type. Further investigations may reverse the present, not very extensive, data. But for the present, the exaggeration of the protein catabolism must be regarded as secondary, so to speak compensatory, and not primary. This conclusion carries with it, obviously, the contradiction of the hypothesis of a primary overproduction of sugar as a factor in diabetes, at least so far as the protein source of the glucose is concerned.

The case may be different in diabetic coma, of which no investigations have been made, if, indeed, exact investigations are possible. Here we are concerned with an acute auto-intoxication, which might easily be assumed to carry an exaggeration of protein catabolism in its wake.

The Formation of Glucose from Protein.-It will be necessary in order to discuss this very important phase of the metabolism of diabetes to anticipate the consideration of the qualitative processes in the catabolism of protein. When the molecule of protein is hydrolyzed in the metabolism, the amino-acids may be subjected to one of two main chemical procedures; the amino-acid may be deaminated, the nitrogen converted into ammonia (urea), and the fatty acids burned; or the carbonous fraction of the amino-acids may be converted into glucose, and the nitrogen converted as before into ammonia (urea). In the opinion of the writer, both processes occur side by side in the body. The subject is one very difficult of quantitative treatment, since the conditions surrounding the necessarily prolonged experimentations resist control and are not susceptible of unequivocal interpretation. It may be stated here that isolated experiments with different aminoacids have indicated that in the liver glucose is formed from them; and viewed as a total process in the normal animal metabolism, glucose is formed from catabolized protein. For the discussion of the state of affairs in diabetes, it is not now whether glucose be formed from protein, but how much glucose is formed from protein. Assuming 55 per cent. as the maximum content of carbon in the protein molecule, since four parts of carbon corresponds to ten parts of glucose, it is obvious that were all the carbon in the molecule of protein converted 
into glucose, 1 gram of protein would yield 1.4 gram of glucose, and the ratio of the nitrogen of the protein to the glucose derivable from it would be $1: 8$. Were all the carbon of the molecule of protein converted into sugar, this would leave the nitrogen, or ammonia, without carbon to form urea. While it is true that the ammonia of the protein catabolism would combine with the $\mathrm{CO}_{2}$ of the blood to form ammonium carbonate, to be converted into urea, yet it has always seemed natural to assume that the carbon of urea like the nitrogen was derived from the molecule of protein directly. If this be true, then the available carbon in the molecule of protein would form 1.3 grams of sugar; and the glucose : nitrogen ratio would be $7.8: 1$. So much for the quantitative possibilities. Now the best experimental data indicate that in the catabolism of protein the glucose : nitrogen ration is under $4.5: 1$. This means that from 1 gram of protein 0.75 gram of glucose is formed. The remainder of the carbon must be burned directly, without conversion into sugar. On this basis of reasoning, if a diabetic body had lost the power of burning and storing glucose entirely, all the sugar formed from protein would be recovered in the urine. And if the animal were on a protein-fat diet, free of preformed carbohydrate, the urine should contain 4.5 grams of glucose to every gram of nitrogen. If under controlled conditions, other things being equal, less glucose were recovered in the urine, it would be proper to infer that the difference had been burned or stored. It is clear, therefore, that if the simple relation stated holds for the interpretation of the glucose: nitrogen ratio, this should be of determining importance in the study of diabetes.

When, however, we come to inspect carefully the glucose:nitrogen ratio, analyse the conditions surrounding it and measure the controls that may be experimentally maintained in its determination, it will become clear that no such dependence as above suggested can be placed on it. The exaggerated importance placed on this ratio in some schools of physiology is in no way better illustrated than in the fact that the ratio is often calculated to the second place of decimals, which is unwarranted by the conditions of the investigation. What are the conditions that are presupposed in the determination of this ratio in the study of diabetes?

(a) It is assumed that the diabetic does not store carbohydrate at all. This assumption is in most cases of diabetes unwarranted. Many cases are able, in some stages of the disease at least, to store such amounts of glycogen as to modify the ratio to quite an extent. The longer the period of observation, the less the error liable to lie in this assumption.

(b) It is assumed that the diabetic does not store nitrogen. This also is unwarranted, in many cases of diabetes at least, and for rather long periods of time. Estimations of the nitrogen output should always be accompanied by estimations of the input. And to make the results certain the observations should be extended over many days. The 
calculation of the ratio on the basis of a single day's measurement of the nitrogen and glucose of the urine may lead to grave error. A man on a fixed nitrogen input of 15 grams per day may exhibit fluctuations in the output of as high as 3 or 4 grams per day, the retention of one day being followed by a sweeping out on the day or days following. The influence of this behavior on the part of the nitrogen metabolism (observable in health), may be shown in a simple calculation: Let it be assumed that on a fixed protein-fat diet, the sugar of the urine was 50 grams, the nitrogen input 15 grams. On one day the nitrogen output might be 13 grams, on the following day 17 grams. 'The $\mathrm{G}: \mathrm{N}$ ratio for the two days would be 3.8 and $2.9: 1$. Now such variations in the nitrogen output are common. They indicate how useless it is to calculate the ratio into the second place in decimals; and how much more representative the ratio would be if taken in periods of a week, with daily estimation of the input and the nitrogen of the stools. It is known that the sugar appears in the urine long before the nitrogen of the protein from which it was derived; and it has been rightly insisted upon that the collection of urine should last at least twelve hours after the last meal was taken, $i$. e., the day for the purpose of the collection of urine should last until the second morning. But this, while correct and in the right direction, is not enough. The $G: N$ ratio should be determined in periods of days, the more the better; and the nitrogen balance for the period should be determined. Many of the notorious fluctuations in the $\mathrm{G}: \mathrm{N}$ ratio reported in clinical diabetes have been undoubtedly due to brevity of the time of observation.

(c) It is assumed that all the glucose in the urine comes from the diet, and that none is derived from stored glycogen. This assumption is in many cases of diabetes entirely unwarranted; in severe and prolonged cases on the other hand, it is substantially correct.

(d) It is assumed, in certain quarters at least, that the body is burning no glucose. This assumption is unwarranted in all cases; as will later be detailed under the discussion of the respiratory quotient, it is quite certain that there is some combustion of glucose in nearly all cases of diabetes except in terminal coma, and often a goodly utilization indeed. Obviously the real ratio is commonly higher than the observed ratio, as a rule, higher in degree directly proportional to the severity of the case.

(e) It has been assumed that protein is protein in the diet, so far as the sugar-forming capacity is concerned; and that, therefore, we need only calculate from the nitrogen output, without inquiring where -it came from. Now this is incorrect, proteins do not yield glucose in any fixed relation to the nitrogen; 100 grams of nitrogen in the forms of gelatin, casein, and edestin, for example, would not yield, under the terms of a perfect experiment, the same amounts of glucose. This is due, as will be elsewhere explained, to the fact that the different amino-acids are not equally available as sources of glucose, and the different proteins contain different amounts of the several amino- 
acids. It is, therefore, necessary to bear a caution in this regard, though to date we do not know how to classify the proteins even approximately on the basis of their glucose-yielding properties. Naturally, it is necessary to employ in the diet a protein free, or nearly free, of preformed carbohydrate, casein and codfish being the most available.

$(f)$ It is assumed that there is no further source of glucose than the protein. Upon no warrant is the glycerol of the fat undergoing combustion to be disregarded. That this glycerol is available for conversion into glucose is chemically certain; that the glycerol of the body is converted into sugar and burned as such, is highly probable. The amounts involved would not be negligible. Fat contains 10 per cent. of glycerol; 200 grams of fat are a usual ration of fat for a diabetic. From the glycerol of this amount of fat the body could form 20 grams of glucose. When it is recalled that few diabetics, on a protein-fat regimen, eliminate over 50 to 75 grams of sugar, the error introduced into the interpretation of the G : N ratio by 20 grams of glucose formed from glycerol is obvious.

Glucose-nitrogen Ratio.-Coming now to the use that has been made of the glucose-nitrogen ratio in the development of the theories of diabetes, we meet with a most unsatisfactory situation. On the one hand, it having been assumed that the diabetic burns no sugar, the ratio has been used as an index of the formation of glucose from protein. On the other hand, using the current physiological ratio $(4.5: 1)$, as representing the potential formation of glucose from protein, the ratio found clinically has been used as an index of the loss of the power of burned sugar. To prove each point it is necessary to assume the other. It is not possible to assume that the glucose : nitrogen ratio represents accurately the relation of the formation of glucose from the protein undergoing catabolism. It is possible, but only in a general sense, to measure the combustion of glucose in the body by the determination of the glucose: nitrogen ratio, they being held roughly to be inversely proportional.

One additional fact tends to invalidate still more the conclusions drawn from this ratio. When the body of the diabetic, in partial or complete fasting, maintains its body heat by the combustion of its own protein and fat, it forms from the unit of its own protein less glucose than from a similar unit of foreign protein ingested as food. This fact alone makes it clear that the ratio currently used in physiology to represent the amount of sugar formed from protein $(4.4: 1)$ cannot be employed as the rigid basis of measurement in diabetes.

In the $\operatorname{dog}$ (in whom the physiological ratio of $4.4: 1$ was determined), the ratio in pancreatic diabetes will usually be found about $2.8: 1$ on an average. In some dogs higher ratios will be found, in other animals lower ratios. But by comparison with the ratios obtained in cases of human diabetes, the ratios observed in the dog are very constant. In phloridzin intoxication in dogs the most common ratio is about $3.7: 1$, though it may be as high as the normal ratio for the 
dog or even higher, up to $5: 1$, the highest figures usually only during the first few days. The high glucose: nitrogen ratio of the first few days of a phloridzin intoxication has been commonly attributed to the washing out of the stored carbohydrate. This may be true in some instances. But in other carefully studied instances, the glucose output was constant from the first day, and the fall in ratio on the third or fourth day was due to a rise in the elimination of nitrogen. When we consider the great difficulties that attend the fixing of the physiological ratio, this we may set at $5: 1$ as a maximum. From this it may be inferred that the diabetic dog eliminates on an average only a little over half the glucose formed from protein; the balance must have been stored or burned. The phloridzinized dog apparently eliminates a larger proportion of the sugar formed from protein than does the diabetic dog, in the usual case eliminating over three-quarters of the glucose formed. It is most suggestive to note that in diabetes in the dog and in phloridzin intoxication in the dog, the ratio observed after the first days is never higher than the ratio determined physiologically for the same animal. Assuming, therefore, that in the physiology of the $\mathrm{dog}$, the body normally forms five units of glucose from the protein containing one unit of nitrogen ( 1 gram protein $=0.8$ gram glucose), it is clear that if the control of the experiments be complete, so long as we infer that the faculty of formation of glucose from protein is a constant, it follows that the diabetic dog, as a rule, retains the power of burning from a third to a half of the sugar produced from the physiological ration of protein, while the phloridzinized dog, whose powers of combustion of glucose are normal, burns less simply because the glucose is swept out of the system before the muscles have opportunity to burn it. When we limit our inductions to the careful experiments in the dog (those in which it may be fairly assumed that the body was neither giving up stored sugar nor storing sugar, storing nitrogen nor giving up stored nitrogen, where the experiments were prolonged and controlled and where preformed carbohydrate of all kinds was excluded) it is certain that the glucose: nitrogen ratio cannot be used as a measurement of the formation of glucose from protein. But on the assumption that diet for diet this is a fixed faculty, a constant, the glucose : nitrogen ratio can be used as an approximate indication of the loss or retention of the power of burning glucose. As will be later pointed out, this conclusion is supported by the study of the respiration quotient.

If one sweeping assumption be made, it is possible on the basis of the experimental data to regard the glucose-nitrogen ratio as representing the ratio of formation of glucose from protein. This assumption is that amino-acids build sugar only via lactic acid, and the availability of amino-acids depends upon their convertibility into lactic acid. The earlier physiological ratio of $4.4: 1$ is unquestionably too high, since determined early in the tests. When fully intoxicated with phloridzin, the dog burns very little sugar, as may be shown in experiments by direct administration. In such a dog, the ratio is 
usually about $3.7: 1$. On the basis of the stated assumption, only one molecule of lactic acid would be formed from each molecule of aspartic and glutamic acids and from leucin and lysin; the remainder of the carbons would be burned. If now we calculate the amount of lactic acid that could be derived from a protein on the basis of this assumption, we obtain values that lie close to the ratio observed in the nitrogen and glucose outputs of the phloridzinized dog. The aminoacids in casein as estimated by the ester method make up only about 50 per cent. of the original weight; there is great loss in the procedures. Let it be assumed that the figures at hand represent the relative values and these we will double to secure the total. When now these different values for the several amino-acids are calculated into terms of lactic acid and this then into glucose, we obtain for the gram of casein the figure 0.56 gram of glucose, corresponding to the ratio $3.5: 1$ - a very close approximation. The aromatic amino-acids, including histidin, must be included in the calculation. The assumption is rather hazardous; but the concordance is certainly striking.

When we come to the analyses of cases of clinical diabetes, we find no such simple, or relatively simple, state of affairs. This must be due, in large part or even entirely, to the fact that it is not possible to make the determinations of the glucose: nitrogen ratio under such conditions of control as are possible in the dog. We cannot be sure that the patient is not eliminating glucose from stored carbohydrate, or even that he is not at the time storing glycogen. We cannot be sure, except under the conditions of a metabolic balance experiment, that the patient is not retaining nitrogen or losing nitrogen, or catabolizing his own protein. We are not able to so govern the diet as to be certain that preformed carbohydrate is excluded; it is not possible to feed a human diabetic on casein or codfish flesh for a period of days: The results would be less uncertain if we were able to make the determinations of the ratio while on a protein-fat regimen after said constant regimen had been previously persisted in for a week, so that the influence of previously ingested carbohydrate could be eliminated; but it is rarely possible to so diet a diabetic. Under all these circumstances it ought to be foreseen that the glucose: nitrogen ratios obtained in the study of clinical diabetes must be exposed to wide variations that ought not to be ascribed to the disease or made the basis for inference.

It has been often assumed as proper that a limited known amount of carbohydrate may be added to the diet of a diabetic when the ratio is being determined, this amount to be subtracted from the sugar of the urine before the ratio is calculated, on the obvious assumption that all the added sugar is eliminated. This practice cannot be countenanced, as it adds a second unknown whereby the certainty of the ratio determined is still further reduced. If the respiratory quotient were shown to be unchanged by the addition of the sugar, and this were known to be recovered completely, as compared with a period without it, the procedure might be permitted; but then it would have no purpose, 
since the desired ratio would have been obtained in the fore-sugar period.

The glucose:nitrogen ratios observed in clinical diabetes run from $1: 1$, seen in mild cases, up to 12 or even $14: 1$. 'The ratio is usually in direct relation to the severity of the disease, though to this there are striking exceptions. It will be best, in the discussion of these ratios, to make of them three subgroups: Those running from 1 to $5: 1$; from 5 to $8: 1$; and those ranging above $8: 1$. The majority of cases of diabetes present ratios running below $5: 1$. There are very few cases as low as $1: 1$, though as stated there are typical cases of diabetes that have no glucosuria on a protein-fat diet. Ratios of 2 to $3: 1$ may perhaps be said to be the most common, and one of the best students of diabetes has termed the ratio $3.7: 1$, when determined under the conditions of a protein-fat diet, as the "fatal ratio," higher ratios being, of course, understood to be equally fatal.

Let us place these ratios and their meanings in concrete terms in a table. Let it be assumed that the subjects are in nitrogen balance, on a diet of fat 200 grams and protein 150 grams a day, corresponding to 125 grams glucose. The protein of the diet may be set in round figures 25 grams per day. Under these circumstances, for the following series of ratios, we have the following values:

\begin{tabular}{|c|c|c|c|}
\hline Ratio & Nitrogen & Glucose in urine & Glucose burned or stored \\
\hline $1: 1$ & 25 grams & 25 grams & 100 grams \\
\hline $1.5: 1$ & 25 & 37 & 87 \\
\hline $2: 1$ & 25 & 50 & 75 \\
\hline $2 . \overline{5}: 1$ & 25 & 62 & 63 \\
\hline $3: 1$ & 25 & 75 & 50 \\
\hline $3.5: 1$ & 25 & 87 & 38 \\
\hline $4: 1$ & 25 & 100 & 25 \\
\hline $4.5: 1$ & 25 & 112 & 13 \\
\hline $5: 1$ & 25 & 125 & " \\
\hline
\end{tabular}

\begin{tabular}{|c|c|c|c|}
\hline & & & $\begin{array}{c}\text { Glucose derived in body } \\
\text { outside of diet }\end{array}$ \\
\hline $6: 1$ & 25 grams & 150 grams & 25 grams \\
\hline $7: 1$ & 25 & $175 "$ & 50 \\
\hline $8: 1$ & 25 & 200 & 75 《 \\
\hline $9: 1$ & 25 grams & 225 grams & 100 grams \\
\hline $10: 1$ & 25 " & 250 " & 125 " \\
\hline $11: 1$ & 25 ، & 275 & 150 \\
\hline $12: 1$ & 25 & 300 & 175 " \\
\hline $13: 1$ & 25 " & 325 & 200 \\
\hline
\end{tabular}

There is no question that, as a rule, the severity of the disease runs parallel to the inability to burn glucose and to the height of the glucose: nitrogen ratio. Assuming that on the average these cases of diabetes are not storing large amounts of glycogen or losing large amounts of glycogen previously stored, that they are in nitrogen balance and that the diet prescribed is the diet actually taken, it might be fairly concluded that when below $5: 1$ the ratio is a general index of the power of the 
body to burn sugar; that the amounts of sugar stated in the table (on the basis of the physiological ratio of $5: 1$ ) to be derivable from the protein in the diet are so derived; and that the amounts in the urine represent the fraction not burned, the difference the fraction burned. If we could be certain of all these factors, the glucose-nitrogen ratio would be a very useful index of the state of the chief defect of the diabetic, the power of burning sugar.

Many cases, however, present much higher ratios. There is in the table a group including 6,7 and $8: 1$, that are grouped by themselves. Though these are above the ratio stated as regarded as the normal physiological ratio $(5: 1)$ they are still within the ratio that would be possible if all the carbon of the molecule of protein were regarded as converted into protein. By some students of diabetes it is held that the diabetic organism displays the pathological faculty of converting into glucose the full carbon content of the molecule of protein, this being regarded as a facultative defect of the disease. We shall later refer to the facultative hypothesis in another connection. If the highest ratios fell within $8: 1$, it might be of some purpose to invoke such a hypothesis; but so long as many cases present ratios greatly in excess of this for which explanations must be found outside of the protein molecule, there is no purpose in this hypothesis for the middle group of cases. The extra amount of sugar involved in these cases is not large and may be reasonably accounted for by reference to the storage capacities of the diabetic body, or to inexact control of the experiment.

Now a few cases exhibit decidedly higher ratios, running up to $12: 1$. How are these to be interpreted? Here the excesses of sugar are large. Assuming that the diet has been controlled, is it possible to derive these in any way from the stored carbohydrates of the body or from protein? Or are we to assume this sugar to have been derived from fat? We face here again the situation that confronted us when the question of the origin of glucose from protein was up for determination. It was there necessary to so set the experiment as to recover such amounts of glucose as to make its derivation from the stored carbohydrates of the body quantitatively impossible. Here it is necessary to so set the experiment as to recover such amounts of glucose as to make its derivation from the stored carbohydrate of the body and from the protein catabolism quantitatively impossible. The experiment has been so carried out as to make it absolutely certain that the body produces glucose in excess of all possible derivation from carbohydrate; and it has been possible to prove, quite positively, its origin from protein under the circumstances. Has it been possible to recover such large amounts of glucose in cases of diabetes as to make the carbohydrates and protein both unequal to its origination, leaving only the fat to serve as the source of the glucose? This question, the origin of glucose from fat, is today the burning question in diabetes.

The question of the formation of sugar from fat as a physiological process will be discussed in another chapter. It will be sufficient to 
state here that it has not yet been proved or disproved. If sugar were formed from fat normally, we would reasonably infer that the process holds in diabetes. If the formation of sugar from fat could be proved in the diabetic, that would furnish strong grounds for the inference that such a process occurs in health. It is a safe rule, when applied to the main streams of metabolism, that chemical processes are fixed and not facultative. And while a normal chemical reaction may be lost in disease, a new chemical reaction of so fundamental a character as the formation of sugar from fat (or from protein) is hardly to be gained in disease. It may be possible, in a disease, to uncover a reaction or process that in health is not available to experiment. If, therefore, it should be proved in diabetes that fat is converted into sugar, this would have a fundamental bearing upon our physiological conceptions.

The sugar found in the urine in the cases of diabetes with high ratios may have come from several sources. These must be known and controlled in any attempt to decide the derivation of the stated sugar. It may, in the first place, have come from preformed carbohydrates of the diet. We insist that the diet of the diabetic must consist, during the period of the test, of fat and protein. This standard at once rules out a large number of reported cases. More than this, to be rigid, the test ought to have been preceded by a number of days of the fat-protein regimen, and the test should run over not one but several days. This would eliminate many more of the reported cases with high ratios.

Could the amounts of sugar involved be derived from tissue glycogen? The human body, when rich in glycogen, may contain as much as two kilos of glycogen, corresponding to two and a half kilos of glucose. The diabetic is always poor in glycogen, and it may be assumed that no diabetic in the active stage of the disease has a kilo of glycogen in the body. Unlike the depancreatized dog, who travels steadily the downward course, human diabetics have their ups as well as downs, periods in which glycogen is stored being followed by periods in which the carbohydrate is swept out of the tissues. It is clear that such a sweepingout of the glycogen, in the form of sugar, could explain a high glucose: nitrogen ratio for a number of days, possibly for a week. By this fact others of the reported cases of high ratios are made uncertain in their meaning. Conversely, if the ratio were determined in a period of storage, a very low ratio would be found, fictitious again if applied to the factor of the combustion of sugar. Theoretically, the determination of the respiratory quotient ought to place us in the position of deciding whether the body is burning or storing the retained carbohydrate; but the practical clinical use of this quotient has not yet been placed on a solid experimental basis.

In the opinion of the writer, the amount of glucose that can be calculated as derivable from the glycerol of the fat undergoing combustion ought in all cases to be subtracted from the figure for glucosuria. This would rule out some of the reported cases. 
It should be known in all cases that there is no nitrogen retention. This has been neglected in many eases, which are to be excluded. The accurate methods of the metabolic experiment are nowhere more in place than in the study of diabetes, in which periods of nitrogen retention, on a heavy protein input, are of frequent occurrence. A slight retention of nitrogen would result in a marked increase in the ratio.

When now we eliminate from the literature the cases of high glucose: nitrogen ratios in which the necessary controls have not been done, are there any instances left in which the sugar recovered from the urine was in such amount as to exclude all other sources of derivation outside of fat? The question must be answered in the negative. The writer has theoretical preconceptions in favor of the formation of sugar from fat. But it is certain that the proof has not been accomplished in the study of diabetes. The figures do not raise a reasonable presumption in favor of the origin of glucose from fat. What the data really do is to enable us to state the question concretely; they teach us how the investigations must be done and controlled. The decision must be left to future measurements in which the shoals of past misfortunes will be avoided.

Upon the other hand, we must realize that the arguments that are commonly advanced against the hypothesis that sugar is derived from fat in the diabetic are not valid. When fat is given to a diabetic in excess, the glucosuria is not increased; in a word, there is no parallelism between the fat ingestion and the sugar output, as there is so often between the protein input and the glucosuria. But the objection is based upon a misconception. The catabolism of protein is largely exogenous, it is a function of the input; the combustion of fat and sugar is not exogenous, not a function of the input, but solely of the body needs. One cannot stimulate the combustion of sugar by giving sugar, or of fat by giving fat; the excess is stored for future use. Since utilization of fat, as well as of sugar, is not dependent upon the input, there would be no reason to expect the glucosuria of the diabetic to be influenced by the ingestion of fat, even were sugar derived from fat.

The fact that refrigeration and work do not result in increase in the glucosuria in diabetes and in phloridzin intoxication, though they increase the fat catabolism, has also been urged as an argument against the origin of sugar from fat. A like error in reasoning lies here concealed. Work does not increase the acetone bodies in the urine of the diabetic; but will anyone now contend that these are not derived from fat? Even though sugar be formed from fat-as the acetone bodies certainly are-it would not necessarily follow that sugar and the acetone bodies would be increased when the combustion of fat is increased by work.

Upon the part of those who incline to regard the ratio of glucose to nitrogen as an index of the capacity or act of formation of glucose from protein, an explanation of the higher ratios has been suggested 
as a facultative process, $i$. $e$., the diabetic forms more sugar from the unit of protein than does the normal. This explanation is too lame to travel far. There is, however, a school of physiologists who are convinced that the formation of glucose from protein in toto is a pathological process peculiar to diabetes. In this sense, the diabetic would suffer from a primary overproduction of sugar. According to this view, the body normally burns directly the carbon moiety of the protein molecule; in the diabetic this is, so to speak, side-tracked to glucose, which being incombustible is eliminated. This explanation has no proper direct foundation and is opposed-entirely apart from work on diabetes-by three sets of facts with which it cannot be well reconciled. The normal blood contains a fore stage of glucose, which in the normal liver is converted into glucose; this fore stage we may reasonably regard as amino-acid. The perfusion of the normal liver with certain amino-acids results in the formation of glucose. And finally, the formation of glucose from protein in the dog with phloridzin intoxication-which is certainly not diabetes-speaks directly against the hypothesis. The mass relations speak strongly against such a view; in diabetes we have hyperglucemia, in the phloridzin intoxication we have hypoglucemia - yet in each case we are supposed to have a pathological facultative formation of glucose from protein. The hypothesis is too far-fetched. That the acts of digestion have nothing to do with the process is shown by the fact that when the products of a predigestion of protein-amino-acids-are given to the dog with phloridzin intoxication, the formation of sugar occurs just the same.

It has been common practice in the treatment of diabetes to watch the sugar elimination when on carbohydrate-free diet, and mark its concordance with the protein of the diet. While very instructive in many cases, it was early known that striking exceptions occur. The use of the glucose:nitrogen ratio, when determined under conditions of control, is without question of much greater reliability, and should form a part of the study of every case of diabetes. The influence of different proteins upon the formation of sugar in the case may also be studied in this manner. While studies on the adaptability of different proteins to the formation of glucose are physiologically very difficult, the practical application in diabetes of such proteins as yield the least sugar is made possible by the use of the glucose-nitrogen ratio. Plant proteins seem to yield less glucose per unit than animal proteins; and egg albumin often yields less sugar than muscle or casein. Such studies must be carried out in extenso with diabetics, since there are other factors of importance that can only be fixed by thorough study. The time of digestion is undoubtedly of importance; the slower the processes of digestion and assimilation required by a certain protein, the less sugar-other things being equal — the body will form from it, since more time will be afforded the powers of glycogenesis and of combustion to dispose of the resulting sugar. The simple result of less sugar to the unit of protein does not, therefore, mean that the 
protein contains intrinsically less sugar-forming amino-acids; it may mean simply that the body has more time to handle the products of digestion-quite in the same sense that in the normal individual sugar in quantity can always produce glucosuria while starch can do so in no quantity.

When the diabetic loses in part or entirely the sugar formed from protein, the body loses by that much the heat value of the protein. After subtracting from the caloric value of protein the caloric value of the urinary products of the protein catabolism, the heat value of protein in the body is about 4 Calories per gram protein. Using the physiological ratio of 5 grams glucose formed from protein to 1 gram nitrogen, 1 gram of protein would yield about 0.8 gram sugar, of a heat value of 3 Calories; if all this sugar were lost in the urine, the protein in such a body would have only the heat value of $1 \mathrm{Cal}$. per gram. This, of course, represents an extreme case. But instances are common in which at least half the sugar is lost, so that the caloric value of protein becomes reduced to $2-2.4 \mathrm{Cal}$. per gram. The diabetic body is quite in the situation of a boiler, built with a wide grate for the burning of large-size coal, being fired with very fine coal, much of which falls unburned into the ash-box. The catabolism of protein is under all circumstances attended by a heat loss through the operation of the factor of the specific dynamic action of protein. This heat is lost in the body and is not available for tissue uses, $i$. e., not convertible except for chemical regulation of body temperature and for work. This heat is, however, not a total loss in diabetes, as under the conditions of the total metabolism it warms the body and possibly permits the combustion of fat to be reduced by that amount. At least this is the interpretation to be placed upon some recent estimations of the heat production in diabetes; it seems that the heat production due to the specific dynamic action of protein is not in diabetes the total loss in the resting state that it is in the normal metabolism. The conversion of protein into sugar is, as a chemical reaction, attended with a loss of heat to the body, it is an endothermic reaction. If all the carbon of the molecule of protein were converted into sugar, this would yield about 1.4 grams of a heat value of 5.1 Calories, while the molecule of protein from which it was formed has in the body the value of only 4.4 Calories at the best. This is an argument against the conversion of all the carbon of the molecule of protein into glucose, so long at least as one inclines to the view that the chemical processes of the body proceed along the simplest lines.

The Formation of Sugar from Fat.-A brief resume will suffice here. There are today no adequate grounds for stating or rejecting the hypothesis of the origin of sugar from fat, attractive as it is on biological and chemical grounds. The failures of attempts to increase glucosuria in the diabetic by massive ingestions of fat prove nothing, since the combustion of fat is not a function of the input. Whether lying in the fatty depots or regularly ingested in the diet, the body 
utilizes of fat only what is needed, not what is presented. This statement is supported by the observation that the respiratory quotient is not influenced by the added administration of fat to a normal diet. It is doubtful if the question can ever be settled except by quantitative experiment-the recovery in the urine of the diabetic of more sugar than could have been derived outside of fat. The older figures for the occurrence of the acetone bodies offered a suggestive hint of the origin of sugar from fat. If a diabetic were to eliminate 200 or 300 grams of acetone bodies daily, in terms of beta-oxy-butyric acid, and each molecule of the acid were to represent one molecule of higher fatty acid, it would follow that the body under those circumstances was catabolizing 600 to 800 grams of fat per day-something contradicted by the known figures for the heat production. This discrepancy could have been explained by the conversion of a portion of the fatty acid into sugar. The newer figures for the amounts of acetone bodies are far lower than those previously stated; amounts of over 100 grams per day are not nowadays reported, and 50 grams per day is a high output. With these figures, the discrepancy between the heat production of the body and the amount of fat catabolized (judged by the elimination of the acetone bodies) is not striking, even in the cases of highest acidosis. The point, however, deserves concrete investigation. Let an illustration be cited. If one molecule of beta-oxy-butyric acid be derived from one molecule of higher fatty acid, then to form 100 grams of beta-oxy-butyric acid, at least 280 to 300 grams of fat must have been catabolized. This fat has a heat value of about 2800 Calories. Subtracting now from this 450 Calories, the heat value of the beta-oxy-butyric acid lost, we have 2350 Calories, to which must be added the Calories produced in the inevitable catabolism of protein, bringing the total up to or more than 2600 Calories, over 35 Calories per kilo per day for a body of 70 kilos. This is rather a high figure, somewhat in excess of the figures noted in direct estimations in diabetes. But when we consider that the elimination of 100 grams of beta-oxybutyric acid is also very exceptional, the figures do not more than suggest a formation of sugar from protein, nor is the suggestion striking.

\section{THE COMBUSTION OF FAT IN DIABETES}

In all cases of established diabetes the combustion of fat is quantitatively excessive, and in most cases qualitatively defective, just as in the case of protein. The defects in the metabolism of fat may not appear early in the course of the disease, but eventually they may be said usually to dominate the situation. We saw that the total heat production of the diabetic per kilo per minute is in excess of the normal. Glucose can be burned to but a limited extent; the loss of the glucose derived from protein reduces greatly the heat value of the protein. With more heat produced and with sugar and protein yielding but a 
fraction of the normal amount of heat, the burden falls upon the catabolism of fat. If this could but be relied upon in diabetes, the situation of the unfortunate subject would be hopeful. But the catabolism of fat breaks down, so that not one support of the tripod remains intact.

Why does the catabolism of fat break down in diabetes? Is it simply a result of overwork, or is there some essential defect in the chemical mechanism? It cannot be simply the result of the enforced exaggeration of function. The average diabetic is fully supplied with fuel when given 100 to 150 grams of protein and 200 to 250 grams of fat per day. Now it is certain that a normal individual could take such a diet indefinitely with no other untoward result than a slight acetonuria. Many barbarian tribes in the colder regions live almost entirely on protein and fat. Of course, the situations are not comparable; for the normal individual on such a diet utilizes the sugar derived from the protein, while the diabetic does not in large part. But it is clear that the defect in the catabolism of fat in diabetes is not the result of the excessively fatty diet or of the abnormal dependence placed upon the combustion of fat as the chief source of body heat.

This brings us to the real question. Is the defect in the combustion essential and primary in the fat metabolism, a direct instance of metabolic defect as is the loss of power of burning sugar? Or is it a secondary result, like the exaggerated catabolism of protein, secondary to the defect in the combustion of glucose? This is the second crucial question in diabetes.

In the normal oxidation of fats, these are first hydrolyzed to the fatty acid and glycerol, the latter converted into sugar. The oxidation of the normal fatty acids with chains of carbons in even numbers is probably effected at the beta atom of carbon, and results in the splitting off of the two lower carbons of the fatty acid, the alpha and the carboxyl groups. Thus the chain is made two carbons shorter. This process is then repeated, down to butyric acid. This scheme holds strictly for palmitic and stearic acids, and for the purposes of this argument, for oleic acid also. From this point on the scheme is uncertain, as will be detailed in another section. If the oxidation were accomplished by attack on the alpha carbon, we would have propionic acid formed from butyric acid, then acetic acid, then formic acid and complete combustion. If the oxidation be by attack on the beta carbon, then beta-oxy-butyric acid would be formed from butyric acid, then diacetic acid. Diacetic acid could be converted into acetic acid, and this into formic acid as before. There is no question but that the latter is the course in diabetes, any question pending concerns the normal course. Reserving this for later discussion, in diabetes either the body forms beta-oxy-butyric acid from butyric acid as an abnormal reaction, that then naturally leads to diacetic acid and acetone (the acetone group); or the diabetic has lost the normal power of burning. beta-oxy-butyric acid and diacetic acid. Whatever the course, the diabetic metabolism produces and contains beta-oxy-butyric and 
diacetic acids and acetone in large amounts, and this is the basis for the acidosis of the disease.

Is the metabolic defect confined to the lower stages of the process of oxidation of the fatty acid, to and below the butyric acid? In the earlier days, when the methods for the estimation of these bodies were imperfect, such large amounts of acetone bodies were supposed to be recovered in cases of diabetes as to make the assumption natural that more than one molecule of butyric acid might be produced from higher fatty acid - unless one were to invoke the formation of sugar from fat. Nowadays we know that the amount of the acetone bodies formed in diabetes is not so large as to make such an assumption proper. It may, therefore, be regarded as certain that in diabetes but one molecule of butyric acid is formed from one molecule of higher saturated fatty acid; the defect in the combustion of fat lies below the stage of butyric acid. The exaggeration of the fat metabolism is made evident in the excess of fat to be found in the blood, liver, and muscles in diabetes, in striking contrast to the paucity of glycogen in the same tissues.

In the section dealing with the fat metabolism will be found a detailed discussion of the question of the derivation of the acetone bodies. While there is evidence that these might be derived, in small amounts at least, from glucose and protein, it is certain that in diabetes-and probably under all other conditions where the clinical complex occurs - they are derived from fat. The quantitative relations alone make this conclusion necessary. Many cases of diabetes eliminate amounts of the ketonic bodies that could not possibly have been derived from glucose or protein. One case has been reported in which a diabetic, ingesting no carbohydrate, eliminated 97 grams of acetone bodies and 40 grams of sugar, with nitrogen in the urine corresponding to a protein catabolism of only 90 grams. This is, of course, an extreme case. But instances are common in which the acetone bodies could not possibly have been derived from the glucose and protein, but must have been derived from fat. In the diabetic we will, therefore, regard it as settled that the ketonic bodies, the acidosis, are of fatty origin.

When the substances concerned are administered to the diabetic, we obtain interesting results. Butyric acid is almost quantitatively converted into beta-oxy-butyric acid. When beta-oxy-butyric acid is administered, it is not oxidized but is eliminated. Propionic acid and acetic acid are oxidized as in the normal. Diacetic acid is in part oxidized, in part eliminated, in part converted into acetone. Acetone is not oxidized in the body, normal or diabetic, to any notable extent. Whether the diabetic organism converts diacetic acid into acetone to a greater relative or absolute extent than does the normal metabolism is not known. The normal body oxidizes beta-oxy-butyric acid very well, diacetic acid also.

Elimination of Acetone Bodies.-The amounts of the acetone bodies that are eliminated in diabetes are subject to great variations in total and in the relative proportions. When the total amount of the acetone 
bodies is low, acetone may be the only member present, and is always the predominating one. As the total output increases, the diacetic acid and the beta-oxy-butyric acid become more and more prominent, especially the latter. In the massive eliminations, the beta-oxy-butyric acid is always in largest amount, the diacetic acid and acetone in small, or even nominal amounts. For instance, there may be 50 grams of beta-oxy-butyric acid, 10 grams of diacetic acid, and 5 grams only of acetone. This is due to the fact that the capacity of the diabetic body for the distoxication of diacetic acid by conversion into acetone is limited; and the capacity of the diabetic body for oxidation of the beta-oxy-butyric acid to diacetic acid is limited. The total figures are difficult of exact estimation, partly because some of the acetone is eliminated in the expired air and some of both acids in the perspiration, but also because of difficulties in the analytical methods. The amount of acetone in the expired air has been greatly overestimated. It is also important to know that the breath in diabetic coma may present a fruit-like odor that can be shown not to be due to acetone. A total ketonic elimination of 20 grams per day is common, eliminations of over 50 grams are uncommon, up to 100 grams very rare and seen only in diabetic coma. The administration of alkali often seems to result in a sweeping out of the substances, though sometimes this is not observed; and in particular it is on the two acids and not on the acetone that the effect is apparent. In other words, the binding of the acids with external alkali has no effect upon the powers of conversion into acetone. Occasionally one will see a case of diabetes with little glucosuria, a low protein catabolism and a total elimination of 30 to 40 grams of acetone bodies, with few symptoms, the subject remaining in this state for a long time.

There are two methods of distoxication of the acids (since oxidation fails): by conversion into acetone and by neutralization. Neither is perfect, since the acetone is somewhat toxic, and the salts of beta-oxybutyric acid are also toxic. As stated, the amount that can be converted into acetone is limited. The function of neutralization is not limited; the amount of ammonia that is to be derived from the protein catabolism is, in mass, always sufficient to combine with all the acids ever produced in diabetes. The difficulties lie in the affinity constants of the different cations, and in the strength of combination of the fixed cations in the tissues. If the acids were but eliminated entirely as ammonia salts, all would be well. But as they circulate they abstract from the tissues sodium, potassium, and calcium and thus disturb the equilibrium of the cations in the tissues. Obviously the correct therapeutic measure is not to administer an alkali of one cation-as sodium bicarbonate-but a mixture of sodium, potassium, and calcium, in order that all these may be present in abundance in the tissues and the equilibrium of the cations in the central nervous system thus maintained. To simply give sodium is little better than what the body does, offer only ammonia. This proposition is based upon the law 
of mass action, and has the support of much experimental work in general biology. The place to test it in diabetes is not in the attack of coma-when the damage is done-but in the daily administration of alkali, to such an extent that the ammonia of the urine is reduced to normal. A case is on record in which a diabetic had taken sodium bicarbonate daily for years. Three days after the cessation of the drug, an attack of coma occurred. Obviously, through all this time while sodium was being given, potassium and calcium were being abstracted from the tissues, since no amount of sodium can prevent the potassium and calcium from participating in the mass reaction of neutralization. The results of alkali therapy would without question be more certain, if all the cations are administered. It must be recalled that potassium and calcium, outside of the blood stream, outweigh sodium; especially potassium, which in some organs is present to four hundred times the amount of sodium.

The ammonia of the urine in subjects not taking alkali stands as a general index of the amount of ketonic acids. The ammonia of the normal, rarely over 0.5 gram per day, is often exceeded; the output may be 2 or 3 grams, 5 or 6 in severe cases, up to 10 or 12 . There is no question that a high figure for ammonia is a danger signal. Each gram of ammonia represents about 6 grams of beta-oxy-butyric acid. And as the estimation of urinary ammonia is comparatively easy, the estimation of the ketonic acids may be conveniently accomplished in this way. The action of administered alkali may also be measured in this way. When fixed alkali is given, the cation will not replace directly and at once the ammonia in molecular relations. If we administer 1 gram mol. of sodium bicarbonate, it will not displace 1 gram mol. of ammonia from the urine (one part ammonia to six parts sodium bicarbonate). Usually much less ammonia will be driven from the urine, though in some cases, after a few days, nearly the theoretical amount may be displaced. Sometimes the amount of ammonia that is displaced is very low; thus the daily administration of 20 grams of sodium bicarbonate may lower the urinary ammonia only 1 gram. We deal here with mass relations that are not yet definable. Two explanations suggest themselves. One is that a part of the fixed alkali administered is held in the tissues to replace cations that have been abstracted by the acidosis. This is surely true, but it cannot explain the gross results. The second explanation is that the alkali is given in a few large doses, often with the meals. It is quickly absorbed, for a short time the circulation is flooded, it is eliminated and there are long periods in the day and during the night when none of it is in operation and the ammonia alone is available. If the fixed alkali were to be administered often, say in hourly doses during the day, with a heavy dose on retiring, a better action might be anticipated. Beyond this, it must be expected that some ammonia would remain simply as an incident of circulation. To drive out the ammonia from urine-to render the normal urine ammonia-free-requires not less than 90 grams of sodium bicarbo- 
nate per day. It may be assumed that when fixed alkali is administered, it will be localized more in the tissues than in the circulating blood; that is, in any moment, the blood would have a lower binding power for it than have the tissues. It may be taken for granted that the formation of the ketonic acids occurs in the liver, where occurs also the formation of ammonia; the combination of the two occurs in situ. A part of the blood streaming from the liver would go from the heart directly to the kidney, and from this we would expect the ammonium oxy-butyrate to be eliminated. The larger portions would go to the tissues, there to meet with greater amounts of the alkali, with the result of a binding of the fixed cation with the acids, the ammonia being set free to return to the liver and be converted into urea. And, lastly, it must not be supposed that the different cations would act as they would if placed with the fatty acids in a test-tube. There are especial affinities of cations for tissue components, ion-protein combinations, that must play an important rôle.

Tissues combine with acids as well as with cations. It is, therefore, not to be denied that the beta-oxy-butyric acid might be in combination with tissue components, and these might be difficult of prompt dislodgement. There is no evidence that the salts in the blood are bound. The normal neutral reaction of the blood is not altered in the most severe cases, though naturally its $\mathrm{CO}_{2}$ content is reduced. In the coma of acidosis an acute acapnia occurs. This is due in part to the presence of the ketonic acids in the blood, in part to hyperpnea, the disturbance in respirations being caused by the acetone in the circulation.

The study of the urine in untreated cases has shown wide variations in the salt content from the normal. Calcium and magnesium have been found in marked excess. For potassium no data are at hand. It has been usually assumed that the calcium was abstracted from the bones. But there is evidence that the calcium of the nervous system is in a state of more labile combination than in bone; and the abstraction of a small amount would be expected to lead to pronounced symptoms. A finding that is very suggestive in this connection is that of increase in the endogenous purin, indicating an exaggerated nucleic catabolism. Since it has never been possible to show a primary abnormal catabolism of protein in diabetes, it is to be inferred that we have here a specific nuclear disturbance, which might well be associated with a disturbance in the equilibrium of the cations in the nucleus. It has been stated that the phosphoric acid of the urine is in excess in many cases of diabetes; this could be derived from either nuclear catabolism or from the bone. Upon these statements no interpretation may be placed today, since observations on the urinary phosphoric acid, without knowledge of the input and of the fecal output, have little meaning except to mislead.

Apart from the three bodies that we group under the term of "acetone bodies," the urine contains volatile acids, and these are usually also 
increased in diabetes. Whether they come from the fat catabolism or whether they indicate the failure of the body to oxidize the volatile acids elsewhere derived, is not known. Fatty acids above acetic acid increase the acidosis in diabetes; and it is possible that the fatty acids resorbed from the intestine (due to bacterial action on carbohydrates) are eliminated in part as ketonic bodies; in part, however, unchanged.

Butter, on account largely of its content of butyric acid, increases notably the output of the acetone bodies. As elsewhere stated, triolein yields a larger amount than do tripalmatin and tristearin. Under these conditions butter and the liquid fats, like olive and cotton-seed oil, are not so adapted to the diet in diabetes as the fats of beef and mutton flesh. But the attempt to induce the diabetic to consume daily, for any length of time, 200 grams of beef or mutton fat is likely to arouse a revulsion to the diet, so that it may be necessary to include butter and olive oil, even though they yield more beta-oxy-butyric acid, oleic acid yielding three molecules of butyric acid.

A large number of substances act antiketonically, $i$. e., depress the formation of the acetone bodies. All substances that can yield glucose in the metabolism do so act as a rule. Thus starches, sugars, protein, glycerol, pentoses, and lactic acid all so act. Some substances act in this way that are not known to form sugar in the body; thus ethyl alcohol, citric acid and d-gluconic acid do so. The sugars and sugarbearing substances act not in proportion to their ingestion, but in proportion to their combustion. The ingestion of fat in excess of the needs of the body does not have any effect upon the acetone bodies. The ingestion of protein is usually followed by a reduction in the acetone bodies, the increased catabolism of protein sparing the combustion of fat. The ingestion of ethyl alcohol, while it reduces the acidosis, in some manner seems to reduce also the toleration for glucose.

To the usual findings there are some striking exceptions. (a) Some diabetics exhibit a good toleration for sugar up to 100 grams per day, with heavy and continuous acidosis. (b) Some diabetics have little or no toleration for glucose, with little acidosis over such long periods of time as reasonably to exclude the action of the body glycogen. (c) In some cases of diabetes, carbohydrates display little or no antiketonic action, even though there is evidence that some of the ingested sugar is burned. (d) Some cases of diabetes display no increase in the acetone bodies when carbohydrates are cut out from the diet. (e) In some cases of diabetes, with a constant output of nitrogen and of glucose, the output of acetone bodies may fluctuate up and down in the most irregular manner. ( $f$ ) In some cases of diabetes, improving under diet and treatment, the acetone bodies persist after the glucosuria has disappeared. $(g)$ In some cases, exercise does not have the usual reciprocal influence on the combustion of sugar and of fat, as revealed in the acidosis.

The rule is that acidosis exists in inverse relation to the combustion of glucose; the less the power of burning glucose, the greater the acidosis. 
Or, in dynamic words, the less the power of burning glucose, the less the power of burning fat. Since according to our present conceptions, it is only from the stage of butyric acid that the difficulty lies, we may state the situation more concretely; the less the power of splitting glucose, the less the power of burning butyric acid. How are we to interpret this remarkable relationship?

The current interpretation is that the combustion of butyric acid is directly related to the combustion of glucose. In the language of one of the German writers, "Fat is burned only in the fire of carbohydrate." A mechanical illustration will make this hypothesis more tangible. Crude oil will not burn directly. When, however, it is sprayed with a jet of steam, it burns well. Here it is a question probably of physical state only. In the case of the combustion of fat, we are to infer that at some stage in the oxidation of butyric acid there is a fixed relationship to some stage in the cleavage of glucose; if the latter be not present, the former is not accomplished. In many instances, the acidosis seems related less to the momentary inability to burn ingested sugar than to the depletion of the glycogen of the tissues; and certainly the extreme depletion of the body glycogen places the diabetic in danger of an explosion of acidosis, of diabetic coma.

The exceptions to this rule of direct relationship are, however, so frequent and sometimes so glaring, that this hypothesis of intercombustion is not accepted by all. The facts could be explained by the assumption of a direct enzymic defect in the combustion of butyric acid, just as in the case of the combustion of glucose. Under this interpretation the action of the combustion of glucose becomes simply one of a division of burden. Anything that exaggerates a defective metabolism makes the defect more apparent. Anything that spares a defective metabolism makes the defect less apparent. When no sugar is burned, the entire burden falls upon the combustion of fat, and the defect (the acidosis) becomes exaggerated; when some sugar is burned, the combustion of fat is spared, and the defect (the acidosis) becomes less apparent. Obviously, under such an interpretation, other factors than the combustion of sugar could influence the combustion of fat and modify the diabetic defect in this catabolism. The numerous exceptions to the usual rule quoted above, are not out of harmony with this hypothesis. In particular, one other behavior deserves attention. It has been observed in severe cases of diabetes that the ingestion of increased amounts of protein, instead of lowering the acidosis, may increase it. And it seems that in severe diabetes, anything that increases the glucosuria, the introduction into the body of glucose that cannot be utilized, tends to depress the combustion of fat and to increase the acidosis. In one reported instance, an attack of diabetic coma followed directly the introduction of carbohydrate into the diet. This is easy of comprehension if we assume a fermentative defect in the fat catabolism. Then any injury would naturally exaggerate the defect, and the combustion of fat would be injured by 
the hyperglucemia just as the combustion of glucose is injured by the hyperglucemia. Harmonious also to this view is the action of combustible substances that spare fat combustion but do not form sugar. Thus ethyl alcohol, simply by sharing the burden of heat production, reduces the acidosis without in the least aiding the combustion of glucose or being itself converted into glucose. Other objections to the view that acidosis occurs in diabetes only because fat cannot be burned except in the fire of carbohydrate, will be stated in the discussion of the whole subject of acidosis, in the chapter devoted to the fat metabolism.

Associated with the acidosis of diabetes is usually found an excessive lipemia. The highest figure for the total fat content of the blood, under conditions when the fat catabolism is worked to the normal maximum (in starvation and in the use of a fat-protein diet) is not over 1 per cent. In severe diabetes we may find six to ten times as much, the fat coming either from the diet or from the fat depots of the body. Normally, the blood can handle $(i$. e., convert into the soluble state and deposit in the tissues) more fat than the diabetic blood contains. In some way in diabetes the mechanism of the solution of fat in the blood serum and its transportation through the body is disarranged. The blood has a singular capacity for the solution of fat at neutral reaction, the component factors of this solubility being not understood. We may assume the fat in solution to be the metabolic state, i.e., it is the fat in solution and not the fat in suspension that is utilized in metabolism. Now in the normal the blood carries little of the fat in suspension, it is promptly carried to the fat depots and deposited. In diabetes, this is not the case; the blood is loaded with a vast excess of fat in suspension, which we do not believe has any metabolic function, but is there simply as dead weight because it has failed of deposition or has been withdrawn from the depots in excess of requirements. Not only is the fat in suspension increased, the fat in solution seems to be reduced. It is possible that the defective lipogenesis noted in diabetes is associated, in part, with the reduced content of soluble fat and the increased content of suspended fat in the blood. In any event, these states suggest a more primary defect in the fat catabolism than simply direct dependence upon the combustion of glucose. When one contemplates the metabolism of an advanced case of diabetes, one must realize that it is quite the same as in the case of the one-horse shay.

\section{THE RESPIRATORY QUOTIENT IN DIABETES}

The respiratory quotient $\left(\frac{\text { Expired } \mathrm{CO}_{2} \text { c.c. }}{\text { Inspired } \mathrm{O}_{2} \text { c.c. }}\right)$ is a matter of great importance in diabetes, because it affords indications as to the type of materials undergoing combustion. Of importance in research it now is; of great clinical interest it would surely be if it could be readily determined. The quotient for the combustion of sugar is 1 ; that for 
the combustion of fat is 0.71 ; that for the combustion of protein may be set at $0 . S$; though it is doubtless different in different proteins, since the carbon content of proteins vary from 50 to 55 per cent., and the oxygen content from 18 to 24 per cent. Obviously, if the simplest relations were assumed to hold and we knew the protein and carbohydrate content of the diet of a diabetic, by a comparison of his respiratory quotient with that of the normal subject on the same diet, we could determine the utilization, or rather combustion, of the carbohydrate. This cannot be done by the estimation of the glucose in the urine, because we cannot separate glucose burned from glucose stored. That it cannot be done directly with the use of the glucose: nitrogen ratio has been already stated. In a similar manner, the determination of the quotient in a diabetic subject on a fat-protein diet would, by comparison with the quotient of a normal body on the same diet, indicate to what an extent the body could burn the sugar derived from protein. It is clear, however, from the present, already extensive data, that these simple relations do not hold. And the interpretations, although they follow approximately the lines indicated, cannot be made as direct as we would wish. There are several factors that tend to invalidate the direct interpretation of the respiratory quotient.

(a) The quotient for the combustion of fat is set at 0.71 . This is in itself a rounded sum, since the quotient must be a little higher for triolein than for tristearin and tripalmatin. But in diabetes a new factor enters. In the figure 0.71 the glycerol is regarded as burned; in diabetes, however, this glycerol after conversion into glucose is only partially burned, and may indeed often be regarded as entirely eliminated. This would reduce the quotient to possibly 0.69 .

(b) The quotient for protein is set at 0.8. This is under the condition that the glucose derived from it is burned. But in diabetes this is the case only in part, in part this sugar is lost. The quotient for the combustion of protein following the subtraction of the amount of sugar represented in the glucose:nitrogen ratio of $5: 1$ would be as low as 0.6. Depending upon the degree of retention of the power of burning the sugar derived from protein, this quotient would vary from 0.6 to 0.8. This indicates at once that in diabetes we may expect sometimes to find the respiratory quotient below 0.7 , below the lowest figure for the physiological combustion of any foodstuff.

(c) The respiratory quotient may be influenced by the diet of the day previous. Now it is not practicable to have diabetics fast over a whole day, so as to reduce this disturbing element. It will usually be found necessary to determine the quotient with not over twentyhour fast. If it could be assumed that the diabetic had lost entirely the power of storing glucose as glycogen and then yielding it to combustion during the fasting period, this error would be small and possibly negligible. But this assumption is not tenable. 
(d) The formation of sugar from protein and of the acetone bodies from fat, and their elimination and consequent withdrawal from the end products of the gas exchange, disturb the quotient. Subtracting the gross equation of butyric acid from the equation of palmitic acid, the quotient of the remainder which is actually burned in the diabetic body is 0.66 . If all the fat were burned only to the stage of butyric acid, the respiratory quotient for fat in the diabetic would be something like 0.66. But this defect is not total, only partial; some of the fat is usually completely burned. Therefore, for the diabetic the respiratory quotient lies somewhere between 0.66 and 0.69 ; just where is not exactly determinable, though the greater the amount of acetone bodies the lower the quotient. When butyric acid is converted into beta-oxybutyric acid, oxygen is added; when this is converted into diacetic acid, oxygen is added and water extruded; when this is converted into acetone, $\mathrm{CO}_{2}$ is split off. Thus when one molecule of butyric acid is converted into acetone, one molecule of oxygen is added and one molecule of carbon dioxid split off, and the respiratory quotient of this total process is 1 . It will be recalled, however, that with high formation of the ketonic bodies, the amount of acetone formed is relatively low. Therefore, the total process involves the binding of oxygen that has been inspired, and the quotient is lowered.

On the basis of the glucose : nitrogen ratio of $5: 1,1$ gram of protein would yield 0.8 gram of sugar containing 0.32 gram of oxygen, while the gram of protein from which it was derived contained at the most only 0.25 gram of oxygen. This oxygen could have been derived either from the inspired air or from water. But as the molecule of protein contains enough hydrogen (after combining with all the nitrogen to form amino groups) to form this amount of glucose, we are justified in the conclusion that gaseous oxygen is bound in this formation of sugar from protein. One gram of protein contains about 0.075 gram of hydrogen; of this not over 0.025 would be needed to combine with the 0.165 gram of nitrogen, leaving 0.05 gram of hydrogen to enter into the formation of glucose with the available carbon and oxygen plus gaseous oxygen. Just how much oxygen is thus bound, cannot be stated. But it is not a small amount, and the result is to lower the respiratory quotient. Assuming the oxygen requirement to be 500 to 600 grams per day, if a diabetic were to form from 150 grams of protein 120 grams of sugar and of this half were to be eliminated in the urine, some 30 grams of oxygen that had been inspired would be eliminated in a fixed state. If, as may be the case, butyric acid is also formed from amino-acids (though this must be a very secondary matter in diabetes), this too would involve the binding of oxygen. Thus the formation of sugar from protein and of beta-oxy-butyric acid and diacetic acid from fat lower the respiratory quotient of the diabetic. And the total result of these cannot be much opposed by the high quotient of formation of acetone from diacetic acid, since this occurs to such a small extent. 
To summarize: The respiratory quotient of protein, 0.8 in the normal, may in the diabetic, because of the binding of oxygen in the glucose that is eliminated in the urine, be as low as 0.6 ; the respiratory quotient of fat, 0.71 in the normal, may in the diabetic through failure of combustion below butyric acid and through binding of oxygen to convert butyric acid into beta-oxy-butyric and diacetic acids be as low as 0.66 . It is necessary to bear these facts in mind when comparing the quotients found in diabetes with those obtained in normal individuals on the same diet.

If now a normal individual, assumed frced of glycogen, were to be placed upon a protein-fat diet (75 to 125 grams of protein and 200 to 250 grams of fat), the respiratory quotient would run between 0.75 and $0.7 \mathrm{~S}$, depending upon the quantitative relations of the protein and fat. In such a subject, the quotient for fat is 0.71 , for protein 0.8. A respiratory quotient of over 0.71 indicates, therefore, that some carbohydrate is being burned. On the other hand, a quotient of below 0.71 does not prove that no sugar is being burned. A part of the sugar formed from protein may be burned; but if a larger portion be eliminated, the quotient will still be lower than the figure for the pure combustion of fat. When to this is added the effect of the non-combustion of butyric acid (which reduces the fat quotient to 0.66) and the oxidation of this acid to beta-oxy-butyric and diacetic acids, it is clear that there may be a considerable combustion of sugar with a reduction of the respiratory quotient below 0.7 . If the acidosis be low, reduction of the quotient below 0.7 indicates, of course, more plainly the defect in the combustion of sugar from protein. If the acidosis be heavy, the higher the quotient above 0.07 the more strongly this speaks for the retention of the faculty of the combustion of glucose. If the fat catabolism were normal, the respiratory quotient ought to be inversely proportional to the glucose:nitrogen ratio; but when the fat catabolism is badly deranged, this operates to obscure this relationship.

Coming now to the actual quotients observed in diabetes, they vary from 0.64 to 0.76 , the average for cases of ordinary severity being 0.73. As a rule, the quotient is lower the more severe the case; but to this statement striking exceptions occur in each direction. All the cases with a quotient above 0.7 burn sugar to greater or less extent. A quotient of 0.7 in a diabetic with high acidosis speaks for a greater sugar utilization than the same quotient in a diabetic with low acidosis. But even the diabetics with quotients below 0.7 burn some little sugar. The quotient for protein, where 0.8 gram of glucose is formed from the gram of protein and eliminated entirely, would be about 0.6. The quotient for the combustion of fat, when the glycerol is converted into sugar and lost and where the fatty acid is only burned to the stage of butyric acid, is 0.66 , and even this will be lower when the binding of oxygen in the oxidation of butyric acid is considered. Obviously, therefore, we cannot necessarily infer that a diabetic burns none of 
the sugar formed from protein or from glycerol unless the quotient is below 0.63 . Such very low quotients are very rare, and indicate how uncommon it is to have the defect in the combustion of glucose absolute. In the past, too exclusive attention has been given to the glucose: nitrogen ratio in the interpretation of the respiratory quotient; the fat catabolism as expressed in the acidosis deserves equal attention. With a protein input of 125 grams of protein per day and fat in abundance, a quotient of 0.73 corresponds probably to a combustion of 25 to 50 grams of glucose per day. By calculation also, we can show that the combustion of 20 grams of glucose per day results in an increase of the quotient of about 0.03 points. There is some evidence that sugar formed from protein is burned better than glucose ingested; but this conclusion is made uncertain by our inability to fix the amount of glucose derived from the unit of protein.

\section{CONSIDERATION OF THE TOTAL METABOLISM IN DIABETES}

The comparison of the total metabolism of the diabetic with that of the normal individual is very difficult, for the same reasons (though with greater force), as in the case of comparisons between different normal individuals. When the total metabolism of normal individuals is measured, very striking variations are found. These are related to age, weight, size, type, and temperament. The total weight of the body ought not to be the unit of metabolism, as the fat and bones are inert in this sense. The heat production we relate to the body surface. But when a man wastes, the ratio of weight to surface may or may not be maintained. If an individual were to waste in muscle and gain in fat, the unit of actual metabolism would be reduced, though the surface of radiation remains unaltered. In particular, the protein metabolism is subject to variations. Then, again, individuals differ greatly in muscular repose, even in enforced rest. And as the values sought to be determined are to be regarded as those of the metabolism of the state of rest, this causes great fluctuations. Finally, the methods of determination, the type of respiration apparatus or calorimeter employed, the training of the subject in the apparatus and the adaptation of the nervous type to such experiences, introduces another set of variables. It is, therefore, not possible to make strict comparisons between individuals, but only between averages of the values obtained with diabetics and with normal subjects chosen to correspond as closely as possible with the sick.

In the beginning of this discussion of diabetes, it was stated that the total metabolism (heat production) of the diabetic was in excess of the normal, compared as fairly as was possible. This excess, for the different directions of measurement, may be tabulated as follows, according to researches of the Carnegie Nutrition Laboratory. 
Excess over normal of:

$\begin{array}{ccc}\text { Heat production } & \text { O-absorption } & \mathrm{CO}_{2} \text {-elimination } \\ 15 \text { per cent. } & 20 \text { per cent. } & 7 \text { per cent. }\end{array}$

The apparent discrepancy between the great excess in the O-absorption and the $\mathrm{CO}_{2}$-elimination is due, in all probability, to the factor of oxygen combined in fixed state in sugar and with butyric acid to form the ketonic acids. Indeed, the discrepancy between the absorption of oxygen and the elimination of carbon dioxid, coupled with the absolute increase in the latter, may be termed characteristic of diabetes. The formation of $\mathrm{CO}_{2}$ is relatively and absolutely higher in severe than in mild cases. This holds true also for the oxygen absorption and heat production, though to less extent. The more severe the acidosis, the more marked the higher values in oxygen absorption, $\mathrm{CO}_{2}$ elimination and heat production. This at once suggests that the loss occurs in the fat catabolism. It was at first assumed that these increased values might be simply the expression of the specific dynamic action of the augmented ingestion of protein; a calculation of this possible factor, however, indicates that this cannot be the case, it is not large enough. This increased heat production is all the more striking when one considers that the formation of glucose from protein is an endothermic process, so that the heat production is really greater than it is actually measured to be. In a crude sense, this loss of heat in the diabetic metabolism may be compared to the loss of heat by friction in a journal with ill-fitting bearings.

The defects of the diabetic metabolism as affecting the utilization of the input may be well shown in tabular form. Let us arbitrarily define four degrees of severity: mild, moderate, severe, and extreme. Let the diet be the same for all-125 grams of protein and 200 grams of fat, with a total heat value of 2300 Calories. The mild case will be assumed to have no glucosuria on the diet, the moderate case one of 25 grams, the severe case one of 75 grams, the extreme case one of 100 grams (a total glucosuria on the glucose:nitrogen ratio of $5: 1$ ). The mild case will be assumed to have no acidosis, the moderate case one of 20 grams, the severe case one of 50 grams, the extreme case one of 100 grams. The following amounts of heat in Calories would then be secured from the diet in each of the following degrees of severity.

\begin{tabular}{|c|c|c|c|c|c|c|}
\hline & & & Mild & Moderate & Severe & Extreme \\
\hline Protein & . & . & 500 & 400 & 200 & 100 \\
\hline Fat & . & . & 1800 & 1700 & 1550 & 1300 \\
\hline Total & . & . & 2300 & 2100 & 1750 & 1400 \\
\hline
\end{tabular}

To secure the heat of 2300 Calories, the three last groups would need to use fat and protein from the body, or else the ingestions would need to be increased, as follows: 


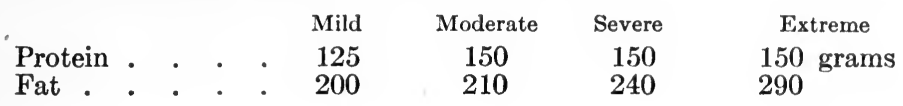

This all under the assumption that the extra ingestions of protein and fat are regarded as completely burned. In short, an extreme case of diabetes may require 150 grams of protein and 300 grams of fat to secure the heat that a normal body can derive from 125 grams of protein and 200 grams of fat. In addition to this excess of metabolic work, the diabetic has to contend with a heavy hyperglucemia due to inability to burn the glucose formed from the protein, and a heavy acidosis due to inability to burn the higher fatty acids beyond the stage of butyric acid. From the extreme down to values quite within the normal are to be found all shades and degrees of severity in diabetes; in some the defect in the protein catabolism being most prominent, in others, the defects in the acidosis being most exaggerated. The figures in the above tables are made intentionally rounded, but they illustrate the facts. The dynamic action of protein has been disregarded, since the maximum ratio for the production of glucose from protein has been employed. Thus at the worst, the diabetic has lost entirely the power of burning sugar, the caloric value of protein is reduced three-fourths, and the caloric value of fat one-third. Even in this state, the diabetic could adapt himself to the situation, were it not for the hyperglucemia and the acidosis. It is not alone what the diabetic has lost in faculties of combustion; it is much more than that. If the sugar and butyric acid were mere losses, compensation could be attained. But they both establish states of intoxication within the body, and it is to these that the diabetic usually succumbs, unless by intercurrent disease. In diabetes the antibactericidal properties of the blood plasma seem to be lowered, possibly as the result of the action upon the cells of the hypertonic concentration of glucose. The idea that the diabetic serum is merely an improved culture medium on account of the increase in glucose is too crude to serve as the explanation of the increased tendency to bacterial infections displayed in this disease.

It is clear, therefore, that the coefficient of utilization of the calories of the foodstuffs may fall to 60 in diabetes. This, however, would not seriously hamper the life of the diabetic, though it naturally limits greatly his function of adaptation. It is not loss of metabolism, it is autointoxication that is the menace of diabetes. 


\section{CHA PTER V}

THE FAT METABOLISM

Fat plays two rôles within the body. Fat represents the ultimate form of the storage of fuel, and the depot fats are quite the most inert and dead of any of the body structures. On the other hand, fats joined with protein and in complex combinations of still unknown composition, represent the most essential structures in cellular protoplasm, cell membranes, and in the central nervous system. 'The subjects of fat in its cellulometabolic relations and fat in the heat metabolism are almost as distinct as though different substances were under consideration. Our information on the two subjects is not equal; we know much concerning fat as fuel; we know little concerning fat in cellular structure.

The fats concerned in a discussion of human metabolism are the glycerids of palmitic, stearic and oleic acids alone. It is true that small amounts of butyric acid and traces of caproic, caprylic, and capric acids exist in milk. It is also known that in the fats of other animals, especially in fishes and in the marine mammals, other fatty acids are present and also other alcohols than glycerol. But for the fat metabolism of the domesticated animals and man we deal essentially only with tristearin, tripalmitin and triolein. That mixed esters occur, in which the three hydroxyl groups of the glycerol are replaced by different fatty acids, is not to be doubted, either for the depot fats or for the lipoids of the central nervous system.

Sources of Fats.-The fats of the body have two sources: The fats ingested with the diet; and the fats formed in the body from sugar. In the one instance the body is passive; in the other the body exercises selection in synthesis. The fats of the diet are not altered in the acts of digestion; they are split, the glycerol and fatty acids absorbed, recombined to their original states and added to the fats of the body. Obviously, therefore, body fat thus derived will vary in the relative proportions of tripalmitin, tristearin and triolein precisely in accordance with the relations of the three fats in the diet. Fat that the body synthesizes from sugar, however, has a constant composition with respect to the relative amounts of the three glycerids. This state of facts may be expressed in the law that body fat derived from diet fat is specific to the diet, body fat formed from sugar is specific to the species. Horses, cattle, sheep, swine, dogs, fowl, and men will from a uniform protein-sugar diet form body fats that differ widely in the relative amounts of the three glycerids; the fat of cattle and sheep containing a high amount of tristearin, the fat of swine, dogs, fowl, 
and man containing less tristearin and more triolein. The melting point of fat may be regarded as the point in temperature where the amount of triolein present is able to dissolve the amounts of tripalmitin and tristearin present. From sugar, therefore, different animals form the different triglycerids in different amounts. Once formed, however, the body apparently cannot alter the triglycerid; it cannot, for example, convert tristearin into triolein or vice versa. Therefore, when fats are ingested, they are, following resorption, simply added unchanged to the fats of the body. It is thus possible, experimentally, to modify the body fat of an animal. The fat of the dog formed from sugar contains a high proportion of triolein and is fluid at the temperature of the body. If a dog be starved until emaciated and then fed exclusively on mutton or fat beef, the mutton-or beef-fat will be deposited unchanged in the tissues of the dog, giving to the dog an unnatural solidity of subcutaneous tissues. If a female dog so dieted and transformed into a "mutton dog" becomes parturient, the milk will be found to contain mutton fat instead of the natural fat of the dog's milk. In a word, the body places its stamp upon the fat it forms from sugar. It can form any one of the three triglycerids from sugar. But once formed, either by itself or received in the diet, the body cannot convert any one of the three triglycerids into another. Therefore, in an omnivorous animal, like the domesticated dog and man, the composition of the body fat depends upon the varying extent to which the body fat has been derived from the diet or formed from sugar. The fat of the carnivora will depend largely upon the composition of the diet; the fat of the herbivora will be most specific to the species, since their diet consists largely of carbohydrate and little performed fat. We may experimentally feed a foreign fat, such as erucaic acid $\left(\mathrm{C}_{22} \mathrm{H}_{44} \mathrm{O}_{2}\right)$ and have it deposited unchanged in the tissues. Lower fatty acids, such as are ingested in milk, are certainly convertible into fat. It is, however, doubtful if any fatty acid below butyric acid is directly convertible (if at all) into fat; it must first be converted into sugar.

Formation of Fat from Sugar.-The mechanism of the formation of fat from sugar is unknown from the experimental point of view. It is quite certain that when the fats are burned in the body, they are gradually built down to butyric acid. It is on the contrary equally certain that butyric acid can be built up to fat. We may, therefore, assume, and this is also the line of least resistance chemically, that butyric acid is the stage in the series of fatty acids at which the transfer of the sugar into fatty acid occurs. The conversion cannot be a direct one, the glucose must first be split. Now lactic acid represents the commonly accepted cleavage product of glucose. Lactic acid is a three-carbon chain, while butyric acid is a four-carbon chain. It might be assumed that the lactic acid would be converted into propionic acid and this then built up to butyric acid. Thus: 


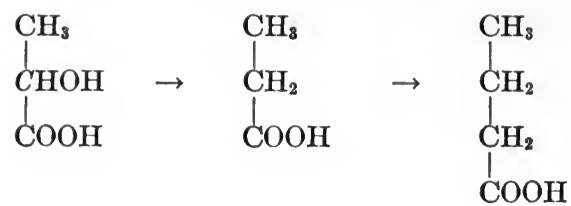

This scheme has the advantage of simplicity. And if it be objected that we have no analogy for the direct addition of a $\mathrm{CH}_{2}$ group, it may be rejoined that since all the schemes for the formation of the higher fatty acids from butyric acid rest upon such additions of $\mathrm{CH}_{2}$, there can be no reason to deny this in the case of propionic acid. Experimental work, however, has given a hint in another direction. When sugar is digested in the autolyzing aseptic liver, lactic, acetic and butyric acids are formed, accompanied by the evolution of hydrogen. From this it may be inferred that the lactic acid is split into acetic aldehyd and formic acid, two molecules of acetic aldehyd then converted into b-oxybutyric aldehyd, which would pass in turn into butyric acid. Thus:



It is clear that unless the formic acid were again utilized in the synthesis of sugar, this scheme entails the loss of one-third of the carbon content of glucose in its conversion into fat. This we cannot believe to be the case, it seems quite certain that the conversion of glucose into fat is complete so far as the carbon is concerned. Further uncertainty is added when we realize that acetic aldehyd when ingested is burned, and not condensed into beta-oxy-butyric aldehyd. Obviously, both schemes present difficulties, yet chemically these are the most feasible that we possess. However the steps, the fundamental fact stands that the body can convert glucose into fat in almost unlimited amounts and without loss of energy.

The site in which the reaction of the conversion of sugar into fat is accomplished is not known. It probably does not occur in the muscles, in which the sugar is burned. It is probable that it occurs in the areolar tissues. It is finally possible that it occurs in the liver. That the velocity of the formation of fat from sugar depends normally upon relations of concentration of glucose in the body, was stated in another connection. Apparently the body forms fat from sugar, to a high degree at least, only when the capacity of the body for the storage of glycogen is fully utilized.

When fatty acids are formed from glucose, glycerol must, of course, be provided for them. Glycerol is derived also from glucose. When 
fatty acids are ingested, glycerol is promptly formed to combine with them; and in fact, the formation of glycerol from glucose seems very easy of accomplishment. The reaction probably proceeds thus:

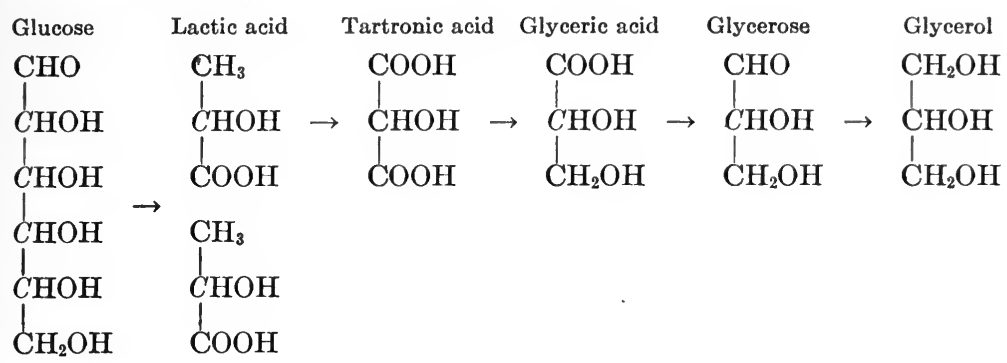

As previously stated, the reaction for the formation of glycerol from glucose is reversible, and there can be little doubt that glycerol is normally catabolized via sugar. The site of the formation of glycerol from glucose is held to be the liver.

When the fat of the diet reaches the lymph spaces of the submucosa of the intestine, it is believed to be entirely in the neutral state, $i$. $e$., the recombination of the glycerol with the fatty acids is completed in the act of resorption. The fat is then collected in the branches of the lacteal system and transported to the thoracic duct. In the contents of this duct the fat is largely in a state of emulsification. The processes of fat digestion and resorption are relatively slow and are probably not completed in less than eight hours after the ingestion of a meal; and thus fat is being poured into the venous circulation through the thoracic duct during some five or six hours. Since the fat of a meal will rarely exceed 75 grams, even where fat replaces the carbohydrates of the diet, it is clear that under the most extreme conditions not over 0.25 gram of fat will be poured into the circulation per minute. This fat, as a rule, is not carried in the blood in a state of emulsification, as it exists in the fluid of the thoracic duct. The blood contains about 1 per cent. of fat, as a rule; it can hold double that amount in solution. The fat exists in the blood plasma in some complex chemical combination, (quite certainly not with protein), in which state it is not only soluble but apparently diffusible. It is clear, therefore, that since the blood can hold at least 1 per cent. of fat in this state of solution, the maximum fat ration of a meal can be carried in this state in the blood in any one moment. This explains why lipemia (the appearance of a fat emulsion in the blood plasma) is, as a rule, not observed following even excessive ingestions of fat. Upon what chemical substance this capacity of the blood plasma for the solution of the neutral fat depends, is not known. 'The fact is, however, crucial to our conception of the fat metabolism. We assume that this soluble state of fat in the blood represents the reaction state of fat. We assume that fat is thus transported to the areolar cells, through 
whose walls it diffuses; the combination is there split and the neutral fat deposited as depot fat. Whenever fat is burned, we assume that this state of solution is the state of reaction. When the fats are needed and must be recalled from the depots, we assume that they are again converted into the state of solution through this complex combination and transported to organs or tissues of combustion; or if fat be burned in the areolar tissues, it is first converted into the soluble state before being available for the processes of combustion. This state of combination, of solubility and diffusibility, is in a word the conditio sine qua non of chemical reaction on the part of the neutral fats. The blood and the tissues, especially the liver, may contain traces of free glycerol and fatty acids. The fats in the depots are within cells, in the strict sense of the word, as truly as in the case of fatty infiltration of the liver. When depot fat is drawn upon, it is that stored in the liver that is first utilized, precisely as in the case of glycogen. The conversion of the neutral fat of the depot cells into the soluble complex we relate to the chemical functions of these cells, just as we relate the conversion of glycogen into sugar to the chemical functions of the liver cells. From this point of view it is obvious that the cells of the areolar connective tissues occupy a position of high metabolic importance.

\section{THE ANABOLISM OF FAT}

Four lipoid groups are included in the anabolized fats. These are the protein-fat complex; the phosphatids; the sterins; and the cutaneous lipoids. Future investigations may indicate that this separation into groups is not based upon a chemical foundation. And even today the classification is largely one of convenience, though certain chemical differentiations seem to exist. All the anabolized lipoids, in whatever form and wherever present, are derived from the neutral fats. We assume that the neutral fats, in soluble and diffusible state, are carried to the specialized cells of the body, there to be transformed into the complex combinations that play so important a rôle in physiological, pharmacological and bacteriological reactions. These lipoids are present in cell membranes, in protoplasm, in nuclei, in the circulating fluids and in the secretions. It is doubtful if they are ever to be regarded as excrementa, even in the secretions of the sebaceous glands or in the bile. They exist in plants to quite as widespread an extent as in animals. Some of the lowest members of the lipoids are relatively simple.in their makeup; others are extremely complex and of these we possess very little real information. Some lipoids, even unassociated with protein, seem to possess biological specificity. They are all more or less pronouncedly colloidal, and present to a striking degree the phenomenon of adsorption. 'To their fatty nature and to their colloidal properties are due in large part the difficulties of a ehemical study of these lipoids. 
A general survey of the chemical and physico-chemical properties of the lipoids, as applied to their cellular relations (in the membrane and protoplasm), indicate two directions in which they might be expected to (and demonstrably do) influence fundamental processes of function. The presence of lipoids in a cell membrane is associated with the quality of semipermeability; and the presence of lipoid in protoplasm brings into operation the law of partition. We have many illustrations of the property of semipermeability in cell membranes, whereby certain substances or elements are permitted to pass and others are restrained; and we are able to prepare inorganic membranes that present these phenomena to a striking extent. So far as living or dead cells are concerned, this matter is not yet upon a sound experimental basis; but we will not go far wrong when we relate this property to the lipoid complex in the cell membrane. With regard to the applicability of the law of partition to biological relations, we are on more explored ground. Protoplasm is a two-phase system, consisting of water in which are suspended protein-lipoid complexes and in which are dissolved salts. Salts and ions are not only dissolved in the water but are also combined with protein; sugar and other crystalloid substances are present, both free and combined. But in so far as the law of partition is concerned, it is the lipoids that dominate the scene. This protoplasm, by virtue of the presence of lipoids, becomes a solvent to substances that are in themselves insoluble in water. It is just as though we had a saturated solution of ether in water. Such a solution will dissolve many substances that are insoluble in water. A great many pharmacological substances, and apparently many biological and bacteriological substances, are lipo-soluble, $i$. $e$., will be taken up by a lipoid or by a water or protoplasm containing lipoid. It is for this reason that neutral fats themselves are taken up by protoplasm. In the intricate physiology of the cell these reactions undoubtedly play a leading rôle. And from the broadest point of view, the modifications that the colloidality of a system impose upon the purely chemical reactions in the system ought always to be kept in mind.

(a) The Fat-protein Combination.-Cells contain apparently neutral fat combined with protein. If a gland, like the kidney, be completely extracted with fat solvents and then digested with trypsin, a subsequent extraction will yield a goodly amount of fatty substance. Some of these are phosphatids and sterins, but a portion consists of neutral fat. The amount of such fat in combination with protein is not large. Nor is the combination confined to the cells, but exists in the blood serum. Beyond these few facts, nothing is known of the fat-protein complex.

(b) The Phosphatids.-These are the best known of the lipoids. Some that are well known contain an amino group. In their simplest form they may be defined as lipoids in which two molecules of a higher fatty acid are combined with glycerol-phosphoric acid, to which is 
bound an amino body. Glycerol-phosphoric acid is a stereoisomeric substance and has the formula:

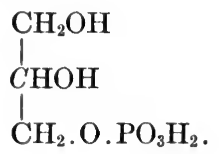

Therefore, the general formula for the simplest monamino-monophosphatid would be:<smiles>[R]O[P+]([O-])(O)OCC(CO)CO</smiles>

The best-known representative of this type is lecithin, widely prevalent in animal tissues. In lecithin may be found two molecules each of stearic, palmitic or oleic acid or any two of the three; thus we have, for example, a distearyl lecithin, a stearyl-oleyl lecithin, etc. The amino base combined with the phosphoric acid in common lecithin is cholin,<smiles>CC(C)OCCO</smiles>

Cholin is tri-methyl-oxy-ethyl-ammonium hydroxid, and the structure is made clear by the equation:

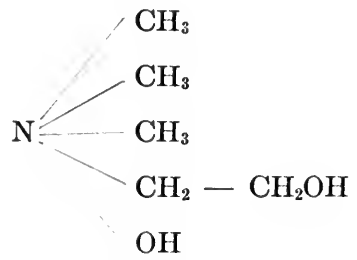

Cholin is formed by the union of glycol,

with tri-methyl-amin,

$$
\begin{aligned}
& \mathrm{CH}_{2} \mathrm{OH} \\
& \mathrm{CH}_{2} \mathrm{OH}
\end{aligned}
$$

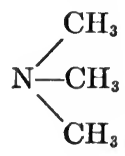


and is found free in some plants, in which lecithins are very common. Thus the equation of distearyl lecithin would be:

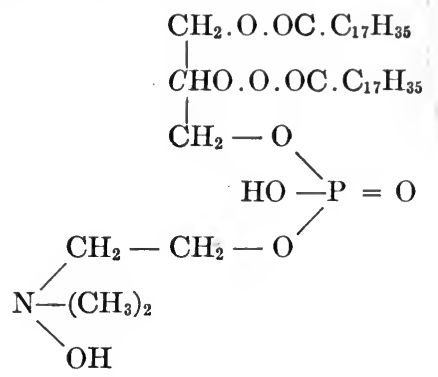

In the lecithins of plants other bases related to cholin and probably derived from it, may replace it. Whether neurin, which has been recovered from brain substance and which is probably trimethylvinyl-ammonium hydroxid, exists performed in lecithin is very doubtful. All the lecithins in the higher animals seem to contain cholin. Yet lecithins exist with only one methyl group. Thus b-kephalin, recovered from the human brain, is dioxy-stearyl-monomethyl lecithin.

More complex lecithins are, however, known. Thus there is evidence for the existence of monamino-diphosphatid, diamino-monophosphatid, and of diamino-diphosphatid. In these larger complexes moreover, greater differentiation occurs in other directions. Firstly, fatty acids other than those of the normal series or oleic acid enter, some resembling the linoleic and linolenic acids. Secondly, sugars enter into the complex, especially d-galactose. These more complex forms of phosphatid are found largely in the central nervous system and of them d-galactose seems to be an invariable component, though d-glucose may also be met with. One of the most complex diphosphatids known has been isolated from heart muscle. It is the complexity of these phosphatids and also the occurrence side by side of different phosphatids, aided by their colloidal and lipoidal properties, that renders the subject of the chemical study of the brain substance so unsatisfactory.

To what extent the body forms its own cholin and glycerol-phosphoric acid and whether it is at all dependent upon these substances derived from the alimentary tract is not known. The body can form glycerol-phosphoric acid, and it is scarcely to be believed that it is dependent upon the diet for its cholin.

(c) The Sterins.-Under this name we group all the lipoids that resemble the cholesterols. Like the phosphatids, sterins are very widely distributed in plants and animals. So far as the higher animals are concerned, however, while all the body cells and fluids contain sterin, it seems to be in but one form, cholesterol. The cholesterol isolated from nervous tissue, from gallstones, from red corpuscles, and from the blood plasma is identical with that isolated from the yolk 
of egg. Related bodies are to be found in the sebaceous secretions and in the wool fat of many animals. 'The common form of cholesterol is a monovalent, unsaturated secondary alcohol, with a ring structure resembling that of the terpens. There is but one hydroxyl group. The substance has a formula something like the following:

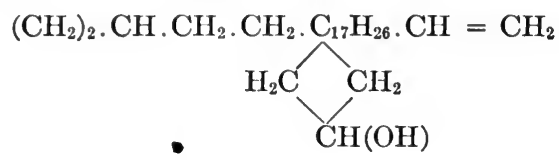

It combines with fatty acids to form esters. In the body both the free alcohol and esters are to be found. 'Thus in the bile and red blood cells it is free; in the brain substance, however, it is usually combined with a fatty acid. That cholesterol is formed from the higher fatty acids in the body is scarcely more than a theoretical, though a reasonable, assumption. It is, however, possible that it is derived from the diet, passing unchanged through the alimentary tract.

(d) The Sebaceous Lipoids.-In the secretions of the skin and scalp are many lipoidal bodies, including both phosphatids and sterins. These are formed as specific secretory products of glands, and are to be regarded as metabolic products, and in nowise as excrementa. Though of greater importance to the fur- and feather-bearing animals than to man, yet in all they have the same function-to enable the body to shed water and to reduce radiation of heat.

\section{THE CATABOLISM OF FAT}

The catabolism of the fats includes the building down of the complex lipoids that bear such important functions in the body, and the combustion of neutral fat and fatty acids. Of the modus operandi of the catabolism of the phosphatids and sterins we are not well informed. From all that we know the phosphatids are split by lipase into their component fractions, each then to go to the fate of its group: the fatty acids are burned; the d-galactose and glycerol are converted into $\mathrm{d}$-glucose; the phosphoric acid is eliminated as an inorganic salt; and the cholin is probably split into its components, glycol and tri-methylamin, the first of which would be burned while the second is apparently eliminated in the urine, the source of the traces of tri-methyl-amin normally present there. As to the fate of cholesterol, we know less. Human feces contains a substance termed koprosterin, apparently a dihydro-cholesterol; horse feces contain a similar substance termed hippo-koprosterol. These are supposed to represent reduction products of cholesterol of the bile, the results of bacterial action. Recent work has made it probable, however, that these substances are derived from the diet and not from the cholesterol of the bile. Human feces contain unchanged cholesterol, and some is certainly resorbed in the 
small intestine. That the cholic acid of the body, present in the biliary acids, is derived from cholesterol, is very probable, as elucidated in the discussion of the bile.

The Combustion of Fat.-The catabolism of fat resolves itself into combustion. Initial to combustion is hydrolytic cleavage of the neutral fat into fatty acid and glycerol. In the case of the complex fats of the anabolic series, we assume that in the autolysis of the cells containing them, these complex combinations are split into their several component parts. In the case of the lipo-protein complex, in the autolysis of the cells these, too, undergo cleavage. For these hydrolytic cleavages, in the velocity in which they occur in the body, we hold the lipases responsible. In the case of the depot fats, we assume that the inert fats are first converted into the soluble and diffusible state. Whether they are then carried to the liver to be acted upon by the fat-splitting ferment, or whether this enzyme acts upon the fat in the areolar tissues, is not known. But under all circumstances, the reaction of cleavage precedes the reaction of combustion.

The fraction of glycerol is not burned directly, we believe, but is converted into sugar and joins the glucose content of the body. The glycerol of $1 \mathrm{gram}$ of neutral fat will form over 0.1 gram of glucose. The combustion of the fatty acids of the saturated series is now fairly well understood. It will be recalled that the carbons in the chain of such a fatty acid are designated from below upward, above the carboxyl, in the order of the letters of the Greek alphabet, alpha, beta, etc. Now in the oxidation of such a fatty acid, the point of attack is the beta carbon, the second one above the carboxyl group. The oxidation at this point, passing through two known intermediary reactions, results in the splitting off of two atoms of carbon, water and carbon dioxid being formed, with the consequent shortening of the chain by two carbons. And this process is repeated until butyric acid is reached. Oxidations attacking the alpha carbon are known, but apparently they do not occur in the normal combustion of fats. Obviously, only fatty acids containing an even number of carbons in the chain could be thus carried to butyric acid. And valerianic, pelargonic and margaric acids, which the body can burn, must accordingly be burned in some other way than to butyric acid. This scheme of combustion deserves a detailed illustration. Thus for stearic acid: 


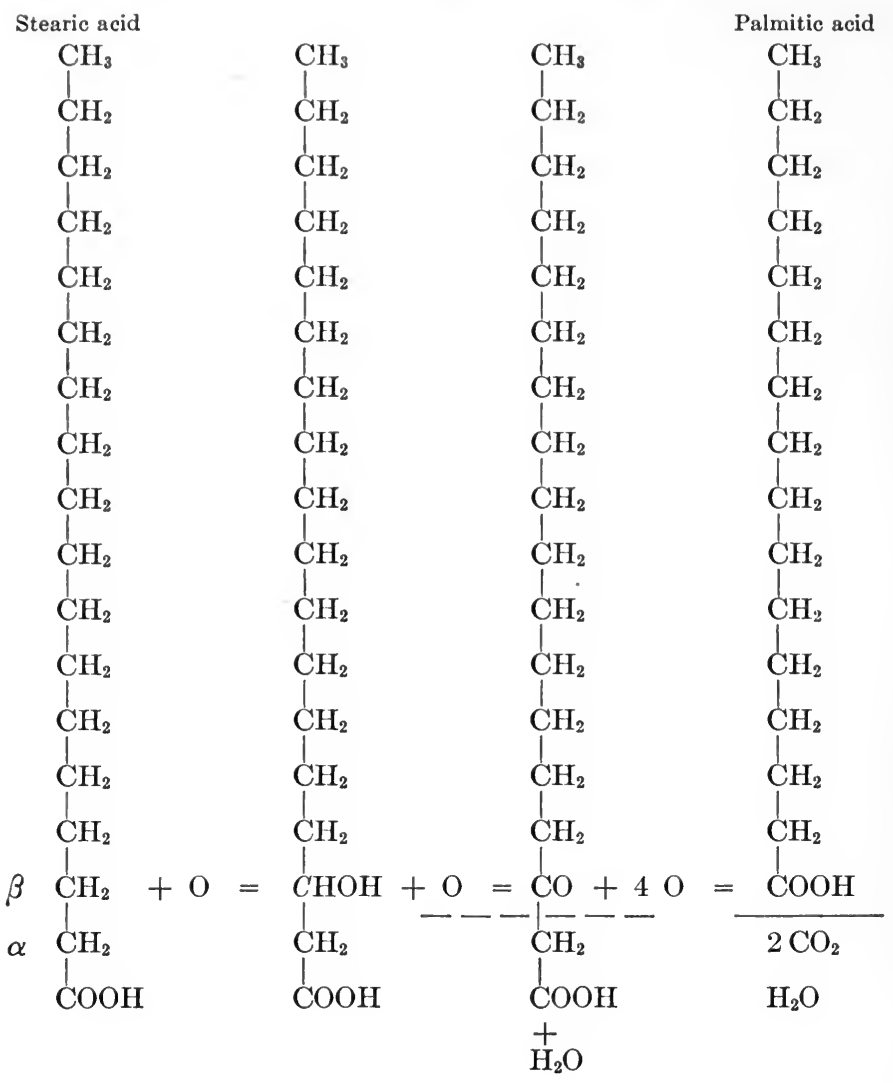

In like manner palmitic acid yields myristic acid; this yields lauric acid; this in turn furnishes capric acid; the next formed is caprylic acid; this yields caproic acid; and this finally, on oxidation of its beta carbon yields butyric acid, as follows:

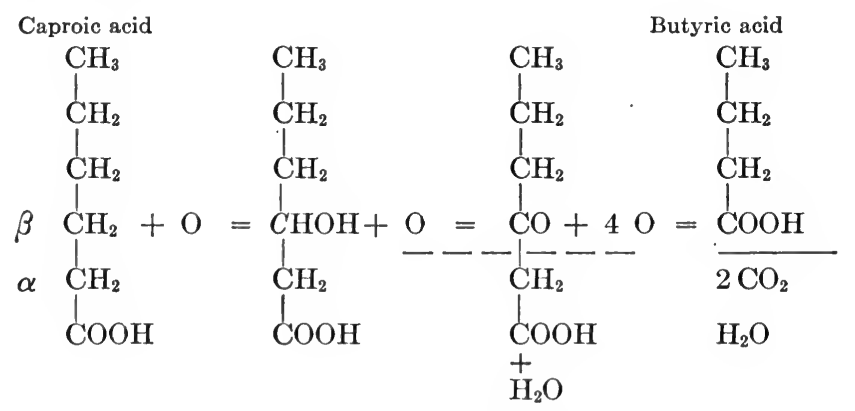

At this point the process suffers a deflection, and this is a matter of great importance, involving the pathogenesis of acidosis. The 
butyric acid is partially oxidized at its beta carbon, to form beta-oxybutyric acid; this substance is further oxidized at the same point, so that the beta carbon is now completely oxidized and aceto-acetic acid is formed. This is then, by the addition of water, split into two molecules of acetic acid, which through formic acid is easily oxidized to carbon dioxid and water. At the same time a side reaction occurs normally to a slight degree, whereby aceto-acetic acid is reduced to acetone; (di-methyl ketone) through the splitting off of a molecule of carbon dioxid. Thus:

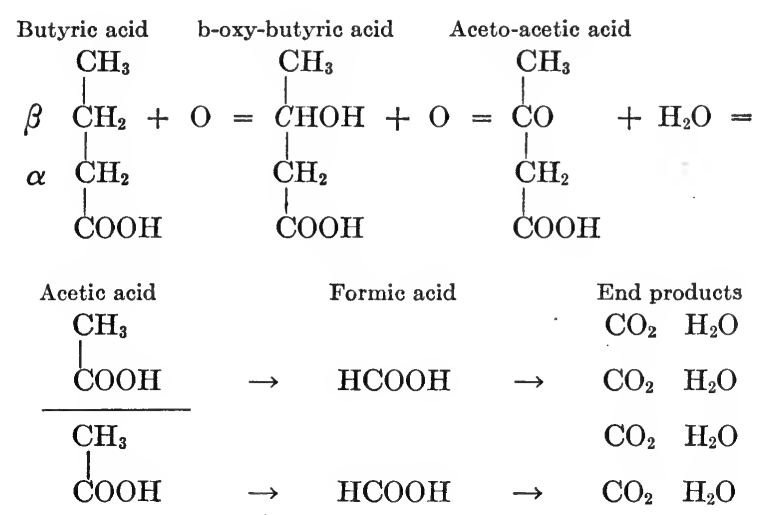

A side reaction occurs, with the reduction of aceto-acetic acid to acetone:



Under normal conditions, there is but a trace of the product of this side reaction in the urine, and none in the expired air. As the amount of fat burned by the body is augmented this side reaction increases, but in greatly more than direct proportion, possibly in the proportion of the square of the unit of increase in the fat combustion. For illustration, if a body burn but 50 grams of fat per day, there will be only a trace of acetone formed; if now the body burn 200 grams of fat, the acetone formed will not be four times the amount recovered when the body burned 50 grams, but rather sixteen or more times. To this general rule there are exceptions, in normal individuals as well as in diabetics. But for the most part the rule holds, that increase in the fat combustion is associated with a very disproportionate increase in the side reaction, the formation of acetone.

An earlier and somewhat more simple conception of the oxidation from the stage of beta-oxy-butyric acid ran as follows: 


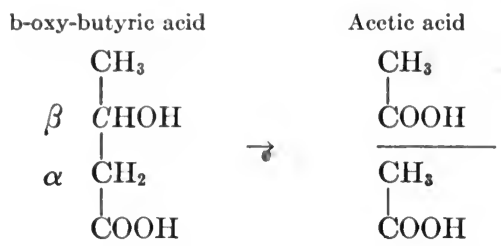

Obviously, according to this scheme, oxidation occurred at the beta carbon and reduction at the alpha carbon, with cleavage between them. According to this scheme aceto-acetic acid and acetone would occur never in the normal combustion of fat. Since they are always present, acetone in substance and aceto-acetic acid necessarily preceding it, the scheme first stated would receive the preference, unless it could be demonstrated that the trace of acetone present in normal urine might have been otherwise derived. This will be discussed under the acetone complex.

The oxidation of triolein is less well understood, and chemically is not so simple. It will be recalled that oleic acid has the equation:<smiles>CCCC=CCCC(=O)O</smiles>

Now when unsaturated fatty acids are oxidized by chemical agents, they tend to be split at the double linkage between the two carbons, and this would form from oleic acid one molecule of pelargonic acid $\left(\mathrm{C}_{3} \mathrm{H}_{17} \mathrm{COOH}\right)$, and one of azelaic acid $\left(\mathrm{COOH} . \mathrm{C}_{7} \mathrm{H}_{14} \cdot \mathrm{COOH}\right)$; one a saturated fatty acid, the other unsaturated, each, however, with an uneven number of carbon atoms in the chain. In the body, however, judging by experiments with the liver, no such reaction is done, but instead caproic acid is formed. It is, therefore, to be inferred that the organism is able to shift the location of the double linkage to such a point that the cleavage in the reaction of oxidation will yield caproic acid. This could be done as follows:

$$
\left.\begin{array}{r}
\mathrm{C}_{8} \mathrm{H}_{17} \\
{ }_{\mathrm{H}}
\end{array}\right\rangle \mathrm{C}=\mathrm{C}\left\langle\begin{array}{l}
\mathrm{H} \\
\left(\mathrm{CH}_{2}\right)_{7} \mathrm{COOH}
\end{array} \rightarrow \begin{array}{r}
\mathrm{C}_{5} \mathrm{H}_{11} \\
{ }_{\mathrm{H}}
\end{array}\right\rangle \mathrm{C}=\mathrm{C}\left\langle\begin{array}{l}
\mathrm{H} \\
\left(\mathrm{CH}_{2}\right)_{10} \mathrm{COOH}
\end{array}\right.
$$

Cleavage at the site of the double linkage with oxidation of the two carbons would yield caproic acid $\left(\mathrm{C}_{5} \mathrm{H}_{11} . \mathrm{COOH}\right)$, and $\mathrm{COOH} .\left(\mathrm{CH}_{2}\right)_{10}$. $\mathrm{COOH}$, which by reduction and cleavage would form two further molecules of caproic acid. This caproic acid would then be open to oxidation at the beta carbon, with the formation of butyric acid. 'This may not be the exact scheme but some such series of cleavages must be carried out, since we know that in diabetes oleic acid yields more acetone than do palmitic and stearic acids, and it must, therefore, pass through the stage of butyric acid, 
These reactions we assume are the results of the activities of oxidation ferments. It seems reasonable to separate the series before the stage of butyric acid from the reactions subsequent to that stage. Apart from the reasons for this separation suggested by the equations, there is a positive fact in its favor, namely, that in diabetes there is no difficulty in the oxidation of the higher fatty acids to the stage of butyric acid, but great difficulty in the oxidation of butyric acid. As to the site of these oxidations, it is certain that they occur actively in the liver, but it is in nowise demonstrated that they occur there alone. There is, however, some evidence that these reactions do not occur in the muscles. If they occur outside of the liver, therefore, it must be in the areolar tissues of the body. And under such an interpretation, the areolar tissues act not only as the depots for the storage of fat, but also as the site of their combustion.

Important from many points of view is the fact that the body does not burn much fat so long as glucose is available. Not until the glycogen stores of the body become reduced does the body burn its fat. This is in a direct sense the converse of the situation in the formation of fat, which does not occur until the glycogen depots are stocked.

The Formation of Sugar from Fat.-In the discussion of the combustion of fat, no mention was made of the possibility that fat might be burned via sugar. All the chemical and experimental data bearing on the utilization of fat in the higher animals indicate that it is burned as outlined; and though in some points the demonstration is not positive, yet on the whole the scheme is satisfactory. The reasons for an assumed conversion of fat into sugar rest upon two wholly unlike propositions. One is the general rule that in the plant world the reaction sugar $\leftrightharpoons$ fat is reversible. The second is that in clinical diabetes sugar seems sometimes to be eliminated in amounts so large as to make difficult its derivation from protein and carbohydrate in the diet and tissues of the subject. The biological argument for reversion does not apply to a concrete question, attractive as a general rule always is; the formation of sugar from fat must be demonstrated experimentally. All efforts to demonstrate this reaction physiologically have failed. If it should prove to be true in diabetes (the question is fully discussed under that heading), that with the subject on a carbohydrate-free diet, subsisting on known rations of protein and fat, the elimination of so much glucose in the urine occurs as not to be derivable from the protein undergoing catabolism, and such elimination could be shown to be maintained so long as to exclude positively any stored carbohydrates in the body - then obviously the conclusion would follow certainly that the diabetic had formed sugar from fat. And if this were to be shown to hold for the diabetic, the writer would regard the reaction as holding for the physiological state. But this positive demonstration has not yet been made. The theory of reversion is attractive; but it must not be used to force the interpretation of an experimental result. The high glucose:nitrogen ratios that have been reported in 
occasional cases of diabetes have not been so controlled as to exclude all sources of error. About such an experiment, involving so much for the theory of metabolism, nothing but the most rigid controls can be tolerated; these have, to date, not been applied.

The Formation of Fat from Protein.-The question of the formation of fat from protein has been one of the stock problems of pathology and to some extent of physiology. For physiology, it was recognized that the solution depended upon the determination of the formation of sugar from protein. Could sugar be formed from protein, then there could be no further question of the formation of fat from protein, since fat is formed from sugar, however derived. For physiology, therefore, the demonstration of the formation of sugar from aminoacids carried with it the conclusion that a living organism could form fat from the amino-acids of protein via sugar. The question of the formation of fat from protein was to pathology, however, an entirely different matter; and widespread misconception of the actual problem at issue has resulted in an enormous volume of misdirected research. The proposition of the formation of fat from protein, as contained in the usual definition of fatty degeneration, ran to the effect that in the degenerated cell the protein of the protoplasm was converted into fat in situ. It is plain that the demonstration of the formation of fat from sugar derived from protein in an organism, in nowise supports the thesis that in a diseased cell the protein of the protoplasm is converted into fat. Yet this misconception pervades most of the writings of pathologists. The data are now in hand for definite decision of the question of the origin of the fat in degenerated cells; and the facts teach us that this fat is not formed from the protein of the protoplasm of the same degenerated cells.

It must, in the first place, be realized that one of the experiments long interpreted to support the origin of fat from protein in the sense of the pathologist, is actually irrevalent to the question at issue. The eggs of the fly contain little fat; blood serum contains little fat. If the eggs of the fly be hatched out upon blood serum and the larvæ developed thereon, the bodies of these larvæ will on analysis be found to contain fat in excess of the fat of the eggs and of the food. This simply proves that the organism of the larvæ can form sugar from protein and fat from this sugar. It in nowise indicates that a degenerating cell converts the protein of its protoplasm into fat.

Equally important is it to realize that the fatty appearance of degenerated cells need not mean fat-content in the chemical sense. In many extreme fatty degenerations of the cells of certain organs, fat is not present in amount greater than in the normal organs. The histological appearances are very deceptive, and even the so-called specific stains for fat are not to be relied upon for quantitative interpretations. Fat is present in normal cells in two states: free, or at least so loosely combined as to be made visible by fat stains; and combined in the protoplasm, in such state that fat stains do not react with 
it. In general the fat that stains is extractable; the fat that is combined and will not stain is not extractable. If the tissue be digested with trypsin, this combined fat is set free, it becomes demonstrable by stains and is extractable. In a fatty degeneration, autolytic processes that resemble tryptic digestion split the fat-protein combination and render the fat stainable. It is, therefore, clear that the mere presence of more stained fat may be simply the expression of the setting free of fat that was in the normal cell not stainable because in complex combination. Apart from this fat, however, it may be shown that all the fat contained in degenerated cells is deposited from the outside.

Three lines of investigation have made the facts and relations clear. If a starving animal (frogs are best, though small animals and birds may be used) be subjected to an intoxication that leads to marked fatty degeneration, as phosphorus poisoning, it will be found that the total fat of the body is not increased. It is either not affected at all, or it is reduced to a slight extent, as might be expected since no food is ingested for the support of the metabolism. Obviously, there could have been no formation of fat unless this was covered by a greater fat catabolism. This, in the frog, can be shown not to have occurred.

If the different organs of such an animal, poisoned with phosphorus while starving, be subjected to quantitative analyses for fat, it will be found that the increases in the fat of certain degenerated organs, especially the liver, is covered by the lessened fat-content of the rest of the body. In other words, the liver has been filled with fat drawn from the other tissues; the process has been one of fat migration and infiltration.

Finally, if foreign fats be used in the experiment, it may be shown that these are heaped up in the degenerated organs. If a starved dog be fattened with mutton, it will be found that apart from a small amount of dog fat formed from the sugar derived from the protein catabolism, the fat of the dog's body is mutton fat and not dog fat. If such a "mutton dog," as they are called in the laboratory, be poisoned with phosphorus, the fat in the fatty degenerated liver will be found to be mutton fat, not dog fat. Obviously, the accumulation of fat in the degenerated liver is due to deposition of mutton fat from the fat depots of the body, and not to the formation of fat from protein in the protoplasm of the liver cells. The same result may be attained by feeding cream to a cat with cantharides nephritis; butter fat will be packed into the diseased renal cells. Other foreign fats may be employed and the identical result secured.

One further fact makes easier the application of these considerations to diseased organs. The liver is of all organs in the body of higher animals, the one most subject to fatty infiltration; infiltration with fat and glycogen are indeed to be regarded as functional in the liver. Now it is precisely in degenerations of the liver that excesses of fat over the normal are to be found. In degenerations of the kidneys, the amount of fat present is usually not in excess of the normal. The 
liver is physiologically a fat depot. When diseased and degenerated, it remains, or indeed becomes still more, a fat depot. Apparently, the very degeneration of the hepatic cells limits its powers of control over the fatty depositions, makes it more passive, less able to throw off or utilize the deposited fat.

It is, therefore, certain that the difference between fatty infiltration and fatty degeneration lies in the normality or abnormality of the protoplasm; it does not lie in the fat-content or in the postulated formation of fat from the diseased protoplasm. A cell with fatty infiltration is a healthy cell containing an excess of fat deposited from the outside. A cell with fatty degeneration is a diseased cell (whose fatprotein complex in the protoplasm has been in whole or part split), containing an excess of fat deposited from the outside. The difference lies in the normality or abnormality of the protoplasm and nucleus, not in the amount of fat and most certainly not in the derivation of the fat, which comes from the fat stores of the body and is not derived by synthesis from the protein of the protoplasm of the cell involved.

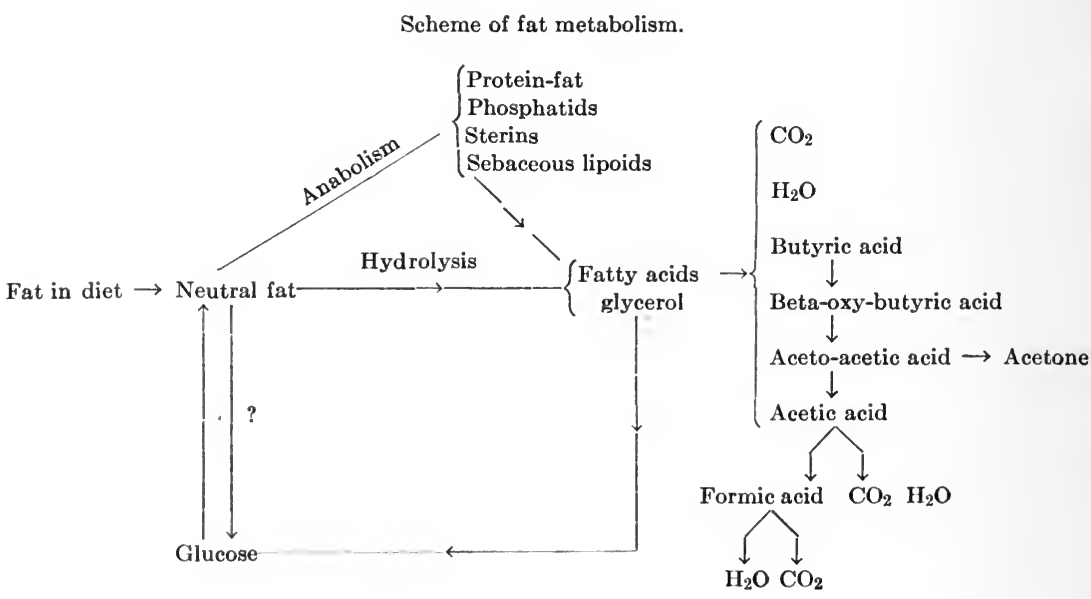

\section{THE PATHOLOGY OF FAT METABOLISM}

So far as known there are but two pathological deflections in the metabolism of fat. The first lies in the failure of the diabetic body to form fat from glucose as in the normal, which condition was discussed under diabetics. The second lies in what may perhaps best be termed acidosis or the acetone complex. Under this term we include the anomaly wherein fats, instead of being burned completely, are in part eliminated as acetone, aceto-acetic and beta-oxy-butyric acids.

The careful investigations of the past decade have made three points clear in relation to this subject. (a) 'The acetone bodies are not derived from carbohydrate at all. (b) The acetone bodies may be derived 
from butyric acid and also from several amino-acids, the products of protein catabolism; in short the acetone bodies may be derived from either fat or protein. (c) In the amounts and under the circumstances under which the complex occurs as a pathological variation, the acetone bodies must have been derived from fat and not from protein.

It will perhaps be best first to discuss the origination of these bodies from protein. The foundation for this derivation rests upon an experimental basis. When different amino-acids are mixed with the blood in perfusion experiments with the dog's liver, some are noted to lead to the formation of acetone, others do not. All the amino-acids have not been tested, but there is evidence that from the following acetone may be formed: leucin, alanin, phenylalanin, tyrosin, histidin, and aspartic acid. The probable reactions whereby acetone is formed from these products of the catabolism of protein follow.

Leucin is an amino-isobutyl-acetic acid. The reactions through which it must pass to reach the acetone group are several:

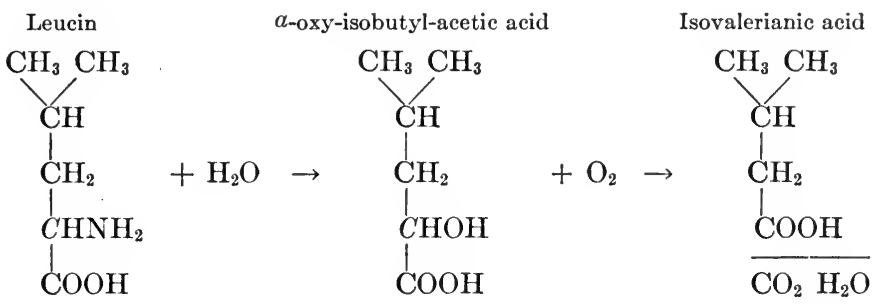

From this point, two possibilities are open. Either the isovalerianic acid is oxidized at the beta carbon, and the two lower carbons split off as $\mathrm{CO}_{2}$ and $\mathrm{H}_{2} \mathrm{O}$; or by substitution of the one methyl group by an hydroxyl group beta-oxybutyric acid would be formed.

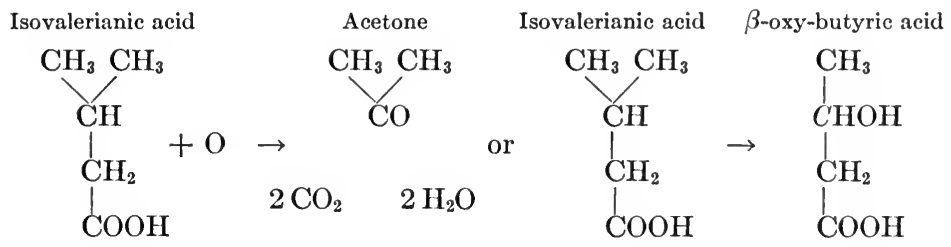

Alanin, phenylalanin, and histidin are all derivatives of propionic acid. Now lactic or propionic acid cannot be converted directly into beta-oxy-butyric acid. Since the stated experiment, however, indicates that acetone is formed from them, some indirect route must be sought. The argument here concerns alanin alone, since what will hold for it need not hold for the two aromatic derivatives, for it is known that when the benzol ring is split in the body, the fatty acid to which it was attached need not follow the normal course of oxidation. What holds for alanin ought, however, to hold for the nearly related serin. The relations between alanin and acetone are seen in the equations: 
<smiles>CC(C)C(=O)O</smiles>

The intermediary stages are unknown.

For phenylalanin, tyrosin, and histidin it is probable that the transformation follows another course, it having been made quite certain that when these substances are converted into aceto-acetic acid, the four carbons of the acid are derived two from the propionic group and two from the nucleus, the aromatic ring being thereby ruptured and the alpha carbon oxidized to the carboxyl, after deaminization. The following illustration will elucidate the relations in the molecules; tyrosin is not illustrated as the equation practically accords with that of phenylalanin:
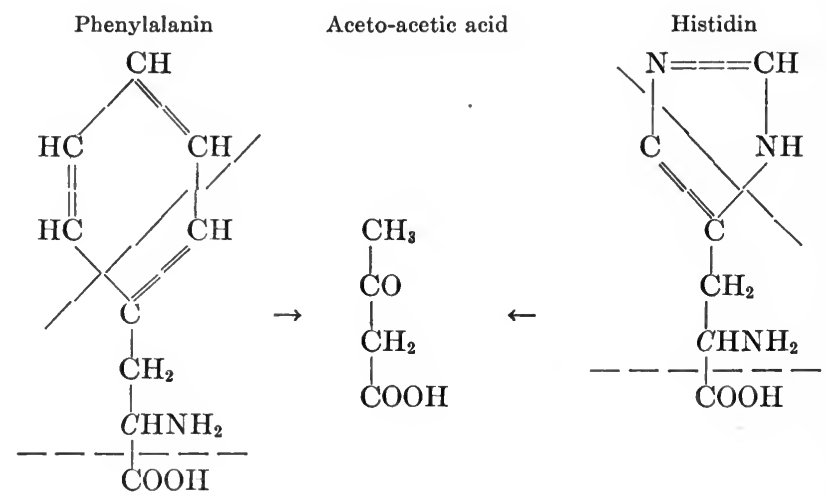

Aspartic acid has the equation:

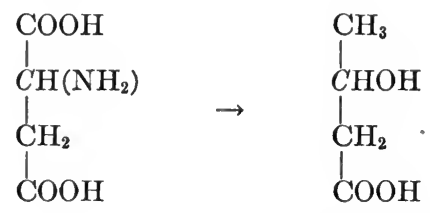

By reduction of the upper carboxyl to a methyl group and the oxidation of the beta carbon after deaminization, beta-oxy-butyric acid would be formed.

Incidentally remarked, alanin, aspartic acid and leucin are being overworked. For these are among the very amino-acids that experimentally have been found to form glucose. For diabetes at least, we cannot be in a quandary which to choose. The glucose we can scarcely relate to any other factor in diabetes than to the amino-acids. And in the quantities in which sugar and the acetone bodies respectively appear in the diabetic, contrasted with the total protein catabolism, 
we have no alternative than to relate the sugar to the amino-acids and the acetone bodies to the fats.

Furthermore, it has been suggested that beyond the stage of the amino-acids, the beta-oxybutyric acid might be formed from ammonium aldehyds. Thus, when the liver is perfused with acetaldehyd ammonia, acetone is formed. This is probably accomplished through the intermediary stage of aldol.

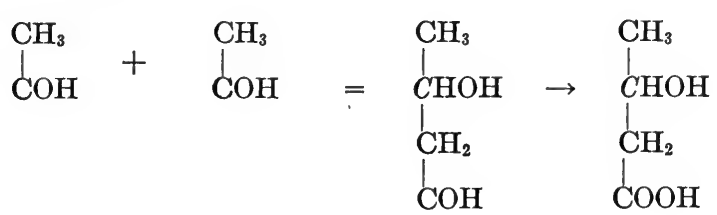

We are not, however, aware that aldehyd ammonia is formed in the body in the manner predicated in this hypothesis.

Granted, then, that for the diabetic the amounts of acetone bodies often present are by their very quantitive relations precluded from derivation from the protein and must be referred to the fats, are there facts known for the non-diabetic instances of the acetone complex that suggest that in them the bodies in question are derived from protein and not from fat? So far as the writer is aware, all the facts speak to the contrary; they plead in all forms of acetone acidosis for the derivation of the ketonic bodies from the fats. Whether this, however, is true of the normal trace of acetone, may be possibly questioned. In the equations given on pp. 353 and 354 to illustrate the combustion of butyric acid, in the one scheme the processes of oxidation were carried from beta-oxy-butyric through aceto-acetic acid to acetic acid; in the other illustration the process was carried from beta-oxybutyric acid directly to acetic acid. According to the first scheme, acetone in traces would be regarded as a normal product of the combustion of fat, a side reaction. According to the second scheme, acetone would not be formed at all in the normal combustion of fat. If now we could relate to the protein catabolism the normal trace of acetone in the urine, we could regard the second scheme as representing the course of the burning of butyric acid. This being assumed, we could then lay our finger upon the point of failure, the defect in the burning of fat that in the clinical instances of acetone complex leads to the formation of these bodies in excess. Special reference will be made to this after we have considered the different types of ketonuria, under which term we include the elimination of the three acetone bodiesbeta-oxy-butyric and aceto-acetic acid, and acetone.

Interesting relations exist between these three bodies. The faculty of reduction of aceto-acetic acid to acetone is very limited, and therefore no mass relation holds between aceto-acetic acid and acetone. Between beta-oxy-butyric acid and aceto-acetic acid, however, an equilibrium seems to exist. The administration of one to the subject 
of acidosis, results also in increase in the other; it makes no difference which one is administered. The addition of either one to liver pulp results in the appearance of the other. Clearly, therefore, where the aceto-acetic acid is not removed by oxidation or converstion into acetone, we have the relation: beta-oxy-butyric $<=>$ aceto-acetic acid. For practical purposes, therefore, a test for one of these bodies is a test for both.

The urinary acetone complex is observed under widely varying circumstances. Definite are the following types:

Diabetes.- This is perhaps the most common, as it is also the most important. It has been discussed under diabetes. Acidosis occurs typically in phloridzin intoxication.

Starvation.-Here we have one of the best-studied instances of ketonuria. A few days after food is withdrawn, acetone appears in the breath and urine. Within a few days, aceto-acetic acid appears, to be soon followed by beta-oxy-butyric acid. The amounts vary widely with different individuals, from a few grams to 50 per day. The higher the output, the more marked the elimination of the acids as compared with the acetone. In some starving subjects eliminations are met with quite as large as in severe diabetes. With the persistence of the starvation, the acidosis may maintain its full individual range. The administration of 100 grams of sugar will be followed by a pronounced drop in the acidosis. Indeed, by the daily administration of this amount of sugar to an otherwise starving subject the acetone complex will be inhibited, while at the same time the losses of flesh and of fat continue, since 100 grams of sugar is but a small fraction of the fuel requirements of the body. This is the most striking of the experiments indicating the relationship of sugar to the acetone complex; it is not starvation, but sugar-starvation that causes the acidosis. The experiment can be performed in another way, by withdrawing all carbohydrate and giving full rations of fat with the minimal ration of protein. At once a marked acidosis will result. Curiously enough, however, this is only temporary; after a time the body becomes accustomed to the diet and the acetone bodies slowly retreat to the normal output. In conditions of subnutrition due to poverty, acidosis is rarely present; this is due to the fact that meats and fats are first sacrificed and carbohydrates, the cheapest foods, are retained. The occurrence of the acetone complex may often be of warning to the physician; thus, in the treatment of a case of obesity, it will, so to speak, mark a line of caution; and in the long-continued infections, it will serve to indicate the degree of tissue starvation and suggest change in the feedling. Starvation does not provoke acidosis in herbivora as markedly as in man and dog.

Infectious Diseases.-Many infections, especially those of the alimentary tract, diphtheria and streptococeus, present a marked acidosis. It is not parallel to the fever, but rather to the intensity of the intoxication, though sometimes high acidosis is seen in mild infections and 
little acidosis in severe infections. The administration of sugar may result in a diminution. But in many cases the diet is not low in carbohydrate and the administration of sugar has no effect. Striking reduction in the acidosis in diphtheria has been reported following the use of antitoxin. Children, in general, display infectious acetonuria to a more marked degree than do adults.

Gastro-intestinal Disturbances.-In children especially, gastro-intestinal disturbances, often of sudden onset, provoke a moderate or even pronounced acidosis. Individuals seem subject to recurrent attacks. There are clearly two groups of these cases; one, in which some indiscretion in diet, some decomposed foodstuff, provokes a gastro-enteritis, with acidosis as one of the symptoms; and the second, in which the acidosis and the gastro-intestinal symptoms appear as results of a common cause, not in the diet. The cases of the last group are most interesting. 'The attacks usually occur in children. They are liable to recur at more or less regular periods; in one case with the details of which the writer is acquainted, from four to six attacks occur per year. The attacks usually begin with vomiting, which is persistent. At the first examination of urine, acidosis is present. The recurrent vomiting lasts for two or three days, and then gradually quiets. The acidosis ceases, for the most part, as soon as the paroxysmal stage is passed. As a matter of close observation it is known that the child eats well of all food and especially including carbohydrates up to the sudden onset. The administration of sugar has no effect upon the attacks, though the test is worth little on account of the persistent vomiting. Glucosuria is not present. The use of large doses of sugar as soon as the stomach will tolerate food, has no effect in cutting short the attack, which always ends by lysis. Excessive dieting with sugar between attacks has no influence upon the occurrence of the same. There is undoubtedly something sui generis in these attacks. Similar severity of vomiting in pregnancy, hysteria, migraine, etc., lead to no such acidosis as is seen in these cases. The tendency to convulsive seizures and to coma is also a feature indicating their peculiar nature. In sucklings with enterocolitis acetonuria is common, and here it is the result of carbohydrate subnutrition; but here the peculiar features of the attacks of periodic recurrent vomiting are lacking. In carcinoma of the stomach associated with pyloric obstruction, or in any case of pyloric obstruction, the subject may present an acetonuria due to carbohydrate starvation, the result of the loss of the ingested carbohydrates through fermentation in the stagnated stomach.

Eclampsia.-In some of the reported cases of eclampsia acidosis has been recorded. Since it does not occur in all cases, it cannot be held to have any causative association. It might, however, be suggested that in the eclamptic woman, the occurrence of acidosis as a result of carbohydrate insufficiency might be the incidental cause of the onset of convulsions. 
Phosphorus Poisoning.-Acetonuria is to be observed early in the course of phosphorus poisoning. It remains a fixed feature in the condition, and is not modified by the ingestion of carbohydrates, of which it is obviously independent, since it occurs before the body can have become poor in glycogen.

Narcosis.-The postoperative acidosis of anesthesia is now well known. Uncommon, it is to be seen much more often with the use of chloroform than following the use of ether. There are three groups of cases. In the first group is found only the presence of acetone bodies in the urine. This does not occur nearly as often as is commonly stated; the exaggeration is due to the fact that crude tests for acetone have been usually employed. The iodoform test is often found positive in the urine when the keton test is negative, though what the substance may be that provokes the formation of iodoform from iodin is not known. In the second group of cases, few in number and most often seen in children, the urinary findings are associated with moderate symptoms, nausea, vomiting, headache or somnolence, passing off within a few days. The conditions in the third group of cases are serious in the extreme. There is heavy acidosis, often convulsions, coma, rapidly developing jaundice, low temperature or high fever, and death in the larger proportion of cases in from three to five days. At postmortem a profound degeneration of the liver is found, a chloroform necrosis in many ways comparable to phosphorous necrosis. The urine contains, in addition to the acetone bodies, ammonium lactate and various amino-acids, the products of the autolysis of the liver. Here also the condition is not one associated with the carbohydrate metabolism, it is a result of the necrosis of the liver. There is no glucosuria, and no evidence that the body does not burn sugar.

In many other conditions occasional instances of acidosis are encountered. These are incidental and may reasonably be attributed to reduction in the carbohydrate metabolism.

The current theory of acidosis is that the failure in the combustion of fat is secondary to failure in the combustion of glucose, either because there is no glucose to burn or because the body has lost the power of burning glucose. This is expressed in the statement that fat can only burn in the fire of glucose. Just as crude oil will not burn alone but will burn in a spray of steam, so fat is held to burn only when glucose is also burned. From this point of view it is apparent that in every instance of acetonuria the combustion of glucose must be faulty; in every case where the combustion of glucose is faulty, one must expect an acidosis. Whenever the combustion of glucose is normal, the combustion of fat should be normal; and whenever the combustion of fat is normal, the combustion of glucose must be normal. These statements represent the current doctrine. The writer cannot share this exclusive view. Opposed to this view are, firstly, clinical and experimental facts; and, secondly, theoretical considerations.

To the rules as above asserted, many exceptions exist, and these 
exceptions are in many instances of crucial meaning. It is true, as a rule, that when the normal body is fed on fat and meat alone, acidosis results. But why should it occur when protein is abundant? Instances have been reported in which acidosis occurred with a diet of 250 grams each of fat and meat; such an amount of protein will yield over 100 grams of sugar. Perhaps it may be urged that such an amount of sugar is not enough to assure the complete combustion of so much fat; but this rejoinder at once makes admission of the existence of other factors that determine the combustion of fat. And in direct experiments, 100 grams of glucose will usually exhibit the maximum antiketonic action. This lack of relationship between the amount of glucose and the extent of combustion of fat is difficult to understand. A small amount of glucose may check a heavy acidosis; a large amount of glucose may have little effect upon a mild acidosis.

The administration of sugar has little or no effect in many of the cases of acidosis in connection with bacterial infectious diseases. The action of diphtheria antitoxin in reducing the acidosis is surely striking enough. Can anyone believe that the diphtheria poison depresses the combustion of glucose (no sign of which is to be noted), and through it the burning of the fat; and when the inhibition of the combustion of glucose is raised by the antitoxin, the combustion of fat is only secondarily relieved? In severe cases of diabetes, the administration of increased protein or of sugar may increase the acidosis instead of lessening it. It may be replied that in these cases the ingested sugar does not burn; but why should its mere presence still further reduce the combustion of fat? There are cases of diabetes with good toleration for carbohydrate who display for months high acidosis, the course of which is quite independent of the carbohydrate of the diet or the glucosuria. On the other hand, there are cases of diabetes with low toleration for carbohydrate who present little or no acidosis. Such cases recall the gradual disappearance of the acidosis in normal individuals who are living on fat and protein; at first the acidosis is marked, then it gradually disappears. To offer as explanation for these cases, that the body has learned to burn fat without sugar is surely a lame procedure. To continue the use of such language, one might say that some diabetics who have good powers of burning sugar have forgotten how to burn fat with it. The plain fact in diabetes is that any factor that aids in the normal course of metabolism (including, of course, the burning of sugar), any factor that increases the health of the body in any way, tends to aid in the proper combustion of fat, and vice versa. That is the reason why alcohol, while sparing the sugar and fat combustions, nevertheless actually lowers the utilization of each. Finally, the acidosis of idiopathic, recurrent vomiting in children, and that resulting from poisoning with phosphorus and chloroform is certainly not dependent upon the combustion of sugar. These attacks strike in the midst of a normal metabolism. There are no indications of any defect in the carbohydrate metabolism, there is no glucosuria. It is 
not possible to believe that any conditions, outside of excessive exercise and refrigeration, could free the body of glycogen within the short time, often only a few hours, in which these forms of acidosis develop. We know now what is required to free the body of storage glycogen. In many of these cases, moreover, the subjects are ingesting carbohydrate. In such cases the contention that sugar is not being burned can be demonstrated in only one way-by the determination of the respiratory quotient. There is not, to the knowledge of the writer, one such determination on record. It is possible that a body might consume sugar and yet not burn it and not eliminate it. But for such a situation to be demonstrated, the respiratory quotient is indispensable.

The support of this hypothesis places us in a quandary when the facts determined for the metabolism of normal individuals on a proteinfat regimen are considered. Acidosis appears at first in all cases, no matter how large the intake of protein. An amount of sugar large enough to check the acidosis in starvation or to modify greatly the acidosis of a diabetic, may be without effect when derived from a heavy ration of protein in the subject on a protein-fat diet. That the body spares its fat and burns sugar whenever it is available is one of the best-known laws of metabolism. The body does not burn its fat until the glycogen depots of the body are depleted and the sugar of the body reduced to the minimum; but then when it needs to burn fat, it cannot, according to this hypothesis, burn it properly because it has not the sugar to burn with it. When now sugar is given indirectly in the form of protein, the body is still unable to burn the fat properly. If time be given, however, the body adapts itself to the function of burning the fat, even if the protein ration be minimal. Certainly it is not possible to explain this train of facts logically and satisfactorily on the hypothesis that the body burns fat only in the fire of sugar.

Theoretical considerations dealing on the one hand with the equations for the burning of sugar and fat, and on the other hand, with our conceptions of fermentations, are sufficient in the opinion of the writer to preclude the acceptance of the hypothesis that fats are burned only in the fire of sugar. It must be clearly realized that inability to explain any set of facts imposes no obligation to accept an unsatisfactory hypothesis. The idea that an hypothesis is like a poor bridge, to be kept and used until a new structure is built, is unscientific; where ignorance exists it must be recognized. That many of the facts of the relations between the combustions of fat and glucose are apparently explained by the hypothesis under consideration is true; that the facts on analysis are not such as to exclude every other explanation is, however, equally true. Many a line of apparent least resistance has been found to lead to an erroneous conclusion. When one contemplates the whole scope of the metabolisms of fat and sugar, one must assume that they have their own systems of enzymic action. In the dog (carnivorous and dependent more upon fat than upon sugar for the body heat), and in the rabbit (herbivorous and depending largely 
upon sugar for body heat), no such dependence of the combustion of the fat upon that of sugar exists as is postulated for man. Interactions occur between the metabolisms, and failure in one might under certain circumstances entrain failure in the other; but that a fundamental metabolism should be absolutely dependent upon another metabolism, that a defect in the one must inevitably result in inhibition of the other, is a situation so at variance to our general conceptions of metabolism and of physiological compensation and adaptation as to be a priori very doubtful.

When one contemplates the reactions whereby butyric acid may be burned, one is still more at loss to understand why the combustion of sugar should control the combustion of butyric acid. So far as is known, in all cases of acidosis, the molecule of higher fatty acid is burned normally down to butyric acid. Disregarding as improbable the direct transformation of butyric acid to propionic acid by oxidation of the alpha carbon, there are two possible routes.



The side reaction from aceto-acetic acid to acetone we may disregard, since it has only the same meaning as the aceto-acetic acid. If the lower scheme be correct, the oxidation of the beta carbon of beta-oxybutyric acid is completed, cleavage occurs between the $\alpha$ and $\beta$, and 
two molecules of acetic acid are formed. Aceto-acetic acid would, therefore, be regarded as an abnormal substance. This view is only tenable if we assume that the traces of acetone present in normal urine are derived from the alanin group of amino-acids of the protein catabolism. In the opinion of the writer, the first scheme is the better founded. The defect in acidosis under the first scheme, lies in the loss of the power of oxidizing beta-oxy-butyric acid to aceto-acetic acid, and of oxidizing aceto-acetic acid to acetic acid. The administration of these two acids in conditions of acidosis is followed by their elimination unchanged. If one accepts the second scheme, though it is less probable, the defect consists in the fact that a partial oxidation of beta-oxy-butyric acid to aceto-acetic acid occurs instead of immediate and complete oxidation with cleavage between the alpha and beta carbons. From the standpoint of the modern theories of oxidation, one might assume that the aceto-acetic acid is the normal intermediary suboxid stage, just as it appears in the oxidation of copper in alkaline solution:

$$
\begin{aligned}
& \mathrm{CuSO}_{4}+2 \mathrm{KOH}=\mathrm{K}_{2} \mathrm{SO}_{4}+\mathrm{Cu}(\mathrm{OH})_{2} \\
& \mathrm{Cu}(\mathrm{OH})_{2}=\mathrm{CuO}+\mathrm{H}_{2} \mathrm{O}
\end{aligned}
$$

On this assumption, we would infer that in acidosis something had intervened to check the reaction of oxidation at the intermediary stage, just as the oxidation of the copper can be checked at the oxyhydrate stage. However one regards this, one is unable chemically to understand how the presence of glucose in the system or the simultaneous combustion of glucose would have the power to make or mar this oxidation. Medical writers have suggested the term "secondary oxidation," apparently unmindful of the fact that coupled reactions have long been known. That the presence of one body may make possible the oxidation of another body is well understood. A good illustration is afforded in the following reaction:

Chromic acid + tartaric acid $=$ formic and other acids (very slow reaction)

Chromic acid + arsenous acid $=$ arsenic acid (very rapid reaction)

Now arsenous acid has no action upon tartaric acid. When, however, the two systems are mixed, the rapid oxidation of arsenous to arsenic acid by the chromic acid is accomplished as before, while at the same time the tartaric acid is also rapidly oxidized. The assumption of a dependence of the oxidation of butyric acid upon the oxidation of glucose violates, therefore, no theoretical requirement. We might suggest some such scheme as the following, based upon the assumption that there is an intermediary stage in the conversion of glucose into lactic acid, and that it is this intermediary product that acts as the accelerating agent in the combustion of beta-oxy-butyric acid.

Glucose $\rightarrow \mathrm{p}_{1} \rightarrow$ lactic acid, $\rightarrow \rightarrow \rightarrow \rightarrow \mathrm{CO}_{2}$ and $\mathrm{H}_{2} \mathrm{O}$

beta-oxy-butyric acid $+\mathrm{p}_{1}=\rightarrow \rightarrow \rightarrow \mathrm{CO}_{2}$ and $\mathrm{H}_{2} \mathrm{O}$ 
Obviously, inhibition in the formation of $p_{1}$ would lead to cessation in the combustion of the fatty acid. What makes it difficult of acceptation is the fact that the oxidation of butyric acid to acetic acid can be accomplished by bacteria, by colloidal metals, by different chemical operations and by carnivora and herbivora directly and in the absence of glucose-but not by man.

Two explanations may be suggested for the occurrence of acidosis. These will not account for all cases, and for those thus inexplicable we must await elucidation through research. One is that the ferment of the reaction is lacking; in diabetes the lack of the normal ferment for the combustion of butyric acid goes in many cases hand in hand with the lack of the pancreatic zymo-excitor for the combustion of glucose. The second explanation is that when the entire maintenance of the heat of the body is thrown upon the fat metabolism (suddenly or through a too prolonged period of time), the overload leads to a quantitative incompleteness in the reaction. The body can convert just so much aceto-acetic acid into acetone and no more. The liver can deaminize just so much tyrosin and no more. Possibly a body can burn just so much butyric acid, and when the function is taxed the reaction of oxidation is incomplete. To use a common term, the acidosis would be the result of overwork. It is not alleged that these two explanations will account for all the instances of acidosis; but they will account for many. And while the failure to account for all the instances of acidosis in nowise discredits these explanations, the failure to account for any instance of acidosis is fatal to the hypothesis that all ketonic acidosis is due to defect in the burning of sugar, through which alone the combustion of fat is assumed to be possible. 


\section{CHA PTER VI}

\section{THE METABOLISM OF PROTEIN}

LiKE sugar and fat, protein bears a dual rôle in the body: it is concerned with growth and regeneration of tissues, in the maintenance of the status quo; and it is a fuel. In the case of sugar and fat, though the importance of the anabolized states is fundamental to cellular structure, quantitatively by far the largest portions of sugar and fat used in the body are utilized simply as fuel. In the case of protein the converse is true; in a properly regulated diet the use of protein as fuel is minimal, its utilization is most largely concerned with the processes of cellular anabolism. That meat can be used as the sole food is true. It contains enough fat and sugar (a part from the glucose formed from it) to furnish the fat and sugar needed in the lipoid-and sugar-carrying complexes of the tissues; and it can serve as the exclusive fuel, though a very wasteful form of fuel. It is a general rule that the animal body does not tolerate ingestions of protein in excess of the needs of its anabolism: all excess is not stored but promptly burned. The determination of the line where tissue utilization ends and fuel utilization begins, or in other words, to separate what we term the endogenous and the exogenous catabolisms, is extremely difficult; and misconception of this relationship has brought much confusion into the subject.

The problem of the state of resorption of the products of the digestion of protein resolves itself into two possibilities, if logic and the known facts are alike to be respected. Either the amino-acids and peptids are resorbed unchanged, and transported to the tissues in that state for utilization or storage, as far as needed by the cells; or blood protein is formed in the intestinal wall by synthesis, and the blood protein carried to the cells for utilization. To attempt to occupy a middle ground, that only so much protein is synthesized in the intestine as may be needed in the tissues, the excess of amino-acids deaminated there and the products carried to the liver to be converted into sugar and urea, is only to confuse the situation. To inquire: should all the amino-acids be built back into protein in the intestinal wall when only a fraction thereof will be needed by the tissues? - does not hit the mark. How should the intestinal wall know how much synthesized protein will be needed by the tissues? Either there is total synthesis (except for superfluous amino-acids) or there is no synthesis in the intestinal wall. To the writer, sound reasoning favors the theory of synthesis of protein from amino-acids in the intestinal wall. The derivation and utilization of the blood proteins is incomprehensible 
on any other hypothesis. Nor can one understand how the aminoacids, if absorbed unchanged, could escape deaminization in the liver. It is true that under the theory of the blood proteins as the common substrate of all the protein anabolism of the tissues, intracellular hydrolytic cleavages must precede the synthesis of special proteins. But under these circumstances the different body cells have offered to them all the assortment of amino-acids, which would not be the case if they were dependent upon the products of the digestion of protein of each meal. The problem of the definition of the endogenous and exogenous protein catabolism is identical in either case; in the one case the excess of amino-acids is catabolized; in the other case the excess of blood protein is catabolized, the concept of equilibrium is as applicable to one as to the other. Without further discussion, since an analytical investigation of the question is not yet possible, we shall found our presentation of the metabolism of protein upon the assumption that the products of the digestion of protein are in the intestinal wall converted into the stock proteins of the blood, these serving as the substrate of the anabolism of the special protein.

The alternative proposition runs to the effect that the products of the digestion of protein are absorbed unchanged and transported to the tissues, largely to the muscles, and there stored. Stored they must be, since the processes of anabolism cannot be supposed to follow the habits of eating. How stored? While there can be no doubt that the tissues and muscles can store amino-acids, and recent investigations seem to have greatly enlarged our conceptions of such a storage there, to the writer it does not seem biologically or chemically logical to regard this as the actual state of affairs. There must be a reaction of formation of blood protein; the constancy of the blood in starvation, in hibernation and in the anadromous fishes during the period of sexual migration, and the apparent dependence of the various body processes on the stock of blood protein, make this assumption necessary. Either the stock proteins of the blood are formed in the intestinal wall, or they are formed in the muscles. The fact that the muscles do build down protein is clear in starvation, other cells of the body, of course, sharing the function. That they should store large stocks of amino-acids and release or use them for anabolic processes seems to the writer far less likely than that they should convert these aminoacids into the stock proteins of the blood. Against the idea of the transportation of the amino-acids to the peripheral tissues, apart from considerations of the toxicity of the unchanged products of the digestion of protein, stands the general proposition that specialization at the portal of entrance affords to the organism not only greater protection against deprivation through hepatic combustion, but also a wider choice and scope in utilization than could be afforded by specialization in the peripheral tissues. Future researches may indicate the contrary of this interpretation, and in any event it is proper and necessary to vest in the muscular system full powers for the synthesis of 
protein from amino-acids. But for the present purposes of this work, we shall aceept the modus operandi as stated, and consider the aminoacids of the alimentary tract to be converted into the stock proteins of the blood in the cells of the intestinal wall.

The state of resorption of the products of protein digestion is to be regarded, as stated in the closing discussion of the resorption of protein, as peptids and amino-acids. The wide leeway that is necessary to permit the synthetic functions to form serum albumin and serum globulin from all sorts of ingested proteins, makes it appear certain that the digestion of proteins is continued down to very simple peptids. These peptids and amino-acids are on resorption assumed to be converted into serum albumin and serum globulin. These two proteins of the blood serum are to be, regarded as the stock proteins of the body, they are carried to all the cells and tissues and are the original stock or basic material from which are formed all the special proteins in the body. These stock proteins bear the biological stamp, which is another argument in favor of a complete or nearly complete hydrolysis of the proteins in the act of digestion.

The site of the conversion of the amino-acids and peptids into serum albumin and serum globulin is primarily the epithelial membrane of the intestine (the resorption membrane). We assume that the liver has little or nothing to do with this reaction because of the absence of signs indicating a flooding of the tissues with amino-acids when the blood from the intestines is switched directly into the venous circulation. If the formation of the stock proteins of the blood were in chief the function of the liver, it is not to be comprehended how the establishment of the Eck fistula is followed by so little disturbance in metabolism. It may be proper, however, under all circumstances to accord to the liver such power and to predicate the formation there of the proteins from such amino-acids and peptids as have escaped conversion in the wall of the intestine. But the chief synthesis (apart from the question of the peripheral tissues) must according to our present light be located in the wall of the intestine-just as the synthesis of neutral fat from fatty acids and glycerol must be located in the wall of the intestine. The mechanism of the synthesis of albumin and globulin from the amino-acids and peptids we rest upon the action of an enzyme. One cannot resist the hypothesis of the so-called reversed action of the proteolytic ferments. But special factors must needs be operative here, since from the products of the digestion of many kinds of protein of widely varying composition and construction, the intestinal wall builds serum albumin and serum globulin, and in each species places upon these the biological stamp of the species. We possess, however, no other concept than that of enzyme action under which the synthesis of the blood proteins becomes at all comprehensible. For him who is fond of wearing an intellectual veil, the notion of vital action will here find for the present an easy application. The serum albumin and serum globulin of the blood have been designated as the stock proteins. 
By this it is not to be inferred that they are the sole proteins present in the blood plasma. But the others are there present in the merest traces, and the quantitative relations alone compel us to accord to the named proteins the rôle of stock proteins. These two proteins present very different physical properties, although data have been advanced tending to show that the albumin passes into the globulin. This, however, is made improbable by the fact that serum globulin contains glycocoll in goodly amount, while serum albumin is devoid of glycocoll; and this amino-acid, it will be recalled, is centrally located in a peptone, being one of the components of the resistant polypeptid. We will, therefore, regard them as distinct proteins, and each probably serves distinct purposes in the syntheses of the different body proteins. The quantitative relations of serum albumin and serum globulin in the blood serum are fairly constant for each species; the difficulties in separation and estimation could account for all the apparent fluctuations in the ratio.

These stock proteins we are to regard as the materials from which all the special proteins of the body are formed. They are also the state in which protein is burned as fuel. We possess exhaustive quantitative studies on the protein content of blood serum after meals rich in protein. We may assume that there is for these proteins a physiological limit, just as there is a limit for blood sugar. If more sugar be poured into the blood than is needed, it is converted into glycogen and stored; if much more than needed be poured into the circulation, so that the conversion into glycogen cannot hold the sugar content down to the maximum normal blood limit, glucosuria occurs. Now in the case of blood proteins there is no storage state, nor is there a physiological albuminuria whose effect is simply to reduce the blood content of protein. When an excess of protein, beyond the needs of the body tissues, is poured into the blood, the increased concentration results in increase in protein catabolism, and the excess of protein is split, deaminated, burned and eliminated. This is the fraction of the protein catabolism that we term exogenous, meaning by this that the protein has never been incorporated in the state of protoplasm, but being in the blood in excess, is catabolized. In a certain physiological sense this excess of protein may be regarded as a foreign body, which the metabolism disposes of by hydrolysis, deaminization, oxidation, and elimination.

\section{THE ANABOLISIM OF PROTEIN}

Under the term anabolism of protein we understand the formation of the special proteins of the tissues from the stock proteins of the blood; and also the formation of other special tissues constituents, such as purin and pyrimidin. The special proteins must be formed progressively during the growth of the individual; and the loss by cell death 
must be replaced in the adult body. All cells have a limited life, they die and the number is maintained through cell regeneration; in the growth of the new cells new special proteins are required, since the dead cells are catabolized. There are cells that are lost to the body though not catabolized. The spermatozoa, the horny cells of the skin and the desquamated epithelia of the mucous membranes are lost to the body though not catabolized, just as are the proteins of the hair and nails. The sum total of these losses is small in comparison with the internal destruction of cells, seen in its most rapid phases in the leukocytes and erythrocytes. During the period of growth and during adult years the synthesis of special proteins is, therefore, for the same purpose - the equipment of new cells.

It is, however, not proper to limit the conception of the anabolism of protein to the equipment of new cells. Cells have an intracellular metabolism, protoplasm is used up in the course of the cellular activities, and there must be a regular replacement of protoplasm within the cell just as there is regular replacement of cells in an organism. Bacteria have a metabolism independent of reproduction, and, of course, to predicate any other condition would violate the law of conservation of energy. It is possible in an indirect way to indicate that such a intracellular metabolism exists. Lymphatic tissue contains about 80 parts of protein to 1 part of purin. The muscular and connective tissues contain less, so we can make the calculation on the basis of at least 200 to 1 . Now the average normal endogenous purin output is about 0.3 gram per day. The corresponding figure for this in total nitrogen output, would amount, therefore, to 60 grams of protein catabolized per day-which is higher than the actual figure. This may be interpreted to indicate that there is an intracellular metabolism involving nucleus to a far greater extent than protoplasm, since were the transformations equal in each or the catabolism related only to cell death, the output of the two fractions would be 200 to 1 . Such a calculation is, of course, only crudely approximate. On the other hand, it is easy to show directly that use of a cell need not lead to appreciable wear as revealed in catabolism. Heavy normal muscular exertion does not increase the output of nitrogen or of creatinin; sugar is burned (four-fifths of it being given off as heat and one-fifth appearing as work), but of chemical evidence of wear on the part of the protoplasm of the muscles there is none. It may, of course, have been so slight as not to have been appreciable in our methods. And theoretically this must be so, since we can no more imagine a tissue working without wear, than we can imagine a machine working without friction. This fraction we term the wear-and-tear quotient or fraction, the protein needed to maintain the proplasmic status quo of the living functionating cell.

So far as we know, the anabolism of protein is always intracellular. 'Though the special proteins may be found extracellular, their synthesis is to be regarded as invariably intracellular. In this synthesis we must 
define two steps: the intracellular digestion of the serum albumin and serum globulin; and the construction of the special protein from the building stones of the same. In each cell is performed the whole act of the digestion of protein; and from the component amino-acids the new proteins are synthesized. The divergencies in the constitution of the special proteins from the composition of the stock proteins are so marked that we are forced to assume that the intracellular hydrolysis is a complete one. This intracellular hydrolysis is, of course, an act of fermentation. And judging by their results (they cannot be well studied in experiment) the intracellular proteolytic ferments are of the type of endotrypsin seen in the yeast germ, and able, like trypsin followed by erepsin, to complete the hydrolysis of protein to the individual amino-acids.

Direct analogies are to be observed in the plant world. Bacteria do not utilize directly the proteins of culture media. These they digest, split into amino-acids, and from these amino-acids build the specific proteins needed in reproduction. Some bacteria cannot digest protein; they are dependent upon the amino-acids for their nutrition, and are thus parasitic upon other bacteria that are able to hydrolyze protein. Some germs are very adaptive. Thus Aspergillus niger will build its proteins from nitrate, glycocoll or glutamic acid as the sole source. In general, bacteria will utilize only alpha amino-acids.

The sprouting of plant seeds is another illustration. When a seed sprouts, the proteins (as well as the carbohydrates) are split into their component parts. These amino-acids, or peptids, are then utilized in the formation of the protein needed in the sprouting shoots of the new plant. The storage forms of protein in the seeds are usually not the forms that exist in the growing parts; and to form these, the storage protein must first be hydrolyzed and the amino-acids made available as building stones.

Illustrations will make the relations clear. In some members of the family of Salmo, notably those of the Rhine and those that inhabit the Western coast of this continent, the salmon do not feed after entering fresh water to ascend to their spawning grounds. They enter fresh water in heavy flesh and very fat, with the testicles and ovaries undeveloped. They ascend long distances, against heavy currents and to high altitudes. As they ascend the sexual organs are rapidly developed, and when the fish reach the spawning grounds these glands are very massive, weighing possibly twenty times as much as when the fish entered the fresh water stream. Since the fish do not feed, the material for the growth of the sexual organs must have been derived from other tissues of the body; and for this but one is available, the muscles. When the fish reach the spawning grounds they are very wasted, especially the males. The muscle substance has had to support not only the work of the ascent, but also to furnish the materials for the growth of the ovaries and testicles. The chief constituent of the ripe spermatozoa is a protamin, salmin. It is not formed directly, 
but through an intermediary stage, a histon. Now salmin is 87 per cent. arginin, the rest is serin, prolin, and valin. In the muscle of the salmon are the serin, prolin, and valin in the amounts needed, but there is less than 10 per cent. of arginin. The histon contains about 30 per cent. of arginin. Now unless the cells of the testicle can form arginin from the monamino-acids (of which we have no evidence), it is clear that in order to form one part of protamin ten parts of muscle protein must be destroyed. The muscle protein is assumed to be hydrolyzed in situ and the products then conveyed to the testicle. Whether the muscle protein after hydrolysis is first reconstructed into blood proteins and these then carried to the testicle; or whether the products of the hydrolysis of the muscle protein are earried as such to the testicle, cannot be determined directly. But carried to the testicle the material is. If it be carried to the testicle in the form of amino-acids, the cells of the testicle then use the required arginin, serin, valin, and prolin, and the rest is returned to the circulation to be burned. If the blood proteins are the material, these are then hydrolyzed in the testicle, and the amino-acids utilized as before stated. There are observations tending to show that serum albumin and serum globulin are formed from the muscle proteins; the constancy of these proteins in the blood is best explained on this view, the muscles and organs waste, but the blood proteins remain relatively constant. Now protamin could not be synthesized from muscle material unless the muscle protein were completely hydrolyzed to the individual amino-acids, because in the protamin there is a particular linking; there being six protones (diarginyl-serin, diarginyl-serin, diarginyl-serin, diarginyl-prolin, diarginylprolin, diarginyl-valin), and it is quite certain that no such linkage occurs in the muscle protein.

Collagen. - Collagen is formed in many tissues. Its content in amino-acids as compared with those of serum albumin and globulin is as follows:

Serum albumin Serum globulin Gelatin
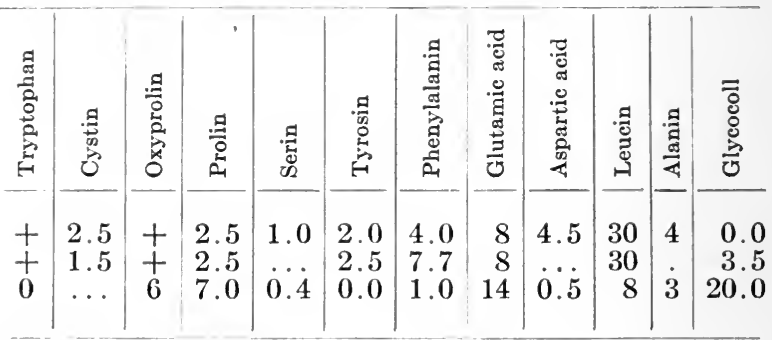

As eompared with the blood proteins, gelatin is high in glycocoll (of which albumin contains none), glutamic acid, and the pyrrols, low in the aromatie amino-acids. Here, again, it is hardly to be believed that collagen could be formed from the proteins except after complete hydrolysis of the latter. 
Casein.-Casein is a highly specialized protein. It is formed from the blood proteins by the lining cells of the mammary glands. Casein contains every known amino-acid found in proteins except glycocoll; apart from this, it resembles serum globulin, being different from serum albumin especially in a lesser content of leucin. But in the intramolecular arrangement of the amino-acids it is very different from the two stock blood proteins; the physical properties are strikingly different, the peptones are different, and the number of $\mathrm{COOH}$ groups is much larger than in the blood proteins. Casein is a perfect protein, capable of supporting the synthesis of all proteins, since the body can form glycocoll de novo or from other amino-acids. The instance is recorded of a man who, until he attained full adult growth, lived solely upon milk.

Hemoglobin.-A highly specialized compound protein is hemoglobin. It is a combination of a globin and hematin, which is a tetra-pyrrol. For the synthesis of this hematin in the red marrow, where the erythrocytes are generated, the pyrrol of the stock proteins of the blood is alone available, so far as we know. In the molecule of protein the pyrrol bodies are intimately bound with the amino-acids. To set them free in order that they may be linked together with iron to form hematin, complete hydrolysis of the protein molecule must be required. Here is an instance where protein is catabolized in order that one constituent of the molecule may be made available for a special synthesis.

Mucins.-The mucins again are very different proteins, though formed from the stock proteins by lining epithelial cells. 'The mucins are difficult of isolation and analysis. It is known, however, that they are poor in arginin, lysin, cystin, aspartic, and glutamin acids, contain usual amounts of leucin, tyrosin, phenylalanin, and tryptophan, and are very rich in glucosamin, from 20 to 30 per cent. This glucosamin is intimately bound with the $\mathrm{COOH}$ group of the normal amino-acids by its $\mathrm{NH}_{2}$ group, it is not localized in one of the peptone groups, but is intimately incorporated in the whole molecule. Here again it is not possible to understand the synthesis of this protein except upon the assumption that the parent cell had the full quota of free amino-acids at its disposal.

Special Proteins.-It is not possible to predicate the derivation of the different special proteins from either serum albumin or serum globulin. Most of the organ globulins resemble closely serum globulin. Naturally a protein rich in glycocoll could not have been deriver from serum albumin directly. Many of the proteins of the connective tissue resemble serum albumin in many ways and there is a general similarity in the chemical and physical properties of these proteins. But some of them are one-fourth glycocoll, while others are deroid of glycocoll. The more the special proteins are studied, the more complex become the findings. And one might as well come to the belief first as last, that in the cells in which these special proteins are 
synthesized, the blood proteins are broken into their individual aminoacids, since this state of affairs alone affords the functionating cells free scope for their reconstructions.

Synthesis of Amino-acids.-An important question concerns the possibility of the synthesis of amino-acids by cells of the higher animal body. If amino-acids can be synthesized, the ultimate dependence of the body upon the proteins of the diet is lessened. For all the aminoacids definite answer cannot be given. It is certain that cystin and the aromatic amino-acids cannot be synthesized in the animal body. The body has not the power to build the benzene ring, nor can it combine this ring with an amino-acid. It is possible that phenylalanin and tyrosin are convertible into each other, but one or the other must be preformed. Nor can the body combine sulphur with an aminoacid, the cystin must be presented preformed. There is also data making it very doubtful whether the diamino-acids can be formed in the body. Chemically it seems possible that lysin might arise from glucose or from the condensation of two molecules of alanin; the body splits arginin into ornithin and urea, but so far as we know cannot unite them. Asparagin spares protein in herbivora, but does not so act in carnivora. This may be interpreted to mean that in herbivora aspartic acid can be converted into other amino-acids, while in carnivora this is impossible. There is no doubt that the body can form glycocoll in large amounts. This is proved directly by the maintenance of body growth on a diet of casein, which contains no glycocoll. And by the administration of benzoic acid it can be shown that the body has the power of making glycocoll available in amounts far in excess of the protein catabolized during the period of the experiment. Possibly in some way, acetic acid and ammonia are united to form glycocoll. It was elsewhere stated that animals may be kept in nitrogen equilibrium with mixtures of amino-acids. This experiment succeeds even if glycocoll be absent, $i$. $e$., the body must synthesize glycocoll. If, in the same experiment, tryptophan or the related tyrosin and phenylalanin are omitted, the experiment fails. On the whole, we may for the present assume that except glycocoll, all the amino-acids must exist preformed in the proteins of the diet in amounts sufficient to offer the needed units. Gelatin, which cannot support the protein metabolism, owing to its lack of aromatic amino-acids, become fully competent as a diet protein when combined with an equal part of other proteins containing the usual amounts of these groups.

It is possible that if we could define exactly the body needs in cystin and phenyl-amino-acids, we could determine the minimal protein requirements of the whole body. No matter in how much excess the other amino-acids may be present, at least that much protein is daily necessary as will furnish the needed thio and aromatic groups. And since different proteins contain different amounts of thio and aromatic amino-acids, the experiments might be so varied as to afford data 
from which could be calculated the amount of protein necessary in the diet to maintain the body in tissue equilibrium.

The possibility of the neo-formation of amino-acids in the animal body by conjugation of $\mathrm{NH}_{2}$ group with fatty acids, has as stated usually been denied (outside of glycocoll), even as a qualitative reaction. Recently, however, experimental evidence has been adduced in favor of such synthesis. The proposition amounts to a reversion, in a circuitous manner possibly, of the reaction of deaminization. When phenyl- $\alpha$-keton-butyric or phenyl-oxy-butyric acid is fed to animals, an acetyl-amino-butyric acid is eliminated. It has been long known that amino-acids may be oxidized in the body to the corresponding $\alpha$-ketonic acid, which is the reversion of the above reaction. Perfusion experiments on the liver have also indicated that alanin is formed from lactic acid, a direct illustration. Such a reaction, if done as a functional act, amounts to the assimilation of nitrogen in the state of ammonia, a faculty that has been hitherto vested solely in plants and lower forms of animal life. Without wishing to deny the occurrence of the qualitative reactions described, it is quite another thing to assume that they illustrate quantitative functional reactions. Our total knowledge of the details of the catabolism of protein is against such a proposition. The mass relations that exist in the liver and tissues must operate against such a synthesis. Were we to administer as food the several fatty acids, it is certain that they would be burned in the liver or converted into sugar, instead of uniting with ammonia to form amino-acids, since the concentration in the liver of ammonia derived from the endogenous catabolism of protein must be extremely low. As a matter of direct interest in questions of metabolism, the qualitative reaction may prove to be of importance. But since a diet does not and cannot contain the appropriate fatty acids, except already preformed as amino-acids in protein, the possibility of ammonia operating in such a closed inner circle - set free in catabolism of tissue, recombined into proteins of blood, anabolized again into tissue-is entirely out of the question, even in part.

Incomplete Proteins and Endogenous Catabolism.-This problem has been approached experimentally from another direction through investigations bearing on the ability of incomplete proteins to sustain the endogenous catabolism. The plan of such investigations is to place an animal upon a nitrogen-free diet of carbohydrate, or carbohydrate and fat, with the necessary salts. When the nitrogenous elimination falls to a constant level, this is taken to represent the minimal basis of endogenous protein catabolism. Incomplete proteins - gliadin, zein, and gelatin have been tested-are then administered in amounts corresponding to the urinary output of nitrogen. Under these circumstances a nitrogen balance is never attained. In the case of gliadin and zein, about one-fourth more nitrogen is eliminated than was contained in the input; in the case of gelatin the inefficiency is much greater; there is 50 per cent. excess nitrogen in the urine. While 
these proteins are all incomplete, they are different; zein lacks glycocoll, tryptophan, and lysin; gliadin lacks lysin; gelatin lacks cystin, tyrosin, and tryptophan. It is clear from the results that these incomplete proteins have always a certain and in some cases a surprising power, in replacing the body protein disintegrated in the endogenous catabolisn. Growth, however, is never to be attained under such circumstances. It has been suggested that these results indicate that the endogenous metabolism for maintenance is different than for growth; and that, furthermore, in the utilization of protein by cells, complete cleavage is not essential. Viewed closely, however, these two inferences are not warranted. It might be possible, in the first place, that one amino-acid could be formed from another, certainly the glycocoll could be so derived. Lysin might be derived from glucose. But it is impossible to assume the synthesis of tyrosin, tryptophan, and cystin in the animal body. However, it is possible to seek another source for these. It is possible that in the excess of nitrogen, eliminated over the input in the protein under investigation, is included nitrogen of body protein catabolized to furnish tyrosin, cystin, tryptophan, and lysin, just as the salmon catabolizes muscle to furnish arginin for the ripening testicle. None of the amino-acids named exist in large amounts in the glandular protoplasm; and without the catabolism of greater amounts of different tissue protein than indicated in the nitrogen deficit, it is possible that the needed particular amino-acids could be obtained wherewith to maintain the endogenous metabolism in accordance with the current conception of protein restitution. There is now a considerable literature on the power of gelatin to spare protein (gelatin replaces protein, being an incomplete protein; it does not spare protein by virtue of the sugar it can yield). Two-thirds gelatin and one-third other protein will hold a dog in nitrogenous balance at the fasting figure. Gelatin spares most largely protein being burned for dynamogenetic purposes; it can be used for anabolism by having added to it the missing cystin, tyrosin, and tryptophan derived by the catabolism of indifferent body protein. If the missing cystin, tryptophan, and tyrosin are added directly as amino-acids, gelatin becomes a complete protein and can sustain nitrogenous equilibrium.

Nevertheless, experiments have shown that it is possible to maintain rats over long periods with food free of tryptophan. This result suggests that it is possible for the body to form tryptophan from the benzene ring introduced in tyrosin and phenylalanin.

Conjugation of Special Proteins.-'The conjugation of the special protein with the other substances that participate in the formation of the compound or conjugated proteins (nucleoproteins, glycoproteins, phosphoproteins, lecithoproteins, hemoglobins, chromoproteins) is accomplished in the particular cells concerned. Some of these conjugated proteins resemble esters, others resemble glucosids. The mechanism of these conjugations is entirely unknown. In some of these compounds the protein seems to functionate as a whole, and cannot be itself hydro- 
lyzed until it has been set free from the other substance. In others this does not seem to hold, the protein within the conjugation is subject to hydrolysis. Pepsin and trypsin split all these conjugations. In the tryptic digestion of some of these, as in the case of hemoglobin at weak alkaline reaction, tyrosin is set free before cleavage into globin and hematin appears to be accomplished. On the other hand, hemoglobin is split by pepsin $\mathrm{HCl}$ before the protein fraction is demonstrably digested. In the case of the lipoid-protein complex of organs, the fat is in firm combination and is not recovered except after a tryptic digestion.

Formation of Special Tissue Proteins.-The formation of the special tissue proteins depends solely on the need, it is apparently not possible to engorge cells with their special proteins, except following periods of starvation. The adaptation is beautifully shown in the case of the gravid uterus. The number of cells in the uterus is increased during pregnancy, and the size of the individual cells greatly enlarged. This is, of course, accomplished through the synthesis of extra muscle protein. Following delivery, during involution, these overgrown cells become reduced to their normal dimensions. This must be explained as due to the hydrolysis of the now excessive protoplasm, the extrusion of the soluble products into the circulation, later to suffer deaminization and oxidation. Here we see a special instance of an intracellular growth, followed later by reversion to the original status. The different cells of the body are of very different orders of magnitude; they may in general be assumed to have an intensity of metabolism directly proportional to their cellular dignity, and a duration of life inversely proportional to their cellular rank. The cells of the common connective tissues can be deprived of blood supply for hours without injury; the cells of the central nervous system die within a few moments when deprived of blood supply. It may be as confidently stated that the cells of fasciæ and tendon have a long span of life, and that the cells of the central nervous system have a short span of life. The anabolic synthesis, cell for cell, will therefore, be greater for the special proteins of the cells of high rank. The growth of the muscular system from birth is growth by enlargement of muscle cells solely, not by multiplication; a certain muscle in the newborn has as many cells as the same muscle at full growth. In hypertrophy of the heart and in the growth of the gravid uterus, however, increase in number of cells is also held to occur. The wasting of the muscles of the salmon during the anadromous migration is not a diminution in number of cells but a shrinking in the mass of the cells. Whether the rule of increase in dimensions but not in numbers holds true for the nervous system has not been determined.

Adaptation in Anabolic Function.-Feeding experiments in growing animals have yielded very interesting results indicative of adaptations in the anabolic functions. When a growing organism is underfed, one of two things may happen: growth may be interrupted or growth may 
be maintained through a shifting of the anabolic functions. If the diet be too insufficient, growth is checked and death will result. But if the diet be so arranged that the animal can maintain weight and nitrogenous equilibrium, growth is continued. In order for growth to be thus maintained, tissue of low grade must be sacrificed to tissue of high grade, just as in the migrating salmon muscle is sacrificed to testicle and ovary. In successful experiments in animals growth is maintained, the skeleton increases in dimensions, the glandular organs are conserved and the central nervous system is held intact; muscle and connective tissue are catabolized and from the products of their hydrolysis the materials are derived wherewith the cellular anabolisms are maintained. With pronounced degree of such underfeeding, the proteins of the blood fall below normal. When later normal feeding is practised, the depleted muscular and connective tissues are restored; and it is remarkable to what extent such processes of restoration can proceed, so that after a time the animals quite equal normal animals. There is, of course, an intensity and a duration of such underfeeding that lead to loss that is irreparable; the animals remain "runts." These facts have been worked out both for carnivora and herbivora, and afford concrete demonstration of the biological impulses in the functions of protein anabolism. The condition in infancy sometimes termed infantilism probably represents such a condition of undernutrition, the result either of improper feeding or, more likely, of reduced digestion and assimilation. While it is true that in young children increase in weight should accompany increase in length of skeleton, it is not equally true that in older children the same rule holds. It is common to find in boys nearing puberty, pronounced increase in height without increase in weight, to be followed later by recovery in weight, the two processes occurring in sequence. Apparently there is at the age of puberty in boys an impulse to grow that may exceed the powers of digestion and assimilation, and under these circumstances tissues of lower order are for a time catabolized to support the growth of tissue of higher order; when the excessive growth ceases, the defects are repaired.

The stock proteins of the blood are also the material for the synthesis of the important pyrimidin and purin bases of the purin metabolism. The direct synthesis of these substances from protein is well illustrated in the hatching of birds. Eggs contain no purin or pyrimidin and all the purin and pyrimidin of the nuclei of the cells of the hatched chicks have been synthesized from albumin. Milk contains no purin or pyrimidin; and from casein, indirectly via the blood proteins, is synthesized all the nucleic material of the growing infant. These matters will be discussed in the proper context under the nucleic metabolism. We also regard the proteins as the parent substance of creatin.

There are no known pathological variations in the anabolism of protein, either qualitative or quantitative. This applies as well to 
the utilization or assimilation of the products of digestion as to the formation of the special proteins and nucleins. There are investigations tending to show that the special proteins of malignant neoplasms are different from those of the prototype cells. In myxedema the skin is infiltrated with a mucoid substance, that has been regarded as different from normal mucins. In the degenerations of tumors of certain types also, proteins of seemingly abnormal appearances occur. While there is every theoretical reason to believe that under conditions of disease such deflections in the qualitative formation of special tissue proteins could occur, the investigations to date have not been carried out with the degree of thoroughness necessary to establish such differentiation.

\section{THE CATABOLISM OF PROTEIN}

Before proceeding to the discussion of concrete acts of catabolism of protein it will be of advantage to elucidate the whole situation. The catabolism of protein may be stated to include five rubrics:

(a) Of this there are two fractions: firstly, the catabolism of protein derived from cells that have died, each cell having like all unicellular organisms a limited term of life; secondly, of protein assumed to have been cast off as the expression of intracellular wear and tear. The first fraction results from the death of the cell, the second (the wear-andtear quotient), is incidental to the life of the cell. This catabolism continues, of course, during starvation.

(b) The catabolism of the amino-acids resorbed from the products of the digestion of protein and not suited to the synthesis of the stock proteins of the blood. When proteins on digestion yield certain aminoacids in larger amounts than can be utilized in the synthesis of serum albumin or globulin, so long as we believe these are not convertible into other amino-acids there is for them no other fate than to be catabolized, probably in the liver. For certain proteins in the diet there must be rather large excesses of certain amino-acids; in all cases there must be some. Thus gliadin contains 36 per cent. of glutamic acid, and elastin 25 per cent. of glycocoll, far more than can be used in the blood proteins. It is possible to assume, as will be elsewhere explained, that in this fraction lies in part the explanation of the specific dynamic action of protein.

(c) The catabolism of superfluous amino-acids derived from serum albumin and serum globulin within the cells of the body prior to the synthesis of the special proteins. All the amino-acids of the stock proteins are not needed in the special proteins. When histons and protamins are synthesized, large amounts of diamino-acids are needed and consequently more monamino-acids will be set free than are required. In the synthesis of all special proteins it may be inferred that more or less of the amino-acids of the stock proteins will be superfluous. These 
amino-acids we believe to be catabolized, but we cannot hope to measure this fraction. Fractions $b$ and $c$, as well as $a$, must be large during the period of growth, when the ratio of protein metabolism to body weight is high. $a+b+c$ represents the endogenous protein catabolism.

(d) The catabolism of the excess of stock proteins of the blood, that quantity over and above the amount needed to support the synthesis of the special proteins. The synthesis of special protein, the anabolism of protein, is not a constant but an adaptable factor, as will be explained. But whatever its extent on a particular day, the needs in amino-acids occasioned thereby are covered by the stock proteins. Obviously $b$ and $d$ have the same meaning, they are superfluous. And all stock protein formed from the diet of one day over and above the needs of the synthesis of that day is promptly catabolized. It is just as though a stream had two outlets and one inlet, the inlet and the one outlet being regulable. 'The outflow from the unregulated outlet will be the difference between the input and the outflow of the regulable outlet. If with a constant input the regulable outlet be raised, more will flow from the open outlet; if the regulable outlet be lowered, less will flow out through the open outlet. If the regulable outlet be constant, increased input will result in increased outflow from the open outlet; if the intake be reduced less will flow out from the open outlet. It is a reciprocal relation between three factors, a relation of equilibrium. It may be illustrated in another way. It is just as though a factory received each day a shipment of perishable material, from which it prepared a conserved product for immediate use. Whatever was left over of the raw material each night was destroyed. If the use of the conserved product rose, then more raw material was worked up and there was less to throw away. If less of the conserved product was used, there was less to make up and more of the raw material to throw away. If with a fixed consumption of the conserved product the intake of raw material was cut down, there was less to destroy; if the intake was increased, there was more to dispose of. Fraction $d$ represents the exogenous protein catabolism.

The maintenance of a constant concentration of protein in the blood plasma under varying experimental conditions indicates the stability of the station of equilibrium. If a $\log$ be bled a third of his blood and the volume replaced with isotonic salt solution, in a surprisingly short time the blood plasma will be found to have recovered its normal content of protein, derived largely from body cells of lesser dignity. The maintenance of the stock proteins of the blood during starvation indicates the same thing, in a slower manner. On the contrary, if dog serum be injected intravenously into a dog, a proportionate excess of nitrogen will be eliminated in the urine in a day or two. And if the dog eat the same excess of protein, the same elimination occurs. In a word, under all circumstances the body endeavors to maintain a fixed concentration of protein in its blood plasma and tissues. The situation in the body is not so rigid as here illustrated, because of the 
faculty of adaptation and compensation, but the fundamental relations are as stated. The body has little storage capacity for protein.

In starvation, another factor of catabolism $(e)$ makes its appearance. Factor $(a)$ persists. When now during the course of a starvation (or in hibernation) the wasting of the most important tissues reaches a certain point, these cells are maintained by syntheses from the stock proteins. These in turn are maintained by syntheses from the less important proteins, those of the muscle and connective tissues. Now in the formation of blood proteins from the protein of muscle and connective tissue, amino-acids of one or another kind will be superfluous, and these will be catabolized.

It will be best first to deal with the questions in a purely qualitative way. The catabolism of a merits first description. We will follow it to the stage of amino-acids. 'Then we will take up the catabolism of rubric $d$. This we will carry to the stage of amino-acids. To these may then be joined $b, c$, and $e$. From this point we will trace the fate of each known amino-acid until the state of excrementation is reached. Thereafter the quantitative relations will be elucidated as far as is possible. The catabolism of nuclein will receive a separate consideration. In some places facts furnish the basis for theory; in other instances, sound, general theory must be used to interpret facts of undoubted occurrence but indeterminate meaning.

There are clearly four reactions concerned in the catabolism of protein: the hydrolysis of the molecule of protein; the formation of glucose from amino-acids; the deaminization of the amino-acids; and the oxidation of $\mathrm{NH}_{2}$ and of the fatty acids.

The Hydrolysis of Protein within the Body.-The hydrolysis of the protein of the cells that are dead presents no difficulties to comprehension. The phenomenon of postmortem autolysis gives so clear an illustration that there can be no doubt of the physiological facts. If a liver be removed from the body and allowed to lie at ordinary temperatures under sterile conditions, it will be found after a few days that the tissue has become softened, the amount of coagulable protein has been greatly reduced, or may indeed be absent, and on analysis the organ will be found to contain large amounts of proteoses, peptone, amino-acids, some urea and ammonia. The autolysis of the liver is not a process confined to the proteins; it involves glycogen, arginin, nucleic acid, and to a slight extent the fats-each carried on, of course, by the appropriate ferment. This self-digestion is thus accomplished through the activity of intracellular ferments which continue to functionate as catalytic agents after the death of the cells. Just as dried and powdered yeast cells contain ferments that will digest the cell residues when placed in solution, so the liver cells contain an endotrypsin which is able to accelerate the hydrolysis of the protein of the hepatic protoplasm to amino-acids. This ferment action does not proceed from the blood, whose influence can be ruled out. Now in the postmortem autolysis of the liver we observe, en masse, the identical 
reaction that we must believe occurs in each liver cell when worn out it dies. Cellular catabolism therefore means, in part, unicellular autolysis. So long as the cell is living its protoplasm remains intact; so soon as it dies, it is digested by the ferments contained within itself.

In the hydrolysis of protein within the body, peptones are, of course, formed, which are obviously not toxic. Peptones formed from foreign proteins in the digestive tract are toxic; those formed in the body from its own proteins are not toxic. This difference between isopeptone and heteropeptone we regard as resident in the intramolecular linking of the amino-acids.

The hydrolysis of the fraction of protein in the protoplasm of the functionating cell, that for reasons of wear and tear must be daily replaced, we must regard as included in the same reaction. There is apparently a difference between protoplasmic protein and mere protein that is to be regarded as a cell inclusion. When the fraction of worn protoplasm is deprotoplasmatized, it becomes subject to hydrolysis. Just how we are to picture to ourselves this condition in protoplasm that renders its protein resistant to digestion is as yet impossible of definition. We can say that it lies in the physico-chemical state of the lipoid-protein complex, but that is a concept and not a definition. Be it what it may, it is the same fact that prevents the unicellular yeast cell from being digested while alive by the ferments that rapidly digest it after it is dead. To term it a vital force is merely to still the question by filling the ear. The concept "anti-ferment" is still largely verbal.

The hydrolysis of the excess of circulating protein, the excess of the input over the needs of the body for syntheses, is not well understood. It could occur either in the circulating blood or in cells. It is difficult to conceive it as occurring in the circulating blood. There is a trypsin in the blood-or at least in the blood corpuscles-and it would not be proper to rule this ferment out because in tests in glass it seems to be of weak activity. But the idea of a ferment action that is so limited, stopping short in a system where the substrate is so concentrated as is protein in the blood plasma, is difficult of entertainment. A less objectionable idea is that the blood unloads its excess of stock protein upon the cells. Within these cells it then would occupy the same position metabolically as the protein of the wear and tear considered in the last paragraph. It would occupy within the cell the same position that glucose occupies in the muscle cell, where it is being burned without being a part of the protoplasm. It has been elsewhere pointed out that when an excess of protein is catabolized, the sugar and urea derived therefrom do not make their appearance in the urine at the same time. This, however, may be an expression of elimination and not of metabolism; and in any event does not give any suggestion as to whether the catabolism of the excess of protein occurs within the cells or without. It will be later pointed out that the catabolism of protein from protoplasm is, so to speak, more effectively accomplished than 
is the case with the catabolism of the excess of protein derived from the diet. This fact ágain does not argue for any site or modus of catabolism of the extra protein. The question must be left open, with the statement that general considerations make it most probable that the reaction occurs within the cells; and of the cells, most likely in the liver. From this point of view we may regard the liver as disposing of the excess of protein by catabolism just as it disposes of the excess of glucose by the formation of glycogen.

Closely related to the question of the site of catabolism of the exogenous protein is the question of the state in which it exists within the body. The distinction between the protein that is a part of the cellular protoplasm and the excess of protein that simply passes through the body, so to speak, in course of catabolism has been the occasion of many famous controversies in the literature of physiology. The former is usually described under the term protoplasmic protein, the protein that in the complex with lipoid and carbohydrate, ion, and salt constitutes the physico-chemical state of the protoplasm of the cell. The excess of protein, commonly termed "dead" or "circulating protein," "storage," "reserve," "labile protein," is not held to exist in such a physico-chemical complex. Where it is stored and how, as well as where it is catabolized, we do not know experimentally. As stated, in the opinion of the writer it is catabolized within the cells, largely in the liver, possibly in the muscles. All opinions are more or less hypothetical; the following, however, seem to the author to follow the line of least resistance.

It is assumed that an equilibrium exists between the cells of the body and the concentration of protein in the blood plasma. There is a certain minimum protein concentration of the blood plasma; if this be reduced, indifferent cells yield their protein to form blood protein for the maintenance of this minimal concentration. If by forced feeding the maximum concentration of blood protein be exceeded, the excess is thrown upon the cells, in the form of inclusions within the cells. The high protein concentration of the blood plasma and the inclusions of protein within the cells, in accordance with the law of mass action, result in the acceleration of the catabolism of this excess of protein by the cells, the reaction continuing until all the excess has been catabolized, and the protein concentration of the cells and blood returned to the normal.

When an animal is given excessive rations of protein, as a rule the nitrogen of the excess is promptly eliminated in the urine. The adult body, in health and full nutrition, tolerates the deposition of fat and glycogen, but does not tolerate the deposition or storage of protein, for which fact the above explanation has been advanced. If the feeding be very forced, a portion of the nitrogen is retained. Assuming that this retention is in the state of protein and not a retention (a nonelimination) of the products of catabolism, this we explain as due to an increase in the concentration of protein in the blood and to inclu- 
sions of protein in the cells, the cellular capacities for the catabolism of protein being exceeded. When the forced feeding is suspended, the retained protein is catabolized within the following few days, and the relations return to the normal. The capacity for such retentions varies with different animals and with different individuals and at different ages. In youth retention means, in part at least, growth, a true fleshening. And youth extends, so far as the growth of muscle is concerned, up to some twenty-four years. Following periods of subnutrition or illness, retentions occur that indicate restitution. But with some normal adult individuals a certain degree of retention occurs that apparently represents a toleration of the cells or a true increase in the protoplasm of the cells. The coefficient of protoplasm we are inclined to hold rather invariable; and yet it is possible that in some individuals at least the body cells, especially the muscle cells, may exhibit a tendency to variations, and may increase or decrease their protoplasm within certain limits depending upon the states of nutrition. But, all in all, the distinction between the states of endogenous protein and the exogenous protein is fundamental.

In the qualitative sense the catabolisms of endogenous and exogenous protein are different. The catabolism of exogenous protein leads only to urea and ammonia, possibly to a small rest of amino-acids. The catabolism of the protoplasmic protein leads to more specific products. Some urea is produced, possibly only in the glandular organs. But creatinin is the end product of the catabolism of the protoplasm of the muscle cells; and it is probable that it alone, and not urea or ammonia, is here produced. The nucleic acid, derived from the endogenous protein by synthesis, yields purin bases and uric acid. It has been suggested that the strictly endogenous protoplasmic catabolism might yield no urea, which would therefore be referable to the exogenous protein alone. This idea is not to be entertained; urea is one of the end products of the catabolism of protoplasmic protein, possibly only of the glandular organs. The elimination of urea under conditions of nitrogen starvation with full carbon nutrition, where no protein is burned as fuel, indicates that urea is one of the end products of endogenous protein catabolism. This fraction of urea is very small, corresponding to not over 2 grams of nitrogen per day. With this small figure we must contrast the 15 or even 20 grams of urea nitrogen observed in the urine of heavy eaters of protein. This excess of protein the body rejects; it is hydrolyzed, and the nitrogen eliminated as urea. This process of disintegration is, of course, not a total loss to the body, since the fatty acids are either burned or converted into sugar; but, as will be pointed out later, it is a very uneconomical method of obtaining sugar.

While it is true that the intracellular proteolytic ferment must be credited with the power of hydrolyzing protein to the final aminoacids, it is at the same time certain that this is not fully accomplished. The urine contains normally a substance termed oxyproteic acid, 
together with several derivatives thereof. This is a polypeptid which may be isolated and yields on acid hydrolysis glycocoll, alanin, phenylalanin, leucin, glutamic, and possibly aspartic acid. In addition to these, the substance contains sulphur; and although cystin has not been isolated, it may be assumed that the sulphur is present in the state of cystin. A goodly fraction of the rest-nitrogen and the neutral sulphur of the urine are contained in this body and its derivatives. Its presence in the urine might, on the one hand, be regarded as an incidental elimination, a slipping through the kidney before cleavage could be completed. On the other hand, it might be derived from some special protein in which these amino-acids were so combined as to form a group resistant to the action of the endotrypsin. It is not known that the amount of the substances exhibits any variations in disease, though could such be determined, light might be thrown on the ultimate source.

There are known variations in the elimination of the rest-nitrogen (retentions); but we haxe no knowledge of variations in the amount formed in metabolism under different conditions. Certainly it is not a variable of the total.

In connection with severe infections, internal suppurations and sometimes in subjects with advanced leukemia or malignant neoplasms, peptone or proteose is eliminated in the urine. Whether these represent incompletely hydrolyzed protein of the protein catabolism or are the result of abnormal enzyme reactions on the part of the infections, is not clear. They are usually not accompanied by an excess of aminoacids in the urine. Foreign peptone is highly toxic; but in these cases it is not possible to separate the symptoms of the disease from such as may have been caused by the peptone.

As stated, the aseptic postmortem autolysis of organs represents apparently en bloc what occurs in individual cells following their death. The cause of the hydrolysis is to be attributed to intracellular proteolytic ferment present in the cells at the time of the removal of the organ from the body, which continues to be active and digests the cells themselves. In certain conditions of disease we observe digestions that are in every apparent respect identical with this postmortem autolysis. Thus in the resolution of croupous pneumonia, in the liquefaction of exudates, in the inspissations of hemorrhages, in the liquefaction necrosis of neoplasms, and in the involution of the uterus after labor we have conditions that are in all probability comparable to postmortem autolysis. If one could deprive an area of the liver of its circulation, it is certain that the liver tissue within this area would be digested just as is the liver tissue in postmortem autolysis, though with a difference of velocity. In tuberculosis large masses of exudate are sometimes accumulated and these do not liquefy in the same manner as the tissues above named. Direct experimentation with the cheesy material of tuberculosis has shown that it is not digestible by ferments of the trypsin type; and with this finding the resistant behavior of tuberculous exudations in disease is explained. 
The Formation of Glucose from Amino-acids.-That glucose is formed in the body from protein has been established by three experimental facts. If a dog, freed of glycogen by starvation, be fed with a carbohydrate-free protein, like casein or codfish flesh, and the eliminations of nitrogen and carbon checked up against the inputs, it will be found that there has been a notable retention of carbon in the body, if the protein input was liberal and the animal was not worked. This carbon could scarcely have been retained in any other form than sugar or fat. Since there is no evidence that fat can be formed from protein except via sugar, the fat may be disregarded. Analyses of the tissues of such a dog will show rich deposits of glycogen, of which the tissues were previously freed by work and starvation. Unless the glycogen and sugar were derived from fat, they must have been derived from protein.

A dog with pancreatic diabetes, if carefully maintained, will eliminate an amount of sugar in such excess of the possibly carbohydrate content of the body and the diet as to make certain a derivation of sugar from protein, unless again it could have proceeded from fat.

A dog with phloridzin intoxication, freed of glycogen by work and starvation, and fed on carbohydrate-free protein (or predigested protein) will eliminate amounts of glucose that are far in excess of any possible storage of carbohydrate in the body of the animal. 'This glucose must have come from protein, unless it could have been formed from the fats. In all three experiments, it is clear that derivation from the fat is not chemically or experimentally excluded. But in the absence of any direct proof of the origin of glucose from fat, and in the possession of direct proof that amino-acids can be converted into sugar, we will regard it as established that in these experiments sugar is formed from protein. No interpretation will hold for all three experiments unless it regards this formation of glucose from protein as a physiological process, not an artificial adaptation or a pathological variation. We will, therefore, regard it as established that in the normal metabolism of the body, glucose is formed from protein.

Direct experiments having shown that glucose can be formed from amino-acids, for the present certainly we must regard this as the only modus for the derivation of glucose from protein. There was once much said of the nitrogenous moiety and the carbohydrate moiety of the protein molecule; there is no such thing, there are only collections of amino-acids in protein. It cannot be maintained in advance that glucose can be formed from all amino-acids. Each amino-acid resident in protein must be tested, and for each amino-acid the demonstration must be exacted. For the present there is from this point of view no way of stating how much sugar can be formed from the products of the catabolism of a unit protein.

How are we to picture this formation of sugar from amino-acids? The most natural point of view, possibly, would be to assume first a reaction of deaminization, whereby the $\mathrm{NH}_{2}$ group or groups would be split off, following which the body would form sugar from the 
hydroxyl-fatty acid. But at once an obvious question arises. Does the body form sugar from these derivatives of fatty acids when they are introduced into the body? For illustration, glycocoll is known in experiment to be a sugar builder. Glycocoll is amino-acetic acid, and when the $\mathrm{NH}_{2}$ group is split off, it would leave glycolic acid, $\mathrm{CH}_{2} \mathrm{OH}$.COOH. But does the body form sugar of glycolic acid when it is administered? Administered to rabbits, glycolic acid is converted, in part at least, into oxalic acid. It is clear that from the simple fatty acids no sugar can be formed. But we have evidence that from the alpha hydroxyl-acids glucose may be formed. The relation is best seen in the case of propionic acid. This fatty acid is burned directly in the organism; but its alpha hydroxylacid, lactic acid, is convertible under favorable conditions of concentration into glucose. If the deaminization of the amino-acid occurs directly the trend of the reaction would, therefore, be in the direction of sugar. But for some amino-acids, as for phenylalanin, it is believed that the corresponding simple fatty acid is produced, and this is not favorable to the formation of sugar, since these simple fatty acids are burned directly in the body. An illustration will make the point clear. Viewing deaminization as a direct reaction with the addition of water we would have for alanin:

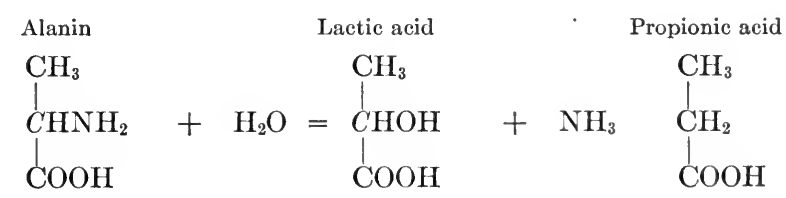

Now phenylalanin is known on bacterial deaminization to form not lactic acid but propionic acid; which in turn becomes phenylacetic acid, following which the fatty acid is completely burned, the aromatic ring being converted into benzoic acid, phenol or cresol, or burned.

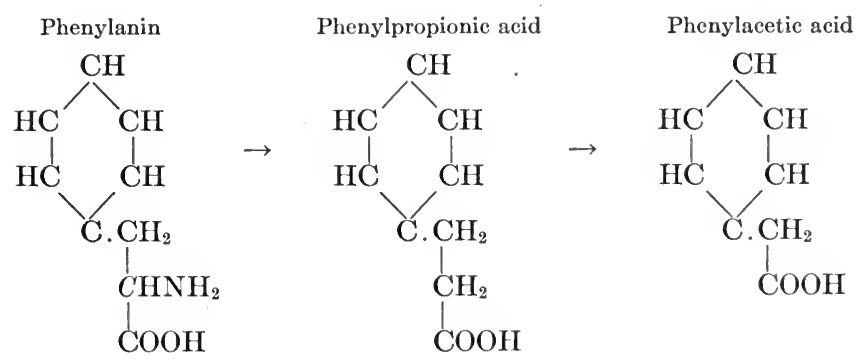

Possibly it is the presence of the phenyl group that makes the difference in the results of the reaction of deaminization.

Unless we can believe that the deaminizations take the course depicted above for alanin, it would be much more logical to assume that the amino-acids are first converted into amino sugars and these then deaminated. The composition of lysin and its resemblance to glucosamin 
gives the clue to what may be regarded in some instances as the correct interpretation. The equations will make the point clear.
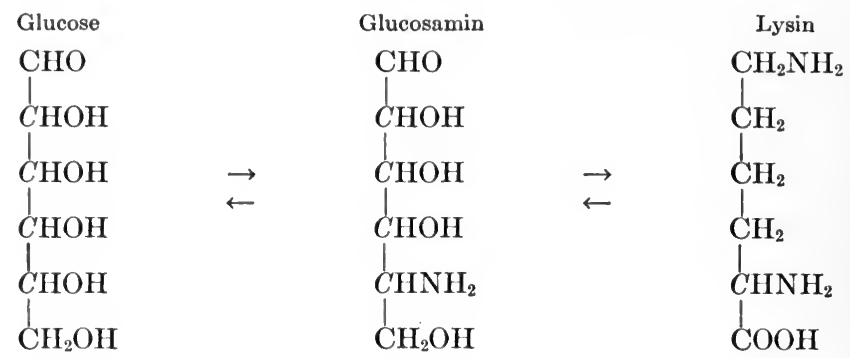

The amino-acid is converted into the amino sugar. Lysin has not been tested for its sugar building powers.

Close relations exist also between alanin and its derivatives and glycerose and lactic acid.

\begin{tabular}{|c|c|c|c|c|}
\hline Glycerose & Glyceric acid & Alanin & Serin & Lactic acid \\
\hline $\mathrm{COH}$ & $\mathrm{COOH}$ & $\mathrm{CH}_{3}$ & $\mathrm{CH}_{2} \mathrm{OH}$ & $\mathrm{CH}_{3}$ \\
\hline$C \mathrm{HOH}$ & $\mathrm{CHOH}$ & ${ }_{1} \mathrm{HNH}_{2}$ & $\mathrm{C} \mathrm{HNH}_{2}$ & $\mathrm{OH}$ \\
\hline $\mathrm{CH}_{2} \mathrm{OH}$ & $\mathrm{CH}_{2} \mathrm{OH}$ & $\mathrm{COOH}$ & $\mathrm{COOH}$ & $\mathrm{COOH}$ \\
\hline
\end{tabular}

Serin is obviously an amino-glyceric acid. Alanin has been tested directly on the depancreatized dog and found to yield glucose. We are justified in assuming that the amino-acid derived from alanin (serin) will react as does alanin. For histidin, phenylalanin, and tryptophan on the other hand (they have not been tested), the same could hardly be true. It seems quite certain that tyrosin, phenylalanin, and tryptophan do not yield their fatty acid for the synthesis of glucose. Nevertheless, recent investigations suggest that histidin, true to its lactic acid, yields glucose in the body, in part at least or under certain circumstances; and possibly future studies may reverse the present opinion for tyrosin, phenylalanin, and tryptophan. Here we now believe occurs deaminization with a later combustion of the fatty acids. This has been made fairly clear by our knowledge of the catabolism of the phenylamino-acids as revealed in alcaptonuria. It is fairly certain that cystin is not a source of glucose. Aspartic acid gives positive results, glutamic acid also. Glutamic acid could become converted into glyceric acid by oxidation at the beta carbon, when it would split into one molecule each of glyceric acid and acetic acid.

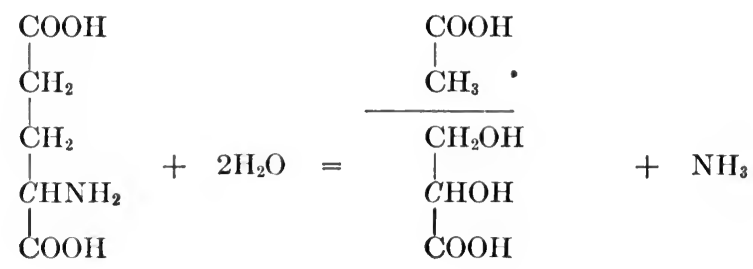


Of glycocoll it is positively known that sugar is formed from it, though the chemical relations to sugar, as stated, are less obvious. It may, however, be converted into glycolic acid, this then reduced to glycolaldehyd, which can be condensed to form glucose.

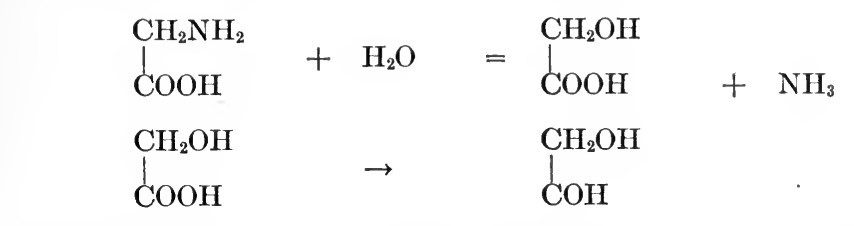

$3 \mathrm{CH}_{2} \mathrm{OH} . \mathrm{COH}=\mathrm{CHO} \cdot \mathrm{CHOH} \cdot \mathrm{CHOH} \cdot \mathrm{CHOH} \cdot \mathrm{CHOH} \cdot \mathrm{CH}_{2} \mathrm{OH}$

For leucin a positive demonstration could not be accomplished. We would not expect ornithin to yield sugar. Prolin we may exclude. Much more extensive investigations of the sugar-building properties of the different amino-acids are necessary before the subject can be considered as clarified; the opinions expressed represent the present state of information. From this all it is clear also that we are not in a position to decide whether the $\mathrm{NH}_{2}$ group is split off preparatory to the formation of sugar or later split off from the amino sugar.

Taking together experimental work and considerations bearing on constitution, we may tabulate the probable relations of the different amino-acids to the formation of sugar as follows:

$\begin{array}{lll}\text { Sugar builders } & \text { Not sugar builders } & \text { Unplaced } \\ \text { Glycocoll. } & \text { Tyrosin. } & \text { Leucin (?). } \\ \text { Alanin. } & \text { Phenylalanin. } & \text { Valin. } \\ \text { Serin. } & \text { Tryptophan. } & \\ \text { Aspartic acid. } & \text { Histidin. } & \\ \text { Glutamic acid. } & \text { Pyrrol. } & \\ \text { Lysin. } & \text { Arginin. } & \end{array}$

Glycocoll becomes glucose via glycolaldehyd; alanin, serin, aspartic and glutamic acids pass into glucose via lactic acid (glycerose); lysin either passes directly through the corresponding amino sugar or might be split into two molecules of lactic acid or glycerose.

Very interesting are the time relations of this process. In the phloridzinized dog, it is assumed that no sugar is burned and that neither sugar nor nitrogen is stored. The appearance of the sugar and nitrogen in the urine of such a dog after the administration of a known ration of protein may be taken as an indication of the relative velocities in the two reactions. When the urine is collected in short periods, it is found that the marked elimination of glucose begins first, and reaches the top of its wave some three to five hours before the elimination of the nitrogen reached the crest of its wave. The eliminations begin about the same time, and are completed at about the same time. But the curves are reversed. The curve of sugar output rises rapidly and 
recedes slowly; the curve of nitrogen output rises slowly and descends rapidly. That this difference is wholly metabolic and in nowise secretory is not fully established.

From this point of view we cannot attempt to make an estimate of the amount of glucose that the body derives from protein in the diet, partly because there are no studies on diets of known aminoacid composition, and partly because the question is not settled for all the amino-acids. Such a study indeed could not today be carried out for the simple reason that we do not know accurately the quantitative content of the several amino-acids in a single protein; in no case (on account of defects in the methods), do the figures for the isolated amino-acids check up to anywhere near the original weight of the protein under investigation. We simply know that glucose is formed from protein, and we regard this process as the result of the conversion of the amino-acids into sugar. An idea of how much glucose can be formed may, however, be obtained in an indirect manner. If we assume that in a dog with complete phloridzin intoxication no sugar is burned but instead all is swept out; if we assume in a dog with pancreatic diabetes that the power of burning glucose is lost and that, therefore, all the sugar formed in the body is eliminated in the urine-then in an experiment extending through many days on a diet free of carbohydrate, we may from the ratio of glucose to nitrogen in the urine calculate the amount of sugar formed. The assumption that in these two experiments no sugar is being burned is not founded; some sugar is burned, though probably not much, and the real ratio is, therefore, higher than the ratio obtained. In the discussion of the glucose: nitrogen ratio in diabetes this was set at $5: 1$ as a maximum, in order to create no prejudice in the discussion of the possible origin of sugar from fat. As a matter of fact, that ratio is too high. One obtains it during the first days of a phloridzin glucosuria, but it is there the expression of a washing-out of stored sugar, or of a later rise in N output. Later the ratio sinks to about $3.7: 1$, never over $4: 1$. While in human diabetes, where the conditions are difficult of control, high ratios are sometimes seen, in the pancreatic diabetes of the dog it is rare to find a ratio over $3: 1$, usually about $2.7: 1$. But as stated, on account of slight combustion of sugar, the real ratio is somewhat higher. In all discussions of the $\mathrm{G}: \mathrm{N}$ ratio with ingested protein and tissue protein, it must be borne in mind that it is possible that the body forms more glucose from a unit of food protein than from tissue protein.

Now when an animal forms from protein 3.7 parts of glucose for each part of nitrogen, obviously this is less than half the amount of glucose that corresponds to the total carbon content of protein. If all the earbon in a molecule of protein were converted into glucose this would amount to $1.3 \mathrm{gram}$, a ratio to the nitrogen in the gram of protein of $8: 1$. If we first subtract the carbon that will later combine with the $\mathrm{NH}_{2}$ to form urea, the ratio will be reduced to $7: 1$. The deaminization of the amino-acids or amino sugars leaves fatty acids 
and sugars; the free $\mathrm{NH}_{3}$ will at once combine with $\mathrm{CO}_{2}$ of the tissue fluids to form ammonium carbonate. In other words, the $\mathrm{NH}_{2}$ groups (except in the case of arginin) do not form urea with any of the carbon of the molecules of amino-acids from which they were split off. 'Therefore, all the carbon in the fatty acids or sugars must be regarded as available for the synthesis of glucose, so far as the $\mathrm{NH}_{2}$ groups are concerned, and a subtraction is not correct. The ratio theoretically is, therefore, $8: 1$; the actual ratio is less than $4: 1$, let us say $3.7: 1$. Does this represent the sugar-forming power of protein? May we infer from this that less than one-half of the amino-acids in the molecule of protein is convertible into sugar? (As elsewhere stated, if the amino-acids form glucose only in proportion to their possible conversion into lactic acid, from a gram of casein about 0.58 gram of glucose could be derived, a ratio of about $3.5: 1$.) The amount that is burned, under the conditions of the experiment may be much higher than supposed, though the respiratory quotient does not lead to such an opinion. The nitrogen cannot be retained in the body in a prolonged experiment, unless the input be very excessive. There is evidence that when a starying phloridzinized animal catabolizes his own protein, there is a better utilization (less sugar is formed) than when subsisting on diet protein. There is also evidence that, within narrow limits, the nearer the protein input is to the known minimal, the less sugar relatively is formed from it. With increases of protein input, the ratio, however, remains the same; doubling the protein doubles the sugar and the nitrogen, the ratio remaining constant. No such proportionality is to be seen in diabetes in man, however. It is in fact partly the differences in the results in animals and in man that make one cautious in defining the sugar formation from protein on the basis of the glucose: nitrogen ratio. That a fraction of the fatty acids of protein is burned directly cannot be doubted. With all the data, therefore, one cannot yet state that the ratio of $3.7: 1$ represents definitely the proportion of carbon that in the catabolism of protein passes into sugar. And this without consideration of the question of the origin of sugar from fat.

The conversion of the amino-acids into glucose is assumed to occur in the liver exclusively, though for this limitation no decisive reason can be offered.

The Deaminization of the Amino-acids. - This we believe occurs in the state of amino-acids for such of these as are to be burned directly; for such as are to be converted into sugar, the $\mathrm{NH}_{2}$ might be split off before or after the state of amino sugar, as previously explained. Many tissues, liver, spleen, and kidneys, contain deaminization ferments. The reactions are of common type, illustrated for alanin:

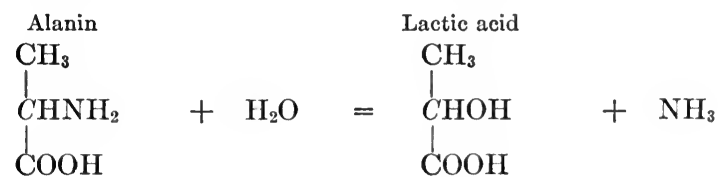


The ammonia combines with the carbon dioxid of the fluids to form ammonium carbonate, the parent substance of urea. Lactic acid would be converted into sugar. In the case of glycocoll, the lower sugar would be condensed to form glucose.

A special form of deaminization ferment is arginase, found in the liver. It splits arginin to form one molecule of urea and one of ornithin.

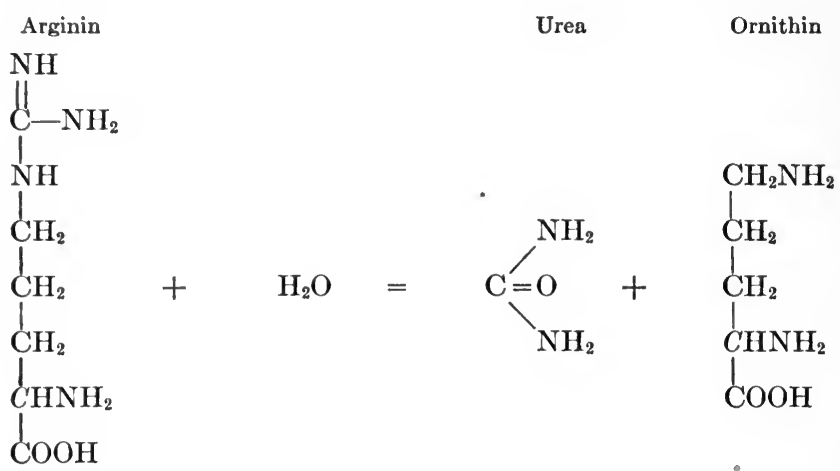

The ornithin is then deaminated like the other amino-acids.

Normal human urine contains but one defined amino-acid, glycocoll; present, however, are traces of other amino nitrogen. If large amounts of amino-acids are introduced into the circulation of an animal, a fraction will be eliminated. In certain severe organic diseases of the liver, as acute yellow atrophy, chloroform necrosis, phosphorus poisoning, eclampsia, and occasionally in severe infections and in diabetic coma different amino-acids appear in the urine, leucin, tyrosin, and cystin being the most prominent. Their presence must be regarded as due to the failure of the deaminization reaction. And the presence of amino-acids in the urine in especial connection with diseases of the liver of acute necrotic type, leads to the inference that it is largely in the liver that the deaminizations of the protein catabolism occur. It is in phosphorus poisoning also that large amounts of lactic acid are eliminated in the urine. This might obviously have come in part from the amino-acids of the alanin type. Apparently the liver fails in part in the deaminizations, and at the same time is not able to utilize the lactic acid.

Oxidation of the Products of Protein Catabolism.- It is in the oxidations that most of the energy of the proteins is set free as heat or made available as work. In the reactions of hydrolysis and deaminization we deal with practically isothermic reactions; there is heat set free but the amount is small. The heat values lie largely in the sugar and fatty acids, since the combining of the $\mathrm{NH}_{3}$ with the $\mathrm{CO}_{2}$ it meets in the circulating fluids is practically an isothermic reaction. If all the carbon in a molecule of protein (52 per cent.) were converted into glucose, this would yield about 1.3 gram, of a heat value of 4.5 Calories, 
against a heat value in the original protein of possibly 4.3 Calories. These are rounded figures and may be regarded as equal. The total caloric value of the same protein in a calorimeter is about 5.7 Cal. When from this is subtracted the heat set free in the oxidation of the two hydrogens of urea (about 1 Cal. for the urea of 1 gram), the small amount of heat evolved in the reactions of hydrolysis and deaminization becomes apparent. This calculation would not hold if but half of the carbon of the molecule of protein were converted into glucose (which apparently approximates the truth), since we do not know the heat value of the fatty acids that are burned directly.

One reads so much in the older literature of the nitrogenous and the non-nitrogenous fractions of the molecule of protein that it is necessary to insist on the radical change in our conception of the catabolism of the molecule of protein introduced by our present idea of hydrolysis to amino-acids and deaminization of the latter. Whether the oxyfatty acids thus set free are converted into sugar or burned directly, whether the formation of sugar occurs from the oxy-fatty acid or from the amino-acids, the fact remains that following the reaction of deaminization the nitrogenous portion is subject to no further oxidations and is not a carrier of energy for the animal body. The ammonia set free in the reaction of deaminization meets in the circulating fluids the carbon dioxid set free in the combustion of fat and sugar. These combine to form ammonium carbonate, which through the intermediary stage of ammonium carbamate is converted into urea. Thus:

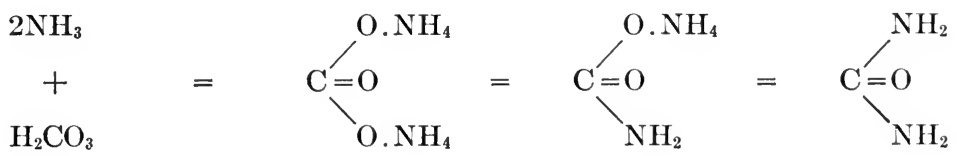

This is not an oxidation; ammonium carbonate contains 50 per cent. of oxygen, and urea only 26 per cent. It is, therefore, not permissible to speak of the oxidation of the "nitrogenous moiety" of the protein molecule; the "non-nitrogenous moiety" (the fatty acids and glucose derived from them in part) alone are available for reactions of oxidation. Under these circumstances the terms nitrogenous and non-nitrogenous moiety were best abandoned, since the cleavage of the molecule of protein is into amino-acids and not into two such fractions at all, and the amino-acids are then split through deaminization into ammonia and oxy-fatty acids.

The mechanism of the oxidation of the glucose has been discussed under the carbohydrate metabolism. The combustion of the fatty acids we regard as a direct building down to acetic and formic acid, with the final formation of water and carbon dioxid. These reactions we attribute to the influence of oxidation ferments. The experimental work on the oxidation ferments in animal and plant tissues is at the present time in a state of apparently inextricable confusion. The 
domains of the catalases, the oxidases and the perioxidases are peopled with contradictions. Oxidations occur apparently in all tissues and in the circulating fluids. Beyond this we actually know nothing.

The Formation of Urea.-Urea is the chemical state in which the largest part of the $\mathrm{NH}_{2}$ of the amino-acids of protein in mammals is eliminated. In birds and reptiles the trend of the reaction is not in the direction of urea; uric acid represents the chief end product. In amphibia and most aquatic animals, however, urea is again the end product. There are but two known and proved reactions whereby urea is formed from amino-acid in the body of the higher animals. And so far as our knowledge extends, the formation of urea from amino-acids is thus adequately and fully explained, and there is no reason or purpose in invoking the many possible laboratory reactions for the formation of urea.

The first of these (though discovered last, stated first because it involves but a small fraction and required no discussion), is the formation of urea from arginin by direct cleavage through the action of arginase in the liver. The reaction is made clear in the equation:

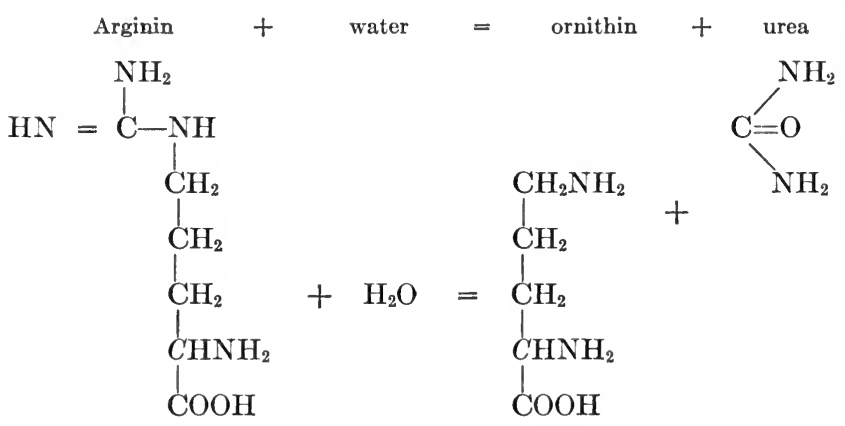

The parenteral introduction of arginin is followed promptly by increase in the elimination of urea corresponding to half the nitrogen of the arginin given. Since arginin does not at the most represent over 10 per cent. of the amino-acids of mixed proteins, the portion of urea thus derived is but a small fraction of the total. But the reaction and its acceleration by a special ferment that operates so far as known largely in one organ, makes the process of particular interest and instruction.

, The $\mathrm{NH}_{2}$ groups from the larger fraction of the other amino-acids (and including the ornithin), with certain exceptions that are later to be pointed out, are split off by deaminization ferments; in the case of such amino-acids as are destined to sugar formation either before the formation of sugar or from the amino sugars, and prior to oxidation in the case as such amino-acids as are destined to direct oxidation. The $\mathrm{NH}_{3}$ thus set free combines in the circulating fluids with the everpresent carbon dioxid to form ammonium carbonate. This is by anhydration held to be converted into urea through the intermediary stage of ammonium carbamate. 

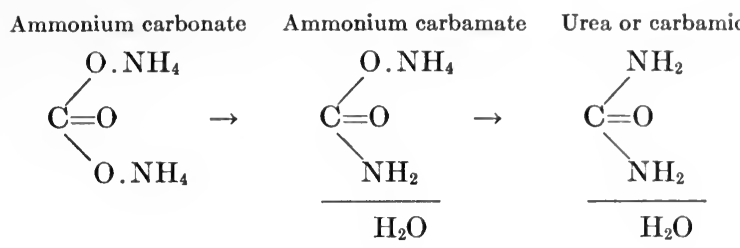

A molecule of water is extruded in each stage, first one nitrogen group being attacked and then the second. The reaction, though it is of course to be termed an anhydration, is in a way both a reduction and an oxidation, since both hydrogen and oxygen are removed, and a unit weight of urea has a higher caloric value than has the same weight of ammonium carbonate. In a general sense any reaction that increases the heat value per gram of a substance may be termed a reduction.

The experimental reasons that prove this scheme of reaction are many. Ammonium carbonate and ammonium carbamate are converted into urea in the living body and in experiments by perfusion or with isolated organs. A fresh liver pulp will form urea from ammonium salts and amino-acids. All ammonium salts that in the body are convertible into ammonium carbonate are eliminated as urea. The blood of the portal vein contains demonstrable traces of ammonia, that of the general circulation does not. This ammonia of the portal vein comes from superfluous amino-acids that have not been used in the synthesis of the stock proteins of the blood in the wall of the intestine, and also from the ammonia set free in the acts of digestion and by bacterial action in the intestine. This ammonia is in the liver converted into urea. The urine contains both ammonium and carbamate salts, the latter in mere traces. By the administration of large amounts of calcium hydrate, the amount of carbamate can be greatly increased. And in the urine of the dog following the establishment of an Eck fistula, demonstrable amounts of carbamate may be found in the blood. These facts point to the existence of ammonium carbonate and ammonium carbamate in normal blood. From all the known facts we may, therefore, regard this as the modus operandi of the formation of urea; the splitting off the $\mathrm{NH}_{2}$ groups of amino-acids to form $\mathrm{NH}_{3}$, the combination of $\mathrm{NH}_{3}$ with $\mathrm{CO}_{2}$ to form ammonium carbonate, and the anhydration of this salt to urea through the intermediary stage of ammonium carbamate.

Recent chemical investigations have indicated a way in which urea might be formed from amino-acid by the direct combination of carbon dioxid with the $\mathrm{NH}_{2}$ groups of amino-acids, in other words, prior to deaminization. This is indicated in the following reactions. When $\mathrm{CO}_{2}$ is passed into a solution of an amino-acid and calcium hydrate, compounds of the type of<smiles>[H][Y](CC(=O)O)C(=O)O</smiles> 
are formed. Illustrations for glycocoll and alanin will make the application clear.

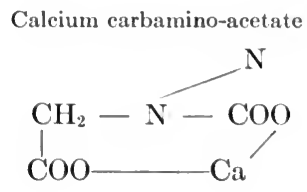

Calcium carbamino-propionate<smiles>[CH]C(=O)NC(C)C(=O)O</smiles>

In other words, by the direct combination of carbon dioxid with aminoacids carbamino acids are formed, whose conversion into urea would naturally follow. In this scheme, which lacks experimental confirmation in animals, the intermediary stage would be the one previously given, carbamate.

Urea is also formed in the body, in small but indeterminate amounts, from creatinin and uric acid. These derivations will be discussed in the connection with the catabolism of these two substances.

The formation of urea is so continuous and the elimination so prompt that the circulating blood is practically free of urea, as it is indeed practically free of $\mathrm{NH}_{2}$ nitrogen. Even with the formation of as large an amount as 80 grams per day, which would be a stupendous protein catabolism, the blood of the entire body would contain in any one second less than ten milligrams of urea. Of course, it is not equally distributed in all the circulating fluids. The larger part would be in the blood poured from the liver into the venous circulation. Urea is indeed formed in other organs and tissues than the liver, or at least may be. Furthermore, the formation of urea occurs in waves. The formation of the endogenous urea may be regarded as a continuous and even process. But the catabolism of the excess of protein in the diet with the formation of the exogenous urea occurs during the few hours following the ingestion of the protein in question. But even at the crest of the wave of urea formation, while the blood drawn from the hepatic vein might be expected to contain demonstrable amounts of urea, it is clear that the blood of the peripheral vessels could not be expected to contain urea in demonstrable amounts. The elimination of urea by the kidneys is apparently a very complete one. In another place, evidence will be adduced to indicate that retentions of urea occur under a number of different physiological and pathological conditions, though it is not certain that the fault lies in the kidney. But under ordinary circumstances, the elimination of urea appears to be one of the most casy, ready and quantitative functions of the kidney.

It was stated above that there is evidence that other organs than the liver can form urea from ammonium carbonate and carbamate. This is true in the sense that splenic pulp, for example, will form small amounts of urea from amino-acids. Urea accumulates in the blood of a dog whose liver and kidneys have been shut out of the circulation, 
proving that the formation of urea from amino-acids continues in the absence of the liver. 'There can, therefore, be no question that as an adaptative function other tissues than the liver can carry on the formation of urea. On the other hand, it is very striking to what an extent the urea-forming function of the liver is retained in profound experimental degenerations of the liver. Whether tissues other than the liver do form urea in the living organism, is another and unproved question. The animal with an Eck fistula is often regarded as an illustration of the formation of urea in tissues other than the liver. This rests upon a misconstruction of the protein catabolism, according to the interpretation here accepted. The portal blood carries to the liver only the amino-acids that have been found superfluous (on the particular days), in the building of serum albumin and serum globulin in the intestinal wall. If an animal were to be fed on the serum albumin and serum globulin of another animal of its own species, even these would not be present to be carried to the liver. This small fraction alone is all the amino-acid that the portal blood contributes to the liver. The larger mass of amino-acids that makes up the urea formation comes from the catabolism of protein within the various body cells (including here the superfluous amino-acids that are set free in the syntheses of the special proteins from the stock blood proteins) and from the catabolism of the excess of protein in the diet, the excess of the two stock proteins formed therefrom. Now the amino-acids of the endogenous catabolism of protein in the various cells of the body are carried to the liver in the arterial circulation. And the excess of the stock proteins of the blood, the result of an excessive ingestion of protein, are of course carried to the liver in the arterial blood; merely switching the portal vein to the inferior vena cava does not keep them out of the liver, it simply results in sending them to the liver via the general circulation instead of through the portal circulation. It is furthermore a priori most likely that at all times the catabolism of excessive protein in the liver follows its deposition there from the arterial circulation instead of from the portal circulation. Be this as it may, it is clear that the establishment of an Eck fistula merely sends the excessive stock proteins to the liver via the arterial circulation instead of via the portal circulation. The liver contains supposedly one-sixth of the blood of the body. And though the portal blood makes up a goodly fraction of this, the circulation through the hepatic artery is a very free and adaptable one. Now because under these circumstances urea is formed as under normal conditions, it is not at all to be assumed that this urea is formed outside of the liver. Surely no one would advance the proposition that the liver can only form urea from protein when it is presented to it by the portal circulation. So far as the data relating to all the different functions of the liver are concerned, including herein the known facts for carbohydrates, fats, proteins and pharmacological substances, it is clear that the liver has the same chemical powers to apply to substances that reach it 
via the arterial circulation as via the portal circulation. We may, therefore, regard the formation of urea in animals with an Eck fistula as occurring in the liver; and although the formation of urea in other organs and tissues may be conceded, to what extent it occurs is unknown. If the liver be not under all physiological circumstances the sole organ for the formation of urea, it is at least the chief organ.

When the total relations of the formation of urea are contemplated, it is clear that there are two fractions in the urea output whose meaning is different and which we ought to keep separated, namely, the endogenous and the exogenous urea. This distinction is of great importance to the theory of metabolism; and the question of the protein needs of the body is only another phase or aspect of this general matter. We shall later observe similar distinctions in creatinin and the purins. For urea the necessity or the advantage of this distinction has only recently become fully apparent. At the same time, it is clear that the quantitative experimental separation of the endogenous from the exogenous urea has not yet been definitely accomplished. And while we know enough to realize that the relations are to some extent adaptable and not rigid, we must expect in the future to secure a more clearcut line of demarcation than we possess today. The urea of endogenous protein catabolism, the catabolism of protoplasmic protein, probably does not exceed 5 to 7 grams per day for a body of 70 kilos when the subject is on a carbohydrate diet covering the needs of the body with just enough protein to establish an equilibrium in nitrogen. It is not related to the creatin of the muscular metabolism. It is derived largely from the catabolism of protoplasm of the cells of the glandular organs, though probably all cells have a slight urea production. The vastly larger mass of urea eliminated under ordinary conditions is derived from the catabolism of exogenous protein, the excess of protein in the diet.

Endogenous Urea.-The urea derived from endogenous sources comprises several fractions. (a) The urea derived from the autolysis of dead cells. It is our conviction that the protein of the protoplasm of such cells is completely catabolized and eliminated. (b) The urea derived from protein catabolized in the wear and tear of living functionating cells. (c) The urea derived from the catabolism of the aminoacids that are found superfluous when the cells of the body form special proteins from the stock proteins of the blood plasma. (d) The urea derived from the catabolism of the amino-acids found superfluous when the body forms the stock proteins of the blood plasma from the products of the digestion of the proteins of the diet. This fraction may be quite large; if a dog were fed on canine blood serum, it would be nil. (e) The urea derived in the conversion of endogenous creatinin into urea. $(f)$ The urea derived in the conversion of endogenous purins into urea. Fractions $(e)$ and $(f)$ have not been found measurable, but on a fixed diet under constant conditions of life, they may probably be regarded as subject to but slight variations. All these derivations of urea are 
subject to slight variations, dependent upon the age of the individual, the requirements for growth, the conditions of general nutrition prior to the experiment, the state of the muscular tissues, the amounts of sugar and fat in the diet, and upon other occasional and individual factors. While it ought to be possible to define a ratio of endogenous urea to total protein content of the body, it is not possible to define an accurate ratio of endogenous urea to body weight, on account of variations in skeleton, connective tissues, and fat-just as it is not possible to define an accurate caloric ratio for body weight.

The ammonia of the urine may be regarded as a portion of both the exogenous and endogenous urea. When the exogenous urea is reduced experimentally, the ammonia fraction falls correspondingly; but if the exogenous urea be entirely withdrawn, urinary ammonia is still present and belongs to the endogenous urea.

The neutral sulphur of the urine tends to hold itself parallel to the endogenous urea, independent of the total nitrogen input and of the total sulphur output.

Exogenous Urea.-The exogenous urea comprises two fractions. (a) The urea formed from exogenous ammonia, amino-acids, creatinin, and purins (these bodies being contained in the diet) and from any other substances in the diet that may be in whole or part convertible into urea. This fraction is usually not large, and it can be excluded by appropriate diet; a milk diet for example, is practically free of such urea-forming substances. (b) The urea formed from the catabolism of protein ingested in excess of the needs of the body for anabolism and replacement for wear and tear of protoplasm. 'This fraction of urea represents superfluity in protein ingestion. The body maintains a fixed concentration of protein in the circulating fluids surrounding the cells. There is no storage state for protein, corresponding to glycogen and fat. To a limited extent the cells of the body can lay on a little protoplasm, just as they can tolerate a certain attenuation. But this state of protein storage is very limited. Whenever the ingestion of protein adds to the circulating fluids protein above the needs of the body, the body maintains constant the protein concentration of its circulating fluids by the prompt catabolism of the excessive protein. The result of this operation is exogenous urea. If, on the other hand, less protein be ingested that corresponds to the needs of the tissues, to preserve constant the concentration of protein in the circulating fluids, the body transforms protein of tissues of low grade into blood proteins. Adaptations there are. In youth the processes of anabolism may display a sort of wasteful excess; under conditions of subnutrition and in old age the processes of anabolism are very effective and economical. These variations are, of course, indirectly reflected in the figure for exogenous urea, since the exogenous urea is all urea above that present in the urine when the body is in exact minimum nitrogenous equilibrium under the conditions of the particular experiment. The elimination of oxidized sulphur in the urine tends to run parallel, to 
the exogenous urea, being very low on a minimal protein input and rising as the input of protein is increased. The curve of the oxidized sulphur elimination resembles that of the elimination of nitrogen, but it is completed in less time. While the cystein of protein is not all split early in the course of digestion, some of it being contained in the final fractions of peptone, nevertheless the exogenous oxidized sulphur is very quickly eliminated.

The elimination of the exogenous urea follows a fairly definite course. The curve of elimination rises, beginning at about three hours after the ingestion of the protein, reaching its height by the sixth or seventh hour (by which time half of the nitrogen will usually have been recovered) and then falls away, reaching the base line at from twelve to twenty hours. If the ration of protein be excessive and especially if repeated regularly, the completion of the elimination of the exogenous urea may be delayed for several days. Different proteins are catabolized with sometimes strikingly different velocities. Thus the nitrogen of casein is quickly recovered; that of egg albumin is not entirely recovered for several days.

Taking into consideration all the varying factors, it is possible to give an approximate estimate of the endogenous urea. In an adult individual (over twenty-five years) in good natural nutrition, living under normal conditions of work, the experiment not having been preceded by a season of hard physical work or of physical illness, the body fat not being excessive and the diet containing enough sugar and fat to cover independently of the protein the maximum heat needs of the body under the conditions of the experiment, the endogenous urea, including the ammonia, may be said to vary from 100 to 170 milligrams urea per kilo per day, these differences expressing individual variations, women inclining to the lower figure, men inclining to the upper figure. Exceptional individuals may present still lower figures; occasional individuals, in apparent health, may seem to require more. All urea in excess of these figures is exogenous urea. Thus the urea nitrogen is commonly 85 to 90 per cent. of the total nitrogen; with the exogenous urea restricted by a low but adequate protein diet, the urea nitrogen will not be over 60 to 65 per cent. of the total nitrogen; the amount will be 6 to 10 grams, instead of 15 to 30 grams. Where climatic, industrial, religious or economic factors operate to lower the consumption of meat, this result of dietetic luxury is less in evidence. In few peoples of the earth, however, does total urea approximate endogenous urea.

Elimination of Urea.-Urea is eliminated largely but not exclusively by the kidneys. There is urea in the secretions of the skin and in the stools, in each instance excrementitious. The variations in these eliminations may be considerable. Profuse perspiration and diarrhea increase largely the urea (nitrogen) output of skin and intestine. Normally probably not over a half gram of urea is thus eliminated per day. But with profuse perspiration, nitrogen corresponding to 
over 2 grams of urea may be eliminated by the skin, and equal figures may be noted in active diarrhea. Whether under conditions of retention of urea, as is sometimes seen in cardiac dropsy and in nephritis, the urea eliminating functions of the skin and intestine are especially active, as has been long assumed by clinicians, is not known. If the concentration of urea in the circulating fluids be increased, increased elimination by the skin and intestine would be naturally expected; and if the kidneys by reason of disease were less permeable to urea, this might show in the nitrogen output of the skin and feces. But no such thing has ever been demonstrated, and the results of purgation and of drug diaphoresis have not been such as to make a so-called vicarious elimination of urea by skin and intestine appear certain. And in any event, the amounts involved cannot be very large. Most of the work dealing with the elimination of urea by skin or intestine has been done in the absence of controls, and with no idea of the amount of urea to be expected from the input of protein; inevitably, therefore, the inferences are not founded upon figures that are to be considered trustworthy.

There is no elimination of urea, or any form of catabolized nitrogen, in the expired air.

Ammonia.-There is a small fraction of ammonia split off in the digestive hydrolysis of protein. Bacteria evolve ammonia from amino-acids in the intestine. This is absorbed, and joins the ammonia formed through the deaminization of amino-acids wherever found. The urine contains from 200 to 600 milligrams of ammonia per day, depending in part upon the amount of protein in the diet. The blood and tissues contain demonstrable traces. The urine of children contains more ammonia than does the urine of adults. The elimination of ammonia in the urine may be regarded as an expression of equilibrium. When exogenous and endogenous acids and all conditions tending to make the urine alkaline are excluded, it is found that the ammonia of the urine bears a general relation of direct proportionality to the urea; if the protein be increased the ammonia output rises, if the protein is reduced the ammonia output is lowered. This we may interpret to indicate that in the blood there is a station of equilibrium in the reaction whereby urea is formed from ammonium carbonate. Both being present in the blood, the kidneys eliminate both. That such a relation of equilibrium exists is indicated also by the very large amount of sodium bicarbonate necessary to reduce the ammonia of the urine to the minimum. If to a healthy person some 90 grams of sodium bicarbonate be given daily, performed ammonia will be practically driven from the urine.

This equilibrium is subject to disturbance, on the one hand by the ingestion of alkali, and on the other hand by the ingestion or formation within the body of acid. The amounts of alkali contained in normal diets are not enough to diminish materially the amount of ammonia in the urine. The amount of acid that may be present in normal diets 
is also not enough to increase materially the elimination of ammonia. When mineral acids are administered, a point will be reached when the ammonia of the urine rises. With some individuals, or at least with individuals on certain diets, a half gram of a mineral acid will cause no rise in the urinary ammonia; in others this amount will cause a rise. With increasing doses, the amount of ammonia tends to increase in general proportion to the amount of acid. The obvious explanation is that the mineral acid combines with the ammonia circulating as carbonate or carbamate and thus withdraws it from the urea formation. Turned about, we say that the ammonia distoxicates the acid and spares the fixed cations of the tissues. As a matter of fact, the acid is eliminated combined both with fixed cations from the tissues (sodium, potassium, and calcium) and ammonia. At first the acid combines with all, there being some loosely bound sodium, potassium, and calcium in the tissues. As the amount of acid is increased, more ammonia relatively and absolutely is eliminated with the acid. When the amount of acid introduced approaches in combining value the total available ammonia, the fixed cations of the tissues are again drawn upon. In general, it has been thought that an acidosis was not of dangerous import so long as the amount was so low as not to draw upon the firmly combined cations in the tissues. This is not, however, strictly true, for there is and under all circumstances must be some abstraction of sodium, potassium, and calcium from the tissues; the law of mass action demands that, it is impossible to conceive of acid in the body combining with ammonia alone. Therefore, long-continued acidosis must lead to dangerous abstraction of cations from the tissues, even though the ammonia elimination be high. Other things being equal, it is immaterial how the acid is introduced. Carnivora bear acid administration by the mouth better than herbivora, on account of the greater content of protein in the diet. If the diet of the carnivora be reduced in protein and the diet of the herbivora increased in protein, the difference in resistance to acidosis disappears. What has been said applies to organic acids as well as to mineral acids. Only organic acids that are incombustible in the body combine with ammonia to be eliminated in combination; otherwise the acid is burned, and the ammonia appears as urea.

From these facts it becomes apparent that increase of ammonia in the urine above the amount usually seen on a particular regimen of protein is to be referred to the existence of an acidosis. And in general we may measure the degree of an acidosis by estimation of the urinary ammonia. This is not strictly true, since as stated fixed cations are always withdrawn in some amount. But since the withdrawal of the fixed cations may in general be regarded as proportional to the acidosis, the estimation of urinary ammonia may still be used as a measurement of acidosis. And this gives to the estimation of urinary ammonia an especial importance in diabetes, in which prolonged acidosis is usually present, giving opportunity for a dangerous abstraction of cations from the important tissues of the body. 
We do not at present believe in the existence of a primary increase in the ammonia of the urine, independent of acidosis, an increase in ammonia due to primary failure of the formation of urea from ammonium carbonate. We regard the urea-forming function as always fully competent to convert all the available ammonium carbonate into urea. Beyond the normal amount, ammonia is eliminated only when withdrawn from urea formation by combination with non-combustible acid. Experiments temporarily excluding the liver from the circulation and resulting in pronounced atrophy of the organ have not been found to have been uniformly followed by high ammonia and low urea; and in any event acidosis has not been excluded. This point of view is obviously based upon the idea that urea is formed in the liver alone; whereas as a matter of experiment, urea is formed in apparently normal amounts in the body of a dog whose liver and kidneys have been shut out of the circulation. Studies in the urine in natural diseases of the liver indicate that in some cases of profound disease of the liver, and also in eclampsia, the ammonia may be high and the urea low. But here, too, acidosis has not been excluded. The writer has personally never seen a case of high ammonia content in urine without being able to demonstrate acid with which it was combined. Future investigations may overthrow this dictum, but at present all the data point in the one direction. The acids usually concerned are beta-oxy-butyric and aceto-acetic acid; but in some degenerations of the liver, lactic acid may appear in the urine combined with ammonia. In some conditions the nature of the acid has failed of elucidation.

Of the ammonia occurring naturally in the urine, what amount is brought out by acids, and what amount represent the direct elimination by the kidneys? When alkali is given in amounts sufficient to make the urine alkaline, ammonia is still present; as stated it requires high doses of alkali to render the urine ammonia-free. Theoretically, the ammonia present in the urine when the reaction of the urine is kept faintly alkaline by the administration of just sufficient alkali, would represent the direct elimination; the amounts over this and up to the figures observed in natural urines represent the fraction related to normal acidosis. The relations would naturally vary with the total nitrogen. In an approximate way, we may perhaps say that the direct elimination of ammonia may amount to from 0.1 to 0.2 gram per day. In other words, probably over half of the ammonia of normal urine has been withdrawn from the urea output by the normal presence of non-combustible acids of metabolic or dietetic origin.

\section{THE CATABOLISM OF SPECIAL AMINO-ACIDS}

The stated scheme of the catabolism of the different amino-acids, while of general application, is with a number of the amino-acids subject to qualitative and quantitative deviations that are of importance. These we will consider here. 
Glycocoll.-A hundred grams of mixed proteins, such as are to be found in an ordinary diet, will contain from 2 to 5 grams of glycocoll. Normally glycocoll is found in the bile in combination as glycocholate. It is frequently found free in normal urine, and is possibly a constant constituent of urine. It occurs in normal urine in combination with benzoic acid as hippuric acid. It can be recovered in the decomposition of the purins, and conversely the purins may be synthesized from glycocoll; but we do not regard either of these reactions as occurring in the body. It exists preformed in the muscle tissue of many invertebrates, but it is not present in the muscle of the higher animals except as a component amino-acid. Ox blood contains free glococoll; the blood of carnivora has not been tested.

The bound fractions in the urine are two: one an undefined combination that is easily split off by weak alkali; secondly, hippuric acid. By the administration of benzoic acid it is possible to show that much more glycocoll is eliminated thus combined than can be accounted for in the normal protein catabolism. It is clear that under such circumstances as the presence of large amounts of benzoic acid, glycocoll is synthesized. This amino-acid, it will be recalled, is in the body convertible into glucose. And conversely, its derivation from glucose might be anticipated. This appears all the more likely when it is recalled that the organism is known to be able to convert $\alpha$-ketonic acids into the corresponding $\alpha$-amino-acids, thus:

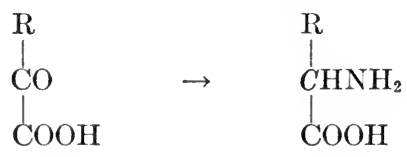

But the facts of elimination in animals receiving benzoic acid speak against this inference. If glycocoll were synthesized from sugar and ammonia, the total nitrogen of the urine would not be altered. As a matter of fact it is increased, and increased much more than the amount of nitrogen in the hippuric acid concerned. When the nitrogen fractions of the urine during the course of the experiment are determined, it is found that the increase in nitrogen output over and above the hippuric acid lies in the rest-fraction, it is not urea. From this two inferences seem justified: the glycocoll is derived from the disintegration of protein somewhere in the body; and this disintegration differs from the normal catabolism of protein, in that the major product is not urea, but more complex derivatives of amino-acids, if not aminoacids themselves. Benzoylated amino-acids (alanin, phenylalanin, leucin, valin, serin, aspartic acid, glutamic acid and ornithin) when administered parenterally are eliminated unchanged-not converted into hippuric acid. The body proteins that contain large amounts of glycocoll belong to the connective-tissue group, which one would most naturally expect to see built down. Beyond this there is but one alternative: glycocoll must be derived from amino-acids higher in the fatty acid scale, possibly in the kidney. How this could be done we do 
not know. Reactions of oxidation and cleavage, attacking the beta carbon, have been suggested. This may be illustrated for alanin:



Such a reaction is purely hypothetical. The animal forms glycocoll from other amino-acids, of that there is little doubt, but we do not know how.

The Aromatic Amino-acids.-These include phenylalanin, tyrosin, and tryptophan. It is the current teaching that these aromatic bodies when set free in the protein catabolism of the tissues are completely burned. All the aromatic acids and other combinations in the urine are held to have been resorbed from the intestinal tract. It is known that under certain circumstances the body ruptures the benzene ring and burns it, and this is what occurs in the cellular metabolism. In the intestinal tract, on the other hand, bacteria carry on different processes with the same amino-acids, the products of which on resorption into the body are not combustible, and are eliminated largely in conjugated form. This is held to apply to all the phenyls, indols, and even benzoic acid: all are held to have been derived from the intestinal tract, none from the tissue catabolism of protein. It is true that many substances that are commonly supposed to act as intestinal antiseptics reduce the aromatic bodies in the urine to a trace. It is true that the restriction of the diet to milk and meat reduces to a minimum the hippuric acid of the urine, since many fruits and vegetables contain benzoic acid in one or another state, and bacterial fermentation of vegetables sets free or forms still another fraction. It is certain that the stools always contain aromatic bodies derived by bacteria from the aromatic-containing amino-acids of the products of digestion. And, finally, aromatic amino-acids introduced into the organism are promptly burned, and do not reappear in the urine. It is, therefore, highly probable that the phenyl, indol, and benzoate-containing bodies in the urine are derived from the alimentary tract, and that the benzene of the amino-acids of protein of tissue catabolism is burned. The evidence for this view is strong, but cannot be regarded as conclusive. The conclusive demonstration would be furnished by benzene-free urine obtained from animals so reared as to have preserved the alimentary tract free of bacteria. In one instance, in guinea-pigs, in which animals have been reared with sterile alimentary tracts, traces of p-oxy-phenyl-acetic and p-oxy-phenyl-propionic acids were found in the urine, but no indol, phenol, kresol, or hippuric acid.

The steps in the rupture of the benzene ring and the reactions of combustion are not well known. The few facts we possess have been derived from the study of alcaptonuria. The substance termed alcapton (homogentisic acid) is dioxy-phenyl-acetic acid (hydroquinone acetic acid). When this substance is introduced into the body of a normal 
individual, it is burned. When introduced into the body of an alcaptonuric, it reappears in the urine in toto. The condition of alcaptonuria, which is a harmless pathological deflection often running in families, has been usually interpreted to be an instance of the checking of a series of reactions at the stage of an intermediary substance. It is assumed that in the normal individual the tyrosin and phenylalanin are burned through dioxyphenyl-acetic acid to the end products, with rupture of the benzene ring. In the individual with alcaptonuria, the reaction does not proceed farther than the stage of dioxyphenyl-acetic acid. This recalls the condition in diabetes, in which the combustion of the lower fatty acids is checked at the stages of b-oxy-butyric and diacetic acids. The relations in alcaptonuria may be illustrated in equations.


In the normal catabolism of dioxyphenyl-acetic acid, the ring is ruptured and both ring and aceto-acetic acid burned; in the alcaptonuric, this rupture of the ring cannot be accomplished and the compound is eliminated unchanged. There are but two points in the scheme that demands discussion. It is clear that in the intestine the tyrosin is converted first into p-oxy-phenyl-propionic acid and this into p-oxy-phenyl-acetic acid. Phenylalanin is built down in the same way in the intestine, and yields phenyl-acetic acid. Now phenylacetic acid will not yield dioxyphenyl-acetic acid in the alcaptonuric, so the processes must be different within the body. There is experimental evidence that phenylalanin is converted into the corresponding 
lactic acid, instead of into propionic acid; and the same may be held to occur with tyrosin. This has been indicated in the equations. The second point concerns the transfer of the acid group from the para position, since in the dioxyphenyl-acetic acid the two $\mathrm{OH}$ groups occupy the para position. This seems to be the rule in certain oxidations of the benzene ring. For example, when paracresol is oxidized by potassium persulphate in acid solution, homo-hydroquinon is formed:<smiles>CC(C)C1CCC(C(=O)O)CC1</smiles><smiles>CC1CCCC(C(=O)O)C1C(=O)O</smiles>

The alkyl group is here seen to have been shifted, just as in the case of the oxidations of the phenyl-lactic and the p-oxy-phenyl-lactic acid to dioxy-phenyl-lactic acid. It is of course possible that thep-oxy-phenyllactic acid is first converted into p-oxy-phenyl-acetic acid and this then converted into dioxy-phenyl-acetic acid; and the same thing would hold true for phenylalanin. But this is made improbable by the experimental fact that in the alcaptonuric, phenyl-acetic acid does not yield homogentisic acid.

Another formulation is, however, possible, and one that has both theoretical and experimental evidence in its favor. Tyrosin and phenylalanin, instead of passing into the stage of lactic acid, are assumed to pass into the state of pyruvic acid, then through an intermediary quinonoid stage into dioxyphenyl-acetic acid.

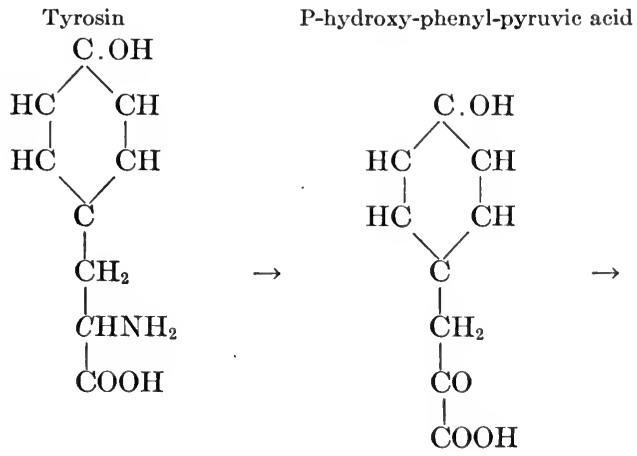

2-5-dihydroxy-phenyl-pyruvic acid<smiles>O=C(O)CC1CC(C(=O)O)CCC1C(=O)O</smiles>

Quinonoin stage



Aceto-acetic acid

Dioxy-phenyl acetic acid<smiles>O=C(O)CC1CCCC(C(=O)O)C1</smiles>

$\rightarrow \quad \begin{aligned} & \mathrm{CH}_{3} \\ & 1 \\ & \mathrm{CO} \\ & \quad \\ & \mathrm{CH}_{2} \\ & \mathrm{COOH}\end{aligned}$ 
Both these formulations have it in common that the stage of dioxyphenyl-acetic acid is normal, and that in the normal organism this homogentisic acid is converted into aceto-acetic acid and then oxidized, the remains of the split ring also burned. Under this point of view the defect of the subject with alcaptonuria lies in inability to complete the series of reactions; and the intermediate stage, dioxy-phenyl-acetic acid, is eliminated unchanged. At the same time it is obvious that the body might split directly the molecule of 2-5-dihydroxy-phenyl-pyruvic acid, with the formation of aceto-acetic acid; and from this point of view the formation of dioxy-phenyl-acetic acid could be considered an abnormal reaction.

Wholely inconsistent with this idea are recent observations to the effect that other simple derivatives of tyrosin and phenylalanin can be burned by the alcaptonuric, provided they are so constituted that they cannot pass through the quinonoid stage. Paramethylphenylalanin and paraoxymethylphenylalanin, and their corresponding pyruvic acids, do not form paraquinonoid derivatives, but are burned by the normal body and also by the alcaptonuric subject. This important observation suggests obviously that the formation of the quinonoid stage and of homogentisic acid are not normal but abnormal reactions, and that the normal reaction follows another course. As formulated to meet the experimental facts, the benzene ring instead of passing into the quinonoid state is split directly, two of the carbons of the ring joining with two of the carbons of the pyruvic acid to form acetoacetic acid. This interpretation has been confirmed by the successful formation of aceto-acetic from histidin. The illustration for tyrosin applies also to phenylalanin.

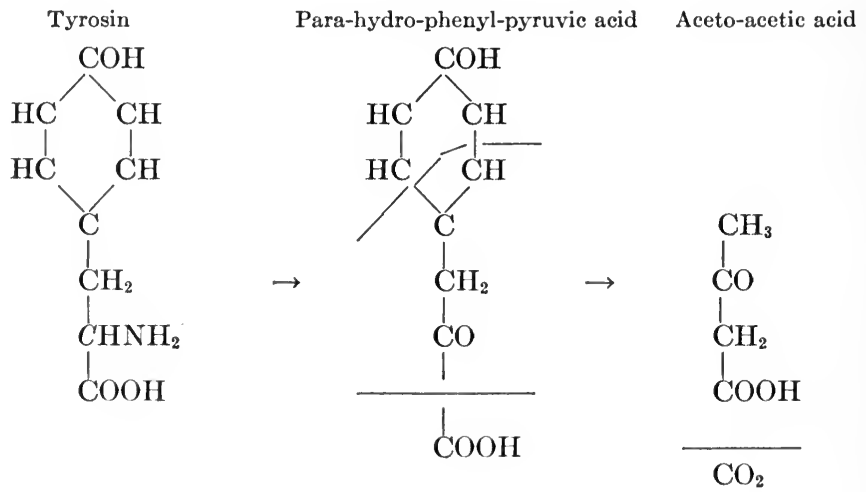

From this point of view the abnormality in alcaptonuria consists in the transfer of the para-hydroxy-phenyl-pyruvic acid into 2-5-dihydroxyphenyl-pyruvic acid, instead of direct cleavage of the benzene ring with the formation of aceto-acetic acid. In addition, however, the alcaptonuric has lost the normal power of burning homogentisic acid. From this point of view, in a word, the normal organism does not form homo- 
gentisic acid from tyrosin but has the power of burning it, while the alcaptonuric does form the substance but has not the power of burning it.

In the individual with alcaptonuria it seems possible that a portion of the dioxphenyl-acetic acid may be derived from partially oxidized tyrosin and phenylalanin absorbed from the alimentary tract. When an excess of protein is ingested, the elimination of alcapton rises; this may be due both to tyrosin and phenylalanin from the protein catabolism and from the alimentary tract. Sometimes the amount of dioxphenyl-acetic acid is more than could have been expected from the protein catabolism, as judged from the nitrogen. Investigations into these cases have indicated that the subjects were consuming proteins rich in aromatic acids. The ratio of the dioxyphenyl-acetic acid to the nitrogen of the urine on mixed diet is usually $4-5: 10$, but in the case of a milk diet, for example, it will be higher. The site of the reaction of the combustion of the aromatic amino-acids is supposed to be the liver.

Epinephrin, the active principle of the adrenal gland, is apparently derived from tyrosin, a transformation suggested by the tyrosinase reaction of the adrenal substance.


Tryptophan. - Concerning the catabolism of tryptophan we know little. The urine of dogs contains normally a derivative of tryptophan, kynurenic acid. This substance is $;$-oxy- $\beta$-chinolin-carboxylic acid. The equations of the two substances are as follows:
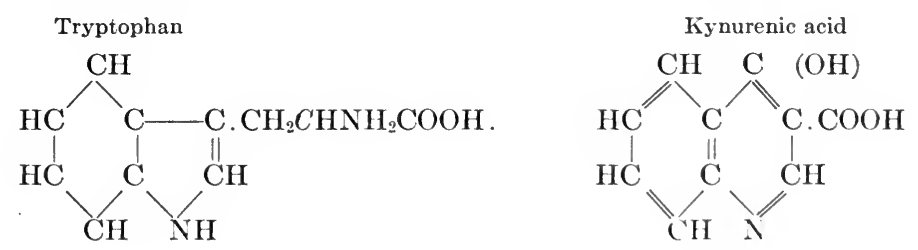

The path of transition is not yet known. The elimination of kynurenic acid in the dog runs parallel to the protein catabolism; but incomplete proteins that do not contain tryptophan, like gelatin, do not provoke elimination. If tryptophan be administered it is recovered in the urine in the state of kynurenic acid. The rabbit, like man, does not 
normally eliminate kynurenic acid and is able to oxidize small amounts of tryptophan; but when large amounts are ingested, a fraction appears as kynurenic acid. This has suggested that normally, in man and in most animals, the tryptophan derived from the catabolism of protein in the tissues is burned through kynurenic acid as the intermediary stage; this reaction, however, in the dog being halted at the intermediary stage. There is as yet no proof that the destruction of tryptophan in the human body is thus accomplished.

Cystin.- The combustion of cystin in the body is always incomplete. There is in the fraction known as the neutral sulphur of the urine unoxidized sulphur that must in large part, if not entirely, have proceeded from cystin. Normal urine may contain a trace of cystin, or cystein. It occurs in the urine following phosphorus poisoning. And conversely, in cystinuria, leucin and tyrosin are often found in the urine. The catabolism of cystin leads to three directions of elimination: in the taurin of the bile; in the neutral sulphur of the urine (part of which comes from taurin possibly, following its resorption in the intestine); and in the sulphates of the urine. There is a small amount of neutral sulphur in the perspiration, but this does not proceed from the cystin derived from the catabolism of protein. Cystinuria is a rare metabolic anomaly, in which the urine, blood and tissues contain cystin, often in large amounts. Leucin, tyrosin and lysin may be present in the urine. Cystin is probably found in the stools no oftener than in normal subjects. The cystin is identical with the cystin obtained in the acid hydrolysis of protein. The condition is often congenital and runs in families. The cystin comes from the protein catabolism, not from the alimentary tract. As a rule, the amount of cystin (plus the other neutral sulphur) in the urine of a typical case represents the full sulphur content of the protein of the diet. Nevertheless, if cystin be given by the mouth, it may be burned, partially or completely. The fraction of neutral sulphur in the urine persists beside the cystin, and from this it is obvious that the cystin is not formed at the expense of other neutral sulphur. In some cases the defect is not complete, and oxidized sulphur (sulphate) is to be found in the urine with the cystin.

The defect consists obviously in a failure of cleavage; the di-cystein (cystin) is not split, and the cystein is, therefore, not available for oxidation. The reason why cystin administered by the mouth is burned lies probably in the fact that it is split in the alimentary tract by bacteria and deaminizated in the intestinal wall; the resorbed products are combustible while the cystin is not combustible.




In cystinuria the amount of cystin in the urine is quite independent of the diet, persists during fasting, and is as stated not exaggerated by the ingestion of cystin. If benzoyl bromide be administered to a normal individual it will be eliminated combined with cystin-in other words, provoke a cystinuria. This is to be explained as due to the fact that the cystin combined with benzoyl resists the processes of combustion. It is not known whether in idiopathic cystinuria any substance abstracts the cystin from the sulphur catabolism in a similar manner.

It would be instructive to study the bile of a case of cystinuria. In this way we could test the hypothesis that the taurin is derived solely from the cystin of the disintegrated erythrocytes, and not from the cystin of the common protein catabolism. 'The occasional occurrence of diamins in the urine of cystinuria is probably not of direct relationship.

Ornithin.-This amino-acid, derived by cleavage of arginin in the liver, is normally burned. In rare cases, usually in connection with cystinuria though not in all cases thereof, there appears in the urine a diamin that has been derived from ornithin, tetramethylendiamin (putrescin). With this diamin is usually found pentamethylendiamin (cadaverin) derived from lysin. It is not invariable to find the two diamins in cystinuria; nor is it only in cases of cystinuria that diaminuria is seen. In these cases the diamins are formed from ornithin and lysin derived from the protein catabolism. In dysentery, cholera and other severe forms of enteritis, these same diamins may be found in the urine, resorbed from the intestinal tract where they were formed from lysin and ornithin by bacteria. The chemical reaction is the same in tissues or carried on by bacteria, it is an enzymic reduction with splitting off of carbon dioxid-a reaction very similar to the formation of acetone from diacetic acid or the formation of taurin from cysteinic acid.
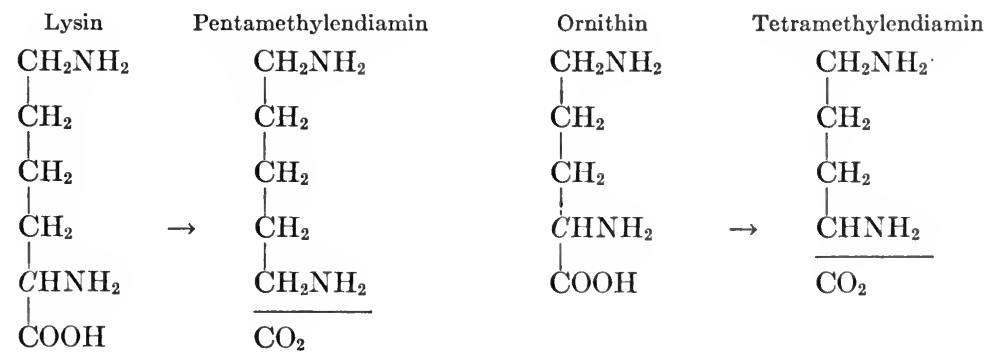

It is certain that these are qualitative deflections, it cannot be assumed that normally these amino-acids are burned through these diamins. When arginin, ornithin and lysin are introduced into the body of the cystinuric, they are eliminated in the form of the stated diamins. When the diamins are introduced into the normal body they are eliminated 
unchanged. The reaction is held to occur in the liver. The association with cystinuria is very obscure, though as stated all cystinurics have not diaminuria. The reactions of cystin and of lysin and ornithin cannot touch each other at any known point. When diaminuria occurs with cystinuria, leucin, tyrosin and lysin may be present in the urine; and evidently there is both qualitative and quantitative desturbance in the catabolism of amino-acids. Enzymic reduction with splitting off of $\mathrm{CO}_{2}$ is a normal reaction for cystein, an abnormal reaction for lysin and ornithin. One might fancy that the relationship of the cystin to the ornithin and lysin might extend back into the protein molecule; but they are not related in the protein molecule, cystin being in large part early and easily split off, lysin and arginin late.

Histidin.-When histidin is administered to dogs, it is burned and the nitrogen eliminated as urea. Recent studies tend to show that the catabolism is accomplished and the ring ruptured as indicated in the following equation, aceto-acetic acid being formed, later in its turn to suffer oxidation.

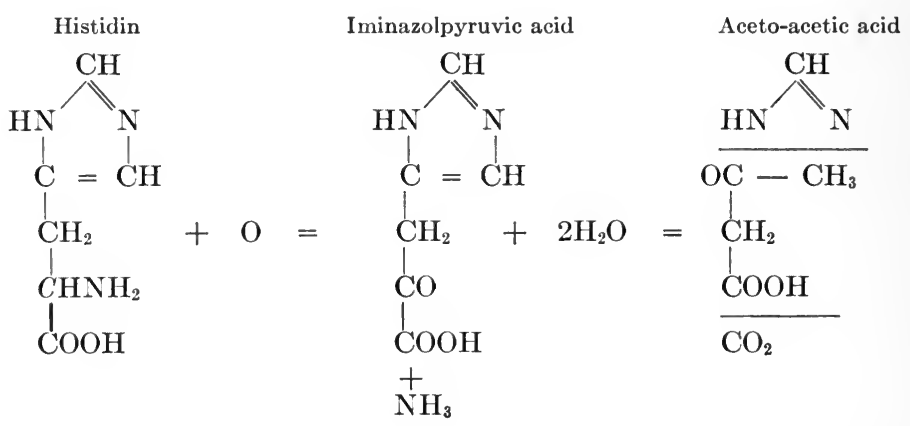

The N-CH-NH group isolated by the rupture of the ring would form urea, as would also the ammonia after combination with the carbon dioxid.

\section{THE REST-NITROGEN}

The partition of nitrogen in the urine is effected by the quantitative determination of urea, ammonia, creatinin, and the purins. When the sum of the nitrogens of these substances is subtracted from the total nitrogen of the urine, the difference is termed the rest-nitrogen. This fraction of nitrogen is only fairly constant in health, but is in particular more or less independent of the total nitrogen input. In this rest fraction are included many different substances, and naturally the amount may be influenced in many different ways. For illustration, hippuric acid is included in the rest-nitrogen and the urine of vegetarians and fruitarians is high in rest-nitrogen on account of the large amounts of hippuric acid in their urine. It is, of course, without 
clear purpose to group these different substances, derived from many sources, under one fraction, since it cannot be a variable in itself. The group is really set apart because of ignorance of many of the constituents.

The amount of rest-nitrogen may vary from 0.4 or even less to 1.5 gram N per day. Since many constituents of this fraction are derived from the common protein, the rest-nitrogen on a milk diet follows roughly the urea.

The following substances are known to be included in the rest-nitrogen: hippuric acid; traces of urinary mucin; traces of diamins; traces of nitrogenous phosphatids; thio-nitrogenous substances derived in the more or less incomplete oxidation of the sulphur-containing cystin, (included also in the fraction of neutral sulphur); traces of mon-aminoacids, glycocoll being most prominent; traces of polypeptid-like substances including oxy-proteic acid, all-oxy-proteic acid, aut-oxyproteic acid and uroferric acid; methylguanidin; allantoin, urochrome, urobilin, hematoporphyrin, and uroerythrin; and other still less defined nitrogen-containing substances. Obviously this group as a group has no meaning in metabolism. The individual substances, of course, have a meaning in metabolism, since they are either end products, eliminated intermediary products, or bacterial products. Individually they are considered in their appropriate connections; as a group they possess no entity.

\section{THE ELIMINATION OF THE END PRODUCTS OF PROTEIN CATABOLISM}

Leaving aside the rest-nitrogen, we may regard the ammonia and urea as representing the output of the protein catabolism. The larger fraction goes out through the urine. A certain fraction is eliminated in the feces. The perspiration contains under ordinary conditions of unconscious perspiration from 200 to 300 milligrams of nitrogen per day. With free perspiration that may rise to as high as a gram per day. It is clear, therefore, that in experiments with nitrogen balance, particularly if the nitrogen input is low and approximating the endogenous demands, the fraction of nitrogen eliminated through the skin is not to be neglected. The growth of the hair and nails is a further loss. A man may lose as much as 100 milligrams of nitrogen per day in the growth of the hair. As stated elsewhere, the nitrogen content of the feces in starvation may run from 300 to 500 milligrams of nitrogen per day. Adding together these three fractions (perspiration, intestinal excrementation, and growth of hair and nails) we are surprised to realize that as much as a gram of nitrogen may be eliminated per day in the extra-renal output, and this mass must always be allowed for in the interpretation of the facts of nitrogenous metabolism.

When the urea-ammonia elimination is followed in hourly estima- 
tions in a subject on an ordinary mixed diet containing more or less exogenous protein, a wave of elimination is observed that reaches its maximum in from five to seven hours after the ingestion of the meal. The more excessive the ingestion of protein, the higher the wave and the more prolonged the downward stroke of the curve; with massive ingestions the time required to reach the crest of the wave may be deferred a couple of hours. If the meal contain considerable fluid, a rise may be noted about two hours after the ingestion of the meal. This is regarded as derived from stored nitrogen, washed out by the fluid; and this wave can be provoked by the ingestion of water alone. The second wave, about the fifth hour, represents the nitrogen of the exogenous catabolism of the meal. With the usual hours of eating, it is clear that the bottom of the curve is not reached before the ingestion of the following meal. Under these circumstances the lowest point of the curve is not found until early on the following morning. It is the exogenous protein that is largely responsible for the sharp ascent of the curve. As the protein input approximates more and more the actual needs of the body, the curve of elimination approaches the straight line, with only a slight rise after meals and a slight depression in the early morning. It is a fair assumption that the slight rise then noted following meals is the result of the catabolism of the amino-acids of the diet that are superfluous in the synthesis of the blood proteins. If, therefore, a dog were fed the exact amount of protein required by his tissues and this input protein were in the form of canine blood serum, we would expect the curve of nitrogen elimination to be a practically straight line just as the curve in starvation is a practically straight line. A contemplation of the curves of nitrogenous elimination emphasizes impressively the difference between exogenous and endogenous protein catabolism, and leads to the conviction that the exogenous protein catabolism is of about as much value to the body as is the blowing-off of steam to a locomotive.

The elimination of nitrogen in the twenty-four hours of a day represents in an approximate way the input of that day. This balance was once regarded as a rigid sequence of cause and effect. That it is a rigid relation over a long time under controlled conditions is true; but it does not hold rigidly for short periods. In ordinary balance experiments, the urine is collected from 8 A.m. to 8 A.m., so that the body has fourteen hours in which to complete the catabolism of the protein of the evening meal. But the day-after-day figures for the nitrogen output will be found to fluctuate rather markedly in a subject on a constant input. If the daily input be 15 grams of nitrogen, the output may fluctuate from 13 to 17 grams; the fluctuations are irregular but in the long run compensatory. For several days the daily balance may be perfect; then for a few days the output may lag behind several grams per day; following this for successive days the output will be several grams in excess of the input. 'Temporary retention is the cause of this fluctuation; there is a lagging, followed by a sweeping-out until the 
balance is attained. Occasionally this may involve as much as onefourth of the nitrogen input. It is different in different individuals, and is most marked in subjects on a liberal input of protein. The fluctuations have sometimes a relation to the total volume of the urine (tending to rise with polyuria), but none to any apparent factor in digestion or mode of life.

It is important to know whether this lagging is in the catabolism or in the elimination. If such lags occur in the protein catabolism, we might expect more marked lags to occur under pathological conditions. If the lags are located in the function of elimination, we might expect more marked lags to occur in pathological conditions involving especially the renal system. There are today no data upon which a decision of this question may be based. If a lag in the protein catabolism occurred prior to the stage of deaminization, we might expect, under very careful conditions of experimentation, to detect it by the estimation of the respiratory quotient. The more natural assumption a priori is that the lag is in the elimination. But when we attempt to test this interpretation in practice, it is very doubtful if it proves valid. We know of three conditions in which similar lags occur frequently. In protein overfeeding, the nitrogen output may fall behind the input. Some times, particularly in young adults, this retention is permanent, and corresponds to a true retention in flesh. But more often, when the overfeeding is discontinued the retained nitrogen is gradually eliminated in the urine, so that after a number of days the extra protein of the input is balanced by the excess nitrogen of the urine. Under these circumstances, was the lag in the catabolism or in the elimination?. The amount of carbohydrate in the diet has a definite effect in the production of nitrogen lag. When glycocoll or asparagin are administered to a dog, it can be shown that the elimination of the nitrogen is definitely retarded, so much so as to have led to the suggestion that possibly amino-sugars were formed under such circumstances. In chronic nephritis, apart from any visible dropsy (as in arteriosclerotic nephritis with polyuria), one may observe a lag in the nitrogen output extending over a number of days, followed by a sweeping-out. Here the lag is independent of the input. Was the lag in the catabolism of protein or in the elimination? In the continued fevers, best seen perhaps in typhoid fever, the patient usually on a low protein input may appear to be in nitrogen equilibrium during the febrile period of the disease. Following the defervescence of the fever, in association with a post-febrile diuresis or independent of it, a large amount of urea may be eliminated. Was this stored in the body in defect of elimination, was uncatabolized protein stored in the body during the febrile period; or was there a large post-febrile catabolism of tissue protein? It seems clear, unsatisfactory as it may be, that no explanation will fit all these various sets of circumstances and the lags that are observed with normal subjects on normal diets. When one considers the great solubility of urea and the ease with which the 
kidney eliminates urea administered parenterally, it seems far-fetched to regard these lags as due to defective elimination. When, on the other hand, one contemplates the almost boundless power of catabolism of common protein possessed by the tissues, including the formation of urea from ammonium salts, it seems far-fetched to attribute the lags to defective catabolism. States of storage of either protein, aminoacids or of urea are not easily assumed, though possible-protein presenting the least difficulty in this regard. The occurrence of dropsy cannot, of course, be excluded, since the body can retain several liters of water without the slightest external sign, and it is very difficult by the control of the body weight or measurements of water input and output to even approximately establish a water balance. But it does not seem possible to assume dropsy, physiological or pathological, for these retentions. A lag is surely present in either catabolism or in elimination.

The following scheme illustrates the main facts of the protein metabolism, including exogenous protein.

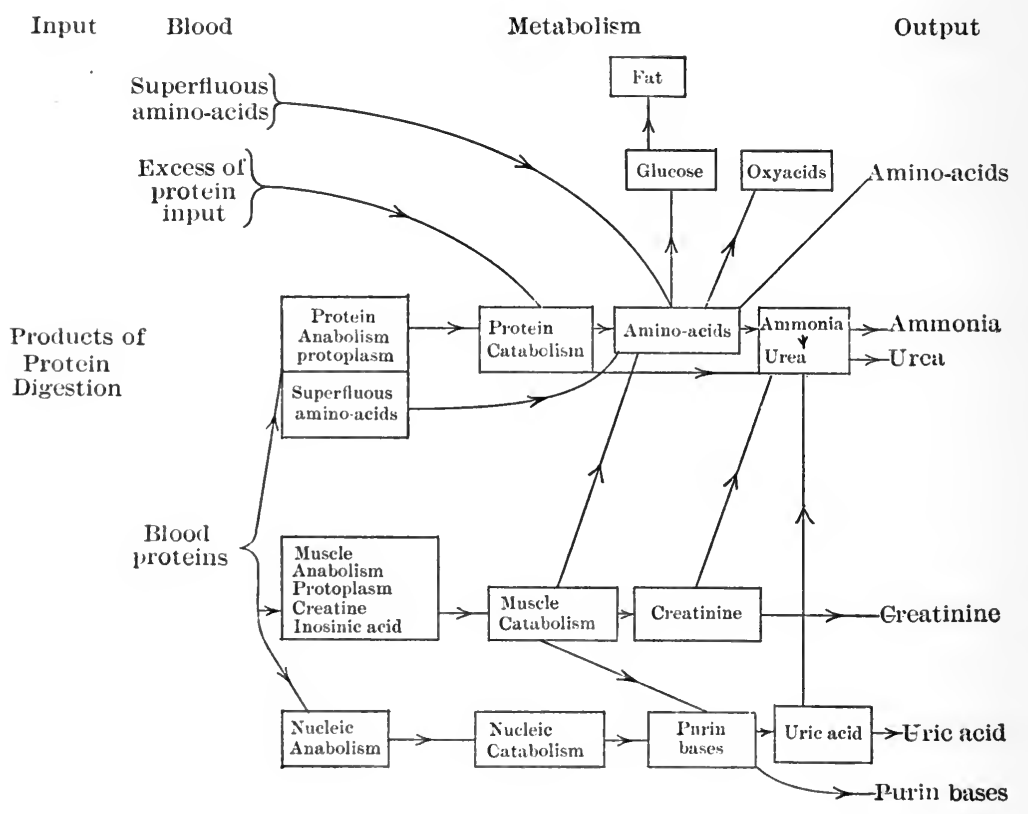

\section{THE METABOLISM OF SULPHUR}

On account of its intimate relations to the protein metabolism, the metabolism of sulphur possesses peculiar interest. Despite the fact that we are dealing with an inorganic substance, we are still far from 
a comprehensive view of the details of the metabolism of sulphur. Sulphur exists in the diet in both organically combined and in inorganic state. So far as known, the inorganic sulphur of the diet has no importance for the processes of metabolism. Inorganic sulphur cannot be utilized in the body for the synthesis of the sulphur combined in protein, this must be ingested preformed. Sulphur exists in organic combination in some of the fats of foods, but so far as known this sulphur is completely oxidized in the body, and is not utilized for organic combination. From all the available data, the only sulphur required in the diet is the sulphur combined in protein; the sulphur combined in fats is oxidized and the inorganic sulphur merely passes through the system. The sulphur combined in protein is largely if not entirely in the di-thio-amino-acid, cystin. This substance is absolutely indispensable to nutrition, and it cannot be formed in the body of the higher animal. A normal and competent protein ration must, therefore, include such proteins as contain the sulphur-bearing amino-acid in ample quantity. When the proteins are digested the cystin is resorbed unchanged, to become an integral component of the different proteins synthesized in the reactions of protein anabolism. From cystin apparently are derived by complex synthesis the sulphured lipoids that are present in the central nervous system, in the bile and in the secretions of the cutaneous system.

The outlets of sulphur are three: in the urine, in the stools, and in the cutaneous secretions. The amount of sulphur eliminated through the skin is considerable, about 30 milligrams of sulphur per day. The sulphur of the stools consists of three fractions apart from ingested inorganic salts; the sulphur compounds of the bile; the sulphur-containing mucin of the alimentary secretions; and the sulphur of the undigested or unresorbed protein of the diet, more or less modified by bacterial action. To these must be added the sulphur of the bacterial bodies. It is not analytically possible to separate these fractions. The sulphur content of the stools varies from 0.2 to $0.3 \mathrm{gm}$. per day. The sulphur compounds of the bile were described under that heading. It is not known to what extent these substances are discharged in the feces, to what extent they are digested or acted upon by bacteria in the intestine, or in how far they may be resorbed.

The sulphur fractions of the urine are three: neutral sulphur, preformed sulphates, and ethereal or conjugated sulphates. The last is really only a portion of the preformed sulphates, conjugated with phenols, cresols, indols, skatols and their derivatives. Whatever amount of these substances (the products of bacterial action on aminoacids in the intestine) is resorbed, this is in the liver conjugated with sulphuric acid, the amount of the preformed sulphates being by that much reduced. It is not possible for so much of these aromatic bodies to be formed as to entirely appropriate the sulphate of the same period. Metabolically the ethereal sulphates have the same meaning as the preformed sulphates. In general, the oxidized sulphur corresponds to 
the urea nitrogen, or better, to that fraction of the urea nitrogen that is derived from the catabolism of exogenous protein. It is tempting to relate this fraction of sulphur solely to the exogenous protein, but the data are not yet sufficiently extensive to warrant this conclusion.

The neutral sulphur is a constant for each individual, being closely parallel to the nitrogen of the endogenous catabolism of protein, independent of the exogenous catabolism of protein. Here, again, it is tempting to relate this fraction solely to the endogenous protein, but the analytical data are too meagre. As an hypothesis, however, it has proper claims to our attention.

Theoretically there should be a balance in the sulphur metabolism. Analytically it is not possible to establish such a balance. This is due to difficulties in determining the sulphur eliminated by the skin, and to inaccuracies in the methods used for the estimation of the total sulphur of the foodstuffs and feces.

Our knowledge of pathological variations in the sulphur metabolism is very limited and fragmentary. Cystinuria, an interesting anomaly of the protein catabolism, has been described under that heading. 


\section{CHAPTER VII}

\section{METABOLISM OF CREATIN-CREATININ AND OF PURIN}

\section{THE METABOLISM OF CREATIN-CREATININ}

Creatin is methylguanidin-acetic acid. The structures of guanidin, of creatin, and of its anhydrid creatinin are as follows:

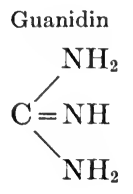



Creatinin

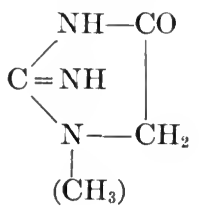

The relationship of guanidin to urea is obvious:<smiles>NC(N)=O</smiles><smiles>NC=C(N)N</smiles>

Urea is easily formed from guanidin by oxidation, it is indeed from a guanidin rest that urea is formed from arginin by arginase.

The subject matter of this metabolism is in a state of confusion, owing to numerous contradictions in work done with different or even with the same methods, and a survey of the metabolism is very difficult. To the author it seems that the following presentation contains the best interpretation of the most clearly established or least contradicted findings.

Elimination of Creatinin.-The elimination of creatinin, as related to its formation, is for each individual a constant related to the mass of muscular tissue, representing the coefficient of muscular metabolism; independent of the formation of urea (into which it may be converted) or of the partition of urinary nitrogen; independent of the endogenous or exogenous catabolism of protein; independent of the protein input when flesh is excluded. If an animal be fed a nitrogen-free diet containing carbohydrate and salts in proper amounts, the urinary nitrogen will after a time maintain a constancy in output and partition which represents the minimal endogenous nitrogenous catabolism. Under such circumstances (tests in swine have given very clear results), the 
nitrogen output varies from 0.025 to 0.035 gram nitrogen per kilo per day. About 18 per cent. of this is creatinin nitrogen (endogenous creatinin), and the figure is very constant; urea nitrogen is about 60 per cent.

There is this endogenous and also a possible exogenous fraction of creatinin in the urine, and therefore an endogenous and an exogenous metabolism. The endogenous metabolism of these substances is an especial cellular metabolism, localized in the muscles. This is made clear by its independence of conditions of increased tissue catabolism outside of the muscles, as in the exaggeration of hepatic catabolism that occurs in starving dogs poisoned with phosphorus. The exogenous fraction is derived from flesh in the diet. It is not derived from the protein of the diet, but from the flesh of the diet. Meat contains one or two parts of creatin in the thousand. Meat extracts contain combined creatin. On cooking, creatin is set free and some creatinin is formed. It is these so-called extractive elements that form the exogenous creatinin fraction. Otherwise, the creatinin is independent of the protein of the diet; it is one of the most constant eliminations, and in every way behaves like the end product of a specific metabolism, just as do the purins. There are moderate variations in the creatinin outputs of different individuals; but in the same individual, if exogenous creatinin be excluded, the elimination is remarkably constant (about 0.02 gram per kilo) and independent of the common protein metabolism.

Derivation of Creatin.-The creatin exists in the muscles in a nondialyzable state of combination, in some complex in the muscle plasma. Whence is this creatin derived? When one realizes that the sole preformed guanidin, known at least, is in the molecule of arginin and that this is converted into urea by arginase, one is led to regard the presence of creatin in the muscle plasma as an act of synthesis. Regarded in this way, we would term creatin a special anabolic product of the muscle cell, formed de novo from protein by synthesis and deposited in the muscle plasma. In what condition of combination creatin exists in the muscle plasma is not known. A complex recently isolated from meat extract offers a suggestion in this direction. This substance, termed vitiatin, has the composition:

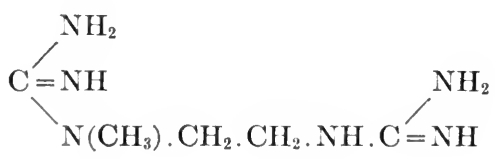

This is obviously a combination of guanidin with amino-acid, and suggests that in muscle protein there is a special polypeptid-like combination of creatin with amino-acid. It is a striking fact that guanidin (creatinin) is recovered most largely in the autolysis of muscle.

What are we to regard as the mother substance of creatin? The amino-acids would most naturally be looked upon as the probable 
source. Guanidin acetic acid is in the rabbit converted into creatin by the addition of a methyl group. This observation, however, does not help us much, for we are not able to trace the way from aminoacid to guanidin. Another view is that creatin is formed from creatinin in the liver, and then conveyed to the muscles. This hypothesis has little experimental evidence in its favor, though there is evidence tending to show that the relation creatin $\langle=>$ creatinin is reversible. In favor of this view may be also the fact that when creatin is administered to an animal or man, it is partly eliminated and partly retained. By retained one could understand conveyed to the muscle (as a food), just as the creatin assumed to have been synthesized from creatinin in the liver would be conveyed to the muscles. This explanation in any event only puts the problem back one step, for whence is the creatinin derived? We are not dependent on exogenous creatinin. If creatinin be the parent substance of creatin, what is the parent substance of creatinin? To the writer, however, the derivation of creatin from amino-acids seems much the more reasonable assumption.

The creatin in the muscle plasma is therefore regarded as the parent substance of the creatinin of the urine. This conversion we regard as due to ferment action, representing a special catabolism of the muscle cell. Autolytic experiments have made such formation of creatinin from creatin in muscle very probable. This formation of creatinin from creatin is a part of the life of the muscle cell, not a result of its function of contraction. Normal muscular movements are not associated with any increase in the elimination of creatinin. Only when the muscle is untrained or the exertion is excessive and accomplished without food, is the elimination of creatinin increased by muscular movements. The heart beating in artificial solutions, however, gives off creatinin to the solution. Electrical tonus, rigor caloris, and rigor mortis result in increased formation of creatin-creatinin.

Administration of Creatin.-When creatin is administered it is partly retained (as food or destroyed), and partly eliminated unchanged; a small amount is converted into creatinin. 'The result of this experiment need not speak against the derivation of creatinin from creatin in the muscle cell. It is possible that this is a special catabolism of the muscle cell, that creatin free in the circulating fluids does not meet the particular conditions necessary for this reaction and present only in the muscle cell. It is clear that this experiment is one in which a positive result proves but a negative result does not disprove. 'Tests by administration of creatin by mouth are not to be trusted, as creatin is subject to bacterial disintegration in the alimentary tract. Parenternal introduction alone is to be relied upon, and the majority of tests with this method have given a positive result, a certain fraction of creatin appears as creatinin. When creatinin is introduced, it is mostly eliminated unchanged, a small fraction is apparently converted into urea.

The daily output of creatinin may rarely be as low as 1 gram or as 
high as 2 grams; average figures run from 1.3 to 1.7 for different individuals. The values are naturally less fluctuating when the exogenous creatinin is excluded, as this is largely eliminated unchanged. In one individual it is common to see the elimination of creatinin (endogenous creatinin) vary no more than 50 milligrams from day to day. Creatin is not usually present in the urine. In birds creatin replaces creatinin in the urine. Creatin is normally present in the urine of sucklings and also of children. At what age of the child it ceases to appear and whether its occurrence is at all dependent on the diet, must be determined by future investigations.

Creatinin Metabolism, a Special Metabolism.-The cardinal feature of this theory of the metabolism lies in the definition of the creatinin metabolism as a specific metabolism. Just as we believe that purin and pyrimidin are synthesized from protein and later bound in the molecule of nuclein, from thence on to constitute a specific metabolism, independent of the metabolism of common protein; so we assume that creatin is synthesized from protein, directly or indirectly, and combined in the muscle plasma, from thence on to constitute a specific metabolism, independent of the common protein metabolism. Here we have limited the rôle of creatin to the muscular system. Possibly this may be a mistake. It is true that other cells than muscle contain traces of creatin; and cells other than muscle will form creatinin from creatin on autolysis. But the trace of creatin in other cells is so small, contrasted with the amounts present in the muscles, that it seems certain that we must accord to the muscular system a particular importance in this metabolism, if not the exclusive rôle. Future investigations will determine this relation.

Creatinin an Endogenous Source of Urea.-Creatinin is one of the endogenous sources of urea. It is, however, very difficult to form an estimate of the amount thus transformed into urea. No direct method is available. If we administer to a man an amount of creatinin equal to the maximum amount in the urine, 2 grams, it will be found that from three-fourths to four-fifths will be eliminated unchanged, the balance will apparently have been converted into urea. 0.4 gram of creatinin corresponds to 0.36 urea; while this is a large portion of the creatinin it is a small fraction of urea. Such an assumption, resting upon the supposition that the reaction with a certain amount of a substance administered by the mouth will be the same as though that substance had originated in the intermediary metabolism, is, of course, very insecure. But it is the only indirect way of attempting to form an idea of the formation of urea from creatinin in the body. The constancy of urinary creatinin indicates that whatever the degree of transformation into urea, this and the formation of creatinin from creatin must both be regarded as processes of striking uniformity.

The urine contains traces of methylguanidin and dimethylguanidin, that are to be attributed to the catabolism of creatinin (or creatin) either in the metabolism or in the alimentary tract. 
Pathological Variations of. Metabolism.-The pathological variations in the metabolism of creatin and creatinin have lately attracted much attention, and are unquestionably of great importance. These variations are in one of two directions: in the direction of excess or reduction in the elimination of creatinin, creatin being absent; or in the elimination of creatin at the expense, so to speak, of creatinin. The data bearing on the increase or decrease in the total elimination of creatinin in conditions of disease are more or less dubious on account of the fact that the diets have usually not been controlled and exogenous creatin and creatinin excluded. Nevertheless, it is probable that the total elimination may be increased under certain conditions of rapid degeneration and wasting of the muscular substance. Such an occurrence with high fever or following prolonged exposure to high external temperature may be attributed to muscular degeneration. On the contrary, late in the progressive muscular dystrophies, the elimination seems to be lowered. It should be pointed out that estimations in states of disease must reveal striking deviations, as individualities in creatinin elimination are rather marked. It must also be determined that the diet is creatin-creatinin-free, and contains an ample input of carbohydrate as well as protein. The weight of the individual must likewise be taken into account, since, as a rule, the elimination of creatinin is proportional to the muscular development or muscular stature of the individual, fat and thin subjects presenting lower elimination.

More interesting, at present at least, are the findings of creatin in the urine in states of disease. In accordance with the interpretation of this metabolism here adopted, the appearance of creatin is usually to be regarded as an instance of arrested transformation; instead of being converted into creatinin, creatin issues unchanged. It is now definitely known that the muscle cells may synthesize creatin in excess; there is in fact evidence tending to show that in starvation the formation of creatin-creatinin is always above normal. But if such overproduction of creatin does occur also in pathological states, future studies will need to fix such occurrence; we shall here assume the appearance of creatin in disease to be at the expense of creatinin. In three groups of conditions especially is creatin to be found in the urine. (a) In inanitions, no matter what the cause, be it starvation, chronic infectious disease, neoplasmic cachexia, intractable vomiting as in pregnancy, exophthalmic goitre, etc. The explanation to be offered in these cases is that muscle is being catabolized to support the glandular and nervous systems. If the carbohydrate input be normal in these cases, creatin may not appear in the urine; if it be deficient, creatin does appear. (b) In acute and rapid degeneration of muscle tissue, such as is to be seen in muscular neoplasms, in dystrophies, in connection with myelitis, following prolonged exposure to extreme heat, during the involution of the uterus in the puerperium, in short, whenever muscle undergoes rapid disintegration. The explanation here to be offered is that it is in the functionating muscle cell that the conversion 
of creatin to creatinin occurs; when the cells degenerate, the creatin is set free and passes in the circulation to the kidneys. (c) In connection with suboxidation of glucose. Recent evidence tends to indicate that the combustion of glucose bears to the conversion of creatin into creatinin the same relation it often bears to the combustion of butyric acid; the reaction is in some way rendered incomplete without it. When there is little sugar burned (either because the body has no glucose to burn or cannot burn it) creatin is eliminated, just as are the ketonic acids. In diabetes and in phloridzin poisoning creatin appears in the urine. Obviously, many of the above instances cited under $(a)$ and (b) may in reality have been due to the absence of sugar combustion. It may be experimentally shown that creatin elimination in nitrogen starvation is checked by the administration of carbohydrate, but continues with the administration of an isodynamic ration of fat. Future investigations will have to determine the exact relations in these three groups of cases, both as to facts and as to their interpretation. In the literature much emphasis has been laid upon the occurrence if creatin in organic diseases of the liver, as in carcinoma, cirrhosis, chloroform degeneration, phosphorus poisoning and in eclampsia. These cases might be ranged under one of the three groups; possibly it may be through disturbances in the glycogenetic function of the liver that the occurrence of unconverted creatin is to be explained. The non-importance of the liver itself in the occurrence of creatin in the urine is shown by the fact that dogs with Eck fistula react just as do normal dogs, which is scarcely to be reconciled with the idea of a predominating influence on the part of that organ. The recently published statement that creatin is a normal constituent of the urine during pregnancy demands confirmation under rigid conditions of control.

\section{THE PURIN METABOLISM}

Structure of Nucleic Acid.-The protein metabolism may be defined in general terms as the metabolism of protoplasm. The nucleic metabolism we regard in the same general way as the metabolism of the nucleus. The nucleus contains, of course, protoplasmic protein: and nucleoproteins exist outside of nuclei. But it is especially with the basic substances of the nucleus, the chromatin, that the nucleic metabolism is concerned. This has a totally different structure and composition: its metabolism is a function sui generis, always to be regarded as independent of the metabolism of common protoplasm. While many of the details of the nucleic metabolism have been long known, it has been but lately that our knowledge of the chemical structure of nucleic acid has been developed to such a point as to enable one to make a survey of the field.

The nucleoproteins are perhaps the most widespread of the compound or conjugated proteins. Existing in every organ and tissue, they vary widely, depending upon differences in the nucleic fraction. 
The proteins with which the nucleic acids are combined in the different organs and tissues are most often to be defined as of the globulin type. But as globulins vary from organ to organ and from tissue to tissue, it were a mistake to attribute the variations in nucleoproteins entirely to the nucleic fraction. It is, however, the variations in the nucleic fraction that are most striking; the variations in the globulins, in the chemical sense at least, are very much less prominent. The structure of nucleoprotein is generally stated to be as follows:

$$
\text { Globulin } \overbrace{\text { Globulın }}^{\text {Nucleoprotein }} \overbrace{\text { Nuclein }}^{\text {Nucleic acid. }}
$$

The amphoteric protein acts as base, the nucleic acids are rather strongly acid. This is not the only form. Thus we may have:

Globulin $\overbrace{\text { Histon }}^{\text {Nucleoprotein }} \overbrace{\text { Nuclein }}^{\sim}$ Nucleic acid.

Histon-nucleic acid and protamin-nucleic acid occur also in nature as such, not combined with higher protein to form nucleoproteins. Combinations with albumin also occur. It seems clear that nucleoproteins differ from nucleins in the matter of the second molecule of protein. One might define them as simple and compound nucleins.

Nucleic acids are compounds of phosphoric acid with sugar and an organic base. The sugar is always a pentose; the base either a purin or a pyrimidin. The best nomenclature has been evolved from experimental work; and since it gives the most clear-cut ideas of definition, we will abandon the older terms. Under nucleosids we understand a glucosid-like combination of pentose with the purin or pyrimidin base; we have, therefore, purin nucleosids and pyrimidin nucleosids. Under the term nucleotid we understand the complex formed by the union of phosphoric acid with a nucleosid. Nucleic acids as they occur in nature are mono- or polynucleotids. Mononucleotids and tetranucleotids are definitely known; the most common of the tissue nucleic acids are tetranucleotids. But there can be no doubt that di- and possibly trinucleotids may exist; and even more groups than four possibly occur in combination. The polynucleotids seem always to contain both purin and pyrimidin nucleosids. In the polynucleotids the bindings between the several nucleotids are effected with the oxygen and phosphorus of the phosphoric acid molecules. In each of these combinations water is extruded; and conversely their cleavage is a reaction of hydrolysis. Thus:

\footnotetext{
Purin + pentose $=$ nucleosid + water

Nucleosid + phosphoric acid $=$ nucleotid + water
} 
The purin nucleosids are far better understood than the pyrimidin combinations.

The purin nucleosids are three in number, depending on which purin base is present. The combination of adenin with the pentose is termed adenosin; the combination of guanin with the pentose is termed guanosin; and the combination of hypoxanthin with the pentose is termed inosin. Xanthin so far as known does not exist combined preformed in a nucleosid. Nor are any of the methyl purins, frequently found in nature, known to exist in the nucleosids of plants.

Recent studies of the nucleosids indicates that the pentose in the known nucleosids of plant and animal origin is d-ribose; d-ribose has the stereoisomeric equation:

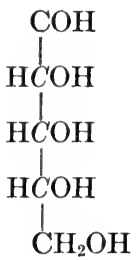

The results of earlier investigations, according to which the pentose in nucleic acids was l-xylose or an arabinose, have apparently been revised to the effect that the pentose in the known nucleosids is d-ribose. They are therefore ribosids.

There are three pyrimidins occurring in nucleosids; and corresponding to the purin ribosids we may assume that in different nucleic acids exist thymin ribosid, cytosin ribosid and uracil ribosid. These nucleosids have not been isolated and studied with the attention given to the purin ribosids.

The combination of purin with ribose to form the nucleosids may be illustrated as follows:

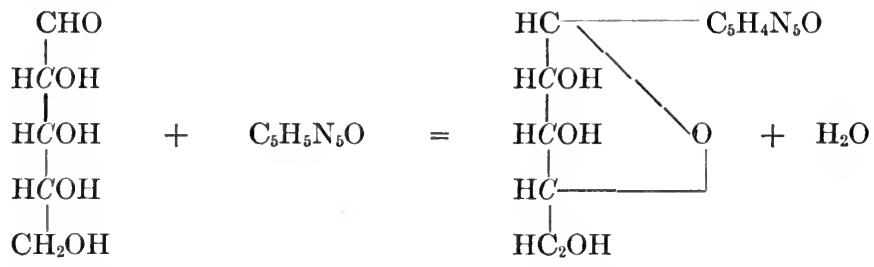

It is known that the purin is attached to the carbon of the aldehyd group, but we do not know where in the purin the attachment is effected. Theoretical considerations, however, suggest that the attachment may be at 7 .

The combination of the nucleosid with phosphoric acid to form the nucleotid may be illustrated as follows:

$$
\mathrm{H}_{3} \mathrm{PO}_{4}+\mathrm{C}_{5} \mathrm{H}_{9} \mathrm{O}_{4} \cdot \mathrm{C}_{5} \mathrm{H}_{4} \mathrm{~N}_{5} \mathrm{O}=\underset{\mathrm{OH}}{\mathrm{O}} \stackrel{\mathrm{O}}{=} \mathrm{P}-\mathrm{O} \cdot \mathrm{C}_{5} \mathrm{H}_{8} \mathrm{O}_{3} \cdot \mathrm{C}_{5} \mathrm{H}_{4} \mathrm{~N}_{6} \mathrm{O}+\mathrm{H}_{2} \mathrm{O}
$$


The bindings that occur to form polynucleotids involve the phosphoric acid groups, of which yeast nucleic acid may serve as illustration.

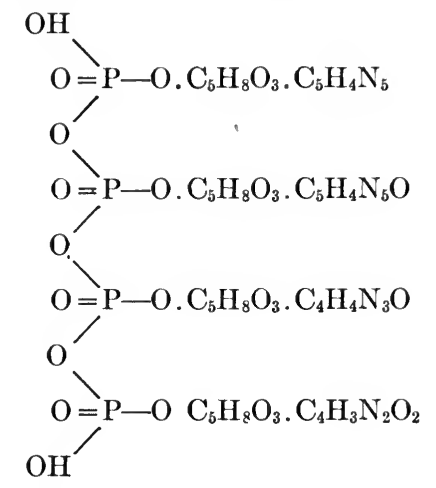

The.purins contained in yeast polynucleotid are guanin and adenin, the pyrimidins are cytosin and uracil. This may be regarded as a representative type, since the nucleic acid of the wheat embryo and of the thymus gland seem very similar if not identical in composition.

The pentose, d-ribose, one of the eight possible pentoses, is scarcely known in nature outside of the plant and animal nucleic acids.

The pyrimidins are derivatives of the pyrimidin ring or nucleus, that is represented as follows, with numbers to indicate the bindings:

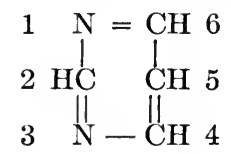

The three pyrimidins that occur in nucleic acids are thymin, cytosin, and uracil. Thymin: 5 methyl-2-6-dioxy-pyrimidin<smiles>CC1=C[NH2+]NC1=O</smiles>

Cytosin: 6-amino-2-oxy-pyrimidin<smiles>Nc1ccnnc1N</smiles>

Uracil: 2-6-dioxy-pyrimidin<smiles></smiles> 
The purin bases are derivatives of the purin ring or nucleus, represented as follows with numbers for the bindings:



The three purin bases that occur in nucleic acids are adenin, guanin, and hypoxanthin.

Adenin: 6-amino-purin, $\mathrm{C}_{5} \mathrm{H}_{5} \mathrm{~N}_{5}$.<smiles></smiles>

Guanin: 2-amino-6-oxy-purin, $\mathrm{C}_{5} \mathrm{H}_{5} \mathrm{~N}_{5} \mathrm{O}$.<smiles></smiles>

Hypoxanthin: 6-oxy-purin, $\mathrm{C}_{5} \mathrm{H}_{4} \mathrm{~N}_{4} \mathrm{O}$<smiles>O=c1[nH]cnc2c1NCN2</smiles>

The Nucleoproteins in Digestion.-The pepsin and hydrochloric acid of the gastric juice split the nucleoproteins into protein and nuclein, and then split the nuclein into protein and nucleic acid. These are simple reactions of hydrolytic cleavage, and once set free the proteins join the other proteins of the diet.

The polynucleotids are entirely resistant to the action of pepsin$\mathrm{HCl}$ in the gastric juice; the nucleic acid is not split into its component mononucleotids. This means that the gastric secretion contains no nucleinase. The mononucleotids are also resistant to hydrolysis in the stomach; phosphoric acid is not split off from combination with nucleosids. This means that the gastric secretion contains no nucleotidase. Nucleosids are resistant to the action of pepsin- $\mathrm{HCl}$, and are not split into their component pentose and base. This means that the gastric juice contains no nucleosidase. In a word, the gastric juice is 
without effect on nucleic acid, nucleotids and nucleosids; it simply splits the protein from nucleoproteins and nucleins.

The pancreatic juice has no greater activities in the direction of these substances than has pepsin. Trypsin will split off the protein from nucleoprotein and from nuclein. It cannot split the nucleic acid into mononucleotids; it cannot cleave from the latter the phosphoric acid and set free the nucleosids; and it cannot split the nucleosids into pentose and base. In other words, the pancreatic secretion is devoid of nucleinase, nucleotidase, and nucleosidase.

The succus entericus, however, contains active ferments for these bodies. Under its action the polynucleotids are split into their component mononucleotids. The intestinal juice, therefore, contains nucleinase. Both purin and pyrimidin nucleotids are split by the intestinal juice into phosphoric acid and the component purin and pyrimidin nucleosids. The secretion, therefore, contains nucleotidase. Here the action stops. The nucleosids are not split into their component pentose and base. The juice contains no nucleosidase. The phosphoric acid combines with ever-present alkali. The nucleosids are resorbed unchanged, so far as the alimentary juices are concerned.

These various experiments have been carried out in glass with digestive juices obtained by fistulæ. When the experiments are carried out in the dog, with appropriate fistulæ, it is found that purin bases are set free. This must be due not to the action of the digestive juices, but to bacteria. By direct test bacteria can be shown to possess nucleosidase. 'To what extent this bacterial cleavage occurs in the normal alimentary tract is unknown. Purin bases are normally present in the stools. Ribose thus set free would become liable to fermentation, as the intestinal bacteria can ferment pentose, at least arabinose. Otherwise, the pentose and bases would be available for resorption, as it is not likely that the purins or pyrimidins would be oxidized in the intestine.

The sum total of our information may be incorporated in the statement that through the action of the digestive juices nucleic acids are split into mononucleotids; these into nucleosids; and these, to an indeterminate extent, are split by bacteria into pentose and bases. Available for resorption, therefore, are nucleosids, pentose, purin and pyrimidin bases. How complete these reactions are cannot be stated. It seems probable that unsplit nucleic acid and unsplit nucleotids could also be absorbed. The feces contain small amounts of purin bases. These proceed partly from the purin set free from the nucleic acid of the diet by bacteria; in part, however, they must be derived from the digestion and bacterial cleavage of dead bacteria and desquamated epithelial cells.

The Fate of the Resorbed Nucleic Acid, Nucleotids, Nucleosids, Pentose, Purins, and Pyrimidins.-The mucous membrane, the membrane of resorption, presents here again evidence of its metabolic functions. Extracts of the mucous membrane exhibit, as does the intestinal juice, 
the power of splitting nucleic acid into the component mononucleotids and transferring these into nucleosids by splitting off the phosphoric acid; this extract also is able to split the nucleosid into the component pentose, purin and pyrimidin bases. Obviously any incompleteness in the digestion of the nucleic acids and nucleotids by the succus entericus may be made good during the process of resorption. And in addition, the nucleosids will be split into their component bodies. How complete are these reactions, cannot be stated. It is, however, a fair assumption, when one contemplates the other digestive functions of the intestinal mucosa, that it is complete. At least until it is proved incomplete we may fairly assume that it is complete. Therefore the products of the digestion of nucleic acids as finally present in the blood of the portal vein are phosphoric acid combined with bases, ribose, free purin bases and free pyrimidin bases. Under ordinary conditions of diet the amount of nucleic acids ingested is not large, since except in especial foods, like sweetbreads, the content of nucleic acids is not heavy.

\section{THE METABOLISM OF NUCLEIC ACID}

This is to the pathologist as well as to the physiologist one of the most interesting of the metabolisms. Presenting to the biologist several of the most striking syntheses in the body, it appeals to the pathologist by reason of the relation of gout to the purins. The study of gout brought into physiology and for years maintained there erroneous conceptions of the nucleic metabolism. And the neglect to separate the exogenous from the endogenous catabolism of nuclein is the reef on which many a thesis of pathology has gone to wreck.

The nucleic metabolism, like those of fat, sugar and protein is to be divided into anabolism and catabolism.

\section{THE ANABOLISIM OF NUCLEIC ACID}

The conjugation of the nucleic acid with proteins to form nucleins and nucleoproteins is comparable to the anabolism of protein. We are here concerned only with the synthesis of the nucleic acids. Included herein are the synthesis of the ribose, purin and pyrimidin bases; the combination of these into ribosids, the nucleosids; the joining of nucleosids with phosphoric acid to form nucleotids; and the union of mononucleotids to form polynucleotids. The native nucleic acids do not all pass through these stages. As previously stated, native nucleic acid may be either mononucleotid or polynucleotid.

The anabolism of nucleic acid presents the most striking illustration of autogenous function. The anabolism of carbohydrate, fat and protein within the body are really little more than reconstructions, for the materials of which (in the sense of definite chemical substances) 
the animal body is dependent upon the diet. But in the case of nucleic acid we observe autogeny. The body is entirely independent of nucleic acid or any one of its components in the diet; it synthesizes its pentose, purins and pyrimidins. The pentose is synthesized from hexose; the purins and pyrimidins are derived from protein, not from preexisting purins or pyrimidins. Not only can the body effect these syntheses, but it is most probable that it always does so; preformed pentose, purin and pyrimidin of the diet are in our present opinion not utilized in the formation of nucleic acid, for which the body relies entirely upon its own synthesized components. It is not known whether there is anything specific, in the biological sense, in the nucleic acids. So far as present investigations go, no signs of such biological specificity have been revealed. The nucleic acids are not colloidal; they are acids, diffusible, and their salts are subject to electrolytic as well as hydrolytic dissociation. There is a certain isomerism possible in the purins. As will be later pointed out, there are two known isomers of uric acid. It is possible that such differences might extend to the purin bases, and in this way the nucleic acids might possess chemical individuality beyond the apparent variations in composition.

That the animal organism forms nucleic acid independently of the input of any of its components preformed, apart from phosphoric acid, is shown in many ways. The eggs of birds and insects contain no pentose, purin or pyrimidin; the larvæ and chicks contain many cells fully endowed with nucleic acid. The young of mammals live on milk which is free of pentose, purins and pyrimidins; normal cellular growth and reproduction are, of course, maintained. The case has been reported of a young man who grew up on milk as his sole diet. In starvation the nucleic metabolism pursues the even tenor of its way. The migrating salmon, who do not feed, form large amounts of nucleic acid in the ripening milt. There is no doubt of the absolute capacity of the body to synthesize to the fullest extent the components of the several nucleosids.

The origin of the ribose is to be sought in the glucose of the body. How this is accomplished we do not know; but that it is accomplished from glucose seems certain. Under certain pathological conditions arabinose is eliminated in the urine. This may, of course, have been derived from the ribose of the nucleic acid. But in some cases of pentosuria, amounts of arabinose are eliminated so large as to seem entirely outside of the possibilities of the known nucleic catabolism, as estimated by the purin output. Under these circumstances the arabinose must have been derived from glucose. That the body forms glucose from pentose is certain; and that it forms pentose from glucose we must also believe.

The purins and pyrimidins are in some way derived from protein, or rather from amino-acids. For the direct derivation of purin from amino-acids we have no scheme or illustration. This statement holds true for the pyrimidin ring. But with the pyrimidin ring once formed, 
we may conceive of the formation of purin by the union of pyrimidin ring and imidazol. The imidazol exists preformed in histidin, an almost invariable component of proteins.<smiles>NC(CC1=CN[CH]N1)C(=O)O</smiles>

When glucose is acted upon by ammonium zinc-hydroxid, methylimidazol is formed.<smiles>Cc1cncnn1</smiles>

From this reaction the direct derivation of imidazol from fatty acids under the action of alkali is not difficult of conception theoretically. But in any event the imidazol group exists in the body preformed in histidin. The formation of purin from the pyrimidin ring plus imidazol is illustrated as follows:<smiles>c1nnc2cc1NN2</smiles><smiles></smiles>

We may in this manner get a general idea of the origin of the purin bases; but of the origin of the pyrimidins we know nothing. None of the methods of laboratory synthesis of pyrimidin throw any light upon its derivation within the body.

Once formed, the purin bases are combined with ribose to form purin ribosids; the pyrimidin bases are combined with ribose to form pyrimidin ribosids. The ribosids (nucleosids) are then combined with phosphoric acid, ever present in the diet, to form the corresponding mononucleotids. These may be combined with protein to form nuclein and the nuclein with protein to form simple nucleic acids. Or nucleotids (four in number usually, two purin and two pyrimidin nucleotids) are combined to form a polynucleotid, which combined with protein forms nuclein and united with further protein forms complex nucleic acid. In a certain sense we may compare the mononucleotid and the polynucleotid with peptone and albumin. These reactions of synthesis we hold are 
accomplished within the cell nucleus. When a cell divides, there must be a marked velocity of this synthesis for the formation of chromatin for the two nuclei; and in the rapid cell divisions of the early embryo, these syntheses are the most striking features. We have spoken of nucleic acids as being nuclear constituents. To this there seems to be one exception. Muscle contains inosinic acid in amounts and under circumstances that seem to preclude its being an intranuclear constituent. We are, therefore, forced to assume that in the case of muscle, the nucleotid (inosinic acid) is in some way combined with the protoplasm, in the so-called muscle plasma.

A number of different nucleotids have been isolated and studied. Guanylic acid (the nucleotid of guanosin, which is the ribosid of guanin) has been recovered from the pancreas, liver, and spleen. Inosinic acid (the nucleotid of inosin, which is the ribosid of hypoxanthin) is present in muscle. The corresponding adenosinic acid has not been isolated. Of the three pyrimidin nucleotids only one has been isolated-cytidin, containing cytosin. It is assumed that they all exist in different simple as well as in complex nucleic acids. Of polynucleotids those occurring in the milt of the salmon and of the herring, in the thymus, in yeast and in the embryo of the wheat have been well studied. These all contain adenin and guanin ribosids. Concerning the pyrimidins the facts are not so definite. In the animal polynucleotids, thymin, cytosin, and uracil are recovered, though but two can exist preformed. The uracil is easily derived from cytosin, and may have been formed from it in the manipulations of isolation. In the triticonucleic acid of wheat, however, only cytosin and uracil are found; either the two existed preformed or only cytosin (ribosid) existed, and the uracil was formed from it in the manipulations of isolation. The same question exists for both hypoxanthin and uracil. They can be formed by oxidation from adenin and cytosin; but there is evidence tending to indicate that they do exist preformed in nucleic acids.

When purins, pyrimidins and pentose are carried to the cells in the blood stream, do the cells use these exogenous substances and of them form nucleic acids, thus sparing the work of synthesis? It will be recalled that the products of the digestion of nucleic acid were nucleosids. We learned further that these nucleosids were split in the wall of the intestine during resorption. The liver contains nucleosidase, so that if any escaped cleavage in the intestinal wall, they would be split in the liver. Therefore, in the circulating blood would be found (in the absence of any oxidation or deaminization), ribose and the purin and pyrimidin bases. Now the liver is active in deaminization, and it will need to be shown as a matter of fact that adenin, guanin, and cytosin pass undeaminizated through the liver into the blood of the vena cava.

Assuming for the sake of argument that these bases could pass through the liver unchanged, is there evidence that the cells use them in the syntheses of nucleic acid? There is no evidence for this view; there 
is also no direct evidence against it. But against this view is the positive fact that exogenous purins appear quickly in the urine. The ingestion of a sweetbread is quickly followed by the elimination of a large amount of purin. That this elimination of exogenous purin is complete in the quantitative sense cannot be stated because we have no reliable way of estimating the amount of purins in the ingested sweetbread. But heavy this elimination is and prompt. The endogenous purins of the urine run remarkably even. When exogenous purins are ingested, the purin output rises in such a manner as to suggest strongly that their elimination is quantitative. That the body cells should put on and lay off the syntheses of purins in such a manner as predicated, seems to the writer improbable, and it will be here assumed that the cells of the body always synthesize the purins, pyrimidins and ribose out of which are formed in the same cells the nucleic acids. Just as in the case of the protein anabolism, synthesis is needed for wear-and-tear, as well as for the construction of young cells.

Pathological Variations.-We have no data whatever tending to indicate that under conditions of disease quantitative or qualitative variations exist in the anabolism of nucleic acid. There are many conditions in which excessive cell multiplication occurs-in leukocytosis, leukemia, in rapidly growing neoplasms-states of cell multiplication in which the excessive growth follows some abnormal stimulation. Of the nature of the abnormal stimuli we have neither experimental nor dynamic conception. In the nuclei of cells formed in such states of abnormally rapid multiplication, histological appearances indicate at times unusual arrangements, deficient or excessive amounts of the chromatic substance. It may, however, be assumed that the fault lies not in the function of anabolism of nucleic acid, but rather in the utilization of the nucleic acid after its synthesis. The defect in rachitis and encalcification lies not in absence or superabundance of lime, but in abnormal utilization of the lime. And in like manner for the present at least, we regard the aberrant relations of the chromatin of abnormal cells to lie not in variations in the anabolism of nucleic acid, but rather in the utilization of nucleic acid.

\section{THE CATABOLISM OF NUCLEIC ACID}

The experimental investigations of the last ten years have disclosed a most interesting and instructive series of enzymic reactions affecting the nucleic acids and their components. When a cell dies its chromatic substance undergoes alterations whose microscopic appearances are best interpreted as indicating solution. 'The strands lose their power of specific staining, the fibrillæ become undifferentiated and revert, so to speak, to the condition of undifferentiated protoplasm. This solution the biologist regards as due to digestion. The reactions that in the death of the cell involve the chromatin in toto, may be regarded 
as involving portions of it in the wear-and-tear of cellular life. The first reactions are hydrolytic cleavages, due to the action of the intracellular proteolytic enzymes, and leading to the splitting off of the two molecules of protein and the setting free of the nucleic acid. This then become available for the catabolic reactions of the nucleic ferments.

Nucleinase, the enzyme that accelerates the cleavage of polynucleotids to mononucleotids, is experimentally present in all organs and tissues. The ferment is specific in the sense that it carries the reaction only to the stage of mononucleotid. It is not specific in this sense: the ferment of an animal body will split the polynucleotids of different plants.

Nucleotidase, the ferment that accelerates the cleavage of the mononucleotids to nucleosids, is also experimentally present in all organs and tissues. It is specific in the same sense as nucleinase is specificits activities are limited to the single reaction.

Nucleosidase, the enzyme that accelerates the cleavage of the nucleosids, the ribosids, into the component ribose and purin or pyrimidin, is likewise experimentally present in all tissues and organs. It is specific in the sense that it will not split other glucosids, though it does cleave the ribosids of purin and pyrimidin.

These three hydrolytic ferments, operating side by side in the tissues, furnish striking illustrations of enzyme action that is purely quantitative but nevertheless strictly limited to a particular substrate class. Nucleic acid is hydrolyzed very slowly in sterile water at ordinary temperature, and these enzymes are pure accelerators. But the field or scope of their accelerating influence is confined to one class of substrate.

These reactions occur exclusively, it is now held, within cells. The result is the setting free of purin bases, pyrimidin bases and ribose. The fate of the latter, except as expressed in the assumption that it is somewhere and somehow converted into d-glucose, is unknown. Before we discuss the enzymic catabolism of the bases, it will be best to state the perfectly demonstrated qualitative reactions through which these substances must pass.

Deaminization and oxidation are the two reactions through which the purin and pyrimidin bases are carried to the end products of their catabolism. These reactions run as follows:

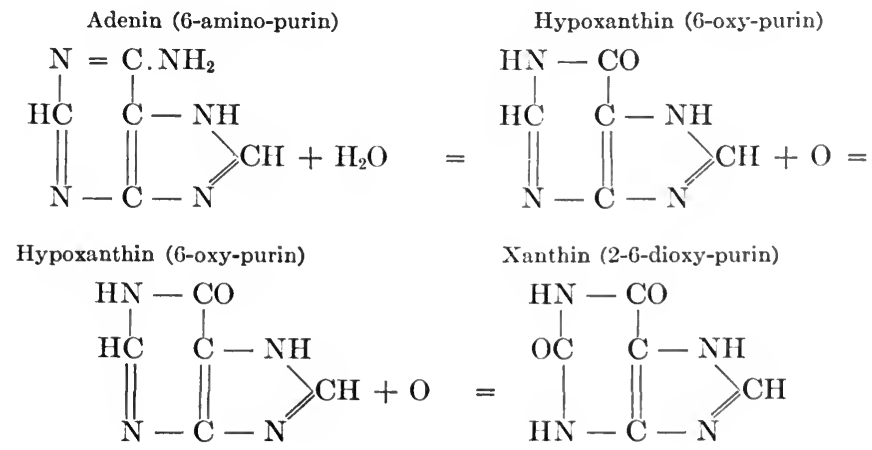



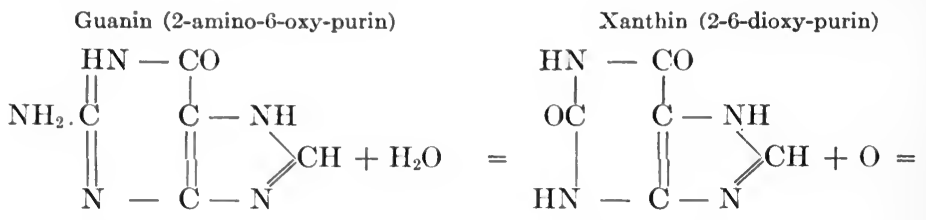<smiles>O=C1Nc2[nH]c(=O)[nH]c21</smiles>

Uric acid (2-6-8-trioxy-purin)

Cystosin (6-amino-2-oxy-pyrimidin)

Uracil (2-6-dioxy-pyrimidin)<smiles>NC1=N[AlH]C=CC1N</smiles>

Thymin contains a methyl group, and it is not known whether the body can split off this methyl group. Otherwise, thymin has the same structure as uracil, and if the body can split off the methyl group, uracil would be formed. The present idea is that monomethylpurins and pyrimidins are not in the body convertible into oxypurins and oxypyrimidins.

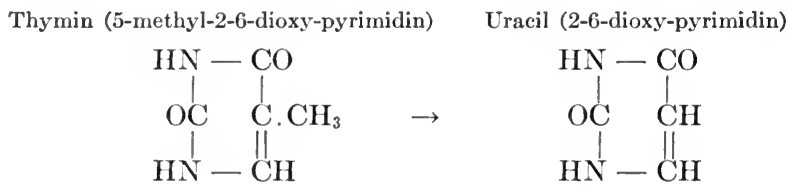

The urine contains a trace of another purin base, epiguanin, which is guanin with a methyl group at 7 . It has never been isolated from a nucleic acid. Yet the analogy with the methylpyrimidin suggests that it occurs in some particular nucleic acid and is eliminated unchanged. As the result of the deaminization of the amino-purins and cystosin, ammonia is set free, to join that formed in the deaminizations of the amino-acids.

When we attempt to fix the conditions of occurrence of these reactions of deaminization and oxidation, we meet with contradictions in the published data. Since these substances occur in all cells and since the nucleinases, nucleotidases and nucleosidases occur in all cells, it might seem to follow that the deaminization and oxidation ferments would also occur in all cells. This does not appear to be the case. Just as arginase is not found in the pancreas but is found in the liver, so some tissues seem to be devoid of the oxidase. The experimental difficulties are marked, and in work of this kind a positive result proves 
more than a negative result disproves. The deaminization ferment seems to be the more widespread, the oxidation ferment the more restricted. In different animals the oxidation ferment has been found in the liver, spleen, kidneys, lungs and in the intestinal mucosa, also in muscle. But the results in animals of different species are not uniform. For the purpose of this treatise the question is not of great importance. Whether all organs accomplish these reactions or whether the purins and pyrimidins have to be transported to certain organs by the circulation, there to undergo the reactions, may for the present remain undecided. That the liver is especially active in the deaminization and oxidation of the exogenous purins is known. It seems possible also that the reaction of deaminization of amino-purin may occur while it is still in the ribosid, in combination with the pentose.

The ferments for the deaminization of adenin and guanin are commonly regarded as identical. Nevertheless, they display variations that have led competent investigators to regard them as different and to name them respectively guanase and adenase. The dualism of these ferments is supported by the occurrence of the guanin gout of swine. It is possible in laboratory experiments to have the reaction of deaminization precede the cleavage of the ribosid. Of the ferments for the deaminization of the cytosin little is known.

The reactions are never complete, $i$. $e$., purin and pyrimidin bases exist with uric acid in all normal urines. This fact is probably to be regarded as an incident of secretion rather than one of reaction. By that we mean that the ratio of purin bases to uric acid in the urine cannot be taken as indicating the relation of reaction. Uric acid, purin, and pyrimidin bases are in the circulating blood that passes through the kidney. Naturally the kidney eliminates. It is, of course, possible that the kidney normally sweeps them all out quantitatively, and in that event the ratio found in the urine might represent the relation of reaction. The ratio of purin bases to uric acid is not constant, it varies in different individuals and with different diets, independent of the exogenous purin. The question is one of importance in the study of gout, but there seems to be no way of reaching an experimental determination of the exact facts and relations.

Are the products of the catabolism of nucleic acid utilized again in the body for the synthesis of new nucleic acid? Our present data speak against such an assumption. It is our understanding that when a subject is on a purin-free diet, the purin output of the body represents the catabolism of nucleic acid. This same conclusion was drawn for the exogenous purins. This is not strictly true in one sense, because uric acid is convertible into urea. But disregarding this fraction, we believe that in the purin output is represented the sum total of the products of the catabolism of nucleic acid. Since the purin and pyrimidin bases are identical, whether derived from the catabolism of nucleic acids of the diet or in the cells of the body, any assumption that the body could utilize the exogenous purins of the diet is tanta- 
mount to conceding a reutilization of the products of catabolism of the endogenous nucleic acids. Now the constancy and individuality of the purin catabolism and the lack of toleration of exogenous purin on the part of the body speak most strongly against the assumption of any utilization of preformed purin and pyrimidin bases in the anabolism of nucleic acid. The anabolism of nucleic acid we regard as always a synthesis de novo, from the amino-acids of protein or from amino-acids and sugar.

Constancy of Catabolism of Nucleic Acid.- Like the creatinin metabolism, the catabolism of nucleic acid is strikingly constant for each individual. Apparently each kilo of cellular substance produces so much purin, pyrimidin and phosphoric acid per day. This ratio of nucleic catabolism to cell content of the body is different in different individuals, varies with age, perhaps with sex, and to some extent with occupation. It cannot be well related to gross body weight on account of the differences in weight of skeleton and fat. But with an individual in normal weight, it is striking how constant the purin output is with a regulated life and with the exclusion of exogenous purin from the diet. Whatever may be the fraction of conversion of uric acid into urea in man, it seems to be quite unvarying though undetermined. The output of purin (we select purin because it is chemically the more controllable) may be interpreted in a direct sense to represent the pace of the nucleic metabolism of the individual. If from the diet of a subject we exclude purins, creatin-creatinin and exogenous protein, the outputs of urea, purin and creatinin (apart from the fractions of uric acid and creatinin that are converted into urea) are independent entities and stand for specific functions of the body. That there are correlations between these three functions is not to be denied. But it is correct to regard them as specific functions; the output of endogenous urea (plus ammonia) representing the catabolism of protoplasm, the output of creatinin representing the catabolism of muscle plasma, and the output of purin representing the catabolism of nucleic acid.

While it is true that the purin metabolism, exogenous purin excluded, is to be regarded as independent of the protein metabolism, it is nevertheless true that with a low protein input that curtails greatly the exogenous protein catabolism, the purin output tends to fall somewhat. It is possible to explain this without any abridgement of the idea of independence of the two metabolisms. It is possible that within certain limits the purin catabolism runs parallel to the work of the cells; and since with greatly lowered exogenous protein catabolism the work of the cells of the liver and kidneys is reduced, this might spare nucleic wear-and-tear. Secondly, it may be due to exaggeration of the reaction of uricolysis; since the urea concentration in the liver must be low, in accordance with the law of mass action the conversion of uric acid into urea would be increased.

If we interpret the purin output to represent the pace of the nucleic catabolism, it is clear that this must be divided among the different 
cells and tissues of the body in proportion to their structure and functions. The glandular cells richest in chromatin are to be regarded as the cells most active in nucleic metabolism. In these we include the liver, the lymphatic organs (spleen, lymph glands, bone marrow, and the circulating leukocytes) the central nervous system, the kidneys, and the pancreas. Muscle plasma contains, however, nucleic acid combined in a special way outside the nuclei, of which more later; the nuclear catabolism of nucleic acid in muscle cannot be large. In a general sense, therefore, those cells are most active producers of purin that contain the largest relation of mass of nuclei to mass of cell; in other words, the largest amount of nucleic acid in relation to weight. This is, of course, just what would be expected from the law of mass action. But there is evidently another factor. Two cells with equal relation of mass of nucleic acid to total mass may not have the same intensity of metabolism; the span of life may be longer for one type of cell, the wear-and-tear less. For example, it seems clear that the pace of life is faster and the duration of life shorter in a circulating leukocyte than in a liver or kidney cell. At least, the purin output in conditions of leukocytosis and in leukemia suggest this most strongly. The purin output of leukemia of the polymorphonuclear type is much greater than in apparently equally intense leukemia of the lymphocytic type. The particular activity of the nucleic catabolism in the leukocyte once led to the erroneous assumption that these cells were practically the single source of urinary purins. This is, of course, untrue; but the lymphatic system is perhaps the most prominent single tissue with respect to the catabolism of nucleic acid.

Extranuclear Derivation of Purin.-That purin bases are not derived in the body outside of nucleic acid is accepted as settled. But that nucleic acid exists in the body outside of nuclei seems to be equally certain. Muscle contains apparently a nucleotid termed inosinic acid, combined with the muscle plasma in some way. On cleavage this mononucleic acid yields phosphoric acid and a ribosid of hypoxanthin. On cleavage of the nucleosid, hypoxanthin and ribose are set free. Perfusion of the muscle of the dog yields uric acid in amounts in excess of the apparent nuclear content of that tissue. When the isolated perfused muscle is tetanized, the elimination of uric acid is much increased and hypoxanthin appears. The source of this hypoxanthin and uric acid is obviously to be sought in part in the inosinic acid; in part, of course, in the catabolism of the nuclei of the muscle cells. But the increase with contractions may be reasonably attributed in large part to the inosinic acid. It is possible, in some individuals at least, to perform this experiment in man. It is well known that muscular work does not affect the twenty-four-hour output of purins in man. If, however, heavy muscular work be done and the urine examined for purins in short periods, it will be found that there is a wave of purin output just after the period of exercise, to be followed later by low elimination. The ratio of purin bases to uric acid rises during the period of 
exercise; later the ratio returns to normal. Apparently the exercise of the muscles brings out rapidly what would otherwise have been brought out slowly, in even distribution. From these observations we may draw the conclusion that there is a regular inosinic catabolism in the plasma of muscle. Heavy exercise does not affect the total transformation of this fraction; but it does alter the distribution in time and also so exaggerates it during the period or actual exercise as to send out the purin bases before they can be oxidized to the extent normally observed. Therefore, with heavy work the urine will contain a little more purin base and a little less uric acid; and the elimination will occur in a wave rather than in a straight line. Just how large is the fraction of this inosinic acid catabolism as compared to the total we do not know; it is apparently not large. The experiment has been reported in which as the result of forced and excessive muscular exercises, the urine became for a time practically purin-free. Although there is no obvious chemical point of contact, one cannot resist the thought that there may be some connection between the inosinic and the creatin catabolism in muscle plasma.

The Extranucleic Origin of Uric Acid.-In all the above discussion the derivation of uric acid has been confined to preëxisting xanthin, this derived from hypoxanthin, guanin and adenin. When guanin (adenin is too toxic for large injections) is injected into animals, it appears in the urine as xanthin and uric acid. In the laboratory it is possible to reach the stage of uric acid from adenin and guanin in other ways than by the steps illustrated in the scheme on pp. 439 and 440. But this fact has no bearing on the question under discussion. Is uric acid derived in the body from any source outside of the purin bases? Before the relations of the purins were worked out, uric acid was supposed to be derived from several sources. Information may be sought in two ways: through laboratory synthesis; and by observation of the uric acid metabolism of lower animals.

Uric acid may be synthesized by heating urea with either glycocoll or trichlorlactic acid, but these reactions offer no information of use. Years ago it was believed that uric acid was derived from common protein, and that excess of protein in the diet (what we now call the exogenous protein fraction) led to increase in the uric acid in the urine. This conception is totally false. Protein has no influence on the formation of purins except through the nuelein and nucleic acids it may carry. Beyond this, the purin and protein metabolisms are independent of each other. A supposed illustration of the influence of protein on the purin elimination has been advanced in the fact that the purin output is lower in starvation than on a normal diet free of purin. The interpretation of the fact is faulty. 'The urea output is also less on a protein-starvation diet than on a protein diet just competent to maintain nitrogenous balance. Apparently, when put to it, the metabolisms cut down their transformations, an expression of increase efficiency of operation under conditions of stress, the factor of wear 
and tear is lessened. During starvation, furthermore, the secretions of the alimentary tract are reduced. (Cf. also page 442.)

In birds and in some reptiles, uric acid is the chief state of output of nitrogen. The amino-acids and ammonia derived from the common catabolism of protein are in the bird converted into uric acid. Ammonium salts, urea and amino-acids when administered to birds are eliminated as uric acid. Perfusion of the liver of the goose with these substances leads to the synthesis there of uric acid. When the liver of the goose is extirpated, the uric acid output falls and the ammonia output rises proportionately. When lactic acid and ammonia are passed through the isolated liver of the goose or mixed with the liver pulp, uric acid is formed. The formation of uric acid in the bird appears to follow the following course: lactic acid is first converted into tartronic acid by oxidation, the lactic acid being derived from aminoacids and from glucose; tartronic acid then adds urea to form dialuric acid, which finally adds another molecule of urea to form uric acid.



When now this scheme is applied to the mammals, it fails. When lactic acid or tartronic acid is fed to mammals with urea, no uric acid is formed even when pyrimidin is added. When perfusion of the liver or experiments with liver pulp are done with tartronic acid and urea, no uric acid is formed therefrom. Even when dialuric acid is used, the results are negative, as they are also with the amids of tartronic acid. The above scheme, while it holds for birds, has no application to the purin metabolism of man.

No other plausible scheme for the direct synthesis of uric acid has been suggested. The data on the derivation of uric acid from the purin bases meets every requirement; and we, therefore, rest all discussion of the rôle of uric acid in physiology and pathology upon the single origination of uric acid from purin bases; and antecedent to this the single derivation of the purin bases from nucleic and inosinic acids.

The endogenous purins vary in normal individuals from 0.15 to 0.40 gram per day, the variations being from individual to individual; in the same subject the output is very constant from day to day. Milk or egg diet is the only known purin-free diet. Of this output, the larger 
part is in the state of uric acid; of purin bases the output may be from 10 to 40 milligrams per day. Usually xanthin and hypoxanthin make up the larger part of the purin bases; adenin and guanin are sometimes present, but only in traces, and guanin in particular is rarely eliminated. The purin output in infancy is high in relation to the weight of tissue - an expression of intense nuclear metabolism. On a purin-free diet the curve of purin output presents the highest point during the morning, the lowest at night.

The Exogenous Purin.-The normal urine of subjects on mixed diets contains more purin of exogenous than of endogenous derivation. The exogenous purins are derived from four groups of ingredients in the diet.

The glands of the animal body are quite rich in purin. The thymus, which is the sweetbread of the table, is very rich in purin, containing to the 100 gram of fresh gland nearly a gram and a half of purin. Pancreas, which is often served as sweetbread, contains about a half gram of purin to the 100 grams of fresh gland. Kidney and liver are about as rich as pancreas; if different, perhaps less. Brain is fairly rich.

The flesh of animals, more commonly used for food than the internal glands, is not rich in purins. In the muscle are two fractions; that derived from the nuclei and that originating in the inosinic acid. The input due to the inosinic acid can scarcely be more than 0.05 gram per day. The flesh of fowl is richer in purin than the flesh of adult beef and mutton; veal and lamb are somewhat richer. As mixed diets go, probably some 200 milligrams of purin are derived daily in this fraction of the exogenous purin.

Vegetables are commonly supposed to be poor in purins, and this is true of many. The legumens, however, are quite rich in purins, as may be observed in analyses of the urine of subjects on a liberal input of peas or beans. The root vegetables are almost purin-free. The grains contain small amounts, so that bread contains about 100 milligrams to the kilo.

The common beverages contain purins. Malted liquors contain more than traces. Coffee, tea and cocoa are rich in purins, since the alkaloids of these plants are methylpurins. Caffein is 1-3-7-trimethylpurin, theophyllin is 1-3-dimethylpurin, theobromin is 3-7-dimethylpurin. In addition to these methylpurins, the extracts of tea and coffee contain amino- and oxypurins, tea being rich in adenin.

In the catabolism of the exogenous purins, it is quite certain that for ordinary inputs the amino- and oxy-purins are eliminated in large part as uric acid, having been deaminizated and oxidized in the liver. There may be a slight increase in the purin bases, but by far the larger part is eliminated as uric acid. For the methylpurins, on the other hand, it is fairly certain that they are not oxidizable to uric acid; the body is able to burn one or two but not the last methyl group, thus eliminating them as a monomethylpurin. From the urine of persons 
accustomed to the use of tea and coffee, four different methylpurins have been isolated-1-methylpurin, 3-methylpurin, 7-methylpurin, and 1-7-dimethylpurin. These are the end products of the catabolism of the methylpurins of the beverages named. It is the present view that methylpurins are always eliminated as methylpurins. But it must not be forgotten that these beverages contain in addition to methylpurins also amino- and oxy-purins. A dose of caffein would be eliminated in the purin-base fraction of the urine; but following the ingestion of a cup of tea or coffee, the uric acid would also be increased. The content of amino- and oxy-purins of tea and coffee, like the content of methylpurins, varies in different samples; and since the strength of the beverages varies widely, the influence of the consumption of tea and coffee upon the purins and the uric acid of the urine must be varied. In the urine of individuals accustomed to the regular use of tea and coffee, the methylpurin bases exceed the natural purin bases in amount.

There is also a difference in the deaminization and oxidation of isolated purins and of purins in the natural state. If adenin and guanin be ingested, it will be found that a large fraction will reappear in the urine; hypoxanthin is usually oxidized in large part to uric acid. But if a sweetbread be eaten whose adenin and guanin content is equal to the amount administered in the experiment stated, there will be little increase in the purin bases of the urine; the increase will be found largely in uric acid. The explanation of this fact is perhaps to be found in the observation that in some tissues the nucleosid is deaminizated before it is split into the component purin and pentose. It is possible that this is the usual order of reaction in the intestinal mucosa and that the deaminization there may be confined to the cleavage of the amino group from the nucleosid. Another explanation would lie in the mass relations of resorption. In the case of the ingestion of the sweetbread, the process of digestion is slow and resorption spread out over several hours. In the case of the ingestion of a dose of adenin or guanin, the whole mass is resorbed in a short time and it might be conceived that the liver could not accomplish a total transformation and bases would pass in part into the blood unchanged, to be carried to the kidneys and eliminated. From this point of view, the difference would be not qualitative but quantitative.

The feces contain more purin bases than does the urine. They are derived in part from the ingested nucleic acid, in part from the cleavage by bacteria of the nucleic acid of the nuclei of the desquamated epithelial cells and of bacteria themselves.

Under varying circumstances affecting the exogenous purins, the total urinary output of purins may vary from less than a half gram to 2 grams per day. As a rule a gram is not exceeded.

The Oxidation of Uric Acid.-The uric acid formed in the body is not eliminated quantitatively; some is oxidized to urea. The magnitude of this fraction is difficult if not impossible of definition and constitutes 
in some respects the crux of the interpretation of the purin output. When the exogenous purins are excluded from the diet, the relations would be susceptible of direct interpretation, certainly in the presence of blood analyses, if there were a balance between production and elimination. But since it is certain that a fraction of the uric acid is oxidized, unless this fraction may be estimated, the foundation of induction fails. In many ways and by many men attempts have been made to measure this uricolysis. If one administers amounts of uric acid up to a gram, from one-fourth to one-half will usually be burned; sometimes the larger part will be burned. The individual factor is again markedly in evidence. If urate of sodium be injected hypodermatically, the same variable results will be secured. In each case the experiment fails to approximate the normal circumstances, since a large mass of uric acid is introduced at one time; and it is possible and indeed probable, that under such circumstances a portion will escape transformation that might have been transformed if it had been evenly distributed through the day. Uricolytic ferments have been demonstrated in the liver, spleen, kidney, muscles and bone marrow. Uricolysis is undoubtedly much greater in the carnivora than in man, since uric acid introduced into the dog is practically all oxidized; in man the fraction is small, usually less than half. But not even an approximate measurement of this uricolysis can be accomplished at present. And the individual factor is so influential in the purin metabolism that it would not be permissible to apply an integral factor obtained as the means of a series of observations; where a mean is the average of a series of widely varying individual figures, we cannot hope to apply it to an individual subject.

Mechanism of Uricolysis. - The mechanism of this uricolysis is not well understood. For the dog and cat the steps of oxidation are fairly well known. Allantoin is a normal constituent of the urine of canines and felines and is said to occur for a few days after birth in the urine of cattle and of infants. As its name indicates, it is present in the allantoic fluid. It is said to occur in the urine of pregnant women and occasionally may be found in normal urine. It must be admitted that most of the reports on the occurrence of allantoin have come down from decades ago, and recent investigations have not been able to confirm the reported findings of allantoin in the allantoic fluid of woman or in the urine of the newborn. The formation of allantoin from uric acid is easily accomplished in the laboratory. The derivation from uric acid may be indicated thus:

Uric acid<smiles></smiles>

Allantoin

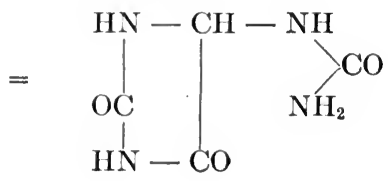


The intermediary steps are unknown. Allantoin is commonly believed to be hydrolyzed to urea and glyoxylic acid.

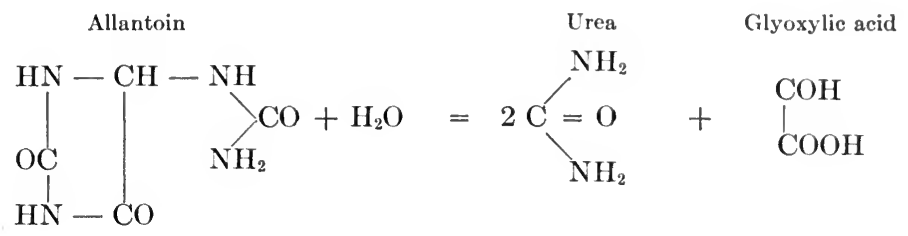

In the carnivora the reaction stops at the stage of allantoin. In the rabbit the ingestion of uric acid is apparently followed by an increase in glycocoll, which could have been derived from glyoxylic acid. In man no indications of this reaction are to be seen. The human tissues do not oxidize allantoin nor do they form allantoin from uric acid, as do the organs of the dog.

In the older literature one finds many references to assumed oxidation of uric acid to oxalic acid; this needs but to be mentioned, since it is devoid of foundation. Despite the researches of four decades we do not yet understand the oxidation of uric acid to urea. We could bear with this ignorance, however, if we but knew to what an extent this oxidation occurs, in any way that it occurs. The writer believes this fraction to be small, but is fully conscious that adequate foundation for the opinion does not exist.

One further result of this situation is that we may make no inference of exogenous purin from the output, since exogenous uric acid like endogenous uric acid is subject to oxidation. Under these circumstances the total exclusion of exogenous purin from the diet in experiments directed to the elucidation of any feature of the purin metabolism is imperative. It will not do to make a subtraction for the exogenous purin.

The curve of the endogenous purin is quite level, being higher during the day than at night, though influenced very little by diet. It is not influenced by sparse or copious water drinking, by acids or alkalies or by alcohol. The curve of the exogenous purins follows in general the periods of ingestion, though it is somewhat prolonged. Normally, the purins of the sweetbread are entirely eliminated within twenty-four hours. Under conditions of health the body no more tolerates a retention of purin than it does a retention of protein.

The only expression of the purin values of the urine are the masses of uric acid and bases. A ratio of uric acid to urea and of purin nitrogen to total nitrogen was once much in vogue. Such ratios are worthless. These magnitudes have independent variables, and cannot therefore bear a ratio to each other. The ratio of uric acid to purin bases has a valid standing, but in our present knowledge we are able to attach little importance to it. It is easy to say that suboxidation would increase the mass of purin bases at the expense of the uric acid. This categorical observation has, however, little value so long as the postu- 
lated variation in ratio does not occur. It is true that in an occasional case of nephritis, the amount of purin bases is high. It is also seen in occasional intoxications, and in some febrile infections. When the uric acid is high one might expect to see the purin bases high, and this is often the case. But increased purin base and reduced uric acid as the expression of suboxidation in the purin metabolism is largely a blackboard proposition.

It is not known in what state the purin bases circulate, whether free or combined. Since their mass is so small, the matter is of little consequence. Uric acid circulates in but one form, as mono-urate. In the blood are ammonium, sodium, potassium and calcium, all are doubtless in combination with uric acid, in proportion to their affinity constants and in relation to their available masses in the system. There is little ammonium in the circulating blood. There is little potassium and a great deal of sodium in the blood plasma; in the blood cells, however, the potassium predominates. Certainly the largest fraction of the circulating uric acid is combined with sodium. Certain misconceptions regarding the state of solubility, combination, and reaction of uric acid have been long current and it is imperative that the correct facts be emphasized, though this is not the place to enter into physico-chemical explanations of the data. Uric acid exists in the blood only as the mono-basic salt; it cannot exist there as the di-basic salt, the carbon dioxid alone makes that impossible. The existence of a quadri-urate is entirely a blackboard proposition, there is no theoretical foundation for it and no experimental proof of it. There is such a salt as a bi-urate, but it does not exist in the circulating fluids or in the freshly voided urine. It is not probable that it exists in tophi or stones. The reaction of a solution of monosodium urate is very faintly alkaline. The reaction of a solution of disodium urate would be more strongly alkaline, but as stated such a salt is not stable in solution in the blood. The solubility of the mono-urates of sodium, potassium and ammonium depends largely upon the temperature, but more upon the form of uric acid.

Tautomeric Forms of Uric Acid.-Uric acid exists in two tautomeric forms, known as the lactam and the lactim states. The lactam state is the less stable and the more soluble; the lactim state is the more stable and the less soluble, and the uric acid in solution is tending always to pass into the lactim state. In the blood both states coexist, as the passage from one state to the other is slow in a colloidal solution. In the urine in all probability the lactim form is alone to be found. 'These tautomeric forms are illustrated in equations.
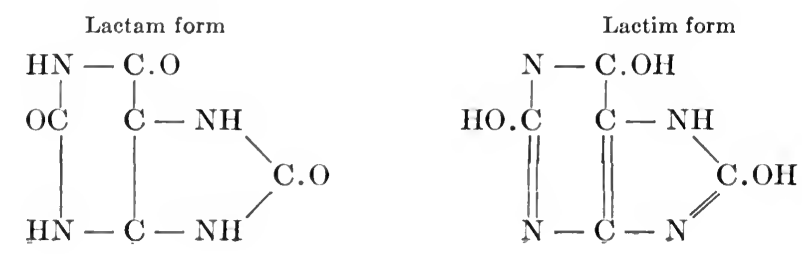
Solubility of Urates.-The solubilities of the three salts at $37^{\circ}$ in the two forms are as follows.

Potassium mono-urate Sodium mono-urate Ammonium mono-urate
Lactam state Lactim state

$\begin{array}{lll}1: 265 & 1: 400 \\ 1: 470 & 1: 710 \\ 1: 1225 & 1: 1850\end{array}$

The marked difference in the solubilities of the three salts is to be noted, and also the fact that the lactim salts are much less soluble than the lactam salts. These solubilities of the salts are, however, very much greater than is the solubility of uric acid, which at $37^{\circ}$ is $1: 15,000$, being $1: 40,000$ at $18^{\circ}$.

Such a solubility means that as sodium urate, 100 cubic centimeters of blood plasma would hold in solution at the temperature of the body only 8 milligrams of the lactim form and 18 of the lactam form. This is not in harmony with experimental facts. One can get ten times as much monosodium urate into solution in blood plasma, combined in such a state at least that it does not settle down or precipitate out. It is possible that this urate is held in physical adsorption by the colloids. It is also possible that it is held in complex combination with the traces of pyrimidins that are present in the blood. With pure solutions in water one may employ physico-chemical methods of measurement to determine whether the urate is in solution; in the blood these methods cannot be well applied with accuracy. On account of the concentration of carbon dioxid, the blood is practically neutral so that the measurement of the electromotive protential leads to no result, as the potential of the urate is covered up. The method of conductivity cannot be used because of the number of other substances there present that are good conductors; and the slight dissociation of the urate is again covered up. But if blood serum be shaken with sodium urate and allowed to stand over night under carbon dioxid pressure, filtered the next day and the uric acid of the clear filtrate determined, it will be found to be ten times as much as water would have dissolved under the same circumstances.

A widespread misconception prevails concerning the possibility of altering the solubility of the urate in the blood by supposed alteration of the reaction of the blood-less alkalinity lowering the solubility, greater alkalinity increasing the solubility. Now there is in the blood no alkaline reaction to lower, and no known way to lower it if it were there to be lowered. There is perforce no alkaline reaction to augment, and no way to increase it if it were there to be increased. The blood is practically neutral and with the greatest tenacity the body maintains this state of neutrality. There is no foundation for the assumption of a lessened solubility of the urate in any state of health or of disease. And there is no foundation for the assumption that the solubility of the urate can be increased therapeutically. There is in the body no other equilibrium, not even the body temperature, so finely adjusted 
and so perfectly regulated as the neutrality of the blood. If the reaction of the blood could be increased or decreased with the ease postulated by the proponents of the uric acid diathesis, life would be impossible.

Slight as is the solubility of the urate in the blood plasma, it is vastly in excess of the usual requirements. When it is realized that on an average not over 1 gram of uric acid is eliminated in the twentyfour hours, it is clear that in any one moment there can be but a trace of uric acid in the total blood of the body. And in the amount that can be secured for analysis, it is very small indeed. This all, of course, is on the assumption that there is no threshold value for uric acid. As a matter of fact, it is very difficult to prove the presence of any uric acid in 200 or 300 cubic centimeters of normal human blood. When this can be done, it is in itself a pathological finding. There are conditions in which the purin output is very greatly increased, as in leukemia; but in leukemia the blood content of uric acid may be so low as again to baffle demonstration-it may, however, be over 0.010 per 100 c.c. of blood. It may, therefore, be assumed that there is normally no threshold value for uric acid; apparently the kidneys attempt with uric acid, as with urea, to effect a complete elimination. This is obviously not a question of solubility, it is a question of the relations of urate to the renal cells. Uric acid is very insoluble, even in the state of the most soluble urate; urea is very soluble. But for both the kidney attempts a complete elimination, so that the circulating fluids are practically free of them.

Eliminating Power of Kidneys for Uric Acid.-While it is apparently true that there is not a threshold value in the kidney for uric acid, and direct tests in nephritis seems to indicate that it is not a particularly irritating substance, yet there appears to be but little leeway in the power of eliminating uric acid, and an increase in the input that must be termed moderate may lead to accumulation in the blood of the normal individual. A meal rich in sweetbread may lead to high values of uric acid in the blood, up to 5 milligrams in the $100 \mathrm{c.cm}$. The accumulation represents a stagnation; the uric acid is poured into the blood faster than the kidneys can eliminate it. There are many pathological illustrations of this form of hyperuricemia. When one analyzes the figures, it becomes clear that the renal power of elimination of uric acid tolerates a surprisingly small overload. If sweetbreads were ingested so as to treble the total amount of output of uric acid, this would be a massive input. To make the relations quantitative let us assume that all the extra uric acid is to be eliminated during six hours following the ingestion, and that during this six hours the total uric acid of the sweetbreads, 2 grams in all, will be gradually poured into the blood. If all this uric acid were to be poured at once into the circulating blood, it would correspond to a concentration of $1: 6000$, far below the solubility of monosodium urate in water even, in which the urate is less soluble than in the blood. 'This 2000 
milligrams would be eliminated during six hours, an average of about 5 milligrams per minute. And this the kidneys, with all the enormous circulation they possess, often cannot accomplish without an accumulation of uric acid occurring in the blood. The amount of blood going through the two kidneys during a minute must be very large and would contain many-fold the amount of uric acid that is eliminated in the minute. While there is not normally a threshold value in the kidney for uric acid, it is clear that the permeability (or rather power of elimination) of the kidney for uric acid is very low and tolerates little overload. It is possible that it is the uric acid in complex combination that resists elimination, but that would really mean an indirect threshold value. Exaggerated input of exogenous purin is the only physiological moment that is known to raise the uric acid content of the blood to the point of certain chemical demonstration, which may be set at a milligram in the 100 c.c. of blood. It must not be inferred that excessive ingestion of exogenous purin is always followed by an accumulation of uric acid in the blood, there are wide individual variations in this regard. Noteworthy also is the fact that marked increase in the concentration of uric acid in the blood does not seem to lead to increase in uricolysis. The accumulated mass of uric acid remains in the blood for a number of hours, during which time the kidneys gradually accomplish its elimination. There is no other known substance in relation to which this peculiar behavior of the kidney is duplicated. And it is important for the correct evaluation of the data bearing on the pathological accumulation of uric acid in the blood and tissues, that this state of affairs should be clearly understood.

The factors in the metabolism of the purins may be illustrated in schematic form as follows, the endogenous purins being in usual type and straight lines, the exogenous purins underscored and in dashed lines.

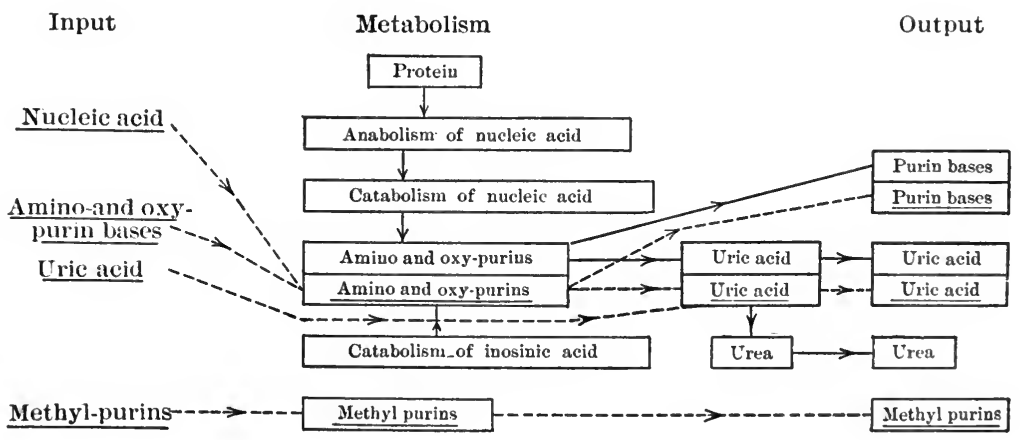

From this chart it is clear that the estimation of the uric acid or the total purin of the urine, cannot be regarded as an index of the total production of purin (even if the exogenous purin be excluded) or of the nucleic metabolism. 
The following schematic chart illustrates the relations of the protein, purin and creatinin metabolisms, the two latter being represented as free of exogenous input, exogenous protein being intentionally retained.

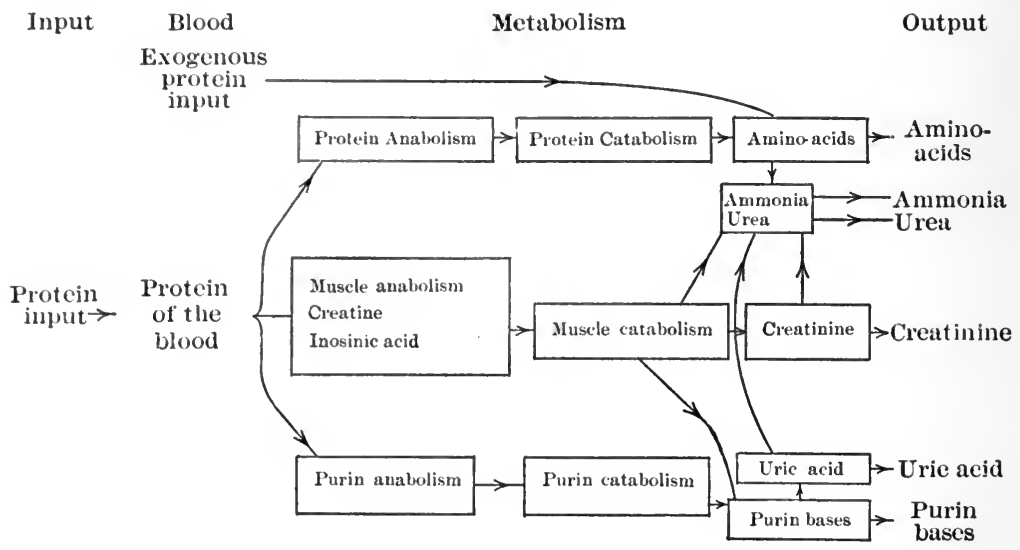

\section{PATHOLOGICAL VARIATIONS IN THE PURIN METABOLISM}

Herein are included variations in purin anabolism; variations in endogenous purin catabolism; variations in the exogenous purin catabolism; accumulation of purins in the blood; variations in uricolysis; variations in the urinary output of uric acid and in the curve of the elimination of uric acid; and deposition of purins in tissues.

Normally there is but one cause of excess of purins in the body, blood and urine--the ingestion of excessive exogenous purins. The results of such excessive ingestions are temporary, and lead to no derangement of the nucleic metabolism, so far as known. Though the renal power of elimination of uric acid is limited, there is no direct evidence that the regular and even excessive ingestion of exogenous purins (the methyl purins excluded) leads to renal or other disease.

There are no known variations in the processes of nucleic anabolism. In the most excessive multiplication of cells, the synthesis of purins is apparently fully competent.

Various in Endogenous Purin Catabolism.-In all conditions in which excessive cell destruction occurs in the body, the catabolism of purins must be excessive. Leukemia, pneumonia, septic leukocytosis, internal collections of pus, rapid degenerations of tissues as sometimes seen in neoplasms, and acute degeneration of the liver lead to excessive purin catabolism. Just as an excess of ingested nucleic acid is catabolized, so an excess of endogenous purins is catabolized. In leukemia we may observe five or more times the normal magnitude of nucleic catabolism. Both uric acid and the purin bases are increased in the urine, the uric acid most of all, though it is not uncommon to find 
the values for purin bases double the normal. The condition is most often temporary, but in leukemia it may last for months. When exogenous purins are added in experiment, they too are elaborated in the usual manner. One cannot resist the impression that the one weak spot in the whole nucleic metabolism lies in the function of elimination by the kidney.

So far as known there are no variations in the endogenous purin metabolism in gout. The term "gout" is here used in its strict sense, without reference or relation to the term "uric acid diathesis." This last-named euphonious expression was widely used for several decades as a cloak for ignorance. But just as fashions in furs change so fashions in the cloak of ignorance change; and the uric acid diathesis has been lately relegated to the closet for old clothes. There is an hypothesis that the total purin metabolism is reduced in gout. This hypothesis is devoid of foundation and is in contradiction to our best knowledge. So far as known the anabolic and catabolic series of reactions of endogenous nucleic acid are normally carried out in the subject of gout. There is a possibility that the oxidation of uric acid is reduced in gout. Uricolysis is, however, not to be regarded as a portion of the catabolism of purins, in the usual sense of the term. If we are to use this term in the sense of including the oxidation of uric acid, then in man the normal catabolism of purin is largely unfinished; and certainly the term ought not to be used in this sense.

The catabolism of the total exogenous purins can be shown to be retarded in gout. Does this carry with it the inference that the catabolism of the endogenous purins is retarded in gout? The possibility cannot be denied. But from the mere fact that an overload cannot be carried, in gout as well as in health, it does not follow that the normal load cannot be carried. The increased concentration of uric acid in the blood in gout would naturally lead one to expect the catabolism of the purins to be retarded. But in other conditions in which equal increase in the concentration of uric acid occurs in the blood, such a retardation is not observed. Admitting, therefore, the possibility of such a retardation in gout, there is no proof of it.

Variations in the Catabolism of Exogenous Purins.-In gout this is distinctly retarded, though qualitatively normal. A sweetbread ingested remains in the metabolism for several days, instead of being completely catabolized in one day as in the normal. This is not to be observed in all cases, but is usual in the event of symptomatic gout. There is no evidence that the catabolism is incomplete; it merely requires more time for completion. This seems to be characteristic of gout.

The Blood Content of Uric Acid.-This is increased as a constant condition in chronic nephritis, in lead poisoning, in gout, and in some cases of leukemia; temporarily, it occurs in pneumonia, sepsis, and following the excessive ingestion of exogenous nucleins or purins. It may be as high as 6 to 10 milligrams in the 100 cubic centimeters 
of blood, but is rarely over 5. But 5 milligrams is ten times the normal concentration. In gout this persists despite starvation, and we may assume that this holds for the other chronic diseases named. The several conditions are to be divided into two groups: those in which the increased concentration of uric acid in the blood is the result of an exaggerated purin catabolism, due either to exogenous or endogenous purins; and those in which there is no increased catabolism of purins. Nephritis, lead poisoning and gout belong to the last group, all the others to the first group.

Since the abnormal concentration of uric acid in the blood in gout, lead poisoning and nephritis is not due to an increased formation of uric acid, it must be due either to a reduction in total elimination, or to renal impermeability. That there is retention of uric acid in gout is true, since tophi are formed, which is not the case with lead poisoning and nephritis. But the amount retained is so slight-10 milligrams per day would account for all the tophi in a gouty body-that this cannot be a reason for the increased concentration in the blood. Observations of cases of gout, nephritis and lead poisoning prove that the total elimination of uric acid is normal in time. In acute gout, as will be later pointed out, there are fluctuations. But these are compensatory. Week in and week out the subjects of gout eliminate the normal amount of uric acid-minus, of course, the trivial retentions in the tophi. There remains but one explanation: the permeability of the kidney is lessened. In other words, a threshold value has been established. When the blood contains, let us say arbitrarily, 3 milligrams of uric acid per 100 c.c. blood (this will vary from case to case as all values do in the purin metabolism), the kidney is able to eliminate the full amount of uric acid formed in a normal manner. A similar increase in the threshold value for sugar seems to occur in diabetes; a lowering occurs in phloridzin poisoning. We are able to give no explanation for this phenomenon. It is not due to nephritis, as the term is ordinarily used, though it occurs in practically all cases of sclerotic nephritis. Gout, like nephritis and lead poisoning, causes a change in the kidneys whereby the threshold value for uric acid is raised. Only when the concentration of uric acid in the blood is increased is the kidney able to accomplish the normal quantitative elimination. The fact is not peculiar to gout nor does it lead to gout; it is rather a result of gout.

Variations in Uricolysis.-Recent investigations tend to indicate that in gout the oxidation of uric acid is reduced, in contradistinction to lead poisoning and nephritis. The demonstration is not complete; it cannot be convincing until we have a procedure wherewith the oxidation of uric acid to urea can be measured. That the catabolism of nucleic acid to the stage of uric acid is in gout retarded in time, is no proof that the oxidation of uric acid is retarded or lessened, since we are dealing with independent reactions. The increased concentrations of uric acid in the blood ought to lead to increase rather than decrease in uricolysis, other factors being constant. And as a matter of fact, in 
many cases of gout the total output is below the normal; and many competent students of gout regard the uricolysis as excessive in these cases, assuming of course, what is generally conceded, that the formation is normal. One must not follow the direct lead of the figure of output. The output of uric acid is often low during the few days before an attack; following the attack it rises, and the excess surpasses the previous retention. Between attacks the figure may be low. Now it will not do to say that the increase following the attack was due to decreased uricolysis, and the low figure between attacks was due to increased uricolysis. The low figure between attacks may have been due to deposition, the high figure following the attack to resolution. The blood content also may vary. In some cases the total added elimination following the ingestion of sweetbread is less than expected; in these cases an increased uricolysis has been invoked. How varying are the experimental data is shown in the fact that in one case of gout the direct injection of urate into the muscles was followed by a normal and quantitative elimination. Possibly increased or decreased uricolysis may be present in different stages of the disease; but of crucial importance they are not. And in any event the stated variations have not been properly established.

Variations in the Urinary Output of Uric Acid and in the Course of its Elimination.-Cases of gout may be grouped under three headings: (a) In many chronic cases there are no abnormalities. (b) In most acute cases there is a drop in the output of uric acid before the attack, followed by a marked rise in elimination after the attack. (c) In some instances there seems to be a slight reduction in the regular output of uric acid. The order of events in relation to the acute cases could be explained by the assumption of an increased concentration in the system prior to the attack or even between attacks, followed by elimination subsequent to attack - the increased concentration having been possibly a factor in the inauguration of the attack, the sweeping-out a factor in the recession. 'The postulated reduction in the output between attacks has been explained as due either to decreased formation, increased uricolysis or deposition within the tissues. As a matter of fact, the figures for this reduced elimination are not convincing. In any event the amounts concerned are small. We cannot assume what is not known-the individual standard of the gouty subject in the state of health. All of the figures quoted in support of this assumed reduction in elimination may be duplicated in normal urines. On the one hand we see the variations in the output of uric acid regarded as the cause of the attack of gout; on the other hand, we might regard them as the result. But it is certain that the same variables occur in the non-gouty, without the production of any of the results supposed to follow in their train in the subject of gout.

The Deposition of Urate in Tissues.-This is the cardinal feature of gout, though it is certain that there is gout without any urate deposition. In some subjects the depositions form gradually, without any 
acute symptoms. In many cases the acute attacks centre about the local deposition, or at least about inflammation surrounding the local deposition. Here lies the crux of the situation in gout. Two facts are certain. Cartilage tends to precipitate urate from solution; and the local deposition in gout occurs in healthy cartilage (in the kidney and liver as well) and not as the result of necrosis or injury. The tendency of cartilage to precipitate urate of sodium can be shown in experiments in glass. Cartilage contains a great deal of sodium; and in accordance with the theory of solution, if saturated solution of sodium urate be combined with a solution of a sodium salt, the urate will be in part, precipitated. Thus a saturated solution of sodium urate is in part precipitated by the addition of sodium chlorid. This factor could not become operative except with saturation of sodium urate in the blood, a condition probably attained in gout. But in leukemia, nephritis and lead poisoning the same degree of saturation of the blood with urate of sodium does not lead to the precipitation of urate in the cartilages. It follows, therefore, that there must be an additional factor. This factor was once sought in necrosis or injury; the latter in part, to explain the selection of certain joints. But it is clear that the local necrosis is the result and not the cause of the attack of gout. The moment or factor that determines the precipitation in cartilage of sodium urate from the blood in the subject of gout, in contradistinction to other diseases in which saturation of the blood with sodium urate is equally present, is not known. There is something specific in gout; the attack of gout is not a mere physio-chemical reaction. Hyperuricemia is probably necessary for the occurrence of an attack of gout, or gout of any kind; but all cases of hyperuricemia are not gouty-there is some specific factor that makes an individual with hyperuricemia gouty. Nor can the difference between the solubility of the lactim and lactam forms of uric acid be regarded as the cause of the precipitation of urate in the cartilages. Equally unknown but especially suggestive of a specific etiology are the toxic symptoms of the acute attack of gout. The fever and other toxic symptoms are not to be explained by matters of concentration of urate of sodium and the deposition thereof in small tophi, nor by the occurrence of small areas of inflammation in cartilage. The fulminating nature of some of the attacks is only another argument for a toxic basis. 'That this lies in the toxicity of uric acid itself, will scarcely be advocater.

It is scarcely necessary to advert to the older view that variations in alkalinity bear a relation to the attack of gout. The postulated decrease in alkalinity would, could it occur, tend to dissolve sodium urate, not precipitate it. As a matter of fact the reaction of the blood and cartilages in gout is neutral, just as it is always neutral in the body. For the diagnosis and therapy of gout two facts are always to be emphasized: that the reaction of the blood and tissues is not altered in gout; and that the amount of uric acid formed in catabolism and the amount 
circulating in the blood cannot be learned by the determination of the uric acid output of the urine.

For the immediate present it is clear that to obtain an idea of the nucleic metabolism, the purin output and the purin content of the blood must be determined in the absence of exogenous purin; and following this the velocity of the exogenous nucleic catabolism should be tested by the administration of sweetbread and the determination of the curve of purin elimination. The total information will often be disappointing; but as yet there is nothing else upon which we may found an opinion as to the state of the nucleic metabolism.

\section{THE FATE OF THE PYRIMIDIN BASES}

The urine contains traces of cytosin and uracil. Otherwise these bases seem to be burned in the body. Of the relations of these substances in the catabolism of nucleic acid, we know nothing. The addition of these bases to a solution of uric acid results in resistance of the uric acid to precipitation, and from this it has been inferred that a complex between the pyrimidin and the purins has been formed. As stated in another section, more uric acid can be gotten into solution in blood serum than in water. This may exist in some such complex; or it may be held in adsorption. An hypothesis for the explanation of the precipitation of urate in gout has been advanced in the suggestion that the occasion for this precipitation lies in the absence of the pyrimidins with which the uric acid forms a soluble complex. Experimental work has not yielded results favorable to this hypothesis. On the other hand, the fact that uric acid will dialyze from a solution in blood serum is not an argument against its existence there in such a state of complex. There would, of course, be an equilibrium in the two states: sodium-urate-in-solution and sodium-urate-in-combination. Dialysis would lower the amount of sodium urate in solution, which would be followed by restoration of the equilibrium by dissociation of the urate in complex combination; and this continuing, urate would dialyze out in excess of the amount present in simple solution. The application of the phase rule and the law of partition would also make possible the dialyzation of uric acid from a system in colloidal adsorption. Adhering, therefore, to the view that sodium urate may be held in the blood in part in chemical combination or physical adsorption, there is no evidence that the defect in gout lies in the lack of these in the subject afflicted.

\section{THE METABOLISM OF PHOSPHORUS}

Phosphorus exists in the body in three important situations: in organic combination in nucleic acid; in organic combinations in the 
lipoids of the central nervous system and elsewhere; and in inorganic combination in the skeleton. Phosphorus is introduced in organic combination in the nucleic acids and phosphorized lipoids of the diet, and as inorganic salts. When ingested in organic combination it is set free in the processes of digestion; and all phosphorus is apparently resorbed in the inorganic state. Phosphoric acid, in the form of the salts of sodium, calcium and magnesium, constitutes therefore the substrate of the anabolism of the phosphorized lipoids and the nucleic acids.

Phosphorus is eliminated through the intestinal tract and kidneys; there is no cutaneous elimination of phosphorus. In the feces are phosphates and residues of organically combined phosphorus. In the urine are phosphates and a small fraction of neutral phosphorus, less usually than 100 milligrams per day of phosphorus. The renal and intestinal eliminations of phosphates are reciprocal. Usually the larger fraction passes out in the urine, but the larger fraction may be eliminated in the stools. The presence of large amounts of calcium and magnesium in the diet tends to deflect phosphates from the urine to the feces. There are no known metabolic relation concerned in the route of elimination.

Assuming that the phosphorus content of the skeleton is stationary and that the phosphorus output of the central nervous system is minimal, the phosphorus output, on a known and controlled input, may be assumed to represent the intensity of the nucleic catabolism. A phosphorus balance in health is easily secured; and it is fairly certain that the amount of phosphorus eliminated daily from the central nervous system is very small and quite constant. As a matter of fact, under controlled conditions, the total phosphorus and the total purin outputs tend to run parallel; and it is possible that adequate and supplementary data may indicate that the total phosphorus elimination is a measurement of the variable of nucleic catabolism-something very much needed. It must be realized that control of the phosphorus input is of crucial importance in such investigations. At present it seems unlikely that with the nucleic catabolism assumed as constant, the elimination of total or neutral phosphorus can be employed as a measurement of the catabolism of the substance of the central nervous system - the amounts involved day by day are far too small. 


\section{CHA PTER VIII}

\section{AUTOINTOXICATION}

THE specific subject of autointoxication does not lie within the scope of this treatise. Nevertheless, reference has naturally been made under different headings to pathological variations definitely known to exist, including autointoxications. It is, however, permissible, in this connection and in the absence of a systematic survey of the subject, to state the point of view in the problem of autointoxication that must be entertained by the student of metabolism. The student of chemical metabolism naturally occupies a more conservative position than the outposts of which the student of organ function has taken possession. He requires a certain association between signs of disturbed functionation and known steps and stages of metabolism. It is for him not enough that the removal of an organ or a particular operative interference should be followed by a symptom-complex, sometimes clear-cut but often very indefinite. It is, in part, because of the failure to attempt to correlate in a specific manner these symptomcomplexes with established data, that the writings on the theory of hormones and the literature on the experimental investigation into the interactions of internal organs present such a mass of contradictions. It is clear that in the near future a treatise on autointoxications will be written on the basis of the internal functions of organs in the light of chemical knowledge of metabolism.

From the point of view of the theory of metabolism, there are a number of ways in which an autointoxication may be established. But in the very beginning it is necessary to define the term, which has been used so loosely as to have almost lost concrete meaning. We may define the term by what it does not contain as well as by what it does contain. Excluded are all forms of exogenous intoxication, such as arise following the ingestion of known or undefined toxic substances and of decomposed food. Excluded are all intoxications due to decomposition of food within the alimentary tract; and all infections and results of bacterial life here. Bacteria in the alimentary tract may in one of three ways injure the organism that harbors them. They may deprive the body of needed components of diet. No instance of such action has yet been discovered, but such action might be conjectured; for example, if bacteria should ferment completely the tryptophan set free in the digestion of protein, the body would be unable to maintain a nitrogenous equilibrium and eventually would fail in protein anabolism. Bacteria might conceivably alter the products of digestion and render them unavailable for the processes of anabolism. Secondly, bacteria may form toxic substances in the alimentary tract from the media upon which they are maintaining their 
existence, just as bacteria form poisons from media in test-tubes. Thus from starch large amounts of butyric acid may be formed. It is known that normal meat undergoes a toxic decomposition in the digestive tract of the animal containing a culture of the bacillus of Gaertner. To what extent such forms of bacterial intoxication occur is not known. Lastly, bacteria may intoxicate through the development of specific infective processes, by the elaboration of specific toxic substances. The infections of the alimentary tract are just as exogenous as are the infections of internal organs; why then are bacterial gastro-intestinal infections termed autointoxications, while typhoid fever and meningitis are called infectious diseases? It is not possible as yet to separate into clinical classes (or even in the laboratory) the two groups of diseases resulting from bacterial action within the alimentary tract; but the fact that none are autointoxications should be realized. The slight bacterial infections of the intestinal tract are, in fact and theory, as truly microörganismal infections as is cholera.

A true autointoxication of the alimentary tract would be related to increase or failure of a secretion; to defect in the digestive processes associated with the formation of a toxic agent; or to the evolution of some toxic substance from the foodstuffs through an abnormal qualitative action of the chemical processes of digestion. Thus, hyperchlorhydria might be termed an autointoxication; and obstructive jaundice is a typical autointoxication by retention of an end secretion of metabolism. We know of no toxic state related to the failure of the processes of digestion, bacterial action being excluded. The resorption of unaltered foreign protein might lead to anaphylactic sensitization. The absorption of the intermediary stages in the digestion of protein, such as peptone, might lead to intoxication if not nullified in the resorption membrane. We know of no abnormal qualitative deflection in the chemical reactions of digestion whereby poisons are formed from the foodstuffs. Future investigations may indicate that such exist. But when one recalls that the processes of digestion are hydrolytic cleavages and lead to the splitting of the large molecules of the foodstuffs into simpler preformed components, it is difficult to understand how any qualitative deflection could result in the formation of toxic substances. In the reconstruction processes in the wall of the small intestine lie, however, possibilities for the origination of toxic substances - a theoretical possibility for which we have no evidence of actual occurrence.

Inside the body the possible modes for the development of true autointoxications are clearly outlined by our knowledge of metabolism. We may state the possibilities under several headings:

Autointoxication by retention of end products of metabolism.

Autointoxication by incompletion of reaction series, whereby an intermediary product becomes an end product or accumulates in the body.

Autointoxication through the failure of reactions of distoxication.

Autointoxication through the formation of a qualitatively abnormal substance in the metabolism. 
Autointoxication through quantitative variation in a normal substance.

While it is clear that many well-known clinical states of autointoxication (such as aseptic fever, the complex that follows extensive cutaneous burns, the condition associated with exposure to high temperature, Addison's disease, the cachexia of thyroidectomy, parathyroid tetany, idiopathic tetany, myxedema, etc.) cannot be elucidated now upon any such basis, illustrations are at hand for each of these classes, either clinical or experimental. These, with the restriction in the use of the data and concepts necessitated by our knowledge of metabolism, will be briefly sketched.

Autointoxication by Retention of End Products of Metabolism.-To the view of the lay mind, this has always been a natural concept of selfintoxication, since with the psychological aversion usually displayed toward the excrementa, the idea that they were of poisonous nature became universal. To the student of metabolism, it was much more logical to assume, for the urine at least, that the processes of metabolism would tend to the final production of end products of innocuous nature. The feces on account of the bacteria they contain are in a sense foreign to the body. As a matter of fact, with one exception we are not now in possession of information connecting any known autointoxication with any end product of catabolism; and with this one exception, the end products of catabolism are known to be relatively if not absolutely innocuous.

The end products of catabolism contained in the expired air are carbon dioxid and water, under normal conditions. The expired air contains no nitrogenous substance. It may contain volatile bodies from the diet-such as alcohol, essential oils from spices and vegetables, aldehyds from fruits-it may contain volatile products of the fat metabolism (such as acetone and probably aldehyd-like substances); but the elimination of end products of metabolism apart from carbon dioxid and water does not occur by this route so far as known. In direct disagreement with these statements are popular ideas on the effects and symptomology of contaminated air. It has been lately shown that the expired air of one species can sensitize an animal of another species following parenteral introduction. This, however, is a specific protein reaction, and can have no relation to the vitiation of air by human beings for human beings. When the effects of the breathing of over-respired air are analyzed, a number of different elements are presented. The air is warmer and the humidity greatly increased. There are the odors of the cutaneous secretions, which rapidly undergo bacterial decomposition. There are the artificial odors of fashion, often even more distasteful. Lastly, there is the pronounced psychic or esthetic effect seen in the refined classes, usually more pronounced in women than in men. Whosoever would rest the influence of over-respired air upon purely chemical grounds must explain in some way the entire absence of the supposedly pathognomonic symptoms in men who, in dense crowds, frequent political meetings 
and drinking quarters. Doubtless reduction of oxygen and increase in carbon dioxid, promoting more rapid respiration, do exaggerate the action of the above-named influences, though there is no evidence of real oxygen hunger or carbon dioxid congestion in the crowded rooms with which we are acquainted in modern life. As to the air of theaters, now so much under discussion, it is certain that vitiation proceeds less from the audience than from the stage. ' It is possible that future studies may demonstrate in the expired air highly toxic substance of volatile nature in extreme attenuation. But for the present we have no metabolic conception of such toxic volatile substances and no chemical data that in any way afford presumption of their existence.

The skin is an organ of elimination of end products of the nitrogenous catabolism. Whether these are only incidentally eliminated, like the salts, in association with the elimination of water; or whether the elimination is a true specific secretory function, is not known. The present data favor the interpretation of elimination as an incidental secretion. The amounts concerned are not large, though they include all the nitrogenous end products. The elimination is not variable except as the output of water is variable; it rises with the water output, falls with the water output, and the usual elimination (0.2 to 0.3 gram N) represents the unconscious perspiration. The so-called vicarious action of the skin for the kidneys has failed of concreted demonstration. Of course the nephritic subject will eliminate more nitrogenous end products on forced sweating; so does the normal subject. That even under conditions of increased concentration of nitrogenous end products of metabolism, exceptionally large eliminations occur through the skin, naturally or provoked, is difficult of demonstration. Crusts of urinary salts have long since ceased to form upon the dry skin of patients with uremia. In any event, an increased elimination would be expected, in accordance with the law of diffusion, from subjects whose blood contains an increased concentration of these substances, both in natural and in provoked sweating. It is possible that future investigations may reveal in the cutaneous secretions highly toxic substances in great attenuation, but we have no data tending to suggest such an elimination. The autointoxication that follows superficial burns of wide extent is not dependent on reduction in the function of cutaneous elimination.

Obstructive jaundice is a perfect illustration of an autointoxication through retention of the end products of metabolism. Whether the toxic action reside solely in the biliary salts and pigment, or is to some extent related to the other bodies contained in bile, as to the lipoids, is not known.

The end products of metabolism eliminated in the urine have long been invoked as the causative factors in autointoxication, especially in uremia. It must be admitted that there are no gaps in the attempts to lay uremia at the door of the end products of the nitrogenous catabolism. In historic succession uremia has been attributed to urea, 
to ammonia, to the purins, to creatinin, and now the attempt is being made to relate it to the rest-nitrogen. It is true that in some of the stages of nephritis, one or all of these may be retained. Such periods of retention are the results of nephritis; there is no evidence that the substances retained provoke uremia, there is indeed the best evidence to the contrary. The rest-nitrogen is apparently the hardest to eliminate, and it is perhaps always retained to some extent in nephritis, apparently with an increased threshold value in the blood. But there is no evidence that the substances included in the rest nitrogen are the toxic causes of uremia. 'The salts of potassium were once incriminated; now it is the chlorion. Of course a toxicity may under forced conditions be dęmonstrated for each of these substances; but such a toxicity may not, with our present clinical, chemical and experimental information on uremia, be invoked as the etiological factor in this intoxication. The retentions and the uremia may go hand in hand, fellow-results of nephritis; but they do not always occur together, there is retention without uremia, there is uremia without retention. To appeal finally to the summation of the toxic actions of these several substances as the cause of uremia, is begging the question. The cause of uremia is as unknown today as in the day of Bright.

Autointoxication by Incompletion of a Reaction Series, Whereby an Intermediary Product Becomes an End Product and Accumulates in the Body.-From the standpoint of theory, this aspect of the question of autointoxication offers much promise. The ketonic intoxication of diabetes, the acidosis of b-oxy-butyric and acetoacetic acid (our bestknown autointoxication) is of this nature. The series of reactions of combustion is incompleted and the body is flooded with the ketonic acids that abstract bases from the tissues and exhibit their own toxic properties. The body attempts to nullify this by the reduction of acetoacetic acid to acetone, and outside of diabetes often and in diabetes for long periods of time succeeds; but this reaction all too often fails, and autointoxication results. Cystinuria represents such a situation chemically, but there is no toxicity of the intermediary product. Under the commonly prevailing view of alcaptonuria, the homogentisic acid is regarded as an unoxidized intermediary substance, again however, without toxicity. One of the most recent hypotheses of the cause of fever attributes to unoxidized purin bases a toxic action upon the heat centre-without justification in the opinion of the writer. Cholin, a very toxic base, is normally set free in the catabolism of the white matter of the central nervous system. It is in some way catabolized, for it is not present in normal urine, at least in more than infinitismal traces. But in conditions of widespread degeneration of the central nervous system, more cholin is set free than can be transformed, and it appears in blood and urine free. Possibly some of the symptoms of these nervous diseases may be due to the toxic action of the excessive amounts of cholin, though this is not demonstrated. The flooding of the blood with lactic acid in phosphorus poisoning represents another instance of the appearance in the system of an intermediary product. 
Hematoporphyrin is the intermediary substance in the formation of bilirubin, and is present in the urine in many states of intoxication. And finally, the symptoms of the $\operatorname{dog}$ with Eck fistula, according to the most recent investigation, may under certain circumstances, be related to an alkalosis, again an incompleted reaction. A reaction series may be checked in one of two ways: either a substance or ferment or other condition necessary for a particular stage is lacking; or some substance enters and by combination with an intermediary body, effects its withdrawal from further reaction. It is after the last-named fashion that acids check the formation of urea from ammonia, that benzoic acid checks the oxidation of glycocoll, that sulphuric acid checks the oxidation of normal phenol. The occurrence of cystinuria, alcaptonuria and of ketonic acidosis cannot be explained on this basis; here some conditions essential to the reaction have failed or become inoperative. It is certain that future investigations will find this a fertile field for studies in autointoxications.

Autointoxication through Failure of Reactions of Distoxication.-This is a most probable cause of autointoxication, though little work has been done. The infectious diseases are recovered from through what amounts to a reaction of distoxication, the reaction of immunity. The body protects itself from innumerable exogenous poisons, when administered by the mouth, by reactions of distoxication. Metals are eliminated distoxicated in the state of some organic combination that is undissociated. Foreign organic substances are combined with metabolic components so as to render them innocuous, good illustrations being the conjugations of phenol and camphor in the liver. Under these circumstances it would seem certain that similar reactions occur within the domain of metabolism. Recent very suggestive investigations on variations in the toxic action of acetonitril indicate extremely close relations between metabolic states and distoxication (or toleration) of toxic bodies. It may well be queried if such are not frequently present between metabolism and product of metabolism. Possibly the interactions of internal organs are, in part, to be elucidated upon some such basis. Why should not the actions of hormones lie in the direction of distoxication as well as in the direction of stimulation? Certainly the field promises much to research.

Intoxication through the Formation of Qualitatively Abnormal Substances in the Body.-When one considers the number of labile organic substances in the body, which is a chemical reaction-system, the wonder is that qualitative deflections do not occur more frequently. Under the most recent interpretation of the origination of alcapton, this substance is not to be regarded as a normal intermediary body, but rather as an abnormal substance. If we regard the pentose in idiopathic pentosuria as derived from glucose, this constitutes a reaction with the production of an abnormal substance, since the normal pentose of the nucleic acid is not the same as the pentose that is formed and eliminated in this disease. We know of no clinical autointoxication in which the formation of a new substance has been definitely estab- 
lished; but the field, for reasons of methods as well as for reasons of view-point, has not been even superficially explored.

Autointoxication through Quantitative Variation in the Production of a Normal Substance.-We have many illustrations of such exaggerations in the quantitative flow of metabolism, but so far no definite autointoxication has been founded thereon. In leukemia there is an enormous exaggeration in the catabolism of nucleic acid; but the presence in the body of the purins even in these amounts does not appear to intoxicate. In a similar manner, in conditions of great exaggeration of the protein catabolism, the end products of this function are strikingly increased. The body evidently tolerates a large overload in normal function and in the concentration of normal substances. Possibly aseptic fever may be ranged with this type of autointoxication, due to huge exaggeration in certain disintegration products of cells.

To estimate properly any concept of autointoxication, the rôle of compensation and adaptation in physiological and chemical functionation must be borne in mind. Organic lesion usually does not produce disturbance in total chemical function; an overload is tolerated, a deficiency is put up with. Just as we must not expect alterations in chemical function with all lesions of the glandular organs; so we must not expect autointoxication with all disturbances in metabolism. Autointoxication may be immediately related to slight metabolic disturbance; it may never appear with a gross variation. So much depends upon the specificity of the particular function, upon the chemical properties and relations of the substance concerned. A chemist contemplating urea would not expect it to be a toxic agent; looking at histidin or cholin he would expect them to be the substrates of reactions whereby highly toxic agents could be easily evolved. This is a point of view necessary to the successful approach to the problem of autointoxication.

There are other forms of apparent auto-intoxications that are not to be classified at present. The secretions of the intestine and of the pancreas are toxic on parenteral introduction, the pancreatic juice particularly so. The action of bacteria can be ruled out, in the infective sense, and possibly in the chemical sense also. The symptoms of the intoxications appear quickly after the introduction of the secretions, so quickly as to make it doubtful if the ill effects could be the results of the manifestation within the body of the enzymic properties of the secretions. Whether these toxic properties are to be related to a metabolic end product or to a specific secretion, has not been determined. What relation the toxic properties bear to the clinical diseases of the alimentary tract is entirely problematical. It is possible that these unchanged secretions could be resorbed under abnormal conditions. It is possible that some results of intestinal obstruction may be due to the resorption of the secretions. It is possible that the symptoms of acute pancreatitis may be due to resorption of the secretion. The available data do not warrant an opinion upon these questions. 


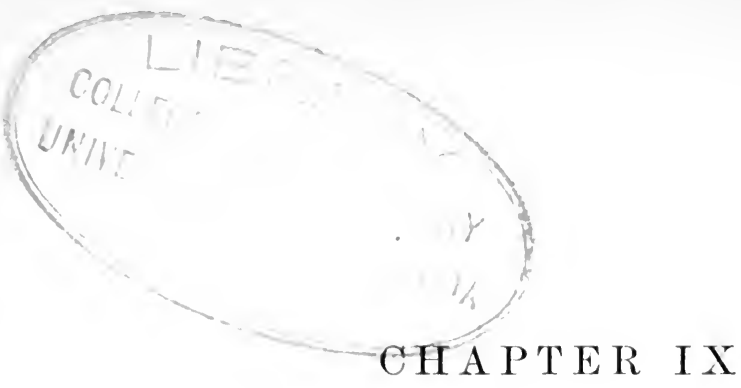

\section{METABOLISM CONSIDERED AS A WHOLE}

\section{GENERAL RELATIONS OF METABOLISM}

Wiren one contemplates the metabolic transformations in animals, it becomes clear that we are concerned with two main factors. There is firstly, a relation of metabolic transformation to mass of tissue, and this relation is one of direct proportionality. There is secondly, a coefficient of metabolic intensity, and this is according to modern conceptions to be related to the factor of the enzyme. The coöperation of these two factors, in different animals, results in the biological condition that different species have widely varying planes of total metabolic transformation. There are animals whose plane of metabolism and growth is tenfold that of man; there are animals whose plane of metabolism and growth is greatly below that of man. Within the species, variations in these two factors bring about individual variations in the metabolic processes. We are not able to determine accurately, or even approximately, in an individual the mass of cellular tissue, since build of skeleton, deposition of fat and water content are widely variable. From this point of view alone, we would not expect two bodies of the same kilo weight or surface area to present identical figures for metabolism. These variations disregarded, however, it is furthermore clear that variations in the coefficient of metabolic intensity must lead to variations in metabolism from individual to individual. Herein lies probably a variable more often and more strikingly operative than variation in the ratio of cellular tissue to total weight. This variation lies in the amount or conditions of operation of the enzymes. Some bodies produce more enzyme, or the conditions of fermentation are favorable; other bodies produce less ferment, or the conditions of enzyme action are unfavorable. In a word, we have represented in individuals the two factors of substrate concentration and enzyme concentration, and must expect to observe in the results the operations of the laws of mass action and of catalytic acceleration.

It is possible in a roughly approximate manner to obtain a notion of the relation of mass of metabolic tissue to transformation in the unit of time. The total energy-content of a body of 70 kilos in normal flesh may be estimater at 150,000 Calories. The basal heat production of such a body would be about 1800 Calories per day, in other words a daily transformation of 1.2 per cent. 'This basal heat produc- 
tion of the fasting and resting body is set free largely, if not entirely, by reactions of combustion in the muscular system. The mass of muscular system in such a body would be not over 30 kilos. Each kilo of muscle substance, therefore, transforms daily into water and carbon dioxid about 15 grams of glucose with the production of 60 Calories of heat. At heaviest work each kilo of muscle can burn per day 100 grams of glucose, with the evolution of some 400 Calories of heat.

The total protein content of a body of 70 kilos may be estimated at 12 kilos. The basal cellular protein metabolism, as will be detailed in this chapter, may be set at 40 grams of protein per day, a daily transformation of $\frac{1}{3}$ per cent.

The total protein content of the muscular system may be estimated at 8 kilos. The creatinin elimination of such a body may be set at 2 grams. The transformation coefficient is, therefore, about 4000:1.

The total nucleic acid content of the body, very roughly estimated from the purin and pentose content of tissues, may be set at 60 grams. The daily purin output of the body on the minimal ration of protein may be set at about 0.3 gram per day, a transformation of a little over 1 per cent. From these figures we may infer that the intensity of metabolism is greatest in the nucleic and least in the creatin-creatinin metabolism.

In all these calculations the conversions of purins and creatinin into urea have been disregarded.

These coefficients of reaction velocity are to be regarded as specific to the particular chemical reaction involved. And the figures, though only approximate, give us some idea of the relations of mass of tissue to metabolic transformation under normal conditions of ferment concentration.

\section{THE SPECIFIC DYNAMIC ACTION OF FOODSTUFFS}

The heat production of an animal depends to a large extent upon the external temperature. There is, of course, as will be later elucidated, a certain production of heat that is maintained even when the body is living in an atmosphere whose temperature equals or exceeds that of the body. But below this point, the production of heat depends in large part upon the difference between the external and the body temperature. If the skin were a perfect insulation, this adaptation would lie within much narrower limits, since then apart from warming the food and drink, the only needs for extra heat production would be for evaporation of water from the lungs and the warming of the inspired air. In certain marine animals the body probably operates upon this basis, which explains their ability to live in cold water whose abstraction of warmth would otherwise make enormous demands upon the production of heat. In the earlier days of metabolic experimentation, the 
reactions of the body of the animal under experimentation were studied without reference to the external temperature. It is now clear that this factor of external temperature is of enormous importance in determining the metabolism of an animal. Under certain conditions the ingestion of food may be followed by, or associated with, an exaggeration of the production of heat, $i$. e., the reactions of combustion are increased on the ingestion of food. The experimental results may be summarized in two statements: there is for each warm-blooded animal a temperature at which the ingestion of food equal only to the starvation catabolism will result in an increased production of heat; when the external temperature is lowered, the amount of food necessary to make evident such increase of heat production is greatly in excess of the starvation requirement. In other words, there is an excess of heat production over that of the fasting organism associated with the ingestion of food under all circumstances; but whether this excess of heat production becomes evident depends, apart from muscular work, upon the external temperature. If the animal be at work or living under a temperature less than $30^{\circ} \mathrm{C}$. (for the dog, this probably varies with different animals depending upon their coat), the extra heat produced on ingestion of food spares heat produced through chemical regulation or exertion, and the curve of heat production does not rise. But if the animal be resting or the external temperature be above $30^{\circ} \mathrm{C}$., the curve of heat production rises following the ingestion of food, and this extra heat is a total loss to the economy. The mechanism of chemical regulation of body temperature is not operative above $30^{\circ} \mathrm{C}$.; below this point it is operative, and the extra heat produced following the ingestion of food is of use to the body, since other combustion is spared to the extent of the extra heat thus set free. The heat production of an animal doing constant work at a constant temperature is uniform and not affected by the ingestion of food; under these circumstances, therefore, the extra heat evolved following the ingestion of food is made use of for support of work.

Heat Production.-Two sets of experiments make the relations clear. Under a constant temperature of $30^{\circ} \mathrm{C}$. a resting dog is found to have a total heat output of 750 Calories. If meat to the value of 750 Calories be administered in a day under comparable conditions, it will be found that the heat output is raised to possibly 1000 Calories. If the input of meat be raised to 1000 Calories, the animal will be in caloric equilibrium. If the same fasting dog be made to do a certain amount of work on an ergometer (22,000 $\mathrm{kgm}$.), so that the heat output is raised to 1000 Calories, it will be found that the ingestion of the same ration that in the resting dog raised the heat output to 1000 Calories will do just the same with the dog at the stated words. In other work, working or resting on a protein diet worth 750 Calories, the heat output of the dog will be 1000 Calories; the heat that is lost at rest is convertible into work under controlled and comparable conditions. The ingestion of the amount 
of protein corresponding to 750 Calories will not place the dog in a carloric equilibrium; nor need the ingestion of the amount of protein corresponding to the nitrogenous elimination of the fasting animal place the animal in nitrogenous equilibrium. When, however, enough protein is administered to the dog to attain nitrogenous equilibrium, it will be found that the output of heat is in excess of the heat production of the animal fasting at the stated temperature. The data with low ingestions of protein suggest the idea that the body is more efficient in the catabolism of its own protein than in the utilization of protein of the diet. It is possible, however, to show by exaggeration of the catabolism of the animal's own protein, a similar excess of heat production. This may be noted in a comparison of the nitrogenous output with the heat output in dogs suffering from phloridzin poisoning; for every unit of excessive protein catabolized, an excess of heat production of about 30 per cent. will be recorded.

When protein is administered in excess of the caloric requirements of the fasting body, with increasing ingestions the excess of heat production tends to become augmented. If a dog, for example, has a requirement of 1000 Calories, and is given meat worth 1500 Calories, the heat output will be about 1200 Calories. If the ingestion of meat be raised to 2000 Calories, the heat output will be some 1400 Calories. In a general sense, therefore, the larger the protein input in excess of the caloric requirements of the body, the greater will be the loss.

The relations are also made clear by the results of the administration of different known amounts of protein to dogs of known weight and caloric output at fixed external temperature. The following tables, taken from the studies of Rubner, make the facts clear:

\section{Calories per Kilo Body Weight per Day}

\begin{tabular}{|c|c|c|c|c|}
\hline $\begin{array}{l}\text { External } \\
\text { temperature. }\end{array}$ & $\begin{array}{l}\text { Fasting } \\
\text { output. }\end{array}$ & $\begin{array}{c}\text { Diet of } 100 \text { grams } \\
\text { meat, } 24 \text { Cal. per } \\
\text { kilo. }\end{array}$ & $\begin{array}{l}\text { Diet of } 200 \text { grams } \\
\text { meat, } 48 \text { Cal. per } \\
\text { kilo. }\end{array}$ & $\begin{array}{l}\text { Diet of } 320 \text { grams } \\
\text { meat, } 81 \text { Cal., } \\
\text { per kilo. }\end{array}$ \\
\hline $\begin{array}{l}7^{\circ} \mathrm{C} . \\
15^{\circ} \mathrm{C} . \\
20^{\circ} \mathrm{C} . \\
25^{\circ} \mathrm{C} . \\
30^{\circ} \mathrm{C} .\end{array}$ & $\begin{array}{l}86.4 \\
63.0 \\
55.9 \\
54.2 \\
56.2\end{array}$ & $\begin{array}{l}\ldots \\
\ddot{5} . \dot{9} \\
55.5 \\
55.6\end{array}$ & $\begin{array}{l}77.7 \\
57.9 \\
64.9 \\
63.4\end{array}$ & $\begin{array}{l}87.9 \\
86.6 \\
76.3 \\
\therefore 3.0\end{array}$ \\
\hline
\end{tabular}

The lessened requirements with rising external temperature are clearly shown. Each input corresponds to a fairly constant output at the different temperatures. When these are contrasted with the figures for fasting, the relation becomes clear. Only the heavy input could cover the needs at the lowest temperature; with each rise in external temperature to $25^{\circ}$, the loss becomes more pronounced. The ration of 200 grams could not cover the needs at $7^{\circ}$, but did cover the needs 
at $20^{\circ}$ (probably also at $15^{\circ}$ ); at $25^{\circ}$ and $30^{\circ}$ there is a heat loss of 20 per cent. The lowest ration could not cover the needs at $7^{\circ}$, certainly not also at $15^{\circ}$; but from that time on did so exactly. The increase in protein input from 100 to 320 grams of meat at the constant temperature of $25^{\circ}$ to $30^{\circ}$, raised the heat production nearly 30 Cal. per kilo per day over the figure for the same $\operatorname{dog}$ at fasting or at input of 100 grams.

Calories per Kilo Body Weight per Day

\begin{tabular}{c|c|c|c}
\hline $\begin{array}{c}\text { External } \\
\text { temperature. }\end{array}$ & Fasting. & $\begin{array}{c}\text { Diet } 275 \text { grams meat } \\
=86.9 \text { Cal. per kilo. }\end{array}$ & $\begin{array}{c}\text { Diet } 550 \text { grams meat }= \\
173.6 \text { Cal., per kilo. }\end{array}$ \\
& & $121.9(-)$ & $133.5(9.0 \%)$ \\
$4.8^{\circ} \mathrm{C}$. & 121.3 & 96.1 & $110.9(10.0 \%)$ \\
$14.6^{\circ} \mathrm{C}$. & 100.9 & $83.7(18 \%)$ & $101.0(42.9 \%)$ \\
$22.1^{\circ} \mathrm{C}$. & 70.7 & $81.7(31 \%)$ & $117.2(89.0 \%)$ \\
\hline $30.7^{\circ} \mathrm{C}$. & 62.0 & &
\end{tabular}

Apart from one aberrant result and with some fluctuations in the lower values, the same relations are here indicated.

This result we ascribe to the specific dynamic action of the protein. Fat and carbohydrate have also a specific dynamic action, but as it is very much less than that of protein, the action of the latter has been chosen as illustration.

These facts possess a peculiar and particular interest to the student of nutrition of the human body. Under modern conditions of life, man is practically independent of the chemical regulation of body temperature. The heating of dwellings and the use of clothing modified to meet the particular conditions of external temperature make us relatively independent of chemical regulation. The temperature of the skin of the clothed portions of the body, under the usual conditions of indoor life, is about $33^{\circ} \mathrm{C}$. Metabolism in a bath at $33^{\circ} \mathrm{C}$. has been shown to be identical with the metabolism of the dressed individual at room temperature. Even out of doors it is doubtful if the temperature of the clothed skin falls below $30^{\circ} \mathrm{C}$. Under conditions of prolonged exposure to extreme temperatures, even despite heavy clothing, it is however certain that the temperature of the skin may fall below this figure; and under these circumstances the mechanism for the chemical regulation of the body temperature becomes operative. But under usual conditions of life, the extra heat production expressive of the specific dynamic action of the foodstuffs must be apparent in the resting state, and must be taken into account in our conception of metabolism.

Careful experimentation on the dog, checked up with data derived from diet experiments on human beings, has made it possible for us to estimate closely the increments of heat that are herein concerned. There are doubtless individual variations, but the figures tend to hold 
for usual normal conditions. The figures are known most accurately for ingestions that correspond approximately with the basal requirements noted during fasting. For protein in particular, with ingestions greatly in excess of the figure for the starvation output in calories, more extra heat is probably set free. For protein it is safe to place the heat eliminated as the result of the specific dynamic action at from 25 to 35 per cent. of the intake, 30 per cent. being a fair average; for fat the figure is about 12 per cent.; for carbohydrate much less, about 6 per cent. In other words, if a resting body at a constant temperature of $33^{\circ} \mathrm{C}$., set free during fasting 2900 Calories of heat, when fed under identical conditions 100 grams of protein, 100 grams of fat, and 400 grams of carbohydrate the heat production would be about 3200 Calories, though the actual heat value of the intake was only 2900 . To meet the difference, place the body in caloric equilibrium and maintain the same ratios between the three foodstuffs, the diet would need to be increased to about 140 grams protein, 114 grams fat, and 425 grams of carbohydrate.

When these considerations are applied to mixed diets the relations must be somewhat modified. The greater the relative ration of protein, the larger would be the specific dynamic action. For mixed diets, from 10 to 15 per cent. more calories must be offered than the body liberates during fasting. It is possible to calculate for different diets about what the input would need to be. Such a calculation is based on the assumption that the specific dynamic action is proportional to the mass of protein catabolized. As a matter of fact, with large ingestions of protein the specific action seems to be more intense, but the difference would not materially affect the figures. For the carbohydrates and fats the contrary is true; if fed in excess of the basal requirements of Calories, the specific dynamic action recedes, the body tends to burn of carbon administered in carbohydrates and fats only what is needed to maintain the heat requirement.

Amounts of food in named mixed diets necessary to maintain Caloric equilibrium in an individual of 70 kilo weight and fasting resting requirement of 2100 Calories per day.

(In each line the bracketed figures represent the amounts that would yield the calories of the basal requirements; below are the actual amounts that would need to be ingested to keep the body in caloric equilibrium. The caloric values have been calculated as rounded figures; proteins, 4; carbohydrate, 4; and fat, 9 . All the figures are given rounded. In the last two diets, free of protein, the production of heat would be augmented over the amounts given by the catabolism of the endogenous protein, about 150 Calories in the first instance and probably 250 Calories in the second instance. These figures have not been included in the totals.) 


\begin{tabular}{|c|c|c|c|}
\hline $\begin{array}{c}\text { Protein } \\
(525) \\
700\end{array}$ & $\begin{array}{c}\text { Carbohydrate } \\
(0) \\
0\end{array}$ & $\begin{array}{c}\text { Fat } \\
(0) \\
0\end{array}$ & $\begin{array}{c}\text { Calories } \\
(2100) \\
2800\end{array}$ \\
\hline $\begin{array}{c}(200) \\
2 S 0\end{array}$ & $\begin{array}{c}(165) \\
175\end{array}$ & $\begin{array}{l}(70) \\
80\end{array}$ & $\begin{array}{c}(2100) \\
2550\end{array}$ \\
\hline $\begin{array}{c}(150) \\
210\end{array}$ & $\begin{array}{c}(250) \\
265\end{array}$ & $\begin{array}{c}(55) \\
65\end{array}$ & $\begin{array}{c}(2100) \\
2500\end{array}$ \\
\hline $\begin{array}{c}(150) \\
210\end{array}$ & $\begin{array}{c}(125) \\
135\end{array}$ & $\begin{array}{c}(110) \\
125\end{array}$ & $\begin{array}{c}(2100) \\
2600\end{array}$ \\
\hline $\begin{array}{c}(125) \\
175\end{array}$ & $\begin{array}{c}(250) \\
265\end{array}$ & $\begin{array}{c}(65) \\
75\end{array}$ & $\begin{array}{c}(2100) \\
2425\end{array}$ \\
\hline $\begin{array}{c}(125) \\
175\end{array}$ & $\begin{array}{c}(150) \\
160\end{array}$ & $\begin{array}{c}(110) \\
125\end{array}$ & $\begin{array}{c}(2100) \\
2475\end{array}$ \\
\hline $\begin{array}{c}(100) \\
140\end{array}$ & $\begin{array}{c}(300) \\
320\end{array}$ & $\begin{array}{c}(55) \\
65\end{array}$ & $\begin{array}{c}(2100) \\
2400\end{array}$ \\
\hline $\begin{array}{c}(100) \\
140\end{array}$ & $\begin{array}{c}(200) \\
215\end{array}$ & $\begin{array}{c}(100) \\
115\end{array}$ & $\begin{array}{c}(2100) \\
2450\end{array}$ \\
\hline $\begin{array}{l}(75) \\
105\end{array}$ & $\begin{array}{c}(300) \\
320\end{array}$ & $\begin{array}{l}(65) \\
75\end{array}$ & $\begin{array}{c}(2100) \\
2375\end{array}$ \\
\hline $\begin{array}{l}(75) \\
105\end{array}$ & $\begin{array}{c}(225) \\
250\end{array}$ & $\begin{array}{c}(100) \\
115\end{array}$ & $\begin{array}{c}(2100) \\
2450\end{array}$ \\
\hline $\begin{array}{c}(60) \\
85\end{array}$ & $\begin{array}{c}(350) \\
370\end{array}$ & $\begin{array}{c}(50) \\
57\end{array}$ & $\begin{array}{c}(2100) \\
2340\end{array}$ \\
\hline $\begin{array}{l}(60) \\
85\end{array}$ & $\begin{array}{c}(240) \\
255\end{array}$ & $\begin{array}{c}(100) \\
115\end{array}$ & $\begin{array}{c}(2100) \\
2375\end{array}$ \\
\hline $\begin{array}{c}(40) \\
55\end{array}$ & $\begin{array}{c}(375) \\
400\end{array}$ & $\begin{array}{c}(50) \\
57\end{array}$ & $\begin{array}{c}(2100) \\
2325\end{array}$ \\
\hline $\begin{array}{c}(0) \\
0\end{array}$ & $\begin{array}{c}(525) \\
555\end{array}$ & $\begin{array}{c}(0) \\
0\end{array}$ & $\begin{array}{c}(2100) \\
2225\end{array}$ \\
\hline $\begin{array}{c}(0) \\
0\end{array}$ & $\begin{array}{c}(0) \\
0\end{array}$ & $\begin{array}{c}(235) \\
270\end{array}$ & $\begin{array}{c}(2100) \\
2425\end{array}$ \\
\hline
\end{tabular}

Under the discussion of the processes of digestion it was pointed out that in the acts of digestion work is performed. The nitrogenous metabolism, measured by the output of nitrogen in the urine, is not increased by mock feeding, though this is associated with active secretion of alimentary juices. A certain heat production is found to be associated with this secretion. It is clear that the specific dynamic action of the foodstuffs as a whole, cannot be related to this work. This is made definite by the fact that the amino-acids derived from a unit of casein display the same specific dynamic action when administered to a dog as the casein from which they were obtained; and by the further fact that when the phloridzinized dog burns his body protein, the same excess of heat production is to be noted. For protein, therefore, it is certain that the specific dynamic action is not related 
to the processes of digestion, but lies within the catabolism. For the carbohydrates and fats, the interpretation is probably different. On the assumption that the fats are resynthesized in the intestinal wall, the chemical reaction of the total fat digestion is isothermic. When starches and disaccharids are split into hexose, from 3 to 5 per cent. of the contained heat is set free. In the case of protein, parenteral introduction is followed by increase in heat production. 'The parenteral introduction of glucose or fat is not followed by a rise in the production of heat. Indeed, the ingestion of 75 grams of glucose by a fasting subject is not followed by any rise in the production of carbon dioxid, the glucose evidently being simply stored as glycogen. And when the body is in caloric equilibrium, the further ingestion of sugar and fat is associated with but very little specific dynamic action. For the sugars this could be explained by their conversion into glycogen. All in all, it seems very likely that for fat and carbohydrate the specific dynamic action is largely related to the work of digestion and assimilation, though for the fats the details are not clear.

Since it cannot be assumed that the specific dynamic action of protein is associated with the acts of digestion and assimilation, could it be related to the elimination of the end products of nitrogenous metabolism? The elimination of urea costs work and heat is set free; this can be shown experimentally. But the amount of heat involved is entirely too small to explain the facts.

Another possibility lies in the combustion of superfluous aminoacids, after the postulated conversion of diet protein into the blood proteins. If this, in part, could be invoked to explain the specific dynamic action of protein, it ought to be greatest in the case of the ingestion of proteins whose amino-acid composition is farthest from that of the blood protein, for example, gliadin; it ought to be lowest after the ingestion of the blood serum of the same species. This suggestion is in harmony with the increase in heat production observed when the starving animal is subjected to phloridzin poisoning; the animal must convert indifferent protein of the connective tissues and after that muscle protein into blood and cell protein, and superfluous amino-acids would be here set free.

However stated, it is certain that the phenomenon is not associated with the anabolism of cellular protein directly, but is a waste that attends this process and also a waste that attends the catabolism of exogenous protein. The total potential energy of protein is not available for cellular or metabolic needs. To say that it is the heat of chemical reaction, explains nothing. There is a difference between the heat value of the metabolizable products that the protein presents to the cells and the heat value in the protein potentially; this appears as free heat and though it can be utilized for work and to spare combustions through chemical regulation of temperature, it cannot be utilized by the cells for anabolic purposes. The total heat value of a gram of protein may be set at 5.7 Calories. Of this about 1.5 Calories 
reappear in the end products in the urine. Of the 4.2 Calories that are then available in the body, about 2 to 2.5 Calories remain stored in glucose, leaving about 2 Calories or a little more still unaccounted for. 'The fatty acids that are not convertible into glucose are burned, and these yield probably not over 1 Calorie, calculating on the basis of the carbon that remains after the extent of glucose formation has been determined. The balance remains unaccounted for (for metabolic needs), and corresponds to the heat set free through the specific dynamic action of the protein. Contemplating the specific dynamic action of protein from this point of view, the moment of loss would seem to lie in the conversion of the amino-acids into glucose, i.e., the glucose formed from a unit of amino-acid contains less heat than did the substrate, and this heat set free is a loss to the metabolism and constitutes the heat of the specific dynamic action of protein. This hypothesis has not been tested experimentally.

The phenomenon is of little practical importance in health, but constitutes a misfortune to the diabetic. Probably the sensation of warmth that follows a meal rich in protein is due to this specific dynamic action of protein; and this suggests that the protein ration be lower in summer than in winter, and should be as low in fevers as may be consistent with the maintenance of nitrogenous equilibrium. For the diabetic the loss of heat is a real misfortune; because with the inability to burn sugar, the energy available to the cells of the diabetic may be reduced to 1 Calorie per gram of protein.

\section{THE RECIPROCAL RELATIONS OF PROTEIN, CARBOHYDRATE, AND FAT}

The Relations of the Non-nitrogenous Derivatives of Protein.-The disposition of the nitrogenous derivatives of the protein molecule was stated under Protein Metabolism. About the fate of the nonnitrogenous fraction has centred in the past the most intense interest, and over this fraction have been waged some of the most determined controversies in the history of physiology. With the demonstration of the origination of glucose from the amino-acids derived from the catabolism of protein, the meaning of the carbon retentions noted in animals on pure protein diets became unquestioned. As stated elsewhere, the exact amount of glucose that the body derives from a unit of protein is not known; and probably it varies with different proteins. It will be safe to assume with mixed diets that a gram of protein will yield 0.5 or possibly 0.6 gram of sugar. Once this sugar is formed, it shares, of course, all the obligations and prerogatives of glucose within the animal economy. And since fat is formed from sugar, in this indirect way fat is, of course, formed from protein. But so far as we know, fat is only formed from protein within the general metabolism; glucose is first formed from the amino-acids and from this sugar, as from all sugar, fat may be formed. When with an excess 
of protein intake glucose is being stored from protein, 1 gram of protein will yield about a fourth gram of fat. To obtain this fat, however, the body must catabolize the protein and eliminate the nitrogenous end products, so that it is a very wasteful method of laying on fat, 5.7 Calories protein input yielding 2.3 Calories stored fat.

When the body is fasting, the concentration of glucose in the blood remains normal, even when it is certain that the glycogenous deposits are quite depleted. This concentration of sugar in the blood under these circumstances is maintained by glucose derived from the catabolism of the body protein.

When protein is being catabolized in excess, it seems in some way to entrain the combustions of sugar and fat. Sugar and fat have a low specific dynamic action. It seems, however, that when they are ingested with large amounts of protein, this action is more pronounced; the excess of heat production inclines to be more than would be observed for the fat and sugar and protein tested separately. In other words, the exaggeration of the protein catabolism tends to exaggerate the combustions of fat and sugar.

Influence of Metabolism of Fat and Carbohydrate upon Each Other.Disregarding the differences in the specific dynamic actions of fat and carbohydrate, these two are isodynamically equivalent, $i$. $c$., they are able to replace each other as sources of energy and heat in the proportions of their caloric values. The ingestion of fat does not materially modify the progress of the metabolism of carbohydrate. But the ingestion of carbohydrate does modify the metabolism of fat. The body burns carbohydrate by preference. If to a body being warmed by the combustion of fat, carbohydrate be administered, the combustion of fat will be lowered or cease, and be replaced by the burning of glucose; and the respiratory quotient will indicate the change. When to a fasting body (being warmed by the combustion of fat) a goodly ration of sugar is administered, the carbon dioxid elimination rises from about 22 to 30 grams per hour. This is due to the combustion of glucose instead of fat, there is more carbon dioxid produced for the same output of heat. Certain quantitative conditions must, however, be present. If the man has fasted long, the rise in carbon dioxid does not occur; instead the body retains the sugar and continues to burn the fat. It was elsewhere explained with what tenacity the body attempts to maintain a certain stage of storage of glycogen; if this has been depleted by starvation, the administration of sugar is utilized by the body for the repletion of this glycogen. But when the reserves of glycogen are relatively restored, even though the subject be burning largely fat, the ingestion of sugar will swing that body from the combustion of fat to that of sugar, with the consequent increase in the output of carbon dioxid. When carbohydrates are ingested in great excess and active conversion of sugar into fat is going on, the respiratory quotient may be higher than 1 , since the conversion of the carbon and hydrogen of glucose into fat sets free oxygen that 
is used in the combustions of the body to the exclusion of atmospheric oxygen. The test is not to be made on all animals, since many"animals will not deposit fat with such rapidity. Respiratory quotients of over 1.3 have been obtained in connection with forced feeding of carbohydrate in the goose and marmot.

The statement that sugar and fat are isodynamic in heat production under comparable conditions has normally no exceptions. But it is a different thing to state that in any individual, carbohydrate and fat may replace each other in the diet in the relation of the caloric coefficients. The digestion may introduce a new factor. A body may require 600 grams of carbohydrate (plus the standard ration of protein) to maintain caloric equilibrium; 250 grams of fat would supply the same heat. But the body may not be able to digest the 250 grams of fat. Occasionally one sees individuals whose powers of digestion of fat are very limited; one meets also with subjects whose powers of digestion of carbohydrates are limited. It is not the ingestion of carbohydrate and fat in the isodynamic ratio, it is the assimilation of carbohydrate and fat in the isodynamic ratio that constitutes the dynamic principle-a point to be borne in mind with the sick.

Comparable as fat and carbohydrate are to each other as mere fuels under controlled conditions, when tested in relation to the protein metabolism they present widely varying properties. Glycogen and fat were defined as the proximate and ultimate states of storage of glucose. In their relations to the protein metabolism, sugar and fat are respectively proximate and ultimate in availability.

The Saving Power for Protein.-Both fat and carbohydrate have the power of sparing protein. But this power is low in the case of fat and very high in the case of carbohydrate. Let the nitrogen output of a fasting object be determined and enough carbohydrate and fat be administered in successive tests to cover the caloric needs of the body. It will be found that on such administration of carbohydrate the nitrogen output will fall to a third of the fasting figure. If the individual be in good flesh, the administration of fat will result in little reduction in the nitrogen output. If the subject be emaciated, however, the administration of fat will result in a reduction in the nitrogen output, though not comparable to that achieved by the carbohydrates. Not only does carbohydrate actually reduce the nitrogen metabolism much lower than fat, it will do this in amounts that do not cover the caloric needs of the body, the balance of the heat being obviously derived from the fats of the body. Under comparable conditions, if feeding with just enough protein and carbohydrate to cover the requirements results in a nitrogenous equilibrium at about the fasting level, the substitution of fat for the carbohydrate will raise the nitrogen elimination about one-half. When a low nitrogen equilibrium is maintained by a ration of carbohydrate just sufficient to cover the caloric needs of the body, a third of the carbohydrate may be replaced by fat without disturbing the balance of nitrogen. 
The sparing power of carbohydrate and fat for protein may be determined in another way. Under certain circumstances the body retains nitrogen. When this is true, such retention is favored by carbohydrate much more than by fat. One reason for the difference lies obviously in the fact that the body contains little carbohydrate and much fat. The further addition of fat could not, therefore, be expected to have much metabolic influence. The mechanism of the protein metabolism (and of the fat metabolism as well), is singularly dependent upon the presence of glucose and glycogen. If these be absent or seriously reduced (and this is easily accomplished), their administration greatly influences transformations. The marked difference in the saving power of fat and carbohydrate for protein makes it impossible to explain this saving as the mere restriction of the protein catabolism to the endogenous, cellular activity, to the exclusion of dynamogenetic utilization. And even within the realm of purely endogenous, cellular protein metabolism, carbohydrate spares and enables the body to maintain a nitrogenous equilibrium on the lowest possible level, far below the level reached with fat ingestions more than covering the caloric needs of the body.

The striking influence of carbohydrate utilization on the catabolism of protein is best shown in deprivation experiments. A fasting man will eliminate, after the first few days when the more or less variable stock of surplus protein is exhausted, from 10 to 15 grams of nitrogen per day. This elimination slowly falls with the continuance of fasting. If now carbohydrates be ingested in amount to insure caloric equilibrium, the nitrogenous elimination will fall directly to 4 or 5 grams and remain there or fall indeed still farther. The substitution of fat for carbohydrate will be followed by a rebound of the nitrogen almost to the earlier figure of the fasting period. By prolonged deprivation of protein with rich feeding of carbohydrate, the nitrogenous output can be reduced to a most surprising minimum, to scarcely more than 2 grams per day. In some recent work on this subject done on swine, in which it was found that the basal protein catabolism was not reached in less than sixteen days, the nitrogen output fell to amounts as low as 1 gram per day for young pigs of 20 kilos weight. In other words, the administration of carbohydrate to the starving body so spares protein as to cut the loss of nitrogen down to a third or even a fourth. The body of the subject in starvation has an abundance of fat; the dynamogenetic combustion of protein is not obligatory by reason of absence of fat; but protein is catabolized instead of body fat to the extent of two-thirds of the protein dissimilated. Fat is, of course, burned; the heat production does not begin to be covered by the catabolism of protein, it is indeed covered largely by the burning of fat. But if the body could contain stores of carbohydrate as it does stores of fat, we would observe the nitrogen output at the low figure that is to be seen in protein fasting with carbohydrate input. This state of affairs constitutes one of the strongest arguments against the hypothesis of the conversion of fat into sugar. 
With the aid of carbohydrates the body may be kept in nitrogenous equilibrium with a total input below the amount necessary to attain caloric equilibrium. Thus a body may be kept in nitrogen balance at, let us say, 7 grams, on a caloric input of 2000 Calories, with a heat production of 2500 Calories. Carbohydrate alone will do this, the substitution of fat for a large fraction of the carbohydrate results in deficit of nitrogen through exaggeration of protein catabolism.

With the aid of carbohydrate the excessive protein catabolism that occurs in the course of prolonged febrile infections may be reduced or indeed obviated. In typhoid fever, for example, by the ingestion of increased amounts of sugar, the total loss of nitrogen that is usually to be recorded for the period of sickness may be greatly minimized, perhaps obviated. In other words, carbohydrate spares pathological as well as normal protein catabolism.

To a certain extent carbohydrate favors the storage of protein with ingestions above the nitrogenous requirements. This is usually not marked in adult life. The idea that carbohydrate spares protein all the way up the scale of increased inputs is incorrect. Usually when we reach the highest level of normal requirements, the ingestion of even large amounts of carbohydrate will not induce the body to spare the nitrogen by retaining the protein. In growing animals it probably does tend to do so, and there is experimental evidence that growing animals will fleshen better on a fixed input of protein plus carbohydrate than on the same protein input plus the isodynamic amount of fat. For a short time, the sudden addition or withdrawal of carbohydrate will have its effect upon the nitrogenous metabolism, but the effect is not continuous. One of the most careful and competent students of metabolism has recorded the following personal observation. When on a mixed diet, with a nitrogen input of 20.5 grams and an output of 19.8 grams (a balance), the withdrawal of 350 grams of carbohydrate from the diet resulted in an increased output of nitrogen, on the second day to 27 grams. 'This observation induced the writer to reverse the experiment. To a diet of a subject in nitrogenous equilibrium at 13 grams was added 300 grams of carbohydrate; the nitrogen of the urine fell over 3 grams. But in neither of these tests could the results have persisted. Both probably represent local disturbances in the liver, rather than in the total metabolism. As a rule, and especially for long periods of time, it is certain that carbohydrate has not the power to save protein in the sense of storing protein. It is very potent in saving protein by checking waste of protein, by checking the burning of protein as fuel, and by aiding the cells to accomplish their endogenous metabolism upon the most economical nitrogenous basis.

Another concrete illustration of the saving power of glucose over the protein catabolism is afforded in recent work on the metabolism of creatin-creatinin. Creatin is one of the most important bodies in the endogenous catabolism; it is set free in the muscle cell and converted into creatinin. In the absence of carbohydrate, this conversion is incomplete and creatin appears unaltered in the urine. 
There is a relation of dependence to carbohydrate exhibited in the combustion of fat, in that while the combustion of the molecule of fatty acid is accomplished down to the stage of butyric acid, below this point retardation tends to occur in the absence of utilization of carbohydrate. The question is discussed under acidosis. There are many exceptions to the rule; there is acidosis with normal combustion of carbohydrate and, on the other hand, sistence of carbohydrate combustion without the development of acidosis. But physiologically the relationship is very impressive. Whenever the body is thrown from the combustion of carbohydrate to the combustion of fat-in starvation or in proteinfat diet, a certain degree of disturbance is to be noted in the combustion of fat, revealed by acidosis and the presence of ketonic bodies in the urine.

In the presence of severe organic disease of the liver, the saving power of carbohydrate for protein may be markedly reduced. Under these circumstances it is open to question whether some of the high nitrogen outputs previously noted in hepatic diseases were not instances of relative carbohydrate starvation with consequent increase in protein catabolism, rather than direct results of the hepatic conditions on the catabolism of protein.

Adequate explanation of the nature of the saving power for protein exhibited by carbohydrate is not at hand. It is, however, apparent that at all points in the scale the cause of the saving power is not the same. There is the saving to be noted with moderate ingestions of protein, as when on an input of 7 grams of nitrogen, a nitrogenous balance is established with an input of less Calories than are necessary to cover the needs of the body. Let us say the heat needs of the body are 2100 Calories and 1800 are given in the diet, protein corresponding to 7 grams (150 Cal.) plus carbohydrate $(1650 \mathrm{Cal}$.). The missing 300 Calories are obtained by combustion of body fat. Now of the carbohydrate in the diet, at least 600 Calories may be replaced by fat without disturbing the balance in nitrogen. Within this range of 1000 Calories the fat and carbohydrate spare protein equally. This saving is due to sparing the combustion of protein for dynamogenetic purposes. The other 1000 Calories covered by carbohydrate cannot, however, be replaced by fat. Within this range sugar accomplishes something that fat cannot do. In part, at least, an explanation for this action is to be found in the concentration of sugar in the blood. In complete starvation the sugar concentrations is preserved in the blood. This sugar can be derived from but one source, after the depots of glycogen have been depleted; from protein. If fat be given instead of carbohydrate - either with protein ingestion or in nitrogen starvation-the body must catabolize protein to secure sugar to maintain the concentration of the blood. How much sugar is used or burned during starvation is not known; but much is not burned, as indicated by the respiratory quotient. The amount of sugar that corresponds to 1000 Calories of heat is 250 grams, and no such amount of sugar 
as this can be needed to maintain the glucose concentration of the blood during starvation. 'To yield 250 grams of sugar 500 grams of protein would have to be catabolized, and the body does not begin to catabolize this much in complete starvation. Those who believe in the reutilization of nitrogen in amino-acids, could invoke a fraction of this sugar to that end; but this does not deserve serious consideration, in the opinion of the writer. For the present we can simply say that the processes of endogenous protein catabolism are more effectively carried on in a body supplied with carbohydrate and supported by it than without it. This is not an explanation at all, but merely a statement of fact. It is true, as often reiterated, that sugar is more soluble and more readily oxidizable than fat; but upon these properties of sugar in the body the peculiar saving power of glucose cannot depend.

There remains finally, the ultimate saving in protein that is attained in nitrogen starvation by the ingestion of amounts of carbohydrate in excess of the caloric requirements. Upon this low plane of metabolism, the maintenance of the sugar concentration in the blood becomes a factor in the catabolism of protein. Under these circumstances, the operations of the endogenous cellular catabolism are reduced to the most minimal plane, less than half that on which nitrogenous equilibrium can be maintained with the aid of carbohydrate. Growth cannot be accomplished under such conditions, but that does not enter into the discussion in adults. We deal here apparently with an expression of the peculiar indispensability of glycogen to tissues. In the discussion of the carbohydrate metabolism and of diabetes, it was pointed out with what marked pertinacity the body protects its store of glycogen, as though it were invaluable. When in a case of diabetes the reserves of glycogen in the tissues are greatly depleted, the organism is apt to collapse like the One Horse Shay. Conversely to this, when the maximum storage of glycogen is attained and maintained in the cells, their endogenous catabolism of protoplasm is accomplished on the very minimal basis. In some way glycogen acts as, in a mechanical sense, a lubricant is related to the wearing parts of a machine; when glycogen is abundant, the wear-and-tear is slight; when it is deficient, the wearand-tear is excessive. The situation here is just the converse presented in the relations of sugar to creatin and fat; in the absence of sugar creatin is not converted into creatinin, and fat is not burned; in the absence of sugar protein is inordinately catabolized. We have no explanation of this in chemical terms. It is possible that we deal here with coupled reactions, the relations of actor and inductor being reversed in the two sets of conditions.

\section{IN ANITION}

It is the usual method of procedure to define the normal state and from this to proceed to the definition of abnormal states. And from 
this point of view it were more logical to discuss the normal diet before taking up the subject of starvation. But so much has developed in the study of different starvations that is directly of service in the discussion of the normal diet, that it will facilitate progress to consider inanition first. Under inanition we understand the withdrawal of nutrients; water and salts are not withdrawn. The term fasting has the same meaning; while as an experimental term starvation in dogs is usually understood to mean the complete withdrawal of ingesta. For the dog water is not required, the processes of catabolism yield enough water to dissolve and carry out the salts derived from metabolism; and the dog on a meat diet can do without water when resting. But this is only the case in such animals as eliminate little or no water from the skin.

Illustrative as fasting experiments have been, in some directions partial hungers have given more crucial information. Such are protein hunger, fat hunger, carbohydrate hunger, or fasting of one kind combined with variations in the feeding with one or other or both of the two permitted foodstuffs. If the metabolism can be shifted in a particular direction by experimental means, as in diabetes, phloridzin or phosphorus poisoning, fasting tests may be made to yield additional information. Our data are not confined to observations on animals, since diet fadists and professional fasters have provided wellutilized opportunities for careful chemical study of inanition in man. And finally, there is pathological material from which valuable information has been derived.

Total Fasting.-By observations on professional fasters largely, the length of time the body can maintain itself without nutriment has been fairly closely determined. Fifty days is the longest fast on record; there have been several fasts of forty and thirty days. Naturally, the length of time the body can conserve itself without food is inversely proportional to the fat and flesh content of the body at the beginning. It is not likely that under the best conditions, a man could fast materially over fifty days. Dogs often die within a few weeks, yet in one case death did not occur before the ninety-eighth day. Fasting as a result of disease is scarcely to be taken as typically illustrative of the state, since the organic conditions underlying the inability to take or assimilate food (obstruction of esophagus or the pylorus, etc.), tend to make the conditions of nutrition unfavorable. On the other hand, the state of nutrition in certain instances of carcinoma of the pylorus is largely due to starvation; and the formation of a gastroduodenal anastomosis has at least the effect of permitting the subject to die of carcinoma after months, instead of dying from starvation after a few weeks.

The state of the body protein has a marked influence on the metabolism during the first few days of fasting, especially in dogs, though the facts would doubtless be found to hold for man. Under ordinary conditions, the nitrogen output is high for two or three days, then 
falls; but if the fasting has been preceded by a heavy protein input, this stage of high nitrogenous elimination will last for two or three days longer. There is a certain degree of protein storage that can be accomplished, and the nitrogenous output of the early days of fasting is related to this variable. Marked also is the influence of stored glycogen on the course of metabolism during the first days of inanition, the result being just the converse of the effect of stored protein. The nitrogen output will be low for from two to five days, depending on the amount of glycogen stored; then it will rise to about the same figure to which it would have fallen in the same individual had the state of nutrition been stored protein instead of glycogen. 'Thus the daily nitrogen output in a subject well stocked with glycogen may be $\delta, 11,12,13,14$ grams; while in fasting following forced protein feeding the daily output of nitrogen would be $18,17,15,14,13$. The respiratory quotient (about 0.7 in established starvation) would be higher in the subject with much glycogen. The figures are, of course, arbitrary, but they represent the general course of the nitrogenous elimination. Nitrogen is lost most rapidly in thin subjects.

The influence of stored fat is twofold. The plane of the nitrogenous output is inclined to be lower in fat individuals; and the middle period of even metabolism that is to be observed in all cases of inanition is greatly prolonged. After a number of days, the body seems to improve in its protein metabolism on the enforced basis of fat minus carbohydrate, and acidosis may not be at all pronounced. This favorable action of abundant body fat on the course of inanition is especially well illustrated in the dog. And in the $\operatorname{dog}$ is recorded the longest survival on record in starvation, one hundred and seventeen days.

The relation of the catabolism of protein to the total heat production is relatively constant under controlled conditions, after the stored protein and glycogen have been exhausted. During the intermediary stage of starvation, the catabolism of protein yields from 10 to 15 per cent. of the total heat production; both fall, but the reduction in the nitrogen is the more marked and its relative participation in the total heat production is lessened. Thus in prolonged fasting, during and after the third week, the catabolism of protein may furnish no more than 5 per cent. of the total heat production.

The curve of total heat production for a prolonged fast has not been determined, though by interpolation with the use of the available data it can be sketched. After the first few days, when the utilization of the stored protein and glycogen provoke irregularities, the heat production is remarkably constant. Most fasting bodies have produced, under controlled conditions of external temperature and muscular work, from 28 to 33 Calories per kilo per day; at complete body rest the figure will fall to 25 Calories. When the oxygen consumption and the carbon dioxid output of the fasting subject are measured from the third to possibly the tenth day, the most marked constancy is exhibited. Then the tendency to lessened heat produc- 
tion becomes apparent. The caloric output per kilo may remain the same; but as the individual is losing weight, the total heat production is falling. This reduction in total heat production will not be over 200 or 300 Calories per day. One might expect that when the body is put to stress for fuel, the mechanism of physical regulation would develop unusual effectiveness in operation to the maximum of the factors that diminished surface radiation; but there is little sign of such an adaptation. There is no reduction in the basal production of heat, even at high external temperature and in extreme emaciation. We have data bearing on under-nutrition in which the total heat production is notably lower than in fasting. Is it here again the presence of sugar that determines the greater effectiveness of the metabolism? Both animals and man tend to become lethargic during prolonged fasting, and the heat output due to involuntary muscular movements is thereby reduced. It is possible that the reduction in total heat production is due to this factor alone.

Elimination of Nitrogen.-The greatest interest in the metabolism of inanition centres about the elimination of nitrogen. The figures in the literature hardly give a fair picture, since usually only the urinary nitrogen is stated. The cutaneous elimination of nitrogen has not been estimated in starvation, but it may be assumed to equal the normal. Including the growth of hair and nails and the loss of epithelium, the total cutaneous elimination of nitrogen cannot be less than 0.3 gram per day on an average. Of the fecal nitrogen in starvation we are better informed. It usually runs from 0.3 to 0.5 gram per day, may, however, rise to 0.7 gram. To be added to the urinary nitrogen we have therefore, from possibly 0.7 to even 1 gram of extrarenal output of nitrogen daily. When it is observed that the urinary nitrogen sometimes falls below 3 grams per day, the magnitude of the correction becomes apparent. There are two defined periods in the nitrogenous catabolism in starvation, apart from the terminal period that will be described separately. The first is the period of nitrogen or glycogen storage, the preliminary stage. As already stated, the nitrogen is relatively high in the one and low in the other. During the second period the urinary nitrogen falls progressively. The daily outputs for a professional faster, Succi, will serve as a typical illustration. They were $17 ; 11.2 ; 10.55 ; 10.8 ; 11.19 ; 11.01 ; 8.79 ; 9.74 ; 10.05 ; 7.12 ; 6.23 ; 6.84$; $5.14 ; 4.66 ; 5.05 ; 4.32 ; 5.4 ; 3.6 ; 5.7 ; 3.3 ; 2.82$, corresponding during the last days to less than 20 grams of protein. A certain amount of protein can be ingested without raising the figure for the elimination of nitrogen, $i$. e., the protein is completely retained to replace loss. Irregularities are present, just as are to be seen on a constant input; but after the first few days a progressive diminution is manifested. The sulphur runs parallel to the nitrogen. The elimination of phosphorus, however, rises relatively, supposedly due to the solution of bone rather than to increased nucleic catabolism, because the calcium output rises. From the data on the elimination of nitrogen in the urine 
we learn, therefore, that the catabolism of the body protein in established fasting falls to less than half the figure known to represent the minimum of equilibrium on a protein-sugar diet covering the caloric needs of the body. This fact holds for man and the dog.

In the rabbit and guinea-pig, however, the elimination of urinary nitrogen remains relatively constant or rises from the beginning of fasting until death.

The partition of nitrogen reveals interesting facts. The greatest reduction lies, of course, in the nitrogen of urea, since all the exogenous urea is excluded. As the nitrogen falls, the percentage of urea $\mathrm{N}$ falls also, so that at the lowest level the urea $\mathrm{N}$ makes up 55 to 60 per cent. of the urinary nitrogen. This figure, however, is often really much lower than it would be were it not for the occurrence of acidosis, which withdraws ammonia and thus lowers the figure for urea. The purin output falls slowly during the period of fasting, but is not strikingly below what may be observed on a milk diet. The creatin-creatinin (both are present) is very constant though well above the figure for endogenous creatinin; the earlier observations on this subject, that read to the contrary, were not reliable. The constancy of this elimination when related to the muscle content of the fasting body is one of the striking demonstrations of the autogeny of this metabolism. The ammonia would be low if acidosis were not present. Sometimes this is the case; in Succi, the urine on the twentieth day contained only a little over 0.1 gram of ammonia. The ammonia output in starvation may be lower than the urinary content in ketonic acids would seem to require. But the urine in starvation contains an excess of potassium, which naturally is available to the ketonic acids. Hippuric acid is absent.

The curve of nitrogen elimination in time seems to be quite near a straight line, lowest at night. This, together with the data on partition and the figures on the total urinary elimination, constitute strong arguments for the rigid application of the distinction between endogenous and exogenous nitrogenous metabolism in normal nutrition.

Metabolism of Carbohydrate.-The metabolism of carbohydrate is reduced to the lowest plane. Little or no sugar is burned, and the glycogen depots are progressively depleted, though at death glycogen is still to be found in the tissues. The concentration of glucose in the blood persists, and this must be derived from protein. When the catabolism of protein yields only 3 grams of nitrogen per day, this would correspond to 10 grams of glucose, an amount that the body could readily burn under the conditions of the experiment, without leaving its mark on the respiratory quotient. As will be later pointed out, in protein hunger with liberal carbohydrate input, the nitrogenous output may be reduced below the lowest figure ever to be seen in starvation; and this difference may be due, in part, to a fraction of protein catabolized in starvation to yield glucose. 
Combustion of Fat.-The combustion of fat is usually defective, in that the ketonic bodies may be eliminated in the urine in rather large amounts. Indeed, starvation is one of the classic illustrations of acidosis. The amount of acetone bodies is often large contrasted with the magnitude of the fat combustion. Thus Succi had often as much as 10 grams of b-oxy-butyric acid when he was burning not over 150 grams of fat, an amount that the normal individual burns with ease. The reason for the difference lies in the paucity of carbohydrate in the body. In some cases, however, the acidosis is practically nil, related possibly to an abnormally persistent retention of glycogen within the tissues. The effect of administering fat to a fasting animal not yet much reduced in body fat is slight; later, it has a more noticeable effect on the metabolism of protein.

Respiratory Quotient.-The respiratory quotient in fasting, after the first days, corresponds to that usually seen when the heat production is maintained by the combustion of fat and protein; 0.74 was repeatedly observed in the studies of one starving man. In other words, it corresponds to that noted in the diabetic who cannot burn glucose and fires with fat and protein. But in the case of the diabetic, the sugar formed from the exaggerated protein catabolism is lost, while in fasting it is utilized.

With the onset of the terminal or premortal period, the metabolic picture changes. The nitrogen in the urine rises sharply, acidosis is usually very pronounced, and the chlorion that has been tenaciously retained, is suddenly increased in amount in the urine. The premortal rise in the protein catabolism is probably due to the extreme depletion of the glycogen; while in many cases it seems directly associated with the reduction of fat, in other cases the animal dies with characteristic symptoms while the body contains considerable fat. Be the cause one or both, the body turns to its cellular protein for dynamogenetic purposes, and with the cells attenuated to the last degree, such inroads cannot be long borne. It is possible that here, as is sometimes the case with pathological autolysis, the total heat production may show an actual rise.

Muscular Work.-When muscular work is accomplished during inanition, it is done on combustion of fat, at least this seems to be the rule in fasting men. Dogs sometimes show a sharp rise in the elimination of nitrogen after exercise, indicating that protein had been catabolized. It is known that a dog can work hard on protein alone. But with choice, doubtless it may be inferred that work will be done on the combustion of fat. In the late stages when fat storage is low, the protein must be drawn upon. It is probable that the efficiency of work (the amount of work accomplished in relation to the heat produced) is lowered during inanition. The body temperature is normal, until the terminal stage, when with falling heat production, even the most efficient restriction of heat dissipation cannot maintain the body temperature and it falls. 
Blood.-The blood in starvation maintains a striking constancy. The concentration of glucose and protein, as well as red corpuscles and their hemoglobin content, remain quite normal. It has been stated that the serum globulin is increased relatively to the serum albumin, supposedly as the result of the abstraction of globulin from the organs. The fat is usually increased, and the liver is well infiltrated with fat. This lipemia is, of course, simply the expression of the transportation of fat from the depots to the organs of combustion. The protein concentration of the blood is maintained by the abstraction of organ, muscle and connective-tissue proteins from their native sites and their transfer into the circulation. But these do not circulate as native proteins; they are converted into serum albumin and serum globulin. As such they are the substrate upon which the essential anabolic processes of the cells, the up-keep and wear-and-tear, are founded. The sugar is also derived from the protein. The persistence with which the blood retains its constituents may be shown in experiment. If a dog be bled the maximum amount of blood that can be taken, and the identical volume of salt solution returned into the circulation, within a half hour a sample of blood will show that the protein and sugar content of the blood have been restored to the normal.

Body Weight.-The body weight is usually greatly reduced at the close of fatal fasting. There is, however, little uniformity in the results in different species and even in different animals of the same species. Sometimes animals die after relatively short fasts and the fat depots and muscles of the body are quite well preserved. In other instances life is preserved until emaciation has become most profound. Evidently the cause of death, even in the absence of demonstrable intercurrent disease, to which fasting animals must be especially susceptible, is not single. The total loss of weight in animals varies from 30 to 60 per cent. of the original weight. The loss in body weight is greatest in dogs that were not very fat at the commencement of starvation. The loss of body protein will vary from 20 to 50 per cent. of the initial content. 'The loss in body fat will vary from 30 to 90 per cent. These marked differences indicate that the course of starvation is very irregular in different animals. In some cases the animals die of pure inanition, life lasts as long as there is any fat to burn and in the last few days excessive combustion of the sole remaining fuel, protein, leads to a marked premortal rise in the nitrogenous output. In other cases certain organs, as the heart, seem to give out long before fuel is exhausted. Possibly the age of the animal may be a factor of influence.

Behavior of Organs and T'issues.-'The behavior of the different organs and tissues illustrates the diverse resistance of the several cells. 'The eyes and skeleton lose the least in weight, not over 5 per cent. The heart, skin and total mass of blood are reduced possibly one-sixth, though the figure for the blood has been ealculated much higher and is obviously difficult of determination. The same figure holds for the brain; for a time the central nervous system resists reduc- 
tion, but later in starvation fat is abstracted in amounts that are really surprising. The glands of the body, as a whole, lose more than the muscles, 50 as against 40 per cent. The loss of weight in the liver is all the more marked when the fatty infiltration is taken into account. The connective tissues are greatly wasted, and furnish to the glands certainly a large amount of protein. In the opinion of the writer, the process of cellular sustenance in fasting is identical with the process of maturation of the sexual organs in anadromous fish; the muscle substance is catabolized, the sexual glands utilize what is needed, and the balance of the amino-acids are burned. So in starvation the least essential cells yield their protein, it is hydrolyzed, the essential cells utilize what is needed for anabolism, and the superfluous amino-acids are burned.

In what may be called successful experiments in fasting, the fat of the body (disregarding the lipoids of the central nervous system and referring to depot fat) is reduced to mere traces. The glycogen becomes reduced to traces but persists to the last in liver and the other glands and in muscle. It is not possible to make an animal body glycogen-free by starvation and voluntary exercise.

The wasting of a fasting body is dimensional and not numerical in the cellular sense. While the individual cubic centimeter of blood is normal in composition, it is still possible that the total mass of blood in the body is reduced. The number of cells remains constant, so far as can be determined; even the cells of the connective tissues that yield most protein are only greatly attenuated, not obliterated. The different degrees of wasting in various tissues obviously expresses the different degrees of intracellular attenuation. When the animal is again fed up, the cells are restored to their original dimensions. The process has been strikingly illustrated in experiments on the expression of substance from muscle cells by hydraulic pressure; under a pressure of some 300 to 350 atmospheres applied to fresh lean muscle, no less than 44 per cent. of the contained protein could be expressed, without any change in the microscopic appearances of the individual muscle cells.

Protein Starvation.-When an animal is deprived of protein and given a liberal ration of carbohydrate, more than enough to cover the heat production of the body, we place the nitrogenous metabolism in a situation in which the powers of adaptation of the function are strikingly displayed. There are a number of such experiments on men, but if the results of more recent studies on swine are applicable to man, the periods of the tests have been too brief. Apparently the protein metabolism does not strike bed rock, so to speak, in less than two weeks, and does not before that time exhibit its full powers of restriction. The striking results are the lowness of the output of nitrogen, the evenness of the curve of urinary nitrogen and the constancy of the elimination of urea (plus ammonia), creatinin and the purins. The total nitrogen in men falls to about 3 grams, and would probably fall 
in a longer test to less than 2 grams. The urea-ammonia make up about 55 to 60 per cent. of the total nitrogen, the creatinin about 17 to 20 per cent. The ammonia is low, as there is no acidosis. Evidently we have here, as clearly as the experiment can reveal them, the basal nitrogenous requirements of the body for up-keep and wear-and-tearfor the regeneration of the cells that are daily undergoing death and the wear and tear of all functionating cells. The amounts are so low as to make it certain that for a long time the body can secure protein for the essential cells from the non-essential cells without approach to the limit of safety. If the input of carbohydrate be reduced below the caloric requirement, the nitrogen of the urine rises. Fat cannot be used to supplant the carbohydrate except to a limited extent. Protein hunger with fat ingestion would be little different from the early stages of fasting, except that the elimination of nitrogen would be a little lower. The maximum saving power of carbohydrate is exhibited in the experiment of protein deprivation with carbohydrate ingestion, and it is under these circumstances that we observe the lowest level to which the protein metabolism has been brought in experiment. Growth is not possible in an animal during the course of such an experiment. If the data be calculated to apply to longer tests, it is apparent that such a diet could sustain life in man for probably one hundred and fifty days, or three times the probable duration of life in complete fasting. If protein be administered to an animal in protein hunger, it will be utilized most effectively the longer the animal has been deprived of protein. If a large ingestion be given, it will be metabolized with great rapidity.

Carbohydrate Fasting. - Carbohydrate fasting amounts to the same thing as a protein-fat diet. If the diet be arranged so as to provide just enough protein to maintain nitrogenous equilibrium, this will be found to be much higher than in the same subject on a proteincarbohydrate diet. The sugar derived from the catabolism of the protein is sufficient to insure the normal combustion of fat (apart from some acentonuria possibly) and to protect the sugar concentration of the blood and the glycogen stores of the tissues. The specific dynamic action of such a diet is high, just as the plane of exogenous protein catabolism is high. It is not to be questioned that such a diet may under certain conditions of life bc termed a normal diet. It is commonly employed in the polar regions and by explorers and hunters in the form of powdered dried meat and fat (pemmican). It is a diet yielding much heat but making heavy demands on the nitrogenous metabolism. It is often employed by physicians to repair depletion of tissues after disease, for which purpose it is not adapted, since with an isodynamic input of carbohydrate and protein, the body would lay on flesh much more rapidly. Fat builds fat truly; but meat does not build meat; in the absence of carbohydrate it simply yields heat and throws upon the body the burden of catabolism of excess of protein and elimination of the end products. 
Fat Starvation.-This is the same state as a protein-carbohydrate diet. Upon such a regimen the body will establish nitrogenous equilibrium on a low level, not over 6 or 7 grams per day. Such a diet is experimentally almost ideal; it yields a large amount of energy with the minimum of metabolic work. The specific dynamic action of the diet is low, for with the saving power of sugar for protein the nitrogenous metabolism is low. Since the body retains nitrogen fully with an abundance of carbohydrate, repair of lost cellular protein and fleshening of every kind is well accomplished. The reaction for the formation of fat from sugar insures the proper storage of fat in the body. The ration of carbohydrate is, however, rather large for some individuals, who suffer from fermentative carbohydrate dyspepsia. In the world of experience outside the experimental laboratory, the absence of fat from the diet is known to carry with it elements of danger. In infants particularly the fat-free or fat-low diet is known to predispose to rachitis and to subnutrition. And in large masses of population subsisting on diets nearly free of fat, a tendency to undernutrition seems certainly to be apparent. Experimentally it is possible to nearly free the animal body of depot fat by feeding with protein and carbohydrate only in quantity sufficient to cover the minimal nitrogenous needs, leaving the caloric wants largely unprovided for. If the experiments are carefully done, the body will be made almost free of depot fat, while the tissues have lost but little nitrogen. This experiment is duplicated in the rigorous reduction of obesity; and experience has taught that danger attends the procedure.

Flesh Diet.-The dog will subsist indefinitely on meat and for experimental periods on protein plus salts. Man also is able to live on flesh alone. It is at first a tax on the organs of digestion, it is certainly a tax upon the organs of nitrogenous elimination; it is at all times a very wasteful and extravagant diet and one that weight for weight does not support heat or work well, but can be tolerated, especially in outdoor life, for long periods of time. The preference of barbarians for the flesh diet has no physiological meaning. The testimony of explorers and sportsmen who have chosen or been compelled to live on flesh is to the effect that once experienced it will never again be the diet of choice, but only of final and inexorable necessity. The greater the heat demands upon the organism, the more onerous and unsatisfactory the diet becomes.

The input of protein required to reach and maintain balance in nitrogen, in the absence of carbohydrate and fat in the diet, is three to four times the amount of protein required in starvation of medium length. In other words, nitrogenous equilibrium would not be established on less than an input of 20 to 25 grams of nitrogen. At the most such a diet would offer no more than 600 Calories of heat, leaving an enormous deficit. If now the input of protein be progressively increased, the figures of nitrogen output will ascend proportionately, more and more heat will be thus derived and the combustion of body 
fat correspondingly reduced. When the input of protein is so augmented as to cover the caloric needs of the body, the nitrogenous metabolism will be in equilibrium on a very high level and the body heat will be supported by the dynamogenetic combustion of protein. If more protein be ingested a certain storage may take place; and in a curious manner, to the excessive specific dynamic action of the enormous ration of protein is added a further tendency to exaggerate the total heat production in a relative manner. If to a diet of excessive protein, carbohydrate be added largely, the body can be induced to retain rather large amounts of the protein, in one state or another-to lose it again so soon as the input is reduced. To cover the caloric requirements of a man of 70 kilos at moderate work living on a flesh diet, not less than 750 grams of protein must be ingested, with a nitrogen content of 120 grams. The striking discrepancy between the specific and the dynamogenetic properties of protein are brought out in these figures. Under the most favorable conditions, protein will support nitrogenous balance on an input of 5 grams of nitrogen per day; but to support the body heat, twenty or twenty-five times as much protein are required. From the sugar derived from this protein the body will easily maintain its stores of glycogen, and some fat may be laid on. But, as a matter of fact, it would be difficult to select another diet that would lay on as little flesh and fat as a pure meat diet. Entirely apart from the consideration of the dynamogenetic aspect of the diet, the enormous labor imposed on the catabolism of protein and the elimination of the end products renders the diet highly undesirable. Even in the pure carnivora, the experience of breeders and trainers of dogs is unanimous to the effect that a pure meat diet is unadapted to the best functionation of these animals, as well as to their best growth and work. A dog will work on a pure meat diet and labor hard if forced. But coursing trainers have long made the experience that "meat gives no wind;" in other words, the maintenance of work is accomplished even in carnivora in the most effective manner on a diet composed of a moderate ration of protein and a large input of carbohydrate.

Forced Feeding.- The ingestion of heavy, excessive rations of sugar and fat lead simply to storage. No exaggeration of the combustion of these substances follows the ingestion of amounts in excess of requirements; nor is the protein metabolism materially affected. On full protein ration, excess of sugar and fat have little effect in saving protein; they have no effect in exaggerating the catabolism of protein or of the total heat production of the body. From this it follows that if the processes of digestion are normal, it ought to be possible to fatten every resting individual. Now rarely an individual is encountered in whom despite excessive resorption of sugar and fat, no fattening occurs. Unless such a result can be explained on the basis of excessive loss of heat through individualism in the mechanism of physical regulation, it is possible to assume that in these subjects the body treats fat and sugar in the same way as all bodies react toward ingestion of exogenous protein-all is catabolized. Experimental data are lacking. 


\section{THE NORMAL DIET}

About the fraction of protein centre the problems of a normal diet. Recent years have contributed greatly to the clarification of these questions. In particular, the definition of the physiological minimum of protein intake has been accomplished. The earlier ideas on the protein ration of a normal diet were derived from statistical observation. The more recent ideas are founded upon thorough physiological experimentation. The earlier standard diets were rich in protein. The experimental diets are relatively poor in protein. Before taking up the consideration of the experimental data, it is necessary to define a diet and then to consider the merits of the statistical method of fixing the components.

Desiderata for Diet.-A diet must fulfil many desiderata, and these are better expressed in sentences than in subdivisions of a sentence. A diet must first of all maintain a nitrogenous equilibrium in the body. It must yield to all the cells of the body such a complete series of the amino-acids and such abundance of these as to enable the anabolic and wear-and-tear processes to be accomplished with the highest efficiency and with the least metabolic exertion. It must provide materials for anabolism of protein in gestation, in lactation and during growth.

A diet must contain energy-bearing foods sufficient to maintain the caloric equilibrium of the individual under his conditions of life. The division of the carbonous foods between fat and carbohydrate will rest to a considerable extent upon the taste and purchasing power of the individual. The law of isodynamic values in these foodstuffs is the foundation of the diet, in the economic sense.

A diet must satisfy the reasonable demands of taste and bulk. It must provide variety in order to satisfy taste, for variety is as essential to appetite as is the most toothsome preparation of food. It must provide a certain bulk, because the physical sensations of satiation are to some extent dependent on bulk; and bulk means also residue for the formation of the feces.

A diet must contain to a certain extent fresh or at least raw foods, upon the absence of which marked disturbances of nutrition, such as scurvey, are known to rest, though the mechanism of these abnormalities is unknown.

A diet should contain a certain fraction of vegetable fiber. Cellulose aids in the physical management of the intestinal contents, prevents caking, renders the intestinal mass more porous and thus facilitates resorption and furnishes a bulk to the stools, of great advantage to regular defecation.

A diet must contain the salts needed by the body, in the qualitative and quantitative sense. 'This is the great advantage of the mixed diet, the salt needed are reliably contained only in such a diet. A diet ought not to contain an excess of salts or of one kind. The use of table 
salt in the diet, as developed under modern tastes, is not physiological but gustatory and is often carried to excessive and injurious extent.

Lastly, a diet should contain extractive substances and flavoring materials that stimulate the secretion of gastric juice. The importance of early and normal secretion of gastric juice to intestinal secretion and digestion has been dilated upon. Bland diets of simple foodstuffs are apt to fail in stimulation of gastric secretion, at least, under modern conditions of life. An excess of extractives, however, is to be avoided, since they tend to replace nutrients. The fault of the modern diet is in many instances that it has flavors but lacks calories.

A diet, in components and in every way, must be adapted to the age, sex, occupation and external climatic relations of life. A serious infraction of this dictum is rarely to be noted, except under cruel exigencies of want or isolation. In modern society, the exaggeration of the point of view of taste and the existence of preconceptions have led to the externalities of the diet being given such consideration over the internalities of the diet as to make many questions of diet financial rather than physiological. The physical and chemical requirements of a diet are nowadays but a small fraction of the food requirements of the diet, using the term in its external sense. It is no exaggeration to state that our people could be physiologically nourished on a fourth of the present financial outlay for the diet. The esthetics of the diet have assumed almost complete control over the energetics of the diet. Subnutrition is not the result, overnutrition rather. But it has led to the overcapitalization of the function of nourishment and has from this point of view established conditions of serious sociological and industrial import.

Amount of Protein Required.-The standard diet defined by the statistical method allows from 115 to 150 grams of protein per day. The theory of judging a diet by the data of the statistical method rests upon the broad foundation that instinct and appetite are spokesmen of physiological needs; and when through generations of racial observation and individual experience, the consumption of food displays a certain mean, it may be taken for granted that this is the best figure in a diet. The modern diet, in a sense, is regarded as an expression of the survival of the fittest. So far so good, and there can be no objection to the general standpoint herein stated. But when we come to analyze the facts, the generalization as stated is seen to lose much of its force. For it is easy to demonstrate that many other factors than physiological experience have been potent in moulding the dietetic notions of a people. Religious rites are closely connected with diets. Will anyone contend that the ritual as related to diet, be it in the savage tribe or in the modern church, is in any way expressive of physiological needs? We have developed from barbarians who followed the chase partly because it was the most easy method of securing food, partly because the instinct to prey was native in the savage breast. Geographical considerations and climatic factors have modified largely 
but irregularly the development of the race from savagery through barbarianism to civilization. In some lands races were compelled to adopt cultivation of the soil, in other places fishery; in some areas, the chase remained long into relative civilization one of the chief methods of securing food. The variations in ethnological development brought about by enforced cultivation of the soil, as contrasted with the state of affairs in a tribe of hunters, are well illustrated in different tribes of our American Indians. Depending upon the method of sustaining the life of the tribe, the standard diet of the tribe varied. Only under modern conditions of transportation have the instincts and tastes of man had opportunity for full choice in diet. Compulsion to some extent and in some degree there has always been. Customs and racial preferences and prejudices are reflected in the diet of a modern people. Dependence on raw and red meat, still to be noted in the diet of the prize fighter, runs back to the days of the chase when red blood and flesh, for man and hound, meant strength, courage and endurance. A survey of the development of taste in foods through the centuries and a correlation of diet customs with the available foodstuffs of the time do not lend much color to the hypothesis that the development of customs in diet have been materially guided by the experience of the race in the concrete physiological sense. According to the best information, the following figures represent in grams the meat consumption per day in the named countries: Australia, 300; United States, 150; England, 130; France, 90; Netherlands, 85; Austria Hungary, 80; Russia, 60; Spain, 60; Italy, 30; Japan, 25. These figures do not afford much support to the idea that the protein consumption of a race corresponds to work or metabolic needs. Practically every native race has discovered alcoholic fermentation. Is this an argument for the physiological value of alcohol?

Leaving the general question, one concrete appeal to the statistical method here concerns us. It is contended that, as a rule, the working classes increase the input of protein when work is increased; that the diet of the working classes is richer in protein than that of the leisure classes. Naturally, the statement is made with the restriction that the working classes concerned must have choice in the selection of the diet. As a matter of fact, it is not true that working classes raise the input of protein when working hard; the world over the price of protein is such as to make choice in protein input very restricted. The German peasant, the oriental coolie, the Italian laborer, the Slav farmer, the cane cutter on the Gulf plantations statistically do not consume much protein; on the contrary, the diet of these classes serves better to illustrate on how little protein men may subsist and work. That undernutrition exists in all these classes is probable; but the cause of this undernutrition cannot be single, it has many elements. It is true, however, that in this country and in England, the popular ideal of diet for the working classes includes a high ration of protein. The American farmer knows the value of fat in the diet, knows it too well 
in fact, since with the conditions under which he works carbohydrates would be in part preferable. The diets of our farming folk are rich in protein. The same is true of the diet of lumbering and construction camps. But at the most the nitrogen input of the working classes does not equal that of the leisure classes-a statement of fact as true here in the land of choice as in Europe where choice in the diet is largely confined to the leisure classes. 'The leisure classes eat meats because the flavors stimulate the jaded tastes and appetite, and because the cuisine of meats is more highly developed than is the cuisine of vegetables. The laborer eats protein heavily simply because he eats heavily of every thing, and naturally consumes a goodly ration of albumin. The only individuals who elect a heavy protein diet with the idea of its supporting qualities are athletes. It is a fair question, since the predilection for a heavy meat ration is confined to Anglo-Saxon athletes, whether this is not the direct descendant of the old red meat and blood notion of the Englishman. Athletes in numbers in our country have demonstrated the perfect reliability of the low protein diet for the most severe athletic contests. Masters of coursing hounds long ago discovered that meat is not the best food for the dog, but that a diet moderate in protein and rich in carbohydrate gives the longest wind and strongest leap.

Protein Content of Milk.-Whe most striking statistical evidence bearing on the question lies in the protein content of milk. This, however, is not in favor of but is opposed to the idea of a high input of protein in the standard ration. There are marked variations in the protein content of the milk of different animals, but perhaps the most striking fact is the low protein content of human milk. The milk of canines and felines is very rich in protein; that of cattle, sheep, and goats is moderately rich, that of horses poor, that of woman probably poorest of all. Yet, of course, these various milks are adapted to the nourishment and growth of the young of each species. It is a fair argument that the amount of protein relatively necessary in the diet of the growing infant may be taken as an amount fully competent in the adult. To the mind of the writer, the sole eseape from this conclusion must rest upon some hypothesis that the dynamogenetic utilization of protein possesses a peculiar and exceptional value. "Blut ist ein ganz besonderer Saft." But unless it can be shown that in the conversion of potential energy into work, protein is peculiarly superior to sugar and fat, the protein input per unit in infancy must be regarded as the maximum coefficient, since at no other time of life are such demands made upon the anabolic functions.

Protein and Anabolic Function.-Physiological experimentation, on the other hand, is based upon the proposition that protein in the body is needed in the functions of anabolism in the regenerative processes and to maintain the status quo of protoplasm, the wear-and-tear of the cells and tissues. Based upon the isodynamic law, the fuel value of protein is judged as fat and sugar are judged. Experiments have 
been carried out in extenso to define the essential cellular fraction of protein metabolism, and to separate it from the dynamogenetic fraction of protein metabolism. It is conceded, on the basis of known powers of compensation and adaptation, that investigations to be reliable must extend over long periods of time, that they must cover every exigency of life, that not only minimal but even unusual demands upon the economy must be fully met, and that in addition a margin of safety is to be permitted. We are now in possession of an enormous literature bearing on the utilization of protein in metabolism; and the definition of the necessary input of protein under varying conditions of life may now be made. 'The conclusions of the physiological investigations have contradicted the inferences drawn from statistical surveys.

Catabolism of Protein in Inanition.-In the study of inanition we learned that the catabolism of protein within the body can be separated into several fractions. (a) Dynamogenetic catabolism for purposes of heat production. This occurs for the first few days in starvation. It occurs in normal subjects when protein is ingested in excess of cellular requirements, the catabolism of exogenous protein is dynamogenetic. (b) Catabolism of indifferent protein to yield material for the anabolism of the essential cells. (c) Catabolism of indifferent.protein to yield sugar to maintain sugar concentration of the blood. (d) The essential protein catabolism, the catabolism associated with the wear and tear and up-keep of the cells of organs and tissues. In a normal diet the input of protein must cover fractions $(b)$ and $(d)$. In nitrogen starvation it may be shown that these two fractions comprise an amount of protein that for a man of 70 kilos is represented by a nitrogen output of not over 3 grams per day. Viewing the matter from the standpoint of protein content, rather than of protein utilization, we have four fractions of protein in the body. (a) The irreducible protein of the cells. There is a shrinkage that is tolerated by every cell, but there is a point in the reduction of protein content that is fatal. (b) The fraction of protein that the cells yield to demands in starvation or nitrogen hunger- the protein between the usual state of the cells and the irreducible protein. (c) There is a fluctuating fraction of cellular protein, possibly advantageous for good functionation, the fraction lost in the first few days of starvation. (d) Lastly, the luxury fraction of protein, the protein that can be induced to go into the cell in protein engorgement, to remain a longer or shorter time after the excessive feeding is discontinued. This fraction is usually small. It is because the body refuses to store proteins that exogenous protein is usually fully and promptly catabolized. Fractions, $a, b, c$, ought to be covered by the protein input of a normal diet.

Protein in Mixed Diet.-Now in a mixed diet, with usual amounts of carbohydrate and fat, what amounts of protein are required to cover the needs of the body and to maintain the normal protein content of the fluids and cells of the body as defined above? From a large number of investigations it is certain that on an average, a half gram 
of protein per day per kilo body weight is sufficient to accomplish this. This would cover the body needs, maintain the normal state of cellular protoplasm, and impose no conditions on the anabolic or catabolic functions of the cells; it would leave little exogenous protein to be catabolized, it would provide little protein for dynamogenetic purposes. The data upon which these statements are based are so extensive and the results in the hands of numerous investigators so unanimous, that there can be no escape from the conclusions.

The crux of the question here appears: Is there warrant or necessity for restriction of the rôle of protein in metabolism to the function of protein in protoplasm? Has protein any advantages as a mere fuel? Does the use of protein as a fuel carry with it any disadvantages? Does the figure stated above carry with it a wide margin of safety, for all ages, sexes, states and conditions of health and disease, for occupational and climatic variations? Is the proposed restriction of protein to the needs of the nitrogenous metabolism a matter of advantage or merely of choice, of indifference to the body?

Excessive Intake of Protein.-Does the use of protein in excess of the cellular requirements (dynamogenetic utilization), possess any advantages? The exogenous protein is hydrolyzed, the amino-acids deaminizated, from a fraction of these sugar formed, the rest is burned. The sugar formed from protein is, of course, identical with the other sugar of the body. If there be any advantage in protein over carbohydrate, it must lie in the fraction of fatty acids from which sugar cannot be formed and which are burned directly. But it passes imagination to ascribe to these simple fatty acids any peculiar and particular functions in the body. So far as we know, the fatty acid fraction of the protein is worth just its caloric content and nothing more. The nitrogen is eliminated as urea and will not be invoked as a valuable substance. When one contemplates the curves in the elimination of nitrogen on a rich protein diet, the curve of production of heat due to the specific dynamic action of the protein and the relatively straight line of elimination of carbon dioxid, one cannot repel the feeling that the metabolism of exogenous protein is a wasteful process.

Has the use of protein for dynamogenetic purposes any disadvantage? Chemically and calorically it is an undesirable fuel. Sugar passes directly from the mucous membrane of the intestine for utilization in the body. 'The products of the digestion of protein must be reconstructed. They must be hydrolyzed, the amino-acids deaminizated, and the nitrogenous end products eliminated by the kidneys. This elimination represents work. The ideal fuel is the one directly available for combustion without preparation. The best diet theoretically is the one that makes the least use of protein as a fuel.

Low Protein Diet.-Is there any evidence that the restriction of protein to the cellular needs carries with it any loss of vigor to the body, any restriction of function, any diminution of adaptation and compensation in any direction? Mystical references to such supposed 
positive properties of a rich protein diet are not absent from the literature; but of concrete statement there is none.

Is the low protein diet adapted to the putting on of flesh, as in youth? This is a point commonly misunderstood. A rich protein diet is not a ration that makes flesh. A pure meat diet forms very little flesh. The protein that is burned as fuel makes no flesh. The protein that is ingested in excess of the cellular needs is catabolized, it is not converted into flesh. Stuffing with protein does not lead to fleshening. It is carbohydrate that has the sparing power for protein. The ideal ration for laying on muscle is a moderate input of protein and a large ration of carbohydrate. This is the natural relation of protein to fat and carbohydrate in the mother's milk; human milk contains only about 0.3 to 0.5 gram of protein per kilo per day; or in terms of calories, the calories of the protein are only about 7 per cent. of the total calories of milk. The suckling has a large surface area and a high coefficient of heat production, but the protein needs are no more than those stated in the figure above. It is the heavy ration of sugar in human milk that makes the low protein so effective as a flesh builder, and this relation holds true in all ages.

Margin of Safety.-Does the figure stated furnish a wide margin for safety? There are, of course, variations in the unit of intensity of metabolism. As a rule, the protein metabolism will be roughly proportional to the mass of protoplasm in the body. But variations occur. Diet contains always more or less preponderance of certain proteins; this is not to be avoided. The nitrogenous balance must always be considered in connection with the total heat transformation. A protein minimum must not represent a minimum on a certain diet. Total-N must not be held synonymous with protein-N. With the use of certain proteins, wide variations would be observed; in direct experiments, proteins and proteins are not comparable. The lowest nitrogen balance on a single ration is to be obtained with potatoes, rice and bread. A diet of peas is much less effective in attaining nitrogenous equilibrium. Thus the protein minimum of a single foodstuff may vary 50 to even 80 per cent.; the minimum on a potato diet is scarcely more than half the minimum on a bread diet. Lower still is a diet of casein and sugar. Theoretically, with abundant carbohydrate blood serum would be the most effective protein in a diet. These observations warn us that the arbitrary establishment of a minimum must include the exact conditions of the test or experiment. The standard protein minimum must also be so ample as to permit of the substitution of a large share of the carbohydrate by fat, at the choice of the individual. Bearing all this in mind, and bearing in mind the fact that a minimum diet ration must in every instance be not only safe but generous when it is allotted to a multitude, we face the quantitative determination of the margin of safety. Certainly 100 per cent. margin of safety must be deemed large enough to cover securely and, indeed, generously every possible exigency. This would make the 
protein input for a diet of mixed proteins, with carbohydrates and fats in the proportion of choice, about 1 gram per kilo per day for a subject of 70 kilos. For a smaller subject, the ration should be relatively a little larger; for a larger individual, it should be relatively a little smaller. The quantitative relation of the different experimental planes of nitrogenous metabolism, including this figure for the normal, are grouped in the following table:

Nitrogenous metabolism for a man of 70 kilos per day:

Catabolism in N-hunger with carbohydrates .

10 to 15 grams

Catabolism in starvation, lowest level

Nitrogenous and caloric equilibrium, with ample ingestion of carbohydrate

Nitrogenous and caloric equilibrium, largely with fat

Normal protein input, safety margin of 100 per cent.

Nitrogenous and caloric equilibrium on protein diet

15 to 20 grams

30 grams

40 grams

70 grams

750 grams

Plant Protein.-Does plant protein fill the requirements of a diet as well as animal protein? So far as the physiologist is concerned, the question of vegetarianism presents no difficulties. Upon minimal diets, a ration of vegetables will need to contain more protein than a mixed diet, simply because so many plant proteins are one-sided in their content of amino-acids. But the amino-acids themselves are identical in plant and animal protein, it is simply a question of all the needed amino-acids being present. With the input of 70 grams, plant protein is fully qualified to maintain the protein metabolism. The problem of availability of a completely vegetarian diet hinges upon questions of bulk, fermentability, digestibility and variety-not upon any question of the utilization of plant proteins.

Such a protein input is certainly sufficient for the needs of the body under all conditions of work and climatic surroundings. Work and external cold demand more carbon, and while this may be furnished in the form of protein, it is preferably furnished in the form of fat or carbohydrate. Granted that the low protein diet is in every way equal to the full maintenance of the body, is the high protein diet to be condemned? Does the extra work imposed on the liver and kidneys constitute an overload of possible danger? In health this overload seems to make no appreciable difference, it is well within the limit of adaptation and compensation. But in many subjects the overload is not well tolerated. And although in modern life it must be recognized that the carrying of overloads is necessary in our complicated civilization, it is hardly to be considered wise for one to deliberately elect to carry an overload that has so little meaning or desirability. In a certain sense, a heavy meat diet is to be classed with indulgence in tobacco and alcohol; in moderation of no demonstrable injury to perhaps the majority, but never to be regarded as a benefit.

Intake of Carbohydrate and Fat.- The relative inputs of carbohydrate and fat depend on the external conditions of temperature, on work, on the digestion of the subject and upon taste. For moderate caloric 
inputs (up to 3000 Calories per day) carbohydrate is certainly to be preferred, though fat should not be excluded. As the needs for heat rise, the input of fat may be gradually increased, so that in hard physical work it is well to have fat furnish a goodly fraction of the energy. There is a certain anti-sugar fad that is more or less prevalent. According to this fad, sugar is the cause of many ills, very loosely defined. There are, of course, instances in which starches are tolerated in the digestion when sugars provoke disturbance. But once digested, sugar and starch are identical in chemical and physiological properties. In the very nature of industrialism, carbohydrate must be the chief fuel of the future, since animal fat is produced only at the expense of maintenance of a costly protein metabolism. Future cultivation of fat-bearing plants may, however, make oils partially available for nourishment of the masses. One of the great problems of the future is to effect reformation of customs and tastes to the end that foods shall be cultivated and utilized on the basis of caloric value per unit of price. Domestic management in this country is grossly extravagant because of ignorance of the nutritional values of foodstuffs in their native and prepared states.

Calculation of Diet Values.-In the calculation of diet values, allowance must be made for the unresorbed fraction. Taking this into account with normal digestion, it is approximately correct to use the following constants as a basis for calculations applied to the known amounts in the diet. The figures in the first column represent the caloric values, in the second column are the caloric values in utilization, per gram.

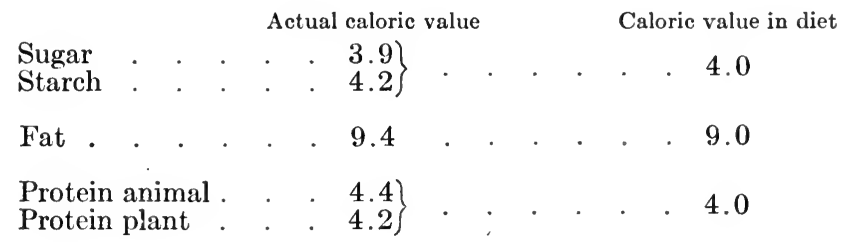

If circumstances compel one to determine the unresorbed residue, the nitrogen of the intestinal secretions must be taken into account, and then the actual values for protein and fat can be used. The following table gives the figures for a number of arbitrary normal diets, ranged for progressively greater demands for heat, the amounts being stated, heat in calories, foodstuffs in grams per day:

$\begin{array}{cccc}\begin{array}{c}\text { Heat requirements } \\ \text { Calories }\end{array} & \text { Protein } & \text { Fat } & \text { Carbohydrate } \\ 2100 & 70 & 50 & 350 \\ 3000 & 70 & 75 & 500 \\ 3500 & 70 & 100 & 700 \\ 4000 & 70 & 150 & 700 \\ 5000 & 70 & 225 & 700 \\ 6000 & 70 & 250 & 850 \\ 6000 & 70 & 400 & 500\end{array}$


These figures represent usual averages of diets of choice (with protein fixed) for the purposes of production of the stated amounts of heat, varying from the state of rest in the first line to very hard manual work in the last line. Usual hard work does not require over 4000 Calories, or at most 5000.

The figures for carbohydrate and fat are in a certain sense arbitrary, representing, however, usual values. Fat and carbohydrate are interchangeable, within certain limits in the diet, in accordance with the isodynamic law, which holds both in heat production and in work. The restrictions of the isodynamic law must be kept in mind. This law does not state that under all conditions protein, fat, and carbohydrate are interchangeable in the diet in the proportions of their caloric values. For protein the law holds only for the dynamogenetic utilization of protein. But even here it fails with large ingestions, since excessive protein input results in an exaggeration in the total metabolism and heat production, which an isodynamic ingestion of sugar or fat would not do. Carbohydrates should be replaced by fats only above the limit of the operation of the saving power of carbohydrate for protein. And finally, the factor of digestion may complicate the situation. Nevertheless, within wide limits and especially for sugar and fat, the law of isodynamic utilization holds.

\section{NUTRITION IN INFANCY AND CHILDHOOD}

This is a subject of great importance for the theory as well as for the practice of nutrition. Before considering the metabolism of the child, it will be instructive to review briefly the influence of gestation and lactation on the metabolism of the mother.

The tissues of the child are derived from the mother and under all circumstance, therefore, must be represented in her metabolism. But during the early months of gestation, the growth of the fetus is so gradual and involves so little material, that any effect upon the metabolism of the mother could not be marked. On the other hand, the pregnant woman is building uterine tissue, mammary gland substance, and laying on flesh herself, largely fat it is true, but nevertheless resulting in an influence on the total metabolism. A retention of nitrogen would, therefore, be expected during the entire period of gestation; during the early months largely in the mother's body, during the last two months of gestation, however, largely in the body of the child. Since in the body of the child a large amount of special proteins are synthesized, there must be superfluous amino-acids to be catabolized. This is probably the cause of the excess of nitrogen to be found in the urine of animals during the early weeks of pregnancy, increased also by the superfluous amino-acids due to the formation of uterine tissue. At the same time, the amount of excess of nitrogen is rather more than could be reasonably accounted for on this basis alone, unless we assume that the processes of anabolism in the early establishment 
of the central tissues are particularly specialized. Later a retention of nitrogen is observed, and corresponds to the building of the new tissues. A pregnant female will retain nitrogen on a diet that previously insured only equilibrium. The amount of retention is not large until the final weeks of gestation, when it rises rapidly and may during the last days reach as much as 4 or 5 grams of nitrogen per day. After delivery, despite the establishment of the secretion of milk (casein formation), the nitrogen of the urine passes from retention to loss, on account of involution of the uterus (a self-digestion that results in the catabolism of a large mass of amino-acids), extending over a number of days, possibly up to ten days or two weeks; when involution is completed, retention for purposes of casein formation is established. It is doubtful if the protein formed in the tissues of the full-term child exceeds that formed in the gravid uterus and hypertrophied breast glands; but the processes are very specialized and probably require more substrate protein.

The consumption of oxygen and the elimination of carbon dioxid at the close of pregnancy will run from 20 to 40 per cent. above the normal. Not all of this is to be attributed to the fetus, since there is extra work of circulation and respiration; and the additional maternal tissues have, of course, their metabolism. In the study of pregnancy in the dog, it has been found that the increased heat production is proportional to the number of young in the litter.

Lactation is a process involving extensive metabolic functionation. The milk sugar is formed in the mammary gland by conversion of d-glucose into d-galactose and the union of these to form the disaccharid lactose. The fats are abstracted from the body fat of the individual, and vary in composition with the body fat; no synthetic work is here done. The casein must be formed by hydrolysis of the blood proteins within the epithelial cells of the gland, followed by the construction of casein from the amino-acids thus offered, the superfluous aminoacids being burned. The work of lactation is much greater than the work of gestation in the unit of time; 100 c.c. of mother's milk, containing a little over 1 per cent. protein, 3 per cent. fat, and 6 to 7 per cent. sugar, has a caloric value of about 60 Calories. A usual secretion during the first weeks of lactation will run as high as 400 c.c. per day with a caloric value of some 250 Calories. Later in lactation when the child is larger, the secretion may be increased to equal 750 Calories per day. This is one-third to one-fourth the heat production of the mother. The child in the first few days of life has probably three times the metabolism that it had in the uterus during the last days of gestation. It is, therefore, clear that from the metabolic standpoint, lactation is a heavier task than gestation.

The composition of milk should biologically be independent of the diet of the mother. As a matter of fact, it is quite independent. Recent investigations have cast much doubt upon the older ideas of modification of the milk by modification of the diet. Starvation makes the 
milk rich in fat; forced feeding with carbohydrate makes the milk poor in fat. The sugar content seems quite constant, and with a normal ration of protein, the casein content is constant; and it is not increased by heavy protein diet. The amount of milk is quite an individual variable - just as in cattle it is a variable related to the breed. The milk of carnivora is very concentrated, that of herbivora much more diluted. Peculiar to human milk is the low content of protein and the high content of sugar.

Proportional to the large relative area of surface of the infant, the per kilo metabolism is very high. For each kilogram of weight in the newborn infant, the heat production is about 100 Calories, as against 30 for the adult. The heat production per area surface is, however, about the same, from 1000 to 1200 Calories per square meter surface area per day. Infants are normally fairly quiet during the first months of life and as they are kept very warm, the heat production of muscular exercise and for chemical regulation is low. Later, of course, it becomes large. The heat production per kilogram falls as the child grows, probably in a curve that resembles the logarithmal curve, the drop at first being rapid, later more and more gradual. At the age of a year the total metabolism probably corresponds to about 70 Calories per kilo per day.

Of the diet necessary to maintain this high intensity of metabolism, only about 7 per cent. is in the state of protein; the rest is divided between sugar and fat in practically equal proportions. A normal growth of an infant corresponds to retention of from 12 to 20 per cent. of the caloric equivalent of the total metabolism. In other words, if a child produces 100 Calories per kilo, the diet will need to contain the equal of from 112 to 120 Calories per kilo per day at least. The retention of protein amounts to from 25 to 40 per cent. of the protein input, illustrating the extremely advantageous utilization of a small ration of protein when ingested with an abundance of carbohydrate.

The same law of growth has been found in all animals tested. Growth is a specific, proportional to the input of calories in the milk, the heat production of exercise being taken into consideration. From this it is apparent to what an extent the daily growth of an infant is dependent upon the slight excess of calories in the food over calories in the heat production; a very little may make or mar the development of an infant. The rate of growth for man is very slow as compared with the rate of growth in most animals, but in each species on its own plane, the law holds. In growing animals; about double the calories of the heat production are contained in the natural diet; about 35 per cent. is retained as growth, the rest is expended in muscular exercise. In the case of infants the retention for growth, which is a specific of the species, is very much smaller and the expenditure also much lower, so that the infant does not ingest food containing double the calories of the heat production of the day. 
As the child becomes older the expenditure for muscular movements increases, while the ratio of growth remains about the same. From infancy to adult life, the daily addition to the body weight is a little more than proportional to the body weight. A disturbing factor is the allowance to be made for physical exercise. This is in children often displayed to the point of exhaustion, and sometimes makes the heat output of the child exceed even the adult output. A resting boy of ten years, should have a metabolism of about 40 Calories per kilo per day. At play, the diet of the child may have to be as high as 100 Calories per kilo per day to maintain caloric equilibrium. 'The diet of a child must, therefore, cover the basal metabolism, the natural increment of growth and the enormous output for physical exercise. It is the inability to judge these fractions correctly that is responsible for so much of underfeeding of children. There are furthermore the additional deprivations so often inflicted on children by the application of fad notions of diet. The relative caloric input of a normal child leading an outdoor life is to be compared to that of a man at heaviest physical work. Protein in excess is not needed, that is clear; but total calories are needed, in the form of sugar and fat. Fat is especially important in the diet of children, though it need never exceed one-fourth or one-third of the total caloric input.

\section{METABOLISM IN OLD AGE}

Metabolism in old age is relatively comparable to that in adult years under similar conditions of inactivity. The heat production per kilo seems to be lower, a couple of calories per kilo per day. The protein catabolism is low, not because the endogenous protein catabolism is much lower than at earlier years, but because the aged consume usually less meat. Less than 0.6 gram per kilo per day has not been recorded for the protein input of the aged; and this is no less than a normal ration when the depletion of the tissues is taken into account, for the tissues of the aged are richer in fat and poorer in protein than in earlier life. There is an increased adaptation to low inputs in the diet, probably because the wear-and-tear needs are low under the conditions of life. In a certain sense metabolism in old age resembles that in chronic subnutrition of mild degree. The retention of protein is least of all possible in old age; and corresponding to the reduced intensity of protoplasmic activity, the sparing power of sugar for protein is very pronounced in old age.

\section{CHRONIC UNDERNUTRITION}

Under conditions of want or associated with disease, a condition of undernutrition is observed that is as interesting physiologically as it 
is important sociologically. The subject subsists on diets obviously below normal requirements, often for long periods of time and naturally with marked loss of flesh. It is rare that the condition approaches starvation; it is much more to be likened to protein hunger, since under conditions of want or famine, protein is the food most lacking. The nitrogen of such subjects may remain stationary at 4 or 5 grams per day. There is nitrogenous equilibrium. When the protein input is raised ever so little, retention of nitrogen is observed; it is apparent that the cells have been depleted and on opportunity retain protein for restitution. The heat production, measured by the consumption of oxygen and the elimination of carbon dioxid, is usually about the lowest normal level, 28 Calories per kilo per day. In a few cases the heat production has been calculated to be as low as 24 Calories per kilo per day. The very lowest values are to be seen in the few days after the defervescence of a prolonged and severe fever. In other words, assuming the accuracy of the observations, these subjects may present as low heat production as the lowest values of normal men in sleep or at complete rest. The subjects of such subnutrition were very lethargic, and doubtless did not exert themselves much. It is doubtful, after allowance is made for muscular activity, if the heat production would fall more than 100 or possibly 200 Calories below that of the normal individual of low caloric output. In other words, the adaptation is largely in the restriction of the protein catabolism to meet a very low input, rather than in any real reduction in the unit of heat production or total metabolism. If the ingestion of carbohydrates be raised, the individual will retain nitrogen on the input to which want has accustomed him. The total heat prodution is lower because the total of body surface shrinks with emaciation. The subjects of subnutrition not the result of disease recover on diets that would be minimal for normal individuals, and later display the usual range of nitrogenous metabolism.

\section{OVERNUTRITION}

Overnutrition means fleshening or fattening, or both. It is sometimes sought by the physician, but is more often a natural occurrence. It is of importance to know how and under what circumstances flesh as well as fat can be deposited. It must be realized in the first place that growth is not completed until about the twenty-fourth year; that in young subjects permanent nitrogen retention (fleshening) is rather easily accomplished. There is, furthermore, a retention due to use. If a person be worked hard and persistently, weight will be lost but muscle will be gained; there will be retention of nitrogen, and after the period of development it will be found that the metabolism per kilo body weight is increased. 'This is the criterion between fleshening and fattening. Tested under conditions of perfect rest, fleshening raises the per kilo unit of metabolism, fattening lowers it. The reason is that metabolism is a function of protoplasm not of fat; 
in fleshening the relative proportion of protoplasm in the body is increased, in fattening it is decreased; when the total metabolism is reduced to the kilo weight of the individual, this is at once apparent.

A seasonal variation in protein, as well as in fat, is sometimes to be observed. The loss of weight in summer and gain of weight in winter may be in part a loss and gain in flesh as well as in fat. Men who have returned to the city to lead a life of leisure after a strenuous summer, are liable, in the gain of weight that occurs, to exhibit for a time a striking permanent retention of nitrogen.

Forced feeding with protein in the presence of carbohydrate has a certain retention of protein as a result, for a time at least. Under usual conditions, the retained nitrogen is lost after the forced ingestions of protein are suspended, especially in subjects over thirty years of age. During the period of stuffing, the heat production of the individual exhibits not only the increase due to the specific dynamic action of the protein, but also a further increase, often not marked but distinct. This is usually assumed to represent the metabolism of the extra-protoplasm that the cells take on under forced feeding. This retention may be in the circulating fluids, as well as partly in the cells, which are known to possess a power of physiological enlargement as well as a marked faculty of attenuation on deprivation of protein. While the concentration of protein in the blood plasma is not materially increased, it is possible that the total mass of blood in the body is enlarged. Analyses of flesh of animals that have suffered forced feeding with excess of protein have indicated that nitrogen is held in non-protein state, yet not to be identified with the extractive nitrogen. Usually this retention is not permanent; if into cells, later they shrink back to the former dimensions; if in the circulating fluids, it is catabolized. In other words, in the absence of protoplasmic undernutrition, the retention of nitrogen accomplished by forced feeding with protein is not permanent. In conditions in which the retention of protein is permanent, the same can be as easily accomplished on a moderate protein input plus liberal carbohydrate.

Fattening cannot occur on a protein diet. Forced feeding with fat in excess of the caloric requirement results in the almost quantitative deposition of the fat in the body. This is unquestionably the most direct and effective method of fattening, if the organs of digestion tolerate the administration of such amounts of fat. When carbohydrates are ingested in excess of the caloric needs, the heat production is not exaggerated; but yet the total excess of carbohydrate does not seem to be deposited in the form of fat. The glycogen stores of the body are under these circumstances filled, and this may account in part for the discrepancy. In part, the discrepancy may be due to fermentation of carbohydrate within the alimentary tract. While fattening with fat is experimentally more directly effective than fattening with carbohydrate, little difference exists practically in the utilization of the two rations. 


\section{OBESITY}

It is a common observation that obesity is the result of overindulgence in food, lack of exercise or both. 'The fact that the tendency to obesity is in some subjects hereditary and very pronounced, has led to the assumption of a pathological endogenous form of obesity. The nature of this state was assumed to lie in a retardation or reduction in the total metabolism or in a suboxidation, since it was soon learned that the protein catabolism was normal. The possibility of such a suboxidation has been elsewhere discussed. In the discussion of undernutrition, it was pointed out that the unit of combustion was not below the lowest normal level. The same statement holds true for obesity. Measured by the consumption of oxygen or by the total heat production, the results are within normal limits. The law of basal heat production remains inviolable. In every chemical and physical way, the subjects of obesity spare heat; and the result in toto is a very efficient and economical organism. 'The obese are fat not because they have suboxidation or retarded metabolism, but because they have a very effective economy. In many instances of obesity the heat production is not low but high; there are marked differences in these individuals. In the obese with high heat production, this will be found to be more than covered by very high input; the obese with low input and low heat production remain still within the limits of normal variations. 


\section{CHAPTER X}

\section{THE PRODUCTION OF BODY HEAT AND THE REGULATION OF BODY TEMPERATURE}

In the cold-blooded animals the velocity of chemical reactions is a function of the body temperature. If we chill the body of such an animal, the chemical reactions of the metabolism are diminished; if we raise the body temperature, the chemical reactions are increased. These animals lack all mechanism for the regulation of the dissipation of heat produced in the chemical reactions of the body. The body of the warm-blooded animal possesses a mechanism for the regulation of body temperature. If, however, the body temperature is raised above the normal, the same law that holds throughout the scale for the cold-blooded animal becomes operative; the chemical reactions are increased as the result of the raising of the body temperature. In cold-blooded animals, as in plants and bacteria, there is a mathematical expression for this increase in chemical reaction as the result of increase in temperature; it is the same relation known to exist for chemical systems in general, namely, that for each 10 degrees increase in temperature (under controlled conditions), the velocity of the chemical reaction is doubled or more than doubled. If we could reduce the temperature of a warm-blooded animal (which the chemical regulation renders impossible so long as function remains) it would be found that the law holds also for warm-blooded animals-just as it has been demonstrated for the warm-blooded cat as clearly as for the coldblooded frog, that the contractions of the heart are a function of temperature. It is the so-called chemical regulation that prevents the law of dependence of reaction velocity on temperature being demonstrable.

There are four separate fractions of heat production in the animal body. These in actual life overlap, but experimentally they may be separated. These are: the basal, irreducible production of heat; the heat of the specific dynamic action of the foodstuffs; the heat of chemical regulation; and the heat of contraction of voluntary muscles.

Basal Heat Production.- The basal heat production represents the heat formed in the fasting animal, at complete rest, at the temperature of $37^{\circ} \mathrm{C}$. Of this heat the body makes no use under these circumstances, since the body temperature would be maintained by the external temperature. As a matter of fact, this fraction of heat production, under modern conditions of life in an enclosed room with appropriate clothing, is the same at $33^{\circ} \mathrm{C}$. The amount of heat herein set free is not a strict constant, but varies in different individuals, at different ages, in the two sexes. We usually express heat production in terms of calories per kilo per day. If the subject be in normal 
flesh, not too thin and not too fat, this approximate mode of designation is satisfactory. A more accurate statement for most purposes would be to relate the heat production to the area of skin surface. That the heat of chemical regulation is directly related to the area of skin, under conditions of temperature lower than that of the body, is obvious; that the basal heat production at body temperature is related to the skin area, is not so certain. This basal heat production we may view as the expression of the reaction velocity of the chemical system of the body.

The amount of heat set free in this fraction is easily determined. It varies from about 24 to 26 Calories per kilo per day, or for a body of 70 kilos, approximately from 1675 to 1800 Calories per day. There are some individual variations, usually in the upward direction. Instances have been known in which by indirect calculation it seemed certain that the basal heat output could not be over 1400 Calories per day. There are probably individuals in whom this heat production rises to 2100 Calories per day. It is difficult to be definite, for the reason that experience and adaptation on the part of the subject is required to enable the operator to exclude all heat production by muscular movements. But on the whole, 25 Calories per kilo per day may be taken as a general average and few exceptions of note will be found. This irreducible basal production of heat continues when none is needed for the maintenance of the body temperature; it persists when with starvation its continuation means death. The body can adapt itself to needs for greater heat production; it has no adaptation to lower the basal heat production. This may be accepted, therefore, as the expression of the reaction velocity of the chemical system of the organized body.

In this heat are several separable fractions. The flow of circulation represents work; the acts of respiration represent work. There is some secretion of alimentary juices, even in fasting. There is work expended in renal elimination. In the processes of cellular repair is chemical work. It is clear, however, that these all do not make up the major portion of this heat production. The largest fraction is undoubtedly due to the combustion of glucose and fat; the former, if the resting fasting body be well stocked with glycogen; the later, in the event of prolonged fasting.

Heat of Specific Dynamic Action of Foodstuffs.-The second main fraction of heat production rests upon the specific dynamic action of the foodstuffs. 'This has been discussed. It may be taken to represent 200 to 300 Calories per day on average mixed diets, lowest when the ration of protein is least.

Heat of Chemical Regulation.--The third fraction is derived through reactions of combustion aroused through chemical regulation of body temperature. When in a resting fasting subject, the external temperature is lowered so that through conduction and radiation so much heat is being lost that the body temperature cannot be held constant on the heat of the basal production, more sugar and fat are cast into com- 
bustion and the heat production brought up to the demands of the moment. At $33^{\circ} \mathrm{C}$. there is no heat production of chemical regulation. It appears with reduction of the external temperature (or in any way whereby the heat dissipation is increased) and becomes progressively greater as the needs increase. How much heat can be produced in this way is not known, as it is very difficult to exclude muscular exercise (shivering) when the body is exposed. This function may be cultivated, and individuals may be so inured to external cold as to tolerate with comfort extreme cold for long periods of time. This is especially seen in swimmers. Any figure must remain a conjecture, but possibly 1000 Calories of heat per day may be derived from the heat production aroused through chemical regulation. This combustion occurs largely in the muscles, associated in part possibly with the muscular tonus.

Heat Associated with Muscular Contraction.-The last fraction of heat production is associated with muscular contractions of the skeletal system. We must distinguish between three kinds: shivering, involuntary restlessness, and motivated movements. Shivering appears so early and is so imperative on chilling the body that very competent students of physiology believe that chemical regulation consists of shivering, evident or hidden. According to this hypothesis, being inured to cold simply means technique in fibrillary shivering. It is certain that this is incorrect. Chemical regulation operates through an exaggeration of the same kind of combustion of carbonous substances that evolves the larger portion of the basal heat production. Possibly the figure allowed for heat production through chemical regulation is far too high; but heat production through chemical regulation is certainly not heat production by muscular contractions. Shivering is very effective in the production of heat, though on prolonged exposure to cold everyone prefers to exercise rather than shiver. Involuntary movements play a prominent rôle in heat production, and are the bane of the experimenter. The very slight muscular difference between resting seated in a chair and resting on a bed may lead to an increase in heat production of no less than 20 per cent. The fact of training in the technique of measurements of body heat on the part of subjects of such experiments makes, therefore, the greatest difference in the results. No two men are equally still when lying on beds as motionless as possible; no two men are equally still when asleep. And no two men are equally in repose under any stated circumstance of life. The marked temperamental differences in physical restlessness we term "nervous" and "phlegmatic" respectively. It is this element that brings the heat production of the sedentary man from the basal production of possibly 1800 Calories to some 2500 or more Calories. To this must be added the heat produced through motivated movements, the doing of work. Work may be classed as light, medium, heavy and maximum. Light work need not involve the output of more than 600 Calories per day; moderate work will involve possibly the production of as much more. Heavy work may involve the pro- 
duction of as much as 2000 Calories per day. And maximum exertion. such as swimming the English channel, heavy logging or participation in a six-day bicycle race, may involve the setting free of as much as 8000 additional Calories in the day. It is, of course, only exceptionally muscular individuals that can accomplish such performances. The average man, even in training for the particular task, can probably not work hard enough to produce a total of 8000 Calories of heat (including all fractions) in the twenty-four hours. The relative efficiency of work, as related to the associated production of heat, is not increased by training; but the numerical possibilities of muscular contractions of a given type are enormously increased through training. Under normal and controlled conditions, all these forms of muscular contractions are maintained through combustion of carbohydrate and fat largely; the protein metabolism need not be increased thereby, nor is muscular work of ordinary and accustomed type followed by increase in the elimination of creatinin, the specific product of muscular metabolism. When properly lubricated, the running machine loses very little metal from its working parts; and the muscle looses very little creatin.

The following table presents in approximate units the heat outputs of an adult under the usual conditions of life at medium activity at a temperature of $15^{\circ}$.

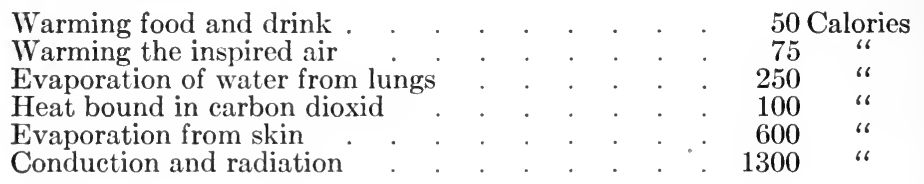

With increased work, the output of heat by radiation and conduction and by evaporation of water from the skin (to a slight extent also from the lungs) will be divided largely according to the individual variable.

\section{THE RELATION OF HEAT PRODUCTION TO METABOLISM}

The law of isodynamic relation of foodstuffs states that carbohydrate, fat and protein may replace each other in the body as fuel when used in the proportions of their caloric values. 'The fixed correlation of heat production and metabolism rests upon this basis. If in the resting, fasting individual, the exact amount of each of the three foodstuff's in the diet be known and the outputs of carbon and nitrogen be known, the heat production can be calculated with considerable accuracy. Much better results are to be obtained, however, if the oxygen absorption, the carbon dioxid output and the nitrogen output are known; from these three data the heat production for the day or the unit of time can be satisfactorily computed. It is also possible from such data to calculate back to the foodstuff's from which the heat must have been derived. Recent developments in the direct estimation of the body heat have made it possible to compare the values determined 
by the use of the respiration apparatus with the direct measurement of body heat; and with greatest care in experimentation, in the exactness of analyses and in the control of the subjects, the constants have been determined with such precision that with the use of the respiration apparatus results are now obtained that agree very well with the findings by direct calorimetry. Since the relative amounts of carbon, hydrogen and oxygen in fat and sugar are not identical, the relations of heat production to oxygen comsumption and carbon dioxid elimination must vary. In other words, the amount of heat as related to the oxygen or carbon dioxid is different in the combustions of fat and sugar, since heat is formed by oxidation of both hydrogen and carbon. Fat is almost free of oxygen; sugar contains enough oxygen to combine with all the hydrogen, leaving the carbon alone to require oxygen. Therefore, far greater amounts of oxygen are required in the combustion of fat than in that of sugar, and this is strikingly displayed in the respiratory quotient. The same argument holds for protein, though to a less extent than for fat. The caloric equivalent of carbon dioxid is obtained by dividing the total heat production in Calories by the grams of carbon dioxid eliminated. The caloric equivalent of oxygen is obtained by dividing the total heat production in Calories by the grams of oxygen absorbed. The caloric value of the protein metabolism corresponds to about 26 Calories for each gram of nitrogen eliminated. The caloric equivalents for oxygen and carbon dioxid, together with the values of oxygen for each gram of the foodstuffs, the products of oxidation of a gram of the same and the respiratory quotients on combustion of each foodstuff, are collected in the following table taken from Benedict and Joslin, from whom are also taken the tables that follow:

Respiratory quotients and caloric equivalents of carbon dioxid and oxygen for protein, fat, and carbohydrates.

\begin{tabular}{|c|c|c|c|c|c|c|c|c|c|}
\hline \multirow{3}{*}{ Foodstuff. } & \multicolumn{2}{|c|}{$\begin{array}{l}\text { Oxygen required } \\
\text { to oxidize } 1 \mathrm{gram} \text {. }\end{array}$} & \multicolumn{4}{|c|}{ Products of oxidation of 1 grain. } & \multirow{3}{*}{\begin{tabular}{|c|}
$\begin{array}{c}\text { Respira- } \\
\text { tory quo- } \\
\text { tient. }\end{array}$ \\
$\mathrm{CO}_{2}$ c.c. \\
$\mathrm{O}_{2}$ c.c.
\end{tabular}} & \multicolumn{2}{|c|}{$\begin{array}{l}\text { Caloric equi- } \\
\text { valent of } \\
1 \text { gram. }\end{array}$} \\
\hline & \multirow[b]{2}{*}{$\begin{array}{l}\text { Wgt. } \\
\text { grms. }\end{array}$} & \multirow[b]{2}{*}{$\begin{array}{l}\text { Vol. } \\
\text { c.c. }\end{array}$} & \multicolumn{2}{|c|}{$\mathrm{CO}_{2}$} & \multirow[b]{2}{*}{$\begin{array}{l}\mathrm{H}_{2} \mathrm{O} \\
\text { grms. }\end{array}$} & \multirow[b]{2}{*}{$\begin{array}{l}\text { Heat } \\
\text { Cal. }\end{array}$} & & & 9 \\
\hline & & & $\begin{array}{l}\text { Wgt. } \\
\text { grms. }\end{array}$ & $\begin{array}{l}\text { Vol. } \\
\text { c.c. }\end{array}$ & & & & $\begin{array}{c}\text { pro- } \\
\text { duced } \\
\text { Cal. }\end{array}$ & $\begin{array}{l}\text { gen re- } \\
\text { quired } \\
\text { Cal. }\end{array}$ \\
\hline Starch . & 1.185 & 829.5 & 1.629 & 829.3 & 0.556 & 4.20 & 1.000 & 2.58 & 3.55 \\
\hline Cane sugar & 1.122 & 785.4 & 1.543 & 785.5 & 0.579 & 3.96 & 1.000 & 2.56 & 3.53 \\
\hline Glucose & 1.066 & 746.2 & 1.466 & 746.2 & 0.600 & 3.75 & 1.000 & 2.56 & 3.52 \\
\hline Animal fat & 2.876 & 2013.2 & 2.811 & 1431.1 & 1.065 & 9.5 & 0.711 & 3.38 & 3.30 \\
\hline Human fat & 2.844 & 1990.8 & 2.790 & 1420.4 & 1.055 & 9.4 & 1.713 & 3.42 & 3.36 \\
\hline Protein & 1.367 & 956.9 & 1.520 & 773.8 & 0.340 & 4.4 & 0.809 & 2.90 & 3.22 \\
\hline
\end{tabular}


The metabolism, energy transformation, and gaseous exchange is variable from individual to individual, though probably a constant in the same individual from time to time. The variations are most marked in infancy and childhood, but are evident in perfect health in adult life. This is, of course, what we should expect, since the intensity factor of protoplasm is a variable. As a rule, the smaller the body weight the larger relatively is the unit of transformation, the greater the production of carbon dioxid and consumption of oxygen. There are also variations between the tall and short, the lean and fat of the same stature. The greatest fluctuations are naturally presented in the figures for output of carbon dioxid. In the comparison of the metabolism of one individual with another, sick or well, these differences must be kept in mind. And variations that are to be regarded as abnormal must be in evidence outside the known ranges of normal variation. The following tables give values for five normal subjects, and give a fair idea of the range of variations in selected individuals.

Average metabolism of normal individuals in experiments without food, in bed calorimeter.

\begin{tabular}{|c|c|c|c|c|c|}
\hline \multirow[b]{2}{*}{ Subject. } & \multirow[b]{2}{*}{ Body weight. } & \multicolumn{2}{|c|}{ Per kilo body weight per minute. } & \multicolumn{2}{|c|}{$\begin{array}{l}\text { Heat eliminated per kilo body weight } \\
\text { per hour. }\end{array}$} \\
\hline & & $\begin{array}{c}\mathrm{CO}_{2} \text { eliminated. } \\
\text { c.c. }\end{array}$ & $\begin{array}{c}\text { Oxygen } \\
\text { absorbed. } \\
\text { c.c. }\end{array}$ & Cal. & (R. Q.) \\
\hline 1 & 83.0 & 2.76 & 3.23 & 1.01 & $(0.854)$ \\
\hline 2 & 74.4 & 2.77 & 3.34 & 0.95 & $(0.800)$ \\
\hline 3 & 67.6 & 2.86 & 3.36 & 0.96 & $(0.851)$ \\
\hline 4 & 66.0 & 2.94 & 3.59 & 1.00 & $(0.819)$ \\
\hline 5 & 49.0 & 3.43 & 4.05 & 1.13 & $(0.846)$ \\
\hline Average &.$\quad$. & . 2.95 & 3.51 & $\overline{1.01}$ & . \\
\hline
\end{tabular}

Apparently, individual and normal variations up to 15 per cent. may be expected in the measurement of the total metabolism and energy transformation of the body.

The following table gives the figures determined with the same individuals studied in the recumbent position with the respiration apparatus. Below the line of averages are the figures for a sixth subject, who displayed rather a marked individualism, and indicate what must be expected in routine examinations of this kind. 


\begin{tabular}{c|c|c|c|c} 
& & \multicolumn{2}{|c|}{ Per kilo body weight per minute. } & \\
Subject. & Body weight. & $\begin{array}{c}\mathrm{CO}^{2} \text { eliminated. } \\
\text { c.c. }\end{array}$ & $\begin{array}{c}\text { Oxygen absorbed. } \\
\text { c.c. }\end{array}$ & (R. Q.) \\
\hline 1 & 82.0 & 2.67 & 3.30 & $(0.810)$ \\
2 & 74.4 & 2.67 & 3.15 & $(0.847)$ \\
3 & 66.0 & 3.06 & 3.68 & $(0.831)$ \\
4 & 65.6 & 2.87 & 3.52 & $(0.816)$ \\
5 & 49.0 & 3.23 & 3.94 & $(0.824)$ \\
Average &. & 2.90 & 3.52 & \\
6 & 59.0 & 3.63 & 4.15 & $(0.874)$ \\
\hline
\end{tabular}

These figures emphasize two considerations. The variations are usually not over 15 per cent., but a subject may be encountered, as in No. 6, who exhibits a more decided variation from the average. The results with the respiration apparatus, the subject recumbent, are so closely concordant with those obtained in the bed calorimeter that the use of this respiration apparatus (that of Benedict) may be safely urged upon clinicians. The measurement of the carbon dioxid is, as a measurement, more accurate and freer of liability of error than the measurement of the oxygen. But the value of the figure for oxygen is much higher for the purpose of calculation of total energy transformation. In diabetes, where little carbohydrate is burned, calculations on the basis of carbon dioxid elimination give very good results; in these cases the respiratory quotient will indicate the state of the carbohydrate combustion.

Under the best of conditions there are rather large opportunities for error in the interpretation of the results of estimations of total energy transformations. This is due largely to the normal variations, and the comparative inability of the experimenter to classify the subject, especially under conditions of disease. Nevertheless, with the nitrogenous metabolism known and controlled and the respiratory quotient determined, the estimation of carbon dioxid output and oxygen intake will enable us to calculate closely to the probably total heat production of the body. It will enable one at least to determine when this is notably increased, and as will be later pointed out, the matter of decrease demands little consideration. A diet should present opportunity for caloric as well as nitrogenous equilibrium; and unless we wish routinely to overfeed carbon, the estimation of the total heat production alone will enable us to classify diseases on the basis of the total transformation of energy and to arrange the input of carbon accordingly. 
Importance of Respiratory Quotient.-The respiratory quotient has an importance beyond that of its use in the calculation of total heat production. It is often of advantage simply to know in what state (fat or glucose) the carbon of the body is being burned. Glucose requires six molecules of oxygen to convert the six atoms of carbon into carbon dioxid; the hydrogen requires no more oxygen than the glucose contains. Fat on the contrary (we are concerned with the fatty acid alone) using stearic acid as an illustration, contains only one molecule of oxygen for 18 atoms of carbon and 36 atoms of hydrogen. To make the comparison clear, let us multiply the molecular weight of glucose by three, or in other words, take three molecules of glucose which has the same amount of carbon and hydrogen as one molecule of stearic acid.

$$
\begin{aligned}
& 3 \mathrm{C}_{6} \mathrm{H}_{12} \mathrm{O}_{6}+18 \mathrm{O}_{2}=\left\langle\begin{array}{l}
18 \mathrm{H}_{2} \mathrm{O} \\
18 \mathrm{CO}_{2}
\end{array} \quad \frac{\mathrm{gO}_{2} \text { in } \mathrm{CO}_{2}}{\mathrm{gO}_{2}}=\frac{\text { c.c. } \mathrm{CO}_{2}}{\text { c.c. } \mathrm{O}_{2}}=\mathrm{Rq}\right. \\
& \mathrm{C}_{18} \mathrm{H}_{36} \mathrm{O}_{2}+26 \mathrm{O}_{2}=\left\langle\begin{array}{l}
18 \mathrm{H}_{2} \mathrm{O} \\
18 \mathrm{CO}_{2}
\end{array} \quad \frac{\mathrm{gO}_{2} \text { in } \mathrm{CO}_{2}}{\mathrm{gO}_{2}}=\frac{\text { c.c. } \mathrm{CO}_{2}}{\text { c.c. } \mathrm{O}_{2}}=\mathrm{Rq}\right.
\end{aligned}
$$

Since the respiratory quotient is the quotient of the volume of carbon dioxid over the volume of oxygen

$$
\frac{\mathrm{CO}_{2} \text { c.c. }}{\mathrm{O}_{2} \text { c.c. }}
$$

it is clear that the quotient (1.0 in the case of sugar) will be much lower in the case of oxidation of fat, about 0.7. The same argument applies to the combustion of protein, except that on account of the nitrogen the calculation is complicated. Mixed protein may be calculated to have a respiratory quotient on combustion of about 0.8 . When now the nitrogenous metabolism is known, as is easily to be determined by the knowledge of input and total output, the respiratory quotient gives directly a good indication of the source of the body heat, whether from glucose or fat. Making now allowance for the nitrogenous catabolism, we can use a table of interpolation values for the determination of the relative degrees of combustion of fat and carbohydrate in a body. Such a table follows, taken from Magnus-Levy.

$\begin{array}{cccc}\begin{array}{c}\text { Respiratory } \\ \text { quotient }\end{array} & \begin{array}{c}\text { Caloric value per } L \\ \text { oxygen }\end{array} & \begin{array}{c}\text { Division of fuel } \\ \text { Carbohydrate }\end{array} & \text { Fat } \\ 1.000 & 5.046 & 100 & 0 \\ 0.950 & 4.986 & 83 & 17 \\ 0.900 & 4.924 & 66 & 34 \\ 0.850 & 4.863 & 49 & 51 \\ 0.800 & 4.801 & 32 & 68 \\ 0.750 & 4.740 & 15 & 85 \\ 0.707 & 4.686 & 0 & 100\end{array}$

The values for each 25 in the scale of respiratory quotients can be calculated if one wishes to secure greater approximation. By the 
use of the data for the metabolism of nitrogen, the carbon dioxid output and the oxygen consumption, we can calculate in a very approximate manner the total heat production and the absolute as well as relative amounts of protein, carbohydrate, and fat that have been catabolized in the body during the period of the experiment. If the normal variations were less and if we could properly classify a sick subject and be able to determine his norm in order to have that as the basis of our comparison of the values determined under conditions of disease (instead of being compelled to compare them with averages of normal values) the method would be one of great dependability for bedside work. With the use of the figure for oxygen, instead of that for carbon dioxid, as the basis for the computation of the heat, the accuracy of the calculation is much increased; so much so that the chief error in the application of the method to the study of the sick lies not in the method itself, but in our inability to form an accurate notion of the normal values with which to compare the findings in the individual case of illness. A safe rule, with the respiratory quotient and nitrogenous metabolism known, would be that the heat values calculated by the use of the caloric equivalents of carbon dioxid and oxygen must display variation of notable degree and in the same direction. The absolute definition of the amount of this variation, as contrasted with normal averages, must then rest upon the value for total energy transformation yielded by the calculation based on the consumption of oxygen. In all investigations of this character it is essential that the digestive organs be resting; in other words, the tests should not be done within at least fifteen hours of the taking of food, and a longer time would be more certain. The best plan is to have the subject eat at six in the evening; and then without breakfast the respiration test is to be made the following morning at eleven o'clock. Such a postponement of breakfast can nearly always be tolerated by any ordinarily sick subject without ill results. A prolongation of the fast for a few hours would give greater certainty of freedom of disturbance by digestion. But in an individual with low glycogen stores, even such a short fast as twenty-four hours might result in a shift to the combustion of fat greater than actually normal in the individual at the time. Such a shortage in store of glycogen would, however, in itself be almost pathological. Muscular repose is imperative.

\section{THE REGULATION OF BODY TEMPERATURE}

Despite the fact that the heat evolved in the four named fractions of heat production vary widely in the diversified circumstances of life, the temperature of the body is quite constant. It is obvious that independently of heat production, the body of the warm-blooded animal possesses an effective mechanism for the regulation of the several factors that influence the temperature of the body. Under 
certain circumstances heat must be conserved; under other circumstances heat must be dissipated. The mechanism for the regulation of the body temperature consists in the co-adaptation of several factors, operating in consonance with or in opposition to the production of heat. These factors belong either to chemical regulation or to physical regulation.

Chemical Regulation.-When the external surface of the body is chilled, increased combustion occurs in the muscles by reflex action; and enough more heat, within reasonable limits, is produced to maintain the body temperature despite the increased dissipation. This is the only operation of chemical regulation. When the external temperature is above $30^{\circ}$ to $33^{\circ} \mathrm{C}$., this regulation is not operative. As the external temperature is lowered and the dissipation of heat from the skin is increased, more heat is set free through increased combustion. What amount of heat may be produced through chemical regulation is not known. As a matter of fact shivering soon sets in, which indicates that the faculty of chemical regulation has usually a limited scope of operation. A body may be inured to exposure to cold and evidently there is adaptation in this function. The heat produced through chemical regulation, when it is operative, is directly proportional to the distance between the reading of the thermometer and $30^{\circ} \mathrm{C}$.-in the absence of air currents and of unusual humidity, both of which exaggerate the radiation of heat from the surface.

As previously stated, the area of the surface of the body is a fundamental variable to which the production of heat is related; in other words, the heat production of the body is proportional to the surface of the body. This law of skin area holds very closely. The surface area of an animal may be approximately calculated by the equation of Meeh:

$$
\mathrm{C}=\frac{\mathrm{S}}{\mathrm{W}^{\frac{2}{3}}}
$$

$\mathrm{S}$ and $\mathrm{W}$ being surface and body weight. The equation in use is therefore, $\mathrm{S}=\mathrm{C} \sqrt[3]{\mathrm{W}^{2}}$. The constant is determined empirically by the use of the equation

$$
\mathrm{C}=\frac{\mathrm{S} \sqrt[3]{\mathrm{W}}}{\mathrm{W}}
$$

for each animal, and for man $\mathrm{C}$ is 12.312. The formula applied is, therefore, $\mathrm{S}=12.312 \sqrt[3]{\mathrm{W}^{2}}$.

The heat production of the resting and fasting body at room temperature, so that the chemical regulation is not operative, varies from 950 to 1200 Calories per square meter surface area. As the external temperature is lowered, the figure is progressively increased. The radiation of heat is proportional to the area; and it is apparent that on external cooling, the surface area determines the heat production, the 
body temperature being kept constant. In the direct sense it is obvious that the basal heat production is related to the surface area of the body. Two men of dissimilar size with a lowered external temperature produce heat in proportion to their surface areas. Even with the external temperature above the body temperature, the law of skin area holds, since the velocity of secretion of perspiration for evaporation of water is proportional to the surface area, other things being equal. Two men of dissimilar size, at $37^{\circ}$ produce heat in proportion to their areas, although there is no radiation. It is important to know whether the relation of surface area to body weight changes with states of flesh after adult dimensions have been attained. The skin is very imperfectly elastic, and with loss of flesh the skin assumes a more or less wrinkled appearance, especially in the aged. Nevertheless, the wrinkled skin falls in to folds, so that the actual radiating surface area is not as large as it was with the same individual when the wrinkled skin was filled out with flesh. If the heat production is proportional to the surface and the surface area in elderly wasted people is increased relatively to the body weight, then we should obtain high values for the relation of heat production per kilo body weight; as a matter of fact we obtain rather low values. The same applies to all conditions of shrinkage of body weight, and thus far we know no need of a correction on account of the factor of loss of flesh. The relation of surface area to weight is much larger in the child than in the adult, and the heat production is proportionately larger.

Physical Regulation.- The two factors in the physical regulation are conduction and radiation of heat, and perspiration and evaporation of water. The body loses heat in small part by warming the inspired air and the food, in large part by radiation and conduction of heat from the surface and by perspiration and evaporation of water from lungs and skin. According to external conditions, the body employs at one time largely radiation and conduction, at another time perspiration and evaporation-most often the former.

The concrete operation of the physical regulation is connected with a series of bodily states and external conditions. These are as follows: the covering of hair upon the skin; the thickness of the skin; the blood supply of the skin; the fat layer beneath the skin; the system of sweat glands; the exhalation of water; the external temperature; the humidity of the atmosphere; the air currents, and type of clothing. While the powers of adaptation are very exceptional, circumstances arise when it is not possible to maintain the normal body temperature, which may be lowered or raised as the case may be.

Hair.-The covering of the hair upon the skin is of very little importance in man, as the hairy covering is slight and is in any event, so to speak, included in the clothing. The different hairy coats of different animals explain their varying resistance to cold; cattle will freeze to death in a temperature easily tolerated by many wild animals. Fur owes its protective action to the stationary air held immovable between 
the hairs (fur consists of about 97 per cent. air and 3 per cent. hair); the hair itself is a poor conductor of heat and the immovable air is a poor conductor. The effectiveness of a fur depends upon the degree to which the hairs imprison the air. When a fur is wet, the hair becomes a good conductor of heat and heat is abstracted on evaporation of the water. The presence of a fatty secretion prevents the natural fur or feathers from becoming really wet, the water is shed and beneath is the same layer of dry stationary air. It is this action of the cutaneous lipoids that enables animals and birds to remain in cold water. The clipping of a fur merely means reduction in the layer of immovable air, and the heat dissipation increases accordingly. The heat production of birds is doubled by plucking the feathers. Rabbits that have been shaved and then covered with starch paste, display a heat production of 150 per cent. over that observed in the native state-simply the increased heat production to meet the exaggerated radiation from the depilated skin. In the spring on the frontier, the boy has his hair cut and the sheep are sheared.

The effect of the coat of hair is to increase the range of external temperature from $30^{\circ}$ downward before the point of chemical regulation is reached. The heat production of chemical regulation in the nude man appears when the external temperature is lowered below $33^{\circ} \mathrm{C}$. But in the $\operatorname{dog}$ this does not appear until about $20^{\circ} \mathrm{C}$. If the $\operatorname{dog}$ be shaved, chemical regulation will become operative at $30^{\circ} \mathrm{C}$. instead of at $20^{\circ} \mathrm{C}$. Man by his habits of dress attempts to surround himself with a tropical skin temperature. He does not wish to employ heat production by chemical regulation; but, as far as possible, by adapting his manner of clothing to the external temperature, extends the range of physical regulation through this factor of cutaneous protection. Practically speaking, apart from the exposed hands, feet, and face, the heat lost from the surface of the skin is constant, in the absence of wind, from $25^{\circ}$ to possibly near zero, through adaptation of clothing. And above $25^{\circ}$, man aims in the other direction, by reduction of clothing, to aid heat dissipation.

Thickness of Skin.-The thickness of the skin is a constant under civilized conditions, apart from states of disease. Barbarian peoples who have inured their bare bodies to external temperature have the skin thickened by exposure and friction, as in the case of the hands and feet in civilized beings. Not an inconsiderable degree of protection may be attained in this thickening of the horny layer of the skin. In myxedema, the skin is thickened by the deposition of an abnormal substance in the skin; and this has the effect of reducing the unit of radiation of heat from the skin of these subjects.

Blood Supply of Skin.-'The blood supply of the skin is a most important variable in man, of less importance in the fur-covered animals. Restriction of heat radiation is accomplished by constriction of the peripheral vessels, so that but little blood courses through the skin in the unit of time; increase in heat radiation is accomplished 
by dilatation of the peripheral vessels, so that much blood in the skin radiates its heat outward. The function of perspiration also is in part a function of the variable of blood supply; dilated capillaries promote perspiration, contracted capillaries depress perspiration. A slight increase in the interior temperature is probably the moment that reflexly results in the dilatation of the peripheral bloodvessels and so increases the radiation of heat as to keep the blood temperature at the normal. The flow of blood to the skin really has the effect of raising or lowering the skin temperature; and as the velocity of radiation of heat from a warm to a cold body is proportional to the difference in temperature, the result is obvious. This is a function that is very variable in disease, and will be discussed from this point of view under fever. It is probable also that there is a paralytic dilatation of the peripheral system resulting in the loss of this function of regulation, with the effect of greatly increasing the production of heat at all but high external temperatures.

The Subcutaneous Fat.- This factor operates to increase the range of physical regulation, just as the coat of fur does. The protecting influence is proportional to the thickness of the layer of fat. The layer of fat operates also in the opposite direction. Vasomotor dilatation is less effective in the fat individual than in the thin, and thus conduction and radiation are more difficult. A current of air at $35^{\circ} \mathrm{C}$. that would have the effect of cooling a thin subject, will have less effect in the case of a fat subject. The cool bath and shower are also less effective. Since at temperatures up to that of the body, heat is dissipated by conduction and radiation as well as by evaporation, the fat man with the restriction of conduction and radiation is thrown more upon evaporation of water, which explains why sweating is more pronounced. When now with high humidity the evaporation of water is made difficult, the reason for the especial discomfort of the fat subject becomes apparent. The exhaustion commonly associated with profuse perspiration is, of course, added to the total effect. There is, however, a further fact, since the two factors named would cease to distinguish the fat from the thin subject at external temperatures equal to or above that of the body. The actual heat production of the fat subject seems to be increased more than in the case of a thin subject; and as evaporation of perspiration alone can remove the heat, the stout subject is at a disadvantage as compared to the spare. Just how the increased heat production of the fat subject is brought about in advance of increase in the body temperature, is not clear.

The System of Sweat Glands.-Dogs and cats possess no sweat glands in the skin, and must remove water by the mouth. In man the system of sweat glands is by no means a constant, but varies from individual to individual. Not a few persons suffer excessively at high temperatures because of sparse perspiration, so that there is little water to evaporate. An occasional individual suffers from congenital absence of sweat glands, and functional inhibition of perspiration sometimes 
occurs. In ichthyosis hystrix the function of perspiration is abolished. When the subjects of this disease are placed in a warm room, the respirations at once become rapid in the endeavor to eliminate water, and the body temperature rises. In the absence of the function of perspiration, a warm atmosphere has the same effect as a hot bath of the same temperature on a normal subject. The evaporation of each liter of water abstracts from the body about 580 Calories; and since the body can discharge several liters of perspiration daily, the individual whose function of sweating is naturally or pathologically reduced is at a serious disadvantage in the regulation of the body temperature. The function of perspiration is normally excited by high body temperature as well as high external temperature, as indicated by perspiration during work. When the external temperature is lowered, the elimination of water from the skin falls to the minimum of insensible perspiration. The skin never ceases to eliminate water, and 500 grams per day probably represents the output of water in ordinary insensible perspiration. Obviously, as the external temperature falls, the loss of body heat by evaporation becomes less and less. A resting or at least inactive individual with a total heat production of 3000 Calories at room temperature, will lose about 700 Calories by evaporation of water, and of this about 450 Calories are lost by evaporation of perspiration. This corresponds to some 750 grams of water. If the external temperature be high, this may rise to 2 or 3 liters, even at rest if the humidity be low. At hard work as much as 5 to 7 liters of perspiration may be eliminated; if the temperature be high and the air dry, the fraction of even the heaviest perspiration that evaporates will be large; if the humidity be high this is, of course, not the case. As air currents increase, the velocity of evaporation as compared with that in a still atmosphere is augmented. But as the influence of air currents on conduction and radiation is still more pronounced, the total effect is to reduce perspiration and evaporation of water. Under the most favorable conditions, three-fourths of the body heat may be dissipated through evaporation of perspiration; under the least favorable conditions less than 5 per cent. will be thus lost. The function of perspiration and the variations in evaporation of the eliminated water are, therefore, of extreme importance in the mechanism of regulation of temperature.

Exhalation of Water. - Since the air of the breath is always warmed to the temperature of the body, the external temperature has no influence on the water content of the expired air, which is normally saturated with moisture. Under usual conditions of respiration, about 400 grams of water will be eliminated in the expired air, corresponding to a loss of heat of about 225 Calories. Forced respiration will raise this to about 600 grams of water, corresponding to 350 Calories of heat. With very rapid respiration, as in extreme exercise, ventilation of the lungs is no longer attended with saturation of the expired air with moisture. From these figures it is clear to what a limited extent only can faulty 
cutaneous elimination of water be compensated for by increased respiratory activity. The elimination of water from the lungs, which is independent of external temperature, is, however, inversely proportional to the humidity; and this fact, under the usual conditions under which a compensation for cutaneous elimination would be desirable, reduces the faculty of adaptation still more.

External Temperature.-Since the velocity of conduction and radiation under constant conditions is proportional to the difference in the temperature between the body and the external medium, the lower the external temperature the greater the loss of heat. Man modifies this factor by clothing to such an extent as practically to keep his body in the state it would be if nude, the external temperature varied only from $25^{\circ}$ to $37^{\circ} \mathrm{C}$. In animals, of course, the influence is of predominating importance. A dog with a moderate coat of hair may be shown by experiment to possess physical adaptation to external temperature from $20^{\circ}$ to $30^{\circ} \mathrm{C}$.; on being shaved, this physical adaptation is lost, and the heat output (and necessarily the heat production) are increased with each degree of temperature lowered from $30^{\circ}$. And yet in man it can be shown that despite clothing, the loss of heat through conduction and radiation may vary 50 per cent. Since customs in clothing are determined by fashions as well as by physics, the influence of external temperature becomes more of a variable under modern condition of life than need be. Under usual conditions of living probably 65 to 80 per cent. of the body heat is dissipated by conduction and radiation.

Humidity of the Atmosphere.-This is of striking influence, since it modifies conduction and radiation as well as evaporation of water. At medium temperature of the external air, say at $18^{\circ} \mathrm{C}$., the influence of humidity is slight. As the temperature is lowered, the influence of increasing degrees of humidity is shown in increased conduction and radiation. Since with lower temperatures there is little perspiration, the reduction of evaporation by increase in humidity is of little consequence. It is at higher temperatures that the influence of humidity is most marked. Here there is little conduction and radiation; the dissipation of heat is accomplished largely by evaporation of water, and this is greatly reduced by high humidity. A dry external temperature of $120^{\circ} \mathrm{F}$. is less oppressive at Yuma than is a moist external temperature of $90^{\circ} \mathrm{F}$. in Philadelphia; exertion is less dangerous and the tendency of the body temperature to rise less pronounced.

The influence of the bath at different temperatures is dependent on the relations to conduction and radiation on the one hand, and prevention of evaporation of water on the other. The cold bath, in proportion to the lowered temperature of the water, abstracts more and more heat by radiation, so that if the body temperature is to be kept up, heat production must be stimulated by chemical regulation and by shivering. The reduction of the body temperature by the cold bath is based upon the hypothesis that the water will lower the febrile body temperature by abstraction of heat without provoking heat 
production by chemical regulation or shivering. As a matter of fact, in practice the heat is abstracted so rapidly that chemical regulation and shivering are before long provoked; but the loss of heat is greater than the increase in combustion, so that the end result is a lowering of body temperature. Of course, if the water is cold enough and the submergence prolonged, the body temperature will be reduced despite chemical regulation and the most marked muscular movements. This is the cause of failures to swim the British channel; the loss of heat by conduction and radiation is so pronounced, that after many hours the powers of heat production fail, the body temperature begins to fall and the swimmer must abandon the attempt. Coating of the skin with vaselin lowers the loss of heat; but even under the best of conditions it is apparent that the heat production in the twenty and more hours that the swimmer must be in the water probably exceeds the largest heat productions ever measured, 10,000 Calories per day. The few men who have accomplished this task in swimming have probably done so largely on account of unfavorable conditions for radiation, such as thick skin and subcutaneous fat. The hot bath checks both radiation and evaporation of water, just as the steam room of the Turkish bath; and as the dissipation of body heat is so restricted, the body temperature rises very quickly. The influence of moisture alone is well realized in the different reactions in the dry hot, and moist hot rooms of the Turkish bath; the dry room is tolerable at very high temperature, the steam room is soon intolerable at a temperature very little above that of the body.

Air Currents.-The influence of air currents is to intensify conduction and radiation and evaporation of water. The lower the temperature the more marked the first of these results. With a fixed air current, increase in humidity results in increasing conduction and radiation with falling temperature. At high temperature, high humidity reduces the evaporation of perspiration by an air current. A wind of a certain velocity has little influence on a moist hot day on the Atlantic seaboard; a wind of the same velocity at the same temperature will necessitate the use of a wrap in southern California. A still cold carries little danger for stock on the western plains; the same cold with strong wind means the death of stock by freezing. A wind of a velocity of a half meter per second is perceptible; and even so slight an air current will have a marked influence on conduction, radiation, and evaporation of water as compared with the results in a still atmosphere. In a still atmosphere, the body has about it a zone of warmed air, slowly rising, but acting in a sense as a blanket, since it is almost stationary; air currents serve to continue the maximum difference between external and skin temperatures. A slight wind can easily raise the heat dissipation, and consequently the heat production, to a goodly extent, as shown in the following figures taken from Wolpert: 


$\begin{array}{ccc}\mathrm{CO}_{2} \text { output per hour } & \mathrm{CO}_{2} \text { output per hour with } \\ \text { wemperature } & \text { calm } & \text { M. per second } \\ 10 \text { to } 15^{\circ} & 25.1 & 30.0 \\ 15 \text { to } 20^{\circ} & 24.1 & 30.0 \\ 20 \text { to } 25^{\circ} & 25.0 & 28.0 \\ 25 \text { to } 30^{\circ} & 25.3 & 24.4 \\ 30 \text { to } 35^{\circ} & 23.7 & 21.6\end{array}$

The subject wore usual summer clothes, yet the effect was marked. Under modern conditions of life, we attempt to obviate or utilize the influence of air currents by adaptation of dress. We permit access of air currents to the skin when at high temperature evaporation of water is desired; we exclude air currents when at low temperature conduction and radiation would be exaggerated. The dictates of fashion frequently lead to disregard of physical regulation. Women wear summer garments in winter. When one reviews the data relating to the production of carbon dioxid at fixed temperatures with varying weights of clothing, and then contemplates the present flimsiness and scantiness of women's apparel and the exposure of children's arms and legs at low external temperature, one will realize that at least a goodly caloric price must be paid.

Type of Clothing.-Fiber, weave and weight modify the physical properties of clothing and permit the reasonable individual to adapt this covering to the exigencies of nature and the laws of physics. With a fixed fiber, a dense weave excludes air currents, lowering conduction and radiation in cold weather and lowering evaporation in warm weather. Dense weaves absorb water poorly, a matter of little importance with the slight perspiration at low external temperature, but of great importance in warm weather, since ready absorption means rapid evaporation. A loose weave permits of free ventilation, chilling in winter but affording in summer rapid absorption of perspiration and evaporation of the water. Variations in fiber are of less consequence than variations in weave; and variously current opinions on this and that fiber are largely arbitrary, though as a rule, animal fibers absorb water less rapidly than plant fibers, even when the weave is identical. This suggests plant fiber for clothing next to the skin and animal fiber for external clothing, at least under conditions when conscious perspiration is likely to occur. Fads dominate clothing to the partial exclusion of hygiene. The reasonable individual will follow the golden path between forced inurement to the elements and complete protection from the elements.

The result of the co-operation of the forces of heat production and heat dissipation, under the control of the heat centre, is that with few exceptions the body temperature is maintained fairly constant, even under very different conditions. There is a diurnal variation of a half of a degree or so, largely individual. The mean of body temperature in different individuals may vary a degree. It is clear that even on the basal heat production, the body temperature could rise above the normal if the adaptations for the regulation of the dissipation of 
heat were disturbed. On the other hand, it is clear that with increased heat production, the body temperature could fall if the adaptation for the regulation of heat dissipation were disorganized. When the conditions for the production of heat are unfavorable and those for the loss of heat favorable, body temperature may fall. When the conditions for heat production are favorable and those for heat dissipation are unfavorable, body temperature must rise. The phenomenon of fever, therefore, to which we must next turn our attention, cannot be regarded as a constant effect of a constant cause. Abnormal lowering of body temperature is uncommon; fever is very common. In shock there is subnormal temperature, as there are in some very grave infections and intoxications. These are all probably due to serious reduction in heat production aided by excessive heat dissipation. But we have no reliable analytical data on this question. Investigations of the important question of shock have not followed the direction of the measurement of heat production and heat dissipation under constant conditions, which indeed in the very nature of the circumstances of shock, infection, coma and intoxication are difficult if not impossible of realization.

\section{FEVER}

When one considers the frequent occurrence of fever, the paucity of our information relative to the production of heat and the regulation of temperature in clinical conditions of fever is amazing. In truth, however, the reason has been in part, due to methods. Until very recently the methods of indirect calorimetry have been cumbersome and also inexact. The use of the respiration-chamber type of calorimeter has been out of the question. Added to this was the impossibility, and often undesirability, of placing the patient under the strict control of diet that would be required in an exact metabolic and calorimetric experiment. The recent improvements and simplification of the respiration apparatus in the Carnegie Nutrition Laboratory, and the more accurate definition of caloric coefficients, have now placed it within the power of every well-equipped hospital to conduct investigations into the metabolism of patients afflicted with infectious and other febrile diseases. The literature on fever is enormous, and in part filled with contradictions in matters of fact as well as divergencies in point of view. An exhaustive discussion of the subject of fever cannot be here undertaken. But it is possible within the space here available to correlate the known facts of fever with the known facts of the physiology of heat production and temperature regulation. It will be best to discuss the matter under specific headings.

Under what conditions does fever appear in the normal body? We know of two physiological or experimental forms of fever; the fact that fever of these types may exist naturally does not make the designations improper. When the body is placed in a bath at $40^{\circ} \mathrm{C}$., the body 
temperature must rise because with loss of heat through radiation and evaporation excluded, the continuance of the basal heat production necessarily raises the temperature of the body. The various forms of heat stroke represent this type of fever in nature. When a puncture is made in the corpora striata, the temperature rises promptly. If the liver be freed of glycogen before the puncture is done, no fever is provoked. In some way, therefore, the puncture has caused a sudden, almost explosive combustion of the glycogen in the liver. No glucosuria appears. This combustion is shown to occur in the liver by the fact that the temperature of the liver and blood under these circumstances becomes higher than the temperature in the muscles; whereas normally it is in the muscles, where the usual combustion of glucose occurs, that the temperature is highest. Just how large is the increase in heat production is not known. It is assumed, quite without experimental reasons, that the sudden excess of production of heat is so pronounced that the full normal mechanism for the dissipation of heat is impotent to cope with it for a time; and as a result of excess of heat production transcending the powers of heat dissipation, the body temperature rises. It is possible that the sudden high fever sometimes seen in injury of the base of the brain, and also in lesions of this region, may be due to irritation or injury of the so-called heat-puncture centre.

When all the other instances and forms of fevers are analyzed, it seems possible to place them in one of two groups: (a) Fever due to disintegration within the body of tissue separated from its nutrition, tissues in aseptic necrosis. (b) Fever due to the presence and functionation of microörganisms, bacteria or protozoa. The number of instances of the first type of fever is small, the last-named form comprises practically all the clinical states of fever. In all, the causation of the fever is to be related to chemical substances elaborated in these cells, bacteria or protozoa, or formed in association with their life or death within the body.

Production of Heat in Fever.-Since the production of heat is primary and the dissipation of heat secondary, investigation naturally approaches the former first. Is there increase of heat production in fever? In many instances of fever, yes; in some fevers there is no increase in heat production. The latter must be due solely to derangement in regulation. In the instances in which there is increase in heat production, is there also increase in nitrogenous catabolism? Does the increase in nitrogenous catabolism account fully for the increase in heat production, or is there increase in combustion of glucose and fat? In normal individuals, would the amount of heat concerned in the increase in heat production observed in febrile disease lead to increase in body temperature, is it such an amount of heat as to tax the mechanism of regulation of body temperature? Does such a degree of increase in the nitrogenous catabolism under all circumstances lead to fever? Have we knowledge of a febrile exaggeration in nitrogenous catabolism? These questions we will consider seriatim. 
There is increase in heat production in many cases of fever. When the data are analyzed, it becomes clear that, as a rule, there is little increase in heat production in fever of usual degree, up to $39^{\circ} \mathrm{C}$.; but that in high fever and apparently proportional to the fever, the production of heat is increased. It is quite certain that we deal with two increases in heat production, of different origination. In the one, the increase in heat production is primary; whether it be the cause of the fever or not, it is associated with the infectious or toxic condition that is at the basis of the disease. In the second, the increase in heat production is secondary to the fever itself; it is the expression of the increased velocity of reactions in the body at higher temperature. When the body temperature of a normal subject is raised by lying in a hot bath, the heat production is increased also, purely as a result of the increased body temperature. It makes no difference what may be the cause of the fever; fever itself, in accordance with the general law of chemical reaction velocity, leads to increase in reaction velocity in the organism, to increased heat production. This should effect both the catabolism of protein and the burning of carbohydrate. These two elements of increase in heat production doubtless overlap. The first is a constant, presumably of the infective process, but may be exaggerated by higher body temperature. The second does not become measurable at slightly febrile temperature, but increases progressively as the body temperature rises. Obviously these two increments of increase in body heat only add to the problem of heat dissipation.

The amounts of heat involved in the increased production are difficult to define, since they are usually determined by calculation of the carbon dioxid, and the diet and other conditions camnot be controlled. By interpolation and the use of data obtained on animals, it seems certain that the amount of extra heat will not usually exceed a few hundred Calories; it is doubtful if the extra heat production in a febrile disease in man ever reaches a 1000 Calories a day over the normal output of the individual under identical conditions. Experimental infections in animals have yielded increase in heat production of as high as 50 per cent. (6 to 7 per cent. increase for each degree C. fever); and this marked increase is covered by the allowance of a 1000 Calories. The resting subject of 70 kilos (in the sense of rest as a febrile subject would rest) on light diet would develop about 30 Calories per kilo per day, or 2100 for the total. An increase to 45 Calories per kilo per day with a fever would be very unusual; but even then the total would be only 3200 Calories.

The increase in heat production is not a unit; in it are included in different cases and under different circumstances, at least four fractions of heat. One is due to toxic exaggeration of protein catabolism. A second is due to exaggerated catabolism due to depletion of glycogen. The patients in the first few days of fever do not eat, the combustions deplete the glycogen (demonstrably low in the tissues in febrile infections) and under these circumstances the subject presents the combus- 
tion of the first few days of starvation, protein catabolism high on account of lack of glycogen. A third, present in some cases, is due to muscular contractions, the shivering in the state of chill in such infections as are attended with chills. The end capillaries are forcibly constricted, the skin becomes cold and shivering sets in, as always, when the skin is chilled. Lastly, we have the fraction of heat due to the excess of catabolism caused by the higher temperature of the body.

Is there increase in nitrogenous catabolism? In many cases there is increase in the catabolism of common protein, in many cases increase in purin, and in some cases possibly in creatinin. The loss in nitrogen (in uncontrolled cases it must be conceded) may be as high as 10 grams per day above the normal. Usually it is under 5 grams. In some cases of febrile infection it is not demonstrable. The cases with high fever, other things being equal, tend to have the highest catabolism of protein.

Increase in Protein Catabolism.-This exaggeration of protein catabolism is in part of toxic origin, the direct result of the action of bacterial products on the cells, especially of the muscles and glands. The increase in protein catabolism may antedate the onset of fever. The hyaline degeneration of muscle cells seen in some fevers suggests itself as the morphological counterpart of the toxic action of the bacterial products, of which the increased catabolism is the chemical result; fatty degeneration in the liver cells is another evidence. Yet it is surprising how slight this exaggeration of protein catabolism may be when the diet of the subjects of infectious fevers is probably controlled. Many cases of fever suffer from glycogen hunger, with the attending increase in the catabolism of protein, due to absence of the saving power of carbohydrate. The subject of an infectious fever has not lost the power of sparing protein with glucose; and by the administration of large rations of sugar, the nitrogen output may often be held to the normal. This is not true of all infections, however, but it is a very important fact in the treatment of fever.

In high fever the heavy increase in protein catabolism is to a goodly extent due simply to increase in the reaction velocity as the result of increased temperature. A bath of $40^{\circ} \mathrm{C}$. will cause as much increase in nitrogenous catabolism in the unit of time as is to be seen in many cases of fever. Under these circumstances, with an adequate input of carbohydrate, the more marked the excess of protein catabolism at lower degrees of fever, the more certain we are that it is a toxic result; the more dependent the excess on the height of body temperature, the more likely it is that it is due to hyperthermia rather than to specific intoxication. The amounts of catabolism of protein that are involved are usually not large; 20 per cent. above the normal with body temperature under $40^{\circ} \mathrm{C}$., may be an average. Increases up to 50 per cent. have, however, been observed. Usually it is easily possible to establish a nitrogenous equilibrium. In some severe cases, however, this does not seem possible, even with liberal rations of carbohydrate; the out- 
put remains above the input, no matter how the input is advanced. It must be confessed, however, that in the state of disturbed digestion and with the lack of control that seems almost a humane necessity in dealing with these subjects, the tests are not entirely convincing.

Does the increase in the catabolism of protein account fully for the increase in heat production, or is there increase in the combustion of sugar and fat? In some cases the mere heat of the specific dynamic action of the protein catabolized is enough to account for the increase in heat observed. When the ingestion of carbohydrate is adequate, the heat production will sometimes be found greater than is to be accounted for by the catabolism of protein, assuming that this is accurately represented by the output of nitrogen. In infections, typhoid fever especially, there is sometimes a post-febrile diuresis in which large amounts of nitrogenous substances are eliminated that have apparently accumulated in the body; and when these are taken into account, what seemed to be a balance for the period of fever is converted into a deficit. Daily observations are, therefore, not enough. But apparently there are many cases in which the excess of nitrogen in the urine is not as large as the excess of heat production. In experimental work on animals, the fat combustion has also been found increased, though there is a general idea that in man the combustion of fat is not increased in fever except with underfeeding with carbohydrate. It is, however, believed that overcombustion of glucose often occurs, even with adequate feeding of carbohydrate and protein.

Do such increases in the catabolism of protein as are to be observed in even the most extreme cases lead per se to fever? 'This question must receive a decided answer in the negative. It is possible to obtain increase in heat production through exaggeration of protein catabolism far in excess of any ever observed in human or experimental fever, without the slightest rise in body temperature.

Inadequate Dissipation of Heat.-In the normal body, would the amount of heat concerned in the increase in heat production observed in fevers lead to elevation of body temperature? Certainly not. When one recalls the ease with which under the external conditions that surround the sick subject, the normal mechanism for the regulation of body temperature is able to dissipate 4000 to 6000 Calories in the day, the failure of the febrile individual cannot be ascribed to overproduction of heat, to an excess of heat production taxing the normal powers of heat dissipation. All the data lead to the conclusion that the cause of fever is not abnormal production of heat. At the most, such abnormal production of heat as has been recorded in the subjects of fever could only constitute an increment, and usually a small one. The cause of the fever lies in failure of adequate and regulated dissipation of heat.

This statement is capable of direct demonstration in animals. When rabbits are injected with pyogenic microörganisms, it can be shown that during the period of oncoming fever, the actual dissipation of 
heat falls to about 95 per cent. of that of the controls. If under these circumstances the heat production had been only normal, this difference must have led to fever within a few hours. With this fact established, two questions arise. Wherein lies the defect in heat dissipation, represented in the 5 per cent. in rabbits? When heat production and heat dissipation are in equilibrium at the higher level, what conditions in the mechanism of regulation operate to hold the level above the normal?

The defect may lie in restriction of the functions of conduction and radiation of heat from the skin. The defect may lie in suppression of perspiration and of evaporation from the skin. None of the minor avenues of heat dissipation can enter into account. There is no such thing, with quiet respiration, as reduction in the volume of water discharged in the exhaled air. There is evidence that both conductionradiation and perspiration-evaporation are in part crippled in fever. It is only a question of diminished adaptation; lost these functions are not, as will be seen. Be the heat production normal, increased or even subnormal, the functions of dissipation do not operate to maintain the equilibrium at the normal level.

Perspiration and Evaporation.-An adult subject, at rest in bed at ordinary room temperature, may eliminate by perspiration about 500 to 600 grams of water. Let it be assumed that heat production and loss of heat by conduction and radiation are constant. A toxic agent operates to reduce the perspiration of water one-half; and as this reduces heat dissipation by 175 Calories, unless another avenue of heat dissipation is able to assume the extra burden, the heat remains in the body. Now 175 Calories represent raising the temperature of 70 kilos of water (the weight of the adult body) about $2 \frac{1}{2}^{\circ} \mathrm{C}$. And the regular reduction of the function of perspiration to the extent named, unless compensated for by another avenue of heat dissipation, would maintain an adult body in fever. There is usually a close connection between the function of cutaneous circulation, which is the chief factor in conduction and radiation, and that of perspiration. And the toxic agent that would operate to depress perspiration would tend to reduce cutaneous circulation. Thus the heat retained in the body by lack of the normal evaporation of perspiration would not be removed by radiation. If not removed by increased expiration, which would hardly be possible, fever would inevitably result. In ichthyosis hystrix the function of conduction and radiation is very impotent between $30^{\circ}$ and $37^{\circ}$, the slightest alteration in room temperature between these two points is reflected in the body temperature. And we may be sure that this represents about the normal situation in the function of conduction and radiation. Under the ordinary conditions of life, therefore, a marked reduction in the function of insensible perspiration would probably lead to fever. At low external temperature this, factor would not alone be effective. But as a rule, with heated houses and clothing, we keep our bodies within 
the range of temperature where the function of water evaporation is a very important fraction of the heat dissipation. And, furthermore, the two functions are inclined to act together and defect in one is fairly certain to be associated with defect in the other.

Is fever associated with reduction in perspiration and evaporation from the skin? In rabbits the ratio of heat lost by evaporation of water and by conduction and radiation is constant in normal and febrile animals. The direct experimental demonstration that the normal plane of insensible perspiration is lowered in fever, has not been accomplished. It is demonstrable, however, that the faculty of increase in the evaporation of water is limited in fever. The evaporation of water is subnormal during the rise of fever, but at the height of fever is identical with the evaporation at normal temperature. The usual increase of perspiration after the ingestion of food is less marked in the febrile state. A normal subject whose temperature is raised by staying in the steam room of the Turkish bath will give off much more sweat than will be eliminated by the febrile subject with the same body temperature. Apparently, therefore, in fever a positive reduction of the normal degree of perspiration need not occur; but the normal faculty of free perspiration at higher temperature is lost or greatly restricted. In the fevers of sudden rise with chill, it seems certain that the insensible perspiration is reduced during the period of rise of the fever.

Reduced Conduction and Radiation of Heat.- The direct demonstration of reduced conduction and radiation of heat from the skin has not been accomplished experimentally in a satisfactory manner. There is no question that during the period of rise of fever the skin is cold; the vasomotor constrictor influences are operative and the skin is quite bloodless. The temperature of the skin remains below what it would be normally under the same circumstances of external temperature, especially in the extremities. The phenomena that attend the cold bath are evidently reversed in fever. The action of cold water is at first to cause a peripheral vasomotor constriction, which drives the blood from the skin and leads to a rapid rise of temperature; this is reduced by the loss of heat in the skin that attends the glow of the reaction. In fever the impulse for vasoconstriction proceeds from the inside, and is continuous. The fact that glow and warmth of skin as well as perspiration attend the defervescence of fever, suggests strongly that the opposite states belong to the rise of fever. When one realizes that an increase of heat corresponding to but a tenth of the daily normal basal heat production is enough to maintain a moderate fever, it is apparent that fever might be induced by slight inhibition of the two chief functions of heat dissipation. Investigations into the so-called neurogenetic fevers have indicated that here the heat production is either normal or subnormal; the defect lies solely in the disturbance of the mechanism of dissipation.

At the point where the febrile body temperature is, for the time 
being, stationary, the heat production and dissipation are equal. This is easily explained. The radiation of heat from a warm to a cold body is proportional to the difference in their temperatures. When the body temperature is raised above the normal, with fixed external temperature, the distance between the temperature of the body and the external temperature is increased; and of course the velocity of radiation from the warm body is increased. A point will be reached when, despite lowered conduction through restriction of circulation, the heat radiation will equal the heat production. It is in fever just as though one were to insert a fourth lining in a three-walled thermostat. With fixed external temperature and constant heating, the temperature of the thermostat would rise until the velocity of heat radiation under the conditions of the fourth lining equals the heat production. The situation is in a sense the converse of that which occurs when the hibernating animal holes in. The heat production falls; the body temperature will, therefore, fall until the radiation at the new plane of production equals the production-when the body temperature remains constant during the winter sleep.

The relation of the surface of radiation to the development of fever explains the great liability of children to fever. The surface of the child, as contrasted with the mass of metabolic tissue, is very large. Disturbances in the functions of radiation from this large surface must disproportionately influence the body temperature, when contrasted with the relations in adults. Just as the child chills very easily, so must the child fever easily.

Pathological variations in the functions of conduction and radiation occur in the opposite direction, and in perspiration also. There are vasomotor derangements in which constant sensible and even profuse sweating occurs at room temperature. Evaporation of this water must lead to a marked increase in heat dissipation, and must necessitate a corresponding increase in heat production. In exophthalmic goitre, in the severe stages at least, the peripheral bloodvessels are widely dilated, the circulation of the skin is very free and the dissipation of heat by conduction and radiation is very marked. The heat production of the subject is also very high. Which is primary - heat production or heat dissipation-and which secondary, is not known definitely; there are reasons for believing that the exaggerated heat production is the primary fact, the result of hyperactivity of the thyroid body.

To summarize therefore: no increase in heat production can result in fever if the mechanism of heat dissipation is normal; the essential abnormality in fever lies in defective dissipation of heat, through disturbance of conduction, radiation and perspiration; heat production may be primarily increased to a moderate extent in fevers; in high fever, the production of heat is secondarily augmented; clinical fevers may be associated with normal production of heat, with subnormal production or with excessive production of heat; a moderate reduction in the faculty of heat dissipation is sufficient to produce fever in a body presenting only the basal minimal production of heat. 


\section{THE PROCESSES OF OXIDATION, SUBOXIDATION, SUPEROXIDATION}

The processes of oxidation in the body, viewed as a total, possess an interest that is historical rather than experimental. The several qualitative reactions of the oxidation of the end products of the different metabolisms have been indicated in their appropriate sections. It is a law that the components of the body are not subject to oxidation in the states in which they circulate. Protein, sugar, glycogen and fat-all must be split before they are subject to oxidation. Protein must be hydrolyzed to amino-acids, these must be deaminized, a fraction converted into sugar, a fraction subjected to direct oxidation. Fat must be split into fatty acid and glycerol, the glycerol is converted into sugar, the fatty acids are subject to oxidation in stages. Glucose must be split into lactic acid, which in the opinion of the writer is then converted into carbon dioxid and ethyl alcohol, which is oxidized in stages. Everywhere is the sequence: cleavage $\rightarrow$ oxidation. The oxidation of sugar and probably of fat occurs largely in the muscles; the oxidation of the end products of the catabolism of protein occurs largely in the liver, where also some oxidation of fat undoubtedly occurs.

The actual modus operandi of the processes of oxidation within the body is not well understood. Oxidation ferments are necessarily concerned, but experimental investigation with these super-labile enzymes is very unsatisfactory. It is clear that concerned in oxidations in tissues, animal and plant, are ferments of three types: oxidases, peroxidases, and catalases. The present conception of oxidation in the world of organic and inorganic matter regards oxidation as a series of reactions in successive oxid stages. While these successive oxid stages are to be regarded as intermediary peroxidations, it is certain that activated oxygen in the sense of ozone is not essential. It is certain, in the broadest sense, that the body oxidations are not related to atmospheric ozone. The very function of the oxidation ferments is to attach atomic oxygen to the ubstances to be burned.

In the chain of processes whereby oxygen is brought to the tissues are many opportunities for abnormal inhibitions. The oxygen must first be taken from the air and gotten to the pulmonary lining membrane; it must then be absorbed through the pulmonary lining membrane; it must then be taken up by hemoglobin; it must then be transported to the tissues; in the tissues it must react with the substances to be burned; the products of the oxidation must be removed from the tissues; the carbon dioxid must be carried to the lungs; from the lungs finally the carbon dioxid must be transferred to the expired air. In this long chain of processes are certainly opportunities for lags and breaks. The general concept of suboxidation, really rather as a term than as a quantitative notion, has long been prominent in 
medical writings. And in a great many diseases concerning organs that have a rôle in one or other of these stages, the idea became general that suboxidation was a result of the disease. This idea is incorrect. While it cannot be stated that a total suboxidation never occurs in the body, it is certain that a total suboxidation must be the rarest phenomenon and not, as usually inferred, a common one. The absorption of oxygen does not provoke metabolism, nor is metabolism modified by variations in the oxygen content of the blood under conditions of respiration; it is the metabolism that determined the absorption of oxygen.

Suboxidation.-Qualitative suboxidations, of course, occur. The diabetic cannot burn glucose, and cannot burn $\beta$-oxy-butyric and aceto-acetic acid. The cystinuric cannot burn cystin. The alcaptonuric cannot burn homogentisic acid. But qualitative oxidation is not what is understood in the idea of suboxidation that is current in the medical profession.

Now the fundamental fact of metabolism is that the oxidation processes depend upon the oxidizable substances and not upon the oxygen. We may sketch the general reaction as follows:

Oxidizable substrate + oxygen + oxidation ferment $=$ oxidation products + free ferment. Despite superficial appearances to the contrary, the analysis of clinical data and experimental evidence alike indicates that there is no failure on the part of the oxygen; failure if such occurs, lies with the substrate or the ferments. It is a common idea that respiration of air poor in oxygen fails to sustain the normal plane of total oxidation; this is an error. There is, of course, a degree of rarefication of oxygen that leads to failure in the reactions, but such is never to be observed in ordinary life. It is a common idea that in diseases of the lungs the entrance of oxygen into the blood is difficult, or indeed only partially accomplished. Cyanosis does occur, and labored respirations are often necessary. But the total oxidation of such subjects, whenever it has been tested, has been found to be normal, if indeed it was not above normal. Whether the use of compressed oxygen in pneumonia does actually accomplish the introduction of extra oxygen is a mooted question. But in any event, it is known that the total oxidation in pneumonia is supernormal. This does not deny the efficacy of inhalations of oxygen in pneumonia; it merely indicates that this supposed favorable action of compressed oxygen cannot rest on the removal of a condition of suboxidation, since none such existed. The normal individual absorbs no more oxygen from an atmosphere of pure oxygen than from natural air. It has been believed that in certain chronic indurative conditions of the lungs the exchange of gases is retarded and incomplete. The oxygen may have difficulty in passing the aveolar mucous membrane and entering the capillary spaces; but it is accomplished, because the total oxidation is normal. In connection with valvular diseases of the heart, when one considers the difficulties in the maintenance of the normal blood pressure and velocity of the 
flow of the blood stream, it is obvious that defective transportation of oxygen to the tissues might occur. Probably it does occur as a very acute situation, since under conditions of sudden failure of the circulation, large amounts of lactic acid appear in the urine, and lactic acid is known to appear with deprivation of oxygen. As an acute condition suboxidation probably occurs in sudden dilatation of the heart, in sudden pneumothorax, and in surgical and traumatic shock; but it is clear that the condition camnot be more than transient. In the usual case of valvular disease, labored though the efforts of the circulation may be, they accomplish the transportation of oxygen in a competent mamner, for the total oxidation of the body is normal. In profound anemia the hemoglobin content of the unit of blood (either by reduction in individual cells or by reduction in number of cells) may fall to a fifth of the normal. Yet this amount of hemoglobin is able to carry to the tissues of the resting or inactive patient all the oxygen needed by the body. Than this, there is no better illustration of the faculty of compensation and adaptation, the capacity for overload. What under all the named conditions may be lost and in fact often is lost, is the capacity for overload. Under all circumstances with which we are acquainted, the tissue capacity for oxygen is normal. Thus in diseases of all kinds-acute and chronic pulmonary disease, valvular and muscular heart disease, the essential anemiæ, in tuberculosis, in cachexiæ, in the wasting diseases, in carcinoma, in muscular dystrophies-whenever and however examined, the total combustions of the body are either normal or above the normal. 'The unfortunate diabetic, who cannot burn sugar and who obtains from fat and protein only part of their energy, still has a total combustion of at least the normal and in many instances certainly above the normal, though to secure this heat he must burn incompletely a greatly increased quantity of protein and fat. The machine that is out of adjustment required more power to run it; so does the human function when out of adjustment - if there be any change it is in the direction of loss. Under these circumstances, the idea of total suboxidation has no standing in modern pathology. A current idea of suboxidation as related to distoxication of hypothetical metabolic substances, represents simply a verbal explanation of a hypothetical situation, and has no experimental foundation. What is needed in the study of distoxication is experimentation, not speculation.

There are three chronic conditions in which the gross data have suggested a low plane of total oxidation-possibly not abnormal but certainly low. These are obesity, myxedema, and castration. To assume for any one of these a total suboxidation is to assume a reduction in the basal production of heat, for which we have otherwise no illustration. In the discussion of the total basal production of heat, it was pointed out that this is in part an individual variable; and that in some cases, very low values had been found. Such a subject would naturally incline to obesity. 'This is one thing. But it is a very different 
thing to believe that in a particular individual, following the establishment of obesity or myxedema or following castration, the body changes its plane of basal heat production to a lower level. A great deal of misconception has been due to the low heat production per kilo in the obese. But it is in just these instances that the defect of this method of calculation is pronounced. A man weighs at full manhood 70 kilos. Later in life he grows very fat, the weight increases even to 140 kilos. The heat production is related to the metabolic tissues, under conditions of control, not to the mass of the fat depots. 'This same man with the normal heat production per unit of surface area would when fat present little more than half as much heat in terms of Calorie per kilo as in his natural state. The real question is: What is the basal heat production of the body? Is it below the amount seen in normal individuals with low basal heat production? There is no evidence that this is ever the case. There is no evidence that an animal has ever changed his basal heat production through myxedema or after castration. The three conditions named all tend to minimize the other fractions of heat production. The subjects are inactive in every sense. Castration and thyroid extirpation convert a nervous into a phlegmatic constitution. The diets are usually low, under the conditions of experimentation or observation, and the heat of the specific dynamic action of protein is low. Cretins and the subjects of myxedema have a low nitrogenous metabolism. With a heavy layer of fat and thickened skin and subcutaneous infiltration in myxedema, physical regulation of temperature is extremely effective, and heat production is spared in this way. In other words, these very subjects present the best opportunities for the measurement of the basal heat production. So far as we know, the basal heat production of each body is a constant to that body, to the mass of cells of that body and to the surface area; and this law is inexorable. The body has marked faculty for increase in heat production; but there is no adaptation for reduction in the basal heat production.

There are three conditions in which most extreme exaggeration in heat production and total metabolism may occur, cellular in origin and not due to muscular movements. These are exophthalmic goitre, malignant disease, and sepsis. In the severe instances of these diseases (in the so-called fulminating cases of exophthalmic goitre, in the stage of rapid growth of malignant tumors and proportional wasting of the body, in profound bacterial intoxications) it is impossible to secure either a nitrogenous or a caloric equilibrium, no matter what the input in the diet. It is true that the powers of digestion and assimilation are often low. But the actual transformations in the metabolism, the output of nitrogen and of carbon dioxid, are very high; and food only seems to feed the flame. Evidently we deal in these cases with great exaggeration of enzymic accelerations, due to direct action of poisons developed in these diseases. The thyroid gland is known to stimulate the several metabolisms. In direct experiment it is demon- 
strable that thyroid substance increases the nitrogenous catabolism and the combustions in the body. In exophthalmic goitre we have evidently an abnormal and excessive production of the substances that so act upon these metabolic processes. Future investigations with the respiration apparatus will doubtless reveal to us many new manifestations of exaggerations of one or the other metabolism, and indicate new points of relationship. When one recalls the direct connection of metabolism with diet, there can be no question that such knowledge is certain to have practical bearings.

\section{THE RELATIONS OF WORK. TO METABOLISM}

The unit of work used in physiology is the kilogrammeter, the work required to lift one kilo one meter against the force of gravity. The kilogrammeter is equal to about 97.63 megaergs and to about 7.2 foot-pounds. It corresponds to about 0.00236 Calorie of heat. The maximum work that a trained man can do does not probably exceed 1800 kilogrammeters per minute, and this cannot be long sustained. For the whole day, it is doubtful if more than one-third that can be accomplished. Calculated for the whole day, that would correspond to the production of over 20,000 Calories of heat, even with the mechanical efficiency of the work being set at 30 per cent. We have no data tending to show that more than half this work can be accomplished in a day by a trained man. The work of walking on the level at moderate pace may be roughly stated to equal one Calorie per kilo body weight per mile.

The source of the energy converted into work lies in the combustions of the body. None of the heat of the basal production of heat is convertible into work; if work be done, more heat is set free. The heat of the specific dynamic action of the foodstuffs is, however, convertible into work. In other words, an amount of work whose doing is associated with the production of some 300 Calories of heat can be done by the individual whose diet possesses that value in specific dynamic action, without there being any additional heat production; working up to that amount or resting, the heat production of the body will be the same.

The heat of chemical regulation is convertible into work. If an individual inured to external cold be exposed to a certain outside temperature, his heat production in the resting state will rise, let us say for a certain experiment, 600 Calories. Now if under identical circumstances, an amount of work be done that is associated with the production of 600 Calories of heat, the total heat production will be found not to have risen above the figure for the first experiment. The needed heat is produced by work and spared from chemical regulation.

Work, in the usual sense of the term, involves so. large an amount of energy transformation as to exceed the heat of the specific dynamic action of the foodstuffs and the heat of chemical regulation. There- 
fore, work usually implies an extra production of heat. The mechanical efficiency of muscular contractions varies usually from 20 to 25 per cent., $i$. e., of the total heat value of the fuel burned to support work, 75 to 80 per cent. appears as heat, and 20 to 25 per cent. is transformed into work. This is about the efficiency of a good steam engine. Higher values are not recorded in trained individuals, but are noted for certain forms of work. Thus in walking, in a trained individual about 35 per cent. of the heat value is converted into work.

Light work entails the combustion of food worth about $\$ 00$ Calories; medium work represents the combustion of food worth about 1200 to 1500 Calories; heavy work represents about 2400 Calories; and excessive or maximum work as high as 8000 Calories per day. This may be shown in a table taken from Benedict and Carpenter.

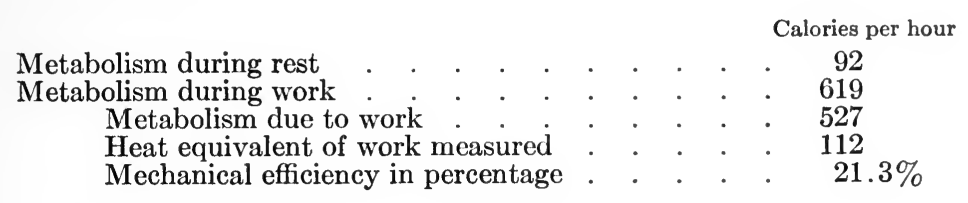

This experiment was done on a professional bicycle rider, and these riders often ride at such a pace for twenty-two or twenty-three hours per day. If this subject had ridden twenty-three hours per day, the values would have been:

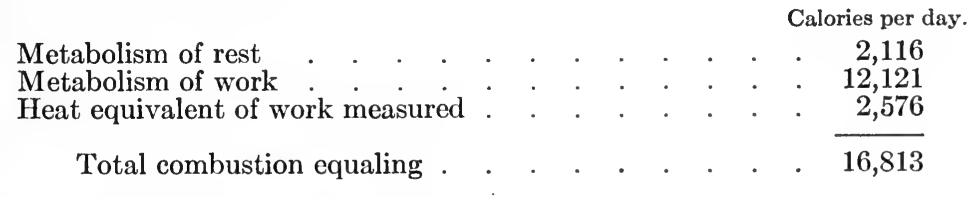

To support such an output, the body must have burned an amount of food that in isodynamic figures would read something like this:



No such amount of food could be ingested; it is burned from the tissues of the individual. Mixed diets have been measured as actually consumed by men in severe work as follows, in rounded figures: protein, 500 grams; carbohydrate, 500 grams; fat, 850 grams, in total representing over 11,000 Calories. These are very extreme figures. The average normal individual cannot accomplish half as much work as above stated, or eat half as much food.

The mechanical efficiency is little increased by training, if at all. Training enlarges the powers of the circulation and respiration, of digestion and assimilation. It increases the number of a particular movement that can be done in the unit of time and extends the period 
of time through which work can be sustained. But it does not materially increase the mechanical efficiency of a particular movement.

The combustions for the support of work first attack the carbohydrates of the body; and if these be present or supplied up to the need, work will be supported by combustion of carbohydrate alone. The actual efficiency of work done on combustion of glucose is little if any greater than in the case of the combustion of fat. But the body burns sugar in preference to fat if choice be presented. The respiratory quotient is high in the beginning of a forced march; late in the march it falls, indicating the combustion of fat. If sugar be supplied (it is now included in the forced-march ration of many armies), the quotient remains high. The sugar reaches the tissues of combustion in thirty to fifty minutes after ingestion; and the sugar ration has advantageously replaced the grog ration of our forefathers. With normal work, especially if uniform and done by trained muscles, the respiratory quotient is the same during work as at rest; but with excessive work, especially in spurts to the point of the fatigue or with untrained muscles, the respiratory quotient will often rise for a short time, later to fall.

In the absence of sugar, the body will burn fat (of the diet or fat from the body depots) for the support of work, though the efficiency is a little lower than in the case of sugar. For extreme exertions, however, fat is the better food, since it is possible to ingest in a day more calories in the state of fat than in the form of sugar. The diet should, however, always contain enough carbohydrate to avoid acidosis and to save the catabolism of excessive protein.

In the presence of carbohydrate, the catabolism of protein is not modified or exaggerated by muscular work. Very excessive work, and especially work with untrained muscles, may lead to increase in total nitrogenous catabolism and an increase in creatinin, but this is an abnormal reaction. Normally, work does not affect the protein catabolism, it does not affect the nucleic or creatin-creatinin metabolism. In a word, physical work under normal conditions does not noticeably affect the up-keep and wear-and-tear of the cells. In the absence of carbohydrate and fat, heavy work can be supported on the combustion of exogenous protein alone, but it is an extravagant process. And under such circumstances, the endogenous protein catabolism is also exaggerated.

At high altitudes, the mechanical efficiency of work is reduced and the capacity for work is also lowered. On the other hand, the resting metabolism is greater than at sea level. These results are not effects that are developed pari passu with each meter of ascent, but they appear when the oxygen content of the air approaches a certain low figure. The altitude at which metabolism in the resting individual is increased is about 12,000 feet, though it is lower for some than for others. Some individuals tolerate an atmosphere so rare as to contain only 10 per cent. of oxygen, better than others are able to endure an atmosphere with over 12 per cent. of oxygen. 'The pressure of oxygen within the 
alveoli of the lungs at sea level varies from 100 to $110 \mathrm{~mm}$. $\mathrm{Hg}$.; at an altitude of 15,000 feet it is reduced one-half. 'This has its effect, of course, upon the combination of hemoglobin with oxygen. At sea level the hemoglobin of arterial blood is about 90 per cent. saturated when absorption is complete; in actual life it is not over 80 per cent. At 15,000 feet altitude, the degree of saturation would be reduced to 65 per cent. The fact that the actual total combustion at 15,000 feet is increased by some 15 per cent. indicates, however, that oxygen reaches the tissues in abundance. From the total data one cannot resist the conviction that the causes of the decreased mechanical efficiency and lowered capacity for work noted at high altitudes are physical rather than chemical.

There is no thermic equivalent for mental work. Resting in the calorimeter without mental work, a subject displays the same output of heat as when engaged in the solution of a mathematical problem. Our measurements have of course a certain range of error, plus and minus; and it is possible that a small production of heat would not be noticed. The fact is striking enough, in view of the variations in circulation that have been assumed to attend mental effort. The fact has, however, no bearing on the application of the law of the conservation of energy to biological processes; any inference to that effect rests upon a misconception of thermodynamics.

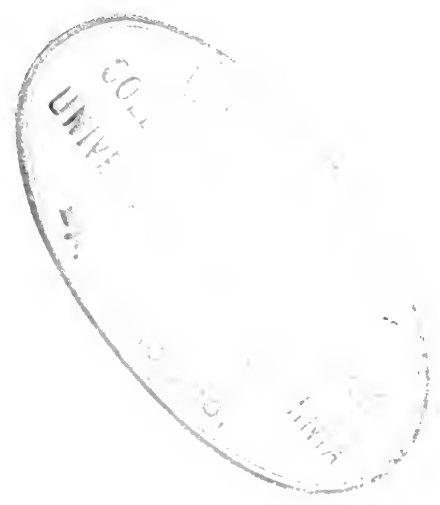





\section{INDEX}

\section{A}

AcApnia, glucosuria, 286

Acetic acid, combustion of, 353

Aceto-acetic acid, combustion of, 353 conversion into acetone, 68

derivation from histidin, 360, 416

from phenylalanin, 360

in combustion of aromatic amino-acids, 410, 411, 412

oxidation of, in diabetes, 328

source of acetone, 353

Acetone bodies, derivation from protein, 359

elimination of, in diabetes, 329

origin from fat, 361

complex, 358, 465

after narcosis, 364

derivation from alanin, 359

from histidin, 359

from leucin, 359

from phenylalanin, 359

from tyrosin, 359

formation of, in diabetes, 328

origin from aceto-acetic acid, 353

polymerization into di-acetone

alcohol, 57

Acrolein, formation from glycerol, 101

Acromegaly, relation to diabetes, 297

Acidosis, 358, 369, 465

distoxication, 330

in diabetes, 362

in eclampsia, 363

in infections, 362

in phloridzin intoxication, 362

in phosphorus poisoning, 364

in starvation, 362

ketonuric, 330

mechanism of, 406

neutralization by bases, 320

relations in tissues in diabetes, 332 to combustion of glucose, 364

to glucose toleration in diabetes, 333

Acids, volatile, in urine in diabetes, 332

Adenin, conversion into hypoxanthin, 439 structure of, 432

Adenosin, 430

Adrenal bodies, relation of ablation to hypoglucemia, 285
Adrenal bodies, relation of, to glucose combustion, 265

Air currents, relation to heat dissipation, 524

expired, toxic proportions of, 463

Alanin, 30

conversion into lactic acid, 395

in oxyproteic acid, 389

source of acetone, 359

of glucose, 391,392

of glycerol, 274

of lactic acid, 268

Alcaptonuria, 410, 411, 412, 413, 467

Alcohol, addition to amino-acids, 31 ethyl, acetous fermentation, 64

Aldehyd, conversion into paraldehyd, 57

Aldohexoses, description of, 20

Aldoses, definition of, 18

Alkalinity of blood, relation to gout, 458 of intestinal contents, 154

Allantoin, cleavage into urea and glyoxylic acid, 449

in urine, 417

relation to uric acid, 448

All-oxy-proteic acid, in urine, 417

Altitude, influence on mechanical efficiency of work, 541

on metabolism, 541

on work, 541

Amino-acid, addition of alcohol, 31 of formaldehyd, 31

content in proteins, 38

in proteins, 31

Amino-acids, bacterial action on, in alimentary tract, 218

biuret reaction of, 31

catabolism of, 407

deaminization of, 67,395

within intestinal tract, 193

in feces, 214

in peptic digestion, 139

in urine, 396,417

in diabetes, 314

linkage of, in molecule of protein, 39

list of sugar builders, 393

new formation of, 379

relation to specific action of protein, 475

resorption of, 192

source of creatin, 424

of glucose, 385,390

synthesis of, 378 
Amino-acids, synthesis of, to protein in intestinal wall, 194

Amino-butyric acid, derivation from $a$ keton-butyric acid, 379

Amino-purin, deaminization of, 440

Amino-purins in beverages, 447

Amino-pyrimidin, deaminization of, 440

Amino-sugar, 391

Ammonia, derivation of, 385

formation by bacteria in intestine, 405

by deaminization of aminoacids, 405

by intestinal deaminization, 405

in hydrolysis of protein, 405

in protein, 37

in urine, 405

in acidosis, 321

primary, 407

relation to acidosis, 405,406

to alkalosis, 405

Ammonia-urea, equilibrium, 405

Ammonium aldehyd, conversion into beta-oxy-butyric acid, 365

carbamate, 399

carbonate, source of urea, 399

Amphoterism, in proteins, 27

Amylase, in intestinal secretion, 168 in pancreatic secretion, 115,157 in succus entericus, 115 salivary, 119

Anabolism of fat, 346

of nucleic acid, 434, 441

of protein, 373

adaptation in, 381

Anemia, oxidation in, 536

Anthracin, relation to dianthracin, 57

Antiseptic action of bile, 179

Antiketonic substances, in diabetes, 333

d-Arabinose, 231

l-Arabinose, 231

Areolar tissues, formation of fat, 259

Arginase in liver, 396, 398

Arginin, 37, 41

cleavage of, 396

hydrolysis of, 62

Aromatic amino-acids, 409

bodies in urine, relation to bacterial action, 409

to metabolism, 409

Arsenic trioxid, conversion into cacodylic oxid, 66

Asparagin, 35

reduction of, 66

Aspartic acid, 35

reduction to ammonium succinate, 67

source of beta-oxy-butyric acid, 360

of glucose, 393

synthesis of, 378

Assimilation of carbohydrate, limit of, 249

Autointoxication, 461
Autointoxication by formation of abnormal substances, 466

by intermediary metabolic products, 465

by overload, 467

by retention of end products of metabolism, 463

gastro-intestinal, 462

relation to alimentary secretions, 467

through failure of distoxication, 466

toxicity of cutaneous secretions, 464

Autolysis in involution of uterus, 389

in pneumonia, 389

in tuberculosis, 389

of cellular protein, 385

Aut-oxy-proteic acid in urine, 417

\section{B}

BACTERIA, action on foodstuffs in alimentary tract, 217

on nucleosid, 433

assimilation of protein, 375

digestion without, 49

Bacterial conditions in alimentary tract, abnormal result on digestion, 204, 206

intoxication, gastro-intestinal, 461

Balance, nitrogen, 418

Basal heat production, 509

Benzene bodies, relations of reaction to configuration, 106

ring, mode of rupture in body, 409

Benzoic acid, derivation from phenylalanin in intestine, 219 , 226

from tyrosin in intestine, 219

Benzol combustion in diabetes, 305

Bernard puncture, 249, 283

Beta-oxy-butyric acid and aceto-acetic acid, equilibrium between, 361

combustion of, 353

in diabetes, 307

derivation from aspartic acid, 360

from leucin, 359

formation from ammonium alde-

Bile, 170 hyd, 361

antiseptic action of, 179

bilirubin, 175

cholesterol in, 178

cholic acid, 172

functions of, 179

glycocoll in, 173

in feces, 216

influence on peristalsis, 179

in stomach, 146

intestinal digestion in the absence of, 181

lipoids of, 177 
Bile, nitrogenous bodies in, 178

pathological variations, 180

phosphorus compounds of, 177

proteins in, 171

quantitative components of, 170

relation to cleavage of fat in intestine, 180

to digestion of carbohydrate, 179

of protein, 179

to resorption of fatty acids, 180 , 181

to solution of fat in intestine, 180

secretion of, 178

sulphur compounds in, 171

taurin, 172

zymo-excitor action on lipase, 159, 180

Biliary acids in feces, 174

Bilirubin in bile, 175

constitution of, 175

derivation from erythrocytes, 175

Biliverdin, formation from bilirubin, 176

Birds, purin output in, 444

Biuret reaction in proteins, 31

Blood content of urea, 400

in fasting, 488

pressure, factor in intestinal resorption, 202

protein, concentration of, 384

state of fat in, 344

Body heat, conduction and radiation, 529 production of, 509

surface, determination of, 518

temperature, regulation of, 509, 517 chemical, 518

physical; 519

weight in fasting, 488

Bread, purin content of, 446

Butter in diet, relation to ketonuria in diabetes, 331

Butyric acid, combustion of, 352 chart of, 367

derivation from triolein, 354

formation from lactic acid, 343 oxidation of, in diabetes, 328

relation of combustion of, to glucose, 367

\section{C}

Cadaverin, derivation from lysin, 415

Caffein, 446 glucosuria, 284

Caloric content of body, 468

equivalents of carbohydrate, 513

in diabetes, 300

of fat, 513

of protein, 513

B values of diets, 474, 502

Calorimeter, bed, metabolism in, 514

Cane sugar in metabolism, 241
Caproic acid, combustion of, 351

origin in oleic acid, 354

Carbamino acids, 399

Carbohydrate, caloric equivalents, 513

digestion within resorption membrane, 190

fasting, 490

fuel for work, 540

influence on protein catabolism in fever, 480

limit of assimilation of, 249

metabolism, 230

in diabetes, 291

in fasting, 484,487

ration in diet, 500

reciprocal relation to fat in diet, 472 , 476

resorption of, 189

respiratory quotient, 513,516

saving power for protein, 478

Carbohydrates, bacterial action on, in intestine, 218

caloric value of, 23

description of, 17

Carbon asymmetric, 19

dioxid, addition to amino-acids, 31 elimination in diabetes, 340

in metabolism, 514,515

monoxid, relation to formation of formaldehyd, 65

Carcinoma of stomach, ferment in, 170

Cardiac disease, oxidation in, 536

Cartilage, relation of composition to urate deposition, 458

Casein, anabolism of, 377

Castration, oxidation in, 536

Catabolism of dead cells, 383

of exogenous protein, 384

of fat, 320

of protein, 383

chart of, 420

in fasting, 385

of protoplasm, wear and tear, 383

of special sugars, 237

of superfluous amino-acids, 383

Catalase, 534

Catalysis, application of laws of, to fermentation, 70, 78

in heterogeneous system, 87

Catalyzer, inactivation of, 76

relation to order of reaction, 75 to reaction, 74

to station of equilibrium, 75

Cells, autolysis of, 385

content of glycogen and glucose, 244

regeneration of, 374

wear-and-tear of, 374

Cellulose, hydrolysis of, 60

in diet, 493

Cerebron, galactose content, 234

Cerebronic acid, 234

Chemical nature of ferments, 94 regulation, 518 heat production, 510 
Childhood, metabolism in, 505

Chloroform, necrosis of liver, 364

Chlorophyl, sugar formation by, 18

Cholesterol, 349

catabolism of, 350

in bile, 178

in feces, 350

Cholic acid, derivation from erythrocytes, 173

in bile, 172

Cholin, 348, 465

Chondroitin, 236

Chymosin, individuality as ferment, 144 in gastric secretion, 144 variations in disease, 144

in intestinal secretion, 169

pancreatic secretion, 160

Circulation, heat production of, 510

of skin, relation to heat dissipation, 520

Clothing, relation to heat dissipation, 525

Coagulation of proteins, 28

Cocoa, purin content of, 446

Coffee, purin content of, 446

Collagen, anabolism of, 376

Colloidal nature of proteins, 24

Combined sugar in tissues, 230

Combustion of fat, 351

of glucose, 261

in muscles, 255

Combustions in support of work, 540

Conduction and radiation of body heat, 529

in fever, 531

p-Cresol, derivation from tyrosin in intestine, 219

oxidation of, 410

Creatin, abnormal appearance in urine, 427

administration of, 425

catabolism, relation to metabolism of glucose, 428

-creatinin metabolism, 469

derivation of, 424

elimination in diabetes, 428

in inanition, 428

in muscular degeneration, 428

metabolism of, 423

relation to amino-acids, 424

source of creatinin, 425

Creatinin, coefficient of muscular metabolism, 423

conversion into urea, 400,426

derivation from creatin, 425

elimination of, 423

endogenous, 424

exogenous, 424

metabolism of, 423

chart of, 454

specificity of, 426

in work, 540

output in urine, 425

pathological variations in elimination of, 427
Crystallization of protein, 27

Cystein, 414

derivation from erythrocytes, 173

in bile, 173

source of glycerol, 274

of lactic acid, 268

Cysteinic acid in bile, 173

Cystin, 33, 41

anabolism of, 421

catabolism of, 414

cleavage in intestine, 414

in urine, 396

oxidation of, 414

relation to sulphur metabolism, 421

requirement of, 378

Cystinuria, 414, 465

relation to diet, 415

Cytidin, occurrence of, 437

Cytosin, conversion into uracil, 440

in urine, 459

occurrence of, 437

structure of, 431

\section{D}

Deaminization ferment, 440

of amino-acids, 385,395

of amino-purins, 439

of amino-pyrimidin, 440

within intestinal tract, 193

Denaturation of proteins, 29

Dextrinuria in diabetes, 308

Diabetes, acidosis in, 362

ammonia as index of acidosis in, 331

caloric equivalents in, 300

carbon dioxid elimination in, 340

combustion of glucose, 297, 299, 300 of various bodies in, 305

carbohydrate metabolism in, 291

definition of, 291

derivation of glucose from protein, 315

elimination of acetone bodies, 329 of creatin in, 428

etiology, 291

experimental, 291

failure of formation of fat from glucose in, 358

fat combustion in, 327

formation of fat from glycogen, 311 of sugar from fat, 326

fructosuria, 235

$\mathrm{G}: \mathrm{N}$ ratio in, 316, 320, 321

heat production in, 340

value of protein in, 326

hepatic glycogenesis in, 309 glycolysis in, 310

hypothesis of direct combustion of glycogen, 304

influence of fever on combustion, 302

lipemia in, 335

masked in obesity, 311

mixed melituria in, 308 
Diabetes, muscular glycogenesis and Digestion, functions of, 113

glycolysis in, 311

O-absorption in, 340

overproduction of sugar in, 306

oxidation in, 536

of butyric acid in, $32 \mathrm{~S}$

pancreatic, 298

protein metabolism in, 313

relation of acidosis to utilization of glucose, 333

of glucose to lactic acid, 292

of work to combustion of glucose, 302

renal retention of sugar, 307

respiratory quotient in, 335,337

salt of the urine, 332

toleration for different sugars, 303

total metabolism in, 339

utilization of calories of diet in, 340

volatile acids in urine in, 332

Diabetic coma, amino-acids in urine in, 396

Dialuric acid, conversion into uric acid, 445

Diamins in urine, 417

Diazo-amino-benzole, conversion into pamino-azobenzol, 58

Di-brom succinic acid, conversion into bromo-maleic acid, 59

Diet, bread, mass of, 184 bulk of, 493

carbohydrate ration in, 500

cellulose in, 493

definition of complete, 49

desiderata, 493

energy content, 493

fat ration in, 500

fresh food in, 493

mass, relations of, 181

milk, mass of, 183

normal, 493

protein, 493 mass of, 184

salts in, 493

vegetable, mass of, 185

Diets, caloric values in, 503

Diffusion, factor in intestinal resorption, 201

of protein, 26

Digestion, abnormalities due to defect in resorption, 204, 205

to qualitative chemical variations, 204,205

disturbances due to abnormal motor functions, 204, 205

to abnormalities in secretion of digestive juices, 204,205

to bacterial conditions, 204 , 206

toimpropermastication, 204

to quantitative chemical variations, 204, 205

to toxic ingesta 204 in vitro, 114

in vivo, 118

of protein, scope of, in stomach, 139

of nucleo-protein, 432, 436

postmortem, 385

relations of heat and energy, 206

resorption of products of, 186

salivary, 119

in vitro, 115

without bacteria, 49

work of, 474

Dihydro-cholesterol, 350

2-5-Dihydroxy-phenyl-pyruvic acid, 411

o-Dioxy-benzene, derivation from tyrosin

in intestine, 219

Dioxyphenyl-acetic acid, 410

lactic acid, 410

Disaccharids, formation from monosaccharids by ferments, 87

hydrolysis of, 61

in metabolism, 241

Disease, gastric, variations of hydrochloric acid in, 135

Distearyl lecithin, 349

Distoxication, 466

Duodenal contents in stomach, 146

\section{E}

Eck fistula, intoxication in, 466 urea formation with, 401

Eclampsia, acidosis in, 363

Elimination of end products of protein catabolism, 417

Emulsin in intestinal secretion, 168

in pancreatic secretion, 159

Endogenous protein, elimination of end products of, 418

purin, catabolisin in gout, 455

Energy relations in digestion, 206

Enterokinase in intestinal secretion, 161

Epiguanin, 440

Epinephrin, glucosuria, 285

relation to tyrosin, 413

Equation, differential, of monomolecular reaction, 71

for determination of body surface, 518

relation of mass of catalyzer to reaction constant, 75

Equilibrium in reaction of bimolecular order, 72

in reaction system, 77

in system of fermentation, $\mathrm{S}$

relation of catalyzer, 75

Erepsin, digestion in vitro, 117 in intestinal secretion, 169

Esters, formation by ferment action, 86 hydrolysis of, 62 racemic, fermentation of, 109

Ether, formation of, from alcohol, 101

Ethyl acetate, hydrolysis of, 62 
Ethyl alcohol, product of combustion of lactic acid, 263

Exhalation of water, relation to heat dissipation, 522

Exogenous ferments in alimentary tract, 169

intoxication, 461

protein, elimination of end products of, 418

External temperature, relation to heat dissipation, 522

to metabolism, 471

\section{F}

Fasting, blood in, 488

body weight in, 488

carbohydrate, 490 metabolism, 484, 487

fat metabolism in, 484,487

heat production in, 484

muscular work in, 487

partition of urinary nitrogen, 486

protein metabolism in, 484, 485

respiratory quotient in, 487

total, 483

wasting of organs in, 488

Fat, alimentary secretions in feces, 215

anabolism of, 346

caloric equivalents of, 513 value of, 24

cleavage in intestine, relation of bile, 180

combustion of, 351

in diabetes, 327

content of feces, 215

catabolism of, 350

derivation of sugar from, 240

description of, 23

formation from glucose, 258, 358 chemistry of, 260

in diabetes, 311

variations in disease, 259

from protein, 356

from sugar, $326,343,355$

relation to glucose concentration of blood, 259

hydrolysis of, 351

ingested in diet, 242

intestinal digestion in absence of bile, 182

metabolism, 342

chart of, 358

in fasting, 484,487

pathology of, 358

of diet in stools, 215

protein complex, 347

ration in diet, 500

reciprocal relation to carbohydrate

in diet, 476,477

resorbed, route of transmission,

197

respiratory quotient, 513,516
Fat, respiratory quotient of combustion of, in diabetes, 339

saving power for protein, 478

site of, formation of, 259

soluble state of, 345

source of acetone bodies, 361 in body, 342

starvation, 491

state of, in blood, 34

in thoracic duct, 344

synthetized in body, 342

unsplit, resorption of, 196

Fats, bacterial action on, in alimentary canal, 218

resorption of products of, digestion of, 196

solution of, in intestine, relation to bile, 180

Fattening, relation to protein diet, 507

Fatty acids, combustion of, 351 in feces, 215 oxidation of, $328,385,397$ relation of bile to resorption of, 180

degeneration, 356

infiltration, 356

of liver, in phloridzin intoxication, 290

organs, fat content of, 356

Feces, 208

amino-acids in, 214

aromatic bodies of, 221

bacteria of, 212

bile in, 216

biliary acids in, 174

color of, 216

content of cholesterol, 350 .

fat content of, 215

fatty acids in, 215

in starvation, 213

mass of, 208

nitrogen content of, 212,417

odor of, 211

peptids in, 214

phosphorus in, 460

protein in, 213

purin of, 447

bases in, 433

reaction of, 211

residue of alimentary secretion in, 213,216

of digestible food, 212

soaps in, 215

solids of, 212

sulphur content of, 421

trypsin in, 163

urea elimination in, 404

Feeding, forced, 492

with protein, 387

Ferment action, qualitative specificity of, 111

reversion of, 85

specificity of, 104

theory of, 55 
Ferment, chemical nature of, 94 concentration of, in fermentation, 82 hydrolysis of starch, 120 inactivation of, 76

oxidation, in combustion of fatty acids, 355

proteolytic, intracellular, 388

reaction, reversion of, 81

Ferments, exogenous in alimentary tract, 169

Fermentation, application of the laws of catalysis to, 70,77

chemical reactions of, 56

general features of, 68

modus operandi of, 94

of carbohydrates in alimentary tract, 218

of fat in alimentary tract, 218

of racemic acids, 109

of stereoisomeric hexoses, 108

of synthetic methyl glucosids, 109 peptids, 109

relation to ionization, 103

theory of intermediary reactions, 95

Fever, 526

conduction and radiation of body

heat in, 531

cutaneous circulation in, 532

cytolytic, 527

heat dissipation in, 530

production in, 527

hyperthermia in, 527

in children, 533

microörganismal, 527

neurogenic, 527

nitrogen elimination in, 530

perspiration in, 530, 532

physiological, 526

protein catabolism in, 480 metabolism in, 529

relation to combustion in diabetes, 302

vasoconstriction in, 532

Filtration of proteins, 26

Flesh diet, 491

Fleshening, 480, 507

Food, bulk of, in intestine, 200

Foods, specific dynamic action of, 469

Foodstuffs, bacterial action on, 217 composition of, 17

conservation of, 49

definition of, 17

discussion of, 48

inorganic constituents of, 49

relation to complete diet, 49

source of, in relation to utilization, 49

specific dynamic action, 510

tables of, $50,51,52,53$

Forced fecding, 492

Formaldehyd, addition to amino-acids, 31 origin by condensation of $\mathrm{CO}_{2}$ and $\mathrm{H}_{2} \mathrm{O}, 65$

oxidation by hydrogen peroxid, 65

Formate, hydrolysis of, 63
Formic acid, combustion of, 353 origin from formaldehyd, 65

Fright, glucosuria, 283

Fructose, 235

toleration in diabetes, 303

Fructosuria, alimentary, 235, 280

diabetic, 235, 308

hepatic, 235

idiopathic, 235

varieties of, 235

G

Galactase, 190

Galactose, 233

anabolism of, 234

conversion into glucose in intestinal wall, 190

in milk, 233

in liver, 190

in mucin, 234

in nervous system, 234

in tissues, 233

Galactosuria, 233

alimentary, 233

Gastric secretion, 126

amylase in, 145

cephalogenous, 127

cerebrospinal, 128

endogenous, 127

hydrochloric acid in, 129

chemical relation of, 132

formation of, 130

influence on mastication in, 126 of mock feeding, 127 of special senses, 127

lactase in, 145

lipase in, 116, 145

maltase in, 145

mucus of, 149

saccharase in, 145

total relations of, to foods, 128

variations in hydrochloric acid

in disease, 135

water of, 147

Gastro-intestinal acetone complex, 363

bacterial intoxication, 461

Gelatin, utilization of, 378,379

Gelification of proteins, 27

Gestation, metabolism in, 502

Glands, purin content of, 446

Gliadin, utilization of, 379

Glucosamin, 38, 236, 391

Glucose, 21

amount in circulatory fluids, 245

chemical state in circulatory fluids and tissue, 244

cleavage into lactic acid, 263

into methyl glyoxal, 263

combustion of, in diabetes, 297 relation to adrenal bodies, 265 to pancreas, 264 
Glucose, combustion of, without oxygen, Glucuronic acid, conjugated with phenols,

265

concentration in blood, 252

derivation from amino-acids, 385

from alanin, 391

from aspartic acid, 393

from glutamic acid, 392

from glycerose, 392

from glycocoll, 391

from lysin, 391

from serin, 392

direct oxidation of, 261

fat formation from, 258

fermentation of, 59

from lactic acid, 392

formation from amino-acids, 390 relation to specific dynamic

from fat, 326 action of protein, 476 in diabetes, 322, 324

from glycerol in diabetes, 323

from protein, curve of, 393 quantitative, 394

in diabetes, origin of, 313

indirect oxidation of, 263

lipoids, 244

nitrogen ratio, $316,318,394$

in phloridzin intoxication, 319

overproduction of, in diabetes, 306

protein, 244

relation to creatin, 480

to combustion of butyric acid, 368

to lactic acid in diabetes, 298

source in phloridzin intoxication, 289

of fat, 355

of glycerol, 344

Glucosids, definition of, 22

hydrolysis of, 61

synthetic methyl, fermentation of, 109

Glucosuria, 276, 287

alimentary, 280

due to epinephrin, 285

to excess of muscular glycolysis, 286

to excessive hepatic glycolysis, 283

to failure in combustion of glucose, 287

in muscular gycogenesis, 286

to lowered hepatic glycogenesis, 281

hemic, 276,287

in diabetes, relation to diet, 325

renal, 276,287

saline, 284

Glucothionic acid, 236

combustion in diabetes, 306

Glucuronic acid, 269

alimentary, 270

combustion in diabetes, 306
219

conjugations of, 269

derivation of, 270

from glucose, 262

in disease, 270

d-Glucuronic acid, possible source of pentose, 232

Glucuronuria, 262

Glutamic acid, 35 in oxyproteic acid, 389

Glutamin, 35 source of glucose, 392

Glyceric acid, glucose formation, 392 in formation of glycerol, 344 source of glycerol, 274

Glycerids, melting point, 34

Glycerol, 273

combustion of, 351

conversion into glucose, 274 into sugar, 328

derivation, for formation of fat, 275 from amino-acids, 273

from glucose, 273

fate of, in body, 274

formation from glucose, 344

Glycerol-phosphoric acid, 348

Glycerose, glucose formation, 392

in formation of glycerol, 344

source of glycerol, 274

Glycocoll, 32

catabolism of, 408

in bile, 173

in blood, 408

in hippuric acid, 408

in urine, 396,417

origin of, for conjugation to hippuric acid, 227

in decomposition of purin, 408

oxyproteic acid, 389

source of glucose, 391, 393

synthesis of, 378

in body, 408

on administration of benzoic acid, 408

Glycogen, 247

combined in tissues, 248

combustion of, 254

content of muscle, 253

conversion into glucose, 254

formation of, 247

in liver, 249

free in tissues, 248

in blood, 252

of liver, derivation from food, 248 relation to portal hyperglucoemia, 249

relations in diabetes, 304

transportation of, 252

Glycogenase, 254

Glycogenesis, hepatic, relation to glucosuria, 281

Glycogenuria in diabetes, 308

Glycol, source of cholin, 348 
Glycolysis, following Bernard puneture, 249

Goitre, oxidation in, 537

Gout, 455

alkalinity of, blood in, 458

blood content of uric acid, 455

catabolism of exogenous purins in, 455

purin catabolism in, 455

elimination in, 457

toxic manifestations in, 458

urate depositions, 457

uricolysis in, 456

Growth, law of, 504

Guanidin, 423

Guanin, conversion into hypoxanthin, 67 into xanthin, 440 structure of, 432

Guanosin, 430

Guanylic acid, occurrence of, 437

\section{H}

HAIR, nitrogen content of, 417

relation to heat dissipation, 519

Hemoglobin, anabolism of, 377

Hematoporphyrin, 465

in urine, 417

relation to bilirubin, 176

Heat dissipation in fever, 530 relation to air currents, 524

to circulation of skin, 520

to clothing, 525

to exhalation of water, 522

to external temperature, 523

to hair, 519

to humidity, 523

to perspiration, 521

to skin, 520

to subcutaneous fat, 521

of specific dynamic action, 510

output, table of, 512

production, basal, 509

in diabetes, 340

in fasting, 485

in fever, 527

in gestation, 503

in infancy, 504

in metabolism, 514

of chemical regulation, 510

of foodstuffs, 470

of muscular contraction, 511

per unit surface, 518

relation to metabolism, 512

relations in digestion, 206

Helicin, hydrolysis of, 61

Hematin relation to tryptophan, 220 structure of, 176

Hepatic glycogen, derivation of, 248 glycogenesis, in diabctes, 309,310

Heptargia, 282

Heterogeneous system, catalysis in, 87
Hexoses, fermentation of, 108

Hippokoprosterin, 350

Hippuric acid, 225

formation in kidney, 227

hydrolysis of, 62

in urine, 417

relation to vegetarian diet, 227

origin in intestine, 226

Histidin, 34 in metabolism, 226

catabolism of, 416

source of aceto-acetic acid, 360 of acetone, 359

Histon, nucleic acid, 429

Homogentisic acid, 410

Humidity, relation to heat dissipation, 523

Hydrazin, reactions of, 112

Hydrochloric acid, chemical relations in gastric secretion, 132

formation of, in stomach, 130

in gastric secretion, 129 action on secretion of ferments, 132

activation of pepsinogen, 132

free and combined, 134

quantitative secretion, 133

relation of secretion to diet, 134

to peptic digestion, 132

variations in disease, 135

relation to peptic digestion, 140

Hydrogen peroxid, oxidation of, 65 relation to formation of formaldehyd, 65

Hydrolytic cleavage, 60 fermentation, 60

Hydroquinon, derivation from tyrosin in intestine, 219

oxidation to quinon, 63

Hydroxylamin, hydrolysis of, 112

para-Hydroxy-phenyl-pyruvic acid, 411, 412

Hyperammonuria, primary, 407 secondary, 407

Hypercapnia, glucosuria, 285

Hyperglucemia in diabetes, 306 relation to glucosuria in diabetes, 307

Hyperpurinemia, alimentary, 454 in gout, 458

Hypophysis, relation to carbohydrate metabolism, 297

Hypoxanthin, conversion into xanthin, 439

derivation from adenin, 439

origin from guanin, 67

oxidation to xanthin, 64

structure of, 432 
I
ImBiBition, factor in intestinal resorp-
tion, 202

Iminazol-pyruvic acid, 416

Inactivation of ferment, 76

Inanition, 482

elimination of creatin in, 427

protein catabolism in, 497

Indol acetic acid, derivation from tryptophan in intestine, 220

derivation from tryptophan, 220

propionic acid, derivation from tryptophan in intestine, 220

Indoxyl, derivation from tryptophan in intestine, 220

relation to total conjugated sulphates, 221

Induction, theory of, in catalysis and fermentation, 97

Infancy, food requirements in, 504

heat production in, 504

metabolism in, 504

nutrition in, 502

Infections, acidosis in, 362

lag in nitrogen elimination, 419

peptone elimination in, 389

Inosin, 430 protein metabolism in, 529

Inosinic acid, occurrence of, 437 source of hypoxanthin, 443 of uric acid, 443

Iodin, combination with hydrogen, 58

Ionization, relation to fermentation, 103

Intermediary reactions, theory of, in catalysis and fermentation, 95

Intestinal contents, alkalinity in, 154 digestion, in absence of pancreatic juice, 164

pepsin in, 141

mucosa, digestion of nucleotid in, 434 putrefaction, relation to urinary

aromatic bodies, 221

secretion, alkali in, 167

ferments of, 168

mucus in, 167

of pancreas, 166

relation to digestion, 167

water of, 167

Intestine, digestion in, 153

formation of urobilinogen and uro-

bilin from bilirubin, 176

resorption from, 187

Intra-intestinal pressure factor in intestinal resorption, 202

Invertase in succus entericus, 115

pancreatic secretion, 158

Isodynamic law, 512

Isomerism, of proteins, 44
b-KephaLin, 349

Ketohexoses, description of, 21

Ketones, $\mathrm{C}_{5} \mathrm{Cl}_{6} \mathrm{O}, 58$

Ketonic intoxication, 465

Ketonuria in diabetes, 330

Ketoses, definition of, 18

Ketosis, 359 chart of, 367

Kidney, elimination of urea in, 404 fatty degencration of, 357

Kilogrammeter, unit of work, 538

Koprosterin, 350

Kynurenic acid, 413

\section{I}

LACTASE in intestinal secretion, 168

in pancreatic secretion, 115,158

in succus entericus, 115

salivary, 126

Lactation, metabolism of, 503

Lactic acid, 266

bacterial, 268

cleavage into $\mathrm{CO}_{2}$ and ethyl alcohol, 263

conversion into butyric acid, 343 into uric acid, 445

derivation from amino-acids, 268

from carbohydrates, 267

from glucose, 263

formation of, 265

in asphyxia and disturbances in oxidation, 269

in diabetes, 269, 305

in hepatic disease, 269

in urine in phosphorus poisoning, 396

origin from alanin, 395

source of glucose, 392 of glycerol, 274,344

Lactose in metabolism, 243

Lactosuria, alimentary, 280

lactational, 243

Lag in nitrogen elimination, 418

in protein catabolism, 419

Langerhans, islands of, relation to pancrcatic diabetes, 294

Law of isodynamic relations, 512

Lead poisoning, blood content of uric acid, 455

Lecithin, 348

Leucin, 35

in oxyproteic acid, 389

in urine, 396

source of acctone, 359

of b-oxy-butyric acid, 359

of glucose, 393

Leukemia, blood content of uric acid in, 455

elimination of peptone in, 389 
Leukemia, nucleic catabolism in, 454

Levulose, 235

conversion into glucose in intestinal wall, 190

in liver, 191

Lipase in gastric secretion, 116,145 variations in disease, 146

in intestinal secretion, 169

in pancreatic secretion, 116, 159

Lipemia in diabetes, 335

Lipoid, relation to law of partition, 347

Lipoids of bile, 178

relation to semipermeability of cell membrane, 347

sebaceous, 349

Liver, amino-acids in urine in diseases of, 396

antagonism of fat and glycogen, 251

arginase in, 396

combustion in, 255

degeneration, nucleic catabolism in, 454

disease, influence on carbohydrate and protein metabolism, 480

fatty degeneration of, 357

infiltration of, 357

formation of glycogen, 249

of urobilin in aseptic autolysis of, 177

relation to nucleic catabolism, 437

storage capacity for glycogen, variations in disease, 257

Lungs, oxidation in diseases of, 535

Lysin, 37

catabolism of, 415

source of cadaverin, 415

of glucose, 391

synthesis of, 378

\section{M}

MALIC acid, fermentation of, 59

Malignant neoplasms, oxidation in, 537

Maltase in pancreatic secretion, 115, 158 in succus entericus, 115,168 salivary, 124

Malted liquors, purin content of, 446

Maltose in metabolism, 242

Maltosuria, alimentary, 242 diabetic, 243, 308

Mass of diets, 181

of tissue, relation to metabolism, 468

Masses of diets, standard and various, 183

Mastication, imperfect, result to digestion, 204

influence on gastric secretion, 126

Melting point of fats, 24

Melituria, 276

alimentary, 271, 280

mixed, in diabetes, 308 relation of liver to, 308

Mental work, thermic equivalent, 539

Metabolism at high altitude, 541
Metabolism, carbohydrate, 230

carbon dioxid elimination, 514, 515

considered as whole, 468

O-consumption, 514, 515

general considerations, 228

heat production in, 514

in childhood, 505

in gestation, 502

in infancy, 504

in obesity, 508

in old age, 505

in overnutrition, 506

in undernutrition, 505

of creatin-creatinin, 423,469

of fat, 342

of lactation, 503

of protein, 370,469

of purin, 428,469

of sulphur, 420

oxidation in, 534

relation to external temperature, 471 to heat production, 512

to mass of tissue, 468

to work, 538

respiratory quotient, 514,515

suboxidation in, 534,535

superoxidation in, 534

total in diabetes, 339

with work, 539

Metals, oxidation of, 64

Methylguanidin acetic acid, 423

in urine, 417,426

Methylimidazol, synthesis of, 436

Methylpurins, oxidation of, 446

Methylpyridyl ammonium in urine, 225

Milk diet, mass of, 183

production, relation to diet, 503

protein content of, 496

Mixed diet, mass of, 184

Mock feeding, influence on gastric digestion, 127

Monochloracetic acid, hydrolysis of, 63

Motor functions of digestion, disturbances due to abnormalities, 204,205

of stomach, 150

variations in disease, 151

Mucin, anabolism of, 377

galactose, content of, 234

in urine, 417

Mucus in gastric secretion, variation in disease, 149

Muscle, autolysis of, 424

functions of combustion, 255

purin content of, 446

Muscles, formation of glycogen in, 253

glycogen content of, 253

site of combustion of glucose, 255, 256

Muscular contraction, heat of, 511

degeneration, elimination of creatin in, 427

glycogenesis and glycolysis in diabetes, 311 
Muscular work in fasting, 487 relation to purin output, 443 supported by fat, 256 by protein, 25

Myxedema, oxidation in, 536

\section{$\mathbf{N}$}

NAILs, nitrogen content of, 417

Narcosis, acetone complex after, 364

Narcotics, glucosuria, 284

Neoplasms, nucleic catabolism in, 454 peptone elimination in, 389

Nephritis, blood content in uric acid, 455

lag in nitrogen elimination, 419

Neurin, relation to cholin, 349

Neutral sulphur in urine, 414,417

Nitrobenzol, reduction to anilin, 67

Nitrogen balance, 418 content of feces, 212 cutaneous elimination, 464

elimination, in fever, 530 influence of carbohydrate, 419 lag in, 418

in perspiration, 417

rest in urine, 416

retention, 419

urinary, partition in fasting, 486

Nitrogenous bodies in bile, 178

end products in urine, relation to uremia, 465

Normal diet, 493

Nucleic acid, anabolism of, 434, 436 hydrolysis of, 439 metabolism of, 434 pathological variations in anabolism, 438

resorption of, 433 structure of, 428

metabolism of different tissues, 443 relation to work, 540 specificity of, 442

Nuclein, 429

Nucleinase, 432, 433, 439

Nucleoprotein, 428 digestion of, 432, 436 tryptic digestion of, 433

Nucleosid, anabolism of, 436 bacterial cleavage, 433 cleavage in intestinal wall, 434 digestion of, 432 resorption of, 433 structure of, 429 varieties of, 430

Nucleosidase, 439

Nucleotid, anabolism of, 436 cleavage in intestinal wall, 434 digestion of, 432 resorption of, 433 structure of, 429

Nucleotidase, 432, 433, 439

Nutrition in infancy, 502
OAtmeal, toleration in diabetes, 303

Obesity, 508 masked diabetes, 311

oxidation in, 536

Oleic acid, combustion of, 354

Order of reaction, relation to catalyzer, 75

Organs, wasting in fasting, 488

Ornithin, 35

catabolism of, 415

origin from arginin, 396

source of putrescin, 415

Overnutrition, 506

Oxalic acid, 272

alimentary, 272 endogenous, 272 origin from carbohydrate, 272 from creatin, 272

from glycocoll, 272

from purin, 273

relation to urea, 449

Oxaluria, 273

Oxydase, 534

O-absorption in diabetes, 340

O-consumption in metabolism, 514, 515

Oxidation fermentation, 63

ferments, 534

in combustion of fatty acids, 353

in anemia, 536

in cardiac disease, 536

in castration, 536

in diabetes, 536

in diseases of lungs, 535

in exophthalmic goitre, 537

in malignant neoplasms, 537

in metabolism, 534, 535

in myxedema, 536

in obesity, 536

in sepsis, 537

in shock, 536

of products of protein catabolism, 396

of purin bases, 439

of uric acid, 448

relation to oxygen, 535

O-phenyl-ethylamin, derivation from tyrosin in intestine, 221

p-Oxy-phenyl-acetic acid, derivation from tyrosin in intestine, 219

p-Oxy-phenyl lactic acid, 410

p-Oxy-phenyl-propionic acid, derivation

from tyrosin in intestine, 219

Oxyproteic acid, 388

in urine, 417

Ozone, relation to formation of formaldehyd, 65

to oxidations in body, 534

\section{$\mathbf{P}$}

Pancreas, internal secretion of, 166 relation to diabetes 296 
Pancreas, relation to sugar combustion, 264 secretion of, 153 self-digestion of, 165

Pancreatic diabetes, formation of glucose from amino-acids, 390

digestion of starch in vitro, 115 juice, toxicity of, 467 secretion, amylase in, 115, 157 chymosin, 160

digestion in intestine in absence of, 164

emulsin, 159

in stomach, 146

invertase in, 158

lactase in, 115,158

lipase in, 116, 159

maltase in, 115,158

pathology of, 163

relations to gastric secretion, 153 trypsin, 160 variations in, 154

Paraldehyd, origin from aldehyd, 57

Pentamethylendiamin (cadaverin), 415

Pentose, 231

content of tissues, 231

conversion into glucose in intestine, 191

Pentoses, description of, 20

Pentosuria, 467

alimentary, 231

idiopathic, 231

in diabetes, 309

nucleic, 231

Pepsin, action within intestinal tract, 141 on nucleosid, 432

on polynucleotid, 432

digestion in vitro, 116

in gastric secretion, 138

relation to hydrolysis of protein, 138

Pepsinogen, 138

Peptic digestion, amino-acids in, 139 peptones in, 138

proteoses, 138

relation to hydrochloric acid, 140

to tryptic digestion, 139

Peptid, 40 variation in disease, 141

binding, 39

in urine, 417

Peptids in feces, 214

resorption of, 192

synthetic, 48

fermentation of, 109

Peptone, elimination in infections, 389

in leukemia, 389

in neoplasms, 389

in peptic digestion, 138 resorption of, 192

Peristalsis, 209 influence of bile, 179

Peroxydase, 534

Perspiration in fever, 530, 532
Perspiration nitrogen content, 417 relation to heat dissipation, 521

Phenylacetic acid, derivation from phenylalanin in intestine, 219

Phenylaceturic acid, 219

Phenylalanin, 32

catabolism of, 409, 410, 411, 412

combustion of, in body, 219

in oxyproteic acid, 389

oxidation of, 391

putrefaction of, in intestine, 219

source of aceto-acetic acid, 360 of acetone, 359

synthesis of, 378

Phenylethylamin, derivation from phenylalanin, in intestine, 221

Phenyl lactic acid, 410

Phenylpropionic acid, derivation from phenylalanin in intestine, 219

Phenol, conjugation with sulphuric acid, 219

derivation from tyrosin in intestine, 219

Phenols, conjugated with glucuronic acid, 219

Phloridzin, acidosis in, 362 glucosuria, 287

intoxication, formation of glucose from amino-acids, 390

Phosphatid, 347 $\mathrm{C}: \mathrm{N}$ ratio, 319

catabolism of, 350

in urine, 417

Phosphorus balance in metabolism, 460 compounds in bile, 178

elimination of, 460 cutaneous, 460

glucosuria, 284

in feces, 460

influence of $\mathrm{Ca}$ and $\mathrm{Mg}$ on elimination, 460

in urine, 460

metabolism of, 459

poisoning, acidosis in, 364 source of fat in tissues in, 357

Physical regulation of body temperature, 519

Pneumonia, autolysis in, 389

oxidation in, 535

Polynucleotid, equation, 431 structure of, 429

Polysaccharids, definition of, 22

Precipitation of proteins, 28

Production of body heat, 509

Products of reaction, concentration of, 73

Prolin, 35 source of glucose, 393

Protamin, construction of, 43

formation by ferment action, 87 nucleic acid, 429 synthesis of, in milk, 376

Protein, amphoterism, 27 anabolism of, 373

pathological variation in, 382 
Protein, anabolism of, special, 377 assimilation of, 370

blood plasma, synthesis of, 372

caloric equivalents of, 513 value of, 48

catabolism of, 383 chart of, 420

in fasting, 385

in fever, 480

in inanition, 497

oxidation of end products of, 396

the elimination of end products, 417

chemistry of, 30

coagulation, 28

colloidal nature of, 24

concentration in blood, 384

concept of molecule of, 44

construction of, 45

of molecule, 42

content in amino-acids, 38 in milk, 496

crystallization, 27

degradation of, 45

denaturation, 29

derivation of sugar from, 237

diet, 491

mass of, 183

digestion by trypsin, 161

endogenous ratabolism of, 388 hydrolysis of, 386

equilibrium between cells and fluids, 387

excessive input, 498

exogenous catabolism of, 388 hydrolysis of, 387

filtration and diffusion, 26

forced feeding with, 387

fuel for work, 540

gelification, 27

heat value of, in diabetes, 326

hydrolysis of, 61,385

by pepsin, 138

in organism, 385

in diet, 493

in feces, 213

incomplete utilization of, 379

isomerism in, 44

metabolism of, 370,469

chart of, 453

in diabetes, 313

in fasting, 484,485

in fever, 529

in gestation, 502

in work, 540

relation to purin metabolism, 442,444

minimal ration, 498

molecular weight, 24

molecule, schematic representation of, 48

needs for anabolism, 496

origin in plants, 29
Protein overfeeding, 507

plant, availability, 500

precipitations, 28

ration, statistical, 494

relation to fat and carbohydrate in diet, 476

requirement in diet, $494,497,499$

resorption of, 192, 370

respiratory quotient, 513,516

of combustion in diabetes, 336,337

saving power of fat and sugar, 476 , 481

solutions, tension of, 27

source of acetone bodies, 359

of glucose in diabetes, 315

of purin, 382

special, formation of, 381

specific dynamic action of, 469

storage of, 387

sugar content, 38

utilization in sprouting of plant seeds, 375

Proteins in bile, 171

biuret reaction of, 31

conjugated, 380

description of, 24

relations to hydrolysis, 381

putrefaction in alimentary tract, 218

Proteolytic ferment, intracellular, 388

Proteose in peptic digestion, 138

Protoplasm, physico-chemical constitution of, factor in intestinal resorption, 202

Putrefaction of phenylalanin in intestine, 218

of protein in alimentary tract, 218

of tryptophan in intestine, 220

of tyrosin in intestine, 218

Purin, anabolism of, 435,454

bases, in feces, 433

in urine, 445

oxidation, 439

relation to fever, 465

to uric acid, 449

state of circulation, 450

catabolism, excessive, 454

in gout, 455

content of blood, 446

of eocoa, 446

of eoffee, 446

of glands, 446

of malted liquors, 446

of muscle, 446

of tea, 446

of vegetables, 446

deaminization of, in liver, 437

elimination in gout, 457

endogenous, 442,445

exogenous, 442,445

utilization, 437

extranuclear derivation of, 443

-free diet, 445

metabolism, 428, 469 
Purin metabolism, chart of, 452, 453 pathology of, 454

relations to protein metabolism, 442,444

test of, 459

nucleosid, 430

nucleus, 432

output, curve of, 449

in starvation, 444 relation to muscular work, 443

relation to urea, 449

resorption of, 433

synthesis of, 436

from protein, 382

total oxidation of, 441

Purins, endogenous, catabolism of, 447

exogenous, catabolism of, 447

of feces, 447

relation of urinary purin to purin metabolism, 449

Purity of reacting substances, 77

Putrescin, derivation from ornithin, 415

Pyrimidin, anabolism of, 435

bases, fate of, 459

nucleosid, 430

relation and solubility of uric acid, 459

resorption of, 433

ring, 431

synthesis from protein, 382

Pyrrol ring, in bilirubin, 175

\section{Q}

Quinonoid reaction, 411

\section{$\mathbf{R}$}

Reaction of fermentation, measurement of, 80

Reactions of fermentations, 56

Reduction fermentation, 66

Regulation of body temperature, 509, 517

Renal glucosuria in disease, 290

in phloridzin intoxication, 288

retention of sugar in diabetes, 307

Rennet in gastric secretion, 144

Reptiles, purin output in, 445

Resorption, defects in, results on digestion, 204, 205

gastric, 149

in intestine, 187

in stomach, 186

of carbohydrate, 189

of fat, 196

of products of digestion, 186

mechanism of, 200

of protein, 192

relation of mass of food to velocity of, 199

space relations, 199

relation to feces, 214
Respiration apparatus, metabolism in, 515

heat production of, 510

Respiratory quotient, 516

for combustion of fat, 336

of glucose, 335

of protein, 336

in diabetes, 335

in fasting, 487

in metabolism, 514,515

in mixed diets, 516

of carbohydrate, 513

of fat, 513

of protein, 513

Rest nitrogen, 389, 416

Reversion of ferment action, 85 reaction, 81

Rotation of monosaccharids, 21

d-Ribose, 231

Ribose, anabolism of, 435

in nucleic acid, 430

resorption of, 433

\section{S}

Saccharase in intestinal secretion, 168 salivary, 126

Saccharic acid, combustion in diabetes, 306

from glucose, 262

Saccharosuria, alimentary, 242, 280

Salicylic aldehyd, oxidation to salicylic acid, 63

Salivary digestion, 119

amylase, 119

pathological variations in, 122

in vitro, 115

maltase, 124

saccharase, lactase, 126

Salmon, growth of milt in migration, 375

Salts in diet, 493

Saving power for protein, 476,481

Seasonal variation in body weight, 507

Sebaceous lipoids, 349

Secretin, action of, 155

Secretion, heat production of, 510

Secretions, digestive, abnormal, result on digestion, 204, 205

intestinal residue in feces, 216

Selenous acid, reduction of, 66

Senses, special, influence on gastric secretion, 127

Sepsis, nucleic catabolism in, 454 oxidation in, 537

Serin, 33 source of glucose, 392

of glycerol, 274

of lactic acid, 268

Shoek, oxidation in, 536

Side reaction in catalysis, 100

Sinigrin, fermentation of, 59 
Skatol, derivation from tryptophan in Sulphate, preformed in urine, 421 intestine, 220

Skin, elimination of urea, 404 relation to heat dissipation, 520 secretion of, 464

Soaps, in feces, 215

Specific dynamic action of foodstuffs, 207, 469

Specificity of ferment action, 104 qualitative, 111 quantitative, 104 relation to intermediary reactions, 104

Starch, hydrolysis of, 60 of ferment, 120

Standard ration, protein, 494

Starvation, acidosis in, 362 fat, 491

feces in, 213

protein, 489

purin output in, 444

Stearic acid, combustion of, 351

Stereoisomerism, definition of, 19

Sterin, 349

Stoicheiometric relations in fermentation, 102

Stomach, digestion in, 126

duodenal contents in, 146

general consideration of functions of, 152

motor functions of, 150

resorption by, 149

Subcutaneous fat, relation to heat dissipation, 521

Suboxidation in metabolism, 534, 535

Substrate, concentration in fermentation, 78

of reaction, initial concentration of, 73

Succus entericus, amylase in, 115 digestion of nucleosid, 433 of nucleotid, 433 of polynucleotid, 433 erepsin in, 117

invertase in, 115

lactase in, 115

lipase in, 115

maltase in, 115

toxicity of, 468

Sugar, colloidal, 244

combined in tissues, 230

concentration in blood, relation to combustion, formation of glycogen and fat, 246 renal level of, 245

content in protein, 38

conversion into fat, 343

derivation from fat, 240

from protein, 237

free in blood, 244

of blood, derivation of, 237

synthesis of, 18

by chlorophyl, 18

Sulphate in urine, 414 relation to urinary nitrogen, 421

Sulphur balance in metabolism, 422 combined in metabolism, 421 compounds in bile, 171 conjugated in urine, 421 dioxid, oxidation of, 58 in cystin, 421

in feces, 421

in oxyproteic acid, 389

in perspiration, 414,421

inorganic in metabolism, 421

in urine, 421

metabolism of, 420

neutral in urine, 414, 421

relation to urinary nitrogen, 422

Sulphuric acid, conjugated, 219 amount per diem, 221

Superoxidation in disease, 537

in metabolism 534

Surface tension, factor in intestinal resorption, 202

$\mathbf{T}$

TARTronic acid, conversion into uric acid, 445

in formation of glycerol, 344

source of glycerol, 274

Taurin, derivation from cystin, 414

from erythrocytes, 173

in bile, 172

Tea, purin content of, 446

Tellurous acid, reduction of, 66

Temperature, influence on reaction velocity, 74

Tetramethylene diamin (putrescin), 415

Theobromin, 446

Theophyllin, 446

Theory of ferment action, 55

$\beta$-Thiolactic acid, 414

Thoracic duct, state of fat in, 344

Thymin, conversion into uracil, 440 occurrence of, 437 structure of, 431

Toleration for glucose, relation to acidosis in diabetes, 333

Traumatism, glucosuria, 283

Trimethylamin, 348

Triolein, combustion of, 354

Trypsin, action on nucleotid, 433 digestion in vitro, 116

in feces, 163

pancreatic secretion, 160

scope of digestion of proteins, 162

Trypsinogen, 160

Tryptic digestion, relation to preceding peptic digestion, 140

Tryptophan, 34

catabolism of, 409, 413

protein metabolism without, 380

putrefaction of, in intestine, 219

Tyrosin, 33 
Tyrosin, bacterial action on, in intestine, 218

catabolism of, 409, 410, 411, 412

combustion in body, 219

in urine, 396

source of acetone, 359 of epinephrin, 413

of lactic acid, 268

synthesis of, 378

Tyrosinase, fermentation by, 107

Tuberculosis, autolysis in, 389

\section{$\mathbf{U}$}

UNDERNUTRITION, 505

Uracil, derivation from thymin, 440

in urine, 459

occurrence of, 437

origin from cytosin, 440

structure of, 431

Urate, bi-, 450

deposition in tissues, 457

mono-, 450

quadra-, 450

Urates, reaction of solutions of, 450

solubility of, 451

Urea ammonia, elimination, course of, 417 equilibrium, 405

blood content of, 400

cutaneous elimination, 464

derivation from arginin, $39 \mathrm{~S}$

from creatinin, 426

from uric acid, 441

elimination of, 400, 404

endogenous, 402

amount of, 404

exogenous, 402

amount of, 404

formation of, 398, 399

from ammonium carbonate, 397

from creatinin, 400

from guanidin, 423

from uric acid, 400

outside of liver, 400

hydrolysis of, 62

origin from arginin, 396

relation to purins, 449

Uremia, causation of, 465

Uric acid, blood content in disease, 455 in gout, 455

conversion into urea, 400, 411

derivation from inosinic acid, 443

from lactic acid, 445

from tartronic acid, 445

diathesis, 455

elimination of, 452

end product of protein catabolism in birds, 445

extranuclear origin of, 444

lactam, 450

lactim, 450

origin from xanthin, 64,440
Uric acid, oxidation of, 447

relation to allantoin, 448

to oxalic acid, 449

of solubility to reaction of blood, 452

ratio to purin bases, 449

solubility of, 451

in blood plasma, 451, 452

state of circulation, 450

synthesis from glycocoll, 444

threshold value in gout, 456

Uricolysis, 442, 448

in gout, 456

Urinary sulphate, 414

Urine, amino-acids in, 396

ammonia content of, 405

aromatic bodies, relation to intestinal putrefaction, 221

cytosin in, 459

elimination of end products of protein catabolism, 417

glycocoll in, 396

metabolic end products in, 465

phosphorus in, 460

purin bases in, 445

uracil in, 459

Urobilin, fate of, 177

formation in aseptic autolysis of liver, 177

in urine, 417

relation to bilirubin, 176

Urobilinogen, formation in intestine, 176

Urochrome in urine, 417

Uroerythrin in urine, 417

Uroferric acid in urine, 417

Uterus, autolysis in involution of, 389

\section{V}

VALIN, 35

Vasoconstriction in fever, 532

Vegetable diet, mass of, 185 protein in dict, 500

purin content of, 446

Vitiatin, composition of, 424

\section{W}

WATER, formation of, $5 \mathrm{~S}$

of gastric secretion, variations in disease, 148

Wasting in fasting, 489

Work at high altitude, 541

combustion in, 540

for support of, $53 \mathrm{~s}$

equivalents of, 539

mechanical efficiency, 539

mental, thermic equivalent, 539

relation to basal heat production, 538

to combustion of glucose in diabetes, 302 
Work, relation to creatinin metabolism, Xanthin, derivation from hypoxanthin, 540 to heat of chemical regulation, 538 of specific dynamic action, 538

to metabolism, 538

to nucleic metabolism, 540 units of, 539

to protein metabolism, 540 439

origin from hypoxanthin, 64

oxidation to uric acid, 64

1-Xylose, origin from d-glucuronic acid, 232

Xylose in nucleic acid, 430

\section{$\mathbf{Y}$}

YEAST, autolysis of, 385

X

$\mathbf{Z}$

Xanthin, conversion from uric acid, 440 derivation from guanin, 440

ZeIN, utilization of, 379 


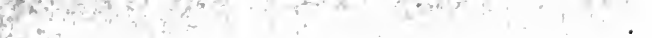







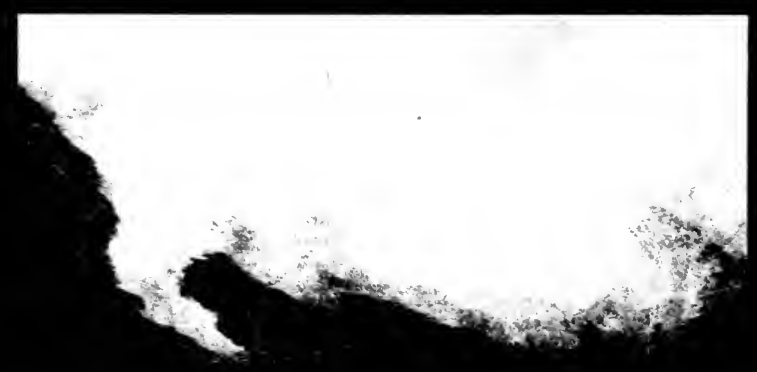




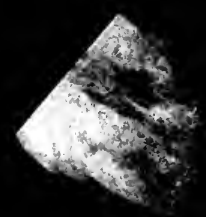

QP141 Taylor, A.E.

D1112

T23

Dipestion and metabolism.

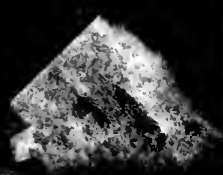

\section{2 \\ c. 3}
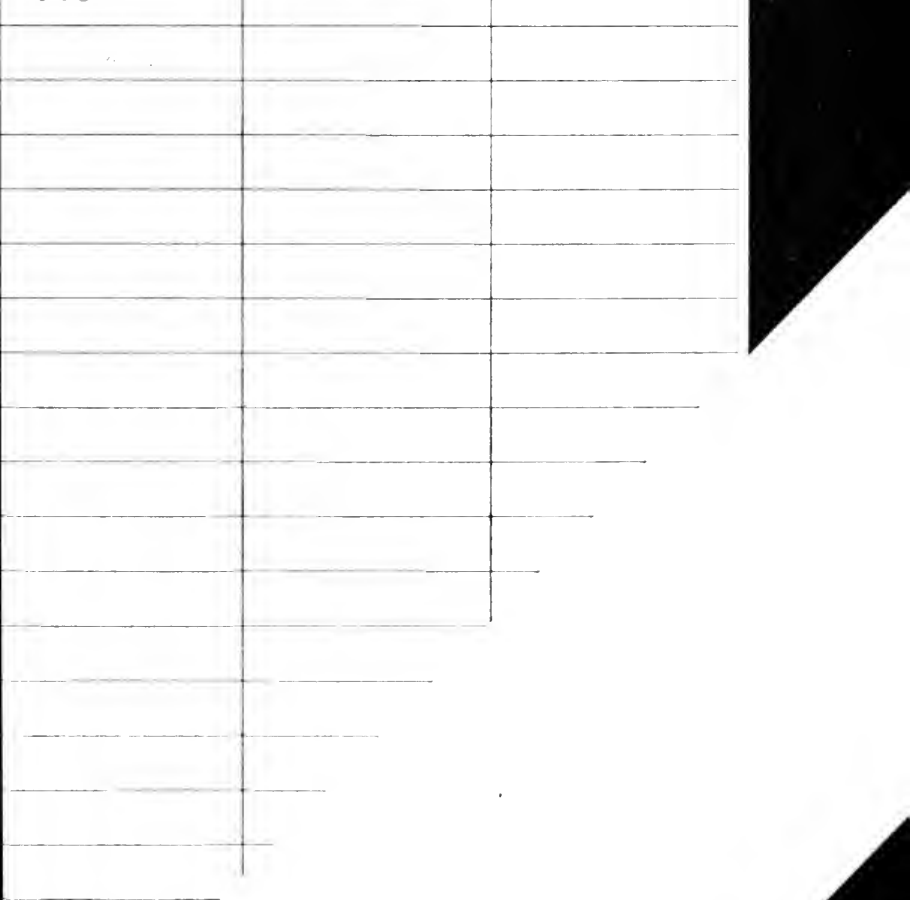

UNIVF 


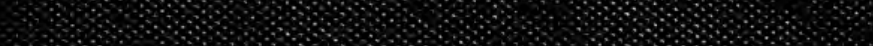


SW $\because *$



